Transmission et circulation des savoirs scientifiques et techniques

Dominique Barjot (dirs)

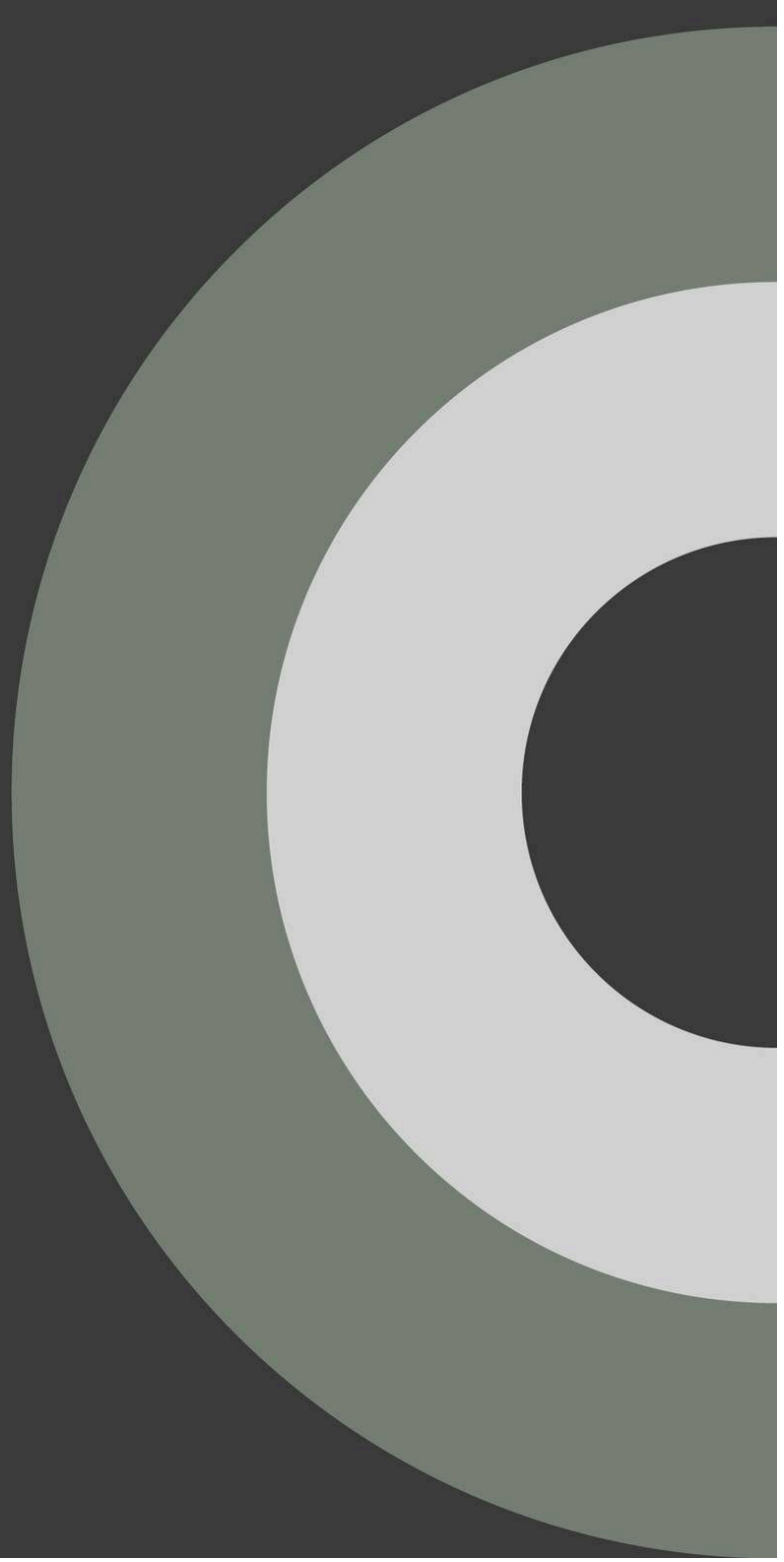




\section{Transmission et circulation des savoirs scientifiques et techniques}

Dominique Barjot (dir.)

DOI : $10.4000 /$ books.cths. 13553

Éditeur : Éditions du Comité des travaux historiques et scientifiques Année d'édition : 2020

Date de mise en ligne : 22 septembre 2020

Collection : Actes des congrès nationaux des sociétés historiques et scientifiques

ISBN électronique : 9782735509010

\section{Sbooks}

http://books.openedition.org

\section{Référence électronique}

BARJOT, Dominique (dir.). Transmission et circulation des savoirs scientifiques et techniques. Nouvelle édition [en ligne]. Paris : Éditions du Comité des travaux historiques et scientifiques, 2020 (généré le 10 décembre 2020). Disponible sur Internet : <http://books.openedition.org/cths/13553>. ISBN : 9782735509010. DOI : https://doi.org/10.4000/books.cths.13553.

Ce document a été généré automatiquement le 10 décembre 2020.

(c) Éditions du Comité des travaux historiques et scientifiques, 2020 Conditions d'utilisation:

http://www.openedition.org/6540 
À l'ère de Facebook et de Twitter, du débat sur les fake news, la question de la transmission des savoirs et de la connaissance n'a jamais revêtu autant d'importance. À une époque où nous sommes submergés sous le flot des informations, il est de plus en plus difficile d'identifier ce qui constitue un savoir ou un savoir-faire vrai. De récents travaux ont révélé l'importance des sciences du vivant, notamment pour l'essor de l'agriculture, mais aussi de la circulation de l'information scientifique et technique, notamment dans la mesure où celle-ci s'accompagne ou même, parfois, ouvre la voie à des transferts de technologie. Un axe de recherche que les auteurs ici réunis explorent à travers une série d'exemples, depuis la fin $\mathrm{du} \mathrm{XVI}^{\mathrm{e}}$ siècle jusqu'à nos jours.

Le Congrès national des sociétés historiques et scientifiques rassemble chaque année universitaires, membres de sociétés savantes et jeunes chercheurs. Ce recueil est issu de travaux présentés lors du $143^{\mathrm{e}}$ Congrès sur le thème « La transmission des savoirs ».

\section{DOMINIQUE BARJOT}

Professeur émérite d'histoire économique contemporaine, Sorbonne Université Lettres, Renmin University of China, membre de l'Académie des sciences d'outre-mer, président du Comité français des sciences historiques 
SOMMAIRE

\section{Introduction}

Dominique Barjot

Transmission et circulation des savoirs scientifiques et techniques : quelles problématiques?

Transmission et circulation des savoirs scientifiques et techniques : des sciences du vivant à

l'agriculture

De l'information scientifique et technique aux transferts de technologie : un défi pour notre temps

In memoriam : Nagwa Abou El Maaty épouse Khalil (1954-2019)

Dominique Barjot

Transmission et circulation des savoirs scientifiques et techniques : des sciences du vivant à l'agriculture

\section{De la médecine à la biologie : un espace privilégié pour la transmission des} savoirs et des savoir-faire

La transmission du savoir et du savoir-faire chez les apothicaires de Montpellier à la fin du $\mathrm{XVI}^{e}$ siècle

Élisabeth Manouvrier

L'apprentissage et le compagnonnage

Évolution de la transmission du savoir médical et pharmaceutique

Les médecins parisiens et la diffusion du savoir médical au XVIII siècle : des savants

pédagogues

Isabelle Coquillard

Un savoir objet de la formation continue des docteurs régents

Orchestrer la circulation des savoirs médicaux dans l'espace du champ médical

Contrôler la diffusion du savoir à l'échelle du royaume : la censure royale des livres de médecine

Les voyages et lettres de correspondance de John Ray, ou l'émergence des sciences naturelles dans l'Europe du XVII siècle

Sabine Kraus

Les années d'études et d'explorations en Grande-Bretagne : du savoir populaire traditionnel au savoir universitaire

Les voyages et correspondances, une géographie de l'histoire

Missions des naturalistes Peyssonnel et Desfontaines dans la régence de Tunis au XVIII siècle Geneviève Falgas

Les conditions de voyage

Les collectes

La transmission des savoirs

Les herbiers artificiels de Marie Fortier : un type méconnu de modèles botaniques

Florence Tessier

« Herbiers artificiels » et modèles de botanique

Plantes artificielles et botaniques

Marie Fortier 
Le réseau français des collaborateurs du Cabinet zoologique de Varsovie pendant la seconde moitié du XIX siècle

Piotr Daszkiewicz

Origines du Cabinet zoologique de Varsovie

Władysław Taczanowski et l'âge d'or du Cabinet

Les contributeurs : mécènes, exilés et naturalistes voyageurs

Le Cabinet et la France

La Maison Verreaux et l'établissement Deyrolle

Les naturalistes français

Le Muséum d'Histoire Naturelle de Paris et le réseau des collaborateurs du Cabinet zoologique de

Varsovie

\section{L'agriculture : entre assimilation des acquis de la science et transmission des savoir-faire}

La transmission des savoirs agronomiques vers la fin du XVIII siècle d'après les travaux des apprentis savants de la société d'agriculture de Blaison en Anjou Antoine Follain

Une expérience décalée, courte, mais spécialement riche

Les modèles des thesmophores

Les sources des thesmophores

De l'importance des relations personnelles

Science, art ou pratique? La place de l'agriculture dans les formes de transmission du savoir au XIX ${ }^{e}$ siècle en France

Christiane Cheneaux-Berthelot

Du sommet à la base : l'agriculture, un vaste terrain d'étude

Des moyens d'enseigner le progrès agricole : de l'initiative privée à une politique nationale

La transmission du savoir-faire agricole dans les fermes-écoles créées par la France en

Afrique du Nord et en Syrie au début du $\mathrm{XX}^{e}$ siècle

Nagwa Abou El Maaty

Les fermes-écoles d'Algérie : un appui à la colonisation?

La création des fermes-écoles sous le protectorat

Bouka à Lattaquié, œuvre des Pères Bénédictins sous le Mandat

Raymond Février et la transmission des savoirs agronomiques

Sébastien Pivoteau

Répondre aux besoins socio-économiques du moment

Orienter les politiques agricoles et de recherche agronomique en France, en Europe, dans le bassin

méditerranéen et les pays en développement 
De l'information scientifique et technique aux transferts de technologie : un défi pour notre temps

\section{L'information scientifique et technique : quels vecteurs?}

De la diagraphie à la numérisation: deux artisans d'une transmission de savoir (CharlesLouis Marle aîné, Louis-Georges Marle) (1829-2017)

Anne Marle-Kolle

Deux artisans de transmission du savoir

Charles-Louis Marle dit Marle aîné

Louis-Georges Marle

L'implication sociale : le SNE (Syndicat National de l'Édition)

Les laboratoires de recherche européens Kodak et la circulation interne du savoir scientifique durant l'entre-deux-guerres

Nicolas Le Guern

La création des trois principaux laboratoires de recherche d'Eastman Kodak

Le savoir scientifique : les rapports de recherche et leur contenu

Circulation interne du savoir scientifique

Petre (Pierre) Sergescu (1893-1954) : historien des sciences et promoteur de la discipline Alexandre Herlea

2018 : cent vingt-cinq ans depuis la naissance de Petre Sergescu

Petre Sergescu : le mathématicien

Petre Sergescu : l'historien des sciences

Petre Sergescu à Paris : le principal artisan de la collaboration internationale en histoire des

sciences après la Seconde Guerre mondiale

Autres activités de Petre Sergescu dans les sciences, leur histoire et diffusion

Petre Sergescu et la Roumanie

Le souvenir laissé par Petre Sergescu

Le « moment vulgarisateur français » : enjeux et modalités de la diffusion du savoir scientifique à la Libération

Robert Belot

Les savants répondent à une volonté de savoir

La vulgarisation par les non-savants

Le Plan comme vecteur de transmission des connaissances sur l'économie : l'exemple des commissions de modernisation à l'époque des quatre premiers plans (1946-1965)

Chunhua Chu

Organisation et fonctionnement des commissions

La vertu de la participation des groupes sociaux

L'action du plan : persuader et informer 


\section{Importance des transferts de technologie}

Transmission des savoirs technologiques de la France à la Russie : le cas de l'industrie textile au XIXe siècle

Olga Melnichenko

Le contexte socio-économique international et les acteurs principaux de transfert des connaissances dans l'industrie textile

La transmission des savoirs technologiques de production

Théodore Watremez, teinturier de Cambrai

Transmission des savoirs dans les constructions industrielles

La transmission des savoirs technologiques par les constructeurs de machines textiles modernes

L'URSS et le CoCom (Comité de coordination pour le contrôle multilatéral des exportations) : transferts de technologie et guerre froide économique

Irina Sheveleva

Le modèle d'emprunt des technologies occidentales par l'URSS : l'Occident a-t-il acheté la paix ou plutôt « vendu la corde »?

CoCom : guerre économique ou sanctions économiques?

Transferts de technologie entre l'URSS et la France (1954-1991)

Boris Vinogradov

Avant le transfert de technologies, laborieux rétablissement de relations commerciales (1950-1966)

Débuts et approfondissement du transfert des technologies (1966-1979)

Vers la crise du transfert de technologies françaises à destination de l'URSS (1979-1991)

Le transfert de savoirs technique et industriel français au Pérou dans les années 1950

et 1960 : l'exemple de la sidérurgie de Chimbote et de la centrale hydroélectrique du Canyon del Pato

Getsiva Cayo Durand de Geist

La coopération technique franco-péruvienne : un domaine privilégié

La construction du Canyon del pato : le rôle de la corporación peruana del santa (CPS)

L'usine sidérurgique de Chimbote : « le début de l'ère de l'acier au Pérou » 


\section{NOTE DE L'ÉDITEUR}

Les articles de cet ouvrage ont été validés par le comité de lecture des Éditions du Comité des travaux historiques et scientifiques dans le cadre de la publication des actes du $143^{e}$ Congrès national des sociétés historiques et scientifiques tenu à Paris en 2018. 


\title{
Introduction
}

\author{
Dominique Barjot
}

1 À l'ère de Facebook et de Twitter, du débat sur les fake news, la question de la transmission des savoirs et, à travers eux, de la connaissance, n'a jamais revêtu autant d'importance qu'aujourd'hui. À une époque où nous sommes submergés sous le flot des informations, il est de plus en plus difficile d'identifier ce qui constitue un savoir ou un savoir-faire vrai. La question se pose notamment à propos de la transmission et de la circulation des savoirs scientifiques et techniques. Les travaux récents des historiens du champ ont révélé l'importance des sciences du vivant, notamment pour l'essor de l'agriculture, mais aussi de la circulation de l'information scientifique et technique, notamment dans la mesure où celle-ci s'accompagne de ou même, parfois, ouvre la voie à des transferts de technologie.

\section{Transmission et circulation des savoirs scientifiques et techniques : quelles problématiques ?}

2 La notion même de transmission des savoirs requiert d'emblée quelques observations de nature théorique. La transmission des savoirs implique des transferts dans le temps ou dans l'espace. Dans le temps, d'une époque ancienne à une autre, moderne, elle peut s'effectuer de manière régulière, continue, voire organisée, à partir d'institutions de conservation des savoirs ou, au contraire, avec des moments de perte, d'oubli et de redécouvertes. Dans l'espace, elle peut s'exercer d'un pays à un autre, d'une aire culturelle à une autre. Se pose donc la question des connexions, aujourd'hui l'une des sources majeures de renouvellement de l'histoire mondiale (histoire connectée).

La transmission est rarement perpétuation à l'identique. Les savoirs transmis sont presque toujours modifiés, remodelés, enrichis, appauvris. Toutefois, il existe différent cas possibles. D'abord, la transmission des savoirs peut favoriser la sacralisation de la tradition, ériger des autorités au poids écrasant et engendrer des blocages. Ensuite, elle peut aussi nourrir le progrès et l'innovation. Émerge ainsi la question : quel est l'objet véritable de la transmission? Il peut s'agir d'un corpus de savoirs élaborés, découverts ou redécouverts, c'est-à-dire une base de connaissances communes à partir de laquelle 
progresser, des outils heuristiques ouvrant la voie à la découverte, à l'invention, à l'innovation, un certain esprit philosophique ou scientifique (voire des mentalités).

Quelles sont, à ce stade, les modalités concrètes de la transmission? Deux voies se dégagent: d'une part l'imitation pure et simple, la reproduction à l'identique, génératrice de routine; de l'autre, l'enseignement qui implique une certaine distance critique, une reformulation pédagogique, voire une remise en ordre ordinaire des savoirs. Consécutivement, cette reformulation peut être le fait de l'émetteur (transformation des savoirs), du récepteur (adaptation des savoirs à ses capacités ou à ses besoins). Enseignement scolaire et apprentissage (professionnel ou familial) peuvent évoluer entre ces deux extrêmes.

5 La transmission des savoirs est un phénomène multiforme. Elle peut prendre des formes multiples, être le fait d'acteurs spécialisés, s'exercer de manière diffuse dans la pratique sociale, recourir à des vecteurs divers, être prise en charge par des cadres institutionnels, se faire tout au long de l'existence dans la vie familiale et sociale, l'activité économique et la pratique religieuse. Par-delà ses multiples dimensions, la transmission des savoirs relève de trois interrogations majeures : quels savoirs? Quels acteurs, facteurs et vecteurs? Quels demandeurs, cibles ou publics, pour quels usages?

\section{Quels savoirs?}

6 Ces savoirs, quels sont-ils? Nés avec les sociétés humaines, ils passent, tôt ou tard, par les sciences et les techniques. Ils ne se résument pas aux connaissances théoriques. Enfin, leur transmission n'est pas un objectif en soi, ce qui pose la question des enjeux du savoir et de sa divulgation. La question des savoirs naît avec les sociétés humaines, avec le langage lato sensu. Les savoirs se constituent en partant de l'observation des choses et des faits et en leur appliquant une interprétation, une explication. Cette interprétation repose soit sur un système philosophique, soit sur des croyances transmises par la tradition. Mais la tradition ne s'oppose pas nécessairement à la science.

\section{Savoirs, Sciences et techniques}

7 Les savoirs, tôt ou tard, passent par les sciences et les techniques, avec des différences selon les époques, une place essentielle des disciplines et d'étroites relations entre l'art et la science. Chaque époque a sa propre classification des disciplines scientifiques, bâtit sa hiérarchie depuis les savoirs spéculatifs (ou connaissances) jusqu'aux savoirs à finalité pratique. Les sciences couvrent un nombre toujours plus grand de champs. La science et le savoir impliquent des connaissances spécifiques ouvrant la voie à des compétences déterminées, d'où une double opposition recherche fondamentalerecherche appliquée et sciences hypothético-déductives et sciences expérimentales.

8 La notion majeure réside dans l'existence de disciplines. À la base de l'enseignement tel que pratique dans le système scolaire et universitaire, ces disciplines ne sont pas étanches, même si elles obéissent à leurs règles propres. En effet, elles progressent surtout sur leurs marges, d'où le succès de notions comme pluridisciplinarité, interdisciplinarité et transdisciplinarité. L'une des questions essentielles tourne autour des relations de l'art et de la science. Elle se pose depuis longtemps dans les champs des 
sciences naturelles (botanique, anatomie) et de la technologie (arts industriels des expositions universelles du XIX ${ }^{e}$ siècle).

\section{Une transmission des savoirs pas limitée aux connaissances théoriques}

9 La transmission de savoirs ne se limite pas aux connaissances théoriques. En effet, l'importance des savoirs empiriques et traditionnels persiste, notamment à travers les savoirs populaires. D'autres relèvent d'une reconstruction. Cette observation apparaît tout à fait claire pour la technologie. Elle se définit comme un ensemble de savoirs techniques requis par le système productif. Elle voit notamment l'opposition entre deux modèles: celui de l'université allemande, donnant une priorité absolue à la science pure, et celui de l'école française d'ingénieur à vocation polytechnique (polytechnique, Centrale, Arts et Métiers). Mais cette opposition n'exclut pas des interactions en France (création de l'assistanat par Pasteur), en Allemagne (Hochschulen, Fachhochschulen) et aux États-Unis (Massachussetts Institute of Tehnology, West Point).

10 La formation technique requiert un savoir-faire, mais aussi un savoir-vivre (rites initiatiques des écoles d'ingénieur). Depuis les origines, la cellule de base est la famille native ou celle qui lui fait fonction de substitut (rôle de l'apprentissage). Les modalités de transmission des savoirs font, de manière probable, appel à des apprentissages par imprégnation, voire par enseignement. Dès lors, le rôle décisif des structures familiales, mais aussi celui des comportements démographiques favorables à l'éducation, notamment celle de l'enfant, se comprend mieux. La société globale joue son rôle dans l'acquisition des savoirs, savoir-faire et savoirs vivres spécifiques. Tel est le cas des groupes d'âge ou des groupes d'expérience. Le travail tient, à coup sûr, une place importante, à travers le compagnonnage, mais aussi à travers les stages, les retours d'expérience, l'évaluation des tâches ou le travail à flux tendu. Par ailleurs, la transmission des savoirs ne constitue pas un objectif en soi. La problématique de la transmission des savoirs s'inscrit dans des moments et dans des politiques. Ces politiques associent la quête de prestige et de puissance, les concurrences internationales, la démocratisation et la popularisation des savoirs. Par ailleurs, avec le temps, se développent les revues scientifiques et les auteurs de vulgarisation.

\section{Acteurs, facteurs et vecteurs de la transmission des savoirs}

11 Les acteurs, ce sont d'abord les individus. Mais important aussi est le rôle des éducations familiales, lignagères et népotiques.

\section{Quels acteurs?}

12 Les institutions peuvent être de formation (transmettre un savoir) ou de recherche (créer ou perfectionner un savoir). Se trouve ainsi posée la question de l'école. Les collèges professionnels et religieux ont joué un rôle fondamental dans la formation des cultures méditerranéennes. En matière de formation, l'école constitue, depuis longtemps, l'une de bases fondamentales de l'Antiquité au Moyen Âge, puis aux Temps modernes et à l'époque contemporaine. Avec le temps, s'est créée une hiérarchie d'institutions. Il s'agit de l'école primaire chargée des apprentissages fondamentaux, 
puis des établissements secondaires, enfin les institutions d'enseignement supérieur : universités, grands établissements, grandes écoles d'ingénieurs ou de commerce.

Les chercheurs manifestent depuis un moment déjà un intérêt particulier pour les universités. Ils ont étudié d'abord leur genèse au Moyen Âge, en Occident, leur rôle du $\mathrm{XIX}^{\mathrm{e}}$ siècle à aujourd'hui. Elles garantissent la qualité et l'orthodoxie de la transmission des savoirs. Elles canalisent prudemment les possibilités d'innovation. Elles peuvent cohabiter avec des institutions étrangères.

Les chercheurs se sont beaucoup intéressés aussi à l'étude des institutions de recherche. En effet, à côté des universités, il existait et existe encore d'autres lieux propices à l'épanouissement et à la diffusion des sciences : ainsi les sociétés savantes, plus tard le Comité des Travaux Historiques et Scientifiques (CTHS). Ces institutions ouvrent un vaste champ à la recherche, surtout si l'on remonte aux origines anciennes (Muséum, ENS Ulm). Il s'agit d'un domaine prédisposé aux comparaisons internationales, surtout si l'on y intègre les fondations (Ford, Volkswagen, Prince de Monaco, Herbert Hoover). S'y rattache, au Xx siècle, l'étude des grands organismes de recherche, dont la France fournit les archétypes, qu'ils soient polyvalents (CNRS), spécialisés (INSERM, INRA, INRIA, IRD, etc.) ou à vocation industrielle et commerciale (CEA).

15 Se trouve ainsi posée la question de la complémentarité entre recherche publique et recherche privé. Elle se pose au niveau des financements (en France, une part très élevée incombe à la recherche publique), mais aussi des institutions publiques et semipubliques, professionnelles ou d'entreprises. Pour les entreprises, la recherche s'exerce dans des laboratoires, certaines ont une longue histoire (Compagnie du Gaz de Paris, Châtillon-Commentry, Lafarge), d'où sont issues de nombreuses innovations, tant de produits que de procédés, mais aussi par filiales interposées (recherche-développement ou $\mathrm{R} \& \mathrm{D}$ et ingénierie) ou encore par l'exploitation de brevets et de licences d'exploitation. Dans les périodes de crise, les grandes entreprises préfèrent ne prendre de risques qu'une fois les inventions éprouvées, d'où le rôle des Business Angels, mais aussi le problème de la propriété industrielle : brevets, dessins et modèles, marques de fabrique, qui, à l'époque contemporaine reprennent la pratique des privilèges.

\section{Formes, canaux et instruments de la transmission des savoirs : d'abord l'écrit}

Les institutions scolaires, universitaires et de recherche ne constituent pas les seules formes, canaux et instruments de la transmission des savoirs. L'apprentissage joue en effet un rôle fondamental. Il est nécessaire de prendre en compte les multiples formes d'apprentissage. Aux origines lointaines de la socialisation des individus se retrouve le rôle de la famille. Le concept d'apprentissage revêt d'ailleurs une importance majeure dans les sciences sociales. Sont concernés les corporations, les compagnonnages comme le travail des enfants. Ce concept a été bien développé par les économistes, à partir des apports de la psychologie cognitive : learning by doing (Kenneth Arrow) ; path dependency (Roland Coase, Giuseppe Dosi, Richard R. Nelson et Sidney Winter). Par ailleurs, l'apprentissage est de tous les temps. Il peut être le substitut ou le complément d'une éducation scolaire (formation générale et professionnelle dans les milieux ruraux et artisanaux). Il permet l'adaptation des praticiens (notaires, juges, avocats, médecins, chirurgiens, barbiers, apothicaires, etc.), après une formation initiale aux exigences 
concrètes de leur vocation sociale. En effet, une grande partie des savoirs techniques n'a été transmise que par oral.

La transmission des savoirs s'effectue cependant d'abord par l'écrit. Si l'écriture n'a pu exister que parce transmise, la révolution du livre et de l'imprimerie constitue cependant un tournant tout à fait majeur. En dépit des découvertes chinoises et coréennes, Gutenberg demeure le seul à l'origine d'une révolution. En effet, l'extraordinaire développement du livre au XVII , et surtout aux XVIII et XIX siècles, a été bien mesuré par Emmanuel Le Roy Ladurie, Robert Chartier et Jan Luiten Van Zanden. Enfin, il a été rendu possible grâce à l'alphabet. Deux éléments majeurs méritent d'être notés à ce sujet. À côté du livre, les périodiques ont apporté une contribution décisive. Si cette diffusion se heurte à de nombreux obstacles, notamment la censure de l'Église et de l'État, en revanche, un certain nombre de facteurs positifs ont joué : l'apparition et le succès de la presse people ou le développement de la presse officielle et administrative.

L'édition scientifique a occupé une place spécifique, en même temps qu'émergeait un marché de la traduction. D'une façon plus large, se posent des questions toujours pertinentes. Elles sont de nature économique : concentration, édition électronique ouverte, modes de validation. Mais elles relèvent aussi du champ de la science. Ainsi la question de l'expertise scientifique recouvre à la fois la problématique de la figure et du magistère de l'expert, celle de la réception et de la contestation de son discours, celle enfin de son indépendance ou non. S'y rattachent les interrogations autour du rôle de la controverse scientifique, du couple information-désinformation (par exemple la propagande de guerre), et du musée (muséologie, médiation culturelle, collections scientifiques entre étude et patrimoine).

\section{Quels autres médias?}

La transmission peut s'exercer par d'autres moyens. Il s'agit d'abord du son (théâtre, chant individuel ou collectif, musique). Le chant, par exemple, a tenu et tient une place fondamentale dans la transmission et la perpétuation des normes sociales. Elle est parallèle à celle occupée par la musique instrumentale. Créatrice de savoir et de savoirfaire, celle-ci constitue un puissant vecteur de transmission des savoirs, en raison notamment d'importants progrès techniques (électronique, informatique). Le son devient d'ailleurs, de plus en plus, un puissant vecteur des savoirs, grâce au télégraphe, au téléphone, à la radio et aux techniques d'enregistrement.

L'image est de plus en plus associée au son. Elle constitue également un vecteur majeur et, peut-être aujourd'hui, dominant. Elle passe par le support de toutes sortes de documents. Elle met en évidence le rôle des arts plastiques. Les images concernent les livres scientifiques, mais, sans exclure un savoir savant, elles cherchent aussi à transmettre un message, d'où leur utilisation éducative et comme instrument de propagande. Ces images ont pris aussi d'autres formes : carte géographique ou affiche, moyen d'information utilisé par le détenteur d'un pouvoir politique, social ou de marché. L'affiche possède d'ailleurs un rôle spécifique, de substitut à l'écriture. Se trouve posé ainsi le problème de la vulgarisation, par transposition ou traduction en vernaculaire. L'image bénéficie d'un pouvoir didactique fort.

21 D'une manière plus large, Marshall Mac Luhan a mis en évidence l'opposition entre média chaud et média froid. Un médium froid favorise la participation de l'audience 
parce qu'il livre plus d'informations (affiche, TV); un médium chaud, au contraire, fournit beaucoup d'informations, mais favorise la passivité (presse, radio, cinéma). Il est donc possible de mener une histoire des médias sous l'angle de la transmission des savoirs: documentaires, émissions d'histoire, émissions scientifiques, informations quotidiennes, si elle obéit aux règles de la déontologie professionnelle. Cela conduit à s'interroger sur le pourquoi du désintérêt pour le cinéma et la télévision (par exemple l'excès de la téléréalité ou de la pornographie), mais aussi de sa manipulation (l'image et le son comme outil de propagande). Comme le cinéma et la télévision, l'internet, puis les réseaux sociaux apparaissent, à l'instar de la langue d'Ésope, comme « la meilleure et la pire des choses». Les réseaux sociaux deviennent ainsi la source principale d'information et de contournement des médias traditionnels. À l'inverse, ils constituent un instrument redoutable de diffusion de la rumeur et d'un qu'en dira-t-on planétaire. Notamment, pour les époques anciennes, les nouvelles technologies de l'information (NTI) ont fait faire à la science d'immenses progrès (reconstitution $4 \mathrm{D}$ en archéologie).

D'autres questions méritent d'être posées. En premier lieu, il y a celle de la formation professionnelle et de ses méthodes. Elle repose sur la recherche, mais aussi la formation des maîtres. Le résultat en a été le développement de disciplines ou sous-disciplines spécifiques. En second lieu, la réflexion porte aujourd'hui, non seulement sur les contenus, mais sur les outils. En troisième lieu, la diffusion du savoir requiert aussi la vulgarisation, à laquelle contribue la presse grand public, mais aussi une presse spécialisée (science, arts, histoire, aujourd'hui philosophie et psychologie). Tout le monde sait la place que tiennent aussi la radio et la télévision. D'une façon plus large, la télévision a conquis une place privilégiée en tant qu'instrument de diffusion de l'information immédiate.

\section{Rôle permanent de la transmission orale}

À côté de ces nouveaux moyens de transmission des savoirs, la tradition orale a joué un rôle essentiel depuis les origines des sociétés humaines. La transmission des savoirs s'exprime tout autant par les gestes que dans l'écrit. L'apprentissage n'est pas nécessairement institutionnalisé (débats sur sa crise, en France, des années 1880 aux années 1990). Il implique une formation sur le tas. Très développée aujourd'hui, elle a une origine ancienne (le séjour en Angleterre au XIxe siècle pour les banquiers et les ingénieurs français). Elle peut en outre s'opérer dans le cadre de collaborations internationales: consultants américains des années 1950 et 1960, missions de productivité des années 1940 aux années 1960. Ils ont en leurs correspondants au XVIII ${ }^{\mathrm{e}}$ siècle, vers l'Angleterre et l'Écosse, avec le soutien, en France, des rois Louis XV et Louis XVI. À l'époque, les missions envoyées outre-Manche incluaient l'espionnage industriel (copies, exportations illicites, débauchage à haut prix). À l'ère de l'intelligence économique, l'espionnage industriel n'a rien perdu de son actualité (Apple contre Samsung, montée des chinois Huawei, Xiaomi, Oppo et Lenovo).

Une large partie des savoirs techniques a recouru à l'oralité (textile, travail des métaux). Les lieux non institutionnels (chantiers, ateliers, navires) demeurent centraux avant l'apparition des cours et des écoles spécialisés. De ce point de vue, la mobilité des professionnels revêt un intérêt essentiel : ainsi pour les verriers italiens sous l'Ancien Régime ou pour les ouvriers des constructions navales. Ces lieux et ces mobilités ouvrent la voie à des hybridations originales entre savoirs et savoir-faire. Les voyages, limités dans le temps, ou l'expatriation constituent un moyen essentiel de transfert des 
connaissances. À cet égard, déterminant est le rôle des traditions locales d'émigration (métallurgistes catalans, verriers italiens, maçons de la Creuse et du Piémont se rendant à Lyon, Paris, Marseille ou Grenoble, ramoneurs auvergnats, bonnes bretonnes). Elles ouvrent la voie aux migrations étrangères des $\mathrm{xIx}^{\mathrm{e}}$ et $\mathrm{xx}^{\mathrm{e}}$ siècles, venues de Belgique, d'Italie et d'Espagne, puis d'Afrique du Nord, du Portugal, de Yougoslavie et de Turquie vers l'hexagone. À chaque fois, les migrants, quand ils reviennent au pays, y rapportent des savoir-faire et des savoirs d'un grand intérêt pour leurs compatriotes.

\section{Transmission et circulation des savoirs scientifiques et techniques : des sciences du vivant à l'agriculture}

Tenu à Paris, du 23 au 26 avril 2018, le $143^{\text {e }}$ congrès du CTHS s'est beaucoup intéressé à cette question de la transmission des savoirs. Les sciences du vivant offrent un champ pour la transmission et la circulation de ces savoirs scientifiques et techniques, en raison des progrès de la biologie et du développement d'une agriculture scientifique.

\section{De la médecine à la biologie : un espace privilégié pour la transmission des savoirs et des savoir-faire}

Depuis longtemps, la médecine et la biologie constituent un espace privilégié de la transmission du savoir et du savoir-faire.

\section{Apothicaires et médecins}

Les apothicaires de Montpellier dès la fin $\mathrm{du} \mathrm{XVI}^{\mathrm{e}}$ siècle, en fournissent un bon exemple ${ }^{1}$. Au Moyen Âge, sous l'impulsion des immigrés juifs, chrétiens et sarrasins, Montpellier voit se développer un centre médical et savant au rayonnement brillant. La cité tire avantage de la présence de médecins réputés, de l'abondance des épices et matières premières importées pour développer le commerce des dragues et leur transformation des médicaments. Ainsi naissent les épiciers-apothicaires, eux-mêmes à l'origine de la profession d'apothicaire, corps de métier libre, mais soumis à des règles spécifiques et pas toujours écrites. Fin $\mathrm{XvI}^{\mathrm{e}}$ siècle, l'École de médecine s'ouvre aux apothicaires. À cette époque, l'art de la pharmacie, parce que relevant des arts mécaniques, s'acquiert à travers l'apprentissage, puis le compagnonnage. Bénéficiant de la réputation de son école de médecine et de la compétence des apothicaires locaux, Montpellier attire les aspirants, de France ou de l'étranger. Néanmoins la tradition orale et technique révèle de plus en plus ses limites. Deux hommes jouent un rôle majeur dans cette évolution. Bernardin II Duranc, apothicaire, à l'origine du droguier de l'École de Médecine (1588), et Pierre Richer de Bellaval, médecin, créateur du premier jardin botanique de France. Ces deux initiatives pédagogiques témoignent d'un renouvellement des savoirs : développement de l'observation et de l'expérimentation, parution de livres plus pratiques et de plus rédigés en plus en français. Sous l'effet d'initiatives comme celles de Bernardin II Duranc et P. de Richer, la constitution de collections contribue aux progrès de la pharmacologie, de la médecine et de la botanique, tandis que les aspirants apothicaires complètent de plus en plus leur 
apprentissage par des cours dispensés à l'École de médecine. La révolution scientifique du XVII ${ }^{\mathrm{e}}$ siècle est en marche. médecine au sein de la société française ${ }^{2}$. Ayant suivi un cursus complet à la faculté de médecine de Paris, leur titre de régent leur confère le privilège d'enseigner l'Art de guérir et de pratiquer la médecine à leur guise. S'ils cherchent le plus souvent à s'informer des nouveautés en matière de traitement, la nécessité pour eux d'opérer une veille scientifique entre de plus en plus en concurrence avec l'exercice libéral de la médecine. Leur formation s'opère notamment par l'entremise des traductions scientifiques (surtout de l'anglais, un peu de l'italien, peu de l'allemand), qui permettent un transfert de connaissances venues de l'étranger. Mais elles ne résolvent pas tout, d'où la préférence des docteurs régents pour la presse médicale professionnelle. Celle-ci exerce un effet structurant sur la formation du groupe professionnel des médecins incluant les docteurs régents. Ces derniers s'efforcent de contrôler les flux nouveaux d'information, de préserver leur prépondérance au sein des professionnels de la médecine et de ne pas livrer les personnes charitables à l'influence de praticiens illégaux : tel est l'enjeu de manuels de médecine charitable. De par leurs fonctions, les docteurs régents peuvent contrôler l'adéquation des connaissances diffusables avec la doctrine médicale de la Faculté, mais aussi communiquer à l'extérieur l'image de la médecine qu'ils ont constituée et à laquelle ils adhèrent, en tentant de combiner logique corporative et logique professionnelle.

\section{Les sciences de la nature, au cœur de la transmission des savoirs?} moyens essentiels de la circulation des savoirs : c'est ce que montre l'exemple de John Ray (1627-1705), éminent naturaliste, fondateur de la botanique et de la zoologie modernes ${ }^{3}$. Celui que l'on a surnommé le «Pline anglais » ouvre la voie aux travaux ultérieurs de Jussieu (1699-1777), Linné (1707-1778), Buffon (1707-1788) et Cuvier (1769-1832). La correspondance de John Ray, en partie traduite en français par William Derham (1657-1735), témoigne du réseau d'échanges collaboratifs et amicaux qui constituent la "République des Lettres» de l'époque moderne. Des voyages et correspondances de J. Ray se dégagent deux conclusions essentielles: d'abord les botanistes de cette «République des lettres » sont des précurseurs de la communauté scientifique internationale moderne, dont ils ont pu être le noyau cristallisateur; ensuite, la vision de la nature par ces naturalistes dépasse notre conception contemporaine, l'étude du « Grand Système de la Nature » inclut en effet l'ensemble des activités humaines, l'homme étant un animal parmi d'autres.

Les voyages ne visent pas seulement les échanges entre savants. Ils constituent en soi une démarche scientifique, à l'instar des missions des naturalistes Peyssonnel et Desfontaines dans la Régence de Tunis au XVIII ${ }^{e}$ siècle ${ }^{4}$. Tandis que certains naturalistes travailleurs dans leurs cabinets de curiosité ou dans leurs bibliothèques, d'autres vont sur le terrain, comme ces deux savants partis au xviII ${ }^{e}$ siècle du Jardin Royal, futur Muséum d'Histoire Naturelle de Paris. Tous deux médecins, mais nés respectivement en 1694 et 1750, leurs récits et correspondances sont publiés en 1838 par Dureau de La Malle, à la fois historien et géographe. Ces documents révèlent comment les naturalistes d'autrefois ont rapporté les pièces qui font aujourd'hui la richesse des collections des Muséums. Peyssonnel et Desfontaines sont au premier rang de ces 
savants naturalistes, qui, en dépit de multiples difficultés, ont rapporté de leurs missions la connaissance du monde, qu'ils ont partagée avec leurs contemporains au sein des académies et sociétés savantes, avant que les publications et l'enseignement scientifique ne la diffusent au sein du plus large public.

La botanique "science aimable", est souvent considérée comme plus ouvertes aux femmes que d'autres disciplines ${ }^{5}$. Si la féminisation des sociétés de botanique de France et de Belgique s'est effectuée de façon tardive, au début du xxe siècle, il a bien existé des pionnières, telles que Marie Fortier. Dès l'origine, à côté des plantes vivantes, la botanique scientifique s'est appuyée sur les herbiers de plantes sèches, les illustrations ou les modèles en trois dimensions fabriqués tantôt en cire, tantôt en papier mâché, tantôt en tissu, ces dernières inspirées des fleurs artificielles pour le mode. Entre 1885 et 1914, M. Fortier, une entrepreneuse parisienne, commercialise des herbiers naturels en tissus, comme matériel pédagogique à destination de l'enseignement primaire et secondaire. Grâce à ses liens avec la Société nationale d'Horticulture de Paris et la Société botanique de France, elle fournit les écoles primaires de Paris durant une décennie. Ces herbiers en tissus, parce que plus fragiles, plus proches des arts décoratifs et de la mode, ont connu moins de succès que ceux en cire ou en papier mâché. Marie Fortier n'a donc pas réussi sa percée, faute d'activité scientifique propre mais aussi parce qu'elle était femme et pas issue d'un milieu social très favorisé.

Cet intérêt pour la botanique ne s'est pas limité à l'Europe occidentale, ainsi que le montre l'exemple du cabinet zoologique de Varsovie ${ }^{6}$. Durant les années 1860, ce cabinet, qui n'était jusque-là qu'un petit musée de province, devient, en Europe centrale, l'un des plus importants centres de recherche sur la faune et une référence pour les collections. Fondé en 1818, il a entretenu, dès l'origine des liens étroits avec le Muséum national d'histoire naturelle de Paris. Néanmoins, son essor doit beaucoup à Władysław Taczanowski (1819-1890), ornithologue et arachnologue réputé, qui le dirigea à partir de 1862. Sachant trouver des mécènes et s'appuyer sur les émigrés en Amérique du Sud, il développe une collaboration privilégiée avec les scientifiques français. Son réseau de correspondants et de collaborateurs français, constitué de savants du MNHN, mais aussi de musées provinciaux, d'entreprises privés et de nombreux naturalistes non professionnels permet un transfert massif de connaissances concernant à la fois le travail sur le terrain et l'acquisition des spécimens, la description et la publication, la préparation des expositions et la formation des collaborateurs.

\section{L'agriculture : entre assimilation des acquis de la science et transmission des savoir-faire}

Si l'agriculture offre un exemple archétypal de l'importance de la transmission des savoir-faire, parfois issus des temps les plus anciens, elle a suscité depuis longtemps l'intérêt des savants, auteurs de traités, mais aussi des grands propriétaires, voire des élites touchées par l'agromania. À cet égard, les archives de la Société des Thesmophories de Blaison en Anjou présentent un intérêt exceptionnel ${ }^{7}$. En effet, si les sociétés royales ont été bien étudiées, il n'en va pas de même des petites sociétés savantes. Cette Société des Thesmophories s'intègre tout à fait, notamment à travers l'activité du bureau d'agriculture d'Angers, dans les grands débats européens du XVIII ${ }^{\mathrm{e}}$ siècle autour de l'agriculture et de l'économie. Sans avoir lu beaucoup, ses membres 
sont assez pénétrés d'esprit scientifique pour justifier leur besoin de reconnaissance sociale et culturelle.

En effet, l'art de l'agriculture tend, au XVIII ${ }^{e}$ siècle, à se transformer en savoir rationnel et raisonnés. Longtemps jugée "malthusienne" par comparaison avec celle de l'Angleterre ou de certaines parties de l'Allemagne, l'agriculture française se transforme cependant, notamment à partir des années 1840, sous le double impact des avancées de la science et de la conjoncture économique générale. Les recherches de Louis Pasteur (1822-1895) ont de nombreuses retombées agricoles: travaux sur la fermentation, les maladies du vin et du bétail, la mise en évidence des microorganismes dans la destruction des vers à soie, puis les recherches sur la lutte biologique à base de bactéries. Toutefois, ce sont surtout les grands fléaux de la fin du XIX ${ }^{\mathrm{e}}$ siècle (oïdium, phylloxéra et mildiou de la vigne introduits des Amériques) qui constituent le facteur déclencheur majeur. Entre 1884 et 1889, se créent de nombreux syndicats agricoles voués à la défense des intérêts des agriculteurs, mais à l'achat d'engrais pour le compte de leurs membres. Faisant appel à l'expertise des stations agronomiques, ces achats collectifs poussent à faire appel à la science agronomique et, donc à coupler pratique et théorie.

Telle était déjà l'ambition des fermes écoles`. Créées en métropole dès 1830 par des intérêts privés, en vue de diffuser le progrès technique et de former des aides ruraux qualifiés, elles représentent, à partir de 1840, l'enseignement élémentaire agricole. Désormais, elles reposent sur une association entre exploitants éclairés et l'État. Ce dernier finance les dépenses de formation, les exploitants assurent le risque économique de l'entreprise. Offrant un modèle d'agriculture performante, ces fermesécoles forment de jeunes ruraux pauvres aux méthodes de l'agriculture raisonnée que préconisait Mathieu de Dombasle. Entre 1922 et 1930, le modèle est introduit en Afrique du Nord et en Syrie. Faute de chefs d'exploitation éclairés, d'entrepreneurs et de formateurs, les deux écoles supérieures agronomiques d'Algérie et de Tunisie ne favorisent pas une évolution comparable à celle observée en métropole, mais leur action n'a pas été totalement négligeable comme en arboriculture ou en matière expérimentale, ainsi au Maroc ou en Syrie.

De 1946 à 1978, Raymond Février a été un acteur majeur de la transmission des savoirs ${ }^{10}$. C'est ce que révèle le fonds versé aux Archives nationales en 2012. Ce fonds recouvre trois grands ensembles documentaires : des dossiers de travail constitués dans le cadre de ses fonctions de chercheur, d'inspecteur général, puis de directeur général de l'Institut national de la recherche agronomique (INRA); des archives issues des missions qu'il a remplies, notamment dans les années 1970-1980, auprès de l'Université de Corse, du Centre du Machinisme agricole, du Génie Rural, des Eaux et Forêts (CEMAGREF) et du Centre International des Hautes Études Agronomiques (CIHEAM) ; un certain nombre de pièces plus personnelles relatives en particulier à son engagement durable au parti socialiste. Il en ressort deux caractéristiques dominantes : un souci permanent de diffusion de connaissances à finalité pratique et immédiate auprès du monde agricole et industriel; la volonté d'influer sur les politiques structurelles en matière agricole et de recherche agronomique. 


\section{De l'information scientifique et technique aux transferts de technologie : un défi pour notre temps}

La transformation des savoirs implique donc la diffusion de l'information scientifique et technique, d'où l'importance d'en identifier les vecteurs. Néanmoins, pour permettre le développement économique, cette information doit s'accompagner de transferts de technologie, notamment des pays industrialisés avancés vers ceux en voie de développement ou émergents.

\section{L'information scientifique et technique : quels vecteurs ?}

L'information scientifique et technique se place évidemment au centre de la diffusion des savoirs. Toutefois, elle emprunte des voies multiformes: individuelles ou collectives, par exemple dans le cadre de laboratoire, variables en fonction des époques et de l'action des institutions.

\section{De l'innovateur et du développeur}

S'appuyant sur des archives familiales, Anne Marle-Kolle oppose deux approches de la transmission des savoirs ${ }^{11}$. Le premier, Charles-Louis Marle (1799-1864 ?) est un précurseur, visionnaire, bref un innovateur. Fils d'un instituteur de Saône-et-Loire, il s'engage en politique contre la Restauration, transmettant ses convictions à ses deux fils, Louis et Jérôme, condamnés, à la suite du coup d'État du 2 décembre 1851, à la déportation en Algérie. Convaincu de l'enjeu formidable que constitue l'apprentissage de la lecture et de la nécessité d'une réforme de l'orthographe, il dirige une école d'enseignement mutuel (fondée par son père) dès 1818, puis, de 1826 à 1829, le Journal de la langue française, grammaticale, didactique et littéraire. En 1829, il publie un ouvrage intitulé Praxigraphie ou recueil d'un nouveau genre pour apprendre par la pratique les règles de grammaire et l'orthographe, puis, surtout en 1839, son Manuel de la diagraphie. Découverte qui simplifie l'étude de la langue par Marle ainé (Paris, Imprimerie administrative de Paul Dupont), avant de lancer un nouvel organe de presse, L'Émancipation de l'enseignement paru entre mai 1848 et novembre 1849. Quant à LouisGeorges, il fait plutôt figure de développeur. De formation scientifique (Lycée Louis le grand, Maths Sup à Fénelon, études d'Histoire à La Sorbonne), il entre aux Belles Lettres, devient responsable des sciences humaines chez Albin Michel, avant de prendre en charge, en 2010, les questions de la numérisation au Syndicat national de l'Édition, en se consacrant au moyen de diffuser le savoir et la culture auprès d'un public à la recherche du confort de lecture (liseuse, tablette, smartphone, restitution orale des textes numérisés).

\section{Diversité des modes de circulation du savoir scientifique}

40 L'institution de grands laboratoires de recherche au sein des entreprises a joué un rôle majeur dans l'accélération et la montée en puissance de la circulation du savoir scientifique. Ce qu'a bien analysé Joseph Schumpeter se retrouve dans le cas des laboratoires de recherche européens Kodak entre les deux guerres ${ }^{12}$. Le groupe américain s'est doté, dès 1912 et sous l'impulsion de son créateur, George Eastman, d'un laboratoire de recherche au sein de Kodak Park, à Rochester, près de New York. Il en 
fait de même en 1928, à Harrow en Grande-Bretagne, pour accroitre l'efficacité commerciale de sa filiale anglaise, Kodak Limited. À cette époque, le groupe vient de créer en France Kodak Pathé, suite à la vente par Charles Pathé, grand concurrent de Kodak, de son usine de films de Vincennes. À la fin des années 1930, Eastman Kodak décide d'accroitre la capacité de recherche industrielle de son groupe en ouvrant deux laboratoires de recherche additionnels (celui d'Harrow et celui de Vincennes, hérité de Pathé). D'une étude conjointe des archives de Kodak Ltd et de Kodak-Pathé, il ressort que les chercheurs conservaient le savoir scientifique produit au sein de rapports de recherche réservés en interne à une liste restreinte de responsables. Les archives françaises éclairent aussi sur les modalités des savoirs scientifiques contenus dans les rapports circulant entre les trois principaux laboratoires de Kodak : très codifiée, cette circulation révèle que, pour Kodak, toute technologie d'avenir représentait un atout potentiellement exploitable.

41 Les processus collectifs de diffusion des savoirs ne périment pas les transmissions plus classiques du maître à l'élève et du chercheur à sa communauté scientifique. La chose paraît très claire dans le cas de Petre Sergescu (1893-1954), grand historien des sciences et promoteur de la discipline ${ }^{13}$. Né en Roumanie, il fait une partie de ses études en France, à laquelle il reste toute sa vie très attaché : licencié en mathématiques et philosophie de l'Université de Bucarest, diplômé du Conservatoire de Musique de cette ville, il complète sa formation à l'ENS et à la Faculté des Sciences de Paris, pour les mathématiques, et au Collège de France, pour l'histoire des sciences. Ayant soutenu une agrégation et un doctorat de mathématique à Bucarest en 1923, il devient professeur suppléant à l'Université et à l'Institut polytechnique de Bucarest, puis professeur à l'Université de Cluj (1926), enfin professeur à l'Institut polytechnique en 1943, date de son admission en tant que membre de l'Académie des Sciences. Il s'impose durant cette période comme un spécialiste renommé de l'Histoire des Sciences (participation à l'Académie Internationale d'Histoire des Sciences) et des Mathématiques (revue Mathematica).

Souvent invité à l'étranger, notamment en France et en Pologne, mais aussi en Suisse et en Tchécoslovaquie, sa vie se trouve bouleversée par la Seconde Guerre mondiale. Élu recteur de l'Institut polytechnique en janvier 1945, il doit se réfugier à Paris dès août 1946. Ses conditions de vie y sont difficiles, puisqu'il n'intègre le CNRS qu'en 1952, jouant un rôle éminent dans le renouveau des congrès internationaux d'Histoire des Sciences, relançant la revue Archives Internationale d'Histoire des Sciences, animant l'Association Française pour l'Avancement des sciences et contribuant à la diffusion de l'information scientifique (conférences radiodiffusées sur Radio France), tout en soutenant la diaspora roumaine en France.

\section{Science et technique comme facteurs de modernisation}

La Libération de la France, en 1944-45, marque l'avènement d'un nouveau rapport à la science et à la technique, vue comme la clé de la renaissance et du progrès ${ }^{14}$. L'atome symbolise tous les espoirs: il s'agit de démythifier, de populariser et de magnifier la nouvelle énergie. Nouveau directeur du CNRS, puis Haut-Commissaire à l'énergie atomique exprime publiquement cette confiance en «la science, malgré l'horreur». À l'époque, le savant veut transmettre, populariser, faire aimer la science, d'où le succès des ouvrages de vulgarisation, voire des bandes dessinées scientifiques. L'année 
1945 constitue bien un «moment vulgarisateur» pour la diffusion du savoir scientifique.

En France, la science est l'une des clés de la modernisation prônée par le Plan Monnet. À travers ses commissions de modernisation, il constitue, entre autres, un vecteur de transmission de connaissances sur l'économie ${ }^{15}$. Imaginé par Jean Monnet et soutenu par le général de Gaulle, à partir de 1946, le Plan se fixe pour objectif de reconstruire et de moderniser le pays, puis de mieux répartir les fruits de la croissance. Dans cette perspective, il vise à informer les agents sur les grandes orientations économiques du pays et sur les prévisions de développement à moyen terme. Ce rôle repose en grande partie sur les commissions de modernisation groupes de travail réuni auprès du Commissariat général du Plan. De composition tripartite (administration, employeurs et salariés), elles deviennent autant des sources d'information pour le Plan que les instruments d'une pédagogie à inculquer aux responsables de l'économie et, à travers eux, au public. Grâce à ces commissions, la planification souple et incitative, "à la française ", a permis de transmettre des connaissances plus larges sur l'économie, comme, par exemple, à propos de l'importance des transferts de technologie.

\section{Importance des transferts de technologie}

Au cours du XIX ${ }^{e}$ siècle, les Français ont joué un rôle déterminant dans la transmission des savoirs technologiques nécessaires au développement de l'industrie textile russe.

\section{L'industrie textile russe au $\mathrm{XIX}^{\mathrm{e}}$ siècle : transferts technologiques depuis la France}

C'est ce que montre bien Olga Melnichenko ${ }^{16}$. Ces transferts ne se sont pas opérés dans le cadre classique des acquisitions de brevets ou de licences d'exploitation. Ils résultent plutôt de multiples initiatives individuelles émanant d'ingénieurs, d'entrepreneurs et de constructeurs de matériels ou de bâtiments tirant avantage de la diffusion en Russie de la "mode de Paris ». L'action de ces ingénieurs, de ces entrepreneurs et de ces constructeurs a été beaucoup moins étudiée pour le textile que pour la banque, les chemins de l'industrie lourde ou les travaux publics. Néanmoins, les sources existent, révélant des figures de premier plan comme Jules Watremez, Claude Giraud (fondateur des usines du même nom et numéro 1 à Moscou) ou des sociétés aussi importantes que Simonnod et $C^{\text {ie }}$ (principal concurrent moscovite de C. Giraud). Apportant leur savoirfaire de contremaîtres et d'ingénieurs, ils ont jeté les bases d'une industrie textile moderne et de grande dimension.

\section{Les transferts de technologie franco-soviétiques à l'époque de la Guerre froide}

Après la révolution russe, les transferts de technologie sont devenus plus difficiles entre la France et la Russie, devenue URSS, notamment à l'époque de la Guerre froide. Le contrôle par les alliés occidentaux des exportations en direction du bloc soviétique relève du Comité de coordination pour le contrôle multilatéral des exportations $(\mathrm{CoCom})^{17}$. Ce dernier a été très mal étudié à la fois en raison de son caractère secret et de l'autorité accordée à l'étude de Gunnar Adler-Karlsson, Western Economic Warfare, datant de $1960^{18}$. Depuis les années 1990, avec la chute de l'URSS, un intérêt nouveau s'est manifesté pour les particularités du CoCom. L'exemple de l'industrie métallurgique montre que l'URSS a beaucoup dépendu des technologies occidentales, 
en dépit d'efforts acharnés, d'où le recours à des transferts illégaux de propriété industrielle (copie de procédés ou de produits). Les progrès économiques de l'URSS dépendant en grande partie de l'aide technologique occidentale, la guerre économique organisée autour du CoCom a beaucoup affaibli le pays. Ces transferts de technologie correspondent à :

«Un processus par lequel des innovations (nouveaux produits ou procédés)

réalisées dans un pays sont transmises dans un autre pour y être utilisée..$^{19}$ "

La confrontation des archives du Ministère Français de l'Économie, des Finances et de l'Industrie, des archives présidentielles françaises ainsi que de celles du Ministère des Affaires étrangères de l'URSS permettent d'y voir plus clair ${ }^{20}$. Première conclusion, le transfert de technologie entre la France et l'URSS a bien existé durant la guerre froide, en dépit des aléas politique et au profit des deux pays. Outre les revenus importants qu'elle a tirés de la vente de ses produits et de ses technologies, la France a orienté l'industrie soviétique vers la technologie française. De fait, plusieurs années après les livraisons et le montage d'équipements français, l'URSS a poursuivi l'achat de pièces de rechange en France et à inviter des spécialistes français pour l'entretien des machines. C'était le prix à payer par l'URSS pour demeurer compétitive. Seconde conclusion, des contacts permanents se sont établis entre les industriels russes et leurs homologues français. L'industrie automobile en constitue un bon exemple : grâce à de tels contacts établis dans les années 1950 et poursuivis jusqu'aux années 1980, Renault a réussi à s'implanter en Russie dans les années 1990 à 2010, avec beaucoup de succès.

\section{Les transferts de technologie : un instrument au service du développement ?}

Les années 1950 et 1960 ont vu s'imposer la question du développement des pays les moins avancés (à l'époque désignés comme "pays sous-développés » ou "en voie de développement»): l'ONU a fait d'ailleurs des années 1960 «la décennie du développement", grâce notamment à l'action de la Banque mondiale. Il est donc pertinent de s'interroger sur la réalité et l'effet éventuel des transferts de technologie en direction des pays du Tiers-monde : tel est l'objet de la contribution de Getsiva Cayo $^{21}$. La question du transfert de know-how entre la France et les pays d'Amérique latine demeure largement à étudier, d'où l'intérêt de deux grands chantiers menés au Pérou par la France ; la construction de l'usine hydroélectrique du Cañyon del Pato et celle de l'aciérie de Chimbote. Il en ressort trois conclusions.

Bien que les deux pays n'aient pas été des partenaires commerciaux majeurs, ils ont entretenu des liens privilégiés en matière de technologie. Les gouvernements et leur administration ont créé les conditions favorables à l'introduction du savoir-faire français au Pérou. Néanmoins, le facteur majeur a résidé dans l'expertise des ingénieurs, techniciens et entrepreneurs français, d'autant plus qu'ils ont su associer au projet des collaborateurs péruviens.

51 Ces deux chantiers n'ont pas seulement engendré d'importants échanges de services, mais ils ont provoqué un mouvement d'industrialisation. Donnant naissance à «l'ère de l'acier » au Pérou, l'aciérie de Chimbote a un impact considérable au sein de la société péruvienne, notamment pour la vielle de Chimbote.

52 L'expérience n'a pas été totalement satisfaisante, en raison des obstacles financiers, politiques et techniques s'opposant au transfert de savoir-faire français, notamment la perpétuation $\mathrm{du}$ fonctionnement de l'usine sidérurgique après le départ au français 
(approvisionnement en électricité, formation insuffisante du personnel péruvien). Mais le bilan apparaît néanmoins positif, ouvrant la voie au développement de la coopération technique entre les deux pays, au profit de transferts accrus de technologie.

\section{NOTES}

1. E. Manouvrier, « La transmission du savoir et du savoir-faire chez les apothicaires de Montpellier à la fin du Xvi ${ }^{\mathrm{e}}$ siècle ».

2. I. Coquillard, "Les médecins parisiens et la diffusion du savoir médical au XVIII ${ }^{\mathrm{e}}$ siècle : des savants pédagogues ».

3. S. Kraus, «Les voyages et lettres de correspondance de John Ray, ou l'émergence des sciences naturelles dans l'Europe du XVII ${ }^{\mathrm{e}}$ siècle ».

4. G. Falgas, « Missions des naturalistes Peyssonnel et Desfontaines dans la régence de Tunis au XVIII ${ }^{\mathrm{e}}$ siècle ».

5. F. Tessier, « Les herbiers artificiels de Marie Fortier : un type méconnu de modèles ».

6. P. Daszkiewicz, «Le réseau français des collaborateurs du Cabinet zoologique de Varsovie pendant le seconde moitié du xix siècle ».

7. A. Follain, "La transmission des savoirs agronomiques vers la fin du XvIII siècle d'après les travaux des apprentis savants de la société d'agriculture de Blaison en Anjou ».

8. C. Cheneaux-Berthelot, «Science, art ou pratique ? La place de l'agriculture dans les formes de transmission du savoir au XIXe siècle en France ».

9. N. Abou El Maaty, «La transmission du savoir-faire agricole dans les fermes-écoles créées par la France en Afrique du Nord et en Syrie au début du xxe siècle ».

10. S. Pivoteau, «Raymond Février et la transmission des savoirs agronomiques ».

11. A. Marle-Kolle, «De la diagraphie à la numérisation, deux artisans d'une transmission de savoir : Charles-Louis Marle aîné, Louis-Georges Marle ».

12. N. Le Guern, "Les laboratoires de recherche européens Kodak et la circulation interne du savoir scientifique durant l'entre-deux-guerres ».

13. A. Herlea, « Petre (Pierre) Sergescu (1893-1954), historien des sciences et promoteur de la discipline ».

14. R. Belot, «La "moment vulgarisateur français" : enjeux et modalités de la diffusion scientifique à la Libération ».

15. C. Chunhua, «Le Plan comme vecteur de transmission de connaissance sur l'économie: l'exemple des Commissions de modernisation à l'époque des quatre premiers Plans (1946-1965)».

16. O. Melnichenko, « Transmission des savoirs technologique de la France à la Russie : le cas de l'industrie textile au XIX ${ }^{e}$ siècle ». 
17. I. Sheveleva, «Modèle d'emprunt des technologies occidentales par l'URSS et le CoCom (comité de coordination pour le contrôle multilatéral des exportations) ».

18. G. Adler-Karlsson, Western Economic Warfare: a case study in foreign economic policy, (Guerre économique occidentale: une étude de cas en politique économique étrangère), Stockholm: Almqvist \& Wiksell, 1968.

19. M. Borstein, Transferts de technologie entre l'Est et l'Ouest: le transfert de technologie occidentale à l'URSS, Paris, OCDE, 1985, p. 162.

20. B. Vinogradov, « Transferts de technologie entre l'URSS et la France, 1954-1991 ».

21. G. Cayo Durand de Geist, « Le transfert de savoirs technique et industriel français au Pérou dans les années 1950 et 1960. L'exemple de la sidérurgie de Chimbote et de la centrale hydroélectrique du Canyon de Pato ».

\section{AUTEUR}

\section{DOMINIQUE BARJOT}

Professeur émérite d'histoire économique contemporaine, Sorbonne Université Lettres, Renmin University of China, membre de l'Académie des sciences d'outre-mer, président du Comité français des sciences historiques 


\title{
In memoriam : Nagwa Abou El Maaty épouse Khalil (1954-2019)
}

\author{
Dominique Barjot
}

1 Madame Nagwa Khalil, née Abou El Maaty nous a quittés le 12 juin 2019, à l'âge de soixante-cinq ans. Elle était veuve et mère de trois enfants $(1980,1983,1984)$. Ayant obtenu un baccalauréat scientifique en 1972, elle s'engagea à l'Université du Caire, dans des études d'ingénieure agronome, option sociologie rurale et développement agricole, obtenant son diplôme (mention bien) en 1976. Elle exerça ensuite les fonctions de professeure d'anglais en collège, à Dekernis, en 1976-1977, puis de biologie, dans la même ville, mais en lycée en 1977-1978. Puis, de 1979 à 1983, elle travailla en tant qu'ingénieur agronome au service de planification de l'Université de Mansoura, toujours en Égypte. Prise par ses obligations de mère de famille, notamment la prise en charge d'un enfant grand handicapé, elle renoua un temps avec son métier d'ingénieur: d'avril à juillet 1989, elle effectua un stage à la société TECHNA (TECHnique de Nutrition Animale), une entreprise de service conseils à Coüeron (LoireAtlantique).

2 En 1995, elle soutint avec succès (mention bien) un DEA d'Études Méditerranéennes, option Civilisation, à l'Institut National des Langues et Civilisations Orientales (INALCO). Il se constituait d'un mémoire dirigé par Henri Laurens portant sur Les réformes agraires égyptiennes (1942-1970). Puis, après un temps d'interruption, elle s'inscrivit en thèse de doctorat d'histoire économique à l'Université Paris-Sorbonne (Paris IV), sous la propre direction de Dominique Barjot. Cette thèse fut soutenue, avec la mention très honorable, le 4 juin 2007. Elle portait sur La scolarisation de l'apprentissage agricole en France: les fermes écoles au service de l'agriculture et de son enseignement (XIX siècle-début du XXe siècle), thèse alors très neuve.

3 Pratiquant l'anglais, l'arabe et le français, Madame Nagwa Khalil s'est illustrée par son engagement bénévole: aide aux tuberculeux et à leur famille, dans le cadre de l'Association "Amélioration de la santé » (1977) et enseignante bénévole en cours 
d'alphabétisation et de remédiation, toutes matières (au collège), en sciences et grammaire arabe (lycée) toujours à Dekernis (1976-1978). Elle renoua plus tard, en 1997-1998, avec le bénévolat : aide aux devoirs bénévole pour des élèves en classe de soutien, au collège Libertaire Rutigliano, à Nantes.

4 La charge de son fils handicapé et le décès de son époux n'empêchèrent pas Madame Nagwa Khalil de publier un article de revue à comité de lecture: «La fabrique des instruments agricoles de la ferme-école des Trois-Croix ", Histoire \& Sociétés Rurales, vol. 21, 2004, p. 115-132, et une contribution aux actes de congrès du CTHS : «L'action des directeurs exploitants des fermes-écoles au sein de la viticulture locale (xIx-début du $\mathrm{Xx}^{\mathrm{e}}$ siècle ", dans Les Hommes de la vigne et du vin. Figures célèbres et acteurs méconnues, Paris, Éditions du CTHS, 2011, p. 11-24. S'y ajoutèrent quatre communications à congrès ou colloques : «Sur les traces de Mathieu de Dombasle : Jules Rieffel et le défrichement des landes de Grand-Jouan ", Le Travail et les hommes, $127^{\mathrm{e}}$ congrès du CTHS, Nancy, 15 au 20 avril 2003 ; celle publiée en 2011 et présentée lors du Congrès de Bordeaux, Les hommes de la vigne et $d u$ vin, 20 au 25 avril 2009, "Une instruction professionnelle agricole gratuite pour la petite paysannerie au XIX siècle: les élèves apprentis des fermes écoles formés par des notables, colloque Les "petites gens» de la terre: paysans, ouvriers et domestiques du Néolithique à 2014, MRSH de l'Université de Caen, 8-10 octobre 2014; celle, posthume, à paraitre dans le présent volume, «La transmission du savoir-faire agricole dans les fermes écoles créées par la France en Afrique du Nord et en Syrie au début du $\mathrm{xx}^{\mathrm{e}}$ siècle », La transmission des savoirs, $143^{\mathrm{e}}$ congrès du CTHS, Paris, 23 au 26 avril 2018. Tout cela ne donne que plus de regret à ce que la thèse, pourtant acceptée par les Presses Universitaires de Rennes, n'ait pas été publiée.

5 Madame Nagwa Khalil laissera l'image d'une chercheuse scrupuleuse et passionnée.

\section{AUTEUR}

\section{DOMINIQUE BARJOT}

Professeur émérite d'histoire économique contemporaine, Sorbonne Université Lettres, Renmin University of China, membre de l'Académie des sciences d'outre-mer, président du Comité français des sciences historiques 
Transmission et circulation des savoirs scientifiques et techniques : des sciences du vivant à l'agriculture 


\section{De la médecine à la biologie : un espace privilégié pour la transmission des savoirs et des savoir-faire}




\title{
La transmission du savoir et du savoir-faire chez les apothicaires de Montpellier à la fin $\mathrm{du} \mathrm{XVI}{ }^{\mathrm{e}}$ siècle
}

\author{
Élisabeth Manouvrier
}

1 Montpellier, au bord du bassin méditerranéen, voit dès le Moyen Âge, sous l'impulsion des immigrés juifs, chrétiens, sarrasins, se développer un centre médical et savant d'une activité incomparable. La présence de médecins réputés, l'abondance des épices et matières précieuses importées, font de cette ville un lieu d'élection pour le commerce des drogues et leur transformation en médicaments. Les "épiciersapothicaires", riches boutiquiers, se livrent à la préparation de ces formules magistrales; ils sont à l'origine de la profession d'apothicaire, corps de métier libre, mais subordonné à des règles sinon écrites du moins traditionnelles. À la fin $d u X V I^{e}$ siècle, l'École de médecine accepte en son sein l'intervention des apothicaires. La transmission du savoir, à la fois aux aspirants apothicaires et aux médecins, va se modifier.

\section{L'apprentissage et le compagnonnage}

$2 \mathrm{Au} \mathrm{XVI}{ }^{\mathrm{e}}$ siècle, l'art de la pharmacie est considéré comme un art mécanique, aussi la formation au métier d'apothicaire, régie par des statuts, comporte deux stades: l'apprentissage et le compagnonnage.

\section{L'apprentissage}

3 L'apprentissage, qui va permettre la transmission du savoir, a lieu exclusivement au sein de la boutique (fig. 1). La connaissance dont disposent certains individus relevant d'un groupe social donné est alors partagée avec d'autres qui intègrent ce groupe pour permettre l'apprentissage. Celui-ci repose sur l'idée de savoirs déjà constitués, 
l'apprenant étant moins savant que celui qui enseigne. Il est reconnu une asymétrie entre l'élève et l'enseignant, autrement dit l'autorité.

Fig. 1. - Boutique d'apothicaire, gravure Leroy.

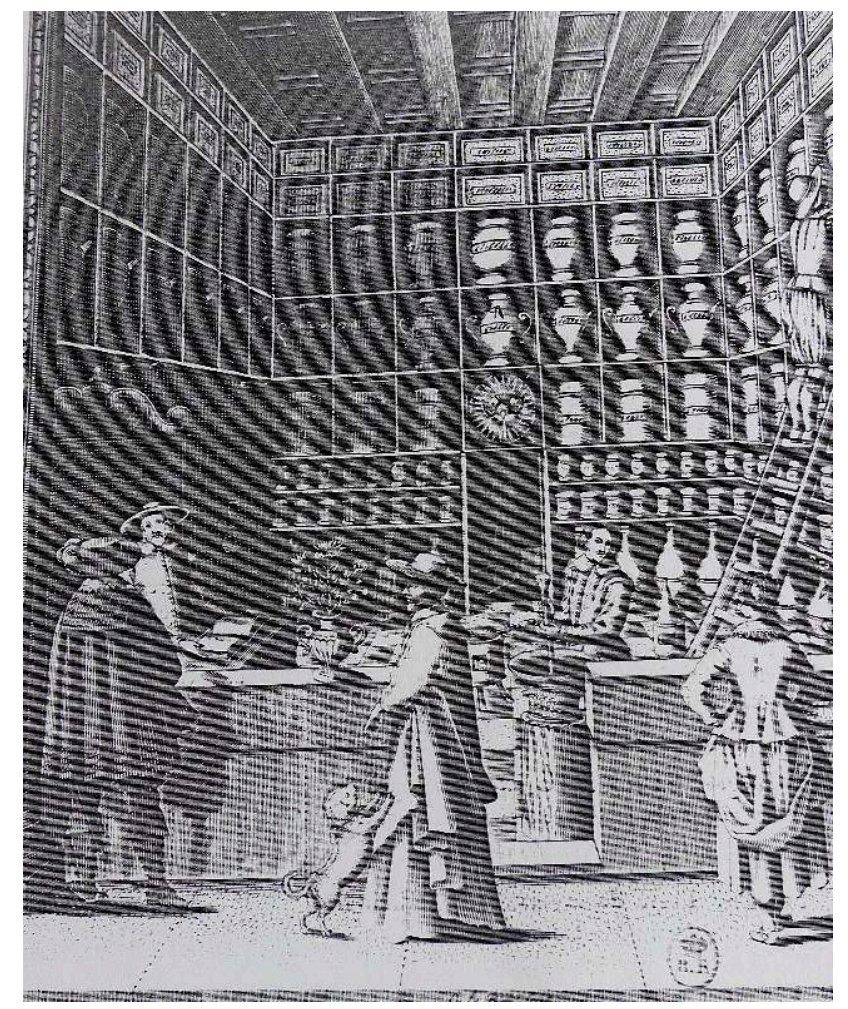

Bibliothèque nationale, Cabinet des estampes, Paris, cliché É. Manouvrier.

Une ordonnance de 1514 précise que l'aspirant apothicaire ne peut être reçu qu'après avoir subi un examen constatant qu'il a fait des études, qu'il sait suffisamment de latin pour "entendre les livres servant à l'art » et qu'il est capable d'apprendre la pharmacie. Dans son ordonnance de 1560, François II exprime le souci de mieux contrôler la formation.

«Il est plus que nécessaire que celui qui traite de la vie des hommes comme le marchant apothicaire espicier, par drogues, médecines, espices et autres choses entrans en corps humain, soit expérimenté par examen et que nul, de quelque estat ou qualité ne s'ingèrent de vendre des dites médecines. ${ }^{1}$ "

En 1596, Guillaume Grès passe devant le notaire royal Maitre Rancoule ${ }^{2}$ en présence de sa mère afin d'être mis en apprentissage pour une durée de deux ans chez Pierre Rougier apothicaire de La Grasse (Aude) qui promet « de lui bien montrer et apprendre le dict art, d'honnêtement le nourrir et entretenir, l'apprenti promettant le bien et loyalement servir ». Le contrat conclu, l'élève verse une somme au maître apothicaire. Il est à noter que les candidats doivent avoir un bon niveau en grammaire et en latin. Le long apprentissage imposé et régi par les statuts de la profession (sept ans selon les statuts de 1572) est sanctionné par un examen et l'élaboration d'un chef-d'œuvre.

6 Ouvriers, apprentis, compagnons, vivent avec le maître apothicaire au sein de la même maison. Tout en étant paternelle la formation est parfois rude, le maitre pouvant punir par voie de correction, non seulement les larcins, mais aussi les manquements au respect et à l'obéissance. 
7 La transmission d'un savoir-faire prédomine et les inventaires notariés des officines de l'époque illustrent à quel point sur le plan technique le rôle initiatique du maître d'apprentissage est essentiel. Le nombre d'instruments à manipuler est impressionnant. Il est courant de trouver dans une moyenne officine de ville à la fois nombre de mortiers et leurs pilons, balances avec leurs poids, cornues, alambics, spatules, chaudières en cuivre, bain-marie, fontaines, fourneaux en cuivre, presse de bois, réchaud, coupons de toile fine, destinés à la fabrication, et une quantité conséquente d'ustensiles de conservation tels les chevrettes, cruches, pots, fioles de verre, petits vases de terre, boites, bouteilles, pots à clystères. Sous la direction vigilante de son maître le jeune élève doit se familiariser avec ce matériel (fig. 2). Des opérations telles que les pesées, les triturations, les distillations, les filtrations sont une partie importante de son cursus. Une fois le geste maîtrisé, le maître enseigne la connaissance et la reconnaissance des matières premières. À l'époque concernée, le règne végétal est largement prépondérant (plus des $2 / 3$ du stock), les règnes animal et minéral étant mineurs dans les préparations. Sens de l'observation, mémoire et ordre vont être les qualités que le maître doit favoriser chez l'élève.

Fig. 2. - Apprenti, aquarelle G. Gravenbroch.

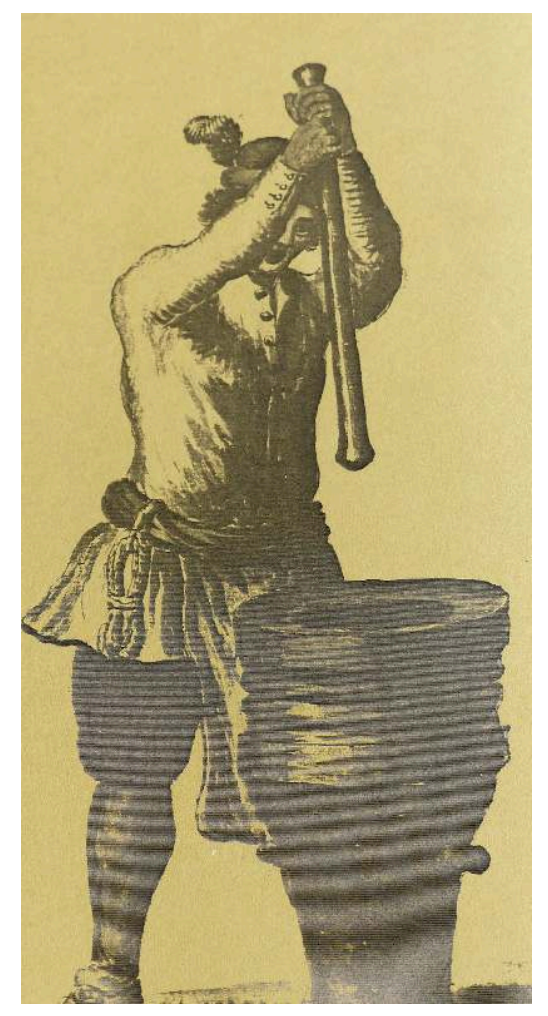

Musée Correr Venise, cliché É. Manouvrier.

8 L'étape suivante de la formation comporte l'élaboration du médicament selon la prescription du médecin : reconnaissance des drogues entrant dans la composition et tour de main «selon l'art». Au nombre des préparations, l'apprenti doit savoir confectionner confitures, sirops, distillats, pour les biens portants, et pour les malades une grande variété de remèdes, aussi bien des poudres, que des troschiques, pilules, sucs, eaux, emplâtres, huiles, électuaires, extraits, confections, tablettes, sirops... 
comme le montre cet exemple de préparation dont le façonnage demande une grande pratique :

«Populeum onguent. Prenez une livre et demie de boutons de peuplier noir bien écrasée et quatre livres de graisse de porc mâle; mettez-les dans un pot de terre verni mêlant le tout ensemble et l'ayant couvert; gardez-le à la cave jusqu'au mois de mai ou juin. Alors ayant placé le même pot ou sont les matières au bain bouillant, ajoutez-y les feuilles écrasées de violier, de nombril de Venus, de ronce, de pavot noir, de mandragore, de jusquiame, de morelle, de laitue, de grande et petite jobarde et de la grande bardane, de chacun quatre onces. Ayant continué la décoction de toutes ces herbes dans le même pot jusqu'à ce que toute l'humidité soit consumée, vous coulerez ensuite et exprimez le tout, purifiant l'onguent que vous garderez en lieu tempéré pour le besoin. »

Par opposition à la formation pratique, la formation théorique parait bien mince pour les jeunes aspirants. Les maîtres apothicaires se réfèrent à des manuscrits médiévaux ; ils consultent les herbulari, thesauri, les pharmacopae, les antidotaires, étudiés dans les facultés de médecine, issus d'ouvrages anciens grecs ou arabes, mais aussi de nouvelles recettes, des traités, des recueils secrets merveilleux. À Montpellier, chaque pharmacie doit détenir l'antidotarium nicolai. En outre la bibliothèque de l'apothicaire comporte des ouvrages religieux, de droit et certains d'entre eux commencent à constituer des herbiers, des cabinets de curiosités. Le papier est cher, l'imprimerie progresse mais la spécialisation des écrits scientifiques est lente, et malgré les traductions en français, la langue officielle de la boutique demeure pour longtemps encore le latin.

\section{Le compagnonnage}

10 Après l'apprentissage, la pratique du compagnonnage impose l'exercice dans différentes officines. Il permet de maîtriser parfaitement le métier et de stimuler les échanges de connaissances, de recettes, de tours de main, d'astuces personnelles.

11 Jean Magnol, protestant âgé de 23 ans, arrive de sa ville natale ardéchoise en 1585 à Montpellier chez Gabriel II Sanche, apothicaire réputé de la ville. Il aide le maître apothicaire chez qui il loge et perçoit un salaire. Ce n'est qu'en 1599 qu'il présente sa maitrise devant quatre maîtres signataires des statuts de 1572. Savoir-faire et savoir sont exigés et sont l'aboutissement d'une longue formation. Les épreuves se déroulent sur deux années et les chefs-d'œuvre réalisés par le candidat sont complexes :

«Le premier chef-d'œuvre porte sur l'électuaire Hyacinthe composé de vingt-neuf substances ; métaux, pierres précieuses, argiles, ivoire, corne de cerf, cochenille de kermès, aromates, roses rouges, le tout dissous dans du sirop de limon. Le compagnon doit savoir préparer cette composition utilisée contre la peste dont la formule se trouve dans l'antidotarium nicolai. L'examen se déroule chez Jacques Catelan.

Le deuxième chef-d'œuvre est le sirop de rhubarbe composé; vingt-deux composants obtenus par décoction et réduction, traitant « les opilations du foie » et ayant une action purgative ; c'est la formule de Nicolas Florentin.

Après le sirop, le candidat doit faire un emplâtre "promatrice ", formule de Nicolas Provost, médecin tourangeau, avec vingt substances: résines, oléorésines, cire, écorces et fleurs. La préparation s'effectue chez l'apothicaire Louis Bosc.

En dernier, Jean Magnol doit réaliser l'onguent d'Arthanita, formule de Mésué, qui comporte du cyclamen dont on utilise la racine souterraine fraîche pour ses vertus laxatives et drastiques. Le maître apothicaire Jean Anchies surveille l'élaboration de la préparation. ${ }^{3} »$ 
Ensuite devant la compagnie des apothicaires le candidat subit l'épreuve dite « acte des herbes » durant laquelle il doit reconnaître les plantes présentées et faire un exposé sur leurs propriétés. Une dernière épreuve consiste à émettre un avis sur l'intelligence des recettes et des médicaments en usage. En 1601, Jean Magnol reçoit la maîtrise en faisant le serment solenne :

« De faire et de composer toutes poudres de bonnes et saines épices..., de bien et loyalement faire tous les ouvrages du dit métier sans y empirer ou mettre aucunes fournitures non pertinentes. ${ }^{4}$ " Son savoir reçoit une reconnaissance publique, la remise de maîtrise s'effectuant en grande pompe avec défilé en présence des autorités médicales de la ville.

Grâce à la réputation de son école de médecine et à la compétence des apothicaires locaux, Montpellier attire de nombreux aspirants même étrangers. Malgré tout, à la fin $\mathrm{du} \mathrm{XvI} \mathrm{e}^{\mathrm{e}}$ siècle, avec le progrès des sciences rationnelles, on se rend compte que la tradition orale et technique ne peut suffire et qu'il faut utiliser d'autres vecteurs de transmission du savoir sans lesquels il n'y a pas d'évolution possible.

\section{Évolution de la transmission du savoir médical et pharmaceutique}

15 À Montpellier, deux hommes et deux dates marqueront le point de départ de cette évolution. En 1588, l'apothicaire Bernardin II Duranc est à l'origine du droguier de l'école de médecine ; en 1593, le médecin Pierre Richer de Belleval crée de son côté le premier jardin botanique de France. La réalisation de ces deux outils pédagogiques va amorcer une période de transition dans l'acquisition du savoir pour les étudiants carabins et apothicaires.

\section{Le jardin botanique « Hortus regius Monspellensis »}

16 À la demande d'Henri IV, sous l'impulsion du professeur de médecine, ichtyologiste et botaniste Guillaume de Rondelet, Pierre Richer de Belleval crée officiellement le jardin botanique en 1593 (fig. 3). Champenois, médecin de formation, il vient perfectionner son art à Montpellier en 1590. Comme il est jugé digne d'appartenir à ce corps de métier, on crée pour lui une chaire associant l'enseignement de l'anatomie et de la botanique. Mais la lutte avec ses collègues est vive, « il doit enseigner l'anatomie par devoir et la botanique par inclination ${ }^{5}$ ». Le professeur est passionné de botanique qui lui parait nécessaire à cause de sa nouveauté, et il n'a qu'un seul but, le soin de ses plantes au jardin des simples et l'arrangement d'un vaste jardin dont il a tracé le plan (fig. 4). Il abandonne l'anatomie et se consacre à son embellissement. Ses intérêts sont confondus avec ceux du jardin du Roi dont il a l'intendance et la direction et, en attente fréquente de fonds, il se montre lui-même d'une générosité rare. Il organise son jardin d'une manière fonctionnelle, introduit de nouvelles techniques, reproduit l'habitat naturel des plantes. Il herborise fréquemment, recherche inlassablement des spécimens du pays ou étrangers, instruit et forme les jardiniers dont les connaissances se limitent alors aux plantes potagères. 
Fig. 3. - Pierre Richer de Belleval.

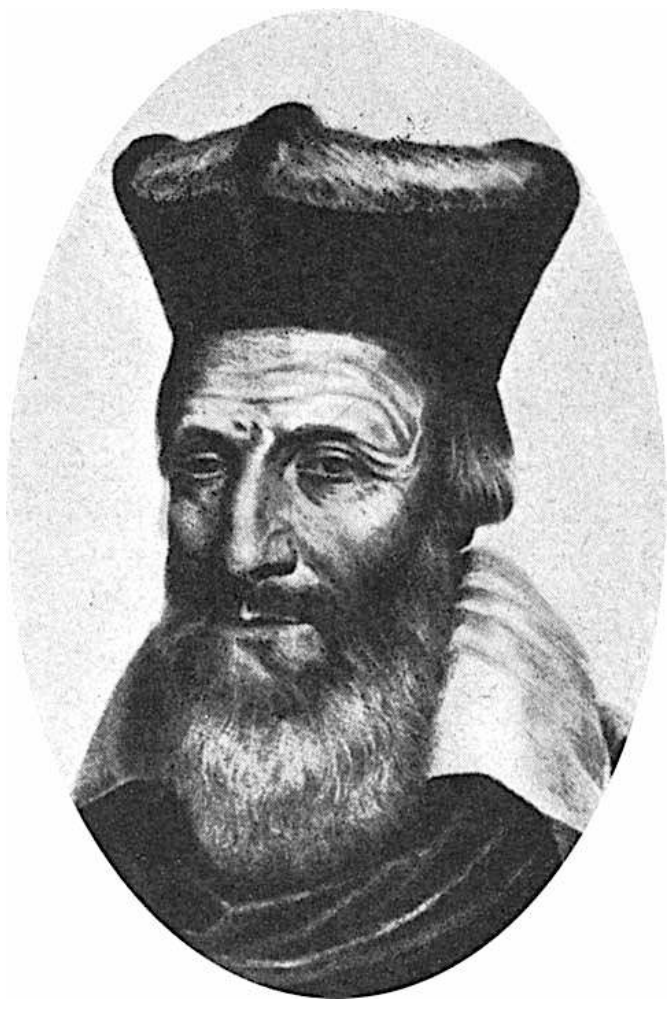

Domaine public.

Fig. 4. - Le jardin des plantes à Montpellier.

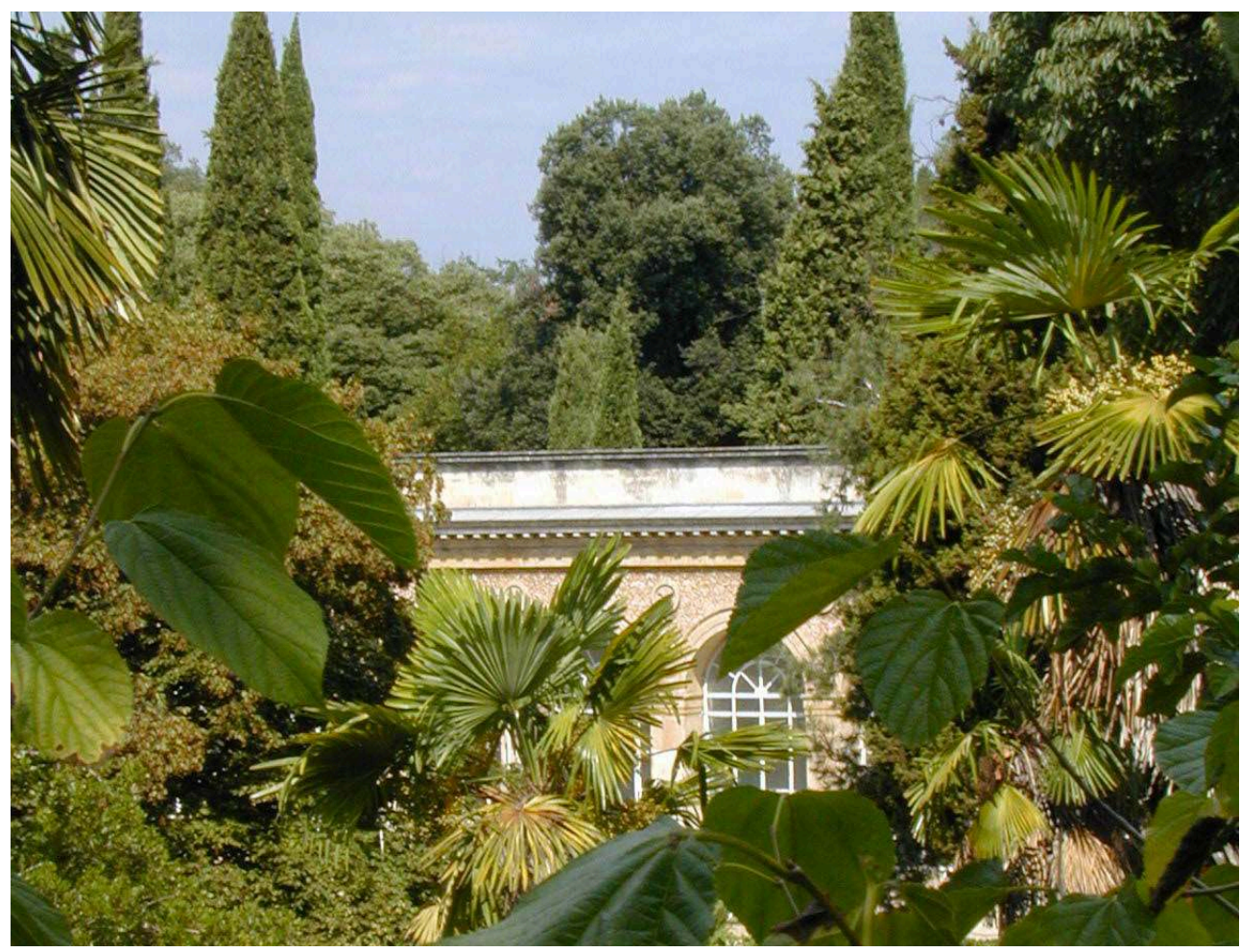

Doc. É. Manouvrier (c) 
« La botanique est à son aurore en France, Richer de Belleval en accélère la lumière.

${ }^{6} »$ grecque ou latine, étymologie, description de la forme, temps de floraison, lieu d'habitation, propriétés, représentation. La collection est nécessaire au botaniste et un dépôt public fournit des échantillons des productions naturelles de la nation. Les témoignages montrent combien l'enseignement de Richer de Belleval est agréable et instructif. La botanique n'est pas une science sédentaire : on peut étudier les plantes dans les herbiers, les livres, mais l'étude en cabinet ne sert que de récapitulations et de confrontations avec les observations faites à la campagne. Les plus habiles botanistes sont ceux qui « courent » et le professeur doit connaitre la topographie des plantes de son pays. Au jardin, on range dans une grande allée dite «le médical» les plantes pour leur démonstration. Richer de Belleval est entouré d'étudiants en médecine, jeunesse bien née et ardente à s'instruire. Les jours d'herborisation sont attendus comme des jours de fête, on en revient plus instruit, avide d'acquérir de nouvelles connaissances dans les courses suivantes. On observe au cours des saisons, la manière d'être des plantes, leur port, leur naturel, leur habitat. On remarque néanmoins les difficultés de cet enseignement à l'air libre. Les assemblées sont plus tumultueuses et le jardin botanique ressemble aux écoles des philosophes grecs.

Il se construit un grand bâtiment destiné à l'analyse des plantes, aux expériences ou à la composition de remèdes; ce qui nécessite un professeur en titre et un démonstrateur royal. Les gradins du laboratoire sont souvent combles, bien que :

"Certains médecins peu éclairés ont l'injustice de regarder ces cours comme futiles tandis qu'ils connaissent l'obligation indispensable dans leur état de distinguer les simples les plus ordinaires que la pharmacie emploie. ${ }^{8}$ "

L'enseignement de la botanique devient plus que livresque, on valorise l'œil du savant, l'observation d'après nature. Le savoir des anciens est trop méditerranéen, on élargit la connaissance à de nouveaux végétaux et les simples deviennent un enjeu de santé publique. On collecte, on conserve, on classe, on crée des herbiers et on édite des livres qui favoriseront largement la diffusion de la botanique, fondée sur l'image plus apte à transmettre les connaissances descriptives. Il existe une grande qualité des gravures. On exige que les imprimeurs aient vu la plante avant de la reproduire. Malheureusement de nos jours une partie de ce travail de reproduction a disparu. Des plaques de Richer de Belleval ont été retrouvées à Grenoble et en Pologne.

21 Pour l'instant on s'en tient à une classification alphabétique, on ébauche des rapprochements par ressemblance mais les critères manquent de précision. Bénéficiant de ces pratiques nouvelles, de nombreux étudiants carabins ont suivi assidûment les 
cours de botanique. Les témoignages sur la présence des aspirants pharmaciens sont plus discrets: Richer de Belleval connaît Bernardin II Duranc à qui il fournit des échantillons. Donc il apparaît des échanges entre les deux communautés et les futurs pharmaciens ont certainement fréquenté les cours de botanique pour compléter les connaissances déjà acquises à la boutique par le biais de l'usage des drogues. Lors de troubles, le jardin botanique est dévasté en 1622. Consciente de son importance pédagogique la communauté médicale participe à sa restitution, le nombre de spécimens s'accroît, une serre est créée.

\section{Le droguier}

22 En 1588, un apothicaire renommé de Montpellier, Bernardin II Duranc, né à Aubenas, a l'idée de créer un droguier au sein de sa boutique, rue de l'Aiguillerie. Celui-ci sera à l'origine du droguier de l'école de médecine.

23 L'originalité de l'initiative est de présenter les produits en état de délivrance à l'officine. Les trois règnes y sont représentés : végétal, animal, minéral. Les plantes qui constituent 80 à $90 \%$ des stocks de l'officine de l'époque ne sont plus simplement présentées aux aspirants pharmaciens sous forme exclusive d'herbiers mais en état d'usage thérapeutique. Par cette action Bernardin II Duranc entend mieux former ses étudiants, apporter une classification plus rigoureuse des produits utilisés, améliorer leur qualité, contrôler leur origine et bien mentionner leurs propriétés pharmacologiques.

Un témoignage du $\mathrm{XVI}^{\mathrm{e}}$ siècle laisse à penser qu'une effervescence de la connaissance de la science règne dans les boutiques des apothicaires de l'époque :

« Rue de l'Aiguillerie j'ai remarqué une belle boutique couronnée d'une enseigne. Le pharmacien est assis au milieu de ses jolis pots émaillés, de ses jolis coffrets peints et dorés; il se donnait une attitude un peu ridicule de docteur régent. Il est en entretien avec un questionneur... Que de science, que de science! Que le livre de la pharmacie est épais, nos boutiques, nos arrières boutiques sont en proportion avec les progrès de l'art... Nous voulons aujourd'hui de bonnes classifications, de bons systèmes ou les diverses parties aient entre elles un agencement nécessaire; publication des logiques, des philosophies médicales rationnelles... tel métal, telle herbe, tel animal pour telle maladie, nomenclature des instruments, indication du temps propice pour donner les médicaments... L'apothicaire ne doit pas être un homme commun, bon anatomiste, bon chimiste, bon botaniste. Toutes les langues lui sont nécessaires, pas uniquement le latin. On peut être apothicaire distillateur dans la journée et alchimiste le soir... ${ }^{9}$ »

Ces propos permettent d'imaginer ce qui se déroule dans l'officine de Bernardin II Duranc. Les aspirants apothicaires étudient les échantillons classés et rangés dans des bocaux de verre ou dans des boîtes assurant la conservation de leurs qualités organoleptiques (fig. 5). Chaque échantillon comporte ses caractéristiques sur une fiche manuscrite mentionnant le nom français, le nom scientifique latin, l'origine, le nom du donateur, la date d'entrée, la partie utilisée, les propriétés pharmacologiques et parfois quelques renseignements scientifiques (fig. 6,7). Cette instruction est complétée par des lectures publiques effectuées par des médecins que les futurs apothicaires ont l'obligation de suivre. Démonstrations et anatomie sont au programme, les séances étant fixées par l'université. Observation et expérimentation sont les mots clefs de l'époque et l'on peut supposer que les arrières boutiques servent de laboratoires à la chimie naissante. 
Fig. 5. - Droguier Montpellier, armoire de présentation et de classement.

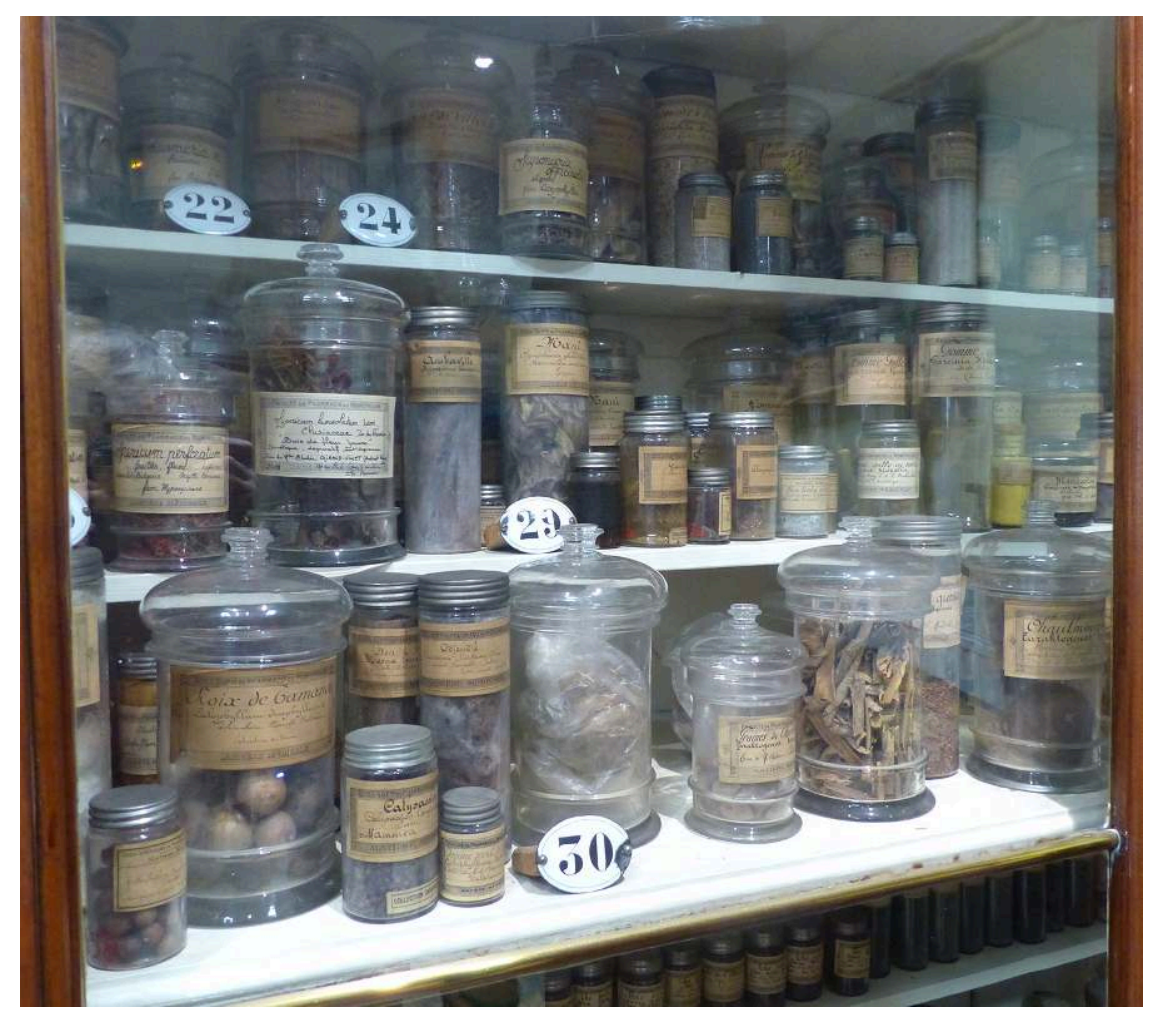

Doc. É. Manouvrier (C).

Fig. 6. - Droguier Montpellier, détail étiquette bocal.

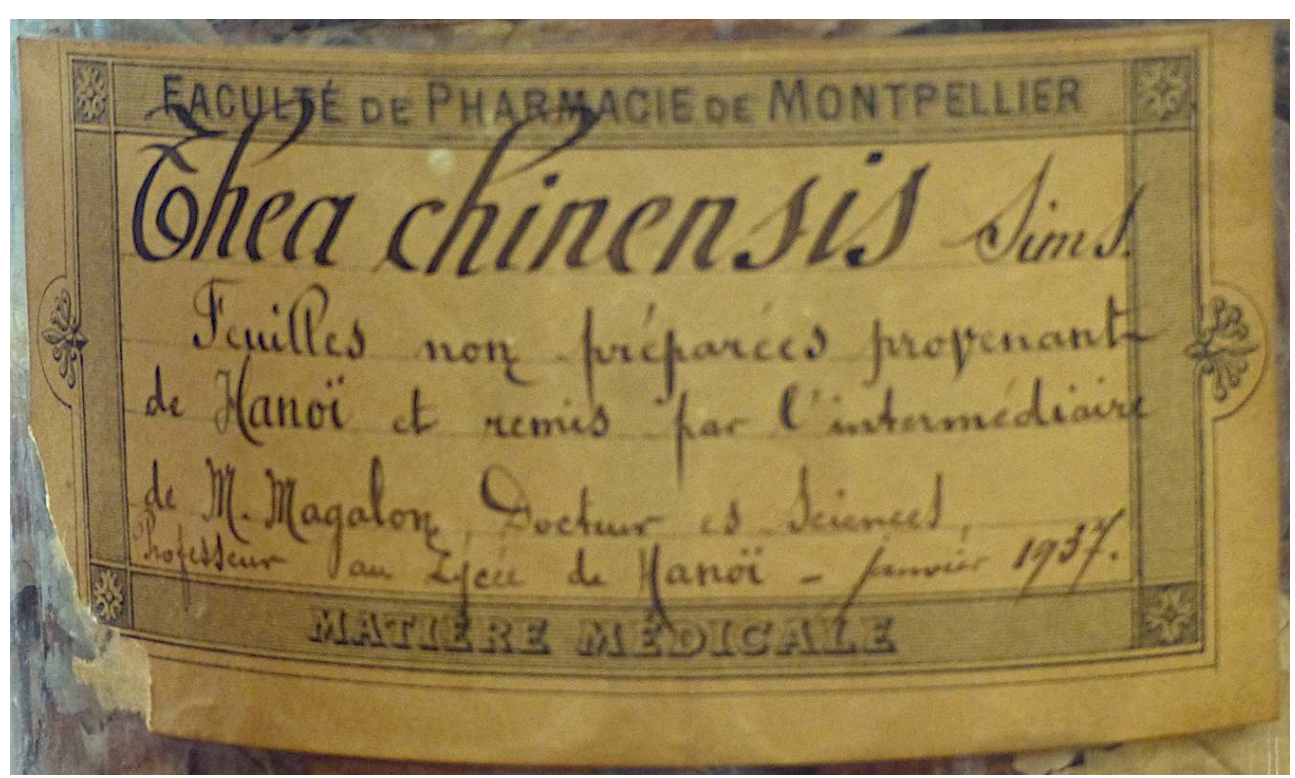

Doc. É. Manouvrier (․ 
Fig. 7. - Droguier Montpellier, gravure.

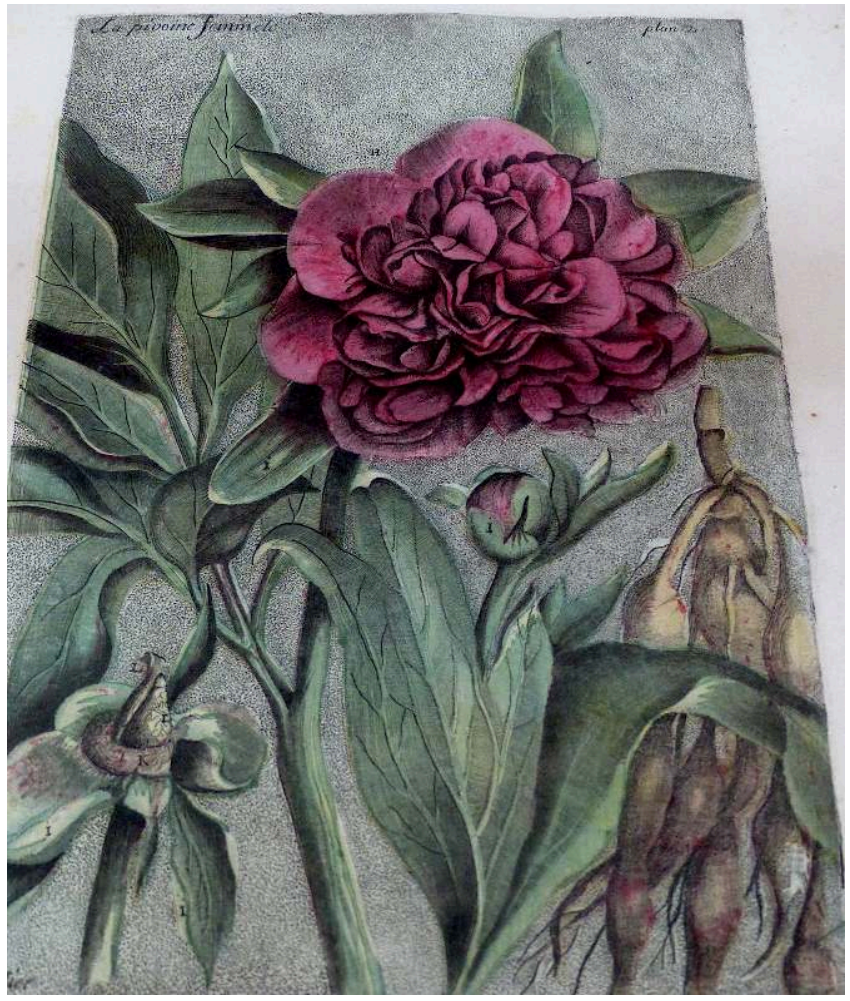

Doc. É. Manouvrier (C).

Toutes ces initiatives suscitent l'attention de l'université; Bernardin II Duranc est invité à présenter ses échantillons au collège de médecine. Bien que reconnue depuis Louis XII comme profession indépendante, les apothicaires ont beaucoup de mal à trouver leur place dans la communauté médicale et les conflits avec les médecins sont fréquents. Cette intervention destinée aux escholiers carabins se révèle primordiale. Bernardin II Duranc est l'un des premiers apothicaires à remplir une charge universitaire, ce qui marque un tournant dans l'histoire de la profession. L'apothicaire reconnu jusque-là comme simple boutiquier, ne connaissant que la pratique, voit s'ouvrir les portes de l'Université et une occasion encore timide de transmettre ses connaissances en botanique et pharmacologie. Il est invité trois fois par an (à la Saint Michel, à Noël, et durant la Semaine sainte) pour présenter sa collection aux futurs médecins et apothicaires, dans l'amphithéâtre d'anatomie. Il s'y rend en robe noire et fait des démonstrations de drogues.

Cette démarche marquant les prémices d'une collaboration entre apothicaires et médecins cesse en 1600, suite à des problèmes relationnels entre Bernardin II Duranc et le collège de médecine. Les démonstrations sont alors confiées à un autre apothicaire Laurens III Catelan, qui constitue un nouveau droguier avec la participation d'annuités versées par le corps des apothicaires montpelliérains.

De nos jours, le droguier, classé monument historique, riche de ses 9500 échantillons et depuis 2002 grâce à la mobilisation des étudiants, voit son rôle éducatif et culturel renforcé: on y enseigne la phytothérapie, l'aromathérapie ainsi que la pharmacognosie. Les échanges avec des étudiants étrangers (chinois, indiens) permettent de confronter les connaissances et d'enrichir les collections. Le jardin des plantes subit une restauration depuis 2012. Classé lui aussi monument historique, on 
peut y consulter 2200 espèces et 1000 en serre: organismes de recherche, d'enseignement supérieur, savants, médecins, botanistes, pharmaciens le fréquentent. La nouvelle transmission du savoir, par la numérisation prévue de l'ensemble des collections aussi bien au droguier qu'au jardin botanique, va permettre une diffusion plus large de ce patrimoine. Ces deux outils pédagogiques n'ont pas encore révélé tous leurs secrets.

Au travers de ces deux initiatives éducatives, on constate que la communauté médicale adhère au renouvellement des savoirs scientifiques et par la manière de les transmettre. Les savoirs des Anciens sans être totalement abandonnés, sont remis en cause : les pratiques sont nouvelles. On se base sur l'observation, l'expérimentation et les phénomènes provoqués afin d'infirmer ou de confirmer l'hypothèse de départ. Des méthodes critiques rigoureuses sont mises au point. Le livre devient de plus en plus pratique, les textes en français sont plus nombreux, des lieux du savoir commencent à se créer (laboratoires, jardins, cabinets de curiosité) favorisant la vulgarisation des dernières recherches, et, malgré la résistance de l'Église, la modernisation de la pensée scientifique s'impose.

L'apothicaire, loin de la caricature qu'en donnera Molière, participe à cette évolution. $\mathrm{Au}$ sein de l'officine, on constate que la tradition est encore pesante, la connaissance du corps humain est complexe, les nouvelles découvertes en botanique ouvrent un champ thérapeutique inexploré, mais les hésitations sont discernables et l'on est confronté à une remise en cause totale. Les initiatives de Pierre de Richer et de Bernardin II Duranc, la constitution de collections qui ouvrent l'horizon sur des matières comme la pharmacologie, la matière médicale et bien entendu la botanique, permettent d'envisager une formation différente pour les aspirants apothicaires; ils pratiquent encore beaucoup l'apprentissage mais la durée de celui-ci sera réduite au bénéfice des cours de botanique et de pharmacologie dispensés dans les écoles de médecine.

\section{BIBLIOGRAPHIE}

BOUMEDIENE Samir, La colonisation du savoir, une histoire des plantes médicinales du Nouveau Monde (1492-1750), Vaulx-en-Velin, Édition des mondes, 2016.

BRIOIST Pascal, « les savoirs scientifiques », Revue d'histoire moderne et contemporaine, $\mathrm{n}^{\circ} 49,2002$, p. 5.

CHARLOT Colette, « un compagnon apothicaire au XVI ${ }^{\mathrm{e}}$ siècle à Montpellier : Jean Magnol », Revue d'histoire de la pharmacie, $\mathrm{n}^{\circ}$ 351, 2006, p. 313-320.

Cirad, Dictionnaire des sciences animales, dico-sciences-animales.cirad.fr DEMOUY Isabelle, « Des droguiers d'hier aux médicaments d'aujourd'hui, le droguier Menier (XIX siècle) ", thèse doctorat pharmacie, Reims, 2010. 
DUPUY Edmond, Cours de pharmacie tome 1, Paris, Éd. Bataille, 1894.

GUITARD Humbert Eugène, « Histoire sommaire de la littérature pharmaceutique ; les traités de pharmacie privés au XVI ${ }^{\mathrm{e}}$ et XVII ${ }^{\mathrm{e}}$ siècles ", Revue d'histoire de la pharmacie, ${ }^{\circ}$ 94, 1936, p. 297-313. IRISSOU Louis, « Deux contrats de louage de services concernant des compagnons apothicaires (Montpellier 1342 et 1343) », Revue d'histoire de la pharmacie, $\mathrm{n}^{\circ}$ 132, 1952, p. 299-301.

IRISSOU Louis, «La pharmacie à Montpellier avant les statuts de 1572 », Revue d'histoire de la pharmacie, $\mathrm{n}^{\circ} 85,1947$, p. 217, 265,321.

IRISSOU Louis, "Contrat d'apprentissage au XIII ${ }^{\mathrm{e}}$ siècle ", Revue d'histoire de la pharmacie, $\mathrm{n}^{\circ} 118$, 1947, p. 218-220.

Kartable, Un nouvel esprit scientifique (XVI ${ }^{e}$-XVIII ${ }^{e}$ siècles), rubrique diffusion des sciences, www.kartable.fr

LAFONT Olivier, VAN ROBAYS Catherine, «La pratique de l'art de l'apothicaire au XVII ${ }^{\mathrm{e}}$ siècle :

information fournie par deux inventaires après décès ", Revue d'histoire de la pharmacie, $\mathrm{n}^{\circ} 295$, 1992, p. 453-456.

LESPINASSE René, Les métiers et corporations de la ville de Paris, épiciers apothicaires, Paris, Imprimerie nationale, 1886, p. 447.

MANOUVRIER Élisabeth, «L'apothicaire des XVII ${ }^{\mathrm{e}}$ et XVIII ${ }^{\mathrm{e}}$ siècles : représentations graphiques et picturales ", thèse doctorat de pharmacie, Tours, 1983.

MONTEIL Amans Alexis, Histoire des Français des divers états au XVI siècle, Bruxelles, Éd. Wouters, Raspoet, 1843.

SARCoS O., « À propos d'un contrat d'apprentissage d'apothicaire en 1596 », Revue d'histoire de la pharmacie, $\mathrm{n}^{\circ} 5,1913$.

WAROLIN Christian, «La création de l'école de pharmacie de Paris 1803 », Revue d'histoire de la pharmacie, $\mathrm{n}^{\circ} 339,2003$, p. 453-474.

\section{NOTES}

1. O. Sarcos, « À propos d'un contrat d'apprentissage d'apothicaire en 1596 », p. 65.

2. Registre déposé chez Maitre Escarguel à Carcassonne.

3. C. Charlot, « un compagnon apothicaire au $\mathrm{XVI}^{\mathrm{e}}$ siècle à Montpellier, Jean Magnol », p. 315-317.

4. O. Sarcos « À propos d'un contrat d'apprentissage d'apothicaire en 1596 », p. 66.

5. P.-J. Amoreux, recherches sur la vie et les ouvrages de Pierre Richer de Belleval, Avignon, Jean Albert Joly, Imprimeur-Libraire, 1786. catalogue BnF 12148/cb. p. 5.

6. P.-J. Amoreux, ibidem, p. 8.

7. P. Morisot, les plantes dédiées à la ville de Montpellier, p. 1.

8. P.-J. Amoreux, Recherches sur la vie et les ouvrages de Pierre Richer de Belleval, p. 29.

9. A. A Monteil, Histoire des français des divers états au XVI ${ }^{\mathrm{e}}$ siècle, p. 93-95. 


\section{RÉSUMÉS}

$\mathrm{Au} \mathrm{XVI}{ }^{\mathrm{e}}$ siècle, les maîtres apothicaires transmettent leur savoir à leurs apprentis au sein même «de la boutique "; transmission empirique et traditionnelle. En 1593, à la demande du roi Henri IV, Pierre Richer de Belleval crée le premier jardin botanique au monde à Montpellier. . En 1588, Bernardin II Duranc, apothicaire à Montpellier, regroupe les principales plantes préconisées à des fins thérapeutiques: c'est l'origine du droguier. Se développe alors une nouvelle dynamique de la transmission des savoirs qui devient plus collective et ouvre pour la première fois les portes de l'Université à un maître apothicaire. Jardin botanique et droguier développent de nouveaux moyens pédagogiques.

\section{AUTEUR}

\section{ÉLISABETH MANOUVRIER}

Docteur en pharmacie 


\title{
Les médecins parisiens et la diffusion du savoir médical au XVIII ${ }^{\mathrm{e}}$ siècle : des savants pédagogues
}

\author{
Isabelle Coquillard
}

1 Les docteurs régents ont suivi un cursus complet à la faculté de médecine de Paris, le titre de régent leur donnant le privilège d'enseigner l'Art de guérir et de pratiquer la médecine où bon leur semble (selon l'édit de Marly de 1707). Au cours de leur carrière professionnelle, ils assurent seuls leur formation continue afin de s'informer des innovations en matière de traitements (nouveaux composants, nouvelles méthodes de préparation des remèdes, modification des modes d'administration). Toutefois, la nécessité de s'astreindre à une veille scientifique entre en concurrence avec l'exercice libéral de la médecine, car elle exige du temps pour compulser les diverses parutions, sélectionner les données utiles, les répertorier pour en faire un usage ultérieur. La question de la diffusion de nouvelles connaissances implique de prêter attention à leurs contenus, à leurs formes mais aussi à leurs espaces de circulation et à la diversité des publics visés. Finalement, quels sont les enjeux de cette circulation des savoirs et informations médicales pour les docteurs régents? Comment tentent-ils de l'organiser et de la contrôler?

2 Dans un premier temps, nous nous intéresserons aux moyens mis en œuvre pour assurer la formation continue des médecins, puis nous verrons comment s'organise la circulation des connaissances dans le champ médical. Enfin, nous analyserons la manière dont les docteurs régents tentent de surveiller la diffusion des savoirs médicaux à l'échelle du royaume.

\section{Un savoir objet de la formation continue des docteurs régents}

3 La formation continue des docteurs s'opère notamment par le biais des traductions d'ouvrages scientifiques. 


\section{Les traductions : des véhicules d'un nouveau savoir}

Entre 1707 et 1789, les deux tiers des ouvrages traduits le sont de l'anglais au français, ce qui correspond au mouvement général d'échanges scientifiques entre la France et la Grande-Bretagne. En 1755, le docteur Ambroise Hosty gagne Londres pour observer, dans les hôpitaux et les maisons particulières, un total de 252 inoculations. Jacques Barbeu Dubourg entretient une correspondance avec Benjamin Franklin, sur ce même sujet $^{1}$. De nombreux docteurs régents sont membres de la Société Royale de Londres. Puis viennent les langues italienne et allemande. Une modeste partie des ouvrages est encore traduite du latin, qui reste la langue des échanges scientifiques, mais aussi celle de la terminologie en botanique et en médecine. Toutefois, leur présence disparaît à partir de 1780. La faible place de la langue allemande est à relativiser : les Allemands rédigent directement leur texte en latin.

Entre 1707 et 1789, trente-trois docteurs régents font des traductions (soit $7 \%$ du groupe sur l'ensemble de la période 1707-1789). Cette activité est réalisée par de jeunes docteurs ou par des docteurs ayant des activités professionnelles moins intenses. Elle entre dans une stratégie de publicité autour du nom du traducteur qu'elle permet d'associer à celui d'une autorité médicale. Les jeunes docteurs privilégient des sujets de médecine plus théoriques, car ils viennent de les étudier pour obtenir leurs grades. La traduction est aussi une source de revenus complémentaires dans des périodes où la carrière professionnelle peine à démarrer ou tend à stagner. Louis Anne Lavirotte entame cette activité dès qu'il est reçu régent en 1752. En 1753, il s'engage à traduire l'ouvrage de Needam, les Nouvelles observations microscopiques, moyennant 250 livres, somme conséquente équivalente à quatre-vingt-trois visites auprès d'un client aisé ${ }^{2}$. Pour Lavirotte, premier médecin de l'armée du Rhin, les traductions représentent une source de revenus complémentaires entre deux campagnes militaires.

6 Les traducteurs privilégient les auteurs contemporains. Les délais entre la publication originale et sa traduction sont, en moyenne, de quatre années et demie. Les docteurs sont attentifs aux ouvrages combinant la présentation du remède, la manière de conduire les cures thérapeutiques et la description des causes et du déroulement de la maladie. Lorsqu'ils traduisent un texte, les docteurs s'assurent de la possibilité de transférer le remède au contexte français (disponibilité des composants et conformité aux goûts de la clientèle). Ainsi, en 1770, Edme Bourru traduit de l'anglais l'Utilité des voyages sur mer, pour la cure de différentes maladies et notamment de la Consomption, une maladie pulmonaire chronique, aussi présente à Paris à cause des habitudes vestimentaires découvrant la tête et la poitrine.

7 La volonté de diffuser des nouveautés implique aussi une réflexion sur la forme donnée à la traduction. La boutique du libraire est le lieu privilégié où sélectionner le texte à traduire. Claude Fr. Duchanoy trouve chez son libraire Mécquignon l'aîné, rue des Cordeliers, vers 1784-1785, la traduction effectuée en 1780 par le docteur d'Édimbourg, A. B. Beerenbroek, d'un ouvrage de Percival Pott, les Remarques sur cette espèce de paralysie des extrémités inférieures que l'on trouve souvent accompagnée de la courbure de l'épine du dos. La boutique est un lieu de circulation des savoirs. Mécquignon l'aîné a publié, en 1780, l'Art d'imiter les eaux minérales de Duchanoy. Enthousiasmé par le texte de Pott, Duchanoy décide de faire traduire les Remarques sur la paralysie de Pott, mais ne joint pas les gravures de l'édition originale. En effet, la théorie de la courbure de 
l'échine a déjà été analysée par le docteur François Hunauld, dans sa thèse de 1742. Il ne faudrait pas risquer de placer le texte traduit en concurrence avec la production originale des docteurs régents. De plus, la gravure augmenterait le prix de l'ouvrage. La traduction induit également une réflexion sur le lexique scientifique et une appropriation du texte de départ menant parfois à sa personnalisation. Les docteurs régents ne manquent pas d'ajouter des préfaces, avertissements, notes, voire une partie entière aux ouvrages qu'ils traduisent.

8 En 1783, Duchanoy expose, dans le Journal de Paris, le succès de l'une de ses cures grâce au remède de Percival Pott, chirurgien de l'hôpital de Saint-Barthélemy, contre le mal vertébral, et souhaite en faire la publicité. Duchanoy place cette traduction au cœur d'un système d'échanges culturels. Le docteur permet la diffusion d'une connaissance vers l'ensemble des médecins dans une relation de type verticale. En contrepartie, il attend que tous les médecins susceptibles de faire des observations sur ces maux spécifiques l'en avertissent. Ici, la relation devient horizontale et annule la hiérarchie entre les docteurs régents et les autres médecins. Ils ne forment qu'un groupe professionnel dont chacun tente d'améliorer les pratiques. En proposant de diffuser les informations dans les journaux médicaux, Duchanoy souligne leur statut d'organe d'expression professionnelle. Si cette diffusion est d'abord destinée aux médecins, elle est susceptible de toucher la frange des autres professionnels de la santé.

Véhicule de nouvelles théories de médecine pratique, la traduction s'insère dans une stratégie éditoriale pour en renforcer la diffusion. Bertrand Dupuy est docteur en médecine de la faculté de Toulouse ${ }^{3}$. En 1761, il traduit les Nouvelles observations sur le pouls intermittent qui indique l'usage des purgatifs, et qui suivant Solano et Nihell, annonce une diarrhée critique, une dissertation mise au jour par Daniel Coxe, médecin londonien. Avec cette traduction, Dupuy prétend répondre à une lettre adressée par un médecin du comté de Foix, en mai 1760. Dupuy et son interlocuteur sont sensibles à la question du pouls, car ils pratiquent la médecine dans la région d'origine de Théophile de Bordeu, docteur en médecine de Montpellier et régent de Paris, auteur des Recherches sur le pouls par rapport aux crises en 1756. Cette publication fait écho au débat né en Angleterre autour des observations de James Nihell (lui-même s'inspirant en partie des observations du docteur Francisco Solano). Bien que traduit en français par le docteur Lavirotte, en 1748, l'ouvrage de James Nihell reste confidentiel. Selon Dupuy, moins de dix exemplaires auraient été écoulés. Il semble que les médecins français, avant 1750, ne soient pas intéressés par cette question d'où l'échec commercial de la traduction de Lavirotte ${ }^{4}$. Le thème n'est pas ancré dans l'esprit des médecins français.

10 Au contraire, l'ouvrage de Bordeu de 1756 est un succès contribuant à vulgariser les travaux de Nihell et à donner une nouvelle impulsion éditoriale. Un climat d'attente se crée autour de la thématique du pouls comme instrument diagnostic. La thèse de Bordeu est soutenue à l'échelle nationale par le texte de Michel, docteur en médecine de Montpellier, en 1757, formé à la méthode de Bordeu en quatre mois. La technique se veut non seulement efficace mais encore facile à apprendre. Convaincu par l'intérêt de la prise du pouls, Dupuy l'érige en «boussole principale ${ }^{5}$ » et abandonne les méthodes ordinaires. Il veut faire de la méthode de Bordeu une norme de la pratique médicale et cherche à la diffuser et à l'imposer. Il la considère comme une source de « changements dans la pratique et dans la théorie de l'art ${ }^{6} »$. Pour accroître son impact, Dupuy lui apporte son soutien en traduisant l'ouvrage de Daniel Coxe, moyen de souligner l'inventivité de Bordeu. Coxe, qui n'a pu consulter l'ouvrage du docteur régent en 
raison de la guerre, partage pourtant les mêmes idées. L'universalité de la méthode contribue à sa validité. Il faut souligner que Bordeu et Dupuy ont le même libraire, Vincent, installé rue Saint-Séverin, le lecteur pouvant y faire l'acquisition des deux livres simultanément. Vincent participe au processus de circulation de l'imprimé, de sa production et de sa distribution. L'unité de lieu contribue à l'association intellectuelle des deux ouvrages ${ }^{7}$. Somme toute, la diffusion de la théorie du pouls, impulsée par une traduction, se fait dans un temps long, treize années, avec une accélération à partir de publication des travaux de Bordeu, renforcée par une nouvelle traduction.

11 Outre les traductions, la presse médicale spécialisée contribue à la diffusion de recherches originales, de méthodes innovantes offrant au médecin la possibilité de ne pas se limiter à une pratique routinière de son art.

\section{La presse médicale spécialisée}

12 Le nombre de titres de presse médicale augmente après 1750, passant de un à six. Le Journal de médecine est alimenté par les articles envoyés par les médecins de l'ensemble du royaume, puis centralisés à Paris. Outre un large réseau de collaborateurs, le journal dispose d'un vaste espace de diffusion comprenant vingt villes dont les grandes cités universitaires, Versailles (afin d'atteindre le public des médecins en Cour) et une ville étrangère, La Haye. En s'adressant à un maximum de médecins, les docteurs régents peuvent diriger une partie de l'enseignement non officiel de la médecine dans l'ensemble du royaume et connaître les préoccupations des médecins provinciaux.

13 Si le Journal de médecine, chirurgie, pharmacie s'ouvre à tous les professionnels de la santé (y compris les sages-femmes), il s'adresse d'abord aux médecins, en accordant une large place aux observations médicales dûment sélectionnées et dont il entend ordonner la rédaction. Publier pour un groupe de professionnels suppose de s'astreindre à certaines règles. Celles-ci sont dictées par les docteurs régents, tirant leur légitimité de la valeur de leur grade et, probablement, de leur position prééminente au sein de la rédaction du journal. Ils postulent une spécificité du discours médical contribuant à renforcer leur position d'expert et agissant comme un moyen de distinction.

14 L'essor du journalisme médical conduit à s'interroger sur les stratégies de lecture et de travail mobilisées pour actualiser les connaissances. Les annonces et extraits de livres, vecteurs d'informations et de publicité de la production médicale, facilitent la sélection des ouvrages les plus utiles à consulter sans ignorer l'ensemble de la production médicale imprimée. Les notices constituent autant de fiches de lecture prêtes à l'emploi pour des docteurs en quête permanente de temps. Outils de perfectionnement et/ou d'accroissement des savoirs, elles permettent aussi d'avoir accès au livre quel que soit le lieu où l'on se trouve. De plus, à défaut de compulser les ouvrages, leurs annonces permettent de se constituer une bibliographie actualisée de tout ce qui concerne la santé. Les périodiques deviennent des instruments de travail avec des index, des tables, permettant une consultation analytique et des rapprochements entre les différents textes grâce à une organisation similaire à celle d'un dictionnaire ${ }^{8}$, source d'un gain de temps. Ces outils favorisent les transferts de connaissances, les journaux devenant des " agents de construction des savoirs ${ }^{9}$ ».

15 Si la lecture de la presse médicale n'est pas explicitement recommandée par la Faculté, elle ne semble pas s'y opposer. Dans le rapport du 20 janvier 1780 sur la thèse de pathologie d'Antoine Fourcroy traitant des abus et de l'usage que l'on peut faire de la 
chimie dans la guérison des maladies, les rapporteurs, Jean Descemet, Joseph Philip et Alexandre Bacher contestent le caractère pathologique du sujet. Ils remettent en cause la présentation de Fourcroy en insistant sur son impossible méconnaissance des « sages réflexions de Messieurs les auteurs du Journal de médecine ${ }^{10} »$. Une injonction implicite est faite aux docteurs régents et aux étudiants en médecine de lire la presse médicale spécialisée. La transmission des savoirs médicaux s'adresse d'abord aux différents acteurs du champ de la profession médicale.

\section{Orchestrer la circulation des savoirs médicaux dans l'espace du champ médical}

Pour réaliser leur projet de médicaliser l'ensemble du royaume, les docteurs régents ont besoin de connaître les préoccupations des médecins de province et les innovations pratiques qu'ils peuvent avoir mises en place.

\section{Au sein du champ médical : des échanges avec les médecins de province}

17 Cela suppose une circulation des savoirs entre la Province et Paris dont un des vecteurs est la Gazette de Santé. Fondée en 1773 par le docteur Joseph Jacques Gardanne, la Gazette de Santé est une feuille destinée aux médecins et aux sociétés savantes (y compris étrangères), reposant sur un système de correspondance ${ }^{11}$. L'économie interne de la Gazette de Santé est le reflet de cette organisation faisant de la feuille un espace de dialogue entre professionnels, indépendamment de leur lieu d'exercice et de leur grade. Le premier article donne toujours des nouvelles de villes étrangères; les deux suivants viennent de province; puis suit une observation ou une nouvelle de Paris. S'adressant à des médecins exerçant à la campagne, les articles concernent des difficultés locales.

\section{Atteindre les marges du champ médical : une mise sous tutelle des savoirs et pratiques des personnes charitables}

Les docteurs régents cherchent à faire du recours à un médecin gradué une pratique générale, concernant tous les groupes sociaux, aussi bien les gens des campagnes que les pauvres des villes. Rappelons que dans le cadre de la pratique libérale, chaque client potentiel compte. À la campagne, le manque de professionnels de santé et le coût élevé de leurs services contraignent les malades les moins fortunés à s'adresser aux personnes se consacrant à des actions charitables (curés, sœurs de Saint-Vincent-dePaul, seigneurs et notables locaux, dames de Charité...).

En dépit de l'absence de grade, celles-ci ne sont pas considérées comme des illégaux ${ }^{12}$. En effet, le cadre charitable interdit toute rétribution. Relais des médecins dans des espaces où la présence médicale est rare, elles participent au mouvement de médicalisation orchestré par les professionnels. Ceux-ci rédigent des ouvrages de médecine charitable ${ }^{13}$, destinés aux non professionnels de l'art, alors transformés en intermédiaires culturels. En contrôlant les savoirs des personnes charitables, les médecins cherchent à orienter leurs pratiques et discours auprès des plus faibles sur 
lesquels elles peuvent exercer une certaine influence et veulent combattre les erreurs populaires. L'entreprise pédagogique frôle parfois la propagande médicale, les personnes charitables contribuant à la diffusion de la médecine officielle.

Afin d'être accessible à tous, les manuels de médecine charitable sont rédigés en français, leurs prix modiques et leur format réduit. Ce sont de véritables succès d'éditions comme La Médecine, Chirurgie et Pharmacie des pauvres du docteur Philippe Hecquet (1740), qui connait une seconde édition dès 1742, puis une troisième en 1749. Hecquet y aborde tous les aspects de la médecine des pauvres ainsi que les maladies professionnelles des artisans. Ces ouvrages proposent deux types de médecine: une "médecine soignante", en détaillant des recettes de remèdes pour soulager, et une "médecine préventive », en diffusant des conseils sanitaires. Les manuels indiquent la maladie et les conditions dans lesquelles il faut les employer. Les remèdes indiqués répondent à une triple exigence : être peu coûteux, simples à préparer, et intégrer des drogues communes, faciles à se procurer. Il est nécessaire de proposer plusieurs médicaments pour une même maladie afin de satisfaire tous les utilisateurs quelles que soient leurs régions (prise en compte des ressources de la flore locale) et leurs capacités techniques. De plus, il faut qu'ils puissent convenir à tous les tempéraments des malades. Enfin, les remèdes doivent être faciles à prendre.

Bien que dans un premier temps, les manuels de médecine charitable soutiennent les efforts des docteurs régents dans la lutte contre ceux qu'ils considèrent comme des illégaux, ils sont, dans un second temps, critiqués, notamment par le docteur Antoine Laurent de Jussieu lors d'une séance publique de l'Académie royale des sciences de Paris, en 1788. Ce revirement est dû à leur large diffusion, résultat d'une modification du marché du livre et à un engouement pour les sciences. Facilement accessibles aux bourgeois, les manuels de médecine charitable sont détournés de leur fonction initiale pour devenir des guides d'automédication. Il semble que les particuliers leur empruntent des recettes pour leur propre usage, attirés par leur simplicité et/ou leur faible coût. Par exemple, un juge de Saint-Pastour en Agenais les utilise bien qu'il soit l'un des plus riches propriétaires de sa province ${ }^{14}$. De sorte que l'usage que le lecteur fait du livre en transforme radicalement la destination. L'Avis au peuple sur sa santé de Samuel Augustin Tissot est détourné de son usage. Les maréchaux-ferrants et vétérinaires de Picardie emploient les remèdes nommés pour soigner les chevaux atteints par des rhumatismes ${ }^{15}$.

L'extension donnée aux espaces de réceptions des savoirs médicaux nécessite d'en contrôler la teneur pour des docteurs régents soucieux de préserver leur première place dans la hiérarchie médicale.

\section{Contrôler la diffusion du savoir à l'échelle du royaume : la censure royale des livres de médecine}

Depuis la promulgation du Code de la Librairie de 1723, pour publier, les libraires reçoivent du Bureau de la Librairie, un mandat de censure et portent leurs manuscrits au censeur désigné. Celui-ci paraphe toutes les pages et les envoie, avec son rapport d'approbation ou de désapprobation, au Directeur Général de la Librairie.

Le roi accorde le pouvoir de censure à des individus qu'il nomme et pensionne. Les censeurs doivent fournir une approbation écrite après l'examen d'un livre jugé 
conforme aux principes de la religion et de la morale. La médecine exige une surveillance accrue d'autant plus qu'elle concerne la survie des populations et les mœurs. Avec l'exercice de la censure se pose la question de la limite entre le subversif et le tolérable pour la faculté de médecine de Paris.

\section{Les trente-sept docteurs régents censeurs royaux pour la médecine}

Les listes des censeurs royaux sont publiées dans l'Almanach Royal à partir de 1742, répartis en fonction de la thématique à laquelle appartiennent les ouvrages contrôlés (théologie, jurisprudence, médecine et chimie, histoire naturelle, chirurgie et anatomie, mathématiques, belles-lettres, géographie, navigation, voyage, peinture, sculpture, gravure et architecture, cette dernière catégorie étant apparue en 1761) ${ }^{16}$. Les censeurs en médecine (dont la dénomination est figée en 1746 sous l'expression, " censeur pour l'histoire naturelle, médecine et chimie ») et chirurgie sont les plus nombreux et représentent $18 \%$ de l'ensemble des censeurs en $1763^{17}$. En devenant censeurs royaux, les docteurs régents sont reconnus comme des experts au service de l'État.

Les docteurs régents sont nommés censeurs environ onze années après leur réception à la régence, moment où chacun a pu faire ses preuves, achever son installation dans la carrière, être libéré des obligations particulières imposées par la Faculté et avoir affiché publiquement son domaine d'expertise scientifique. Ainsi, les nominations de Jean Guettard et de Jean Descemet sont dues à leur connaissance de la botanique, celle d'Henri Tessier et de Jean-Jacques Paulet à leur expertise en agronomie. Cette spécialisation accrue répond à la nécessité d'intégrer des hommes capables de suivre les évolutions de la publication d'ouvrages portant sur des domaines très pointus de la science médicale. Aussi, parmi les censeurs, se mêlent des docteurs régents âgés et plutôt conservateurs (Andry, Burette, Astruc) et des hommes plus ouverts aux nouveautés (Charles Vandermonde, Poissonnier, Colombier). Au-delà de quinze années de réception, il s'agit d'honorer une brillante carrière dans la pratique libérale (cas de Bouvard et de Vernage) ou dans le service de l'État (cas de Boyer luttant contre les épidémies ou de Baron, auteur de formulaires pharmaceutiques pour les armées).

Le choix des censeurs se porte de façon privilégiée sur des docteurs ayant déjà exercé des fonctions professorales (cas d'un peu plus d'un docteur censeur sur deux). Celles-ci laissent légitimement supposer une parfaite maîtrise des savoirs médicaux. Le recrutement des censeurs s'effectue dans tous les espaces d'enseignement afin de ne pas privilégier une institution par rapport à une autre et de garantir la représentativité de toutes les sensibilités médicales. Les censeurs doivent être en contact avec la sphère de la recherche, ce que permet la participation aux travaux des institutions et sociétés scientifiques. Si l'Académie royale des sciences et la Société Royale de Médecine dominent (avec douze censeurs chacune), les docteurs régents sont aussi présents dans les académies étrangères (pour au moins huit d'entre eux), comme la Société Royale de Londres (Malouin), l'Académie de Stockholm et Turin (Macquer). Les censeurs ayant eu un contact avec le monde de l'édition (Lavirotte, Vandermonde) sont recherchés, car ils ont eux-mêmes éprouvé la censure. Les docteurs régents peuvent être nommés censeurs ponctuellement comme Bernard de Jussieu en 1752 et 1759, pour juger du contenu des Transactions philosophiques de la Société Royale de Londres, et de l'Essai sur 
l'histoire économique des mers occidentales de France. Tous les censeurs ne sont pas inscrits sur la liste des censeurs royaux ${ }^{18}$.

Il nous semble qu'un jeu sur la complémentarité des spécialités médicales entre censeurs existe. Les médecins censeurs ont le droit d'examiner les livres relevant du domaine de la santé, donc ceux des chirurgiens et des apothicaires. De sorte que les médecins hospitaliers et des armées sont sensibilisés aux questions de chirurgie par leurs fonctions, les docteurs régents spécialistes de la chimie (Bucquet, Macquer, Fourcroy...), à la pharmacie. Cette spécialisation permet peut-être de compenser le faible nombre de censeurs en chirurgie (ils ne sont que trois en 1757). En outre, on peut l'interpréter comme une stratégie pour que les questions de santé restent sous le joug des docteurs régents, donc de la faculté de médecine de Paris.

Les censeurs royaux ${ }^{19}$ ont pour mission de garantir le respect de la doctrine de la Faculté, d'éviter la publication de toutes sortes d'opinions et préceptes sur l'art de guérir. Pour publier un livre de médecine, il est nécessaire d'obtenir l'approbation des censeurs royaux, dont les fonctions sont le maintien nécessaire "des dogmes, des mœurs et des droits du prince ${ }^{20} »$. Toutefois, la Faculté conserve son droit de regard en tant que "Juge né de tout ce qui concerne l'Art de guérir", car les ouvrages qu'elle censure ont une utilité dans l'enseignement. Les censeurs royaux autorisent la publication des auteurs particuliers dont la production n'est point contraire à ce qui est établi. Au contraire, la Faculté émet un jugement qui doit faire loi pour l'exercice de la médecine, c'est-à-dire qui détermine la doctrine sur la nature et le traitement des maladies, sur la préparation et composition des remèdes. De manière générale, les livres sont répréhensibles lorsqu'ils comportent des attaques contre des particuliers ou l'autorité souveraine, contre les mœurs et la religion.

L'intensité de l'activité de censeur varie en fonction des individus, certains semblant en faire une de leurs activités principales. Tels sont les cas des docteurs Jean Guettard et Achille Le Bègue de Presle (avec presque 200 ouvrages censurés par chacun). La majorité des docteurs régents censeurs (quinze), se contente de juger moins de dix livres durant la totalité de leur temps d'activité, sachant que huit d'entre eux se satisfont de deux à trois livres. Ici, l'objectif est clairement d'associer son nom à une sommité scientifique et de s'afficher comme partageant ses idées, l'approbation du censeur figurant toujours à la fin de l'ouvrage.

31 À la recherche constante d'une conformité des écrits avec la doctrine de la faculté de médecine, la censure dessine les contours de la culture médicale à travers les éléments qu'elle rejette. En étant censeur, le docteur régent joue le rôle de filtre entre la production médicale imprimée et le public, et fait la promotion d'un discours allant dans l'intérêt du groupe. L'impératif du respect de la doctrine médicale de la Faculté conduit au rejet de certains manuscrits allant à son encontre. En juillet 1763, Antoine Casamajor refuse la réédition d'un ouvrage de Pierre Abraham Pajon des Moncets ${ }^{21}$, pourtant aussi docteur régent, dont la thèse est opposée à l'inoculation ${ }^{22}$. Or, l'auteur estime que par ce jugement, Casamajor use de ses fonctions de censeur pour défendre les positions de la Faculté décidée à approuver publiquement l'inoculation médicale ${ }^{23}$. En 1757, la Faculté étant contre ce procédé, Casamajor avait approuvé la publication du Tableau de la petite vérole d'André Cantwell, un de ses fervents opposants ${ }^{24}$. Le verdict du censeur l'emporte puisque l'ouvrage de Pajon des Moncets n'est pas réimprimé. L'esprit de corps de la Faculté prime sur le service au roi. Henri Michel Missa approuve l'impression du Médecin des Dames ou l'art de se conserver en santé, en 1771, car l'auteur 
prend garde de recommander le recours, dans les cas graves, aux « conseils éclairés et salutaires d'un médecin expérimenté25 $»$.

C'est bien le groupe des médecins qui décide du contenu et de la forme de diffusion du message dans le cadre institutionnel offert par la Librairie. Le groupe professionnel impose sa propre norme de communication.

\section{Usages et enjeux de la censure des livres médicaux}

Les censeurs doivent pouvoir justifier leurs décisions. Ils apprécient la clarté et la précision du propos, l'utilité de l'ouvrage. De sorte qu'en 1785, Paulet juge la traduction des Institutions de médecine pratique de Cullen par Pinel, "utile surtout aux jeunes médecins ${ }^{26} »$. Ce dernier point est cher aux docteurs régents, car le même argument est utilisé par Descemet en 1786 pour approuver le Traité de dysenterie de Zimmerman traduit de l'allemand par Lefebvre de Villebrune ${ }^{27}$. En 1768, Missa souligne l'intérêt de la nouvelle méthode pour nourrir les enfants trouvés et ceux des pauvres des provinces, présentée par Raulin dans le tome second de De la conservation des enfants ${ }^{28}$. Les censeurs doivent parfois déjouer les ruses des auteurs. Pour contourner la censure de Missa, Lefebvre de Saint-Ildephont distribue une feuille contenant deux annonces : l'une sur le chocolat antivénérien approuvé par Missa comme étant la même chose que le traitement de Van Swieten, l'autre sur un remède à base d'arsenic pour guérir le cancer, n'ayant jamais été lue par le docteur. En les plaçant sur la même feuille, Lefebvre de Saint-Ildephont espère créer une confusion et faire croire à l'autorisation des deux textes ${ }^{29}$.

Si les docteurs régents censeurs tentent de faire respecter la doctrine médicale de la Faculté, d'autres n'hésitent pas à la remettre en cause, de même que ses membres. Garant de l'unité du corps des docteurs régents, chargé d'assurer sa défense, mais aussi celle de sa conception de la médecine, le doyen de la Faculté n'hésite pas à s'engager personnellement lorsqu'un des docteurs régents est l'objet d'attaques dans des publications. Dans une lettre au directeur de la Librairie, d'août 1780, le doyen Jean Baptiste Boyer exprime son mécontentement quant aux propos que le censeur du journal l'Avant-Coureur a autorisé à faire imprimer. Un des articles critique le cours du docteur régent Louis Claude Bourdelin, «placé comme un automate qui ne dit depuis vingt-cinq ans que la même chose ${ }^{30} »$. Boyer s'insurge aussi contre la critique du Codex de la Faculté servant pourtant de référence en matière de confection de remèdes à tous les apothicaires de Paris. Boyer reproche au journal de ne pas avoir consulté la Faculté en ce qui concerne les articles traitant de médecine, contrairement à ce que prend soin de faire le rédacteur des Annonces, affiches et avis divers. Boyer entend effectuer une censure préalable en tant qu'expert en la matière. Par cette action, il souligne une des difficultés de la censure. Les ouvrages étant répartis en fonction des titres, certains, à l'intitulé peu explicite, peuvent échapper à la censure des médecins. La réponse de Malesherbes n'a pu être retrouvée mais le rédacteur nie les attaques envers Bourdelin et précise avoir relevé que quelques doses du Codex sont mal indiquées dans les cours de chimie du Jardin des apothicaires ${ }^{31}$.

Finalement, les traductions d'ouvrages médicaux autorisent un transfert de connaissances venues de l'étranger. Cependant, elles ne traitent que d'un unique sujet et leur forme livresque impose une lecture cursive afin d'en saisir réellement le fond. 
De sorte que les docteurs régents se tournent vers un moyen de diffusion plus souple : l'organe de presse médicale professionnelle.

Les origines diverses des nouveaux savoirs médicaux (la Province mais aussi l'étranger) et le réseau d'échange construit autour d'eux contribuent à la formation du groupe professionnel des médecins incluant les docteurs régents. Ceux-ci tentent de contrôler les nouveaux flux d'informations afin de conserver leur première place au sein de l'ensemble des professionnels de la médecine et de ne pas laisser les personnes charitables aux mains de ceux qu'ils jugent être des praticiens illégaux. Bien qu'ils n'aient pas de vocation scientifique, les manuels de médecine charitable sont les vecteurs de la promotion d'une médecine utile.

En tant que censeurs royaux pour la médecine, les docteurs régents ont l'opportunité de vérifier l'adéquation des connaissances potentiellement diffusables avec la doctrine médicale de la Faculté dont ils sont les garants. De plus, ils s'assurent de la diffusion d'une image de la médecine conforme à celle qu'ils ont construite et à laquelle ils adhèrent. Si les docteurs régents souhaitent assumer la formation médicale continue des médecins et l'éducation des personnes charitables aux premiers secours médicaux, ils veulent contrôler les contenus et leurs modes de diffusion. Les docteurs régents tentent de mener de front logique corporative et logique professionnelle.

Il serait intéressant de poursuivre la réflexion, ici amorcée, en s'intéressant au phénomène de transmission des savoirs médicaux auprès des néophytes et aux objectifs et usages de la vulgarisation médicale.

\section{BIBLIOGRAPHIE}

BLANGONNET Catherine, « Recherche sur les censeurs royaux et leur place dans la société au temps de Malesherbes (1750-1763)», Paris, thèse de l'École des Chartes, 1974-1975.

CANTWELl André, Tableau de la petite vérole, Paris, Hérissant, 1758.

Commentaires de la Faculté de Médecine de Paris, 1777 à 1786, Paris, G. Stenheil éd., 1903.

COXE Daniel, Nouvelles observations sur le pouls intermittent qui indique l'usage des purgatifs, et qui suivant Solano et Nihell annonce une diarrhée critique, Paris, Vincent, 1761.

DESESSARTZ Jean Charles, Très respectueuses représentations de la Faculté de Médecine en l'Université de Paris au roi contre la Société Royale de Médecine, Paris, s. d.

HANLEY William, « Une réflexion de l'époque sur le nombre de censeurs royaux en place au XVIII ${ }^{\mathrm{e}}$ siècle », Revue d'Histoire littéraire de la France, vol. 105, 2005/1, p. 207-214.

HERMANN-MACARD Nicole, La censure des livres à Paris à la fin de l'Ancien Régime (1750-1780), Paris, Presses Universitaires de France, 1968.

Journal des Savants, avril 1772.

LAFONT Olivier, Des médicaments pour les pauvres. Ouvrages charitables et santé publique aux XVII et XVIII ${ }^{e}$ siècles, Paris, Pharmathèmes, 2010. 
LAGET Mireille, « Les livrets de santé pour les pauvres aux $\mathrm{XVII}^{\mathrm{e}}$ et XVIII ${ }^{\mathrm{e}}$ siècles », Histoire, Économie et Société, $\mathrm{n}^{\circ}$ 4, 1984, p. 567-582.

NEGRONI Barbara de, Lectures interdites. Le travail des censeurs au XVIII siècle : 1723-1774, Paris, A. Michel, 2014.

NICOLI Miriam, Les savants et le livre. Autour d'Albrecht von Haller et Samuel Auguste Tissot, Genève, Stlatkine, 2013.

PAUTHIER Céline, L'exercice illégal de la médecine (1673-1793). Entre défaut de droit et manière de soigner, Paris, Glyphe et Biotem, (Histoire médecine et société), 2003.

PAJON des MONCETS Pierre Abraham, Dissertation sur la petite vérole dans laquelle on prouve, que cette maladie n'est pas dangereuse et dans laquelle, on donne en même temps les moyens de prévenir les dommages qu'elle fait à la beauté, Paris, A. Boudet, 1758.

PEIFFER Jeanne, VITTU Jean-Pierre, « Les journaux savants, formes de la communication des agents de la construction des savoirs (XVII ${ }^{\mathrm{e}}$-XVIII ${ }^{\mathrm{e}}$ siècles) », Dix-huitième siècle, $\mathrm{n}^{\circ}$ 40, 2008, p. 281-313.

Prospectus d'une Gazette de santé contenant les nouvelles découvertes sur les moyens de se bien guérir quand on est malade par un docteur régent de la Faculté de Médecine de Paris, Paris, V ve Ballard, 1773.

SETH Catriona, Les rois aussi en mouraient. Les Lumières en lutte contre la petite vérole, Paris, Desjonquères, (L'esprit des Lettres), 2008.

VIAUD Jean-François, « Recettes de remèdes recueillis par les particuliers aux XVII ${ }^{\mathrm{e}}$-XVIII ${ }^{\mathrm{e}}$ siècles. Origines et usages », dans Histoire, médecine et santé, Remèdes, nº 2, automne 2012, p. 61-73.

VITTU Jean-Pierre, « Du catalogue au dictionnaire, l'évolution des tables de périodiques littéraires à l'époque de l'Encyclopédie ", Dix-huitième siècle, n 25, 1993, p. 423-431.

\section{NOTES}

1. C. Seth, Les rois aussi en mouraient. Les Lumières en lutte contre la petite vérole, p. 51 et p. 130.

2. Archives nationales, MC/ET/VII/319, Inventaire après décès de Louis Anne Lavirotte, 21 avril 1759.

3. Archives nationales, MC/ET/XVII/1045, Inventaire après décès de Bertrand Dupuy, 17 septembre 1787.

4. Il est possible que le style de Lavirotte rebute à la lecture de sa traduction.

5. B. Dupuy, «Préface ", D.coxe, Nouvelles observations sur le pouls intermittent..., p. XXXVI.

6. Ibid.

7. L'hypothèse d'une stratégie commerciale du libraire semble envisageable.

8. J.-P. Vittu, « Du catalogue au dictionnaire, l'évolution des tables de périodiques littéraires à l'époque de l'Encyclopédie », p. 423-431.

9. J. Peiffer, J.-P. Vittu, « Les journaux savants, formes de la communication des agents de la construction des savoirs (XVII ${ }^{\mathrm{e}}$-XVIII ${ }^{\mathrm{e}}$ siècles) », p. 281-313.

10. Commentaires de la Faculté de médecine de Paris, 1777 à 1786, p. 459. 
11. Prospectus d'une Gazette de santé contenant les nouvelles découvertes sur les moyens de se bien guérir quand on est malade par un docteur régent de la Faculté de Médecine de Paris, p. 2.

12. C. Pauthier, L'exercice illégal de la médecine (1673-1793). Entre défaut de droit et manière de soigner, p. 383-384.

13. O. Lafont, Des médicaments pour les pauvres. Ouvrages charitables et santé publique aux $\mathrm{XVII}^{\mathrm{e}}$ et XVIII ${ }^{e}$ siècles. M. Laget, "Les livrets de santé pour les pauvres aux $\mathrm{XVII}^{\mathrm{e}}$ et $\mathrm{XVIII}{ }^{\mathrm{e}}$ siècles ", p. 567-582.

14. J.-Fr. Viaud, « Recettes de remèdes recueillis par les particuliers aux $\mathrm{XVII}^{\mathrm{e}}$-XVIII siècles. Origines et usages ", p. 70-71.

15. M. Nicoli, Les savants et les livres. Autour d'Albrecht von Haller et Samuel Auguste Tissot, p. 255.

16. W. Hanley, « Une réflexion de l'époque sur le nombre de censeurs royaux en place au XVIII ${ }^{e}$ siècle », p. 209.

17. C. Blangonnet, «Recherche sur les censeurs royaux et leur place dans la société au temps de Malesherbes (1750-1763) », p. 58-59.

18. N. Hermann-Macard, La censure des livres à Paris à la fin de l'Ancien Régime (1750-1780), p. 43.

19. B. de Negroni, Lectures interdites. Le travail des censeurs au XVIII siècle, 1723-1774.

20. J.-Ch. Desessartz, Très respectueuses représentations de la Faculté de Médecine en l'Université de Paris au roi contre la Société Royale de Médecine, s.d., p. 13.

21. P. A. Pajon des Moncets avait obtenu le droit à une première impression en décembre 1757, mais nous n'avons pas retrouvé le nom de son censeur. Il est absent du registre des privilèges et permissions simples de la Librairie et de la fin du livre qui mentionne uniquement l'existence de cette permission et sa date.

22. P. A. Pajon des Moncets, Dissertation sur la petite vérole dans laquelle on prouve, que cette maladie n'est pas dangereuse et dans laquelle, on donne en même temps les moyens de prévenir les dommages qu'elle fait à la beauté, 1758 .

23. Bibliothèque nationale de France, ms. naf. 3345, «Lettre de Pajon des Moncets à Malesherbes, 8 juillet $1763 »$, fol. $27 \mathrm{r}^{\circ}-29 \mathrm{v}^{\circ}$.

24. A. Cantwell, Tableau de la petite vérole, p. VI.

25. Journal des Savants, avril 1772, p. 463-464.

26. BnF, ms. fr. 22 016, Archives de la Chambre syndicale de la Librairie et Imprimerie de Paris, aux XVII et XVIII ${ }^{e}$ siècles. Rapports des censeurs sur les ouvrages soumis à leur examen pour l'obtention des privilèges ou permissions, 1769-1788, fol. 65.

27. BnF, ms. fr. 22 015, Archives de la Chambre syndicale de la librairie et Imprimerie de Paris, aux XVII et XVIII siècles. Rapport des censeurs sur les ouvrages soumis à leur examen pour l'obtention des privilèges ou permissions, 1769-1788, «Approbation du 20 octobre 1786 ", fol. $35 \mathrm{v}^{\circ}$.

28. Ibid., fol. 300-301.

29. Gazette de Santé, 7 décembre 1775, « Lettre de Missa du 4 décembre 1775 », p. 197.

30. BnF, coll. Anisson-Duperron, ms. fr. 22 135, LXXIII-LXXV Gazettes, petites affiches, almanachs, journaux, etc., sous M. de Malesherbes, 1744-1763. LXXV Années 1760-1763, « Lettre du doyen Boyer du 10 août 1760 à Malesherbes ", fol. 304. 
31. Ibid., « Lettre de Michel Lambert, imprimeur libraire à Paris, à Malesherbes, 15 août 1760, de Paris ", fol. 295.

\section{RÉSUMÉS}

$\mathrm{Au} \mathrm{XVIII}^{\mathrm{e}}$ siècle, les docteurs régents forment le Corps de la faculté de médecine de Paris et le groupe professionnel des médecins. Cette double appartenance les autorise à enseigner l'art de guérir et à l'exercer où bon leur semble mais les contraint également à l'auto-formation continue. Si les traductions d'ouvrages médicaux autorisent un transfert culturel, elles ne traitent que d'un sujet unique. Leur forme livresque impose une lecture cursive amputant le temps de pratique libérale. Aussi les docteurs régents se dotent d'organes de presse médicale professionnelle. Ils tentent de contrôler les flux d'informations afin de conserver leur première place au sein des professionnels de la santé et de ne pas abandonner les personnes charitables aux mains des «charlatans ». Censeurs royaux, les docteurs régents vérifient l'adéquation entre les nouvelles connaissances et la doctrine médicale de la Faculté dont ils sont garants, tentant de lier les logiques corporative et professionnelle.

\section{AUTEUR}

\section{ISABELLE COQUILLARD}

Doctorante en Histoire Moderne, Université Paris Nanterre 


\title{
Les voyages et lettres de
} correspondance de John Ray, ou l'émergence des sciences naturelles dans l'Europe du XVII siècle

\author{
Sabine Kraus
}

1 L'un des moyens essentiels de transmission des connaissances est la circulation des savoirs à travers les voyages et les correspondances des savants. Professeur de grec et de mathématiques à Cambridge, prêtre anglican, éminent naturaliste né à l'aube de la révolution scientifique, John Ray (1627-1705) a lié amitié et commerce épistolaire avec de nombreux savants de l'Europe occidentale. Considéré comme le fondateur de la botanique et de la zoologie modernes, il est le premier à proposer une définition précise de la notion d'espèce en la rattachant à une communauté d'origine. Ray apporte ainsi une méthode nouvelle de classification selon des critères purement biologiques. L'œuvre de John Ray, surnommé le " Pline anglais ", formera la base des travaux à venir de Jussieu (1699-1777), Linné (1707-1778), Buffon (1707-1788) et Cuvier (1769-1832).

La correspondance prolifique de John Ray nous fait entrer dans la dimension relationnelle de la science. Elle constitue ainsi un témoignage direct de la construction collective des sciences naturelles au $\mathrm{xvII}^{\mathrm{e}}$ siècle, à travers un réseau d'échanges collaboratifs et amicaux qui fait exister de manière concrète la «République des Lettres » de l'Europe moderne ${ }^{1}$. De son vivant, John Ray publie un nombre considérable d'ouvrages scientifiques en latin, lingua franca de la communauté scientifique de cette époque. Par contre, la plupart de ses lettres, comme ses carnets de voyage, sont rédigés en anglais. Une partie de sa correspondance sera publiée en 1718 par son disciple et ami médecin, le naturaliste et théologien William Derham (1657-1735), sous le titre, traduit en français : Recueil de Lettres Philosophiques, écrites par feu Mr. Ray, et d'autres Personnes, avec qui il entretenoit correspondance, tant en Angleterre que dans les Pais étrangers. On y a joint celles de Mr. François Willughby. Ce recueil contient plusieurs Découvertes curieuses de l'Histoire des Quadrupèdes, des Oiseaux, des Poissons, des Insectes, des Plantes, des Fossiles, des Fontaines, etc., ${ }^{2}$. De nombreux extraits sont aussitôt traduits en français et publiés 
l'année même dans la Bibliothèque angloise, ou Histoire littéraire de la Grande-Bretagne, périodique français imprimé à Amsterdam et fondé par deux huguenots exilés en Angleterre, Michael de La Roche, et Armand Boisbeleau de La Chapelle. La Bibliothèque anglaise participe à cette époque à une active diffusion de la culture anglaise sur le continent. Elle est largement ouverte aux sciences, et publie de nombreux articles des Transactions Philosophiques de la Société Royale de Londres, dont Ray est membre depuis $1667^{3}$. Ainsi, les sociétés et journaux de la République des Lettres jouent également un rôle important dans la diffusion des connaissances en histoire naturelle. C'est à travers ce réseau cosmopolite que se constitue la botanique, science naissante que l'œuvre de John Ray va influencer de manière décisive.

\section{Les années d'études et d'explorations en Grande- Bretagne: du savoir populaire traditionnel au savoir universitaire}

3 Contrairement à ses amis et collaborateurs, John Ray n'a pas étudié les plantes en devenant médecin. Les jardins botaniques, créés dans les universités où était enseignée la médecine, avaient pour objet l'étude des vertus médicinales des plantes. Ray a une tout autre approche, ce qui est nouveau à une époque où les plantes étaient simplement listées dans des catalogues et des herbiers. Il sera le premier naturaliste à proposer un système taxonomique cohérent, applicable aux plantes et aux animaux, en utilisant l'espèce comme unitét. Ce qui lui vaut d'être un pionnier de la science botanique, c'est le fait d'avoir acquis ses connaissances à travers un savoir populaire traditionnel transmis par ses parents.

\section{L'enfance et l'éducation de ses parents}

4 John Ray est né dans le petit village de Black Notley dans le comté de l'Essex. Son père Roger est le forgeron du village. Sa mère Élizabeth est une herboriste qui exerce également la médecine et la chirurgie. Femme d'une grande piété, elle est d'un secours précieux pour tout le voisinage, à qui elle prodigue ses soins ${ }^{5}$. Ce qui requiert de sa part une excellente connaissance pratique de la botanique. Du reste, les plantes utilisées par les apothicaires et les médecins sont collectées par des femmes à cette époque ${ }^{6}$. C'est en accompagnant sa mère pour la collecte des plantes médicinales dans la campagne que John montre dès son plus jeune âge un vif intérêt pour la nature et ses merveilles. Le jeune Ray passe également des heures à observer son père forgeron, et par conséquent habile artisan. C'est là qu'il apprend l'importance de la relation entre la structure et la fonction des pièces fabriquées par son père. Plus tard, John appliquera cette compréhension à son travail de botaniste, en identifiant les caractéristiques structurelles comme le trait déterminant dans la différenciation entre les espèces ${ }^{7}$.

\section{Les années à Cambridge et le début des explorations}

Durant ses années de collège à Braintree, John Ray est remarqué pour son intelligence, et se voit octroyer une bourse d'études à l'Université de Cambridge en 1643, à l'âge de 16 ans. Ray acquiert de grandes compétences en grec, en latin et en hébreu. Après l'obtention de son diplôme en 1648, il devient membre de Trinity College et chargé de 
cours l'année d'après. Par la suite, il est nommé professeur de grec en 1651, de mathématiques en 1653, puis lecteur en humanités en 1655. L'histoire naturelle n'est pas enseignée à Cambridge. L'étude des plantes, comme dans les autres universités à cette époque, est strictement liée aux études de médecine, et elles sont la source principale de ce que l'on nommait alors materia medica, c'est-à-dire la pharmacologie. Des catalogues de plantes sont tenus par des médecins professeurs de Trinity College, notamment par John Pratt, qui cultive également un jardin botanique dans lequel il convie ses étudiants ${ }^{8}$.

6 John Ray va reprendre les expéditions botaniques de son enfance lors d'une maladie qui l'atteint en 1650. Son premier intérêt pour la botanique devient alors une passion. Profitant de sa convalescence, il commence à faire de longues randonnées équestres dans la campagne autour de Cambridge, et collecte des plantes. Ray renoue ainsi avec sa curiosité pour la beauté variée des plantes, et l'artisanat habile de la nature. Une fois rétabli, Ray continue à parcourir les bois et les plaines marécageuses des environs de Cambridge, durant ses vacances d'été. Quatre ans plus tard, ses expéditions deviennent également familières aux élèves et amis qui l'accompagnent, et qui partagent avec lui une passion pour les choses de la nature. Parmi eux, Francis Willughby (1654-1672), un aristocrate fortuné arrivé à Cambridge en 1652, montre un enthousiasme pour l'étude de l'histoire naturelle qui sera très stimulant pour John Ray. C'est le début d'une collaboration zélée, ainsi que d'une profonde amitié, qui ne prendront fin qu'avec la mort prématurée de Willughby en 1672, à l'âge de 37 ans. Le premier fruit de leurs efforts communs est le Catalogue des plantes de Cambridge, publié en latin en 1660. Résultat de six années d'herborisation, ce catalogue est d'autant plus remarquable que, lorsque paraît deux siècles plus tard la nouvelle Flore de Cambridge, listant 950 espèces, 700 l'étaient déjà dans le catalogue réalisé par Ray9.

\section{De l'observation de terrain au savoir universitaire : le Catalogus plantarum circa Cantabrigiam nascentium}

7 Pour écrire un tel ouvrage, Ray doit d'abord se familiariser avec la littérature de ses prédécesseurs. Il constate qu'il n'y a aucun accord sur la terminologie, et qu'aucune histoire générale des plantes n'a été publiée depuis celle de Jean Bauhin (1541-1613), médecin, apothicaire et botaniste. Ce dernier est le fils d'un médecin réformé qui doit quitter la France et se retirer en Suisse. Né à Bâle, Jean Bauhin étudie la botanique sous Leonhart Fuchs (1501-1566) à Tübigen et Conrad Gessner (1516-1565) à Zurich. En 1561, il étudie la médecine à Montpellier et suit les enseignements de Rondelet (1507-1566), dont Fuchs et Gessner avaient également été les élèves. Et tout ce petit monde entretenait une correspondance soutenue. L'Histoire universelle des plantes de Jean Bauhin, publiée en 1650, va être la source fondamentale du travail de Ray. En effet, c'est une compilation de tout ce qui était connu de son temps. Vaste encyclopédie botanique, L'Historice décrit plus de 5000 plantes et comporte plus de 3500 illustrations, la plupart empruntées à Fuchs. Là où auparavant demeurait une grande confusion dans la nomenclature des plantes, Ray va entreprendre un travail de classification méthodique, afin de fournir une identification exacte de chaque plante, menant à la confirmation du nom de l'espèce et de sa description complète, fournissant des détails précis sur la morphologie, les périodes de floraison, la distribution géographique, les usages médicinaux ${ }^{10}$. Comme le présente P.H. Oswald and C. D. Preston dans leur édition 
anglaise du Catalogue de Cambridge de John Ray, publiée en 2011 par la Ray Society, la première flore d'une province britannique n'est pas seulement un catalogue botanique, mais elle inclut également, au bénéfice des débutants, un index des noms anglais et des lieux, ainsi que les significations des noms des plantes et des termes botaniques utilisés ${ }^{11}$. John Ray, bien qu'auteur de cet ouvrage, a bénéficié de l'assistance de ses amis, notamment Willughby. Après avoir fini la rédaction de l'ouvrage pour publication début 1659, Ray a l'idée d'étendre son étude à toute l'île britannique. Dans sa lettre à Willughby, en date du 25 février 1659, il lui fait part de son intention, et de sa méthode. Dans ce but, il commence à écrire à tous ses amis et connaissances ayant des compétences en herboristerie, pour leur demander d'explorer leur région durant l'été, et de lui envoyer un catalogue des plantes qu'ils ont trouvées dans leurs provinces, ainsi que l'endroit où elles poussent. Ce qui va donner lieu à une correspondance qui durera durant toute la vie de John Ray.

8 Alors qu'il enseigne à Cambridge, les premières expéditions de John Ray se déroulent durant les vacances d'été. Or, comme ce fut le cas pour Jean Bauhin, ce sont des événements politiques et religieux qui vont être pour lui l'occasion de se consacrer entièrement à l'étude de la nature. Au XVII ${ }^{\mathrm{e}}$ siècle, le monde académique et l'Église sont étroitement liés. En 1660, lorsque la République de Cromwell prend fin et que la monarchie est rétablie, l'Université exige que tous ses membres soient des ecclésiastiques. C'est à ce moment-là que Ray se fait ordonner prêtre. En 1662, Charles II (1630-1685) oblige tous les ministres du culte à signer l'acte d'uniformité restaurant les rites catholiques après la période calviniste sous Cromwell (1599-1658). Ray ne peut s'y résigner, comme environ 2000 autres ecclésiastiques ${ }^{12}$. Il est alors évincé de l'Université et se retrouve sans ressources. C'est grâce à la générosité de ses amis, notamment Francis Willughby, que John Ray va pouvoir s'adonner pleinement à l'œuvre qu'il est en train de construire. De plus, c'est sur l'incitation de celui qui est devenu son « patron » que Ray va étendre son travail de classification à tous les êtres vivants. Les deux amis s'entendent sur le fait que pour le naturaliste, l'étude de spécimens de musée et de la littérature doit être subordonnée à l'observation personnelle et à la connaissance de l'organisme dans son habitat naturel ${ }^{13}$. Par conséquent, dans le but de réaliser leur grand projet de "Système de la Nature ", Willughby et Ray embarquent pour un "Grand Tour " de l'Europe qui durera trois années. Deux élèves de Ray, Nathanael Bacon et Philip Skippon, sont également du voyage.

\section{Les voyages et correspondances, une géographie de I'histoire}

9 Le 18 avril 1663, les quatre naturalistes britanniques s'embarquent à Douvres pour le continent.

\section{Le " Grand Tour », de Douvres à Montpellier, capitale de la botanique}

10 Ils visitent les Pays-Bas espagnols, la Principauté de Liège, la Prusse et la Suisse en remontant le Rhin, puis l'Autriche jusqu'à Vienne. À la fin de l'année, ils arrivent en Vénétie et y passent l'hiver. En avril 1664, après avoir visité Naples et le Vésuve, leur route se sépare. Willughby, désirant aller explorer l'Espagne, remonte vers le nord en 
compagnie de Bacon. Ray et Skippon eux, continuent vers la Sicile et Malte. Après un hiver romain, ils se dirigent vers la Suisse pour arriver à Genève le 20 avril 1665. Ils sont en France fin juillet, et arrivent à Montpellier à l'automne, pour n'y rester que quelques mois d'hiver, malheureusement. En effet, en janvier 1666, la France déclare la guerre à l'Angleterre, et le $1^{\text {er }}$ février, Louis XIV promulgue un édit sommant tous les Britanniques à quitter le royaume dans les trois mois. En avril 1666, Ray et Skippon sont donc de retour en Angleterre, en compagnie de deux de leurs compatriotes rencontrés à Montpellier ${ }^{14}$.

$11 \mathrm{Au} \mathrm{XVII}{ }^{e}$ siècle, Montpellier est un grand centre de vie intellectuelle qui attire de nombreux étrangers. La ville est particulièrement appréciée par les naturalistes anglais, en raison de la renommée de son illustre faculté de médecine, et sa réputation pour la pratique de la botanique de terrain, inaugurée par Rondelet. Par ailleurs, Montpellier était un bastion du protestantisme, et la faculté est étroitement identifiée avec les Huguenots et Henri IV, qui, en 1593, avait doté l'école de médecine du premier jardin botanique de France, ainsi que d'une chaire d'anatomie et de botanique. Celle de chirurgie et de pharmacie vit le jour quatre ans plus tard ${ }^{15}$. Dans ses carnets de voyage publiés en 1673, John Ray notera d'ailleurs que :

« Le nombre d'apothicaires dans cette petite ville est pratiquement incroyable, il y a

130 boutiques, et pourtant tous trouvent quelque chose à faire. ${ }^{16}$ "

De fait, la cité languedocienne est au XVII ${ }^{e}$ siècle la capitale de la science botanique naissante. Durant leur séjour, Ray et Skippon vont rencontrent un certain nombre de leurs compatriotes, venus étudier à Montpellier. Parmi eux, c'est en particulier avec Martin Lister, inscrit à l'école de médecine depuis 1663, et Francis Jessop, naturaliste et mathématicien, que des liens solides et amicaux vont se lier.

Tous ces amoureux de la nature se forment auprès de Pierre Magnol (1638-1715), diplômé de l'école de médecine en 1659. L'enseignement officiel lui étant refusé, car il est protestant, Magnol n'en continue pas moins à faire des herborisations, entouré d'un nombre imposant d'élèves français autant qu'anglais, sur qui il fait forte impression. C'est d'ailleurs l'occasion d'échanges féconds entre Ray et Magnol au sujet de leurs nouveaux concepts de classification: l'espèce pour Ray, la notion de famille pour Magnol ${ }^{17}$. Quelque vingt ans plus tard, Pierre Magnol formera Joseph Pitton de Tournefort et Antoine de Jussieu, ainsi que Tankred Robinson et Hans Sloane, amis britanniques de John Ray qui viendront ensemble étudier la médecine à Montpellier en 1683. À leur retour en Angleterre, Ray, Skippon, Lister, Jessop, et Willughby vont devenir de grands amis et entretenir une correspondance régulière, à laquelle contribueront bien sûr Robinson et Sloane, tous portés par la volonté de travailler collectivement au progrès de ce qui s'appelait alors « la nouvelle science », c'est-à-dire l'histoire naturelle.

\section{Voyages de découvertes botaniques et linguistiques}

Partis avec l'idée d'établir une classification de toutes les choses vivantes, Ray et Willughby rapportent du continent une immense moisson qu'ils commencent alors à organiser. Partout où ils sont allés, ils ont collecté avec zèle des spécimens de plantes, de poissons, d'oiseaux, de pierres et autres curiosités ; ils ont également noté le détail d'antiquités, de coutumes et de dialectes locaux ${ }^{18}$. Les deux naturalistes, comme tous leurs amis, s'intéressent à toutes les choses de la nature. Cependant, chacun va 
s'attacher à produire une histoire naturelle spécifique: une histoire générale des espèces végétales pour Ray, un traité d'ornithologie et une histoire des poissons pour Willughby. Martin Lister, pour sa part, se penche sur l'étude des insectes, négligés jusqu'ici car jugés nuisibles et méprisables. Ils ont tous conscience que les disciplines qu'ils fondent ne peuvent être cultivées par un seul. Le progrès de la science demande un vaste travail collectif et une coopération indispensable entre les différentes branches du savoir. Or, dans le milieu intellectuel de cette époque, on ne conçoit pas de séparation entre les humanités et l'étude de la nature. Ray, comme le fit Gessner au siècle précédent, collectionne des spécimens aussi bien végétaux que linguistiques, et il y a une relation vitale entre ses manières de collecter les deux ${ }^{19}$. Dans son Catalogue des plantes de Cambridge publié en latin en 1660, Ray avait déjà inclus un index des noms anglais des plantes et des lieux, ainsi que la signification des termes botaniques utilisés.

Comme il nous le relate dans ses écrits, ses voyages en Angleterre lui font observer la différence de dialecte et la variété des mots locaux dans les diverses régions parcourues. Ces langues vernaculaires étant difficiles à comprendre pour un étranger, Ray collectionne donc expressions locales et noms vernaculaires pour lui-même, comme autant de plantes natives de la région. Il recueille également des proverbes entendus lors de ses voyages. Ayant observé que toutes ces expressions apparaissent dans les conversations courantes, il engage ses amis et connaissances des différentes parties de l'Angleterre à faire des observations et enquêtes similaires, ce qui lui offre de larges contributions. Observations de spécimens de plantes et d'animaux, de noms vernaculaires et de proverbes locaux se mêlent dans les correspondances. Ray, collectionneur minutieux, naturaliste ayant enseigné les humanités, applique la méthode de l'histoire naturelle pour ordonner le matériel recueilli avec la collaboration de ses amis : il collecte des spécimens linguistiques, et cherche une méthode pratique pour les classer de manière systématique, en se référant à ses observations propres, ainsi qu'aux auteurs. Dans la section «Proverbes et observations proverbiales relevant de la santé, de la diète et de la médecine ", Ray accompagne la plupart des expressions citées par des annotations qui donneront des bases rationnelles au vernaculaire ${ }^{20}$.

La correspondance de Ray fait apparaître l'enthousiasme manifesté par ses amis lors de la publication de sa Collection de Proverbes anglais ${ }^{21}$ en 1670. Cette dernière sera suivie, quatre ans plus tard, d'une publication de Mots anglais qui ne sont pas utilisés de manière générale $^{22}$, le premier dictionnaire anglais de dialectes régionaux des contrées du nord et du sud de l'Angleterre, puis l'année suivante, d'un Dictionnaire trilingue avec des mots communs en anglais, latin et grec à l'usage des étudiants. Les mots sont classés par catégories dont les intitulés reprennent les sections d'une histoire naturelle : du ciel, des éléments et météores, des pierres et métaux, des plantes, des herbes, des arbres et arbrisseaux, des parties des animaux ${ }^{23}$. De fait, les écrits du professeur de langues préoccupé de questions d'étymologie sont sous-tendus par la passion première du botaniste. Toutes ces publications, adressées à un large public, auront de nombreuses rééditions.

En effet, Ray est soucieux de l'utilité de tels écrits, publiés sous format de manuels à l'usage des étudiants et naturalistes en voyage à l'étranger, leur facilitant la conversation avec la population locale aussi bien qu'avec les savants avec lesquels ils sont en relation. Ses collections de proverbes et de mots locaux répondent en outre à la nécessité de comprendre les langues vernaculaires parlées par les habitants des régions explorées, afin de ne pas perdre de précieuses informations transmises par des gens 
ordinaires. Fils de forgeron autant que naturaliste érudit, Ray se souvient qu'il a acquis ses premières connaissances en botanique à travers un savoir populaire transmis en langue commune, ou vulgaire. Il a donc conscience de la richesse et de la valeur des dialectes ainsi que des connaissances qu'ils véhiculent. Aussi, Ray récoltera autant de proverbes qu'il le peut :

«Car les proverbes sont tirés de l'expérience et des observations de nombreux siècles; et ils sont l'ancienne philosophie naturelle du vulgaire qui nous a été transmise, préservée en vieil anglais dans de mauvaises rimes..$^{24}$ »

18 Par ailleurs, la nécessité de préserver et d'étudier les dialectes provinciaux est due au fait qu'ils commençaient à disparaître avec la généralisation de l'anglais standard qui est en train de se construire comme unité linguistique nationale. Diversité des langues vernaculaires et biodiversité des espèces natives vont donc de pair, et sont étudiées avec une ferveur égale par toute la communauté des « curieux de la nature ».

\section{Une communauté amicale et cosmopolite}

Cet effort commun se fait jour dans leur correspondance, qui nous fait entrer dans la fabrique des ouvrages. À travers ces lettres apparaît la vie et le contexte domestique de Ray et ses amis, une pratique sociale regroupant érudits autant qu'amateurs qui partagent tous le même enthousiasme et se sentent appartenir à une même communauté amicale aux liens étroits. Ils tissent ainsi un réseau de communication et de circulation, aussi bien des connaissances que des personnes. Livres, manuscrits, instruments, dessins et observations circulent de main en main. Tous se visitent mutuellement, espérant toujours y retrouver l'un de leurs amis également de passage. Dans ses lettres, Ray nous fait part de ses activités. Nous apprenons qu'il séjourne à Broomhall chez Francis Jessop pour des expéditions botaniques et des expérimentations auxquelles participe également Willughby. C'est dans son domaine de Middleton Hall que Ray met en ordre les notes recueillies lors de leur voyage.

Le décès prématuré de Willughby laisse ses écrits sur les oiseaux et les poissons inachevés. Ray entreprend leur publication dans le cadre d'un travail collectif. Francis Jessop, ainsi que Ralph Johnson, vicaire de Brignall et ami intime de Ray et Willughby, contribuent largement à l'achèvement de l'Ornithologie, en envoyant à Ray nombre de spécimens et d'observations personnelles, ainsi que des illustrations supplémentaires, aidé pour cela par Philip Skippon et Thomas Browne rencontrés à Montpellier lors du «Grand Tour ». Lorsque Tankred Robinson entreprendra le sien avec Hans Sloane, Ray lui écrit à Paris durant l'été 1683 pour lui demander d'aller visiter John Maria Ferro, un apothicaire de Venise qui possède de nombreuses plantes rares séchées, et plus particulièrement un grand nombre de figures de plantes dessinées très exactement par une "curieuse main » en noir. Vivement intéressé par cette belle collection, Ray lui demande de les acquérir afin de les insérer dans son Histoire des Plantes. Outre la nécessité grandissante de dessins précis et exacts, tous sont préoccupés de la réception de leurs livres auprès du public, rendus agréables aux lecteurs par leurs riches illustrations.

21 La vaste tâche que constitue l'identification des espèces requiert continuellement de nouvelles observations. Les spécimens de différentes régions et pays sont comparés non seulement avec les observations propres, mais également avec les sources auxquelles on se réfère. On constate qu'une même plante a des noms différents selon les auteurs. 
Lorsqu'on discute d'un problème particulier, les échanges se font souvent dans les trois jours, et à travers leur progression, on s'aperçoit effectivement de l'imprécision des descriptions antérieures, et l'on se remercie mutuellement de la correction des erreurs. D'ailleurs, lorsque ses amis envoient les catalogues de plantes demandés par Ray, tous prennent l'habitude de faire suivre le nom par son auteur, pour éviter toute confusion. On insiste aussi sur la nécessité d'observer les plantes durant leur croissance, et en toute saison, afin de parvenir à une identification exacte. Dans ces lettres, on discute particulièrement de l'adaptation des espèces à leur environnement. On remarque également l'effet possible des guerres ainsi que de l'accroissement du commerce et de la navigation sur la migration des oiseaux, déviant leur trajet vers une région dans laquelle ils n'avaient jamais été observés auparavant. Dans l'approche vitaliste du «Système de la Nature » partagée par John Ray et ses amis, l'adaptation au milieu de vie est progressivement mise en évidence. Dans ses lettres, Ray insistera sur le fait que, de même qu'il en est pour les animaux, toute la diversité du monde végétal est induite par le climat, le terroir, la nourriture ou d'autres circonstances externes, et relève de ce fait de la formidable plasticité du vivant ${ }^{25}$.

Dans les Lettres Philosophiques, la controverse autour des fossiles est très présente. Le premier à avoir établi l'origine organique des fossiles est le naturaliste danois Nicolas Steno (1638-1686) rencontré à Montpellier en 1666. Ray et ses amis y avaient créé des liens étroits avec Magnol, qui lui fera parvenir son catalogue de plantes de la région de Montpellier. De plus, Ray et Lister reconnaissent leur dette envers Rondelet pour l'achèvement de l'Histoire des Poissons de Willughby ${ }^{26}$. À cette époque, de multiples liens se nouent en effet entre savants français et britanniques. Les échanges scientifiques s'amplifieront encore par le commerce de livres, de graines, de plantes séchées, que Hans Sloane (1660-1753) entretient avec les élèves de Magnol, qu'il retrouve au jardin des plantes de Paris, envoyant des nouvelles de Tournefort à John Ray. Sloane sert également de trait d'union entre l'Académie des Sciences et la Société Royale, promouvant la coopération entre savants français et anglais, et essayant de coordonner les efforts en facilitant les échanges dans le domaine des sciences naturelles, grâce à son zèle et à sa courtoisie ${ }^{27}$.

Deux points essentiels se dessinent à travers les voyages et correspondances de John Ray. D'une part, les botanistes de la "République des Lettres », échangeant lettres et spécimens apparaissent comme les précurseurs de la communauté scientifique internationale moderne. Des travaux récents considèrent d'ailleurs les histoires naturelles de zoologie et de botanique non comme des compléments de la physique et de l'astronomie dominant la révolution scientifique, mais comme en étant véritablement le noyau ${ }^{28}$. D'autre part, les naturalistes du XVII siècle partagent une vision de la nature beaucoup plus vaste qui ne correspond pas à la nature telle qu'on l'entend de nos jours. Surnommé avec raison le « Pline anglais ", John Ray n'oppose pas nature et culture, car pour lui et ses amis, l'étude du grand «Système de la Nature " inclut également des observations sur les activités humaines, l'homme étant alors considéré comme un animal parmi d'autres. La notion de plasticité de la nature, chère à John Ray, est entendue comme la capacité de la vie à se transformer et à s'adapter en 
permanence. Elle regarde donc aussi bien les objets produits par la nature que les objets produits par les activités humaines, le langage en premier lieu.

\section{BIBLIOGRAPHIE}

AYRES Peter, Britain's Green Allies: Medicinal Plants in Wartime, Foreword: a brief historical perspective, Leicester, Matador, 2015.

BIRKHEAD Tim, Virtuoso by Nature : The Scientific Worlds of Francis Willughby FRS (1635-1672), Leiden, BRILL, 2016.

BRYAN Malcom, John Ray (1627-1705) Pioneer in the Natural Sciences, Braintree, The John Ray Trust, 2005.

CONSIDINE John, Small Dictionaries and Curiosity: Lexicography and Fieldwork in Post-medieval Europe, Oxford University Press, 2017.

CRAM David, Francis Willughby's Book of Games: A Seventeenth-Century Treatise on Sports, Games and Pastimes, New York, Routledge, 2016.

LA ROCHE Michel, BOISBELEAU Armand de, Bibliotheque angloise, ou Histoire litteraire de la Grande Bretagne, Amsterdam, chez la Veuve de Paul Marret, Tome quatrième, Première Partie, 1718.

LEWIS Gillian, «The Debt of John Ray and Martin Lister to Guillaume Rondelet of Montpellier ", Notes and Records of the Royal Society of London, vol. 66, issue 4, 2012, p. 323-339.

MAGNER Lois N., A History of the Life Sciences, Third Edition, Revised and Expanded, New York/Basel, Marcel Dekker, Inc., 2002.

MAGNOL Antoine, «Une Vie inédite de Pierre Magnol », La Botanique à Montpellier, Notes et Documents réunis et publiés par J.-E. Planchon, Montpellier, impr. de Boehm et fils, 1884.

MICKel Clarence E., « John Ray: Indefatigable Student of Nature », Palo Alto, Annual Review of Entomology, vol. 18, 1973, p. 1-17.

PICcIотто Joanna, Labors of Innocence in Early Modern England, Harvard University Press, 2010.

RADT Charlotte, «Quelques conférences du Palais de la Découverte », Journal d'Agriculture Tropicale et de Botanique Appliquée, vol. 12, Paris, Laboratoire d'Ethnobotanique, 1965, p. 117-120.

RAY John, Philosophical Letters Between the Late Learned Mr. Ray and Several of His Ingenious Correspondents, Natives and Foreigners. To which are Added Those of Francis Willughby Esq; The Whole consisting of many curious Discoveries and Improvements in the History of Quadrupeds, Birds, Fishes, Insects, Plats, Fossiles, Fountains, etc. Published by W. Derham, Chaplain to his Royal Highness George Prince of Wales, and F. R. S. London : Printed by William and John Innys, Printers to the Royal Society, at the Prince's Arms in St. Paul's Church-yard. 1718.

RAY John, A Collection of English Proverbs: Digested Into a Convenient Method for the Speedy Finding Anyone Upon Occasion; with Short Annotations. Whereunto are Added Local Proverbs with Their Explications, Old Proverbial Rhythmes, Less Known Or Exotic Proverbial Sentences, and Scottish Proverbs, Cambridge, Printed by J. Hayes for W. Morden, 1670. 
RAY John, A Collection of English Words Not Generally Used: With Their Significations and Original, in Two Alphabetical Catalogues, the One of Such as are Proper to the Northern, the Other to the Southern Counties. With Catalogues of English Birds and Fishes; and an Account of the Preparing and Refining Such Metals and Minerals as are Gotten in England. Printed London, Printed by H. Bruges for Tho. Burrell, 1674.

RAY John, Observations Topographical, Moral, \& Physiological; Made in a Journey through part of the LowCountries, Germany, Italy, and France: with a Catalogue of Plants not Native of England, found Spontaneously growing in those Parts, and their Virtues. By John Ray, Fellow of the Royal Society. Whereunto is added A brief Account of Francis Willughby, Esq; his Voyage through a great part of Spain. London : Printed for John Martyn, Printer to the Royal Society, at the Bell in St. Paul's Churchyard, 1673.

RAY John, Dictionariolum Trilingue Secundum Locos Communes, Nominibus Usitatioribus Anglicis, Latinis, Graecis Ordine Parallelos Dispositis. London: printed by Andrew Clark for Thomas Burrell, 1675.

RAY J., DERHAM W., Select Remains of the Learned John Ray, M.A. and F.R.S.: With His Life, by the Late William Derham, D.D. Canon of Windsor, and F.R.S., Published by George scotT, M. A. and F. R. S., London, printed; and sold by Ja. Dodsley in Pall-Mall; and J. Walter, at Charing-Cross, 1760.

Roos Anna Marie, « Naturalia: The History of Natural History and Medicine in the Seventeenth Century », Notes and Records of the Royal Society of London, vol. 66, issue 4, 2012, p. 313-321.

STOYE John, English Travellers Abroad, 1640-1667, New Haven, Yale University Press, 1989.

THOMSON Keith, Before Darwin: Reconciling God and Nature, New Haven and London, Yale University Press, 2005.

WAQUET Françoise, «Qu'est-ce que la République des Lettres ? Essai de sémantique historique », Paris, Bibliothèque de l'École des chartes, t. 147, 1989, p. 473-502.

WILLIAMS Élizabeth, « Medical Education in Eighteenth-Century Montpellier », Centres of Medical Excellence? Medical Travel and Education in Europe, 1500-1789, éd. Ole Peter Grell, Andrew Cunningham, Farnham, Ashgate, 2010.

YALE Élizabeth, Sociable Knowledge: Natural History and the Nation in Early Modern Britain, University of Pennsylvania Press, 2016.

\section{NOTES}

1. F. Waquet, «Qu'est-ce que la République des Lettres? Essai de sémantique historique », p. 473.

2. Philosophical Letters Between the Late Learned Mr. Ray and Several of His Ingenious Correspondents,..., Published by W. Derham. Traduction française dans la Bibliothèque Angloise.

3. http://dictionnaire-journaux.gazettes18e.fr/journal/0146-bibliotheque-anglaise

4. L. N. Magner, A History of the Life Sciences, Third Edition, Revised and Expanded, p. 258.

5. W. Derham, « The Life of Mr. Ray », p. 52-53.

6. P. Ayres, Britain's Green Allies: Medicinal Plants in Wartime, p. 5.

7. M. Bryan, John Ray (1627-1705) Pioneer in the Natural Sciences, p. 10.

8. T. Birkhead, Virtuoso by Nature: The Scientific Worlds of Francis Willughby FRS (1635-1672), p. 79. 
9. M. Bryan, John Ray (1627-1705) Pioneer in the Natural Sciences, p. 15.

10. Ibid., p. 13.

11. John Ray's Cambridge Catalogue (1660) translated and edited by P. H. Oswald and C.

D. Preston, Ray Society.

12. K. Thomson, Before Darwin: Reconciling God and Nature, p. 66.

13. C. E. Mickel, « John Ray: Indefatigable Student of Nature », p. 6.

14. D. Cram, Francis Willughby's Book of Games: A Seventeenth-Century Treatise on Sports, Games and Pastimes, p. 71.

15. E. Williams, « Medical Education in Eighteenth-Century Montpellier », p. 249-50.

16. J. Ray, Observations topographical, moral, \& physiological..., p. 454.

17. A. Magnol, « Une Vie inédite de Pierre Magnol », p. 13.

18. J. Stoye, English Travellers Abroad, 1640-1667, p. 295.

19. J. Considine, Small Dictionaries and Curiosity: Lexicography and Fieldwork in Postmedieval Europe, p. 99.

20. "Proverbs and Proverbial Observations belonging to Health, Diet and Physick ", dans A Collection of English Proverbs, p. 32.

21. A Collection of English Proverbs.

22. A Collection of English Words Not Generally Used.

23. Dictionariolum trilingue: "Of heaven, of the elements and meteors, stones and metals, plants, herbs, trees and shrubs, of the proper parts and adjuncts of animals ", dans E. Yale, Sociable Knowledge: Natural History and the Nation in Early Modern Britain, p. 200.

24. «For proverbs are drawn from the experience and observations of many ages; and are the ancient natural philosophy of the vulgar, preserved in old English in bad rhythmes, handed downe to us ", dans J. Picciotto, Labors of Innocence in Early Modern England, p. 146.

25. J. Ray, Philosophical Letters, p. 346.

26. G. Lewis, «The Debt of John Ray and Martin Lister to Guillaume Rondelet of Montpellier », p. 324.

27. C. Radt, "Quelques conférences du Palais de la Découverte ", Journal d'agriculture tropicale et de botanique appliquée, p. 117-120.

28. A. M. Roos, "Naturalia: The History of Natural History and Medicine in the Seventeenth Century », p. 314.

\section{RÉSUMÉS}

John Ray (1627-1705), éminent naturaliste né à l'aube de la Révolution scientifique, a rencontré et entretenu des correspondances avec de nombreux savants de l'Europe. Considéré comme le fondateur de la botanique et de la zoologie modernes, John Ray, surnommé le " Pline anglais ", 
est le premier à proposer un concept précis de la notion d'espèce. À travers sa correspondance, se dessine un réseau d'échanges collaboratifs et amicaux participant à la construction collective des sciences naturelles. Ses Lettres Philosophiques nous font découvrir le contexte historique et culturel dans lequel ce savoir s'est élaboré, dans le milieu intellectuel protestant où la théologie et la science sont étroitement liées au XVII ${ }^{\mathrm{e}}$ siècle. Bastion huguenot doté du premier Jardin du Roy de France, Montpellier était devenue la « capitale de la botanique » et attirait les naturalistes de toute l'Europe. John Ray et ses amis viendront y rencontrer Pierre Magnol. Membre de la Royal Society, Ray s'engagera également dans la publication d'ouvrages adressés à un large public: collections de proverbes et de mots, manuels scolaires et dictionnaires en langues vernaculaires.

\section{AUTEUR}

\section{SABINE KRAUS}

Doctorante en histoire des sciences et de la médecine, Université de Montpellier 


\title{
Missions des naturalistes Peyssonnel et Desfontaines dans la régence de Tunis au XVIII ${ }^{\mathrm{e}}$ siècle
}

\author{
Geneviève Falgas
}

1 Parcourir les galeries du muséum d'Histoire Naturelle de Paris, ou celles d'autres grandes villes de France, c'est non seulement pénétrer dans des conservatoires de la connaissance mais aussi ouvrir les portes du grand large, et celles ensuite de la transmission des savoirs. En effet, derrière chaque planche d'herbier, chaque roche exposée, chaque vitrine d'insectes ou de papillons, il y a des hommes et leurs voyages qui ont permis ces collectes sur le terrain. Ils rapportaient, parfois dans les pires difficultés, les objets de ces collectes qui étaient mis à la disposition de leurs académies ou sociétés savantes.

2 Puis, au-delà de la matérialité des objets, se profile le long cheminement du travail des savants qui ont répertorié, analysé, classé les nouveaux apports : il a fallu intégrer les résultats de ces découvertes aux savoirs anciens, obligeant à une actualisation permanente qui remet souvent en question des connaissances jusque-là considérées comme acquises.

3 Ainsi, pendant que certains naturalistes travaillaient dans la quiétude des cabinets ou des bibliothèques, d'autres, intrépides et prêts à tout pour remplir leurs missions, s'en allaient à la recherche de connaissances nouvelles. C'est dans ce but qu'au XvIII siècle, les naturalistes Peyssonnel et Desfontaines partirent du Jardin Royal ${ }^{1}$ pour les pays de Barbarie.

4 Les récits et correspondances des deux naturalistes furent publiés en 1838 par l'historien et géographe, Dureau de La Malle². Bien que beaucoup de ces documents aient été perdus, le géographe souligne à la fois le grand intérêt et le côté complémentaire de ces deux voyages pour justifier son choix de les sortir de l'oubli et de les publier ensemble ${ }^{3}$.

5 La publication de Dureau de La Malle, et les nombreuses annexes qu'elle contient, constitue la source principale de l'étude ici présentée. 
6 Jean-André Peyssonnel, qui naquit en 1694, appartenait à une famille de la bourgeoisie provençale. Il reçut un enseignement de qualité, devint médecin et, parallèlement, fut nommé correspondant de l'Académie des Sciences de Paris. En 1724, il obtint du bibliothécaire du Roi, l'abbé Bignon, l'autorisation d'une mission en Barbarie. Il séjourna huit mois dans la régence ${ }^{4}$ de Tunis et autant dans celle d'Alger.

7 De Barbarie, Peyssonnel adressa à l'abbé Bignon, et au surintendant du Jardin Royal des Plantes, Pierre Chirac, des Lettres qui leur rendaient compte de son voyage, des observations et découvertes qu'il y faisait. Ses sujets d'étude furent multiples : lors de l'itinéraire sur les côtes nord de la Tunisie, il étudia le corail, premier à en pressentir la nature animale; il observa les populations et leurs manières de vivre, s'occupa de géographie, d'archéologie, de belles lettres - en relevant quantité d'inscriptions d'histoire aussi.

8 René Louiche Desfontaines, quant à lui, naquit presque soixante ans plus tard, en 1750, au Tremblay en Bretagne, dans une famille de paysans aisés. Il reçut une solide formation humaniste et scientifique, puis fit ses études de médecine à Paris. Là il fréquenta le Jardin Royal, où il rencontra le professeur et premier médecin du roi, Louis-Guillaume Lemonnier. Celui-ci proposa à Desfontaines, alors âgé de 33 ans, d'effectuer un voyage officiel en Barbarie, pour une mission d'histoire naturelle, "y faire des observations sur la géographie, les antiquités, les mœurs des habitants, et particulièrement sur l'histoire naturelle", "dans presque toute l'étendue de ces territoires ${ }^{5} »$.

9 Pendant son séjour en Barbarie, il avait adressé régulièrement à Lemonnier, soit de Tunis, soit d'Alger, « une suite de lettres, dans lesquelles il comptait retrouver plus tard les matériaux de la relation de son voyage ${ }^{6} »$. Malheureusement, comme l'on sait, une partie de ces lettres a été perdue.

\section{Les conditions de voyage}

10 L'un et l'autre eurent des conditions de voyage privilégiées, parce qu'effectuées au nom $d u$ Roi sous la protection des consuls de France et des accords signés en 1532 entre François $1^{\text {er }}$ et Soliman Le Magnifique.

11 Ces accords, appelés Capitulations, constituaient des sauf-conduits pour les Français qui commerçaient en Méditerranée ou partaient au nom du Roi. Comme Desfontaines l'écrivait à Lemonnier, les deux naturalistes n'auraient pu mener à bien leurs missions sans ces accords :

«Il y a ici un botaniste de Danemark qui n'aura pas les mêmes avantages que moi, et qui ne pourra pas voir les mêmes lieux. ${ }^{7}$ "

12 La protection de ces voyageurs officiels était également assurée à l'intérieur du pays, même si parfois les populations voyaient arriver ces étrangers avec méfiance. Lors du voyage de Desfontaines à Sfax, le bey lui donna des lettres de recommandation pour le gouverneur de la ville qui lui témoigna « beaucoup de marques d'amitié ${ }^{8}$ ».

13 Par ailleurs, leur profession de médecin leur donnait de la considération, et ils finissaient par avoir plus ou moins des fonctions auprès des Puissances, c'est-à-dire des gouverneurs de ces pays: longtemps, la médecine fut proche de la botanique alors qu'on soignait par les plantes. 
Les Lettres de Peyssonnel, ou plutôt celles qui ont subsisté, longtemps oubliées dans les archives du Muséum, constituent un journal de voyage délivrant tout d'abord des informations sur les conditions dans lesquelles pouvait s'accomplir un tel voyage, à une époque où tout déplacement relevait de l'aventure. Le naturaliste quitta Paris en avril 1724, "par le coche » précise-t-il, pour un voyage de 15 jours jusqu'à Marseille, où il embarqua à bord d'une " pinque ", mettant 9 jours pour traverser la Méditerranée.

5 À Tunis, il fut l'hôte du consul de France qui, dès son arrivée, le conduisit au palais du bey pour lui être présenté, avec ses passeports et le but de sa mission. Il en fut de même pour Desfontaines : quelque soixante années plus tard, en août 1783, il quitta Marseille à bord d'un petit navire de commerce qui, huit jours après, mouilla dans la rade de Carthage. Suivant l'usage, il se rendit chez le consul qui l'amena aussitôt chez le bey. Ce dernier le prit en considération et l'invita à se joindre à ce qu'on appelait «le Camp ». C'était l'imposante colonne militaire qui s'en allait deux fois l'an recouvrer l'impôt auprès des tribus de l'intérieur. Le Camp d'été parcourait le nord du pays, le Camp d'hiver s'en allait vers les oasis du sud et la frontière tripolitaine.

C'est au sein de la colonne d'hiver que Desfontaines partit, en décembre 1783, pour le plus long de ses voyages à travers la régence de Tunis, dans des conditions exceptionnelles - ayant été investi de la charge de médecin du bey. Il ne tarit pas d'éloges sur la manière dont ce dernier le traita. Au départ, il lui avait offert quatre chameaux qui s'ajoutèrent à deux mules déjà achetées ; il avait aussi loué une tente et pris quatre domestiques à son service : « Voilà mon train ${ }^{9}$ » écrivait-il à Lemonnier.

\section{Le manque de sécurité}

Toutefois le quotidien de ces missions n'était exempt ni de difficultés ni de dangers. Ils se trouvèrent souvent dans des conditions périlleuses qu'ils surent, le cas échéant, affronter avec courage. Parmi tous les détails dont il parsème ses écrits, et qui sont autant de renseignements sur l'état du pays, on peut relever que Desfontaines le juge quelque peu abandonné, et surtout peu sûr :

«Il est bien triste que ce beau pays soit inculte et infesté de voleurs. »

8 Un jour, il faillit être agressé : alors qu'il herborisait avec un seul domestique pour escorte, il fut approché par quatre hommes menaçants :

"Ces cantons sont remplis de voleurs, et de voleurs hardis. ${ }^{10}$ »

Par ailleurs, en écrivant à Lemonnier, Desfontaines ajoutait qu'il fallait toujours être sur ses gardes car :

«Ces méchantes gens [...] feraient encore moins grâce à un chrétien qu’à tout autre.

$11 \gg$

Et d'en expliquer la cause :

"On n'aime pas à voir des chrétiens dans ce pays-ci. ${ }^{12}$ "

Quant à Peyssonnel, il fut aussi parfois en butte à cette hostilité : au cours de son périple dans la régence d'Alger, il note son arrivée mouvementée à Constantine :

«Les habitants sont très ennemis des chrétiens, et j'y ai reçu beaucoup d'injures. ${ }^{13}$ »

Mais les populations ne se montraient pas seulement méfiantes à l'égard des Européens parce qu'ils étaient chrétiens. Ils se méfiaient de leurs intentions : dans la région du Kef, à l'intérieur des terres, la population accueillit Peyssonnel par des insultes et on 
lança des pierres contre la maison qu'il occupait. La rumeur disait qu'il venait chercher de l'or, et qu'il était un espion à la solde des chrétiens pour prendre la ville ${ }^{14}$.

Ainsi pourrait-on relever dans les lettres des deux naturalistes de nombreux autres exemples de cette insécurité qui rendit souvent problématiques certains de leurs déplacements ${ }^{15}$. En particulier les haltes pour la nuit, plus ou moins hasardeuses quand ils ne voyageaient pas avec le Camp du bey, c'est-à-dire la colonne militaire chargée de prélever les impôts à l'intérieur du pays : l'un et l'autre se déplaçaient à dos de mule, couchant sous une tente qu'ils installaient dans les douars, ou bien où ils pouvaient, le plus souvent dans l'insécurité et l'inconfort ${ }^{16}$.

\section{La piraterie}

La course, selon le mot de l'époque, était encore très active du nord au sud de la Méditerranée. Les descentes de corsaires chrétiens sur les côtes de Barbarie - surtout ceux des chevaliers de Malte - étaient fréquentes et redoutées. Les deux naturalistes furent confrontés, l'un et l'autre, à la peur que cette réalité suscitait dans le pays.

Dans le sud du Cap-Bon, où les habitants «n'avaient jamais vu des chrétiens voyageurs ", ils s'étaient imaginé que Peyssonnel et son escorte étaient venus " pour les enlever et les mener en servitude». Ils durent veiller toute la nuit: le bruit s'était répandu que ces chrétiens « devaient donner quelques signaux pour aviser les corsaires de venir enlever le douar ${ }^{17} »$. Et Peyssonnel de conclure :

« De notre côté, nous ne souhaitions rien que de voir la nuit bientôt passée, pour nous retirer et quitter ce pays si dangereux. ${ }^{18}$ »

Desfontaines, en traversant cette même région, avait trouvé les habitants nerveux : ils craignaient l'arrivée des corsaires de Malte ${ }^{19}$. Mais les dangers de la piraterie guettaient les musulmans comme les chrétiens. À Bizerte, Peyssonnel note que «les Turcs y arment beaucoup de galiotes pour aller contre les chrétiens ${ }^{20}$ ». Ces chrétiens qui restent indifférenciés dans l'esprit des habitants de l'intérieur, qu'ils soient Français, Espagnols ou Italiens, et qui sont regardés comme des ennemis :

«[...] excepté dans les ports de commerce, où l'on a pour les nations amies les

égards qu'on doit avoir. ${ }^{21}$ "

Desfontaines signale à plusieurs reprises que les habitants des villes côtières sont plus doux, plus tolérants, ayant de tout temps été habitués à commercer avec les Occidentaux. Le contraste est très vif avec l'intérieur des terres, ou le sud du pays, souvent aride, pauvre, peuplé de tribus agressives ${ }^{22}$.

\section{Des pays inhospitaliers}

Parfois aussi ces régions se révélaient hostiles. Outre les voleurs de grand chemin, les serpents, comme les scorpions noirs aux morsures redoutables, y pullulaient: Desfontaines avouait se coucher chaque soir avec inquiétude, ayant trouvé à deux reprises des scorpions noirs dans sa tente. Peyssonnel fut confronté au même danger ${ }^{23}$.

Quant aux lions et aux tigres, tous deux en parlent également: "ils sont "forts communs" dans ce pays; "heureusement je n'en ai rencontré aucun dans mes promenades ", écrit Desfontaines, qui entendit pourtant une nuit "plusieurs fois le hurlement des lions ${ }^{24} »$. Peyssonnel, aussi audacieux, n'hésitait pas à s'enfoncer dans 
des régions boisées où abondaient, ainsi qu'on l'en avait prévenu, des lions et des tigres qui demeuraient « du côté des montagnes »- bien qu'il conclût :

« Mais nous n'en vîmes point. ${ }^{25}$ "

Le danger lié aux bêtes sauvages n'était pas le seul : la nature même des contrées traversées, dans la régence de Tunis comme dans celle d'Alger, était souvent inhospitalière :

« [...] des ravins profonds au bord desquels il faut marcher avec précaution [...], des montagnes couvertes d'épaisses forêts, des pics arides, des rochers escarpés et des profonds abîmes creusés par les torrents. ${ }^{26}$ "

De même, la rudesse du climat mit à mal leur résistance physique, à l'un comme à l'autre. Alors qu'en plein mois de février 1725, Peyssonnel et son escorte traversaient la région montagneuse de Guelma, dans la régence d'Alger, ils eurent très froid et se trouvèrent pris, un jour, dans des bourrasques de pluie et de grêle :

"Qui se changèrent en neige et en brouillard si fort [qu'ils furent] obligés d'aller

chercher au plus tôt un gite. ${ }^{27}$ "

Par contre, dans ces pays de forts contrastes, il en allait tout autrement en été : quand Desfontaines partit au début du mois d'août 1784 pour le sud de la régence de Tunis, il sous-estima les effets de la chaleur, dont il souffrit beaucoup, en particulier après avoir marché tout un jour dans des sables brûlants :

«[...] je ressentais des douleurs cuisantes aux mains, au visage, et mes paupières étaient si gonflées que j'avais peine à voir. ${ }^{28}$ "

Aussi, les nuits de clair de lune, il lui arriva de partir herboriser bien avant l'aube, parfois au milieu de tribus "indomptées " ${ }^{29}$. Une fois même, il fut "extrêmement tourmenté par la soif ", sa provision d'eau étant finie ${ }^{30}$. À son retour à Tunis, à la fin du mois d'août, il se dit «bien fatigué par les chaleurs excessives qu['il avait] essuyées pendant le cours de [son] voyage ${ }^{31}$ ». Peyssonnel, qui sillonna la région de Constantine en plein mois d'août 1725 , avait noté cette chaleur insupportable ${ }^{32}$ : comme Desfontaines, il se levait en pleine nuit pour aller herboriser :

« Nous partîmes de Zawan [Zaghouan] à deux heures après minuit. »"33

\section{Les collectes}

Pour Desfontaines, ces voyages comblaient le botaniste qu'il était avant tout.

\section{La botanique : joies et déceptions ; difficultés des collectes}

Il éprouvait des joies profondes à aller de découverte en découverte :

«Chaque plante que je trouvais me donnait un sentiment de plaisir inexprimable:

c'était pour moi une nouvelle conquête..$^{34}$ "

Le voyage avec le Camp du bey, qui dura "plus de trois mois et demi", se termina au mois d'avril 1784, et il se prépara aussitôt pour une nouvelle expédition dans la région de Zaghouan, au sud de Tunis :

«Le mois d'avril est ici la saison du printemps. J'herborise du matin au soir. ${ }^{35}$ »

Quand il annonce son voyage vers Sfax, en juillet 1784, il écrit à Lemonnier qu'il espère recueillir «des graines, des insectes et autres productions naturelles, la saison des 
plantes étant alors passée ${ }^{36}$ ». Toutefois s'il allait d'émerveillement en émerveillement, Desfontaines connut aussi des moments plus décevants :

«J'ai éprouvé beaucoup d'ennui à Kairouan parce que nous y avons séjourné plus de quinze jours. Point de plantes, peu d'oiseaux, encore moins de minéraux. Livré à moi-même je comptais les heures et brûlais d'envie de marcher vers des montagnes que j'apercevais dans le lointain..$^{37}$ "

Les confins du Sahara lui laissèrent une impression de tristesse à cause de leur uniformité, car on n'y voyait ni verdure ni habitation humaine : la nature y semblait morte.

Desfontaines n'hésitait pas à exprimer ses émotions, dépassant le côté purement scientifique de sa mission: par exemple, le 15 septembre 1785, il évoque sa tristesse d'avoir dû se séparer d'un ami :

«Cette journée fut bien triste pour moi, écrit-il dans sa relation de voyage, [...] mon

âme ne fut occupée que de lui pendant cette journée. ${ }^{38}$ »

On relève sur ce sujet une différence avec Peyssonnel, qui effectua son voyage beaucoup plus tôt dans le siècle, et dont les relations sont dépourvues d'expression de sentiments personnels, à de rares exceptions près. Desfontaines, lui, s'inscrit davantage dans le courant Rousseauiste ${ }^{39} \mathrm{du}$ XVIII ${ }^{\mathrm{e}}$ siècle, exprimant ses impressions devant les beautés de la nature, souvent émerveillé : à peine à terre à son arrivée à Tunis, il se mit à recenser toutes les plantes qu'il voyait. Sa sensibilité apparait dans la minutie des descriptions : les salicornes " qui tapissaient les lieux bas et humides", les bouquets de pancratium maritime "qui s'élevaient au-dessus des sables brûlants du rivage », ou " les touffes de câpriers chargés de fleurs » qui « pendaient des fentes des rochers ${ }^{40}$ ".

41 Après huit mois d'exploration dans la régence de Tunis, Desfontaines passa dans la régence d'Alger. Au début de septembre 1785, alors qu'il s'apprêtait à quitter le port d'Annaba ${ }^{41}$ pour rejoindre la France, il rencontra un autre botaniste Jean-Marie Poiret (1755-1834), qu'il décida d'accompagner pendant quelque temps. Au cours d'une excursion, ils découvrirent "une très belle espèce d'ipomea » : " une fleur aussi grande que celle du liseron ordinaire, mais du plus beau rouge, s'élevait au-dessus d'une broussaille impénétrable ${ }^{42}$ ». L'acquisition de cette fleur s'avéra une entreprise difficile, qui les laissa " percés de sueur, les habits en lambeaux et les mains ensanglantées ». Sur l'une des planches numérisées de l'Herbier de Desfontaines et de celui de Poiret, au Muséum ${ }^{43}$, figure une « ipomoea sagittata », avec cette mention « récolteur : Poiret avec Desfontaines ", localité originale : La Calle, Algérie, précisant en latin "apud lacuum ripas prope La Calle ${ }^{44} »$. Exactement l'endroit où herborisaient Poiret et Desfontaines, à la frontière algéro-tunisienne, quand ils cueillirent cette ipomée.

Ils passèrent une quinzaine de jours à parcourir les paysages les plus variés, étangs, prairies, bois, montagnes, plaines de sable, où leur marche fut parfois très pénible. Mais pour rendre compte de cette « [une] foule de plantes, d'insectes, d'oiseaux et de reptiles peu ou point connus ", ils étaient prêts à tout braver, à tout supporter.

\section{L'Antiquité romaine}

43 Cependant, on l'a vu, ces deux naturalistes s'intéressaient à beaucoup plus qu'à la botanique, et tout retenait leur attention. L'un comme l'autre, en particulier, font preuve d'un extrême intérêt pour les vestiges de l'antiquité romaine, dont le pays est couvert. Pour Desfontaines, cet intérêt est marqué par une profonde sensibilité au 
souvenir de ce que fut cette civilisation : à son arrivée à Tunis, en août 1783, passant une dernière nuit à bord avant de débarquer, il nota que la proximité des ruines " d'une des plus fameuses villes du monde, au milieu des champs où elle fut autrefois ", le bouleversa au point de lui ôter le sommeil ${ }^{45}$. Il ne manque jamais de visiter des ruines, faisant des croquis, recueillant ce que lui en disaient ses accompagnateurs :

«J'ai pris beaucoup de notes [...] sur les ruines que j'ai rencontrées. J'ai acheté des médailles, copié beaucoup d'inscriptions qui pourront éclairer la géographie ancienne de ce pays. $»^{46}$

\section{Observations et relevés divers}

Pour Peyssonnel comme pour Desfontaines, un autre aspect de l'Histoire naturelle est omniprésent : ce qu'on appelle aujourd'hui « l'ethnographie, [c'est-à-dire] les hommes, leurs manières de table, leur mobilier, leur costume, leurs techniques, les formes sociales et politiques, les croyances religieuses ", pour reprendre la présentation qu'en fait l'historienne Lucette Valensi ${ }^{47}$. Dans sa lettre à Lemonnier du 15 avril 1784, déjà citée, Desfontaines évoque les résultats de son voyage avec le Camp du bey : là aussi il a pris «[...] beaucoup de notes", partout où il est passé, « en particulier, sur les arts, la culture, les mœurs des habitants, les diverses tribus arabes qui habitent le royaume $[\ldots]^{48} »$.

Dans le petit village de Soliman, non loin de Tunis, il remarque une population d'origine espagnole, descendant de Maures andalous chassés d'Espagne au $\mathrm{Xv}^{\mathrm{e}}$ siècle, et " qui conserve[nt] encore la langue espagnole ${ }^{49}$ ». Aussitôt il veut tout savoir sur son histoire. Tous ces renseignements, il les consignait tantôt dans sa correspondance, tantôt dans des notes éparses sur ses carnets de voyage.

Par ailleurs, tout au long de leur cheminement à travers les régences de Tunis et d'Alger, chacun en son temps, les deux naturalistes collectent des renseignements qu'on pourrait qualifier sans exagérer d'encyclopédiques. Rien ne leur échappe, ils notent tout sur les sujets les plus divers : par exemple, pour ne citer que Desfontaines, les eaux thermales, dont l'exploitation dans ces pays remonte à l'Antiquité ; le régime des eaux de pluie; le flux et le reflux des marées; les colonies d'oiseaux aquatiques; l'aqueduc romain de Zaghouan, long de $150 \mathrm{~km}$, qui alimentait Tunis et Carthage dans l'Antiquité. Dans ce même lieu, il s'intéresse à la teinture des bonnets rouges, qui s'exportent dans tout l'Orient : l'eau de source y est si pure que la teinte qui en ressort est sans égale. Il cherche à connaître tous les modes de culture des plantes nourricières observées, avec l'idée de voir si ces plantes pourraient être cultivées en France. Il lui arriva d'empailler des oiseaux, qu'il destinait au Muséum de Paris $^{50}$ : à l'occasion, il étudiait de plus près les échantillons de roches qu'il avait ramassés, comme de la galène, de la calcédoine, du cristal de roche, des fragments teintés de vert qui signalait du cuivre, ou teinté de rouge pour le fer ${ }^{51}$. Desfontaines excursionna avec Poiret jusqu'au mois de novembre 1785, puis il tomba malade et ne put poursuivre son voyage plus loin que Tlemcen, du côté d'Oran. Il se replia sur Alger et quitta définitivement l'Afrique du Nord. 


\section{La transmission des savoirs}

47 Cueillir des plantes, ramasser des échantillons de roche, noter tout ce qu'ils voyaient, ce n'était là qu'une partie de leur mission. L'autre partie, c'était d'en rendre compte, d'abord à leurs commanditaires, comme une courroie de transmission qui remontait du terrain jusqu'aux académies ou sociétés savantes. Ils ne perdaient jamais de vue le but de leur mission : transmettre des savoirs nouveaux.

\section{Circulation des savoirs nouveaux entre académies et sociétés savantes}

Desfontaines rendait compte de l'accomplissement de sa mission au fur et à mesure de ses explorations, ainsi qu'il l'écrivait à Lemonnier dans sa première lettre datée du 15 avril 1784, à Tunis. Il revenait alors des trois mois passés au Camp du bey :

«[...] Je ne vous envoie Monsieur qu'un très court tableau de mon voyage. J'ai pris beaucoup de notes sur les pays où j'ai passé, [...]; mais ce n'est pas en voyageant qu'on peut faire quelque chose de suivi..$^{52}$ »

Aussi compte-t-il sur son retour à Paris pour mettre de l'ordre dans ses notes et commencer à en tirer des enseignements. Sur place, il profitait de moments où il ne voyageait pas, entre deux excursions pour «faire sécher des plantes ${ }^{53}$ ». À l'une de ces occasions, il avait écrit :

«Je me levai le lendemain de très bon matin afin de décrire et préparer les plantes que j'avais trouvées la veille..$^{54}$ »

50 Dans une autre lettre à Lemonnier, du 12 août 1784, il précisait :

«[...] je vais m'occuper maintenant à rédiger les observations que j'ai faites, pour vous en faire part..$^{55}$ »

51 Peyssonnel en son temps écrivait à Pierre Chirac, l'intendant du Jardin Royal :

«J'aurai soin d'exécuter vos ordres au sujet des semences, des graines et des plantes..$^{56} »$

52 À propos desquelles il devait «faire un herbier des plantes inconnues et $\operatorname{rares}^{57}$ ». Malheureusement, les produits de ces collectes n'arrivaient pas toujours à leurs destinataires, ainsi que l'explique Dureau de La Malle dans sa publication de 1838. Tel fut le sort de ceux envoyés par Peyssonnel au surintendant du Jardin Royal :

«Dont la négligente administration aura laissé perdre, non seulement les notes scientifiques du jeune voyageur, mais encore les herbiers et les graines qu'il a dû envoyer, ou rapporter avec lui. ${ }^{58}$ "

53 C'est entre contemporains que circulaient ensuite les découvertes, les observations, les hypothèses suscitées par les collectes faites sur le terrain. Ces hommes en effet qui parcouraient le monde tissaient des réseaux serrés d'échanges avec les diverses académies ou sociétés savantes qui existaient à cette époque. Lorsque Dureau de La Malle publia les récits et correspondances des deux naturalistes, il nota en sous-titre ces multiples relations. Par exemple, Desfontaines fut :

«Membre de l'Académie des Sciences de Paris, membre de la société d'Agriculture de Paris, de l'Académie des Sciences et Belles Lettres de Dijon, de la société de physique de Gottingue (Göttingen aujourd'hui), de la société linnéenne de Londres, etc.etc..$^{59}$ " 
nut de même pour Peyssonnel qui revenait à Paris avec une somme importante d'observations et d'informations, qu'il avait communiquées régulièrement à l'abbé Bignon et au surintendant du Jardin Royal, ainsi qu'aux diverses académies dont il était :

«Membre associé (et non pas titulaire comme Desfontaines) de l'Académie des Sciences, de l'académie de Marseille, de la société royale de Londres, des académies de Rouen et de Montpellier. ${ }^{60} »$

C'est alors qu'on mesure combien la science était objet de partage : les découvertes des uns servaient aux autres comme d'une base pour aller plus loin, ou pour conforter leurs propres observations. Par exemple, Desfontaines mentionne le nom d'un autre botaniste, «l'abbé Teissier, de l'Académie des Sciences ». Ce dernier lui a communiqué "quelques observations sur les usages du henné61 » qui complètent ce que lui-même avait observé sur «cet arbrisseau intéressant »- quand il le découvrit au cours d'une herborisation $^{62}$. Quand Jean-Marie Poiret rencontre Desfontaines qui effectuait alors son périple dans la régence d'Alger, il s'en félicite :

«Ce savant académicien a bien voulu m'associer à ses recherches et me

communiquer ses lumières. »

Il admirait cet homme « envoyé par l'Académie des Sciences pour faire des recherches en Histoire naturelle particulièrement en botanique » (Lettre XII). Peyssonnel, comme Desfontaines ou Poiret étaient en relations étroites avec les naturalistes du Jardin Royal, puis du Jardin des Plantes, ou étudiaient et se succédèrent des savants de grande renommée, comme Buffon (1707-1788) ou les botanistes de la famille de Jussieu ${ }^{63}$, parmi beaucoup d'autres.

\section{Intégration des savoirs anciens aux connaissances nouvelles}

57 En effet, la science ne se transmet pas qu'entre contemporains, elle vient aussi des générations antérieures dont on ne saurait ignorer les découvertes: c'est cette accumulation des savoirs, dans l'épaisseur du temps, qui est porteuse de progrès.

Ainsi Desfontaines se réfère à ses prédécesseurs. Certaines remarques, dans sa correspondance, son récit ou ses notes de voyage l'explicitent : il connaît parfaitement les travaux de Joseph de Tournefort (1656-1708), un des maîtres de la botanique au XVII siècle. Il publia d'ailleurs un complément des études de Tournefort que ce dernier n'avait pu terminer avant sa mort ${ }^{64}$.

59 Mais il remonte beaucoup plus loin dans le temps pour tenter de trouver des réponses à ses questions : il avait apporté des livres pour étudier plus précisément les plantes qu'il recueillait. Herborisant du côté de l'île de Djerba, il essaya d'identifier le lotos, le mythique lotos de l'Odyssée, pour savoir exactement de quelle plante ou de quel arbuste il s'agissait : il avait en main le texte de l'historien grec Polybe où celui-ci mentionnait cette plante, de même que ceux de Théophraste et plus tard de Pline. Il jugea cependant ces textes trop imprécis pour arriver à un résultat ${ }^{65}$.

60 C'est ainsi, au sein des académies, dans ces échanges entre savants, que ces connaissances nouvelles étaient confrontées, étudiées, organisées, jusqu'à ce que des découvertes toujours plus récentes en rendent certaines obsolètes ou en confirment d'autres. 
61 En 1838, quand Dureau de La Malle entreprit la publication des lettres et récits des deux naturalistes, il justifiait de cette manière la publication de ces missions déjà anciennes : en attendant, disait-il, d'autres missions contemporaines ou futures qui apporteraient leurs lots de savoirs nouveaux, il était nécessaire de rappeler ces " sources anciennes ${ }^{66}$ ». Elles fournissent en effet des observations précieuses, à l'instar des travaux de Desfontaines sur lesquels « a été bâti plus tard l'édifice, aujourd'hui si imposant, de la géographie botanique ${ }^{67} »$.

\section{Diffusion de ces connaissances par la publication et l'enseignement}

62 Une autre étape restait à franchir dans cette transmission des savoirs : la diffusion. Cette dernière se faisait par la publication et l'enseignement. Revenu à Paris après ces deux années en Barbarie, Desfontaines entreprit la publication d'une œuvre imposante. Il avait rapporté de ce voyage, entre autres collectes et observations, une matière extraordinaire en botanique qu'il rassembla et classa dans son « Herbier de la Flore Atlantique ». Puis, huit ans après son retour, il publia l'ensemble de cette mission de découvertes, en latin comme c'était l'usage pour la botanique, sous le titre de Flora Atlantica ${ }^{68}$ - Flore de l'Atlas, ou Flore du pays des Atlantes ${ }^{69}$. Flora atlantica ${ }^{70}$ recense quelque 2000 espèces, parmi lesquelles environ 300 espèces nouvelles ${ }^{71}$.

63 L'Herbier Desfontaines, qui remonte à plus de 230 ans, fait aujourd'hui partie de la Collection des Herbiers historiques du Muséum de Paris. Desfontaines ne cultivait pas seulement la botanique pour elle-même ; il chercha constamment à la rendre utile en l'appliquant à l'agriculture. Il encouragea la culture des arbres étrangers en France, rassemblant ses idées dans un livre qu'il publia en $1809^{72}$.

Dans le même temps, il s'employait à traduire les travaux qu'il menait en un langage simple et compréhensible du plus grand nombre. C'est-à-dire qu'il se consacra à l'enseignement, ne réservant pas les fruits de son labeur aux seuls savants avec lesquels il était en relation. Desfontaines avait été nommé professeur au Jardin des Plantes, et il s'était alors occupé avec passion de l'enseignement de la botanique, d'une manière toute nouvelle qui développa le goût de l'étude des végétaux chez ceux qui se pressaient « en foule à ses leçons ${ }^{73}$ ". Ses contemporains soulignèrent la simplicité et la bonhomie de ce maître qui ne songeait qu'à intéresser ses auditeurs, " pour les gagner ensuite à la science ${ }^{74} »$.

Ainsi, pendant plus de quarante ans, près de 1500 personnes vinrent régulièrement entendre Desfontaines au Jardin des Plantes. Dans cette Préface de l'édition de 1838, on peut encore lire :

«Presque tous ceux qui savent aujourd'hui la botanique en France, la savent ou de

lui, ou de botanistes qui ont été ses élèves. ${ }^{75}$ "

Un de ses auditeurs, peut-être fut-il son élève, Adrien de Jussieu - de cette grande famille de botanistes - rédigea un discours qu'il lut aux funérailles de Desfontaines. Les mots employés témoignent de cette empathie que dégageait la personnalité du vieux maître : il y exprimait sa tristesse de voir désormais au Muséum sa place vide ou occupée par un autre :

«[...] Nous étions si bien accoutumés, et nous aimions tant à l'y voir. ${ }^{76}$ » 


\section{BIBLIOGRAPHIE}

DESFONTAINES René, À la mémoire de Joseph Pitton de Tournefort. Choix de plantes du Corollaire des Instituts de Tournefort, publiées d'après son herbier et gravées sur les dessins originaux d'Aubriet, éd. Levrault, Paris, 1808 (œuvre numérisée, Gallica).

DUREAU DE LA MALLE Adolphe, Peyssonnel et Desfontaines. Voyages dans les régences de Tunis et d'Alger, Paris 1838, tomes I (Peyssonnel) et II (Desfontaines), numérisés en un seul volume de 968 pages dans la version Gallica.

JUSSIEU Adrien de, « Funérailles de M. Desfontaines », dans Collection de l'Académie royale des sciences, éd. Didot, Paris, 1833, 12 p. Discours prononcé le 18 novembre 1833, (document Gallica). POIRET Jean-Louis Marie, Voyage en Barbarie ou Lettres écrites de l'Ancienne Numidie pendant les années 1785-1786 sur les religions, les coutumes et les mours des Mores et des Arabes bédouins avec un essai sur l'histoire de ce pays, éd. Née de La Rochelle, Paris, 1799, (2 vol.). 
Jean-André Peyssonnel. Voyage dans les régences de Tunis et d'Alger, Présentation et notes de Lucette Valensi, éd. La Découverte, Paris, 1987, 268 pages.

\section{NOTES}

1. Le Jardin Royal devint en 1793 le Muséum national d'histoire naturelle de Paris.

2. A. Dureau de La Malle, Peyssonnel et Desfontaines. Voyages dans les régences de Tunis et d'Alger, Paris 1838, tomes I (Peyssonnel) et II (Desfontaines), numérisés en un seul volume de 968 pages dans la version Gallica.

3. Dureau de La Malle, ibid. I, Préface, p. IX.

4. Le mot de "régence" appartenait au langage diplomatique français: pour administrer ces provinces de l'Empire, Constantinople détachait des représentants, les pachas, qui étaient, en quelque sorte des « régents».

5. Dureau de La Malle, ibid., Préface, p. VII.

6. Dureau de La Malle, ibid. II, Préface p.XXX: Lemonnier, premier médecin de Louis XVI, les communiquait au roi, « qui se plaisait à les lire ».

7. Dureau de La Malle, ibid. II, p. 80.

8. Dureau de La Malle, ibid. II, p. 124.

9. Dureau de La Malle, ibid. II, p. 80.

10. Dureau de La Malle, ibid. II, p. 67.

11. Dureau de La Malle, ibid. II, p. 76.

12. Dureau de La Malle, ibid. II, p. 196 et 108.

13. Dureau de La Malle, ibid. I, p. 217.

14. Dureau de La Malle, ibid. I, p. 124.

15. Dureau de La Malle, ibid. p. 196 - I, p. 110 - I, p. 298 - I, p. 298.

16. Dureau de La Malle, ibid. II, p. 108.

17. Dureau de La Malle, ibid. I, p. 193.

18. Dureau de La Malle, ibid. I, p. 198.

19. Dureau de La Malle, ibid. II, p. 108.

20. Dureau de La Malle, ibid. I, p. 241.

21. Dureau de La Malle, ibid. I, p. 197.

22. Dureau de La Malle, ibid. II, p. 123.

23. Dureau de La Malle, ibid. I, p. 106.

24. Dureau de La Malle, ibid. II, p. 166.

25. Dureau de La Malle, ibid. I, p. 115.

26. Dureau de La Malle, ibid. II, p. 146-147.

27. Dureau de La Malle, ibid. I, p. 284-285.

28. Dureau de La Malle, ibid. II, p. 110.

29. Dureau de La Malle, ibid. II, p. 132.

30. Dureau de La Malle, ibid. II, p. 114. 
31. Dureau de La Malle, ibid. II, p. 137.

32. Dureau de La Malle, ibid. I, p. 113.

33. Dureau de La Malle, ibid. I, p. 92.

34. Dureau de La Malle, ibid. II, p. 63.

35. Dureau de La Malle, ibid. II, p. 81.

36. Dureau de La Malle, ibid. II, p. 98.

37. Dureau de La Malle, ibid. II, p. 62-63.

38. Dureau de La Malle, ibid. II, p. 200. C'est en quittant Alger qu'il nota ces sentiments dans sa relation de voyage : il n'y a aucune précision sur cet ami.

39. D'après sa fiche, disponible sur le site du CTHS [https://cths.fr/an/savant.php? id=106281], Desfontaines eut Jean-Jacques Rousseau comme élève au Muséum.

40. Dureau de La Malle, ibid. II, p. 5.

41. Annaba, l'ancienne Hippone, patrie de saint Augustin.

42. J.-M. Poiret, Lettres de Barbarie : 1785-1786, p. 157.

43. Planches numérisées, accessibles par un lien communiqué par le service des Herbiers Historiques du Muséum concernant l'Herbier Desfontaines (mars 2018).

44. «Près des rives d'un lac aux environs de La Calle ».

45. Dureau de La Malle, ibid. II, p. 3.

46. Dureau de La Malle, ibid. II, p. 58, Lettre à Lemonnier, 15 avril 1784.

47. Jean-André Peyssonnel. Voyage dans les régences de Tunis et d'Alger, Présentation et notes de Lucette Valensi.

48. Dureau de La Malle, ibid. II, p. 58, Lettre à Lemonnier, 15 avril 1784.

49. Dureau de La Malle, ibid. Peyssonnel avait fait la même remarque : vol. I, p. 84

50. Dureau de La Malle, ibid. II, p. 167.

51. Dureau de La Malle, ibid. II, p. 176.

52. Dureau de La Malle, ibid. II, p. 80.

53. Dureau de La Malle, ibid. II, p. 125.

54. Dureau de La Malle, ibid. II, p. 183.

55. Dureau de La Malle, ibid. II, p. 82.

56. Dureau de La Malle, ibid. I, p. 230.

57. Dureau de La Malle, ibid. I, p. 227.

58. Dureau de La Malle, ibid. I, Préface p. XIX.

59. Dureau de La Malle, ibid. II, p. 533.

60. Dureau de La Malle, ibid. I, p. 7.

61. Dureau de La Malle, ibid. II, p.54. Les observations communiquées par l'abbé Teissier provenaient des notes envoyées par le consul de France à Alexandrie.

62. Dureau de La Malle, ibid. II, p. 53.

63. Les trois frères : Antoine de Jussieu (1686-1758), nommé professeur de botanique au Jardin du Roi en 1709 ; Bernard de Jussieu (1699-1777), également nommé professeur de botanique au Jardin du Roi en 1722 ; Joseph de Jussieu (1704-1779), qui participa à l'expédition au Pérou de La Condamine. 
64. R. Desfontaines, À la mémoire de Joseph Pitton de Tournefort. Choix de plantes du Corollaire des Instituts de Tournefort, publiées d'après son herbier et gravées sur les dessins originaux d'Aubriet.

65. Dureau de La Malle, ibid. II, p. 131.

66. Dureau de La Malle, ibid. I, Préface, p. VIII.

67. Dureau de La Malle, ibid. II, page XXIII : Éloge historique de R. Louiche Desfontaines [...], lue à la séance publique de cette Académie le 11 septembre 1837.

68. "Flora Atlantica, sive historia plantarum quae in Atlante, agro Tunetano et Algeriensis crescunt ", Parisiis 1798-1799 : éd. Desgranges-Blanchon, Paris, 1798-1799.

69. «Flora Atlantica » = «Flore du pays des Atlantes». Il s'agit d'un peuple qu'Hérodote (424-425 av. J.-C.) plaçait dans la partie septentrionale de l'Afrique, s'étendant du Maroc au reste du Maghreb.

70. Dureau de La Malle, ibid. II, Préface page XLIV : Desfontaines publia la Flora atlantica, en 4 volumes, en 1798-1799.

71. Dureau de La Malle, ibid. II, Préface p. XXII.

72. Dureau de La Malle, ibid. II, Préface, p. XXX : Histoire des arbres et arbrisseaux qui peuvent être cultivés en pleine terre sur le sol de France.

73. Dureau de La Malle, ibid. II, p. XXVII.

74. Dureau de La Malle, ibid. II, p. XXVIII.

75. Ibid.

76. Document Gallica : «Discours de M. Adrien de Jussieu, membre de l'Académie, prononcé aux funérailles de M. Desfontaines le 18 novembre 1833, A. de Jussieu, «Funérailles de M. Desfontaines ", dans Collection de l'Académie royale des sciences, éd. Didot, Paris, 1833, 12 p. Discours prononcé le 18 novembre 1833.

77. Dureau de La Malle, ibid. II, p. XXIX.

\section{RÉSUMÉS}

$\mathrm{Au} \mathrm{XVIII}^{\mathrm{e}}$ siècle, les naturalistes Jean-André Peyssonnel (1694-1759) et René Desfontaines (1750-1833) partirent vers la Barbarie, actuelle Afrique du Nord, pour y étudier tout ce qui touchait à l'histoire naturelle de ces pays, la flore en particulier. Sur le terrain, ils connurent souvent, malgré la protection diplomatique dont ils jouissaient, de difficiles conditions de voyage: insécurité, hostilité des populations à l'égard des chrétiens, qui s'ajoutaient aux conditions climatiques de ces pays au relief souvent inhospitalier. Mais rien ne les empêcha d'accomplir leur mission: récolter, rassembler, puis transmettre leurs observations et notes diverses aux académies et sociétés savantes avec lesquelles ils étaient en relation. Ainsi s'élaboraient des savoirs nouveaux, qui étaient diffusés d'abord au sein de la communauté scientifique puis en direction du grand public par l'enseignement et les publications. Peyssonnel et Desfontaines furent de grands acteurs de la transmission des savoirs. 
AUTEUR

GENEVIÈVE FALGAS

Docteur en Histoire, membre associé du laboratoire France, Amériques, Espagne, Sociétés,

pouvoirs, acteurs (FRAMESPA, UMR 5136, université Toulouse - Jean-Jaurès/CNRS) 


\title{
Les herbiers artificiels de Marie Fortier : un type méconnu de modèles botaniques
}

\author{
Florence Tessier
}

1 La botanique, la "science aimable», a souvent été présentée comme une science plus ouverte aux femmes que les autres. L'histoire de la botanique comporte pourtant encore peu de figures féminines, pour des raisons et par des mécanismes qui commencent à être abordés dans l'historiographie récente. Ainsi de 1854 à 1900, la Société botanique de France, acteur majeur de la communauté des botanistes, ne compta que très peu de femmes : 26 sur 1476 membres $^{1}$. Les travaux de Sarah Erman confirment que La féminisation des sociétés de botanique de France et de Belgique (1875-1940) ${ }^{2}$ s'est effectuée tardivement, début $\mathrm{xx}^{\mathrm{e}}$ siècle, et qu'elle avait été plus ancienne et plus massive en Belgique.

2 En listant ces vingt-six pionnières afin d'essayer de comprendre les causes et les ressorts de leur intérêt pour la botanique, l'une d'entre elles attira particulièrement mon attention. L'annonce de son admission parue dans le Bulletin de la Société de 1888 se lit ainsi :

«Mlle Fortier, fabricante d'herbiers artificiels, boulevard Poissonnière, 20, à Paris,

présentée par MM. Duval et Malinvaud. ${ }^{3}$ "

3 Que sont ces herbiers artificiels? Qui était cette $\mathrm{M}^{\text {lle }}$ Fortier, pourquoi s'intéressait-elle à la botanique au point de solliciter l'entrée dans cette société savante ? Telles sont les questions que soulevait cette mention énigmatique.

\section{« Herbiers artificiels » et modèles de botanique}

\section{Herbiers de plantes sèches et herbiers d'illustrations}

4 L'expression «herbiers artificiels " se trouve dans la littérature botanique avec plusieurs significations. Leur point commun est de se référer à un objet conçu comme 
un substitut des herbiers de plantes sèches comme moyen d'étude ou d'enseignement de la botanique.

5 Toute étude, tout enseignement du vivant doit composer avec la caducité et la temporalité de son sujet. La plante vivante suit ses rythmes propres et n'est pas constamment disponible ; le jardin botanique, limité par la place disponible et le climat, ne peut viser à l'exhaustivité. Les botanistes ont dû remédier à ces inconvénients en se basant sur les deux substituts de la plante vivante que sont les herbiers de plantes sèches et les illustrations.

6 Complémentaires, les herbiers et l'iconographie botanique ont évolué en parallèle dès le $\mathrm{XVI}^{\mathrm{e}}$ siècle, partageant souvent les mêmes étagères, les mêmes reliures ou les mêmes liasses, voire les mêmes pages : quelques herbiers anciens voient ainsi leurs spécimens enrichis de dessins précisant forme et couleur des fleurs ou des racines. Ils ont aussi partagé les mêmes dénominations : « herbarium », « herbal » ou " herbier », si bien que la littérature botanique de la fin du XvIII ${ }^{\mathrm{e}}$ siècle doit trouver des termes pour établir la distinction.

«On distingue de deux sortes d'herbiers; les uns, que l'on nomme herbiers naturels, parce qu'ils sont composés de plantes desséchées; et les autres, que l'on appelle herbiers artificiels, parce qu'ils sont composés de dessins, de peintures ou de gravures, coloriées ou non coloriées. ${ }^{4}$ "

7 Le terme d'« herbier artificiel » a ensuite été utilisé spécifiquement pour les techniques d'iconographie ne passant pas par le dessin mais tirées directement de la plante, comme les impressions naturelles ${ }^{5}$, ou au xix ${ }^{e}$ siècle les photogrammes obtenus par exposition de plantes sur du papier photosensible.

\section{Modèles 3D en botanique}

8 Herbiers et illustrations ont aussi leurs limites, soulignées par les botanistes euxmêmes: la plupart des plantes perdent une partie de leurs caractéristiques une fois desséchées. Les couleurs disparaissent, les textures s'estompent et sont difficiles à percevoir, l'organisation spatiale des tiges et branches devient difficile à lire ; certaines plantes se plient difficilement au format d'une planche d'herbier, comme les palmes de palmiers.

9 La modélisation de plantes en trois dimensions a donc été appelée en renfort de l'enseignement de la botanique dès le $\mathrm{xVIII}^{\mathrm{e}}$ siècle. Antoine-Laurent de Jussieu précise :

"Les dessins coloriés sont utiles [...] l'exécution des plantes en relief aura une utilité d'un autre genre et sera surtout recherchée pour les plantes grasses, telles que les ficoïdes, cierges, euphorbes, joubarbes, champignons, etc., dont on ne peut conserver les formes et les caractères dans les herbiers, et que l'on ne parvient même jamais à bien dessécher. Il serait surtout avantageux de fixer par une imitation exacte la forme et la structure des champignons, dont la plupart n'ont qu'une existence éphémère, et qui, dès lors, ne peuvent être présentés avec ordre aux élèves dans les démonstrations botaniques. ${ }^{6}$ "

L'ouvrage d'Anne-Marie Bogaert-Damin ${ }^{7}$ offre un bel aperçu de plusieurs types de modèles botaniques, qui diffèrent tant par les matériaux que par les buts des représentations. Cire, plâtre, papier-mâché, verre ont ainsi été utilisés du XVII ${ }^{\mathrm{e}}$ au $\mathrm{XX}^{\mathrm{e}}$ siècles pour représenter plantes entières, fruits, champignons, et fleurs y compris dans leurs détails anatomiques. 
11 La cire semble avoir été le premier matériau utilisé pour les modèles de plantes, et l'Italie est renommée dès le XVII ${ }^{e}$ siècle pour la qualité de ses sculptures ou moulages de plantes en cire. Bologne, puis Florence furent des pôles centraux de cette fabrication et le musée d'histoire naturelle de l'Université de Florence "La Specola» en conserve aujourd'hui encore une large collection'.

12 En France, la technique est magnifiquement illustrée par le Carporama, une collection de fruits exotiques réalisée vers 1802-1826 à l'île Maurice par Robillard d'Argentelle et maintenant conservée au Muséum national d'Histoire naturelle. Le même MNHN possède également depuis 1825 une collection de cires de champignons de Pinson', inspirées des planches de l'Histoire des champignons de France de Pierre Bulliard $(1752-1793)^{10}$. Des modèles botaniques en cire, représentant fleurs ou plantes entières, ont continué d'être produits tout au long du $\mathrm{XIX}^{\mathrm{e}}$ siècle, en Grande-Bretagne par exemple.

13 À l'instar des modèles en cire, les modèles en verre commandés en exclusivité par l'Université de Harvard en 1886 aux verriers Blaschka, déjà renommés pour leurs modèles zoologiques d'invertébrés, sont de véritables œuvres d'art.

14 Des matériaux plus courants comme la terre cuite émaillée ou le plâtre modelé ou moulé, puis peint, ont également été utilisés pour représenter fruits ${ }^{11}$ et champignons ${ }^{12}$. Avec les modèles en papier mâché de Louis Auzoux, « on atteint une production de type industriel. Ce docteur en médecine se fait connaître à partir de 1822 pour ses modèles d'anatomie humaine qu'il nomme " clastiques » car ils sont démontables ${ }^{13}$ ".

15 Il étend sa fabrication à la botanique à partir des années 1860 , avec des modèles à une échelle très grossie qui présentent la partie cachée des plantes, ou la disposition interne des fleurs ${ }^{14}$. Leur succès est dû au procédé de fabrication qui lui permettait d'obtenir "des modèles légers, résistants et reproductibles en série ${ }^{15}$ ", et moins coûteux que la cire; les archives de l'entreprise permettent de constater des ventes dans le monde entier ${ }^{16}$. Pour l'enseignement de tous niveaux, l'utilisation de ces modèles peut être perçue comme le signe d'une approche pédagogique nouvelle, en concurrence plutôt qu'en complément des supports traditionnels d'étude que sont l'herbier et l'illustration botanique ${ }^{17}$.

Des modèles Auzoux sont actuellement conservés dans de nombreuses institutions du monde entier, dont 32 modèles des années 1870-1880 dans les collections du Musée national de l'Éducation de Rouen ${ }^{18}$; ils ont été présentés dans plusieurs expositions récentes ${ }^{19}$.

17 Ce succès a suscité des concurrents : vers 1900-1910 la maison Émile Deyrolle fils a également à son catalogue environ 150 modèles botaniques similaires, mais qui semblent réalisés en staff ou en cire. Les modèles fabriqués de manière quasi industrielle par Robert Brendel (ca. 1821-1898) et Reinhold Brendel (ca. 1861-1927) ${ }^{20}$ sont également bien connus et largement diffusés. Basés sur ceux réalisés entre 1862 et 1867 par A. Lohmeyer à la demande du Pr. Cohn à l'Université de Breslau, ils se distinguent du procédé d'Auzoux par la diversité des matériaux utilisés (papier mâché mais aussi bois, toile, rattan, pulpe de canne, perles de verre, plumes...), mais sont également démontables. 


\section{Plantes artificielles et botaniques}

Les travaux publiés jusqu'ici sur ces différents types de modèles botaniques ne mentionnent pas l'utilisation des fleurs artificielles en tissu. L'idée existe pourtant depuis au moins le XVIII ${ }^{e}$ siècle. Pierre Bulliard y fait allusion à l'entrée «Herbiers » de son Dictionnaire élémentaire de botanique ${ }^{21}$.

\section{Le cabinet artificiel de Madame de Genlis}

$19{\text { C'est } \mathrm{M}^{\text {me }} \text { de Genlis }}^{22}$ qui nous donne le premier exemple détaillé d'une collection de plantes artificielles en tissu pour l'enseignement de la botanique. Cette pédagogue et auteure prolifique, bien connue pour avoir été chargée par Philippe d'Orléans, duc de Chartres, de l'éducation de ses enfants dont le futur roi Louis-Philippe, est adepte de méthodes modernes; les sciences et les techniques figurent en bonne part dans son programme éducatif.

20 Dans son "Discours de la botanique considérée relativement à l'éducation ${ }^{23}$ ", elle propose comme activité agréable et instructive de faire réaliser par les jeunes filles des herbiers de fleurs artificielles; elle dit en avoir fait un elle-même de 700 plantes exotiques, formant ainsi un « cabinet de botanique très curieux » dans les années 1780 .

21 Dans sa Maison rustique, pour servir à l'éducation de la jeunesse, ou Retour en France d'une famille émigrée..., elle précise :

«L'auteur de cet ouvrage a fait, il y a vingt-trois ou vingt-quatre ans, près de mille plantes artificielles, presque toutes exotiques, qui formoient un cabinet qui a été vu par un grand nombre de personnes; ces plantes, étoient d'une imitation que M. de Buffon trouva si parfaite, qu'il parut regretter vivement de n'avoir pas de fonds pour faire cette entreprise en grand, au Cabinet d'histoire naturelle ; il auroit voulu faire une galerie remplie de ces fleurs, pour servir à l'étude de la botanique durant l'hiver. Pour conserver toujours ces plantes placées dans des caisses, il faut les couvrir de châssis de gaze ou de canevas, le tout recouvert d'enveloppes détachées de toile cirée, et, quand on les découvre, ne pas les exposer au soleil. ${ }^{24}$ »

Cette collection aurait été vendue aux enchères lors des confiscations révolutionnaires.

\section{Le projet de Wenzel}

Cette idée de former une collection de plantes artificielles au Cabinet d'histoire naturelle, devenant en 1793 Muséum national d'Histoire naturelle, est relancée dans le débat public quelques années plus tard. En 1790, le journaliste, pédagogue et naturaliste Louis François Jauffret (1770-1840) publie une brochure intitulée Projet d'établir en France une manufacture de végétaux artificiels qui doit occuper utilement, dans le sein de la capitale, environ quatre mille ouvriers des deux sexes; d'après les nouveaux procédés de T.-J. Wenzel, fleuriste de la Reine.

Thomas Joseph Wenzel, «fleuriste » de la reine Marie-Antoinette, est un fabricant de fleurs artificielles, ayant employé jusqu'à 200 ouvriers et ouvrières. Les débuts de la Révolution et sa promotion d'un train de vie plus sobre et vertueux sont sans doute préjudiciables à son industrie de luxe, et il tente ainsi de mettre son art au service de la science et de l'éducation du peuple. Le journaliste Charles de Villette s'enthousiasme pour la proposition : 
«Représenter la nature telle qu'elle est; construire une plante telle que nous la voyons dans le site où elle croît; saisir avec précision la forme de ses feuilles, ses échancrures et ses fibres; rendre jusqu'au nombre d'étamines qui distinguent chaque espèce : telle est l'entreprise que ce Naturaliste veut créer en France. [...] Le Cabinet d'histoire naturelle de Paris est sans doute une collection magnifique. Mais ne pourrions-nous pas dire qu'il est moins le sanctuaire de la nature, que son vaste tombeau ? [...] Combien nos galeries artificielles seraient plus animées $!^{25} »$ projet aurait été bien accueilli ${ }^{26}$ par Bernardin de Saint-Pierre, le Dr Guillotin, et même Jussieu :

«Aussi des savans distingués par leur goût pour les arts ont-ils donné les témoignages les plus encourageans à $\mathrm{M}$. Wenzel. La feuille du cultivateur reconnoit l'importance de sa découverte pour l'agriculture. M. Bernardin de Saint Pierre, dont le jugement est d'un si grand poids dès qu'il s'agit de peindre la nature, déclare que le projet de manufacture de végétaux artificiels lui paroit mériter d'être favorisé particulièrement par l'assemblée nationale. »

Il ne se réalise pourtant pas. Wenzel conserve une certaine activité comme fabricant et enseignant de fleurs artificielles ${ }^{27}$, mais se lance également comme entrepreneur de spectacles : il donne des fêtes, des concerts et des spectacles de lanterne magique dans le «Pavillon de l'échiquier» qu'il a fait construire sur un terrain du faubourg Poissonnière. À la Restauration, son gendre Jean-Baptiste Prévost reprend l'entreprise de fleurs artificielles sous le nom Prévost-Wenzel, en activité jusqu'en 1844.

\section{Fleurs artificielles pour l'enseignement de la botanique}

La fabrication de plantes artificielles en tissu comme modèles botaniques reste discrètement présente tout au long du XIX $\mathrm{X}^{\mathrm{e}}$ siècle, aussi bien en France où l'industrie de la fleur artificielle pour la mode et la décoration est particulièrement développée, qu'ailleurs en Europe.

Les rapports des expositions annuelles des produits de l'industrie permettent de suivre l'évolution au cours du XIX ${ }^{e}$ siècle de l'industrie des fleurs artificielles, qui occupe à Paris des milliers puis des dizaines de milliers d'ouvriers et se diversifie de plus en plus. À partir de 1839, une catégorie du concours de l'Exposition est réservée aux « fleurs pour la botanique ", à côté des fleurs artificielles pour la mode. Les lauréats restent à peu près les mêmes dans les deux catégories : Constantin est primé en 1844 et 1851; M. et $\mathrm{M}^{\text {me }}$ de Laëre ${ }^{28}$ en $1844,1849,1865$; Mme Fürstenhoff ${ }^{29}$ de Paris, obtient une médaille d'argent en 1849 et 1851 ; $\mathrm{M}^{\text {me }}$ Vény, de Brest, une médaille de bronze en 1849. $\mathrm{M}^{\mathrm{me}}$ Marie-Rose Vény a également essayé d'obtenir la reconnaissance de la communauté scientifique en présentant en 1846 à la Société de pharmacie une collection de plantes artificielles dans le but de servir aux démonstrations botaniques. Le rapport est très favorable et estime que « madame Vény a fait pour l'étude du règne végétal ce que le Dr Auzou a fait [...] pour la science de l'anatomie ${ }^{30} »$. Malgré l'existence de ces produits médaillés, les modèles botaniques en fleurs artificielles semblent être restés assez peu répandus. 


\section{Marie Fortier}

\section{Débuts}

L'entreprise de Marie Fortier entre 1885 et 1914 montre une tentative plus ambitieuse de lancer en France une production de fleurs artificielles pour l'enseignement de la botanique. Quel parcours a pu l'amener à la création de ces supports pédagogiques ? Ses origines sont assez modestes, et éloignées de la communauté scientifique dont elle va chercher à se rapprocher.

Née à Paris le $1^{\text {er }}$ avril 1844, Marie Flavie Fortier est probablement ${ }^{31}$ la fille de Julien Hippolyte Fortier (1816-1896), désigné comme employé, et issu d'une famille de la petite bourgeoisie. De ses parents ${ }^{32}$ morts à Cormeilles-en-Parisis, il a hérité en 1863 une petite succession dont une maison à Paris en indivision avec sa sœur, ses neveux, et son frère. Celui-ci, François Alphonse Fortier (1825-1882), tapissier et négociant à Paris, est connu comme un des premiers photographes. Ayant épousé Anne Siredey, Julien Hippolyte Fortier ${ }^{33}$ en a au moins quatre enfants : deux filles et deux fils ${ }^{34}$, dont Marie Flavie semble être la cadette.

Sans autre éclairage sur ses années de jeunesse, nous pouvons supposer qu'elle a appris jeune la fabrication des fleurs artificielles, peut-être dans un contexte familial: les ouvrières se formaient très tôt, travaillant soit en ateliers soit à domicile. Des hommes travaillaient aussi dans le secteur, plutôt aux étapes de préparation des étoffes et des pièces (apprêt, teinture, découpage à l'emporte-pièce) ou de fabrication des outils spécialisés ; l'assemblage des fleurs elles-mêmes étant plutôt une tâche féminine ${ }^{35}$.

La spécialisation de Marie Fortier dans les modèles botaniques lui a imposé d'étendre son savoir-faire au-delà des fleurs horticoles habituelles et de créer outils et techniques pour un plus vaste choix de plantes; elle demandait donc une longue expérience du métier d'ouvrière-fleuriste. Ses herbiers sont décrits ainsi :

"Ce sont des boîtes à couvercle en verre contenant un certain nombre de plantes

artificielles accompagnées de leur nom scientifique et de leur nom usuel..$^{36}$ ”

Dans une plaquette intitulée " Notice sur les collections artificielles ${ }^{37}$ ", elle en attribue l'idée à « un de nos savants agronomes » qu'elle ne nomme pas; elle se donne comme but d'améliorer la connaissance des plantes utiles ou nuisibles pour les enfants des campagnes, en proposant des modèles qui permettent aux enseignants des écoles rurales de présenter les plantes en toutes saisons.

C'est sans doute pour assurer la promotion de ses herbiers artificiels qu'elle entre à la Société nationale et centrale d'horticulture de France comme "Dame patronnesse », le titre attribué aux femmes membres. Cela lui permet de présenter son œuvre dans les expositions annuelles de cette société où elle est souvent remarquée et primée dans la catégorie Instruction horticole, par exemple pour les années 1883, 1886, 1890 et $1893^{38}$.

Elle expose aussi ailleurs: en 1885, elle obtient une médaille d'or à l'exposition de l'Association horticole lyonnaise pour une « collection fort belle de plantes artificielles pouvant servir à l'enseignement horticole ${ }^{39}$ ". La même année, à l'Exposition du travail au Palais de l'Industrie, ses herbiers sont remarqués par la presse ${ }^{40}$. En 1887, une Médaille d'argent de $3^{\text {e }}$ classe lui est décernée par la Société d'horticulture du département de Seine-et-Oise pour ses «Fleurs et plantes artificielles ou séchées servant aux démonstrations et aux collections botaniques ». 
C'est aussi en 1887 qu'elle essaie une autre approche en se tournant vers le réseau des botanistes : elle suit les exercices pratiques du Laboratoire de botanique pratique du Muséum dirigé par Édouard Bureau qui la cite comme une "élève assidue ${ }^{41}$ ». Elle figure sur le registre des donateurs de l'Herbier du Muséum cette même année pour un " échantillon de feuille pour la parure » qui ne nous est pas conservé.

Quelques mois plus tard, elle entre à la Société botanique de France. Est-ce grâce à l'encouragement de Clotaire Duval, vice-secrétaire de la Société botanique et également membre de la Société nationale d'Horticulture ? C'est en effet lui qui la parraine, avec Ernest Malinvaud, alors secrétaire. Elle semble en être restée membre quelques années, au moins jusqu'en 1894.

\section{Succès et déceptions : les écoles de la ville de Paris et le contrat Hachette}

Dans sa Notice, Marie Fortier présente ses herbiers comme destinés aux écoles primaires et principalement rurales, une orientation confirmée par les articles de presse qui mettent son travail en valeur :

« $\mathrm{M}^{\mathrm{Ile}}$ Fortier [...] l'a conçue et l'a créée pour servir à l'enseignement de la botanique dans nos écoles rurales, et l'idée nous paraît essentiellement pratique. De toutes les connaissances, celle des plantes qui les entourent s'impose en première ligne à nos populations. ${ }^{42}$ "

40 Le matériel pédagogique pour la botanique bénéficie d'une conjoncture très favorable: les lois Jules Ferry instaurant l'instruction obligatoire, gratuite et laïque sont accompagnées d'une réforme des programmes qui fait entrer les sciences, dont les sciences naturelles, au programme de l'enseignement primaire comme une matière obligatoire et non plus facultative. Les sciences naturelles ont également une part accrue dans les programmes de l'enseignement secondaire depuis 1880 : la botanique y est enseignée en classe de cinquième. Pour l'enseignement secondaire féminin (loi Camille Sée de 1880, programme de 1882), le programme est allégé en sciences, mais la botanique reste ${ }^{43}$.

41 Ces réformes scolaires suscitent un important développement de l'offre éditoriale, mais aussi d'un matériel pédagogique varié. Les instituteurs sont en effet encouragés à baser leurs leçons sur les objets davantage que sur les livres, à privilégier les leçons de choses, et à constituer de petits musées scolaires ${ }^{44}$ comprenant roches, fossiles, boîtes d'insectes, animaux empaillés... et herbiers. Ils se voient donc proposer une vaste gamme de matériel illustré : cartes, tableaux pédagogiques, images, par les éditeurs de manuels scolaires ou des marchands spécialisés.

42 Si le marché est favorable, il reste difficile pour une entrepreneuse seule, comme semble l'être Marie Fortier, d'assurer à la fois la fabrication et la commercialisation. Aussi se tourne-t-elle vers un partenaire capable de lui assurer la visibilité dont elle a besoin pour augmenter ses ventes : l'éditeur Hachette, déjà positionné sur le marché de l'édition scolaire et du matériel pédagogique.

43 Le contrat entre Hachette et Marie Fortier ${ }^{45}$ prend effet au $1^{\mathrm{er}}$ janvier 1887 pour une durée initiale de deux ans. Il donne à Hachette « le droit exclusif de vente » (excepté les commandes faites par la Ville de Paris) sur 119 plantes listées en annexe avec leur prix, qui vont de $1 \mathrm{~F}$ (par ex. pour la petite pervenche), à $10 \mathrm{~F}$ (pour la spirée ulmaire), en 
échange de l'engagement de les porter à leur catalogue et d'en soigner «la vente de leur mieux ».

C'est peut-être le soutien de la maison Hachette qui lui permet d'exposer à l'Exposition universelle internationale de 1889, comme le commente une revue suisse destinée à un public d'enseignants :

«Ces herbiers ont l'avantage de représenter la fleur fraîche qui ne se fane pas. La

fleur se prête si bien aux leçons de choses qu'il est utile de pouvoir s'en servir

même en hiver. ${ }^{46}$ "

Primés ${ }^{47}$ et longuement commentés par le jury du matériel pédagogique pour l'enseignement primaire, ils sont aussi mentionnés dans la catégorie dédiée à l'enseignement secondaire, avec la mention « collections de plantes artificielles pour l'étude de la botanique adoptées par le ministère pour les lycées et collèges de jeunes filles ${ }^{48} »$.

Grâce à la visibilité que lui donnent les diverses expositions, Marie Fortier constate que «l'herbier ne peut être demandé que par les personnes qui l'auront $v^{49}$ »; et que la mention au catalogue de Hachette ne semble pas suffisante pour susciter les ventes. Dans un échange de courriers avec l'éditeur, elle explique que ses contacts lui permettent de se faire connaître en province et même à l'étranger, mais qu'il lui est difficile de rediriger ensuite les acheteurs potentiels vers Hachette. Elle demande donc une modification du contrat afin de pouvoir vendre elle-même, parallèlement et au même prix. Au lieu de l'avenant qu'elle souhaite, Hachette lui propose une nonreconduction du contrat qui s'achève donc le 31 décembre 1889.

47 Nous avons dit que ce contrat comprenait une exception à la clause d'exclusivité : si Marie Fortier visait tout d'abord les écoles rurales, c'est à Paris même qu'elle obtient ses premières commandes. Dès 1886, la mairie de Paris achète des exemplaires de son herbier pour six écoles supérieures et dix écoles primaires. Le rapport présenté suite à cette expérience au Conseil municipal en $1888^{50}$ est très favorable : les enseignants qui l'ont utilisé en sont très contents, et de nouveaux achats sont demandés.

En 1891, elle fournit aussi des fleurs (pour 300 F) pour les dioramas du Musée forestier de la Ville de Paris $^{51}$ qui vient d'être créé dans l'ancien pavillon des forêts de l'Exposition de 1889, installé sur l'île de Bercy du lac Daumesnil au bois de Vincennes ${ }^{52}$.

En 1899, elle vend " 10 exemplaires à 121 francs $^{53}$ " pièce ; on retrouve également la trace d'achats en 1902, 1904 et 1906. Sollicitant de nouveau la mairie de Paris en 1907 pour l'acquisition de "fleurs artificielles pour l'enseignement du dessin », elle obtient l'achat de 75 collections de fleurs (série 3 ) et de 30 collections (série $n^{\circ} 4$ ) pour un total de $1275 \mathrm{~F}$ pour les écoles de filles, $600 \mathrm{~F}$ pour les écoles de garçons. Cet achat est renouvelé en 1908 pour $974 \mathrm{~F}$ avec cette fois un rééquilibrage en faveur des écoles de garçons des arrondissements de Sceaux (34 collections) et de Saint-Denis.

On constate ainsi un glissement de l'usage de ses fleurs artificielles, des modèles botaniques aux modèles pour cours de dessin. En 1914, Marie Fortier fait paraître une annonce publicitaire pour ses fleurs « admises pour l'enseignement dans les écoles de la Ville de Paris» dans une revue destinée aux professeurs de dessin ${ }^{54}$, en tant qu' « enseignante à l'École technique de fleurs 9 rue Notre-Dame de Bonne Nouvelle ». Il semble donc qu'elle ait étendu son activité à l'enseignement professionnel du fleuriste artificiel. 
51 Après cette date, je ne retrouve plus trace de son activité ; elle a alors 64 ans. Il ne semble pourtant pas que son travail lui ait permis de s'assurer une retraite confortable : à partir de 1926 au moins, elle réside à Neuilly-sur-Seine dans une maison de retraite de l'Assistance publique ${ }^{55}$. Elle y meurt à 87 ans, sans héritiers, le 19 avril $1931^{56}$.

\section{Modèles en fleurs artificielles dans les collections muséales :}

52 S'ils semblent avoir été uniques en France, les herbiers artificiels de Marie Fortier avaient une concurrence au niveau européen : dès 1884, des plantes artificielles en tissu comme modèles de botaniques étaient produites à Breslau par $\mathrm{M}^{\mathrm{me}}$ Christina Jauch sous le contrôle scientifique du Dr B. Stein, inspecteur du jardin botanique, sous le nom de "Flora artefacta »; cette production bénéficiait du soutien et des achats du ministère de l'Instruction publique de Prusse ${ }^{57}$ et était proposée dans les catalogues de marchands spécialisés à côté des modèles Brendel. L'Université d'Amsterdam en possède une collection de 42 pièces $^{58}$; on en trouve également dans la collection de modèles de l'Université de Coïmbra au Portugal ${ }^{59}$.

Je n'ai pas retrouvé en France d'exemplaires des herbiers de Marie Fortier, pourtant vendus par centaines à Paris. La fragilité de ces fleurs de tissu, même enfermées dans des boîtes à couvercle de verre, en est une explication. La prise de conscience de l'intérêt historique du matériel pédagogique ancien est par ailleurs assez récente, et toutes les collections survivantes n'ont pas nécessairement été identifiées.

Un groupe de recherche espagnol, le CEIMES, a étudié et mis en valeur les riches collections de matériel pédagogique ancien de plusieurs établissements d'enseignement secondaire de Madrid $^{60}$. Pour la botanique, l'Instituto Cardenal Cisneros possède des modèles Auzoux, des modèles Deyrolles, des affiches pédagogiques, et onze boîtes d'herbiers artificiels de Marie Fortier, étiquetés «Hachette et $C^{\mathrm{ie}} . .$. Collection Marie Fortier ». Soigneusement conservés, ils ont probablement été achetés par le Professeur Manuel María José de Galdo López de Neira (1825-1895) lors d'un voyage à Paris, peutêtre pour l'Exposition universelle de 1889. La délicatesse et la fidélité des modèles proposés expliquent les éloges qu'ils suscitaient dans les expositions.

Ainsi peut-on compléter les types de modèles botaniques tridimensionnels par les fleurs artificielles en tissu. Ils semblent avoir rencontré un succès moindre que les modèles de cire ou de papier mâché, et ont plus rarement été conservés. Outre leur fragilité, les raisons pourraient inclure la proximité de cette production avec les arts décoratifs et la mode, et le fait que ses productrices étaient des femmes par cela même plus éloignées de la communauté scientifique.

56 Ainsi, malgré ses efforts pour tisser des liens avec la communauté scientifique, au Muséum ou à la Société botanique de France, malgré l'intérêt suscité par ses herbiers dans les expositions, Marie Fortier ne semble pas s'être insérée dans un réseau fort: elle avait probablement contre elle son manque d'activité scientifique; mais peut-être aussi son genre et ses origines sociales. Ses ventes à la Ville de Paris mais aussi à des acheteurs internationaux lui ont permis de vivre de son art, mais pas d'atteindre une renommée durable. 

fleurs artificielles se sont plus rarement conservés dans les collections subsistantes de matériel pédagogique scientifique, ce qui explique qu'ils soient restés méconnus. Les plantes artificielles ont connu une autre utilisation plus répandue dans les dioramas de musées, un dispositif muséographique lui-même en voie de disparition. nos jours, l'idée de former des herbiers de plantes artificielles n'a pas totalement disparu; elle est cependant surtout visitée par des artistes, fascinés par le double jeu entre l'art et la science, entre la nature et l'artifice.

\section{BIBLIOGRAPHIE}

AMARAL Raquel Filipa de Almeida, «Modelos didáticos na museologia e ensino da botânica na Universidade de Coimbra », mémoire de maitrise en muséologie et patrimoine culturel, Coimbra, 2011.

BALDINAI Enrico, « Pomological models », Il Museo di storia naturale dell'Università degli studi di Firenze. II, Le collezioni botaniche, Firenze, Firenze University Press, 2009.

BELHOSTE Bruno, Les sciences dans l'enseignement secondaire français. Textes officiels 1789-1914, Paris, INRP et Economica, 1995.

BOGAERT-DAMIN Anne-Marie, Voyage au cœur des fleurs : Modèles botaniques et flores d'Europe au XIX siècle, Namur, Presses universitaires de Namur, 2007.

BULLIARD Pierre, Dictionnaire élémentaire de botanique ou exposition par ordre alphabétique, des préceptes de la botanique..., Paris, Didot le Jeune, 1783.

BUREAU Ed., POISSON Jules, FRANCHET A., MAURY M., « Laboratoire de botanique pratique du Muséum d'histoire naturelle (classifications et familles naturelles) », Rapport sur l'École pratique des hautes études, 1887-1888, p. 157-160.

CHANAL Nicolas, «L'anatomie clastique de Louis Auzoux : une entreprise au XIX siècle, thèse de doctorat de médecine vétérinaire ", thèse de doctorat de médecine vétérinaire, École nationale vétérinaire d'Alfort, 2014.

[URL : http://theses.vet-alfort.fr/telecharger.php?id=1742]

CHARPIN André, TIMBAL Jean, « Liste des membres de la Société botanique de France de son origine (1854) à 2003 (150 ans) », Acta Botanica Gallica, 154, 3, 2007, p. 423-492.

CHARPIN André, « Dictionnaire des membres de la Société botanique de France », Journal de Botanique, Société Botanique de France, $\mathrm{n}^{\circ}$ hors-série, 2018.

COCKS Margaret Maria, « $\mathrm{D}^{\mathrm{r}}$ Louis Auzoux and his collection of papier-mâché flowers, fruits and seeds ", Journal of the History of Collections, vol. 26, $n^{\circ} 2,2014$, p. 229-248.

CONIHOUT Isabelle de, Botanica in originali : livres de botanique réalisés en impression naturelle $d u X^{e} I^{e} a u$ XIX $X^{e}$ siècles : exposition, 5 mai-12 juin 1993, Bibliothèque nationale, Paris, Bibliothèque nationale, 1993. 
CUCCUINI Piero, « The fungi of Jean-Baptiste Barla, artist and naturalist », Il Museo di storia naturale dell'Università degli studi di Firenze. II, Le collezioni botaniche, Firenze, Firenze University Press, 2009. DEGUEURCE Christophe, Corps de papier : l'anatomie en papier mâché du docteur Auzoux, Paris, La Martinière, 2012.

EGGINGER Johann-Günther, Belles plantes ! Modèles en papier mâché du $D^{r}$ Auzoux, Réseau Canopé, 2018. Ouvrage publié à l'occasion de l'exposition du même nom au Musée national de l'Éducation, Rouen, du 25 mai 2018 au 25 février 2019.

"L'Enseignement par les fleurs artificielles ", La Nature. Revue des sciences et de leurs applications aux arts et à l'industrie. Journal hebdomadaire illustré. $27^{\mathrm{e}}$ année, $1899,2^{\mathrm{e}}$ semestre, $\mathrm{n}^{\circ} 1358$ à 1383 , p. 155.

FIORINI Graziana, MAEKAWA Luana et STIBERC Peter, « Save the Plants: Conservation of Brendel Anatomical Botany Models », The Book and Paper Group Annual, vol. 27, 2008, p. 25-45. [URL : http://cool.conservation-us.org/coolaic/sg/bpg/annual/v27/bp27-07.pdf] FORTIER Marie, Notice sur les collections artificielles, Paris, Imp. Chaix, 1885.

"L'Instruction publique à l'Exposition universelle de Paris ", Éducateur et bulletin corporatif: organe hebdomadaire de la Société Pédagogique de la Suisse Romande, band 26, 1890, p. 385.

GENLIS $\mathrm{M}^{\mathrm{me}}$ de, "Discours de la botanique considérée relativement à l'éducation », Discours moraux sur divers sujets, et particulièrement sur l'éducation, Paris, 1802.

GENLIS $\mathrm{M}^{\mathrm{me}}$ de, Maison rustique, pour servir à l'éducation de la jeunesse, ou Retour en France d'une famille émigrée..., Paris, Maradan, 1810.

KERAUDREN-AYMONIN Monique, «Le Carporama de L. M. A. de Robillard d'Argentelle », dans Bulletin du Muséum national d'Histoire naturelle, $4^{\text {e }}$ série, t. 1, 1979, Miscellanea, p. 117-149.

KERAUDREN-AYMONIN Monique et AYMONIN Gérard G., « Une œuvre scientifique et artistique unique : le Carporama de L. M. A. de Robillard d'Argentelle », Bulletin de la Société Botanique de France, Lettres Botaniques, vol. 131, nº 4-5, 1984, p. 243-246.

LAËRE L. J. de, La fleuriste des salons : traité complet sur l'art de faire les fleurs artificielles, Paris, Goubaud, s.d.

Exposition universelle internationale de 1889 : rapports du Jury international. Groupe II, $1^{\text {re }}$ partie, Éducation et enseignement, classes 6, 7, 8, et 6-7-8, Paris, Imprimerie Nationale, 1891. OLSZEWSKI Margaret, « Designer Nature: The Papier-mâché Botanical Teaching Models of Dr Auzoux in Nineteenth-Century France, Great Britain and America » Thèse en Histoire et Philosophie des sciences, Cambridge University, 2009.

NEPI Chiara, «I modelli in cera delle piante e delle tavole didattiche », Il Museo di storia naturale dell'Università degli studi di Firenze. II, Le collezioni botaniche, Firenze, Firenze University Press, 2009, p. 216-227.

RUIZ Guillaume, « Les modèles en papier mâché du Docteur Auzoux au musée de l'École vétérinaire d'Alfort », Thèse de doctorat vétérinaire, École vétérinaire de Maisons-Alfort, 2009. [URL : http://theses.vet-alfort.fr/telecharger.php?id=1211]

« Rapport fait le 2 décembre 1846 sur des plantes artificielles présentées par $\mathrm{M}^{\text {me }}$ Vény », Journal de pharmacie et de chimie, volume XI (1847), p. 39.

VILLETTE Charles de, Lettres choisies de Charles Villette sur les principaux évènemens de la Révolution, Paris, impr. de Clousier, 1792. 
VILLETTE Charles de, «Projet d'établir en France une manufacture de végétaux artificiels, qui doit occuper utilement, dans le sein de la capitale, environ quatre mille ouvriers des deux sexes ; d'après les nouveaux procédés de T.-J. Wenzel, fleuriste de la Reine, rédigé par L. F. JAUFFRET, invo de 136 p. A Paris, 1790... ", L'esprit des journaux, francais et étrangers, tome IV, $20^{e}$ année, 1791, p. $152-159$.

WENZEL Thomas Joseph, L'art d'imiter les végétaux : conformément aux modèles exposés dans l'un des portiques du Palais national des Sciences et des Arts, les jours complémentaires de l'an X, d'après les nouveaux procédés, inventions et découvertes de T.J. Wenzel, fleuriste, breveté par la Reine en 1785. Courspratique et complet, en vingt-cinq leçons, proposé par souscription, Paris, 1802.

\section{NOTES}

1. A. Charpin et J. Timbal, « Liste des membres de la Société botanique de France de son origine (1854) à 2003 (150 ans) ».

2. Présentés en 2017 dans le cadre du séminaire "Femmes et savoir » des doctorantes du centre Alexandre Koyré.

3. « Séance du 10 février 1888 », Bulletin de la Société botanique de France, 1888.

4. P. Bulliard, Dictionnaire élémentaire de botanique ou exposition par ordre alphabétique, des préceptes de la botanique..., p. 98.

5. Voir I. de Conihout, Botanica in originali, livres de botanique réalisés en impression naturelle....

6. Cité par C. de Villette, «Projet d'établir en France une manufacture de végétaux artificiels... », p. 156-157.

7. A.-M. Bogaert-Damin, Voyage au cœur des fleurs : modèles botaniques et flores d'Europe au XIX siècle.

8. C. Nepi, «I modelli in cera delle piante e delle tavole didattiche », p. 216-227.

9. André-Pierre Pinson (1746-1828), plus connu pour ses cires d'anatomie humaine.

10. M. Keraudren-Aymonin, « Le Carporama de L. M. A. de Robillard d'Argentelle »; M. Keraudren-Aymonin et G. G. Aymonin, "Une œuvre scientifique et artistique unique : le Carporama de L. M. A. de Robillard d'Argentelle ».

11. E. Baldinai, « Pomological models », p. 229-235.

12. P. Cuccuini, « The fungi of Jean-Baptiste Barla, artist and naturalist », p. 245-249.

13. C. Degueurce, Corps de papier : l'anatomie en papier mâché du docteur Auzoux.

14. M. M. Cocks, « Dr Louis Auzoux and his collection of papier-mâché flowers, fruits and seeds ", p. 229.

15. G. Ruiz, «Les modèles en papier mâché du Docteur Auzoux au musée de l'École vétérinaire d'Alfort ", p. 9.

16. N. Chanal, «L'anatomie clastique de Louis Auzoux : une entreprise au XIX siècle ", p. 55-79.

17. M. Olszewski, «Designer Nature: The Papier-mâché Botanical Teaching Models of Dr Auzoux in Nineteenth-Century France, Great Britain and America ».

18. J. G. Egginger, Belles plantes! Modèles en papier mâché du Dr Auzoux. 
19. Exposition Prodiges de la nature, les créations du docteur Auzoux (1797-1880), févrieravril 2017, Université de Montpellier, commissaires Françoise Olivier et Numa Hambursin.

20. G. Fiorini, L. Maekawa, et P. Stiberc, «Save the Plants: Conservation of Brendel Anatomical Botany Models », p. 25-45.

21. P. Bulliard, Dictionnaire élémentaire de botanique..., p. 98.

22. Stéphanie Félicité du Crest, par son mariage comtesse de Genlis (1746-1830).

23. $\mathrm{M}^{\text {me }}$ de Genlis, "Discours de la botanique considérée relativement à l'éducation », p. 205.

24. $\mathrm{M}^{\mathrm{me}}$ de Genlis, Maison rustique, pour servir à l'éducation de la jeunesse, ou Retour en France d'une famille émigrée..., t. 2, p. 46.

25. C. de Villette, Lettres choisies [...] sur les principaux évènemens de la Révolution, p. $358-361$.

26. C. de Villette, "Projet d'établir en France une manufacture de végétaux artificiels », p. 156-157.

27. D'après sa brochure publicitaire: T. J. Wenzel, L'art d'imiter les végétaux [...]. Courspratique et complet, en vingt-cinq leçons, proposé par souscription.

28. $\mathrm{M}^{\text {me }}$ Louis Jacques de Laëre, née Joséphine Virginie Bouilly (1819-...) est aussi l'auteur du manuel La fleuriste des salons: traité complet sur l'art de faire les fleurs artificielles.

29. Emma Fürstenhoff née Lindengren (1802-1871), issue de la noblesse suédoise mais désargentée, se fit connaitre pour ses fleurs en cire, mais en fabriquait aussi en tissu ; elle se fixa à Paris vers 1850 .

30. « Rapport fait le 2 décembre 1846 sur des plantes artificielles présentées par $M^{\text {me }}$ Vény ", Journal de pharmacie et de chimie, p. 39.

31. L'État civil de Paris disparu dans les incendies de la Commune ne subsiste que sous une forme reconstituée où les filiations ne figurent pas, j'ai dû le recouper avec plusieurs autres sources.

32. Convention d'indivision entre les héritiers de François Jean Fortier et Marie Françoise Boussiard concernant une maison située 9, rue Saint-Georges, MC/ET/CXVII/ 1403, 11 février 1880. Archives nationales, Minutes et inventaires du notaire Lapalme $\mathrm{MC} / \mathrm{ET} / \mathrm{CXVII}$.

33. Né à Paris le 7 avril 1816, décédé le 4 septembre1896, sources: archives de Paris, État civil reconstitué et Gazette du Palais, p. 558, jugement du Tribunal civil de la Seine du 27 octobre 1898 concernant un désaccord entre M. Baradu, fleuriste, d'une part et Hippolyte, Alphonse et $\mathrm{M}^{\text {lle }}$ Marie Fortier d'autre part, sur le lieu de sépulture de $\mathrm{M}^{\text {me }}$ Baradu née Fortier.

34. Anne Marie Joséphine née le 15 septembre 1839 dans le $3^{\mathrm{e}}$ arrondissement, Julien Hippolyte né le 6 septembre 1841, Marie Flavie née le $1^{\mathrm{er}}$ avril 1844, $9^{\mathrm{e}}$ arrondissement ; source : archives de Paris, État civil reconstitué.

35. Les rééditions successives de 1829 à $1901 \mathrm{du}$ « Manuel du fleuriste artificiel... » chez Roret donnent une bonne idée de la profession.

36. «L'instruction publique à l'Exposition universelle de Paris », p. 385.

37. M. Fortier, Notice sur les collections artificielles, p. 1-4. 
38. Journal de la Société nationale d'horticulture de France, séance du 22 octobre 1883 , p. 641 ; ibid., 1886, p.673; "Compte-rendu de l'Exposition de mai 1890 », ibid. 1890, p. 699 ; « Documents relatifs à l'exposition de mai 1893 », ibid., 1893, p. 368.

39. Lyon horticole, Association horticole lyonnaise, 1885.

40. Le Temps, $25^{\mathrm{e}}$ année, $\mathrm{n}^{\circ} 8936,18$ octobre 1885, p. 2, col. 5 .

41. E. Bureau, J. Poisson, A. Franchet, «Laboratoire de botanique pratique du Muséum d'histoire naturelle (classifications et familles naturelles)», p. 157.

42. Le Temps, $25^{\mathrm{e}}$ année, $\mathrm{n}^{\circ}$ 8936, 18 octobre 1885, p. 2, col. 5.

43. B. Belhoste, Les sciences dans l'enseignement secondaire français. Textes officiels 1789-1914.

44. La maison Deyrolle en vend de tout constitués et de toutes tailles au début du $\mathrm{xx}^{\mathrm{e}}$ siècle.

45. Conservé avec un échange de courriers, dossier HAC 23.15, archives Hachette à l'IMEC «Institut Mémoires de l'Edition contemporaine ».

46. «L'instruction publique à l'Exposition universelle de Paris », p. 385.

47. Exposition universelle internationale de 1889: rapports du Jury international, p. 319-322.

48. Ibid. p. 547.

49. Dossier HAC 23.15, Archives Hachette, IMEC.

50. Procès-verbaux du Conseil municipal de Paris, 1888, p. 598 et 1078.

51. Gallica Rapports du conseil municipal de la Ville de Paris, et Rapports et documents, 1892, partie 2, p. 18.

52. Ce musée forestier a fermé dans les années 1920, voir Commission du vieux Paris, Compte-rendu de la séance plénière du 21 décembre 2017, https://cdn.paris.fr/paris/ 2019/07/24/ff51f2d9cca71791b0378c1248dce459.pdf

53. Rapports et documents... Conseil de Paris 1899, p. 199.

54. Le Dessin : organe mensuel de l'Association amicale des professeurs de dessin de la ville de Paris et du département de la Seine, 1914-01, $3^{\mathrm{e}}$ de couverture.

55. La maison de retraite Galignani au 89 bd Bineau accueille « 100 personnes des deux sexes, âgées de 60 ans révolus, respectables et de très bonne moralité ». La moitié des places disponibles est réservée à titre gracieux aux anciens libraires, imprimeurs, savants ou artistes français. Site officiel de la ville de Neuilly sur Seine : https:// www.neuillysurseine.fr/les-edifices-remarquables?BATI_NUM=15.

56. «Par jugement [...] envoi en possession des successions", Journal officiel de la République française, 4 mai 1933, p. 4682.

57. «L'Enseignement par les fleurs artificielles », p. 155.

58. http://academischecollecties.nl

59. R. F Amaral, "Modelos didáticos na museologia e ensino da botânica na Universidade de Coimbra », p. 10.

60. http://ceimes.cchs.csic.es/ 


\section{RÉSUMÉS}

Au-delà de la plante vivante, depuis ses origines la botanique scientifique s'est appuyée sur les herbiers de plantes sèches et les illustrations. Elle a aussi eu recours, particulièrement pour l'enseignement, à des modèles en trois dimensions fabriqués selon divers procédés et matériaux, dont ceux en cire et en papier mâché qui subsistent dans des collections muséales. Un autre procédé existant depuis la fin du $\mathrm{XVIII}^{\mathrm{e}}$ siècle, mais peu connu est la plante artificielle en tissu inspirée des fleurs artificielles pour la mode. De 1885 à 1914, une entrepreneuse parisienne, Marie Fortier, a commercialisé de tels « herbiers artificiels » comme matériel pédagogique pour l'enseignement primaire et secondaire. Elle crée des liens avec la Société nationale d'Horticulture de France et la Société botanique de France, et fournit durant une décennie les écoles primaires à Paris. Un exemple unique de ses œuvres subsiste à Madrid.

\section{AUTEUR}

\section{FLORENCE TESSIER}

Conservatrice des bibliothèques, Bibliothèque de botanique, Direction des bibliothèques et de la documentation, Direction générale déléguée aux collections, Museum national d'Histoire naturelle (MNHN) 


\title{
Le réseau français des
} collaborateurs du Cabinet zoologique de Varsovie pendant la seconde moitié du XIX ${ }^{\mathrm{e}}$ siècle

\author{
Piotr Daszkiewicz
}

L'auteur remercie le professeur Marie Palacio David, le docteur Dominika Mierzwa et le professeur Dariusz Iwan, de l'Institut et Musée de Zoologie de Pan de Varsovie, et M. Lâm PhanTranh des Archives des Frères Lazaristes pour leur aide.

\section{Origines du Cabinet zoologique de Varsovie}

1 Les débuts du Cabinet zoologique de Varsovie remontent à 1818. La décision d'acheter la riche collection zoologique du comte Sylwiusz Minkwitz (1772-1818) de Gronowice (Grunwitz) est à son origine ${ }^{1}$. À la mort de cet aristocrate silésien, la Commission d'Éducation décida d'acheter son cabinet, comptant à l'époque environ vingt mille spécimens, en vue de constituer une collection zoologique nécessaire à l'Université de Varsovie récemment fondée (en 1816); une création devenue possible suite à une courte libéralisation de la politique tsariste envers les Polonais. Dans le but d'évaluer la valeur de cet achat, ainsi que pour veiller au bon déroulement de la transaction et du transport, la commission délégua trois commissaires, Marek Antoni Pawłowicz (1789-1830), Józef Skrodzki (1789-1832) et Feliks Jarocki (1790-1865). Tous trois avaient été formés en France et particulièrement au Muséum national d'histoire naturelle (MNHN) de Paris. Jarocki devint ensuite le responsable du cabinet zoologique et garda d'étroites relations avec les naturalistes français ${ }^{2}$. Rappeler ce fait est important car, au cours de la période qui fait l'objet de cette présentation, la collaboration du cabinet zoologique avec les institutions et les scientifiques français avait ainsi déjà une longue tradition derrière elle ${ }^{3}$. 


\section{Władysław Taczanowski et l'âge d'or du Cabinet}

2 La seconde moitié du XIX siècle fut une période bien particulière pour la zoologie en Pologne. Dans ce pays, privé de son indépendance depuis 1795, les répressions qui suivirent les insurrections de 1830-1831 et 1863-1864 touchèrent fortement les institutions scientifiques. La fermeture des universités, l'interdiction des sociétés savantes, les pillages des bibliothèques, des musées et des collections scientifiques ainsi que les arrestations, les déportations en Sibérie ou encore l'émigration forcée, ont fortement marqué l'histoire de la science en Pologne. Paradoxalement, la zoologie polonaise connut, durant cette période hostile, un véritable âge d'or'.

Władysław Taczanowski (1819-1890) fut le principal artisan de ce succès 5 . Excellent ornithologue et arachnologue, il dirigea, à partir de 1862, le Cabinet zoologique de Varsovie. Fils d'un officier de la Grande Armée, originaire d'une famille appauvrie par les répressions russes, il est partisan du travail à la base, adversaire de la lutte armée pour l'indépendance car, en soutenant ses collègues insurgés, il pensait que cette attitude était condamnée à l'échec. Taczanowski, comme les "positivistes» de l'époque, considérait que c'était le développement de l'économie, de la science, et non la lutte armée, qui étaient nécessaires pour moderniser le pays et assurer son avenir. Taczanowski réussit à transformer le cabinet zoologique de petit musée provincial en une grande collection de renommée mondiale, et en un important centre de recherches scientifiques.

\section{Les contributeurs : mécènes, exilés et naturalistes voyageurs}

Privé de subventions publiques ou institutionnelles, il sut trouver des mécènes privés. Citons parmi eux Konstanty Branicki (1824-1884), riche aristocrate, mais aussi voyageur et naturaliste amateur. Sans sa grande générosité, mais aussi celle de son frère Aleksander, un tel développement de la zoologie aurait été impossible dans les conditions d'occupation russe de la Pologne. Les exilés polonais, et principalement Benedykt Dybowski (1833-1930), devinrent ainsi correspondants de cabinet et constituent une source précieuse d'information sur les animaux de Sibérie. Les émigrés en Amérique du Sud, principalement au Pérou, à l'instigation de Taczanowski, exploraient la nature néotropicale ${ }^{6}$. Parmi ces explorateurs, citons le nom de Konstanty Jelski (1837-1896), qui passa quatre ans en Guyane française et dix au Pérou. Par la voie de sa correspondance et de nombreux autres échanges, Taczanowski réussit ainsi à nouer un réseau de contacts avec un nombre important d'institutions naturalistes en Europe.

\section{Le Cabinet et la France}

5 La France fut pour lui un pays de prédilection. Francophone et francophile, Taczanowski se rendit à plusieurs reprises à Paris. Il y étudia les collections du Muséum national d'histoire naturelle et celle de la "Maison Verreaux ${ }^{7}$ ». Il y prépara une expédition scientifique en Algérie. Quant au comte Branicki, il était propriétaire du château de Montrésor, mais possédait également des biens immobiliers à Paris. Il fonda 
un musée privé d'histoire naturelle, principalement localisé dans ses propriétés en France, afin de préserver ces collections d'un nouveau pillage par les autorités d'occupation russe. Ce n'est qu'à l'indépendance de la Pologne, que la famille Branicki fit le généreux don à l'État polonais. Un autre riche aristocrate, beau-fils par alliance de Balzac, Jerzy Wandalin Mniszech (1824-1881), réunit à Paris l'une des plus importantes collections de coléoptères ${ }^{8}$. Il fut un précieux collaborateur du Cabinet zoologique de Varsovie.

\section{La Maison Verreaux et l'établissement Deyrolle}

6 Taczanowski connaissait de nombreux naturalistes français, dont les frères Verreaux. Rapidement leur entreprise devint un partenaire privilégié du cabinet de Varsovie et de nombreux collectionneurs naturalistes polonais, tant en France qu'en Pologne. Les relations de Jules et Édouard Verreaux avec Taczanowski, d'abord scientifiques et commerciales, se transformèrent en véritable amitié. Probablement, les frères Verreaux furent les plus importants collaborateurs du Cabinet zoologique de Varsovie : la «Maison Verreaux » lui rendait de nombreux services. Ceux-ci ne se limitaient pas aux simples achats et ventes de spécimens. Cette entreprise aidait par exemple à la détermination des espèces, à l'expertise des objets et à l'estimation de leur valeur. Elle s'occupait aussi de la naturalisation d'animaux; la plus célèbre fut celle d'un lion tué par le comte Branicki lors de l'expédition d'Algérie en $1866^{\circ}$. Cet animal fut l'un des objets phares lors de la réouverture du cabinet au public à l'automne 1868. C'était aussi pour les naturalistes de Varsovie un important lieu de rencontres avec les zoologistes travaillant sur les autres continents et particulièrement en Asie et en Amérique du Sud; ils y trouvaient enfin un terrain privilégié pour la préparation de leurs propres expéditions et la détermination de destinations géographiques pour les voyageursnaturalistes polonais. Les frères Verreaux n'hésitaient à avancer à Taczanowski et ses collaborateurs des sommes importantes. Leur «Maison» constitua également un lieu du travail pour les réfugiés polonais, comme ce fut le cas pour Alphonse Parvex ${ }^{10}$.

7 Les frères Verreaux n'étaient pas à Paris les seuls naturalistes-commerçants avec lesquels Taczanowski entretenait des liens tant scientifiques que commerciaux. Les noms d'Henri (1827-1902) et d'Émile (1838-1917) Deyrolle apparaissent fréquemment dans les lettres de Taczanowski. Les naturalistes polonais passant par Paris visitaient toujours le commerce de la rue du Bac. Peut-être le fait qu'Henri Deyrolle travailla pour Mniszech en collectant et déterminant des coléoptères joua-t-il aussi un rôle dans ces contacts?

\section{Les naturalistes français}

8 Le père Armand David (1826-1900) occupe une place bien particulière parmi les correspondants du Cabinet zoologique de Varsovie. Les recherches menées dans les archives des Lazaristes à Paris, où est conservée sa correspondance, ont mis en évidence l'existence de plusieurs lettres de Taczanowski et Branicki. La correspondance avec le cabinet zoologique concerne en majorité des collections ornithologiques, entomologiques et mammalogiques, elle présente aussi des détails sur les expéditions et les séjours des naturalistes polonais à Paris. Les lettres de Taczanowski sur la faune asiatique présentent un intérêt particulier pour l'histoire de la zoologie. Le cabinet de 
Varsovie disposait d'une importante collection d'animaux de Sibérie, collection composée d'objets recueillis par les exilés polonais et particulièrement par Benedykt Dybowski. Ces objets et ces informations étaient complémentaires à ceux d'Armand David, originaires de la Chine. L'échange des informations et des spécimens permit de préparer diverses publications sur la faune de l'Asie Centrale. Le père David devint également l'ami des comtes Branicki. Il accompagna Constantin Branicki dans ses expéditions en Algérie et en Tunisie. Branicki acheta aussi des spécimens asiatiques de David, finança ses voyages et aida à l'édition de ses livres.

9 C'est à Armand David que s'adressa René Oberthür (1852-1944) afin de pouvoir étudier la collection de lépidoptères péruviens récemment apportée à Paris par Jan Sztolcman (1854-1928), un élève et proche collaborateur de Taczanowski. René (1852-1944) et Charles (1845-1924) Oberthür représentèrent ensuite d'importants correspondants du Cabinet zoologique de Varsovie en matière de collections de papillons. Ils furent également éditeurs de l'Ornithologie du Pérou de Taczanowski, une importante monographie de la zoologie néotropicale.

Parmi les correspondants et collaborateurs du Cabinet zoologique de Varsovie, citons encore Félix-Édouard Guérin-Méneville (1799-1874). La Bibliothèque Centrale du MNHN conserve sa correspondance entomologique avec Antoni Waga (1799-1890) et Konstanty Tyzenhauz (1786-1853). Rappelons aussi que Guérin-Méneville militait activement pour l'indépendance de la Pologne. Pierre Joseph Henri Marmottan (1832-1914) fut l'ami et correspondant de Taczanowski. Les lettres des naturalistes polonais révèlent que ces derniers s'inquiétèrent beaucoup pour lui et pour Jules Verreaux lors du siège de Paris en 1870 et durant la Commune. Le contenu du catalogue de la collection ornithologique de Marmottan, ainsi que les lettres, prouvent des échanges très fréquents entre lui et ses collègues de Varsovie. Les noms de Paul Maisonneuve (1849-1927) et Hippolyte Lucas (1814-1899) figurent également parmi les collaborateurs du Cabinet zoologique de Varsovie.

\section{Le Muséum d'Histoire Naturelle de Paris et le réseau des collaborateurs du Cabinet zoologique de Varsovie}

11 Au cours du xix ${ }^{e}$ siècle, le Muséum de Paris joua un rôle bien particulier pour les sciences naturelles en Pologne, tant comme lieu de formation de naturalistes que comme référence dans le domaine des collections. Il n'est donc guère surprenant que ce fût au sein de cette institution que les Polonais se préparaient à l'exploration des faunes exotiques et, de retour en Europe, y déterminaient les spécimens. Parmi les proches collaborateurs du Cabinet zoologique de Varsovie se trouvent des scientifiques de cette institution, dont Achille Valenciennes (1794-1865), Charles-Émile Blanchard (1819-1900), Eugène Simon (1848-1924) et Émile Oustalet (1844-1905).

Le réseau de correspondants et de collaborateurs français (67 identifiés jusqu'à présent) de Taczanowski joua un rôle vital pour le Cabinet. Taczanowski construisit ce réseau lors de ses voyages scientifiques en France, à l'aide de sa correspondance, par sa participation aux sociétés savantes et grâce à ses contacts avec les revues scientifiques 
ainsi que lors de son expédition en Algérie (1866-67). Dans ce réseau, nous retrouvons des savants du MNHN, des musées provinciaux, des entreprises privées et de nombreux naturalistes non professionnels. Ce transfert de connaissances concernait toutes les pratiques naturalistes de l'époque: le travail sur le terrain et l'acquisition des spécimens, les techniques en taxidermie, la détermination taxinomique, la description et la publication, la préparation des expositions et la formation des collaborateurs. Les Français constituent le groupe le plus nombreux et le plus important (loin devant les Britanniques et Allemands) des collaborateurs du Cabinet zoologique de Varsovie au cours de la seconde moitié du XIX $x^{e}$ siècle.

\section{BIBLIOGRAPHIE}

BRZĘK Gabriel, « Złoty wiek ornitologii polskiej », Memorabilia Zoologica, vol. 3, 1959, p. 1-175.

BRZĘK Gabriel, Historia zoologii w Polsce, Kraków, Polska Akademia Umiejętności, 2007.

DASZKIEWICZ Piotr, « La maison Verreaux au XIX ${ }^{\mathrm{e}}$ siècle à Paris, plaque tournante des collections naturalistes mondiales ", Journal d'agriculture traditionnelle et de botanique appliquée, vol. $74, \mathrm{n}^{\circ} 4$, 1995, p. 111-129.

DASZKIEWICZ Piotr, « Quelques remarques sur “ l'Ornithologie du Pérou " - la première monographie de la zoologie néotropicale », Organon, vol. 34, 2005, p. 73-95.

DASZKIEWICZ Piotr, «L'expédition zoologique polonaise en Algérie d'après la correspondance de Władysław Taczanowski à Konstanty Jelski », Organon, vol. 41, 2009, p. 109-120.

DASZKIEWICZ Piotr, « Feliks Jarocki (1790-1865), directeur du cabinet d'histoire naturelle de Varsovie et ami de Frédéric Chopin - en quête de souvenir sur son séjour en France ", Annales du Centre scientifique de l'Académie polonaise des sciences à Paris, vol. 13, 2011, p. 187-192.

DASZKIEWICZ Piotr, « Cabinet du Roi, Jardin des Plantes, puis Muséum national d'histoire naturelle - une institution modèle pour les sciences polonaises de la fin du XVIII et du début du XIX siècle ", Annales du Centre scientifique de l'Académie polonaise des sciences à Paris, vol. 14, 2012, p. 465-467.

DASZKIEWICZ Piotr, « Documents inédits concernant l'histoire et la vente de la collection entomologique de Georges Vandalin Mniszech (1822-1881) », Le Coléoptériste, vol. 16, nº 2, p. 80-82. FEDOROWICZ Zygmunt et FELIKSIAK Stanisław, 150 lecie Gabinetu Zoologicznego w Warszawie (1818-1968), Warszawa. Memorabilia Zoologica, Nouvelle Série 1, 2016.

KOWALSKA Krystyna, MRoCZKowSKA Anna, ZIELIŃSKA Barbara, « Listy do Antoniego Wagi Konstantego Branickiego i Benedykta Dybowskiego », Memorabilia Zoologica, vol. 12, 1964, p. 7-317. 


\section{NOTES}

1. Z. Fedorowicz et F. Stanisław, 150 lecie Gabinetu Zoologicznego w Warszawie (1818-1968), p. 1-271.

2. P. Daszkiewicz, « Feliks Jarocki (1790-1865), directeur du cabinet d'histoire naturelle de Varsovie et ami de Frédéric Chopin - en quête de souvenir sur son séjour en France ", p. 190-192.

3. P. Daszkiewicz, « Cabinet du Roi, Jardin des Plantes, puis muséum national d'histoire naturelle - une institution modèle pour les sciences polonaises de la fin du XVIII et du début du XIX ${ }^{\mathrm{e}}$ siècle », p. 457-467.

4. G. Brzęk, « Złoty wiek ornitologii polskiej », p. 120-127.

5. G. Brzęk, Historia zoologii w Polsce, p. 197-202.

6. P. Daszkiewicz, «Quelques remarques sur "l'Ornithologie du Pérou" - la première monographie de la zoologie néotropicale », p. 73-76.

7. P. Daszkiewicz, «La maison Verreaux au XIX siècle à Paris, plaque tournante des collections naturalistes mondiales », p. 114-115.

8. P. Daszkiewicz, « Documents inédits concernant l'histoire et la vente de la collection entomologique de Georges Vandalin Mniszech (1822-1881) », p. 80-82.

9. P. Daszkiewicz, «L'expédition zoologique polonaise en Algérie d'après la correspondance de Władysław Taczanowski à Konstanty Jelski », p. 109-112.

10. K. Kowalska, A. Mroczkowska et B. Zielińska, «Listy do Antoniego Wagi Konstantego Branickiego i Benedykta Dybowskiego », p. 125, 137, 140, 148.

\section{RÉSUMÉS}

Dans les années 1860, le Cabinet zoologique de Varsovie, auparavant petit musée de province, devint l'un des plus importants centres de recherches sur la faune et une référence pour les collections en Europe Centrale. Władysław Taczanowski (1819-1890) fut à l'origine de cette transformation. Le réseau de correspondants et de collaborateurs français de Taczanowski joua un rôle vital pour le Cabinet. Dans ce réseau, nous retrouvons des savants du Muséum national d'histoire naturelle, des musées provinciaux, des entreprises privées et de nombreux naturalistes non professionnels. Ce transfert de connaissances concernait toutes les pratiques naturalistes de l'époque : le travail sur le terrain et l'acquisition des spécimens, les techniques en taxidermie, la détermination taxinomique, la description et la publication, la préparation des expositions et la formation des collaborateurs. 


\section{AUTEUR}

\section{PIOTR DASZKIEWICZ}

Chargé d'étude du développement du référentiel taxonomique TAXREF (UMS 2006 PatriNat), service du Patrimoine Naturel (OFB-CNRS-Muséum national d'Histoire naturelle), professeur extraordinaire à l'Institut de l'histoire des sciences, Académie polonaise des sciences, Varsovie, membre de la Société polonaise de dendrologie et de la Société polonaise d'acarologie 


\section{L'agriculture : entre assimilation des acquis de la science et transmission des savoir-faire}




\title{
La transmission des savoirs
} agronomiques vers la fin du XVIII siècle d'après les travaux des apprentis savants de la société d'agriculture de Blaison en Anjou

\author{
Antoine Follain
}

1 Le dossier de la « société des Thesmophories » de Blaison en Anjou a été présenté dans un livre sans que nous en épuisions l'intérêt ${ }^{1}$. Créé par huit personnes, ce cercle d'étude est remarquable par le lieu, vraiment très modeste, et par un dynamisme étonnant. De plus, les «thesmophores » ont laissé de leur activité des archives qui ont permis d'en faire une étude assez complète. C'est une toute petite chose mais qui est liée à des plus grandes dans la province, comme l'activité du bureau d'agriculture d'Angers, ainsi qu'à des plus grandes encore, puisque l'agronomie et l'économie sont des sujets débattus partout en Europe, dans les milieux savants et politiques. Mais le nom même de la société est révélateur des limites de ces apprentis savants : «Thesmophories » renvoie à des fêtes agraires antiques et il pouvait convenir, mais ils se sont qualifiés ensuite de «thesmophores» alors que le sens de thesmophorus est « qui apporte la loi ». C'est ainsi qu'il faut comprendre l'usage que Voltaire en a fait pour qualifier Catherine II de «thesmophore du Nord » en pensant à la déesse Cérès, dont l'épithète la législatrice rappelle qu'elle avait amené aux hommes l'agriculture et l'ordre. Les thesmophores de Blaison sont des amateurs, des lettrés non-accomplis. Le sujet du congrès du CTHS « La transmission des savoirs » invite alors à se demander quels modèles et quelles sources ont pu inspirer des petits bourgeois campagnards dans leur pratique associative et dans leurs écrits? Qu'ont-ils reçu, de qui et comment? Qu'ont-ils diffusé à leur tour? 


\section{Une expérience décalée, courte, mais spécialement riche}

2 Tout n'a pas été conservé des archives des sociétés royales d'agriculture au XvIII ${ }^{\mathrm{e}}$ siècle, mais celles qui en ont laissé ont permis que soient publiées des études, après tout suffisantes. Beaucoup de sujets ont été couverts par la somme d'André Bourde sur l'agronomie au Siècle des lumières et bien sûr par des travaux postérieurs ${ }^{2}$. Mais on n'a toujours étudié que les sociétés royales alors que les petites sociétés savantes n'ont tout simplement rien laissé pour travailler sur elles - sauf précisément celle de Blaison.

\section{Une société dans l'air du temps}

3 Avec une création en 1775, et des activités documentées en 1776 et 1777, cette histoire est tardive. L'intérêt des savants et des élites sociales pour l'agriculture est à chercher plus tôt et il y eut même un effet de mode, une « agromanie » moquée par Voltaire dans son Dictionnaire philosophique portatif... (1764) à l'article «Blé». En Anjou, l'activité du marquis de Turbilly remonte aux années $1750-1760$ et la création du bureau d'agriculture à 1761. Plusieurs périodiques cessent même de paraître au début des années 1770. Le mouvement s'est donc essoufflé quand apparait la société de Blaison et les thesmophores sont des tard-venus. Mais peut-être illustrent-ils aussi un décalage entre les élites qui se désintéressent de l'agronomie et les propriétaires et exploitants dans les bourgs et campagnes de l'Ouest, qui sont atteints plus tard et qui seront encore actifs au XIX ${ }^{e}$ siècle.

4 Toute trace d'activité des thesmophores disparaît après 1777. Cet objet historique n'existe que par la conservation de trois liasses qui témoignent de son activité. Si ces documents n'avaient pas été apportés aux Archives départementales par un descendant de l'un des membres de la société, jamais on n'aurait compris ce qu'il y avait derrière certaines annonces parues dans des périodiques. Manque-t-il des papiers pour 1778, 1779 ? La société a peut-être duré en se repliant sur elle-même, après que les thesmophores ont été déçus par l'échec d'un projet d'assèchement, comme on le voit dans un brouillon de lettre ${ }^{3}$.

5 Ces archives témoignent donc de l'existence d'une petite institution savante pendant au moins deux ans et d'une forme de sociabilité et d'une aspiration à être « utiles » qui sont très caractéristiques du XVIII ${ }^{e}$ siècle ${ }^{4}$. Elles attestent de multiples interrogations sur l'agriculture, mais aussi sur l'ordre social et politique ${ }^{5}$. Sous la première cote (aujourd'hui 8D1) se trouvent des lettres et mémoires rattachés à deux projets qui étaient l'assèchement de marais et la création d'une levée pour compléter l'ensemble de digues qui protégeait déjà les basses vallées des crues de la Loire. Plus intéressant: sous la cote $8 \mathrm{D} 2$ ont été rangés les programmes publiés par les thesmophores et les copies de plusieurs annonces parues dans des gazettes. Le meilleur est en $8 \mathrm{D} 3$ où se trouvent des brouillons de lettres adressées par la société à des correspondants ainsi que des lettres reçues par la société et surtout les mémoires qui répondent aux questions posées dans les programmes. 


\section{Les activités des thesmophores}

6 Les membres sont d'abord un groupe de parents, d'amis et de voisins qui ont constitué une société mais qui au lieu de jouer aux cartes et comparer leurs vins, ont donné à cette société une orientation savante et tout un programme d'action, par trimestre et année, en étudiant des « questions » :

«Des personnes que l'amitié rassemble tout les mois, desirant joindre aux agréments de la société celui de leur instruction, se sont proposées d'augmenter le plaisir de se voir, par des questions relatives à leur état : en conséquence elles ont arrêté de discuter les questions suivantes dans leurs entrevues de janvier, février et mars 1776 [...] Y a-t-il dans le produit beaucoup de différences entre la grande et la petite culture ? Deuxième question. Quelle est la différence du coût entre ces deux cultures? Troisième question. La grande culture nuit-elle à la population? En chercher les preuves dans les différentes élections de notre province.

Février 1776. Première question. Dans la classe des prairies artificielles, quels sont les semis auxquels on doit donner la préférence relativement à nos terreins? Deuxième question. Est-il une manière propre à dessécher les récoltes de ces prairies artificielles, pour en faire des fourrages d'hiver?»

7 Les thesmophores ont le plus souvent répondu par eux-mêmes. Ils ont des limites, car ils s'appuient sur ce qu'ils connaissent le mieux et participent aux sujets généraux sous un angle angevin, notamment pour la viticulture, les prairies et l'élevage ${ }^{6}$. Mais ils répondent comme il convient à cette époque, c'est-à-dire avec les nouvelles méthodes d'économétrie diffusées par les grands auteurs. Ainsi, dans le mémoire de Duvau répondant à la première question d'avril 1776 «Les propriétaires des grandes terres auraient-ils plus de profit à en arrenter à bled les terres, que de les affermer ?", lu en séance tenue chez Bréau le 22 avril 1776, on trouve deux séries de calculs sur la « rende» (le profit) et le « produit » selon plusieurs options. D'abord, si l'exploitation est louée complète :

«J'en supose une des meilleurs, composée a peu-près de 60 arpents de terres labourables, 25 arpents de prés et pâtures, et les maisons, granges, jardins etc. Je divise ces 60 arpents de terre en trois tiéres ce qui fait 20 arpents chacque, parce que notre usage est d'ensemencer un tiére en froment, un tiére en orge ou avoinne, et l'autre tiére que l'on tire à gueret affin d'y metre du froment l'année suivante. Cette metairie avec ses dependences peut étre affermée 800 \# (£ivres). Surquoy il convient déduire les grosses réparations qui surviennent aux logements et qui sont à la charge du propriétaire, que l'on peut estimer 20 \# par an. Reste à 780 \#. »

Un calcul par unité de surface aboutit à un résultat différent :

"Ces 60 arpents de terre peuvent être arentée un boisseau de froment par boissellée, ce qui fait 600 boisseaux, qui à raison de $35 \mathrm{~s}$. le boisseau qu'on peut l'estimer année comune. Reviennent à 1050 \#. Les prés et pâtures montant à 25 arpents pourraient être arentées moitié des terres, c'est-à-dire 6 ecuellées la boissellée, qui donneraient $125 \mathrm{bo[isseau]x} \mathrm{f[romen]t} \mathrm{au} \mathrm{même} \mathrm{prix.} \mathrm{Reviennent} \mathrm{à}$ 218 \# 15 s. Les logements et jardins vallent au moins de ferme ou de rente 80 \#. »

9 Ne discutons pas les résultats, observons seulement la méthode. De tels calculs posent de gros problèmes. Ainsi, dans un autre mémoire, un thesmophore confronte les résultats d'une prairie vouée au foin et d'une prairie servant à "herbager " des bestiaux. La base est qu' « une presrie de cinquante arpants peut produire année commune cent charretées de foin [voir plus bas sur ce 100] qui pourront valoir trante livres [la charretée] prise sur [le] lieu ", d'où 60 livres à l'arpent; moins les frais de fauche et de transport estimés à 6 livres par charretée ou 12 livres à l'arpent; reste 
48 livres soit pour 50 arpents 2400 livres «sans courir aucun risque ». La deuxième spéculation consiste à "arbager [les] 50 arpent [pour] metre cinquante bœufs qui pouront donner cinquante livres par bœuf de benefice"; d'où un «capital» de 2500 livres. La difficulté est qu'il faut engager des «avances»: acheter des bêtes à 150 livres la pièce, soit 7500 livres immobilisées. Il retire donc 375 livres «pour l'insterai de l'argent » (qui aurait pu être placé ailleurs et à $5 \%$ ) et ramène le bénéfice à 2125 livres. De plus, il y a un risque de mortalité des bestiaux. D'un côté, 2125 livres et un risque. D'un autre côté, 2400 livres et, affirme-t-il « aucun risque ». Mais comment ignorer que le thesmophore avait d'abord estimé la production à 55 charretées de foin avant de le barrer pour écrire «cent»? Ce n'est pas le seul chiffre corrigé dans les mémoires.

10 Alors où est la vérité si un thesmophore n'est pas sûr de ses comptes et s'il a envie de prouver l'option qu'il préfère ? C'est un problème pour tous les mémoires chiffrés des thesmophores. C'est aussi le cas pour tous les comptes des grands auteurs du XviII siècle, vers la fin des années 1760 , et au début des années 1770 il y eut des disputes dans les Éphémérides du citoyen et le Journal de l'agriculture à partir des tableaux de Quesnay. Or l'argumentation des physiocrates était fondée sur des comptes.

\section{Relations avec les paysans}

11 En plusieurs occasions les thesmophores s'informent de leurs pratiques et de leurs raisons auprès des paysans. Ils rapportent par exemple une réponse remarquable, lorsqu'un cultivateur leur explique qu'il fait une certaine plantation en terre inondable en sachant très bien que le plus souvent il n'aura rien mais une bonne année sur trois lui suffit pour faire un profit.

12 En revanche, dans l'autre sens, important pour le thème de "La transmission des savoirs", rien n'indique que les thesmophores ont transmis quoi que ce soit aux paysans.

\section{Les modèles des thesmophores}

13 Vers 1870, l'archiviste et historien Célestin Port avait commenté le dossier déposé aux Archives d'Angers dont il était directeur'. Il avait imaginé qu'ils lisaient la Gazette d'agriculture et le Mercure, Quesnay, Turgot, Necker, l'Inégalité de Rousseau et l'Ami des hommes de Mirabeau! Mais où sont les preuves que les thesmophores ont connu, détenu et lu ces livres? Selon Célestin Port, les thesmophores lecteurs de la Gazette agissaient aussi comme des correspondants puisqu'ils lui avaient écrit. Or, c'est contredit par les archives, car l'envoi du programme de 1776 à la Gazette n'est pas de leur fait ${ }^{8}$.

Essayons de comprendre honnêtement comment des petits bourgeois de Blaison ont pu se trouver des modèles d'organisation et des sources d'information et d'inspiration. $\mathrm{Au}$ XVIII siècle, les gens de lettres, les savants et la bonne société philosophent et s'informent des questions littéraires et esthétiques, savantes et techniques, voire politiques à partir de périodiques, de livres et dans des institutions. Dans le contexte angevin, une institution savante créée à la fin du XviI siècle cherche à corriger certaines faiblesses et l'autre est toute récente. 


\section{L'académie d'Angers}

15 Les statuts de l'académie royale d'Angers (1685) autorisent 30 académiciens « ordinaires » (titulaires) qui sont les principaux magistrats de la ville et des personnes de qualité supérieure. Le talent y compte moins que la position dans la société. «La science des belles-lettres [...] la parfaite intelligence des poètes et des orateurs [...] fait l'occupation de l'assemblée » et secondairement elle "embrasse la connoissance des langues et de l'histoire, de la philosophie et des mathématiques ». Les statuts prévoient aussi des domaines interdits ou qui ne seront abordés qu'avec précaution. L'académie procède par séances « ordinaires » réservées aux membres et les séances " publiques » sont ouvertes seulement à des « personnes de condition » expressément invitées.

16 Après une période de dépérissement dans les années 1730 et 1740 , la situation est un peu rétablie et, en 1760, elle change enfin son nom en « Académie des Sciences, Belles Lettres et Arts d'Angers ». Les académiciens déclarent maintenant vouloir entendre des conférences relatives aux arts "méchaniques» et «civils». Mais si l'académie s'intéresse aux sciences «naturelles » comme la botanique, elle ignore les sujets trop pratiques. Quant aux membres, l'ouverture à des « associés » dits « étrangers » (à partir de 1747) et « résidents » (à partir de 1762) ne représente qu'une timide évolution. Vers 1776 , si les séances sont dites plus ouvertes que par le passé, il n'y a pas de sources pour comprendre vraiment qui pouvait y assister. À défaut d'entendre et voir, les gens du commun peuvent lire des comptes rendus dans les Affiches d'Angers - nous y reviendrons.

17 Finalement, nous ne pouvons pas savoir si un thesmophore a pu se faire admettre dans le public des séances mais nous supposons que si cela avait été le cas, il se serait empressé d'en rendre compte à ses collègues et il y aurait en $8 \mathrm{D}$ une preuve documentaire.

\section{La Société royale d'agriculture}

18 Le bureau d'agriculture est l'expression même du courant de pensée agronomique et économique en France au XVIII ${ }^{\mathrm{e}}$ siècle. Rappelons qu'une action gouvernementale a été portée par Henri Léonard Bertin, contrôleur général des finances de 1759 à 1763, puis secrétaire d'État surnuméraire et détaché du contrôle général des finances, avec des attributions comprenant entre autres l'agriculture. Une circulaire aux intendants du 22 août 1760 ordonne la création des sociétés d'agriculture. La tutelle des intendants induit une organisation dans le cadre des généralités, alors que celle de Tours comprend trois provinces et villes capitales dont aucune n'est autorisée à créer sa propre société - d'où trois « bureaux ».

19 Les débuts sont prometteurs : le bureau passe des annonces dans les Affiches d'Angers qui rendent compte aussi de certaines séances. Un questionnaire est imprimé et distribué dans toute la province. Les membres se répartissent une vingtaine de sujets d'étude tels que les ustensiles du labourage, les engrais et amendements ou encore les prairies naturelles et artificielles, pour présenter plus tard des rapports. Le bureau a 20 membres et des "associés" en nombre illimité, mais en pratique il n'y a pas d'ouverture à des candidats assez honorables. Au temps des thesmophores, le directeur est Pocquet de Livonnière, conseiller au présidial d'Angers et auteur du Traité des fiefs, puis Rogue, auteur d'un traité de droit commercial, puis un chanoine. De plus, l'enquête 
générale n'a aucun résultat connu, le bureau n'organise pas de concours ni d'expériences, pas plus qu'il n'a de ferme modèle.

Mais bien sûr, aussitôt créés, les thesmophores se mettent en relation avec la société royale et plus précisément avec le doyen Cotelle, un religieux secrétaire perpétuel depuis 1764, qui leur répond:

« Le bureau d'agriculture étably à Angers verra toujours avec une vraye satisfaction des citoyens patriotes et eclairés se reunir en société pour l'instruction des cultivateurs dans les campagnes dans l'économie rural. Et pour les progrès de l'agriculture, il seroit à désirer que celles que vous venez de former, monsieur, trouvast des imitateurs, et quelles se multipliassent dans nos provinces. Le goust de la culture et de tout ce qui y est relatif, en se repandant de proche en proche, deviendroit le goust dominant, et il en resulteroit un avantage reel en faveur du bien public et particulier. Notre société â applaudy, monsieur, au projet que vous avez formé. Elle s'empressera mesme de vous communiquer les memoires qu'elle à répandû dans le public, et qui sont insérés dans les gazettes et les journaux de l'agriculture, ainsy que ceux qui luy ont été addressés par les autres sociétés du royaume et des pays étrangers à la France. Elle recevra avec le mesme plaisir les observations et les mémoires qui seront le fruit de vos assemblées patriotiques et les repandra dans le public lorsqu' ils contriburont à son instruction. »

Le doyen Cotelle rapporte aussi la « surprise » des membres du bureau quand ils ont été informés "en recevant votre prospectus" et si le bureau dit espérer que les thesmophores auront des "imitateurs", il n'est question d'aucune forme d'affiliation ni de coordination d'un réseau - ce qui sera plutôt l'esprit du XIX ${ }^{e}$ siècle.

\section{Les sources des thesmophores}

En France, on retrouve une littérature agronomique originale avec le Traité de la culture des terres de Duhamel du Monceau (1 ${ }^{\text {ère }}$ édition: 1750 ) et surtout ses Éléments d'agriculture (1756 et 1762) portés par des expériences réalisées dans son domaine. Les auteurs et parutions se multiplient, et des gazettes spécifiques sont créées. Les plus grands succès $\mathrm{du}$ XVIII ${ }^{\mathrm{e}}$ siècle sont La Nouvelle maison rustique... (1 ${ }^{\text {ère }}$ édition en $1700,12^{\mathrm{e}}$ en 1798 et $13^{\mathrm{e}}$ en 1804) et les deux livres de Duhamel du Monceau. Dans les mêmes années, l'agriculture est centrale dans la pensée des «économistes» ou "physiocrates »: Du Pont de Nemours, le docteur Quesnay, le marquis de Mirabeau, et au second plan un certain Charles de Butré sur lequel nous reviendrons. L'abbé Baudeau fait paraître en 1765 le premier numéro des Éphémérides du citoyen ou chronique de l'esprit national dont il reste longtemps le principal auteur et qui dure jusqu'en 1772. On pourrait étendre la liste, mais qu'ont lu les thesmophores?

\section{Où l'on attend des livres, sans les trouver}

Faute de citations dans leurs mémoires - et faute d'inventaires après décès, hélas, car nous aurions pu savoir s'ils avaient possédé des livres! - on peut tout imaginer, depuis la possession de vieux livres de "mesnage des champs " et d' " oeconomie rustique » jusqu'à des livres tout récents, en passant par des copies manuscrites personnelles de livres imprimés. Un thesmophore dans le premier mémoire du corpus ( $1^{\mathrm{er}}$ janvier 1776) dit exposer une opinion contraire à celles des "écrits modernes » et "des écrivains célèbres »- mais lesquels? Dans un autre, Malécot est d'abord dans le vague quand il évoque "plusieurs traités de la culture des vignes» (les a-t-il seulement lus ?) puis il 
cite le livre « de Mr. Bidet de l'académie d'agriculture de Florence [...] en deux volûme imprimés en 1759 » et l'on sait aussitôt comment il l'a connu : « monsieur de Longueil à bien voulu me le prêter ${ }^{9}$ ». Dans tout le corpus, c'est la seule référence à un livre.

Ont-ils eu accès à des ouvrages généraux comme l'Encyclopédie de Diderot et d'Alembert ou à des périodiques comme les Trimestres de la Société royale d'agriculture ou le mensuel Journal oeconomique [...] sur l'agriculture, les arts, le commerce...? Les thesmophores n'ont peut-être jamais lu autre chose que des almanachs et le périodique régional.

\section{Les Affiches d'Angers, miroir d'une province}

Imprimées à partir de 1773, les Affiches et toutes les publications de la même espèce ont pour modèle la Gazette de Renaudot ${ }^{10}$. Cette « feuille » hebdomadaire (pliée pour donner quatre pages) est bon marché. C'est d'abord un journal d'annonces pour les gens qui ont de l'argent à placer et qui veulent tout savoir des biens qui se négocient; d'où l'importance d'être abonné. On y trouve aussi des avis de spectacles, des résumés de textes de loi, des avis de souscription et parution, comme le prospectus «du Précis historique des séances de la société royale d'agriculture au bureau du Mans » dans le ${ }^{\circ}$ 23 du 7 juin 1776. Dans le même numéro une rubrique "Avis intéressants " présente une méthode "trouvée [par] un agronome de Nuremberg" pour tuer les «puces de terre [qui] font souvent un ravage étonnant dans les jardins ». Le contenu des Affiches est très varié.

On peut admettre que la lecture des Affiches permette à des gens comme les thesmophores de savoir ce qui se passait à l'académie et au bureau d'agriculture sans être membre et sans assister aux séances. Mais le style des Affiches renseigne davantage sur la forme que sur le fond. Ainsi, dans le $\mathrm{n}^{\circ} 24$ du 17 juin 1774, le compte-rendu de la séance publique de l'académie royale rapporte que «M. Gastineau, professeur en droit [...] fit l'ouverture de la séance par un discours » dont les Affiches disent les intentions, louent la forme «avec autant de vérité dans les principes que justesse \& d'harmonie dans les expressions ", mais ne restituent pas les arguments. Sur un autre orateur, il est écrit «excellente dissertation [...]. La profondeur des recherches \& le stile de ce discours furent admirés des auditeurs", mais rien du contenu n'est rapporté! Quelquefois seulement, les Affiches publient un article assez long et qui donne même des leçons de méthodologie. Ainsi dans le $\mathrm{n}^{\circ} 37$ du 16 septembre 1774, sous un titre «Agriculture » est publié un «Extrait d'un mémoire de M. Gouge, secrétaire perpétuel de la Société royale d'agriculture au bureau de Laon, sur la récolte des avoines ».

Les Affiches ne peuvent pas être regardées aujourd'hui comme une publication de référence, ni savante, ni agricole, mais elles ont pu être la source principale des thesmophores. Ainsi, l'article de Gouge expose un problème posé par des techniques traditionnelles, démontre une erreur de compréhension en Picardie, rapporte l'expérience contraire des Bretons et des Bas-Normands, donne plusieurs autres preuves et rapporte des expériences pratiques et parachève sa démonstration par le rapport de ses propres expériences menées durant trois ans.

Un autre aspect des Affiches est qu'elles permettent d'être imprimé et lu dans tout domaine. En effet, dans les années 1770-1780, l'agriculture n'est pas moins présente dans les Affiches que ne le sont les Lettres. Une condition pour être édité est d'imiter le style des Affiches, c'est-à-dire que le plus souvent le propos est enrobé dans un style 
assez peu supportable aujourd'hui. Un sujet agricole comme ceux traités par les thesmophores dans leurs mémoires avait donc sa place dans les Affiches, à condition d'être rédigé comme on le demandait aux auteurs. Ainsi tel article «sur l'usage trèsnuisible de mener paître les bestiaux dans les prairies » ( $n^{\circ} 19$ du 21 mai 1774) ou celui sur la « manière d'enchausser ou de chauler les grains » ( $\mathrm{n}^{\circ} 40 \mathrm{du} 7$ octobre 1774) aurait pu être rédigé par un thesmophore. Un problème est que les articles sont pour la plupart anonymes, comme dans beaucoup de publications de ce temps ${ }^{11}$.

\section{Les thesmophores publiés}

Nous avons repéré que les thesmophores ont fait paraitre trois fois leurs programmes dans les Affiches et aussi donné collectivement un article. Si l'un d'eux a été publié comme auteur anonyme nous ne le savons pas - mais là aussi nous supposons que cela aurait laissé quelque trace dans les archives de la société pour 1776 et 1777 . La publication des thesmophores dans les Affiches est aussi la seule preuve qu'ils ont un peu pratiqué une agronomie expérimentale et pas été seulement des agronomes de papier ou qui rapportent les expériences des paysans des environs :

«Les personnes qui composent la susdite société, ont découvert une propriété interressante dans la plante, connue sous le nom d'herbe à soie, qui nous a été apportée des Indes, depuis douze à quinze ans. On s'étoit mal-à-propos imaginé que sa soie étoit contenue dans la capsule, ou espéce de cocon qui porte la graine, mais qui n'a pas d'autre propriété que de donner cette graine enveloppée d'un duvet fort court, \& qu'il est fort difficile de filer : un des membres de cette société s'est assuré, par expérience, que la soie que produit cette plante est sur la paille ou chenevotte, comme au chanvre, prodigieusement abondante, \& de la longueur de la plante; elle est magnifique \& on en peut espérer les plus grands avantages en la cultivant avec soin : elle est vivace, on la coupe vers la fin de novembre, \& et on la met rouir à l'eau ou dans l'herbe, comme le chanvre; mais comme l'espece d'écorce charnue qui couvre les fibrilles soyeuses, ne les quitte pas aisément, on la met au débouilli comme la soie, \& l'on obtient la plus belle filasse. »

Cette expérience sur une plante du type Yucca a été publiée dans le numéro du 29 mars 1776. Ni les thesmophores ni le directeur des Affiches ne savaient que ces observations étaient déjà connues des gens qui lisaient les bons livres, voire même seulement l' Encyclopédie.

\section{De l'importance des relations personnelles}

Comme les thesmophores n'ont probablement jamais assisté aux séances de l'académie et du bureau d'agriculture, n'ont pas été non plus de grands lecteurs, sinon des Affiches d'Angers, c'est finalement de personne à personne qu'ils semblent avoir appris le plus.

\section{Monsieur de Longueil, un voisin bienveillant}

Les thesmophores ont d'abord eu une relation privilégiée avec un voisin, le marquis Henri-Charles de Longueil, qui a joué le jeu du programme du $1^{\text {er }}$ trimestre 1776 en communiquant à la société un mémoire "anonyme » auquel ils ont répondu par une lettre « À M. de Longüeil de l'académie des sciences et de la Société roiale d'agriculture d'Angers, à son château de la Gerondiére à Blaison » : 
«M. Malécot le j[eun]e vient de nous lire un mémoire sur nos trois questions de janvier [...] Sans être agronomes et encore moins littérateurs, nous aurion aisément reconu aux recherches et aux observations et au stile de ce mémoire savant, un homme consommé dans tous les genres. Mais notre surprise et notre réconnaissance ont étés sans bornes, lorsque notre confrere nous a apris que vous étiés l'auteur de cet ouvrage. Nous n'osions esperer, monsieur, d'être honorés dès notre naissance, de l'atention d'un homme de qualité couroné des lauriers de Mars ${ }^{12}$ et de l'olive de Minerve, et notre amour-propre ne pouvait être plus délicieusement flaté. Daignés, monsieur, continuer de nous honorer de vos lumiéres pour exciter notre zêle [...] Nous ne cherchions d'abord qu'à nous instruire en nous amusant, mais nos amusement deviendront des ocupations sérieuses, s'ils méritent votre atention... »

La relation était flatteuse. On relève aussi dans une dernière lettre du marquis dans les archives, une réaction à des maladresses de la société, ou seulement de Duvau, qui lui avait fait une réponse un peu sèche sur un sujet agronomique. Le marquis de Longueil leur fait ainsi la leçon sur les codes sociaux, y compris entre savants : même sûr de son résultat, on respecte l'auteur, et d'autant plus selon son rang.

\section{Monsieur de Butré, un « vrai savant »}

Les thesmophores publient leurs programmes pour obtenir des réponses écrites et ils espèrent davantage. C'est pourquoi ils adressent des courriers et des mémoires à des institutions et à des savants et même des lettres « de courtoisie » au contrôleur général du royaume pour le féliciter de sa nomination. On imagine leur joie quand ils ont reçu de lui un mot de réponse - ou plus probablement d'un secrétaire autorisé à signer en son nom. Leur plaisir est évident aussi quand ils répondent en février 1776 à Charles de Butré :

«Il est glorieux pour nous d'être dès notre naissance en correspondance avec un savant, qui veut bien prendre la peine de nous encourager et de nous instruire. »

De Butré va les orienter et c'est lui aussi qui a fait publier leur programme dans la Gazette d'agriculture à Paris :

«J'ai reçu, monsieur, d'hier seulement le paquet que vous m'avez envoyé, sur le champ j'ai fait passé à l'auteur de la Gazette d'agriculture un programme de votre second trimestre afin de rendre publique votre digne association et faire connoitre quelle s'occupe des objets les plus utiles et les plus interressants, et qu'elle puisse servir d'émule à d'autres sociétés, dont il seroit fort avantageux qu'il y en eut de pareilles dans toutes les provinces. »

De Butré est connu des historiens comme disciple des principaux physiocrates. En 1776, il continue de travailler, d'écrire et de correspondre avec des personnages importants, comme Mirabeau, mais il a pris ses distances avec les grandes controverses. Les thesmophores ignorent tout de cela. Pour eux, de Butré est un membre de la Société royale d'agriculture de Tours, où il réside en 1776, ainsi que membre des sociétés de Paris et Orléans. Il encourage les thesmophores, notamment dans sa lettre du 15 janvier 1776 donc au tout début de leurs exercices d'écriture savante, mais il les incite aussi à modérer leurs entreprises ${ }^{13}$ et il leur donne des conseils méthodologiques :

«La société amicale et patriotique que vous venez de former, présente des vues, et des recherches d'une utilité trop réelle pour ne pas mériter l'approbation de tous les citoyens éclairés qui s'occupent du bien public. Les questions que vous proposez sont des plus importantes, car elles intéressent également l'économie rurale et politique. Leur solution doit apprendre à tous les économes de la nation, les moyens 
de s'occuper avantageusement et de se communiquer les objets de leurs travaux, afin de profiter par là des connoissances qui peuvent leurs manifester les opérations les plus fructueuses. Vos trois prémières questions bien traitées, suffiroient pour vous occuper votre $1^{\text {ère }}$ année. Vous y demandez la différence de la grande et petite culture, en produit, avances, et population. C'est assurément présenter l'objet dans son point essentiel. Mais auparavant il seroit nécessaire de bien caractériser ces deux especes de culture, afin de ne leurs attribuer que ce qui est uniquement de chaque genre particulier, et qu'on ne dise point vaguement que la grande ou la petite culture sont plus avantageuses, sans auparavant avoir bien désigné ce qu'on entend sous ces deux diverses dénominations; et il faut tacher de parler un langage uniforme afin de ne pas nous cantonner par provinces. ${ }^{14}$ " qu'ils n'ont pu assister à rien, mais ils ont su quand même imiter le fonctionnement de l'académie et du bureau d'agriculture. Malgré l'abondance de la production de livres, ils n'ont pas lu «grand-chose » - en tout cas pas des lectures de première importance mais par des biais et par une publication secondaire comme les Affiches d'Angers, ils ont eu connaissance de grandes questions sur l'agriculture et ils ont appris comment donner des réponses conformes à la science agronomique et économique de leur temps.

«J'ai donné en 1767 un ouvrage assez étendu sur la comparaison de ces deux cultures qui a été imprimé dans les Éphémérides du citoyen vol. 9, 10 et 11. » complet, où on trouvera les détails les plus circonstanciés sur tout ce qui concerne ces deux cultures ». C'était impressionnant pour les thesmophores mais l'ouvrage ne sera jamais terminé. En fait, les thesmophores ne savent pas vraiment qui est de Butré mais ils pensent avoir noué une relation « avec un savant, qui veut bien prendre la peine de nous encourager et de nous instruire ». Ayant reçu la première lettre, ils répondent le 14 février et se mettent à débattre. De Butré répond poste pour poste. Les thesmophores envoient une petite lettre en mars et une importante le 2 avril.

Malheureusement, le contact est vite perdu. C'est que Charles de Butré n'était pas ou n'était plus un ligérien. Depuis 1774, il était employé en Allemagne dans le margraviat de Bade et c'est son intérêt pour les « provinces de petite culture » qui l'avait amené en 1775 à Tours, d'où il avait écrit aux thesmophores la lettre du 15 janvier dans laquelle il évoquait « des recherches sur cet objet ». Or, il devait finir par rentrer en Allemagne. Sa dernière lettre datée de Tours est du mois de septembre 1776 (archives des thesmophores) puis ses propres archives (conservées à Strasbourg) attestent qu'il a signé début octobre une quittance de blanchisserie à Karlsruhe. De Butré n'est plus jamais revenu en Touraine et ni lui ni les thesmophores n'ont voulu ou pu reprendre la correspondance.

Ainsi, quand ils argumentent avec le marquis de Longueil et surtout avec monsieur de Butré, ils discutent les chiffres et les idées car ils sont assez pénétrés d'un esprit scientifique pour considérer que seuls comptent les faits et non l'identité de celui qui s'exprime - ce qui leur vaut une remontrance du marquis. Il est arrivé aussi que du Cormier et un autre thesmophore (non identifié) donnent des réponses opposées à une même question en mars et avril 1777. La société a donc dû discuter de leurs arguments 
et comptes. Commeau-Delaroche écrit un français simple et fait toujours très court. Mais quand par exemple il répond à la deuxième question de novembre 1776, il donne des réponses très pratiques, bien informées, en même temps qu'il montre qu'il sait calculer comme le veut la science agronomique. Autrement dit: il écrit mal mais il travaille bien. Dans une autre de ses réponses, à la première question de mai 1776, son texte, si mal orthographié soit-il, pose le problème en introduction, développe des arguments et tire une conclusion. Parfaitement construit, son mémoire donne l'essentiel sans effets de style. Mais du coup, il n'écrit pas comme on l'apprécie dans les Affiches, au bureau ou à l'académie. Mais il y a encore plus mystérieux que les thesmophores! À savoir la réponse aux questions de février 1776 sur les prairies artificielles, reçue par la société de Blaison sous la forme d'une lettre anonyme, datée d'Angers, qui est fascinante par l'écart entre l'écriture qui est épouvantable et la pensée qui est très juste ${ }^{15}$. Nous ne savons pas comment des gens ont pu acquérir des savoirs aussi incomplets et une audace qui leur permette d'espérer « en metre quelque chose dans les afiche d'Angers", ce qui "pourois me faire conoitre au cas que je peu aitre de quelque utilité pour l'ar de l'agricultures ou dans l'ar veterinaires »- (sic). Cet anonyme a comme les thesmophores une grande soif de reconnaissance sociale et culturelle et il est amusant de voir qu'il prend les amateurs de Blaison pour plus influents qu'ils ne sont, en espérant être publié grâce à eux.

Les thesmophores ont finalement été déçus dans leur entreprise, car les savants, les gens de qualité, les ont tenus à distance - de Butré excepté. Dans la France dite « de l'Ancien Régime ", en 1776 encore, bien penser ne suffisait pas. Il fallait aussi être bien de sa personne et les thesmophores ne l'étaient pas...

\section{BIBLIOGRAPHIE}

BOURDE André-Jean, Agronomes et agronomie en France au XVIII ${ }^{e}$ siècle, Paris, Sevpen, 1967, 1743 p.

FolLAIN Antoine, « Les comptes fantastiques des Thesmophores de la société d'agriculture de Blaison en Anjou », dans Les fruits de la récolte. Études offertes à Jean-Michel Boehler, Strasbourg, PUS, 2007, p. 119-134.

FOLLAIN Antoine (dir.), Une société agronomique au XVIII siècle : les Thesmophores de Blaison en Anjou, Dijon, EUD, 2010, 280 p. Préface de Daniel RocHE. Le livre comprend l'édition annotée des archives de la société des thesmophories.

LEBRUN François, « Une source de l'histoire sociale : la presse provinciale à la fin de l'Ancien Régime, les Affiches d'Angers, 1773-1789 », Le Mouvement social, n 40, 1962, p. 56-73.

PORT Célestin, « Les Thesmophories de Blaison (1775-1777) », Revue d'Anjou, juillet-décembre 1869, p. 340-353.

ROCHE Daniel, Le Siècle des lumières en province. Académies et académiciens provinciaux 1680-1789, Paris et La Haye, Mouton, 1978, 394 et 513 p. 


\section{NOTES}

1. A. Follain, «Les comptes fantastiques des Thesmophores de la société d'agriculture de Blaison en Anjou » et A. Follain (dir.), Une société agronomique au XVIII siècle... Voir notamment les articles A. Follain et C. Trénit, «Un cercle dans l'air du temps », p. 9-55 et C. Fleith-Schweiger, A. Follain et C. Trénit, "Les activités des Thesmophores... ", p. 139-180 ; sur la structure agraire et sociale B. Maillard, «Les "bêcheurs" ligériens au XVIII siècle ", p. 193-206 et J.-L. Guitteny, «L'espace des Thesmophores. Étude des baux conservés dans les archives du notariat Malécot à Blaison », p. 207-236.

2. A.-J. Bourde, Agronomes et agronomie en France au XVIII siècle... Voir aussi dans notre livre: L. Musset, «Agronomie et sociétés savantes agricoles: une mise en perspective de la société des thesmophores (XVIII ${ }^{\mathrm{e}}$-XVIII ${ }^{\mathrm{e}}$ siècles) », p. 251-266.

3. Voir les sections «Trop d'efforts pour peu des succès » et « Des amateurs sincères ou des assoiffés de reconnaissance déçus de ne pas l'obtenir ? », p. 173-179.

4. «Utilité, le mot est partout repris. Il guide les tâches savantes qu'une frontière mal tracée sépare des techniques [...] Il justifie la conquête agronomique, l'intérêt commercial et industriel, la réflexion réformatrice [...] Cette philosophie utilitaire apparaît très tôt [début XVIII ${ }^{e}$ siècle] concours, publications, travaux, lui font atteindre un public élargi [...] Les finalités sont le bien public [...] et la promotion des talents... » p. 383, dans D. Roche, Le Siècle des lumières en province.

5. Une esquisse des idées politiques et sociales des Thesmophores a été dessinée dans notre livre par Serge Bianchi, p. 267-273, mais le sujet mériterait davantage, cf. les comptes rendus du livre par Patrick Fournier dans Histoire et Sociétés Rurales $n^{\circ} 33,2010$, p. 244-246, Jean-Pierre Jessenne dans les Annales historiques de la Révolution française, $\mathrm{n}^{\circ}$ 367, 2012, p. 220-222, Jean-Michel Boehler dans la Revue d'histoire moderne et contemporaine, 2012, n ${ }^{\circ} 3$, p. 179-181 et Graham Hamish dans French History, volume 27, juin 2013, p. 288-290. Le corpus entier est à la disposition des chercheurs.

6. Voir l'article de T. Mekechvili et B. Musset: "Les Thesmophores et la vigne...", p. 237-250.

7. C. Port, « Les Thesmophories de Blaison (1775-1777)».

8. La Gazette d'agriculture, commerce, arts et finances (sous ce titre de 1769 à 1783) édite en plus un supplément mensuel, le Journal de l'agriculture.

9. Il s'agit de l'ouvrage de Nicolas Bidet, patronné par Duhamel du Monceau : Traité sur la nature et sur la culture de la vigne, sur le vin, la façon de le faire et la manière de le bien gouverner, à l'usage des differens vignobles du royaume de France, effectivement paru en 1759.

10. F. Lebrun, «[...] la presse provinciale à la fin de l'Ancien Régime, les Affiches d'Angers, 1773-1789 ».

11. Un périodique n'avait véritablement qu'un seul « auteur » qui était le directeur de la gazette, lequel s'appropriait tout ce qu'il publiait parce qu'il en portait la responsabilité devant les autorités judiciaires et administratives.

12. Comme tout jeune noble d'épée, le marquis a servi dans l'armée.

13. Leur programme de 1777 a une question par mois, ce qui est plus raisonnable que les trois mensuelles de 1776. 
14. Première lettre de M. de Butré datée du 15 janvier 1776 à Tours, en réponse à la formation de la Société et à ses questions du $1^{\mathrm{er}}$ trimestre, Arch. dép. Maine-et-Loire, $8 \mathrm{D} 3$, pièce 4.

15. Le texte complet est édité dans le livre p. 89-91.

\section{RÉSUMÉS}

Dans un bourg de l'Ouest, Blaison en Anjou, quelques personnes ont créé vers 1775 un cercle d'étude qui n'était pas tout à fait original pour l'époque, mais qui se distingue aux yeux des historiens pour deux raisons au moins. D'abord, par un incroyable dynamisme durant les deux années qui sont documentées. Ensuite, par ses archives, qui jusqu'à preuve du contraire sont uniques en France. En effet, si la moitié à peu près des sociétés royales d'agriculture implantées dans les capitales régionales a laissé des archives, on ne sait rien des sociétés locales qui devaient relayer les nouveaux savoirs jusqu'aux paysans. Comme aucune autre société locale n'a laissé d'archives, celle de Blaison éclaire donc les historiens sur « La transmission des savoirs » et ce qui était parvenu de la science agronomique dans les petites villes et les campagnes, qu'il s'agisse des bons sujets de réflexion ou des méthodes savantes, vers la fin du XVIII ${ }^{\mathrm{e}}$ siècle.

\section{AUTEUR}

\section{ANTOINE FOLLAIN}

Professeur des universités et directeur de l'Institut d'histoire moderne, Université de Strasbourg, Palais universitaire, Faculté des sciences historiques 


\title{
Science, art ou pratique ? La place de l'agriculture dans les formes de transmission du savoir au XIX ${ }^{\mathrm{e}}$ siècle en France
}

\author{
Christiane Cheneaux-Berthelot
}

$1 \mathrm{Au}$ XIX ${ }^{\mathrm{e}}$ siècle, on parlait de l'art agricole, ce qui laisse place à l'esprit de création et en même temps, c'était une pratique, un artisanat si l'on considère la maintenance des instruments agricoles. La science était du côté de l'État ou d'œuvres privées qui semblaient à mille lieues des préoccupations des praticiens des campagnes, selon Daniel Zolla :

«Quand on se contente de la pratique sans se soucier de la théorie qui l'explique, on se condamne à la routine ? ${ }^{1}$ "

2 Parler de "routine", c'est poser le problème du malthusianisme économique des agriculteurs français au xIx ${ }^{e}$ siècle, face aux avancées des Anglais ou des Allemands :

«Il n'y a pas longtemps que l'agriculture anglaise a acquis le haut point de perfection où elle est parvenue. Dans les neuf dixièmes de la France, les bonnes méthodes de culture y sont encore inconnues [...] L'art de la culture est donc bien loin d'être arrivé dans toute la France au point où il peut atteindre. ${ }^{2}$ "

3 Le plan adopté ici s'articule en deux parties. La première partie se propose d'analyser l'étendue d'un vaste terrain d'étude : l'agriculture au xIX siècle. Deux points essentiels sont traités: les déboires d'une pédagogie du progrès en vue de l'éducation des cultivateurs, puis, "de la création à l'innovation: les techniques et le matériel agricole ». Dans une seconde partie, il est question des moyens d'enseigner le progrès agricole en passant de l'initiative privée début de siècle à une politique nationale fin de siècle. Ici encore sont abordés deux aspects majeurs: la difficile mise en place d'un enseignement généralisé vis-à-vis du public et l'investissement des chimistes de laboratoire à la conquête de la science de l'agronomie. 


\section{Du sommet à la base : l'agriculture, un vaste terrain d'étude}

4 Alors que le nombre des ruraux et des actifs agricoles étaient au plus haut niveau ${ }^{3}$, l'agriculture n'avait pas de ministère distinct. En 1881, la création d'un ministère spécial fut l'acte par lequel Gambetta inaugura son arrivée au pouvoir ${ }^{4}$.

\section{Les déboires d'une pédagogie du progrès}

5 À aucun moment les gouvernements n'ont perdu le système de la collecte statistique : dès la Constituante, on s'employa à rétablir les mercuriales, et la tradition de l'enquête annuelle sur les moissons fut reprise par François de Neufchâteau (29 messidor an VIII-18 juillet 1800). Inspiré par le libéralisme économique, il s'attacha à favoriser la renaissance des sociétés d'agriculture, figurant parmi les membres fondateurs de la Société d'Agriculture en 1798. Puis, c'est Chaptal à l'Intérieur en l'An IX, de 1800 à 1804, qui créa le Service de la Statistique ${ }^{5}$. Les préfets devaient adresser trimestre par trimestre un «état de situation » de leur département, notamment, celui des produits agricoles ${ }^{6}$.

En 1812, fut inaugurée la première enquête générale sur les trois "règnes » animal, végétal et minéral. Il s'agissait de faire le bilan de la Révolution par le biais d'une comparaison systématique à un écart de 10 ans pour juger de ses effets par l'artifice d'un double découpage: la coupe chronologique de la décennie révolutionnaire et le découpage en départements ${ }^{7}$. Cependant, les rubriques des questions concernant l'agriculture manquaient de cohérence, elles dénotaient un manque d'étude analytique et rationnelle du monde rural (fig. 1). 
Fig. 1. - La Statistique agricole de 1814, Notices, inventaires et documents.

\section{Chapitre IV_Observations générales_}

1. État de l'atmosphère pendant l'année ; son influence sur la végétation, sur les époques de semailles, des moissons, désignation de ces époques_Observations météorologiques, si l'on est dans l'usage d'en faire dans le département.

2. Circonstances qui ont favorisé ou entravé les travaux de l'agriculture_fléaux qui ont nui aux récoltes_Détails sur les pertes causées.

3. Progrès de l'économie rurale pendant l'année, soit par l'introduction de cultures nouvelles, soit par le perfectionnement des anciennes _ Détails à ce sujet_Entreprises remarquables qui méritent l'attention du gouvernement_Plantations des routes_Leurs progrès_Moyens d'y subvenir_Obstacles qui s'opposent à leur extension.

4. Travaux des Sociétés d'agriculture_Leur composition_Leur influence dans le pays Encouragements qu'elles donnent ou dont elles ont besoin.

5. Artistes vétérinaires_leur nombre__Leur influence__Leurs services_Sont-ils très employés dans le pays ?_Indication de ceux qui sont le plus distingués.

6. Prix des objets les plus nécessaires à la vie, et de la journée de travail_Comparaison des prix avec ceux de 1789 _Augmentation ou diminution pendant l'année.

7. Un grand nombre de propriétaires cultivent-ils par eux-mêmes ?_Quel est le mode le plus usité pour les baux ou à moitié fruit ? _Quelle est la durée des baux ?_Quelle sont les conditions du cheptel ?

8. Mouture économique_Depuis quand est-elle introduite ?_Quels sont les progrès ?

9. Police rurale_Gardes champêtres_Leur nombre_Leur salaires_Leur influence_Leur conduite_Délits ruraux_ Quels sont les délits les plus ordinaires ?_ Sont-ils devenus plus ou moins fréquents ? Comment sont-ils réprimés ? _ Note relative sur les usages dans le département relativement au glanage _à l'enlèvement des chaumes_à l'affouage_au grapillage_Inconvénients qui résultent des usages existants _ les mendiants et les vagabonds sont-ils nombreux dans les campagnes ?_Quel est le résultat des mesures prises pour les réprimer?

Édité par le Ministère de l'Instruction publique et des Beaux-Arts, Paris, F. Rieder et Cie Éditeurs, 1904, p. XIV de l'introduction.

7 Comme le cadastre ne fut terminé qu'en 1834, la statistique de 1837 ne donnait des indications que sur la population et le territoire en général. En 1841, apparurent des informations sur les cultures et les animaux domestiques, toujours rien sur la population agricole. Il faut attendre la réhabilitation des enquêtes agricoles «décennales» (1852, 1862, 1882, 1892), pour avoir des rubriques plus variées, notamment l'enquête décennale de 1862 où 214 pages étaient consacrées aux cultures, animaux, une part étant réservée à l'économie rurale (fig. 2). 
Fig. 2. - Statistique de la France, Agriculture.

\section{Troisième partie_Économie rurale}

a) Des diverses modes d'exploitation du sol : nombre de propriétaires cultivant par les soins d'un maître-valet, d'un régisseur : -nombre des propriétaires cultivant de leurs mains, cultivant à la fois pour eux et pour autrui, comme fermiers, métayers, journaliers ; nombre de fermiers non-propriétaires, des métayers non-propriétaires, des journaliers non-propriétaires ; _nombre des fermes louées par des baux de 3,6,9 ans et au-dessus.

b) Étendue des exploitations rurales (par quotité de superficie depuis moins de 5 jusuq'à 100 hectares.

c) Valeur vénale des prix de fermage, par hectare, des diverses natures de propriétés (terres, près, vignes, bois)

d) Salaires et gages des travailleurs agricoles nourris et non-nourris (salaires ordinaires avant et après la moisson ; _nombre moyen de journées de travail par an et par ouvrier agricole ; _gages et salaires des ouvriers et domestiques loués à l'année et attachés à la ferme).

e) Industries accessoires des travailleurs agricoles.

f) Outillage agricole.

g) Améliorations et faits agricoles divers constatés de 1852 à 1862 (défrichement, ensemencement et plantations de dunes, de montagnes, irrigations, drainages, desséchements).

Résultats généraux de l'enquête décennale de 1862, par son excellence la Ministre de l'agriculture, du Commerce et des Travaux Publics, Strasbourg, imprimerie administrative de Veuve berger-Levrault, 1868, p. 49

8 Néanmoins, l'agriculture était au cœur des préoccupations économiques depuis le milieu du XVIII ${ }^{\mathrm{e}}$ siècle : physiocrates et agronomes voulaient répandre des méthodes plus productives. Dans cette optique, la Société royale d'agriculture de la généralité de Paris avait été créée en 1761. Abolie sous la Convention en 1793, elle ressuscita en 1798 sous l'impulsion de François de Neufchâteau. Sa mission était de rassembler l'ensemble des connaissances de l'époque et de les soumettre à un débat approfondi avant de les diffuser dans le reste du royaume via la création de sociétés d'agriculture régionales ${ }^{8}$. Considérée comme la plus importante du pays, la société nationale d'agriculture publia une série de Mémoires depuis sa fondation en 1761 (tirés à 1000 exemplaires) et un bulletin mensuel de ses séances depuis $1837^{9}$, plus des indications biographiques dans l' Annuaire de la société.

Au lendemain de la Révolution de Juillet, les sociétés d'agriculture se développèrent, encouragées par le gouvernement. Tous les individus qui pouvaient s'intéresser au Progrès agricole étaient vivement encouragés à en faire partie. Selon Gabriel Désert, il faut y voir l'influence des nobles et notables légitimistes, qui, ayant du temps libre et des capitaux pour mettre en valeur leur patrimoine foncier, remplissaient « le rôle de catalyseurs, de diffuseurs de l'agriculture moderne ${ }^{10}$ ». Selon Nadine Vivier ${ }^{11}$, à partir de 1867, les conservateurs adhérèrent massivement à la Société des Agriculteurs de France, puis, après la victoire des Républicains en 1879, ces conservateurs irréductiblement hostiles à la République se replièrent sur les sociétés d'agriculture et bientôt dans les syndicats qu'ils dominaient. Ils façonnèrent alors une image flatteuse de notables exerçant une influence très bénéfique pour le progrès agricole. Leur volonté étant de conquérir les suffrages des paysans, face aux Orléanistes au pouvoir ${ }^{12}$. 
Mais quelles que fussent les raisons des créateurs et membres des sociétés agricoles, le succès de ces dernières encouragea une presse spécialisée.

Pour transmettre les avancées agronomiques aux paysans supposés "routiniers », on envisageait de passer par les grands exploitants, propriétaires ou fermiers, destinés à diffuser les techniques modernes aux petits cultivateurs. Les revues spécialisées s'adressaient à une élite terrienne. Ainsi, on peut lire, dans le Cours d'agriculture théorique et pratique à l'usage des écoles primaire et des plus simples cultivateurs d'Émile Jamet en 1846 :

«On m'a dit plus d'une fois que mes notices, insérées dans le journal hebdomadaire de notre arrondissement, s'élevaient trop au-dessus de l'intelligence des cultivateurs. ${ }^{13}$ »

11 Au début du XIX ${ }^{e}$ siècle, Mathieu de Dombasle, dans ses Annales de Roville ${ }^{14}$ (1824-1832), partit du double postulat d'un «retard français» face aux agricultures anglaise et allemande et que seule la grande exploitation pouvait répondre aux besoins de progrès. De 1810 à 1842, Mathieu de Dombasle publia près de 39 ouvrages, ainsi que de nombreux articles, dont la plupart connurent plusieurs rééditions ${ }^{15}$, mais l'alphabétisation des paysans, très restreinte à l'époque, en a limité l'impact. La pédagogie des Annales donnait des conseils d'économie rurale, ce qui était rare en 1824 : " capital, comptabilité, dépenses et produit ». Quant à son Calendrier du Bon cultivateur ${ }^{16}$, il s'achevait par 70 pages en moyenne d'enseignement destiné aux agriculteurs moins instruits intitulé : «La richesse du cultivateur ou les secrets de Jean-Nicolas Benoît ». Il y présentait un personnage fictif et le faisait dialoguer avec son cousin, lui exposant des expériences réussies de techniques agricoles, sous forme de «leçon de choses». Il semble qu'il y ait eu au final pénétration dans les couches populaires : le «Calendrier [du Bon Cultivateur] est devenu le catéchisme dans lequel les enfants de nos villages ont appris le langage du progrès agricole ${ }^{17}$ ».

Lorsqu'on regarde la composition du comité d'associés-fondateurs qui a donné naissance au Moniteur de la propriété et de l'agriculture, paru à partir de 1835, on mesure à quel point le parrainage venait des élites: pas moins de 11 vicomtes, 20 comtes, 7 barons, 1 chevalier, 5 ducs et 7 marquis sur 60 fondateurs. Cette revue mensuelle visait le «développement des arts agricoles, la propriété du sol et la défense et extension de la propriété » avec le concours des représentants de la grande propriété terrienne. Le Journal d'agriculture pratique ${ }^{18}$ naquit deux ans après, en 1837 : sa devise était :

«Progrès avec prudence, pratique avec sciences.»

13 Néanmoins, la pédagogie de cette revue a mis du temps à être efficace; avant 1854, les informations, présentées mois par mois, faisaient apparaître les articles d'auteurs sans suivi alphabétique et sans classement par thème. Difficile donc, sur plus de 500 pages, de trouver rapidement une information. Malgré la concurrence ${ }^{19}$, le JAP, tirait à 2000 exemplaires en 1849 , et à 10000 exemplaires en 1860 , preuve de son succès ${ }^{20}$ (fig. 3 ). 
Fig. 3. - Table des matières du JAP.

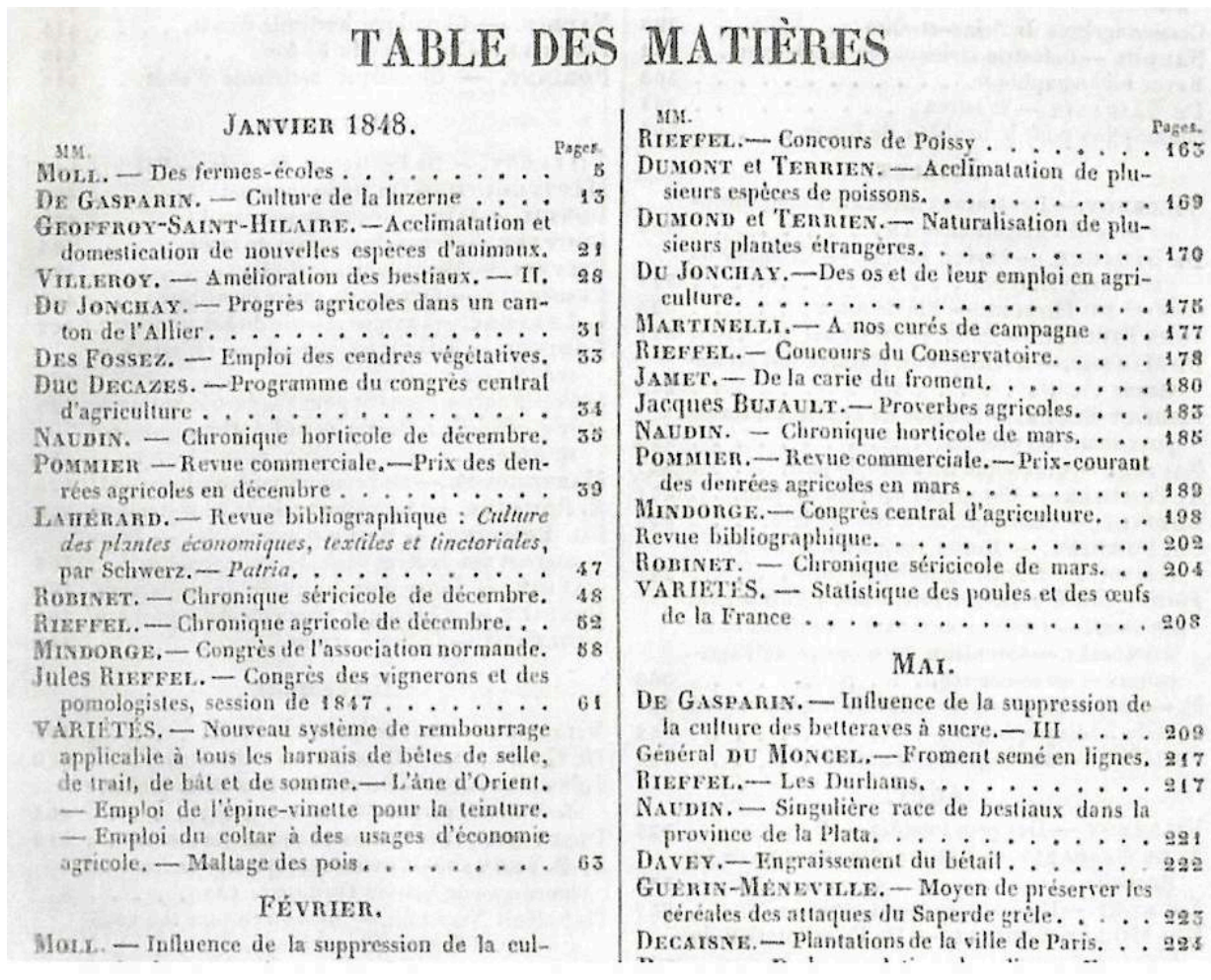

Alexandre Bixio (dir.), Journal d'agriculture pratique et de jardinage et d'économie domestique, Paris,

librairie agricole de la maison rustique, deuxième série, tome $\mathrm{V}$, janvier à décembre 1848.

14 Chloé Gaboriaux a analysé les aspirations de ces « agriculteurs-pratiques ». Contre leurs aînés physiocrates et aussi contre les économistes de leur temps ${ }^{21}$, les agriculteurspratiques refusaient de voir dans le modèle anglais la seule voie de progrès agricole et pensaient que les petites exploitations, majoritaires en nombre, n'étaient pas exclues de la modernisation, en étaient même la condition. Ils défendaient une sorte de modèle de développement spécifiquement français ${ }^{22}$.

C'est en 1788 que s'organisèrent les premiers comices agricoles dans la généralité de Paris. Supprimés à la Révolution, il fallut donc attendre le 31 mai 1833 pour les voir refleurir. Les comices organisaient des concours sur les techniques de labour les animaux les plus performants, ils donnaient des primes. Ces réunions avaient un tel pouvoir d'attraction dans les campagnes au niveau du canton et de l'arrondissement qu'on peut dire qu'elles ont joué un rôle important dans la diffusion des nouveautés. En Bretagne et dans le Nord-Est, les comices cantonaux se multiplièrent par la volonté des propriétaires et des fermiers de la région. Il s'agissait d'échanger leurs expériences afin d'améliorer les procédés agricoles. Ces assemblées jouissaient d'un grand prestige et formaient des pôles d'attractivité économique. Ils avaient chacun entre 100 et 500 adhérents $^{23}$.

\section{De la création à l'innovation : les techniques et le matériel agricole}

L'exemple pris dans le département de la Seine peut néanmoins montrer les différences importantes de machines à cultiver d'un canton à l'autre et les réticences à 
l'innovation, à l'instar de ce que l'on pouvait lire à propos du passage de la faucille à la faux :

«La faux elle-même, cet outil simple, ne pénètre que lentement dans les contrées où son usage n'est pas général depuis longtemps [...] S'il est naturel d'hésiter à employer la faux, parce qu'on doit apprendre à la connaître, combien n'est-il pas naturel qu'un fermier hésite à se faire, dans son pays, le promoteur d'une moissonneuse. ${ }^{24}$ »

Dans la culture des légumes de plein champ, il s'avère que l'on ne donnait à la terre que deux « façons ", à savoir : labour à la charrue et hersage. Cette culture légumière était tellement répandue dans l'arrondissement de Saint-Denis, dans différents cantons qu'on remarquera que le panel d'instruments agricoles était très réduit et se limitait à ces deux types, charrue et herse. Il y avait, dans chaque canton plus de trois cent et jusqu'à quatre cents " charrues du pays ». Et, sauf dans le canton de Courbevoie, moins bien doté en herses, un nombre impressionnant de herses : entre six cents et huit cents herses par canton. Dans l'arrondissement de Sceaux, la culture légumière et fruitière était plus associée aux céréales et aux plantes fourragères, et là, le panel d'instruments de travail était plus varié. À part le canton de Vincennes, il y avait une similitude parfaite dans l'équipement des trois autres cantons: on y trouvait une multitude de " machines perfectionnées diverses »: scarificateurs, extirpateurs, fouilleuses, houes à cheval, buttoirs, semoirs et coupe-racines. C'est que l'emploi des outils dits " cultivateurs » remplaçait "avec avantage », selon Mathieu de Dombasle, un ou deux labours sauf le premier (fig. 4).

Fig. 4. - Tableau de l'outillage agricole en 1862, cantons de Pantin, Courbevoie, Saint-Denis (arrondissement de Saint-Denis) et canton de Charenton, Villejuif, Vincennes et Sceaux (arrondissement de Sceaux).

\begin{tabular}{|c|c|c|c|c|c|c|c|c|}
\hline & & \multicolumn{3}{|c|}{$\begin{array}{l}\text { Nombre d'instruments, } \\
\text { arrondissement de Saint-Denis. }\end{array}$} & \multicolumn{4}{|c|}{$\begin{array}{l}\text { Nombre d'instruments, arrondissement de } \\
\text { Sceaux. }\end{array}$} \\
\hline \multicolumn{2}{|c|}{ Type d'outillage } & $\begin{array}{l}\text { canton de } \\
\text { Pantin }\end{array}$ & \begin{tabular}{|c|} 
canton de \\
Courbevoie \\
\end{tabular} & $\begin{array}{r}\text { canton de } \\
\text { Saint-Denis } \\
\end{array}$ & $\begin{array}{l}\text { canton de } \\
\text { Villejuif }\end{array}$ & $\begin{array}{l}\text { canton de } \\
\text { Charenton }\end{array}$ & $\begin{array}{l}\text { canton de } \\
\text { Sceaux }\end{array}$ & $\begin{array}{l}\text { canton de } \\
\text { Vincennes }\end{array}$ \\
\hline \multicolumn{2}{|c|}{$\begin{array}{c}\text { Charrues du } \\
\text { pays }\end{array}$} & 400 & 382 & 312 & 473 & 121 & 233 & $"$ \\
\hline \multirow{2}{*}{\begin{tabular}{|c|} 
Charrues \\
perfectionné
\end{tabular}} & vec avant-tra & $"$ & 104 & 369 & $"$ & 72 & 10 & 235 \\
\hline & ans avant-tra & $"$ & $"$ & $"$ & $"$ & 2 & " & $"$ \\
\hline \multirow{8}{*}{$\begin{array}{l}\text { Machines } \\
\text { perfectionné } \\
\text { es diverses }\end{array}$} & Scarificateurs & $"$ & $"$ & $"$ & $"$ & 14 & $"$ & " \\
\hline & Extirpateurs & $"$ & $"$ & $"$ & 1 & 10 & 3 & $"$ \\
\hline & Fouilleuses & $"$ & $"$ & $"$ & 1 & 5 & " & $"$ \\
\hline & Houes à chev & $"$ & $"$ & $"$ & 8 & 17 & $"$ & " \\
\hline & Herses & 800 & 180 & 646 & $"$ & 175 & 458 & $"$ \\
\hline & Buttoirs & $"$ & $"$ & $"$ & 5 & 12 & $"$ & $"$ \\
\hline & Semoirs & $"$ & $"$ & $"$ & 1 & 6 & 1 & $"$ \\
\hline & coupe-racine & $"$ & $"$ & $"$ & 39 & 18 & 8 & $"$ \\
\hline $\begin{array}{c}\text { es diverses } \\
\begin{array}{c}\text { Machines à } \\
\text { faner }\end{array}\end{array}$ & & $"$ & $"$ & $"$ & $"$ & 4 & $"$ & $"$ \\
\hline \multicolumn{2}{|l|}{$\begin{array}{c}\begin{array}{c}\text { Machines à } \\
\text { faucher }\end{array} \\
\end{array}$} & $"$ & $"$ & $"$ & $"$ & 4 & $"$ & $"$ \\
\hline \multicolumn{2}{|l|}{$\begin{array}{l}\text { Machines à } \\
\text { moissonner }\end{array}$} & $"$ & $"$ & $"$ & $"$ & 2 & 1 & $"$ \\
\hline \multirow{2}{*}{$\begin{array}{c}\text { Machines à } \\
\text { battre }\end{array}$} & à vapeur & $"$ & $"$ & $"$ & $"$ & 1 & 1 & $"$ \\
\hline & 8 par des anir & $"$ & $"$ & $"$ & 7 & 5 & 6 & $"$ \\
\hline
\end{tabular}

Source: A.N.F./11/2709. Enquête de 1862, séries départementales, questionnaires par cantons. Département de la Seine, arrondissement de Saint-Denis, cantons de Pantin, Courbevoie, Saint-Denis ; arrondissement de Sceaux, canton de Charenton, Villejuif, Vincennes et Sceaux. 
La « charrue du pays » qui était la plus répandue dans les cinq cantons du panel, était la « charrue de Brie » avec avant-train, une charrue particulièrement adaptée aux terres fortes (fig. 5). Les charrues « du pays » et " perfectionnées » étaient plus de deux mille ${ }^{25}$ alors que l'on comptait seulement deux charrues «sans avant-train» dans les cinq cantons. Un rapport de un à mille qui interpelle. La charrue Dombasle était de ces charrues sans avant-train ou charrue simple qui reprenait la conformation d'un araire (fig. 6). Cette charrue datait de 1821, elle faisait figure de progrès et cependant, cinquante ans après sa création, on n'en trouvait que deux dans les cinq cantons précités. C'est que sa diffusion avait fait débat. Avant la charrue Dombasle, les charrues sans avant-train étaient assimilées à des araires archaïques. En 1809, le Nouveau cours complet d'agriculture théorique et pratique critiquait abondamment la charrue sans avanttrain sur pas moins de dix pages ${ }^{26}$. C'est que cette charrue remettait en cause le savoirfaire des laboureurs aguerris et compétents pour ouvrir le champ à des ouvriers moins qualifiés, plus jeunes; c'était une question de chômage pour les anciens laboureurs. C'est pourquoi, Mathieu de Dombasle recommandait de prendre mille précautions avant de confier un nouvel instrument entre les mains des ouvriers, de façon à ne pas froisser leur amour-propre ${ }^{27}$.

Fig. 5. - Charrue de Brie perfectionnée à age oblique.

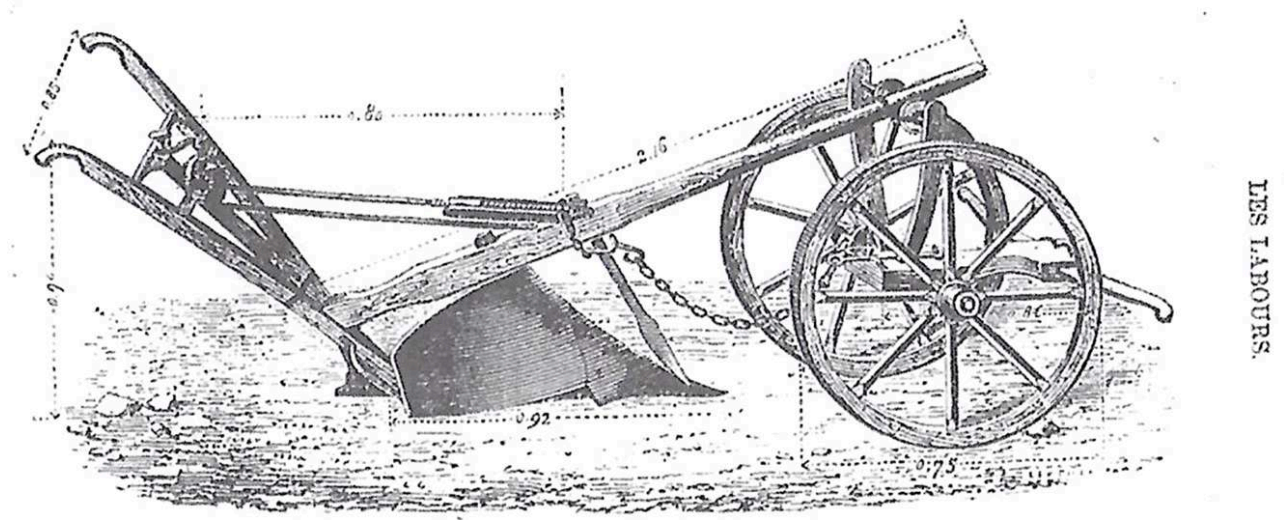

Gustave heuzé, La Pratique de l'agriculture, t. 1, Paris, librairie agricole de la Maison rustique, 1889, p. 125. 
Fig. 6. - Charrue Dombasle sans avant-train.

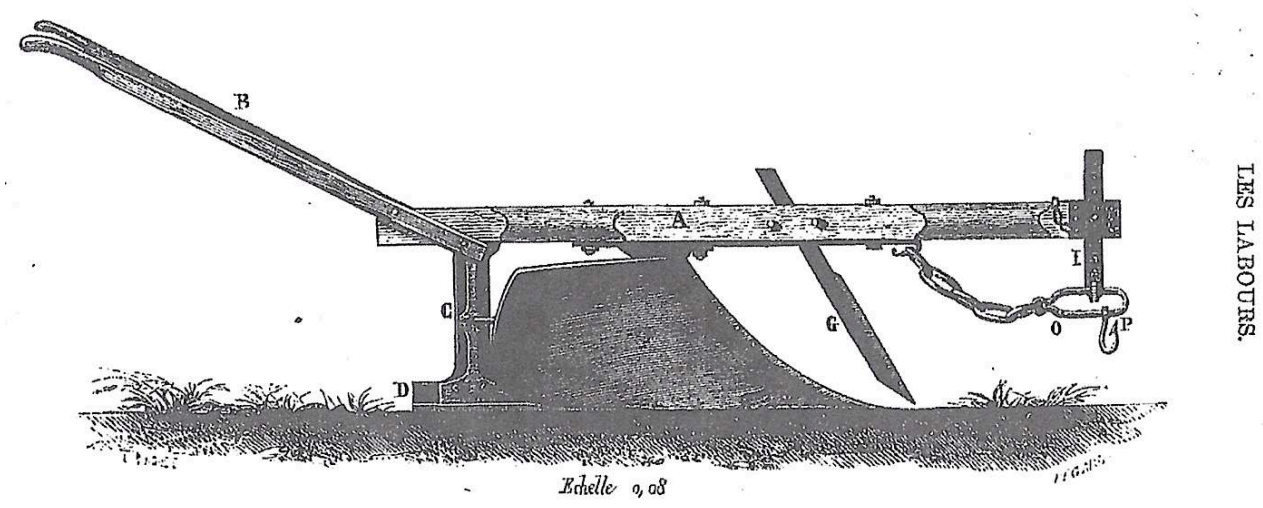

Gustave heuzé, La Pratique de l'agriculture, t. 1, Paris, librairie agricole de la Maison rustique, 1889, p. 125.

19 Au demeurant, en 1832, la charrue sans avant-train de Dombasle reçut un avant-train pour devenir la charrue de Roville à avant-train, et de manière concomitante, les charrues à avant-train avaient été perfectionnées, les deux modèles se rapprochaient. Aucune charrue ne faisait l'unanimité, les cultivateurs, ainsi tous rompus aux travaux manuels, techniciens par nécessité, rajoutaient ou supprimaient des pièces ou changeaient la direction des pièces de leurs charrues. En 1835, il semble que l'usage de la charrue Dombasle se soit généralisé dans tout le royaume, le nombre de fabrique se multipliait, de façon à fournir près de dix milles araires en France, dont un tiers fabriqués à Roville ${ }^{28}$.

\section{Des moyens d'enseigner le progrès agricole : de l'initiative privée à une politique nationale}

Avant 1848, il n'existait aucun enseignement officiel de l'agriculture en France, sauf à l'École d'Alfort créée en 1806, confiée à Victor Yvart ${ }^{29}$, qui exploitait la ferme annexée à l'École. Malgré les vœux de la Société impériale d'agriculture, qui demandait la création d'un enseignement primaire et secondaire d'agriculture, l'administration impériale refusait d'accéder à sa demande.

\section{La difficile mise en place d'un enseignement généralisé.}

Ce que les pouvoirs publics refusaient de faire, l'initiative privée s'en chargea. À partir de 1830, des fermes-modèles ou fermes-écoles avaient commencé à s'organiser. En 1822, Mathieu de Dombasle créa l'École de Roville : il prit à ferme, pour vingt-deux ans, cent cinquante hectares de terres sur lesquelles il substitua la culture alternée, avec suppression de la jachère, avec l'assolement triennal. Il diffusa ses méthodes de culture par les Annales de Roville. Bientôt, l'École reçut des élèves de tous les points de l'Europe. Fondateur de la première fabrique importante de machines agricoles, pionnier de l'industrie sucrière, il lutta pendant vingt ans contre les difficultés financières de cette exploitation ${ }^{30}$. L'agronome, qui ne recevait de l'État qu'une subvention insignifiante, dut abandonner à regret l'œuvre à laquelle il avait consacré sa vie. L'école de Roville 
avait formé environ trois cents élèves et donné une vive impulsion à l'agriculture française.

En 1826, une société par actions fonda l'établissement qui prit le titre d'Institut agronomique de Grignon $^{31}$, près de Neauphle-le-Château, dont la direction fut confiée à Auguste Bella ${ }^{32}$. Le système développé par Dombasle à l'École de Roville en 1823 reposait sur un capital d'exploitation faible ne permettant pas l'emploi des engrais commerciaux faute de moyens financiers. L'Institution de Grignon mettait en œuvre les principes de la " culture améliorante ${ }^{33}$ ", mais avec de gros capitaux et emploi d'engrais commerciaux pour compléter le fumier. À Roville, on pensait «dépense à l'hectare " tandis qu'à Grignon, on pensait « prix de revient à l'hectolitre ${ }^{34}$ ». Avec Grignon, l'école de Grandjouan ${ }^{35}$ et celle de la Saulsaie ${ }^{36}$ formèrent trois écoles régionales.

En 1848, pour la première fois, l'enseignement agricole fit l'objet d'un long débat à l'Assemblée nationale. Certains souhaitaient retenir les populations agricoles sur leurs terres pour éviter le gonflement des banlieues, d'autres voulaient en faire le socle d'une économie moderne. Les conservateurs voulaient laisser libre cours à l'initiative privée, les Républicains étaient pour l'implication de l'État dans cette tâche. Le Ministre de l'agriculture et du Commerce parvint à faire voter par l'Assemblée Nationale le 3 octobre 1848 une loi : l'enseignement en agriculture était reconnu comme un élément essentiel de la politique agricole. Un système de bourses permettait aux meilleurs élèves des fermes-écoles d'accéder aux écoles régionales puis d'entrer à l'INA : dès l'origine l'enseignement agricole est caractérisé par l'attention portée à la promotion sociale de ses élèves.

Les fermes-écoles départementales accueillaient les jeunes d'au moins 16 ans pour en faire de bons ouvriers agricoles et des contremaîtres en 3 ans d'études et travaux pratiques. L'État se contentait de payer les professeurs et de donner à chaque élève 250 francs par an d'entretien, plus un pécule selon le mérite (250 à 300 francs) ${ }^{37}$. La partie pratique se faisait aux risques de l'exploitant de la ferme, le directeur étant soit le propriétaire, soit le fermier. Ceci explique le grand nombre de désirs de création (46 projets en 1849), mais les députés de la Seconde République rognèrent le budget dès 1850 et leur nombre alla décroissant : 70 en 1849, 52 en 1853, 48 en $1865^{38}$.

Quant à l'Institut national agronomique installé à Versailles, il ouvrit ses cours le 2 décembre 1850 et disparut deux ans plus tard. Le décret du 2 décembre 1852 le supprima invoquant des dépenses supérieures aux avantages retirés. À tous ses degrés, l'enseignement agricole périclita; sa dotation fut réduite de moitié en 1870 . Sous l'Empire, les Chambres d'agriculture furent nommées, au lieu d'être élues par arrondissement, le Conseil Général de l'Agriculture ne fut plus réuni, le Ministère de l'Agriculture et du Commerce furent mis sous la tutelle du Ministère de l'Intérieur, l'INA fut fermé. Le Second Empire considérait que si l'économie générale allait bien, elle entraînerait l'agriculture, il n'y avait pas à lui faire un sort particulier.

C'est la loi du 9 août 1876 qui rétablit une grande partie de ces institutions. L'institut agronomique fut reconstitué et de grandes écoles spéciales d'horticulture, de laiterie, des industries annexes, furent aussi créées. La loi du 16 juin 1879 rendit l'enseignement de l'agriculture obligatoire, dans les écoles normales d'instituteurs et dans les écoles primaires. Les stations agronomiques et les laboratoires agricoles vinrent compléter le cadre de l'enseignement ${ }^{39}$. 


\section{Les chimistes à la conquête de l'agronomie} transformations : des chimistes partirent à la conquête d'un territoire de recherches, auparavant dévolu aux agronomes. Le pionnier fut sans doute Jean-Baptiste Boussingault : en 1834, il acquit une ferme à Bechelbronn ${ }^{40}$ à laquelle il adjoignit un laboratoire en 1836 pour analyser le sol, les engrais, semences et végétaux tout au long du cycle de culture. Il en publia un ouvrage, l'Économie rurale, publié en France en 1843-44, traduit en allemand en 1844-45 puis en anglais en 1845. Boussingault soutenait que les minéraux étaient importants dans la nutrition végétale à cause de leur proportion d'azote. L'allemand Liebig, au contraire, dans sa première édition, en 1840, De la chimie et ses applications à l'agriculture et à la physiologie, pensait que c'est leur contenance en minéraux qui faisait la valeur fertilisante des engrais, et que l'ammoniac contenu dans les eaux de pluie suffisait à restituer l'azote emprunté.

Le transfert des agronomes aux chimistes fut surtout sensible à la Section d'Économie rurale de l'Académie des Sciences, où de nombreux chimistes furent nommés à partir de 1840 : Boussingault y entra en 1839. Les correspondants de cette section étaient aussi de plus en plus des chimistes: Kuhlmann ${ }^{41}$, I. Pierre ${ }^{42}$. Mais la chimie agricole n'était encore qu'une de leurs recherches, il a fallu attendre 1850 pour qu'une deuxième génération de chimistes ne se consacra qu'à des questions de chimie agricole. C'est le cas de Dehérain dans sa thèse de 1859 sur l'emploi des phosphates en agriculture, puis de l'ingénieur Millot, ou encore Bobierre ${ }^{43}$. Selon Nathalie Jas, avant le xvIII siècle, l'image du chimiste était plutôt celle de l'alchimiste ténébreux, il lui a fallu conquérir la respectabilité. Puis, au XIXe les chaires et laboratoires de chimie se sont multipliés : il y avait assez de postes à pourvoir et l'intitulé de leurs chaires ne les limitait pas au seul champ défini par celle-ci.

En 1867, Louis Grandeau décida de fonder à $\mathrm{Nancy}^{44}$ une station agronomique de l'Est, sur le modèle allemand; la présentation des stations allemandes à L'Exposition universelle de 1867 l'aurait incité à cette création. Pendant les années suivantes, deux périodiques spécialisés, les Annales agronomiques et les Annales de la Science agronomique française furent fondés. Dans les États germaniques les plus industrialisés, les chimistes agricoles allemands pensaient que les engrais étaient nécessaires à une agriculture performante, elle-même indispensable dans un contexte d'industrialisation et d'urbanisation rapides. Or, le commerce des engrais était intrinsèquement frauduleux et perverti. Donc, à la chimie agricole de distinguer un «vrai » engrais d'un produit falsifié. Cette dialectique fonctionna bien et, au cours des années 1860, les grandes stations allemandes s'imposèrent comme des lieux de recherche et de contrôle performants. Puissantes, reconnues scientifiquement et socialement, elles devinrent les modèles à imiter ${ }^{45}$.

Pour assurer la réussite de sa station d'agronomie, Grandeau ne ménagea pas sa peine. Mais, la station restait une entreprise privée qui ne reçut que des subventions de départ, alors qu'en Allemagne, les stations recevaient des aides annuelles de l'État et des administrations provinciales. En 1877, il n'existait que deux stations agronomiques, celle de Nancy et celle de Mettray. Au cours des années 1870-1880, en France, 25 établissements furent subventionnés par le ministère, mais c'étaient des établissements au statut précaire, ne pouvant guère mener de vraies recherches. Les directeurs de stations agronomiques ne parvenaient pas à imposer, dans le contexte 
français, le système du contrat entre stations et fabricants, système qui avait tant favorisé les stations allemandes. Cependant, elles réussirent à survivre aux difficultés de départ. En 1900, on comptait ainsi une soixantaine d'établissements et en 1921 fut créé l'Institut des recherches agronomiques (IRA).

31 Les recherches de Louis Pasteur (1822-1895) eurent de nombreuses retombées en agriculture. Outre ses travaux sur la fermentation, les maladies du vin et du bétail, sa mise en évidence des micro-organismes dans la destruction des vers à soie constitua le départ de nombreuses recherches de lutte biologique au moyen de bactéries. Mais il semble néanmoins que ce qui a fait progresser la science en matière d'agriculture, ce sont les grands fléaux de la fin du XIX ${ }^{e}$ siècle qui obligèrent la science à progresser dans le domaine de l'agriculture. L'oïdium, puis le phylloxéra et le mildiou de la vigne introduits des Amériques ont dévasté successivement les vignes de l'Ancien monde. Les savants ont été obligés de décrire, d'expliquer et de combattre ces fléaux ${ }^{46}$, car la disparition temporaire ou définitive de certains vignobles dépeuplait des régions entières. Entre 1884 et 1889, 587 syndicats agricoles furent créés; outre la défense des intérêts des agriculteurs, ils procédaient à l'achat d'engrais pour le compte de leurs adhérents. Ces achats collectifs se faisaient en passant par des expertises des stations agronomiques. Ils ont donc eu un rôle actif à la fois dans l'utilisation des engrais chimiques et dans le recours aux représentants de la science agronomique : ils ont ainsi aidé à coupler pratique et théorie ${ }^{47}$.

\section{BIBLIOGRAPHIE}

AGULHON Maurice, DÉSERT Gabriel, SPECKLIN Robert, « Apogée et crise de la civilisation paysanne », dans DUBY Georges, WALLON Armand (dir.), Histoire de la France rurale, t. 3, Tours, Mame imprimeur, 1987.

BOURGUET Marie-Noëlle, Déchiffrer la France. La statistique départementale à l'époque napoléonienne, Paris, édition des archives contemporaines, 1988.

DUGUAY Raymond, L'Enseignement agricole en France à tous les degrés. Écoles et cours d'agriculture, Paris, librairie Larousse, 1903.

FRAISSE Fr., Fêtes du centenaire de Mathieu de Dombasle et concours régional, compte rendu au nom de la société centrale d'agriculture de Meurthe et Moselle, impr. E. Réau, Nancy, 1878.

GABORIAUX Clhoé, « Entre innovations agronomiques et pratiques paysannes. La figure de l'agriculture pratique au XIX ${ }^{\mathrm{e}}$ siècle ", dans Sciences, chercheurs et agriculture. Pour une histoire de la recherche agronomique, BONNEUIL Christophe, GILLES Denis et MAYAUD Jean-Luc (dir.), Paris, éditions Quae L'Harmattan, 2008, p. 45-60. 
GRANDEAU Louis, « Les stations agronomiques aux États-Unis, en Allemagne et en France, leurs ressources et leur développement ", Annales de la Science agronomique française et étrangère, t. 1, 1901, p. 452-460.

GRANDEAU Louis, L'agriculture et les institutions agricoles du monde au commencement du XXe siècle, t. III, Paris, imprimerie nationale, 1906.

JAMET Émile, Cours d'agriculture théorique et pratique à l'usage des plus simples cultivateurs, suivi d'une notice sur le chaulage en Mayenne, Château-Gontier, L. Delaplace éditeur, 1846.

JAS Nathalie, « Déqualifier le paysan, introniser l'agronome, France 1840-1914 », Écologie \& politique, 2005/2, n 31, p. 45-55.

JAS Nathalie, Au carrefour de la chimie et de l'agriculture, Paris, Édition des archives contemporaines, 2000.

KNITTEL Fabien, «La diffusion d'une pédagogie agricole : les écrits de Mathieu de Dombasle », Annales de l'Est, Association d'historiens de l'Est, 2002, p. 131-143.

MANGON Hervé, Traité de génie rural, travaux et instruments et machines agricoles, Paris, Dunod éditeur, 1875.

MATHIEU de DOMBASLE Christophe-Joseph-Alexandre, Annales agricoles de Roville ou mélanges d'agriculture, d'économie rurale et de législation agricole. Tome I à VIII in- $8^{\circ}$. Librairie Huzard, Treuzel et Wurtz, Paris, 1824-1832.

PASSY Louis, Table générale des principales matières contenues dans le Bulletin de la Société Nationale d'agriculture de France depuis la création du Bulletin (1837), jusqu'à l'année 1894, Paris, typographie Chamerot et Renouard, 1896.

PROFESSEURS, ÉCONOMISTES, AGRICULTEURS, MÉDECINS VÉTÉRINAIRES, Cours complet d'agriculture, d'économie rurale et de médecine vétérinaire, $4^{\mathrm{e}}$ édition, tome 7 , Paris, au Bureau du Cours complet d'agriculture, 1846.

VIVIER Nadine, « L'enseignement agricole sous le Second Empire. L'application du décret du 3 octobre 1848 ", dans Les enjeux de la formation des acteurs de l'agriculture, 1760-1945, actes du Colloque ENESAD, 19-21 janvier 1999, p. 145-152.

VIVIER Nadine, Élites et progrès agricole, XVI ${ }^{e}$-XVIII ${ }^{e}$ siècle, Presses universitaires de Rennes, 2009. zoLLA Daniel, Questions agricoles d'hier et d'aujourd'hui, chronique agricole du journal « Les Débats ", Paris, Armand Colin, 1904.

\section{NOTES}

1. D. Zolla, Questions agricoles d'hier et d'aujourd'hui, p. 10-11.

2. Cours complet d'agriculture, d'économie rurale et de médecine vétérinaire, p. 75.

3. Les ruraux représentaient $75 \%$ de la population. En 1850, il y avait $64 \%$ d'agriculteurs dans la population active, et $50 \%$ en 1861.

4. L. Grandeau, L'agriculture et les institutions agricoles du monde au commencement du $X^{e}$ siècle, p. 1-2.

5. Le 18 germinal an VIII (8 avril 1800). 
6. Circulaire du 19 germinal an IX (9 avril 1801). Chapitre 1 : topographie, chapitre 2 : analyse de la population. Chapitre 3 : état de la société hygiène, criminalité, instruction, mendicité, ressources, chapitres 4 et 5 : produits agricoles, activités industrielles, commerce, arts.

7. M-N. Bourguet, Déchiffrer la France. La statistique départementale à l'époque napoléonienne.

8. Société royale d'agriculture de la généralité de Paris (1761-1788); Société d'agriculture du département de la Seine (1798-1814); Société royale et centrale d'agriculture (1814-1848); Société nationale et centrale d'agriculture (1848-1853) puis en 1915 : Académie d'agriculture de France, dont l'action perdure.

9. L. Passy, Table générale des principales matières contenues dans le Bulletin de la Société Nationale d'agriculture de France depuis la création du Bulletin (1837), jusqu'à l'année 1894, p. 10.

10. M. Agulhon, G. Désert, R. Specklin, Apogée et crise de la civilisation paysanne, p. 116-117.

11. N. Vivier, Élites et progrès agricole, $\mathrm{XVI}^{e}$-XVIII ${ }^{e}$ siècle, p. 192.

12. Cette image pouvait d'autant mieux s'imposer qu'elle s'appuyait sur une première image que les légitimistes des années 1830 avaient commencé à forger.

13. É. Jamet, Cours d'agriculture théorique et pratique à l'usage des plus simples cultivateurs, suivi d'une notice sur le chaulage en Mayenne, p. 3.

14. C.-J.-A. Mathieu de Dombasle, Annales agricoles de Roville ou mélanges d'agriculture, d'économie rurale et de législation agricole.

15. F. Knittel, «La diffusion d'une pédagogie agricole: les écrits de Mathieu de Dombasle », p. 131-143.

16. Calendrier du bon cultivateur, ou Manuel de l'agriculteur praticien, Nancy: Impr. de Haener, chez l'Auteur, et Paris: $\mathrm{M}^{\mathrm{me}}$ Huzard, 1821, 1822, in- $12 ; 2^{\mathrm{e}}$ éd., Paris, $\mathrm{M}^{\mathrm{me}}$ Huzard, 1824, in-12, VII-493 p. ; $3^{\mathrm{e}}$ éd. Paris, $\mathrm{M}^{\mathrm{me}}$ Huzard, 1830, in-12, XI-475 p. ; $4^{\mathrm{e}}$ éd., Paris, 1833, in-12, VIII-588 p.; $5^{\mathrm{e}}$ éd. (1838), $6^{\mathrm{e}}$ éd., Paris : $\mathrm{M}^{\mathrm{me}}$ Huzard, 1840, in-12,

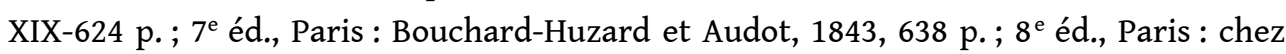
Bouchard-Huzard, et Nancy, 1846, in-12, XXV-660 p.; $9^{e}$ éd., Paris: $V^{e}$ BouchardHuzard, in-12, XLVI-820 p., et 1860, in-12, LI-871 p.

17. F. Fraisse, Fêtes du centenaire de Mathieu de Dombasle et concours régional, compte rendu au nom de la société centrale d'agriculture de Meurthe et Moselle, impr. E. Réau, Nancy, 1878. Cité par F. Knittel «La diffusion d'une pédagogie agricole : les écrits de Mathieu de Dombasle », p. 131-143.

18. J.-A. Bixio (dir.), Journal d'agriculture pratique, de jardinage et d'économie domestique, Paris, bureau de la maison rustique, de 1837 à 1848, puis le périodique continua sous le nom de Journal d'agriculture pratique (JAP), jusqu'en 1879. En 1866, c'est Édouard Lecouteux qui en devint rédacteur en chef. En 1909, le Journal de l'agriculture fondé par Jean-Augustin Barral fusionna avec le JAP; jusqu'en 1937, le journal subit des transformations qui amenèrent sa disparition.

19. Jean-Augustin Barral fut le cofondateur avec Bixio du JAP en 1837. Bixio en fut le rédacteur en chef de 1837 à 1848, puis Barral lui succéda de 1850 et 1866, date à laquelle il fut évincé par le propriétaire du titre. Barral créa alors une revue concurrente, le Journal d'Agriculture. 
20. C. Gaboriaux, «Entre innovations agronomiques et pratiques paysannes. La figure de l'agriculture pratique au XIX ${ }^{\mathrm{e}}$ siècle », p. 54.

21. Le terme désigne alors les théoriciens du libéralisme économique dont la tribune était Le Journal des économistes.

22. Convaincus, dès les années $1830-40$, que les gouvernements ne reviendraient pas sur le partage égal des biens, beaucoup de propriétaires terriens se sentaient menacés par le morcellement. En même temps, ils perdaient leurs privilèges politiques avec l'abaissement du cens en 1831, puis sa suppression en 1848.

23. N. Vivier, Élites et progrès agricole, $\mathrm{XVI}^{e}-\mathrm{XX} \mathrm{X}^{e}$ siècle, p. 196-198.

24. H. Mangon, Traité de génie rural, travaux et instruments et machines agricoles, p. 709.

25. 2233 charrues du pays, « perfectionnées avec avant-train » dans les cinq cantons de Pantin, Courbevoie, Saint-Denis, Villejuif et Charenton.

26. Critiques des charrues sans-avant-train dans le Nouveau cours d'agriculture en 1809. Source : Membres de la section d'agriculture de l'Institut de France (ouv. coll.), Nouveau cours complet d'agriculture théorique et pratique ou dictionnaire raisonné et universel d'agriculture, Paris, Deterville, 1809, p. 369 à 396.

27. Précautions à prendre auprès des ouvriers pour introduire de nouveaux instruments sur une exploitation agricole. Source: C-J-A. M. de Dombasle, «De l'introduction des nouveaux instruments d'agriculture dans une exploitation rurale ", p. 294-295.

28. Catalogue des instruments de la fabrique de Roville. Source : C-J-A. M. de Dombasle, "Catalogue des instruments de la fabrique de Roville", Calendrier du bon cultivateur ou manuel de l'agriculteur praticien, 1830, p. 473-475.

29. Victor Yvart (1763-1831) était un agronome français. Le gouvernement lui confia la direction d'une ferme nationale pour faire valoir des terres de mauvaise qualité, en parties marécageuses, cultivées de seigle. Il dirigea ces terres pendant 30 ans, elles devinrent un modèle pour la culture des plantes alimentaires, et pour l'élève des animaux domestiques. Mais sous le régime de la Terreur, il fut accusé de provoquer la famine en consacrant des terres à blé aux plantes sarclées et aux prairies artificielles dans sa ferme, et il n'eut que la fuite pour éviter la mort. Il professa pendant 10 ans l'économie rurale à Alfort. Il fut l'un des principaux collaborateurs du Nouveau Cours complet d'agriculture (1809). Son neveu, Charles-Auguste Yvart (1798-1873), directeur de l'École vétérinaire d'Alfort, surnommé le "grand moutonnier ", créa dans l'École, à partir de 1840, par le métissage et la sélection, un type de mouton à viande ayant conservé les qualités lainières du Mérinos. Appelée d'abord race d'Alfort, puis race de Grignon à la suite de son transfert dans l'école d'agronomie de même nom, ou race Dishley-Mérinos, elle prit son nom définitif de race d'île-de-France en 1922 à la suite de sa diffusion dans les fermes du Bassin parisien.

30. L. Grandeau, L'agriculture et les institutions agricoles du monde au commencement $d u x^{e}$ siècle, t. III, p. 7.

31. Un traité mit ensuite, à la charge du budget de l'agriculture, toutes les dépenses de l'enseignement à l'école de Grignon, en vue d'en réduire le prix de pension.

32. Auguste Bella (1777-1856), était un militaire de carrière, chevalier de l'Empire, et fondateur avec Antoine-Rémy Polonceau de l'École d'agriculture de Grignon, dont il assura la direction pendant vingt-deux ans. Bella fut soigné pendant la campagne 
napoléonienne de Hanovre (1802-1803) par Albrecht Thaër, médecin du roi et futur directeur de l'École d'agriculture de Moëglin (Brandebourg). Il suivit ses cours d'agriculture pendant près de deux ans et les mit en application à Grignon. Un décret de 1912 créa l'École supérieure d'enseignement agricole et ménager, annexée à l'École de Grignon. En 1971, l'École nationale supérieure d'Agriculture de Grignon fusionna avec l'Institut national agronomique pour devenir l'Institut national agronomique de Paris-Grignon (INA-P.G.).

33. Suppression des jachères, réduction des cultures de céréales, cultures alternées, stabulation du bétail et emploi d'engrais commerciaux en complément du fumier.

34. N. Jas, Au carrefour de la chimie et de l'agriculture, p. 45.

35. Un élève de Roville, Jules Rieffel, entreprit de défricher les landes de Grandjouan, dans la Loire-inférieure, fonda en 1840 la première ferme-école officiellement reconnue et dotée d'un budget sous le nom d'Institut agricole de Grandjouan.

36. Nivière, propriétaire de la Saulsaie, près de Lyon, après avoir assaini ce domaine marécageux, y créa l'école d'agriculture qui prit le nom du domaine.

37. N. Vivier, « L'enseignement agricole sous le Second Empire. L'application du décret du 3 octobre $1848 »$, p. 147.

38. Ibid, p. 148.

39. R. Duguay, L'Enseignement agricole en France à tous les degrés. Écoles et cours d'agriculture, p. 9 à 13.

40. Aujourd'hui, Merkwiller-Pechelbronn dans le Bas-Rhin.

41. Université de Lille.

42. Titulaire de la chaire de chimie à Caen.

43. À Nantes.

44. Louis Grandeau, «Les stations agronomiques aux États-Unis, en Allemagne et en France, leurs ressources et leur développement », p. 452-460.

45. N. Jas, « Déqualifier le paysan, introniser l'agronome, France 1840-1914 », p. 45-55.

46. On peut citer Jules-Émile Planchon (1823-1888) et Pierre Viala (1859-1936) qui menèrent un combat pour imposer l'usage des vignes américaines résistantes au phylloxéra. Louis Ravaz (1863-1937) qui étudia les porte-greffes résistants au phylloxéra et à la chlorose; ses enseignements de 1897 à 1930 contribuèrent à la formation de générations d'ingénieurs agricoles français et étrangers. Alexis Millardet (1838-1902) fut reconnu pour son étude du mildiou de la vigne et la découverte de la bouillie bordelaise en 1885. Victor Vermorel (1848-1937), mécanicien et inventeur, milita dans nombre de comices, sociétés d'agriculture, et participa à la création de la revue Le progrès agricole et viticole en 1879. Il présenta en 1887 le " pulvérisateur Éclair ", qui devint la sulfateuse de plusieurs générations. Source: C. Bain, J-L. Bernard, A. Fougeroux, Protection des cultures et travail des hommes, p. 73 à 83.

47. N. Jas, « Déqualifier le paysan, introniser l'agronome, France 1840-1914 », p. 54. 


\section{RÉSUMÉS}

Alors que l'agriculture était la principale activité économique du pays, mais qu'elle était concurrencée par l'industrie, sa place et sa part dans la transmission des savoirs étaient sujettes à discussion. Longtemps école empirique, l'art de l'agriculture tendait à se transformer en école rationnelle et raisonnée depuis l'époque des physiocrates. Par quelles formes, canaux et instruments la transmission du savoir-faire paysan, qui se faisait de père en fils, devint peu à peu un savoir formalisé dispensé par des organismes privés ou publics, des revues à diffusion plus ou moins large et des ouvrages spécialisés? Quelle place les gouvernements et régimes successifs ont-ils faite à l'éducation agricole? Quel regard les contemporains ont-ils porté sur l'héritage millénaire des techniques agricoles, confronté aux nouveautés apportées par les progrès du XIX siècle?

\section{AUTEUR}

\section{CHRISTIANE CHENEAUX-BERTHELOT}

Docteure en Histoire, Université Paris IV - Paris-Sorbonne, chercheure associée au Centre Roland Mousnier, UMR 8596 Sorbonne Université CNRS 


\title{
La transmission du savoir-faire
} agricole dans les fermes-écoles créées par la France en Afrique du Nord et en Syrie au début du $\mathrm{Xx}^{\mathrm{e}}$ siècle

\author{
Nagwa Abou El Maaty
}

1 Les fermes-écoles furent créées en Métropole dès 1830 par des initiatives privées, afin de hâter le progrès agricole et de former des aides ruraux qualifiés ${ }^{1}$. À partir de 1848 , elles représentèrent l'enseignement élémentaire de l'agriculture (décret du 3 octobre). Dès lors, ces établissements constituaient une association entre des exploitants éclairés, agronomes et agriculteurs, et l'État. Celui-ci prenait en charge les dépenses de la formation. Les exploitants géraient quant à eux les domaines à leurs risques et périls. Ainsi, la ferme-école jouait un double rôle. D'une part, l'exploitation offrait à la localité un modèle d'agriculture performante et fructueuse, d'autre part, elle formait des jeunes ruraux pauvres, âgés de quinze à seize ans aux méthodes de «l'agriculture raisonnée ", préconisée par Mathieu de Dombasle ${ }^{2}$ dans le cadre du système intensif de polyculture-élevage.

2 En tant qu'établissements d'enseignement, les fermes-écoles représentaient un mode efficace de transmission du savoir et du savoir-faire agricole. La pédagogie de l'exploitation-école, dont elles portent le nom, domina tous les niveaux de l'enseignement agricole en Métropole jusqu'au début du $\mathrm{xx}^{\mathrm{e}}$ siècle. Les fermes-écoles étaient, par leur vocation pratique, les plus concernées par cette pédagogie. Elles dispensaient aux élèves-apprentis «un apprentissage sérieux du travail par l'instruction et de l'instruction par le travail ${ }^{3} »$. Cette conception pédagogique répondait à leur but principal qui consistait «à former d'habiles cultivateurs praticiens ${ }^{4} »$. En exigeant des élèves-apprentis d'exécuter les opérations agricoles comme le feraient des travailleurs, on les mettait au fait des nouvelles pratiques. Ce fut 
la mission des fermes-écoles, mission accomplie en Métropole par des agronomes et de grands propriétaires soucieux du progrès agricole, jusqu'au début du $\mathrm{xx}^{\mathrm{e}}$ siècle $^{5}$.

Des établissements furent ensuite créés dans les années 1920-1930 par les Français en Algérie, ainsi que sous le protectorat au Maroc et en Tunisie et sous le mandat en Syrie. Les fermes-écoles outre-méditerranéennes (fig. 1) répondaient aux mêmes objectifs qu'en Métropole. Mais quel était leur statut? Quel était leur fonctionnement par rapport aux fermes-écoles de Métropole? Qui les dirigeaient et comment? Qui les fréquentaient parmi les jeunes des campagnes? Comment les autochtones percevaientils les réalisations de ces établissements? Les renseignements disponibles, aux archives nationales et aux archives d'Outre-Mer d'Aix-en-Provence ainsi qu'aux archives diplomatiques de Nantes, constituent nos sources principales. Il convient en premier lieu d'étudier les fermes-écoles de l'Algérie Française.

Fig. 1. - Les fermes-écoles outre-méditerranéennes.

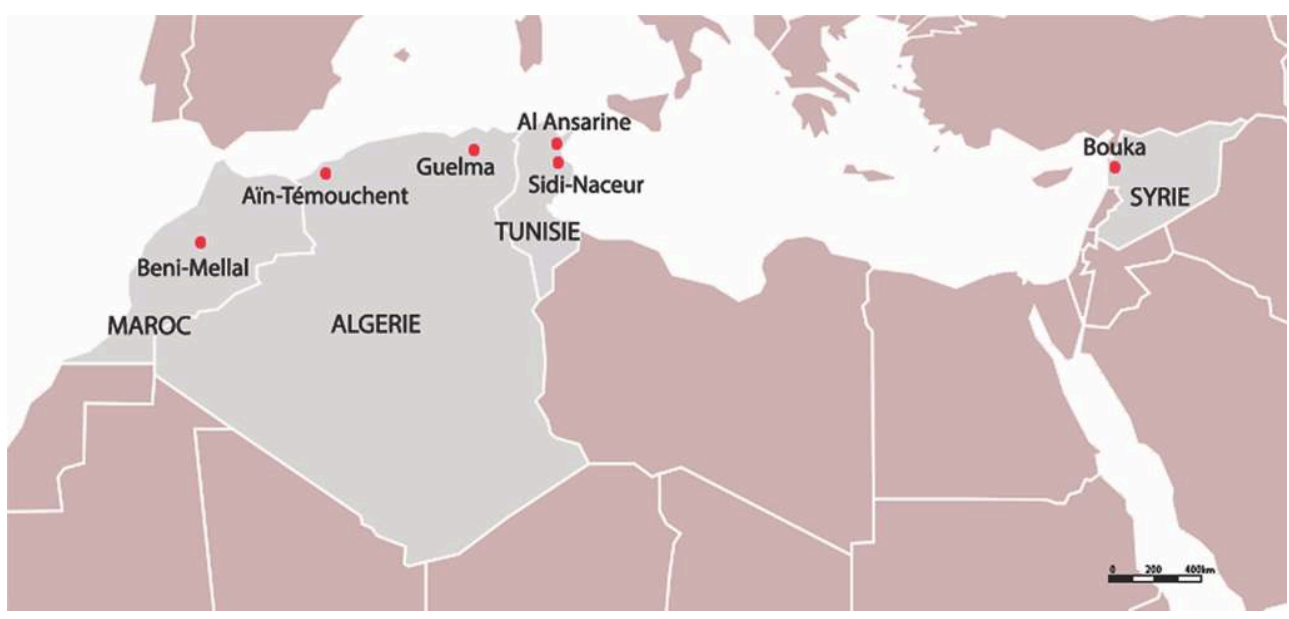

Atlas de France, CDIP Bouffemont, 2003.

\section{Les fermes-écoles d'Algérie : un appui à la colonisation?}

Comme en Métropole, l'Algérie devait bénéficier de la mise en place des fermes-écoles dans le cadre de la loi du 3 octobre 1848. Au-delà des rôles qu'elles devaient jouer au sein de l'agriculture et de son enseignement, ces établissements étaient considérés comme un appui à la colonisation, en réponse aux difficultés de peuplement. Dans une période de crise agricole (1847-1851), des agriculteurs et des agronomes préconisaient une colonisation agricole de l'Algérie. Le but était de fonder en Algérie des fermesécoles recevant des jeunes pauvres de la Métropole afin de concourir à la prospérité de la colonie ${ }^{6}$. Les colons estimaient qu'il était plus avantageux de fonder des fermesécoles en Algérie qu'en Métropole, où les cultivateurs étaient plus aptes à hâter le progrès agricole en l'absence d'établissements de ce type :

«Ce n'est pas chez eux que nos colons peuvent aller rechercher les meilleurs procédés de culture. ${ }^{7}$ "

5 Dans le cadre de la politique d'assimilation sous la II ${ }^{e}$ République, les fermes-écoles ne semblaient pas constituer un moyen rapide de peuplement agricole de l'Algérie. Celuici s'effectuait plutôt par l'installation des colons sur des concessions de terre de deux à 
douze hectares. Cependant, l'échec agricole des premiers colons faisait des fermesécoles un moyen de rationaliser l'agriculture de la colonie. Dès lors, de nombreux projets furent élaborés par des colons et des organisations agricoles locales, ce qui porta le Second Empire à prendre en considération la création des fermes-écoles en Algérie. Cela conduisit à la modification des dispositifs de la loi du 3 octobre 1848 pour l'Algérie. Cette volonté de mettre en place des fermes-écoles en Algérie trouva écho chez l'empereur en 1865. Il décréta la mise en place des fermes-écoles arabes-françaises dans les trois provinces d'Algérie (décret du 25 mai 1865) ${ }^{8}$. Ce fut la première fois que l'on élabora l'instruction agricole des jeunes algériens dans les fermes-écoles. Cette initiative de Napoléon III - qui paradoxalement n'encourageait pas les fermes-écoles et l'enseignement agricole en Métropole - était néanmoins cohérente avec sa politique algérienne entre 1858 et 1865 . Comme l'indique Dominique Barjot :

«L'Empereur hésite entre une politique de développement de la colonisation

européenne et une politique favorable aux Arabes. ${ }^{9}$ "

Sur le plan politique, le décret du 26 mai 1865 coïncidait avec l'expédition du Mexique et les tensions relatives à la question algérienne. Le décret n'ayant pas été appliqué, les fermes-écoles arabes-françaises restaient un projet parmi d'autres établis par les colons dès 1850. Pour ces derniers, ces établissements ne représentaient pas uniquement un moyen de peuplement rural et de qualification professionnelle des travailleurs agricoles. Ils leur garantissaient également une main-d'œuvre permanente au sein de la propriété pour une durée de trois ans. D'un autre côté, certains colons agriculteurs considéraient que l'apprentissage agricole en Algérie ne pouvait pas se réaliser d'une façon libre. En effet, les ouvriers indigènes changeaient souvent d'exploitants et ne mettaient pas tous en pratique de bons procédés de culture et d'élevage :

«Tel colon veut des engrais, laboure, sème, herse, assole avec discernement ; et tel

autre fort insouciant fait de la culture bédouine, sans autre forme de procéder..$^{10}$ "

Il fallut attendre le début $\mathrm{du} \mathrm{xx}^{\mathrm{e}}$ siècle pour fonder des fermes-écoles en Algérie : Guelma en 1922 et Ain-Temouchent en 1926. La création de ces deux fermes-écoles, dans une période de conjoncture économique coloniale défavorable, témoignait des nouveaux besoins de l'agriculture algérienne, tant pour les colons que pour les autochtones. D'une part, le ralentissement de la colonisation agricole, ajouté à la crise économique, nécessitait de la part des agriculteurs européens d'augmenter la productivité du sol, d'autre part, les petits cultivateurs indigènes, encore plus touchés par cette conjoncture, devaient augmenter leurs productions agricoles. Sur le plan de l'enseignement agricole, Guelma et Aïn-Témouchent venaient combler un vide au sein du premier degré de l'instruction professionnelle de l'agriculture.

\section{La ferme-école de Guelma : activités expérimentales et bon niveau d'enseignement}

8 L'établissement de Guelma constituait à la fois une ferme-école et une station expérimentale. Il dut sa création aux collectivités et aux organisations agricoles locales : le conseil municipal de Guelma, le conseil général de Constantine et le syndicat des agriculteurs de Guelma. Ce dernier était chargé de l'administration de la fermeécole sous l'autorité d'un conseil d'administration; la gestion technique de l'établissement était confiée au conseiller agricole local qui prenait la fonction de directeur. L'exploitation-école devenait alors la propriété du gouvernement. Cette 
organisation se rapprochait donc plus des écoles d'agriculture de la Métropole à partir de 1918 que des fermes-écoles gérées "aux risques et périls" des directeurs exploitants. Toutefois, le processus de l'enseignement ne différait en général pas de celui d'une ferme-école. Quant à l'expérimentation agricole, elle constituait une activité obligatoire, au même titre que la production et la formation. Toutes ces activités s'exerçaient sous le contrôle du Gouvernement général; la direction de l'Agriculture avait un droit de regard sur la gestion des agents.

9 En ce qui concerne l'enseignement, la ferme-école de Guelma recevait des jeunes indigènes âgés de quatorze à dix-huit ans, recrutés parmi des fils de cultivateurs familiarisés avec les travaux agricoles. Ils séjournaient à l'internat de l'établissement pendant deux ans, durée fixée pour l'étude, et pouvaient faire une troisième année de perfectionnement. Le recrutement se faisait sans difficultés ; l'établissement avait une capacité d'accueil d'environ trente apprentis. Ces derniers avaient par ailleurs un niveau scolaire satisfaisant. À l'instar des fermes-écoles de Métropole, l'instruction professionnelle de Guelma comprenait des cours théoriques (tabl. 1).

Tabl. 1. - Les cours théoriques et le personnel enseignant de Guelma.

\begin{tabular}{|l|l|}
\hline Cours & Enseignants \\
\hline $\begin{array}{l}\text { Génie rural } \\
\text { Horticulture }\end{array}$ & Le directeur \\
\hline $\begin{array}{l}\text { Agriculture générale } \\
\text { Agriculture spéciale }\end{array}$ & Le conseiller agricole \\
\hline $\begin{array}{l}\text { Sciences générales, Mathématiques, } \\
\text { Sciences appliquées, Arboriculture }\end{array}$ & Un professeur \\
\hline Zootechnie & Le vétérinaire sanitaire \\
\hline Français & Un instituteur \\
\hline Répétitions pendant les études & Un surveillant et un moniteur \\
\hline
\end{tabular}

Compte-rendu de la ferme-école de Guelma, 1936, CAOM 10102.

10 Comme le montre le tableau, l'établissement dispensait un enseignement théorique de niveau élevé pour une ferme-école, avec des cours de sciences générales et de sciences appliquées. L'établissement possédait un laboratoire afin d'y faire les applications de ces cours. Ces disciplines étaient enseignées en français, ce qui ne posait aucun problème quant au suivi des cours par les élèves. De même, l'apprentissage à la ferme se déroulait dans de bonnes conditions. Surveillés par les chefs de culture, les élèvesapprentis exécutaient toutes les opérations de culture. En dehors des travaux agricoles, les élèves participaient régulièrement à l'entretien du matériel agricole de la ferme. Cet apprentissage était considéré comme un complément indispensable à l'apprentissage agricole proprement dit, compte tenu des difficultés de réparation des machines importées de Métropole.

11 Cet apprentissage s'effectuait au sein de l'exploitation de Guelma qui occupait plus de quatre-vingt-dix hectares sur les cent cinquante composant la superficie totale de la station expérimentale. Outre les cultures traditionnelles céréalières et fourragères, l'établissement expérimentait des cultures potagères et possédait un verger expérimental. Les cultures céréalières, principalement le blé (dur et tendre), bénéficiaient des résultats obtenus par la station expérimentale sur le rendement de différentes variétés de semences ${ }^{11}$. La même démarche fut adoptée pour les cultures 
fourragères : vesce-avoine pour fourrage et pour ensilage, trèfle d'Alexandrie, orge et betteraves fourragères. Mais l'arboriculture et l'horticulture vivrière constituaient les deux filières les plus importantes. Les plantes potagères faisaient l'objet d'études et d'essais, afin de déterminer les meilleures conditions permettant d'en augmenter le rendement. Les pommes de terre en particulier bénéficiaient des essais comparatifs quant au rendement de différentes variétés.

En ce qui concerne l'arboriculture, diverses espèces furent cultivées: pruniers, cerisiers, amandiers, abricotiers, pêchers, orangers, mandariniers, citronniers et oliviers. Des cours saisonniers de la taille de l'olivier furent organisés dans la fermeécole pour les apprentis tailleurs de la région ainsi que pour les élèves-apprentis de Guelma. C'étaient en général ces derniers qui effectuaient la taille des arbres, dans le cadre des travaux pratiques de deuxième année. On ignore les voies de commercialisation de ces produits. Néanmoins, Guelma avait une situation géographique favorable à l'écoulement des produits.

13 L'élevage ne connut pas d'améliorations importantes. Les efforts étaient orientés vers la sélection des bovins de la race de Guelma pour la production laitière. Le directeur de l'établissement attribuait la modestie des résultats à l'insuffisance de pâturages. Cela ne réduisait cependant pas le rôle que la ferme-école jouait à travers ses travaux agricoles et horticoles, ainsi que la formation qu'elle dispensait aux jeunes indigènes. Ces derniers y apprirent à rationaliser leurs pratiques culturales et appliquer les méthodes permettant de tirer le meilleur profit du sol en fonction des circonstances naturelles locales. Considérée comme une réussite, l'expérience de Guelma donna lieu à la création d'une autre ferme-école, celle d'Aïn-Témouchent dans le département d'Oran.

\section{Une ferme-école pour les colons d'Aïn-Témouchent}

La croissance de la population d'Aïn-Témouchent, "une région fertile entièrement colonisée " (cf. carte 1) nécessitait la présence de nouveaux établissements d'enseignement, notamment agricoles. Sur le plan de la production, les populations locales n'avaient plus à leur disposition des terres en friches à mettre en valeur. Cela exigeait l'adoption du système intensif dans un contexte de crise économique qui affecta l'agriculture. La création d'une ferme-école répondait donc à ce double objectif. La demande du conseil municipal d'Aïn-Témouchent était à cet égard appuyée par le conseil général et la chambre d'agriculture d'Oran.

La ferme-école d'Aïn-Témouchent fut établie sur environ cent cinq hectares concédés à la commune par le décret du 5 juillet 1883. Tout comme Guelma, cet établissement fut administré par le syndicat agricole de la région, qui désigna une partie de ses membres pour fonder le conseil d'administration de la ferme-école. Ce dernier organisa la mise en place de l'établissement en prévoyant une somme de deux cent soixante-quinze mille francs pour assurer le fonctionnement de la ferme-école au cours de l'année agricole de 1926. Il fallait réaliser des travaux d'alimentation en eau potable et construire des logements pour le personnel et les élèves ainsi que des bâtiments agricoles. En dehors de ces opérations d'aménagement, des travaux de défoncement furent réalisés en vue de créer un vignoble d'expérience sur dix hectares. Douze animaux de trait furent en outre achetés en attendant que l'alimentation soit assurée pour d'autres animaux de rente. 
L'administration de la ferme-école consacrait par ailleurs quarante hectares de l'exploitation, soit près de $38 \%$ de sa superficie, aux cultures céréalières. L'exploitation possédait une moissonneuse-batteuse et de grandes quantités d'engrais dont du superphosphate ${ }^{12}$. Néanmoins, on sait qu'il existait des divergences entre la direction de l'établissement et le syndicat agricole sur la gestion de la ferme. Cela montre l'inconvénient de l'étatisation de l'exploitation agricole, et on comprend dès lors la volonté de l'État de préserver la liberté d'action agricole des directeurs-exploitants des fermes-écoles métropolitaines. Celle d'Aïn-Témouchent connut par ailleurs des difficultés dans le recrutement des élèves-apprentis, "par suite d'un désintéressement de la population rurale pour l'enseignement pratique agricole ${ }^{13}$ ", souligna le gouverneur général de l'Algérie. Il était en effet probable que les fils de colons accordaient leur préférence à un enseignement plus théorique qu'à un apprentissage agricole par le travail dans la ferme-école ${ }^{14}$. L'inspecteur de l'enseignement prit de nombreuses mesures afin de favoriser le recrutement: construire une cinémathèque ainsi que des ateliers de forge et de menuiserie, mettre en place des cours de vinification, etc. ${ }^{15}$. Ces mesures furent plus ou moins efficaces puisque la ferme-école continua de fonctionner pendant encore une décennie, avant de fermer ses portes au début des années 1940, alors que l'Algérie française entrait dans une période d'incertitudes.

\section{La création des fermes-écoles sous le protectorat}

Le développement de l'agriculture, principal secteur de l'économie en Tunisie et au Maroc, était d'une importance capitale aussi bien pour les indigènes que pour le gouvernement protecteur. Par conséquent, il fallait enseigner aux jeunes gens les pratiques culturales permettant de tirer la meilleure partie du sol tout en diminuant le coût de cet apprentissage. Si la ferme-école représentait la voie la plus efficace de l'apprentissage agricole, son coût était considéré comme très élevé. En ce début de $\mathrm{xx}^{\mathrm{e}}$ siècle, alors que les fermes-écoles en France étaient financièrement délaissées par l'État, il ne fallait pas attendre des créations en grand nombre en Afrique du Nord. Parallèlement à de rares créations de fermes-écoles, le gouvernement du protectorat essaya de développer l'instruction agricole pratique sous d'autres formes. En Tunisie, l'administration du protectorat proposa d'organiser l'enseignement agricole des indigènes dans les écoles primaires, ce qui provoqua le mécontentement des délégués tunisiens du comité consultatif de l'enseignement ${ }^{16}$. Ils estimaient qu'une telle organisation s'effectuait au détriment de ces deux enseignements et doutaient de l'intention des hommes du protectorat. Ces derniers, quant à eux, estimaient que :

«Ce n'est pas seulement une élite qu'il convient de former en ce pays, élite intelligente, instruite, capable de diriger : c'est la masse même qu'il faut sortir de son inaction et de sa passivité. ${ }^{17}$ " 


\section{La ferme-école de Smindja : une création mal perçue par les élites du pays} Tunis ", à l'instar des grandes écoles d'agriculture de la Métropole. Elle recevait en particulier de jeunes français installés en Tunisie ou de jeunes autochtones qui voulaient exploiter leur propriété familiale. Une ferme de stagiaires fut par ailleurs fondée à Sidi-Tabet en 1923. Elle servait à l'apprentissage des anciens élèves d'écoles d'agriculture de la Métropole, afin de les familiariser avec l'agriculture de Tunisie. Dans le cadre de ces structures d'enseignement agricole, l'établissement Sidi-Naceur de Smindja représentait par la qualité de son enseignement, une école pratique et par sa vocation, une ferme-école.

L'enseignement de la ferme-école connut deux phases : la première de 1914 à 1922, au cours de laquelle la formation était essentiellement pratique. La seconde commença à partir de 1922, date de la réorganisation de l'enseignement ; la partie théorique vit son niveau s'élever. Le contenu de l'enseignement théorique était comparable à celui de Guelma: des sciences générales et appliquées, avec l'horticulture et l'arboriculture comme disciplines communes à toutes les fermes-écoles d'Afrique du Nord. Il ne s'agissait pas de la qualification d'une main-d'œuvre mais de la formation de futurs chefs d'exploitation, à l'instar des écoles pratiques de la Métropole. L'établissement formait une population de jeunes aisés. Ces derniers payaient chacun sept cents francs pour la pension. Les bourses leur étaient accordées par la Direction d'économie générale de la chambre d'agriculture de Tunisie en fonction de leur classement aux examens, donc au mérite. Les élèves diplômés de la ferme-école avaient le droit à la même majoration de points que ceux des écoles d'agriculture de France pour l'admission à l'École Coloniale d'agriculture de Tunis. Le diplôme leur permettait également l'exemption du service militaire. Ils pouvaient par ailleurs " concourir à l'obtention d'un lot de culture sur les fermes coloniales ${ }^{19}$ ".

21 Dans un article paru dans le journal La Nahda en janvier 1931, intitulé "L'école d'Agriculture de Smindja », l'auteur déplorait la "pitoyable situation» de la fermeécole. Il reprochait au gouvernement du protectorat d'apporter une subvention « dérisoire ». Il critiquait l'insuffisance de l'équipement agricole ainsi que du nombre d'animaux et déclarait un endettement de l'établissement. Il dénonçait enfin une représentation des indigènes non équilibrée avec celle des Européens au sein du conseil de perfectionnement de la ferme-école ${ }^{20}$. Ces accusations furent réfutées dans l'ensemble par la direction de l'établissement, mais elles reflétaient encore une fois l'inefficacité de la gestion des exploitations-écoles, propriétés de l'État. Concernant précisément le domaine de Smindja, il était composé de :

« Un vignoble de 145 ha, une olivette de 4000 pieds, une orangeraie de 2,5 ha. ${ }^{21}$ "

La ferme-école avait également un potager doté d'équipements hydrauliques afin de familiariser les élèves à la pratique des cultures maraîchères et fruitières. En revanche, l'exploitation, orientée vers l'arboriculture, ne disposait pas d'un important cheptel. Ces activités agricoles et horticoles étaient favorisées par de bonnes infrastructures et de bons moyens de communication et de transport. De par sa situation au pied du massif du Djebel Zaghouan, le domaine avait les meilleures conditions d'hygiène et de salubrité. Il était par ailleurs alimenté en eau, électrifié et en liaison téléphonique avec Tunis. Aussi était-il bien desservi par la voie ferrée et par la ligne d'autobus Tunis- 
Zaghouan. Il est difficile d'évaluer l'apport de cette ferme-école sur l'agriculture et surtout sur l'horticulture locale. Néanmoins, des contemporains de l'établissement affirment que :

«Les élèves sortis de la ferme-école de Smindja adoptent résolument les méthodes modernes de culture et donnent les meilleurs exemples dans les milieux agricoles. ${ }^{22}$

»

$\mathrm{Au}$ Maroc, le centre d'apprentissage agricole de Béni-Mellal près de Casablanca fut transformé en ferme-école.

\section{Une mise en place difficile de la ferme-école de Béni-Mellal au Maroc}

Bien que le Maroc fût doté d'un très grand nombre d'écoles d'apprentissage, celui de l'agriculture se limitait à une section au sein de quelques-unes de ces écoles. À la fin des années 1930, les besoins en main-d'œuvre agricole qualifiée dans la région de Casablanca nécessitèrent de faire de l'apprentissage agricole une priorité. Mais la mise en place en 1938 de la ferme-école de Béni-Mellal présenta quelques difficultés. Cellesci étaient en premier lieu liées à la langue française; trois élèves sur quatre ne la parlaient pas. Cela porta l'inspecteur de l'enseignement primaire "musulman » à effectuer le recrutement dans d'autres localités ${ }^{23}$. Une autre difficulté résidait dans la faiblesse des moyens financiers des élèves; la pension était fixée à cent quatre-vingts francs par mois ${ }^{24}$, somme excessive pour ces familles défavorisées. L'importance agricole de cette zone irrigable exigeait de remédier à ces difficultés. L'instruction des élèves-apprentis se limitait aux travaux pratiques; le français n'était plus obligatoire. Proposés par le contrôleur civil, ces décisions n'étaient pas partagées par le directeur de l'établissement. Il estimait qu'il fallait apprendre le français aux élèves-apprentis afin qu'ils puissent lire, comprendre, tenir une comptabilité plus tard et rationaliser les mauvaises pratiques agricoles que l'homme âgé ne changera pas facilement :

"J'ai expliqué à des cultivateurs, démontré que telle façon d'irriguer n'était pas la meilleure, qu'il valait mieux piocher, sarcler, désherber plutôt que de noyer le champ sous une nappe d'eau. Ils m'ont compris, approuvé et remercié mais sitôt le dos tourné un grand coup de sape dans le séguia ouvrait toutes grandes les écluses que j'avais eu tant de peine à faire fermer. ${ }^{25}$ "

Installée sur une trentaine d'hectares, la ferme-école utilisait des bâtiments que l'État français donna à bail à la direction générale de l'instruction publique. À ses débuts, l'établissement ne possédait pas de cheptel ; la direction des services économiques lui céda « quelques ovins et bovins prélevés sur les troupeaux des stations d'élevage de la région du Tadla ${ }^{26} »$. Dans sa première année d'exercice, la ferme disposait d'une récolte de tabac sur pieds et d'une récolte de coton. Or, comme la ferme-école n'était pas un établissement public au sens de la personnalité civile et de l'autonomie financière, l'administration des domaines intervenait dans la vente des récoltes; l'internat manquait souvent des légumes pour l'alimentation des élèves. En dehors des problèmes de langue, qui freinaient la coopération franco-marocaine dans l'instruction des apprentis, cet établissement ne disposait pas des éléments nécessaires à la bonne marche de la ferme-école. Celle-ci fut en effet fondée à l'image des centres d'apprentissage mécanique, sans prendre en considération les principes de l'exploitation-école qui devait offrir un modèle de culture et d'élevage. Cela n'était pas chose aisée étant donné la complexité administrative de sa gestion et de son 
encadrement. En Syrie, la France, État mandataire, se préoccupait des activités pratiques, notamment agricoles.

\section{Bouka à Lattaquié, œuvre des Pères Bénédictins sous le Mandat}

Dans sa session extraordinaire de mai 1930, le Conseil représentatif de l'État des Alaouites passa un accord avec la congrégation des Pères Bénédictins pour installer une ferme-école au sein du centre agricole de Bouka.

\section{Le centre agricole de Bouka : une ferme-école et un orphelinat}

Dans le cadre de leur convention passée entre l'État des Alaouites et la congrégation (15 août 1930), les bénédictins olivétains avaient pour mission d'assurer la direction et le fonctionnement de la ferme-école pendant une durée minimale de cinq ans; l'orphelinat de Bouka pendant vingt-cinq ans. Ces deux établissements devaient " appartenir à la province française de leur congrégation ${ }^{27}$ ». Le domaine concédé était constitué par le bien-fonds. La congrégation était par ailleurs « exemptée de tout impôt sur les constructions, étant considérée comme établissement d'intérêt général ${ }^{28}$ ». Par contre, les terrains étaient soumis aux impôts étant considérés comme propriété privée. Ces conditions favorables à la congrégation traduisaient les attentes de l'État des Alaouites concernant les améliorations agricoles :

«Le gouverneur maintient son point de vue. Il explique que la congrégation va s'installer en vue de mettre en valeur les terrains par des procédés modernes et de dépenser des sommes importantes pour construire toutes les bâtisses dont ils ne peuvent point se dispenser. ${ }^{29}$ »

À partir de 1936, l'Administration française procéda à une réorganisation de l'enseignement professionnel et tenta d'améliorer ses conditions matérielles ${ }^{30}$. Il y eut sous le mandat une prise de conscience du rôle à jouer des activités pratiques en Syrie, pays qui «méprisait l'enseignement technique » où "l'enseignement vivait sur une tradition littéraire et académique ${ }^{31} »$. L'effort de la France dans ce domaine paraissait toucher l'opinion publique syrienne qui y voyait une volonté créatrice. En ce qui concerne l'instruction publique en général, l'action mandataire consistait à développer dans le pays une culture nationale, pénétrée d'idées occidentales ${ }^{32}$; parmi elles, la modernisation de l'exploitation école de Bouka.

\section{La ferme-école de Bouka en Syrie (1930-1942) dirigée par le père Peyrton}

La ferme-école de Bouka fonctionnait comme celles de 1848 en Métropole :

«L'enseignement professionnel de l'École sera conforme au plan d'études actuellement en vigueur et sur la base du programme d'enseignement des fermesécoles françaises. ${ }^{33}$ "

Elle fut dirigée par le révérend père Peyrton, directeur du centre de Bouka. Ce dernier était assisté par un professeur d'agriculture diplômé d'une école française. Les leçons de français étaient données par les religieux. L'enseignement des langues française et arabe était obligatoire au même titre. Il faut rappeler que selon l'art. III du mandat, « le 
mandataire développera l'instruction publique donnée au moyen des langues indigènes en usage sur les territoires de la Syrie et du Liban ${ }^{34} »$. Les élèves étaient recrutés pour la plupart d'entre eux dans les deux sandjaks du gouvernement de Lattaquié. Néanmoins, l'État des Alaouites autorisait la ferme-école à recevoir des élèves étrangers ${ }^{35}$ (tabl. 2).

Tabl. 2. - Les élèves du centre agricole de Bouka, selon leur pays d'origine.

\begin{tabular}{|l|c|}
\hline Pays & Nombre d'élèves \\
\hline Syrie (Lattaquié) & 61 \\
Liban & 4 \\
Palestine & 1 \\
\hline Total centre agricole & 66 \\
\hline Total orphelinat & 38 \\
\hline Total ferme-école & 28 \\
\hline
\end{tabular}

Archives diplomatiques de Nantes, Mandat Syrie-Liban, Beyrouth Ins. Pub 89.

31 La ferme-école de Bouka délivrait un diplôme et un certificat: le premier, intitulé "Diplôme de l'école d'agriculture de Bouka », était obtenu par les élèves ayant une moyenne supérieure ou égale à quinze sur vingt, c'est-à-dire par les plus brillants. Ceux qui obtenaient une moyenne comprise entre treize et quinze sur vingt recevaient le second titre, " certificat d'instruction agricole ». Ces notes étaient obtenues en fonction des résultats d'examens trimestriels portant sur les études ainsi que sur les travaux pratiques réalisés dans la ferme et la station d'essais ${ }^{36}$ de l'établissement.

La formation comprenait les travaux en atelier et des cours théoriques : géométrie, dessin, etc. La durée des études dans cette école était de trois ans. Et afin d'appliquer les travaux de l'école professionnelle artisanale à l'agriculture, ils ouvrirent une section de "ferronnerie et de mécanique appliquées à l'agriculture ». Ces études, une fois terminées, devaient assurer des débouchés aux élèves sortis (art. 35 du cahier des charges). Les agriculteurs qui cherchaient des régisseurs ou des chefs de culture s'adressaient à la direction de l'établissement. Ces anciens élèves-apprentis n'avaient pas de difficultés à trouver du travail, mais nous ne disposons pas de renseignements précis sur leur devenir. En 1941, les bénédictins voulaient établir un établissement de second degré au sein du centre de Bouka. La demande fut rejetée, compte tenu des circonstances politiques ${ }^{37}$. Bouka ferma ses portes quelques années plus tard, marquant l'histoire locale par une formation professionnelle sérieuse. Les élèves-apprentis apprenaient les méthodes agricoles rationnelles et l'utilisation de machines et d'instruments perfectionnés (tabl.3). L'exploitation possédait un matériel agricole complet et nombreux, digne d'une grande ferme-école métropolitaine du $\mathrm{xx}^{\mathrm{e}}$ siècle. 
Tabl. 3. - Les machines et instruments agricoles de la ferme-école de Bouka en 1930.

\begin{tabular}{|l|c|}
\hline Instruments et machines & Nombre \\
\hline Charrue à 4 socs légère & 1 \\
\hline Charrue à 3 socs légère & 1 \\
\hline Charrues à un soc légère & 8 \\
\hline Houes à cheval & 6 \\
\hline Charrue brabant double & 1 \\
\hline Herses canadiennes & 15 \\
\hline Puicheurs canadiens & 2 \\
\hline Panneaux de herse trapézö̀dales & 30 \\
\hline Herse à ćtoile & 1 \\
\hline Semoir cn paquets & 1 \\
\hline Batteuse à manège & 1 \\
\hline Semoirs à 9 rayons & 1 \\
\hline Tarare cribleur & 1 \\
\hline Râteaux mécaniques & 2 \\
\hline Moissonneuse javeleuse & 1 \\
\hline Meule biseautée pour faucheuse & 1 \\
\hline Meule en grés & 1 \\
\hline Tonneau à eau sur roues & 1 \\
\hline Roulcau Gross Vrill & 1 \\
\hline Pulvérisateur & 1 \\
\hline Hache paille & 1 \\
\hline Pulvérisateur vermorel & 1 \\
\hline Rouleau plat & 1 \\
\hline Jar-jars & 3 \\
\hline Charrues araires avec socs & 12 \\
\hline Moissonneuse javeleuse & \\
\hline «Hirondelle » & 1 \\
\hline Tonneaux à eau sur roues & 2 \\
\hline Charrues 2 à 1 soc & 4 \\
\hline Charrue 1 à 1 soc & 1 \\
\hline Charrue tourne oreilles à 2 socs & 1 \\
\hline Petite charrue tourne oreille & 1 \\
\hline Tuyaux en toile & 60 \\
\hline Lances d'arrosage & 3 \\
\hline Raccords & 3 \\
\hline Supports appliqués & 3 \\
\hline & \\
\hline & \\
\hline
\end{tabular}

Archives diplomatiques de Nantes, Mandat Syrie-Liban, Beyrouth Ins. Pub. 8.

Les liens que l'agriculture put établir avec l'économie et la formation professionnelle, au sein des fermes-écoles de la Métropole, étaient moins évidents au cours de la période coloniale en Syrie et surtout au Maghreb. La création tardive de ces établissements influa sur leur organisation qui était alors plus scolaire que celle des fermes-écoles de 1848. Pourtant, l'agriculture et son enseignement dans ces pays étaient confrontés aux mêmes problèmes que la Métropole au milieu du XIX ${ }^{\mathrm{e}}$ siècle. Il s'agissait du point de vue de la formation professionnelle d'un décalage entre la théorie et la pratique. Mais encore fallait-il trouver des chefs d'exploitation éclairés, entrepreneurs et formateurs à l'instar de ceux de la Métropole. À cet égard, les deux écoles supérieures agronomiques d'Algérie et de Tunisie ne constituèrent pas un facteur d'évolution comme ce fut le cas pour les instituts métropolitains. Ni les fils des colons, majoritaires dans ces écoles, ni la minorité des élèves indigènes se préoccupaient de la transmission de leur savoir et savoir-faire agricoles appris dans ces écoles. Néanmoins, cela ne diminue pas l'action agricole de ces fermes-écoles dans certaines filières comme l'arboriculture ou certaines activités comme la démarche expérimentale qui constituait le point commun entre ces exploitations. Enfin, l'histoire de ces fermes-écoles coloniales, en articulation avec celle de l'exploitation-école en Métropole, ouvre une voie de réflexion sur le rôle d'une telle institution dans la vulgarisation de l'agriculture outre-méditerranéenne. Cette notion reste encore synonyme du développement agricole dans ces pays. 


\section{BIBLIOGRAPHIE}

ABOU EL MAATY Nagwa, La scolarisation de l'apprentissage agricole en France. Les fermes-écoles au service de l'agriculture et son enseignement (XIXe-début $\mathrm{XX}^{e}$ siècle), thèse de doctorat en histoire économique contemporaine, Université de Paris IV-Sorbonne, 2007, 3 vol.

BARJOT Dominique, CHALINE Jean-Pierre, ENCREVÉ André, La France au XIX siècle, 1814-1914, Presse universitaire de France, 2002.

BARJOT Dominique et TRANG PHAN Thi Hoai (dir.), Économie et développement durable : héritage historique et défis actuels au sein du monde francophone, Paris, SFHOM, 2016.

DEBBANCÉ J., L'expérimentation agricole et l'enseignement rural au Liban et en Syrie, Beyrouth, 1942. JEGOU Bruno, « Le monde rural français, un ébranlement sans révolution (1914-1929) », dans BARJOT Dominique (dir.), Les sociétés rurales face à la modernisation. Évolution sociale et politique en Europe des années 1830 à la fin des années 1920, Paris, SEDES, 2005, p. 205-227.

ROBINET M., «L'enseignement agricole en Tunisie », Travaux de la journée du centenaire de Grignon, 1926, p. 193-198.

SAUL Samir et PIQUET Caroline, "Les Sociétés coloniales au Proche et Moyen-Orient », dans BARJOT Dominique et FRÉMAUX Jacques (dir.), Les sociétés coloniales à l'âge des empires des années 1850 aux années 1950, Paris, SEDES, 2013, p. 133-143.

\section{NOTES}

1. B. Jegou souligne le rôle des initiatives individuelles dans la modernisation des pratiques culturales des paysans dans les années 1920, «Le monde rural français, un ébranlement sans révolution (1914-1929) », p. 205-228.

2. C.-J.-A. Mathieu de Dombasle, maitre à penser de l'agriculture française du $x x^{e}$ siècle; il forme une première génération d'agronomes, futurs exploitants des fermesmodèles et fermes-écoles, dans "La ferme-exemplaire de Roville ». Son action agricole et sa pensée sont analysées dans la thèse de l'auteur, N. Abou El Maaty, La scolarisation de l'apprentissage agricole en France. Les fermes-écoles au service de l'agriculture et de son enseignement, (XIX ${ }^{e}$ siècle-début $\mathrm{XX}^{e}$ siècle).

3. Archive du ministère de l'agriculture, C528/6087, «Les fermes-écoles ou les écoles professionnelles de l'agriculture », fol.2. Ce fond d'archive a été transféré à la bibliothèque d'histoire rurale, Université de Caen.

4. Archives départementales de Loire-Atlantique, 1M1985, « Note sur l'organisation des fermes-écoles ".

5. La dernière ferme-école, celle de Royat en Ariège, ferma ses portes en 1928.

6. Archives nationales $\mathrm{F}^{10} 1741$, «Lettre de Goëz, élève à l'institut agronomique de Grand-Jouan, adressée à son excellence Monsieur le ministre de l'agriculture et du commerce ", le 10 janvier 1848 .

7. Bibliothèque nationale de France, SP-9536, « Rapport de la commission désignée par la Société agricole de Philippeville, sur la création des fermes-écoles en Algérie », 1851. 
8. CAOM 10 102, « Décret du 26 mai 1865 relatif à la création des fermes-écoles arabesfrançaises en Algérie ».

9. D. Barjot, «La France dans le monde : affrontement avec l'Allemagne et expansion coloniale », p. 543.

10. CAOM 10 102, «Projet de ferme-école présenté par A. Estre en 1866 », fol. 3-4.

11. Le Bidi 17 et le Bidi 3 étaient les deux meilleures variétés de blé dur : ils donnaient 20 quintaux à l'hectare.

12. Cet engrais n'était pas alors utilisé dans les fermes-écoles restantes en Métropole. Les résultats agricoles font défaut aux sources disponibles.

13. CAOM 10 105, «Lettre du gouverneur général de l'Algérie, adressée au préfet d'Oran », le 17 février 1932.

14. En 1935, les candidats à la ferme-école étaient au nombre de 20 dont 5 venaient d'Oran, 5 de Mascara, 10 d'Aïn-Témouchent et de Philippeville; CAOM 1 H 76. Aucun candidat de Sidi bel Abbès où existaient des écoles pratiques.

15. CAOM 10 105, "Lettre de l'inspecteur de l'enseignement dans les fermes-écoles, adressée au directeur de la ferme-école expérimentale d'Aïn-Témouchent», le 17 février 1932.

16. Archives diplomatiques de Nantes (Arch. dip.), 2 Mi 477, «Rapport présenté par Doliveux, directeur général de l'instruction publique et des beaux-arts", séance du 25 avril 1925.

17. Ibid.

18. D'après $M$. Robinet, ce transfert était dû à des fièvres qui sévissaient dans la région, «L'enseignement agricole en Tunisie », p. 193-198, p. 196.

19. Arch. dip. Nantes, 2 Mi 512, « Lettre de sous-directeur de la ferme-école de Smindja, adressée au Résident général de France », Tunis, 1927.

20. Arch. dip. Nantes, 2 Mi 503, « Note sur l'article publié dans le journal Nahda sur l'École d'Agriculture de Smindja ».

21. Arch. dip. Nantes, 2 Mi 512, «École d'agriculture de Smindja », p. 4. Entre 1911 et 1922, 239 élèves suivirent cette instruction horticole pratique, M. Robinet, « L'enseignement agricole en Tunisie ».

22. Ibid, «Rapport de M. Robinet, ingénieur agricole (G), chef des services de l'agriculture en Tunisie ».

23. Arch. dip. Nantes, Protec. Maroc DIP 52, « Lettre de l'inspecteur de l'enseignement primaire musulman Maroc-Nord, adressée au directeur de la ferme-école de BéniMellal, sur l'enseignement agricole à Béni-Mellal Rabat », le 28 février 1939.

24. Ibid, « Lettre du contrôleur civil, chef du territoire d'Oued Zem, au directeur général de l'enseignement », le 4 novembre 1939.

25. Arch. dip. Nantes, Protec. Maroc DIP 52, «Lettre du directeur de la ferme-école au chef du service d'enseignement musulman », le 4 février 1939.

26. Ibid, «Lettre du chef de service de l'élevage, adressée au directeur des affaires économiques ", le 30 novembre 1939.

27. Arch. dip. Nantes, Mandat Syrie-Liban, Beyrouth, $2^{\mathrm{e}}$ versement, Ins. Pub. 89, Décision $n^{\circ} 027$ « portant approbation de la convention passée entre le gouvernement 
de Lattaquié et la congrégation des Pères bénédictins olivétains, pour la cessation du centre agricole de Bouka ».

28. Ibid, "Note $\mathrm{n}^{\circ} 57$ au sujet de la convention du Centre agricole de Bouka, le 19 janvier 1932 ", fol. 15.

29. Ibid, Mandat Syrie-Liban190, «Procès-verbal de la séance du 5 mai 1930 du conseil représentatif de l'État des Alaouites ", fol. 11.

30. Arch. dip. Nantes, Beyrouth Ins. Pub. 168, «Note sur la construction de l'école, service d'enseignement technique au Haut-Commissariat de la République française en Syrie et au Liban ".

31. Ibid.

32. Ibidem.

33. Arch. dip. Nantes, Mandat Syrie-Liban, Beyrouth Ins. Pub. 89, «Cahier des charges du centre agricole de Bouka, École », art. 1.

34. Ibid.

35. Arch. dip. Nantes, Mandat Syrie-Liban, Ins. Pub. 62, «Arrêté $n^{\circ} 1678$ fixant les conditions d'admission des élèves étrangers dans la ferme-école de Bouka.

36. Les détails de ces travaux font défaut aux sources disponibles. L'activité expérimentale, au sein de l'agriculture en Syrie et au Liban, fait l'objet de J. Debbancé, L'expérimentation agricole et l'enseignement rural au Liban et en Syrie.

37. Ce contexte politique pré indépendance est traité par S. Saul et C. Piquet, «Les sociétés coloniales au Proche et Moyen-Orient », p. 133-143.

\section{RÉSUMÉS}

Représentant le premier degré de l'enseignement agricole de la Métropole en 1848, les fermesécoles furent créées en Afrique du Nord et en Syrie entre 1922 et 1930. Ces établissements devaient former des aides ruraux qualifiés et moderniser l'agriculture. Dans cette nouvelle ère agronomique, motoculture, fertilisation, sélection végétale et activité expérimentale constituaient des moyens d'amélioration agricole. En dépit de leur nombre limité - deux en Algérie et en Tunisie, une au Maroc et en Syrie - ces fermes-écoles offraient à l'agriculture locale l'exemple de pratiques raisonnées. Cependant, elles ne présentaient pas aux élèves un modèle de gestion de l'entreprise agricole comme en Métropole. Les établissements d'Afrique du Nord avaient une administration étatique complexe. Toutefois, ces exploitations dispensaient aux jeunes ruraux une formation qui faisait défaut à ces pays essentiellement agricoles. 


\section{AUTEUR}

\section{NAGWA ABOU EL MAATY}

Ingénieure agronome en développement agricole de l'Université du Caire, ancien ingénieur agronome en chef à l'Université de Mansoura (Égypte), docteur en histoire économique contemporaine de l'Université de Paris-Sorbonne - Paris IV 


\title{
Raymond Février et la transmission des savoirs agronomiques
}

\author{
Sébastien Pivoteau
}

1 Cet article s'appuie essentiellement sur les documents donnés aux Archives nationales en 2012 par Raymond Février (né en 1920). Conservé sous le numéro 20170135, ce fonds, classé et inventorié par l'auteur à l'automne 2017, comprend trois grands ensembles documentaires: des dossiers de travail constitués dans le cadre des fonctions de chercheur, d'inspecteur général et de directeur général que R. Février occupe successivement, de 1946 à 1978, à l'Institut national de la recherche agronomique (INRA) ; des archives produites au cours des missions d'administration et d'expertise qu'il remplit notamment, dans les années 1970-1980, auprès d'établissements d'enseignement et de recherche tels que l'université de Corse, le Centre du machinisme agricole, du génie rural, des eaux et des forêts (CEMAGREF) et le Centre international de hautes études agronomiques (CIHEAM); des pièces relatives à son engagement durable au Parti socialiste et à d'autres aspects de sa vie privée ${ }^{1}$. La quasi-totalité du parcours professionnel et politique de l'un des principaux acteurs de la recherche agronomique française de la seconde moitié $\mathrm{du} \mathrm{xx}^{\mathrm{e}}$ siècle se trouve ainsi éclairée. À titre complémentaire, deux autres sources ont été mobilisées: d'une part, le témoignage recueilli par Denis Poupardin en 1996, publié dans la revue Archorales² ; de l'autre, deux volumes de mémoires demeurés inédits, obligeamment communiqués à l'auteur ${ }^{3}$.

2 À partir de ce corpus, il s'agira donc de relire la trajectoire professionnelle et politique de R. Février à l'aune des questionnements soulevés par la transmission des savoirs agronomiques. Il apparaîtra que son souci permanent de diffusion de connaissances à finalité pratique et immédiate auprès du monde agricole et industriel, conforme à la fonction première assignée à un organisme de recherche appliquée comme l'INRA, se double d'une volonté non moins ferme d'orientation à plus long terme des politiques publiques en matière d'agriculture et de recherche agronomique. 


\section{Répondre aux besoins socio-économiques du moment}

Dès ses premières recherches en alimentation animale, menées dans les conditions particulièrement précaires des années 1943-1944, R. Février est amené à nouer des relations étroites avec des éleveurs, ne serait-ce que pour disposer des troupeaux de vaches et de porcs indispensables à ses travaux ${ }^{4}$.

\section{Tisser des liens étroits avec les organisations professionnelles agricoles}

4 Après la guerre et son recrutement à l'INRA nouvellement créé, il est rapidement sollicité par les Centres d'études techniques agricoles (CETA), alors en plein essor. Le fonds R. Février conserve ainsi le texte d'une conférence donnée en janvier 1958 auprès de ces petites coopératives d'agriculteurs férus de modernité, au cours de laquelle il fait la promotion d'un modèle d'élevage porcin reposant principalement sur l'utilisation des produits de l'exploitation (céréales, pommes de terre, betteraves) ${ }^{5}$. Celui-ci procède des observations qu'il a pu effectuer lors de deux missions au Danemark en 1947 et 1953-1954', mais aussi des échanges chaleureux qu'il a entretenus dans les années précédentes avec de nombreux éleveurs, souvent jeunes, des CETA. Car ces organisations se caractérisent précisément par une conception partagée de l'innovation agronomique, à rebours des relations verticales, de maître à élève, qui prévalent encore dans les autres structures de vulgarisation agricole comme les services départementaux de l'Agriculture? .

5 Les actions de formation et d'expérimentation qu'il conduit auprès des CETA amènent R. Février à prendre précocement en compte l'intérêt économique des agriculteurs dans l'établissement des problèmes scientifiques et de leurs solutions. Aussi, à la différence d'autres agronomes pour lesquels toute innovation est «bonne en soi », il manifeste une vive compréhension pour les "résistances" de certains secteurs agricoles vis-à-vis du "progrès technique", dont témoignent par exemple les remarques suivantes, tirées d'un rapport qu'il dresse peu après son départ de la direction générale de l'INRA en 1978 :

«On voit certes encore beaucoup d'exploitations que l'on qualifie de routinières, où le "progrès technique " ne pénètre pas. A-t-on beaucoup réfléchi sur les raisons objectives de cette « résistance»? N'a-t-on pas un peu vite accusé l'appareil de diffusion, et non la pertinence de cette résistance à des innovations qualifiées $a$ priori comme «bonnes en soi »? N'est-il pas frappant de constater que, dans certains secteurs, les innovations se diffusent de façon explosive (ex. céréales, aviculture), alors que dans d'autres, elles pénètrent lentement la masse (élevage). Faut-il conclure que les agriculteurs et les vulgarisateurs, si compétents dans un secteur, seraient retardataires dans l'autre? Et quand ce sont les mêmes? Ne vautil pas mieux se demander si le problème soulevé par l'introduction du "progrès technique » dans certains secteurs, pose des problèmes financiers, de main-d'œuvre et de marchés mal protégés (viande), tels que la prudence conduit, par une logique implicite ou explicite, à refuser le "progrès »..$^{8}$ "

R. Février réfute dès lors une vision linéaire et déterministe du développement pour s'inscrire dans les principes de la rationalité limitée et, plus largement, dans la matrice intellectuelle du constructivisme. 
7 Le mouvement des CETA s'essouffle après la loi d'octobre 1966, qui renforce le poids des chambres d'agriculture dans l'organisation de la vulgarisation technique ${ }^{9}$. Se développent en parallèle des instituts techniques, appelés à former un «trait d'union entre l'échelon départemental de la Vulgarisation et l'échelon, généralement national, de la Recherche ${ }^{10} \%$. R. Février coopère très tôt avec ces organismes: il contribue grandement à la création de l'Institut technique du porc (ITP) en 1961, avant d'être nommé, lors de sa mise en place l'année suivante, président du Conseil scientifique de l'Institut technique de l'élevage bovin (ITEB) ${ }^{11}$. Dans un rapport qu'il rédige en 1970 en qualité d'inspecteur général, il préconise de renforcer les échanges entre les ingénieurs de ces structures et les chercheurs de l'INRA. Les premiers pourraient de la sorte approfondir leurs connaissances, partant affermir leurs propres activités de formation, tandis que les seconds seraient davantage sensibilisés aux préoccupations quotidiennes du monde agricole :

"Il est donc nécessaire que les ingénieurs de ces instituts passent, pour la plupart, 1

à 2 ans dans des services de recherches, pour acquérir leur méthode, leur façon de penser, ce qui leur facilitera ensuite les contacts: la variété de nos activités permettrait d'ailleurs de choisir les secteurs les plus adaptés à ces besoins. Mais parallèlement, il est souhaitable qu'un certain nombre de chercheurs puissent aller dans les instituts professionnels, soit des jeunes qui s'avèrent mieux à l'aise dans des problèmes de développement, soit des plus anciens qui pourraient y occuper des postes plus élevés. ${ }^{12}$ "

8 R. Février s'investit donc promptement et fortement dans la diffusion des résultats de la recherche auprès des professionnels, sans attendre ni se limiter à l'implication officielle de l'INRA dans la vulgarisation, dont les premiers jalons sont posés en 1959 avec la création de la Section d'application de la recherche à la vulgarisation (SARV), remplacée en 1964 par le Service d'expérimentation et d'information (SEI) ${ }^{13}$.

9 La coopération avec les organisations agricoles se concrétise notamment à l'occasion d'événements particuliers tels que les «Journées du Porc» et les concours de porcs abattus. Le fonds R. Février garde par exemple la trace d'un exposé devant quelque 400 éleveurs et techniciens agricoles lors de la «Journée du Porc » organisée à Brive-laGaillarde le 11 juin 1955 :

«M. Février, Directeur de la Station de Recherches sur l'Élevage [au Centre national de recherches zootechniques (CNRZ) de Jouy-en-Josas], parla du problème de l'alimentation des porcs. [...] il expliqua que pour que l'engraissement soit rentable, l'alimentation des porcs devait essentiellement être fournie par les productions végétales de la ferme, auxquelles d'ailleurs le porc offre un débouché intéressant. Lorsque l'alimentation de la ferme ne permet pas de fournir aux porcs une ration équilibrée, il y a lieu de compléter avec des aliments achetés. Entre l'élevage du porc en vase clos, qui pose des problèmes sanitaires et des problèmes de prix de revient, et l'élevage du porc en autarcie, M. Février préconise un compromis qui doit permettre à la fois d'obtenir des prix de revient très bas et une qualité propre à satisfaire le consommateur. ${ }^{14}$ »

Le compte rendu d'où est extrait ce passage fait apparemment l'objet d'une large couverture médiatique puisqu'il est publié ou diffusé auprès d'au moins six organes de presse (parmi lesquels La France agricole, La Revue de l'élevage, Le Porc et Le Moniteur du Progrès agricole $)^{15}$.

11 Au-delà de telles manifestations, R. Février s'efforce de médiatiser largement ses travaux de recherche en alimentation porcine ${ }^{16}$. On en retrouve ainsi la teneur dans de nombreux titres de la presse spécialisée : outre ceux déjà indiqués, peuvent être cités $L a$ 
Tribune du monde rural, le Bulletin technique d'information, ou encore les Cahiers de nutrition et de diététique ${ }^{17}$. Certaines émissions radiophoniques leur offrent par ailleurs un ample écho, comme l'attestent ses interventions sur le rôle respectif du lait écrémé, des pommes de terre, des betteraves et des antibiotiques dans l'alimentation des porcs sur les ondes de Radio Luxembourg et de la RTF en 1956 et $1957^{18}$. La documentation conservée laisse parfois affleurer plusieurs niveaux complémentaires de vulgarisation. En témoigne singulièrement la publication, en avril 1961, dans La Tribune du monde rural, d'une interview de R. Février au cours de laquelle il est invité à revenir, dans des termes jugés plus accessibles, sur « un très important article » qu'il « a écrit récemment dans la Revue des industries de l'alimentation animale» :

«Le niveau scientifique de cet exposé le rendrait peu accessible à la plupart de nos lecteurs, mais nous avons pensé qu'ils seraient intéressés de connaître quelquesunes des idées de base qu'a développées Monsieur Ferru (sic). Nous lui avons donc rendu visite, et publions, ci-dessous, le compte-rendu de notre entretien. ${ }^{19}$ »

\section{Nouer des relations équilibrées avec les industries agroalimentaires}

Les liens de R. Février avec le monde industriel sont restés longtemps ténus, en dépit d'un intérêt précoce pour cet univers : après avoir bénéficié d'une bourse financée par les industriels de l'alimentation animale, il avait songé à travailler pour leurs entreprises, avant de rejoindre le Service de la Recherche et de l'Expérimentation du ministère de l'Agriculture, puis l'INRA, en 1945-1946²0.

13 C'est surtout à partir du milieu des années 1960, et plus encore des années 1970, qu'ils se resserrent durablement, à la faveur de l'impulsion donnée par les gouvernements successifs au développement des industries agroalimentaires (IAA). Dans ses fonctions d'inspecteur général, puis de directeur général, il plaide alors pour l'établissement ou le renforcement de relations équilibrées entre les chercheurs de l'INRA et les ingénieurs ou entrepreneurs de ce secteur.

«La phase de méfiance, observe-t-il en 1970, est en voie de passer, et la collaboration peut s'envisager si les industriels ne justifient plus par leur attitude, également nouvelle, le reproche de "mercantilisme" qui leur a été maintes fois adressé..$^{21} »$

14 À cette condition, R. Février soutient la mise en œuvre d'actions de recherche communes, qu'elles portent sur des « problèmes particuliers » ou bien qu'elles relèvent d'un partenariat plus global inscrit dans la durée. Les premières impliquent pour l'INRA de "pouvoir mettre à disposition d'un industriel, un chercheur, volontaire, pour 1-2-3 ans, en percevant en contrepartie l'équivalent de sa rémunération » ou de " pouvoir recevoir en plus grand nombre qu'actuellement, des ingénieurs de l'industrie qui viendraient collaborer, dans une équipe, à un problème d'intérêt commun ${ }^{22}$ ». Quant aux secondes, elles aboutissent notamment au projet de création, à Lille, en 1966-1968, du Centre de recherche de la technologie des industries agricoles et alimentaires (CERTIA), sous les doubles auspices de l'INRA et des industriels de la région du Nord ${ }^{23}$.

Une coopération raisonnable avec les IAA suppose aussi à ses yeux un partage équitable des fruits de l'innovation, soit des conditions acceptables de cession des brevets déposés par l'INRA : 
«La prise des brevets, ou la conclusion de contrats, comportant des royalties (quand la prise d'un brevet est difficile) doit pouvoir être encouragée avec un " juste retour » au laboratoire inventeur. ${ }^{24}$ " concertation avec des industriels, lorsqu'il est appelé à présider un groupe de travail sur les biotechnologies et leurs enjeux pour les IAA, dans le cadre des Groupes d'études et de mobilisation (GEM) lancés par Édith Cresson. Le rapport remis en août 1992 à Élisabeth Guigou, ministre chargée des Affaires européennes, au nom de ce groupe composé d'une vingtaine de membres issus de l'industrie, de la recherche et de l'administration, prône un certain assouplissement de la réglementation française et européenne sur les organismes génétiquement modifiés (OGM): les mesures de protection des consommateurs, pour légitimes qu'elles soient, ne devraient pas étouffer le développement de l'innovation en Europe, dans le contexte de l'ouverture prochaine du Marché unique européen et de la concurrence exacerbée des industriels américains $^{27}$.

\section{Communiquer auprès du grand public et de la « techno-structure " étatique}

Tout au long de ses années passées à l'INRA, R. Février ressent le besoin de ne pas limiter ses efforts de communication aux seuls utilisateurs de la recherche agronomique, ainsi qu'il en fait état dans un éditorial du Bulletin de l'INRA justement intitulé « ...et le faire savoir » :

«Il n'est pas suffisant, en effet, que les groupes directement intéressés par notre activité soient conscients de notre efficacité : les idées, les opinions qui prévalent lors des décisions, se forment souvent dans des cercles différents, principalement dans les organisations professionnelles non spécialisées, et pour l'État, dans ce que E. Faure désignait récemment sous le nom de «techno-structure ». Or, dans ces milieux, les informations qui concernent l'Inra pénètrent peu, et celles qui y parviennent sont presque toujours biaisées, souvent dans un sens défavorable. [...] Depuis près d'un an, nous avons demandé à B. R. Lévy, ingénieur, attaché à la direction générale, de s'attacher à établir des rapports avec la presse, la radio et la télévision. ${ }^{28}$ "

19 R. Février entend donc toucher "les décideurs, politiques et administratifs, [...] plus sensibles à la grande presse et aux dîners en ville qu'aux documents dont ils sont submergés ", et, au-delà, l'opinion publique. Pour ce faire, il obtient la création d'un poste de public relations, bientôt confié à Bertrand-Roger Lévy, et, suivant une inclination héritée d'un père plusieurs fois ministre à la fin de la III ${ }^{e}$ République, il n'est pas avare en articles et entretiens destinés à la presse d'information générale, qu'elle soit nationale, régionale ou même locale ${ }^{29}$. À cet égard, le fonds R. Février renferme aussi bien un numéro du Figaro agricole de 1969 spécialement consacré à l'INRA, dont il a assuré le pilotage ${ }^{30}$, qu'un article rédigé pour Notre Pays, "petit mensuel» de ses 
Hautes-Alpes natales, alors qu'il est directeur général de l'un des principaux opérateurs de recherche français ${ }^{31}$.

L'enjeu, il est vrai, n'est pas mince. S'assurer l'adhésion de l'opinion et des pouvoirs publics aux réalisations et projets de l'INRA, ce n'est pas seulement se donner les meilleures chances d'obtenir des moyens d'action conséquents; c'est encore faire partager largement une certaine vision de l'agriculture et du développement économique et social, en vue d'influer sur les choix structurels en matière de politiques agricoles et de recherche agronomique.

\section{Orienter les politiques agricoles et de recherche agronomique en France, en Europe, dans le bassin méditerranéen et les pays en développement}

De fait, R. Février n'a eu de cesse de revendiquer pour l'INRA un rôle d'éclaireur, sinon d'aiguillon des politiques agricoles.

\section{Intégrer les savoirs agronomiques à l'élaboration des politiques agricoles}

Peu après sa démission de la direction générale en 1978, du reste motivée par des déceptions répétées dans ce domaine, il ne manque ainsi pas de réaffirmer cette ambition prospective dans le rapport déjà cité :

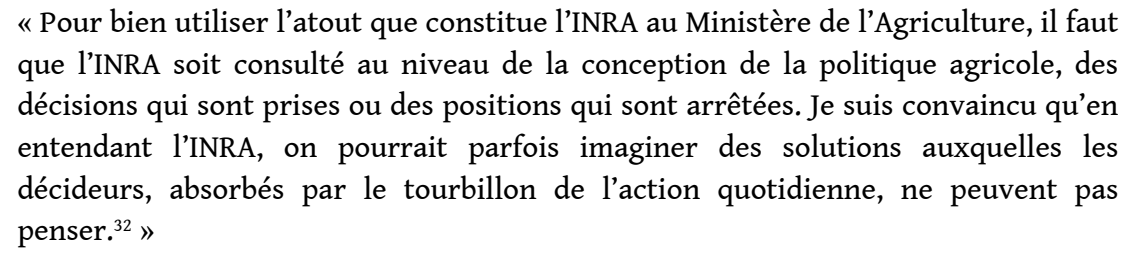
que l'INRA soit consulté au niveau de la conception de la politique agricole, des décisions qui sont prises ou des positions qui sont arrêtées. Je suis convaincu qu'en entendant l'INRA, on pourrait parfois imaginer des solutions auxquelles les décideurs, absorbés par le tourbillon de l'action quotidienne, ne peuvent pas penser. ${ }^{32} »$

23 À l'appui de cette conviction, R. Février évoque volontiers «l'affaire des protéines ». Dès 1966, il alerte en effet le ministre de l'Agriculture Edgar Faure sur les dangers que fait peser la dépendance croissante de l'élevage français vis-à-vis des importations de tourteaux de soja américains. En vain : il faut attendre l'embargo Nixon de 1973 sur les exportations de soja pour qu'une politique volontariste en faveur du colza et du tournesol voie le jour aux niveaux national et européen, dans le sillage des recherches que l'INRA avait entreprises « sans moyens supplémentaires » et « sans directives ${ }^{33}$ ».

Dans le même ordre d'idées, le directeur général R. Février prend publiquement position sur la question des isoglucoses. Dans les colonnes du journal La Croix du 5 août 1977, il regrette ainsi que l'utilisation massive de sirop de maïs bon marché par les fabricants de boissons américains, à la suite de l'embargo sur le sucre cubain, ne donne lieu qu'à des mesures protectionnistes "réactionnaires", sous la pression des sucriersbetteraviers. Non sans susciter l'ire du cabinet du ministre, il appelle de ses vœux le déploiement d'une audacieuse stratégie de production de ces substances sucrées à partir d'amidon de blés ${ }^{34}$.

Un dernier exemple d'orientation politique inspirée par la recherche agronomique peut être mentionné ici : le développement des biotechnologies comme alternative à l'usage intensif des intrants chimiques. Effectivement, avant même le « rapport Poly » Pour une 
agriculture plus économe, plus autonome (1978), R. Février s'inquiète du recours généralisé aux produits fertilisants et phytosanitaires d'origine industrielle, moins d'ailleurs pour des raisons environnementales que pour des considérations économiques et sociales, «la charge qu'ils entraînaient risqua[n]t de rendre dérisoire le bénéfice de cette intensification ${ }^{35} \%$.

"Viscéralement socialiste ", il s'attache constamment à rallier à ses vues les militants et dirigeants de ce parti (Section française de l'Internationale ouvrière jusqu'en 1969, puis Parti socialiste), auquel il adhère dès l'époque du Front populaire ${ }^{36}$. Aussi le fonds R. Février est-il riche en pièces de correspondance, notes et rapports, produits dans le cadre des Commissions Agriculture et Recherche ${ }^{37}$ ou dans un contexte plus informel ${ }^{38}$ : s'y rencontrent notamment un rapport destiné au Premier secrétaire François Mitterrand sur Les protéines: agricoles et industrielles (1979), ainsi que le manifeste Pour une autre agriculture avec les socialistes (1981), qu'il rédige à l'occasion de l'élection présidentielle ${ }^{39}$. Autre témoignage $\mathrm{du}$ «couplage de $[\mathrm{s}] \mathrm{es}$ préoccupations professionnelles et de $[\mathrm{s}]$ on engagement politique, [...] qui sera une constante de [s]a vie active $»^{40}$ : dans les années 1960 , et surtout 1970, il prépare, pour le compte d'un "petit réseau d'une douzaine de parlementaires ", des dizaines de questions écrites au gouvernement, portant sur les moyens alloués à l'INRA comme sur les grandes orientations de la politique agricole. Un exercice qui l'amène parfois à formuler, à la demande du ministère, un projet de réponse à ses propres questions ! ${ }^{41}$

$\mathrm{Au}$ cours des années 1970-1980, R. Février tâche également d'infléchir certaines politiques publiques en dehors de l'INRA et du monde politique français. Dans les différentes institutions nationales, européennes et internationales où il remplit alors des missions d'administration et d'expertise, il s'emploie en effet à consolider les assises de la recherche agronomique en y défendant une recherche fondamentale forte, à la fois largement autonome de la commande publique et étroitement articulée avec les acteurs et les territoires où elle a vocation à être appliquée.

\section{Concentrer et structurer en réseaux les moyens de recherche fondamentale et appliquée à toutes les échelles}

Un tel modèle d'organisation de la recherche est en fait fourni par l'INRA lui-même. Sur fond de rééquilibrage géographique impulsé par le Plan dans les années 1960-1970, l'ouverture de nouveaux centres de recherche en province (à Lille, avec le CERTIA évoqué plus haut, mais aussi à Nantes, Rennes, Orléans, Clermont-Ferrand, Avignon...) lui permet de se rapprocher au plus près des bassins de production et de leurs besoins spécifiques ${ }^{42}$. Dans le même temps, un fonctionnement en réseau doit prévenir une funeste dispersion des moyens, ce que R. Février prend soin de rappeler en 1978 :

«[...] il ne faut pas aller jusqu'à l'excès auquel pousseraient certaines revendications régionales, voire régionalistes. Vouloir disposer dans une région de la quasi totalité des spécialités de l'INRA, aboutirait à doter chacune d'entre elles de moyens si modestes que ces unités squelettiques seraient vouées à la mort intellectuelle. [...] En revanche, l'Établissement doit avoir, dans la plupart des grandes régions agricoles, un Centre de recherches, axé essentiellement sur une ou deux des grandes productions qui constituent la spécialité régionale (arboriculture, grande culture, élevage...), et disposer des moyens (et avoir l'attitude d'esprit) pour répercuter, sur les laboratoires de l'INRA compétents d'autres régions, les problèmes régionaux qu'il ne pourrait pas résoudre par lui-même. ${ }^{43}$ " 
R. Février s'efforce donc d'imprimer une politique similaire dans les autres établissements publics nationaux où il exerce des responsabilités entre le milieu des années 1970 et celui des années 1980. En tant que président du Conseil chargé de superviser la création d'une université en Corse (1976-1980), il promeut ainsi une nette spécialisation dans les études méditerranéennes, comme «l'implantation [à Corte] d'autres activités «intellectuelles » à proximité immédiate, de façon à disposer, un jour, d'un campus vivant, attractif, pour la France et d'autres pays méditerranéens ${ }^{44}$ ». De même, à la présidence du CEMAGREF (1982-1985), il tente de "recentrer [s]es activités [...] sur ses secteurs d'excellence et où son activité ne faisait pas double emploi avec celles de l'INRA ", à savoir l'hydraulique et le machinisme agricole ${ }^{45}$. Toujours en quête de complémentarités fonctionnelles, il propose, en qualité de président du Conseil d'administration de l'Office national des forêts (ONF) (1984-1985), qu'un accordcadre de collaboration avec l'INRA organise le développement conjoint de recherches forestières ${ }^{46}$.

Dans les années 1970-1980, R. Février œuvre par ailleurs au rapprochement entre établissements au sein de plusieurs organes français et européens de coordination des politiques de recherche. Sur le plan national, il prend part, notamment à titre de membre du Comité consultatif de la recherche scientifique et technique (CCRST) (1972-1975), à la définition des "actions concertées » de la Délégation générale à la recherche scientifique et technique (DGRST) visant à décloisonner les disciplines et les organismes ${ }^{47}$. Auprès de la Commission des Communautés européennes, il contribue à la création, officialisée en 1974, du Comité permanent de la recherche agricole (CPRA), qui lui confie plus spécialement l'animation scientifique du groupe «Agriculture méditerranéenne » (Agrimed) (1979-1983) ${ }^{48}$.

31 Son intérêt marqué pour cette thématique le conduit à assumer en parallèle les fonctions de président du Comité consultatif scientifique (1978-1985), puis de secrétaire général (1985-1991) du CIHEAM, dont les activités d'enseignement sont assurées par quatre Instituts agronomiques méditerranéens (IAM) créés successivement à Bari, Montpellier, Saragosse et La Canée. Là encore, il essaie de «mettre en place une certaine spécialisation au profit de quelques laboratoires [des IAM] bénéficiant d'une sorte d'habilitation pour être leaders méditerranéens dans leur discipline ", et tout particulièrement têtes de réseau de la coopération agronomique avec les pays de la rive $\operatorname{sud}^{49}$.

\section{Favoriser l'émergence de systèmes nationaux de recherche dans les pays en développement}

Cette préoccupation pour les partenariats scientifiques avec les pays en développement (PED), R. Février l'a manifestée précocement - au moins depuis une mission effectuée en Tunisie en $1961^{50}$ - et régulièrement, dans l'exercice des diverses fonctions indiquées ci-dessus comme au sein de la commission Tiers-Monde du Parti socialiste ${ }^{51}$. Mais elle trouve sans doute son expression la plus achevée dans les notes et documents de travail qu'il rédige pour Edgard Pisani, commissaire européen chargé du Développement, en tant que conseiller spécial (1981-1983) ${ }^{52}$.

Dans le contexte de l'adoption d'un premier programme-cadre pour la recherche et le développement technologique (PCRD), R. Février s'applique alors à redéfinir la doctrine européenne de coopération scientifique avec les PED, et notamment les pays d'Afrique, 
des Caraïbes et du Pacifique (ACP) signataires des accords de Lomé. Jusque-là, l'essentiel des crédits alloués à ce titre ont bénéficié à des organismes de recherche européens hérités de la période coloniale, tels que l'office de la recherche scientifique et technique outre-mer (ORSTOM) et le Groupement d'étude et de recherche pour le développement de l'agronomie tropicale (GERDAT), ainsi qu'au Groupe consultatif sur la recherche agronomique internationale (GCRAI) dominé par les États-Unis. À l'inverse, dans un document de travail du 12 mars 1982, il leur assigne comme objectif fondamental d'" encourager dans les pays en développement, [...] le renforcement d'un potentiel scientifique et technique endogène ${ }^{53}$ ».

Les raisons qu'il détaille dans une note du 24 février 1982 méritent d'être citées in extenso :

«[...] cette recherche, effectuée sur place, doit être aussi réalisée par des autochtones, pour des raisons techniques, politiques et économiques.

Techniques: car ils sont mieux placés pour définir correctement une bonne problématique, du moins s'ils n'ont pas été saisis du démon de l'imitation.

Politiques: car si on reconnaît à la Science l'importance qu'elle revêt dans le développement, il n'est pas pensable qu'on la confie à des «mercenaires scientifiques ». De surcroît, des autochtones formés à la discipline scientifique fourniront ultérieurement des cadres technico-politiques capables d'orienter la politique scientifique. Ce rôle ne peut être longtemps confié à des étrangers.

Économiques: le rôle de la Recherche scientifique n'est pas seulement de secréter des innovations. Il est de constituer - dans l'appareil des États ou dans les entreprises - des cellules dont la créativité et l'imagination mettent en question les habitudes. Cette fonction de contestation positive est souvent aussi utile que la fonction d'innovation.

En outre, par dialogue avec les décideurs - politiques ou managers d'entreprises -, les chercheurs permettent de concevoir une stratégie plus prospective, en indiquant les voies où l'on peut saisir et exploiter de nouvelles possibilités technologiques.

La recherche ne doit pas être limitée au rôle d'assurer le suivi de décisions stratégiques : elle peut contribuer à leur élaboration..$^{54}$ »

Au-delà de la seule question de la coopération scientifique avec les PED, on retrouve dans ces lignes, en définitive, un condensé des idées de R. Février touchant la transmission des savoirs agronomiques. S'y affirme en effet l'ambition d'une recherche soucieuse des conditions matérielles et humaines de son application, comme de son influence sur les politiques publiques. Une ambition qui, pour "grande" et inlassablement poursuivie qu'elle soit, n'épuise évidemment pas la richesse d'une trajectoire dont l'histoire reste à écrire. 


\section{BIBLIOGRAPHIE}

CORNU Pierre, VALCESCHINI Egizio et MAEGHT-BOURNAY Odile, L'Histoire de l'INRA, entre science et politique, Versailles/Paris, Éditions Quae, 2018.

CRANNEY Jean, INRA. 50 ans d'un organisme de recherche, Paris, Inra Éditions, 1996.

FÉVRIER Raymond, témoignage recueilli par POUPARDIN Denis en 1996, Archorales, tome 6, septembre 2001, p. 9-103.

ROLLAND Léon, « L'histoire de la vulgarisation agricole avant 1966 », Économie rurale, n 159, 1984, p. 11-16.

TUDESQ André-Jean, « Média et monde agricole après 1945 », Économie rurale, n 184-186, 1988, p. 205-214.

\section{NOTES}

1. Nous nous permettons de renvoyer le lecteur intéressé au répertoire numérique détaillé du fonds Raymond Février (1946-2010) accessible dans la Salle des inventaires virtuelle des Archives nationales. Ce travail a bénéficié des recommandations et de l'appui bienveillant de Solange Bidou et d'Henri Pinoteau; qu'ils en soient ici chaleureusement remerciés.

2. Tome 6, septembre 2001, p. 9-103.

3. Respectivement intitulés Mon Dictionnaire. $G$ : D'une guerre à d'autres, 2012 [rédigé pour l'essentiel en 2002-2003], 32 pages, et Politique II. Deuxième partie: Raymond Février, 2015 [daté de mars 2010 sur la dernière page, hors annexes], 124 pages. Qu'il nous soit permis d'exprimer ici toute notre gratitude à Raymond Février pour cette insigne marque de confiance.

4. R. Février, témoignage dans Archorales, p. 13-14, Mon Dictionnaire. G: D'une guerre à d'autres, p. 22-28, et Politique II. Deuxième partie : Raymond Février, p. 9-10.

5. Archives nationales (Arch. nat.), 20170135/4, «Comment produire un porc maigre en utilisant les aliments de l'exploitation ».

6. Arch. nat., 20170135/12.

7. R. Février, témoignage dans Archorales, p. 29, et Politique II. Deuxième partie: Raymond Février, p. 100.

8. Arch. nat., 20170135/8, dossier 7, «L'INRA et ses problèmes », p. 33.

9. P. Cornu, E. Valceschini et O. Maeght-Bournay, L'Histoire de l'INRA, entre science et politique, p. 94; L. Rolland, "L'histoire de la vulgarisation agricole avant 1966 ", p. 15-16.

10. Arch. nat., 20170135/8, dossier 7, «L'INRA et ses problèmes », p. 35.

11. R. Février, témoignage dans Archorales, p. 29 et 66.

12. Arch. nat., 20170135/5, « Réflexions sur l'INRA », p. 9.

13. P. Cornu, E. Valceschini et O. Maeght-Bournay, L'Histoire de l'INRA, entre science et politique, p. 99-100 ; J. Cranney, INRA. 50 ans d'un organisme de recherche, p. 199-200. 
14. Arch. nat., 20170135/3, « Compte-rendu de la Journée du Porc, à Brive », p. 2.

15. Ibid., p. 1.

16. Pour un bref panorama des moyens d'information à disposition des agriculteurs à cette époque, voir A.-J. Tudesq, « Média et monde agricole depuis 1945 », p. 205-214.

17. Arch. nat., 20170135/4 (pour les années 1952-1961) et 20170135/7 (pour la période 1962-1975).

18. Arch. nat., 20170135/4.

19. Arch. nat., 20170135/4, «Comment obtenir un porc maigre », La Tribune du monde rural.

20. R. Février, témoignage dans Archorales, p. 11.

21. Arch. nat., 20170135/5, « Réflexions sur l'INRA », p. 10.

22. Ibid.

23. Arch. nat., 20170135/14.

24. Arch. nat., 20170135/5, « Réflexions sur l'INRA », p. 10.

25. Arch. nat., 20170135/15.

26. R. Février, témoignage dans Archorales, p. 57-58; P. Cornu, E. Valceschini et O. Maeght-Bournay, L'Histoire de l'INRA, entre science et politique, p. 122 ; J. Cranney, INRA. 50 ans d'un organisme de recherche, p. 282-285.

27. Arch. nat., 20170135/35 ; R. Février, témoignage dans Archorales, p. 78, et Politique II. Deuxième partie : Raymond Février, p. 55.

28. R. Février, « ... Et le faire savoir », Bulletin de l'INRA, n 55,1970 , p. 1 (cité dans P. Cornu, E. Valceschini et O. Maeght-Bournay, L'Histoire de l'INRA, entre science et politique, p. 120).

29. R. Février, témoignage dans Archorales, p. 48.

30. Arch. nat., 20170135/6, "Les chercheurs de l'INRA parlent... », Le Figaro agricole, supplément au n² 216, novembre 1969.

31. Arch. nat., 20170135/37, «Vers une nouvelle agriculture », Notre Pays, janvier-mars 1978. La première des trois parties dactylographiées porte l'indication manuscrite " petit mensuel des H[au]tes-Alpes ».

32. Arch. nat., 20170135/8, dossier 7, «L'INRA et ses problèmes », p. 32. C'est R. Février qui souligne.

33. R. Février, témoignage dans Archorales, p. 42, et Politique II. Deuxième partie: Raymond Février, p. 19 et 98.

34. Arch. nat., 20170135/9.

35. R. Février, Politique II. Deuxième partie : Raymond Février, p. 98.

36. Ibid., p. $82-87$ [citation p. 82].

37. Arch. nat., 20170135/38 et 20170135/39.

38. Arch. nat., 20170135/42. Voir en particulier l'abondante correspondance échangée avec Edgard Pisani et Henri Nallet, ministres de l'Agriculture respectivement dans les années 1960 et 1980.

39. Arch. nat., 20170135/38.

40. R. Février, Politique II. Deuxième partie: Raymond Février, p. 14. 
41. Arch. nat., 20170135/41 ; R. Février, Politique II. Deuxième partie: Raymond Février, p. 28.

42. Arch. nat., 20170135/8, dossier 3, 20170135/9 et 20170135/14 ; R. Février, témoignage dans Archorales, p.60; P.Cornu, E. Valceschini et O. Maeght-Bournay, L'Histoire de l'INRA, entre science et politique, p. 105-109; J. Cranney, INRA. 50 ans d'un organisme de recherche, p. 209-211.

43. Arch. nat., 20170135/8, dossier 7, «L'INRA et ses problèmes ", p. 39.

44. Arch. nat., 20170135/19 ; R. Février, témoignage dans Archorales, p. 69-70 [citation p. 69], et Politique II. Deuxième partie : Raymond Février, p. 35 et 107-110.

45. Arch. nat., 20170135/21 ; R. Février, témoignage dans Archorales, p. 75-78 [citation p. 76], et Politique II. Deuxième partie : Raymond Février, p. 116.

46. Arch. nat., 20170135/22 ; R. Février, témoignage dans Archorales, p. 92-95, et Politique II. Deuxième partie : Raymond Février, p. 45-48 et 117-119.

47. Arch. nat., 20170135/18 ; R. Février, témoignage dans Archorales, p. 55-56 ; P. Cornu,

E. Valceschini et O. Maeght-Bournay, L'Histoire de l'INRA, entre science et politique, p. 86-90 ; J. Cranney, INRA. 50 ans d'un organisme de recherche, p. 185-196 et 321.

48. Arch. nat., 20170135/26 ; R. Février, témoignage dans Archorales, p. 87-89.

49. Arch. nat., 20170135/29 ; R. Février, témoignage dans Archorales, p. 95-101 [citation p. 98], et Politique II. Deuxième partie : Raymond Février, p. 49-52 et 121-122.

50. R. Février, Politique II. Deuxième partie : Raymond Février, p. 17-18.

51. Arch. nat., 20170135/39.

52. Arch. nat., 20170135/27 et 20170135/28 ; R. Février, témoignage dans Archorales, p. 82-87, et Politique II. Deuxième partie : Raymond Février, p. 39-43 et 115.

53. Arch. nat., 20170135/27, « Programme de recherche et de développement dans le domaine de la «Science et de la Technique au service du Développement » », p. 3. C'est R. Février qui souligne.

54. Arch. nat., 20170135/27, « Recherche scientifique et développement », p. 3-4. C'est R. Février qui souligne.

\section{RÉSUMÉS}

À partir des documents qu'il a donnés récemment aux Archives nationales et à l'aune des questionnements soulevés par la transmission des savoirs agronomiques, cet article se propose de relire la trajectoire de Raymond Février (né en 1920) qui, entre autres fonctions, a occupé successivement, de 1946 à 1978, celles de chercheur, d'inspecteur général et de directeur général à l'Institut national de la recherche agronomique (INRA). Deux traits la caractérisent entre tous : d'une part, le souci permanent de diffusion de connaissances à finalité pratique et immédiate auprès du monde agricole et industriel; de l'autre, la volonté non moins ferme d'influer sur les politiques structurelles en matière d'agriculture et de recherche agronomique. 


\section{AUTEUR}

\section{SÉBASTIEN PIVOTEAU}

Ingénieur de recherche aux Archives nationales (Direction des Fonds/Département de l'Environnement, de l'Aménagement du territoire et de l'Agriculture) 
De l'information scientifique et technique aux transferts de technologie : un défi pour notre temps 
De l'information scientifique et technique aux transferts de technologie : un défi pour notre temps

\section{L'information scientifique et technique : quels vecteurs?}




\title{
De la diagraphie à la numérisation : deux artisans d'une transmission de savoir (Charles-Louis Marle aîné, Louis-Georges Marle) (1829-2017)
}

\author{
Anne Marle-Kolle
}

Lorsque Charles-Louis Marle aîné édite son Manuel de la diagraphie en 1839, il a déjà publié dix ans auparavant un ouvrage intitulé, Praxigraphie ou recueil d'un nouveau genre pour apprendre par la pratique les règles de grammaire et l'orthographe ainsi que le Journal de la langue française, grammatical, didactique et littéraire. Cent soixante-dix ans plus tard, son descendant Louis-Georges Marle participe à son tour à cette transmission des savoirs en étant responsable des productions numériques aux éditions Albin Michel, après avoir été secrétaire d'édition aux éditions les Belles-Lettres. On se propose de comparer les deux méthodes ainsi que leur devenir ou leur avenir. Les sources utilisées sont les suivantes : pour Charles-Louis Marle aîné, Manuel de la diagraphie. Découverte qui simplifie l'étude de la langue par Marle aîné. Paris, Imprimerie administrative de Paul Dupont et $\mathrm{C}^{\mathrm{ie}}, 1839$, Journal de la langue française grammatical, didactique et littéraire. Paris, 1827-1830, Archives privées; pour Louis-Georges Marle, Les identifiants. International standard text code. Electre in ISTC-SNE Paris, 2015, Groupe normes et standards du SNE, Adéquation du langage. Onix au métier de l'éditeur... Paris, Atelier du SNE, juin 2016, Sources orales 2017.

\section{Deux artisans de transmission du savoir}

2 La vocation de Charles-Louis Marle tout comme celle de Louis-Georges est de transmettre le savoir au plus grand nombre. Si Charles-Louis né en 1799, fils d'un instituteur ${ }^{1}$ de Saône et Loire, s'engage dans un processus politique qu'il transmettra à ses deux fils, Louis et Jérôme ${ }^{2}$ condamnés à la suite de la Révolution de Juin 1848 et du coup d'État du 2 décembre 1851, à la déportation en Algérie ${ }^{3}$, Louis-Georges se consacre uniquement aux moyens de diffuser par le biais de la numérisation le savoir et la 
culture à une population privilégiant le côté pratique de la lecture sur l'outil informatique liseuse, tablette ou smartphone... ou bien à une population handicapée, les textes numérisés étant parfois restitués oralement.

\section{Charles-Louis Marle dit Marle aîné}

3 Lorsque Charles-Louis, publie en 1848 L'Émancipation de l'enseignement ${ }^{4}$, il a derrière lui un long parcours consacré aux moyens de transmettre le savoir. Directeur, avec son frère Lazare Olivier, d'une école d'enseignement mutuel fondée par son père en 1818, il publie en 1822, Charles et Guillaume, les parfaits élèves d'une école d'enseignement mutuel ${ }^{5}$, devient de 1826 à 1829 directeur du Journal de la langue française, grammaticale, didactique et littéraire, "destiné à lutter contre la révolution romantique " écrira Jacques Chadourne en 1979, dans le Bulletin d'information pédagogique de l'Académie de Mâcon après avoir cité Marle :

"C'est bien après une révolution politique qui a accru le domaine de notre néologie et enfanté le dangereux romantisme qu'il convient d'opposer de nouvelles barrières au néologisme. ${ }^{7} "$

Cependant, le début du XIXe siècle abonde en nombreuses méthodes d'apprentissage de la lecture impliquant une réforme de l'orthographe. Marle fera partie de ces réformateurs en publiant Le Manuel de la diagraphie, s'inspirant, en l'améliorant de la méthode de Laffore de Bourrousse, employée dans les écoles primaires, à partir du 22 juin 1829 : la Statilégie, lecture immédiate. Art de lire au plus haut degré de simplicité8. Peu avant la Révolution de 1830, Marle proclame ses idées populistes, qui vont lui valoir les attaques d'une intelligentsia avec laquelle il n'aura de cesse de polémiquer :

«Nous renverserons les préjugés qui tiennent en laisse la portion instruite de la société et garrotent le peuple. Nous voulons éclairer le peuple en masse et en quelques jours seulement. ${ }^{9}$ »

Jacques Chadourne rappelle :

« Si le conservatisme linguistique, l'autorité de la norme et la pérennité de la langue caractérisent ses écrits en grammaire, il n'en est pas de même en ce qui concerne la transcription des phonèmes. Il se lança dans une véritable croisade iconoclaste. ${ }^{10}$ "

6 En effet, ce fut une croisade destinée à réformer totalement l'orthographe par un système de phonèmes, la diagraphie. En 1838, il expérimente sa méthode à Lyon ${ }^{11}$ et Paris $^{12}$. Il décide alors de publier le Manuel de diagraphie, suivi de la grammaire diagraphique, et de la grammaire théorique, pratique et didactique ou texte primitif de la grammaire didactique. Il interrompt sa croisade éducative pour fonder en 1841, à Lyon, le journal quotidien Le Rhône où il va mettre en pratique sa méthode diagraphique en publiant, les célèbres sermons du dominicain Lacordaire. Le 23 mai 1845, dans une annonce faite dans son journal, il propose à ses lecteurs Les conférences complètes de $\mathrm{M}$. Lacordaire à Lyon, exactement recueillies et précédées du portrait et de la biographie du révérend Père. Le résultat fut un succès, mais le Père de l'Ordre des prêcheurs ${ }^{13}$ fondateur de la Congrégation enseignante en 1852, ne pouvant concevoir qu'une éducation chrétienne ne put être dispensée hors d'un établissement religieux ${ }^{14}$, intenta un procès à l'auteur pour ce qu'il considérait comme une contrefaçon ${ }^{15}$. Procès perdu, qui conduit Charles-Louis à la faillite mais ne l'empêche pas de publier de 1848 à 1849, l'Émancipation de l'enseignement ${ }^{16}$ et de février à août 1849, La Constitution. République du Présent et de l'Aveniri ${ }^{17}$. Deux versions existent pour la fin de Charles-Louis. Il aurait vécu 
misérablement à Mâcon jusqu'à sa mort en 1860 ou bien aurait quitté la France pour les États-Unis, puis pour le Venezuela où il serait mort en $1864^{18}$.

\section{La méthode de diagraphie}

«Trois jours suffisent pour établir la diagraphie dans une classe. Elle est un guide incessant de bonne prononciation - elle permet à l'élève dans la même situation que si un bon maître lui dictait perpétuellement un bon livre - elle économise le temps consacré aux dictées - elle réunit sans en avoir les inconvénients tous les avantages de la cacographie et des autres genres de devoirs d'orthographe - elle fait réfléchir les enfants, elle exerce leur jugement et féconde leur intelligence ${ }^{19}$. »

\section{Le manuel de diagraphie : découverte qui simplifie l'étude de la langue ${ }^{20}$}

7 L'ouvrage de 71 pages comporte 25 pages consacrées à la méthode et 44 aux rapports, procès-verbaux, avis. L'idée générale du système consiste à définir les sons ou voyelles par 19 signes.

Fig. 1. - Valeur des 19 signes précédents et de leurs modifications d'après les trois règles.

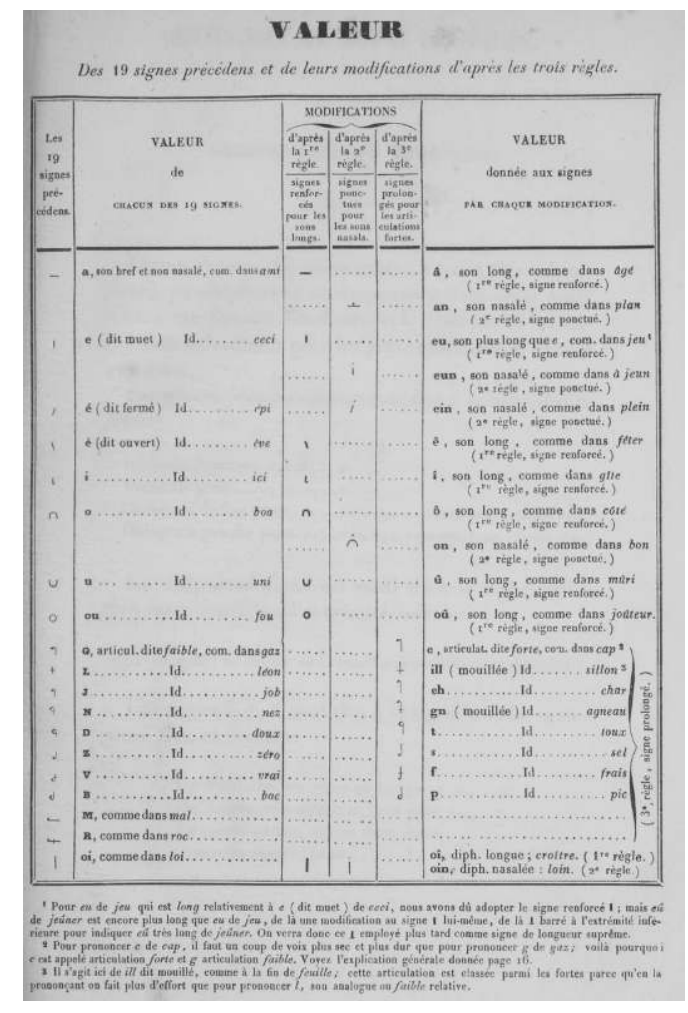

Charles-Louis Marle, Manuel de de la diagraphie, np.

Chaque signe peut être modifié selon sa valeur : â (son long) =-, an (son nasalé) =surmonté d'un point, eu =I, ein = I surmonté d'un point, on (son nasalé) $=\mathrm{u}$ inversé surmonté d'un point, puis l'articulation des consonnes représentées par des petits traits munis de crochets ou de petites croix.

\section{Le système}


Fig. 2. - Observations sur tout ce qui précède. Simplicité du système.

Lignes droites, lignes courbes, à traits fins ou renforcés, ou simplement accompagnées d'un point, voilà toutes nos figures. Étudions-en le système et les caractères individuels pour les graver facilement dans la mémoire. fine,

Les quatre premiers signes sont dus à la ligne droite

Horizontale pour..... - ..a

Verticale pour....... 1...e

Oblique à droite pour. 1 $\ldots e \dot{e}$ (le signe, ressemble un peu a l'accent aigu. $)$

Oblique à gauche pour $1 \ldots . \dot{e}$ (le signe I ressemble un peu i l'acent grave.)

Le cinquième signe est aussi dù à la ligne droite fine, mais courbée à l'extrémité inférieure :

t....i (ce signe ı ne diffère de l'i usuel que par l'absence du point.)

Les signes $6,7,8$ sont dus à la ligne courbe également fine, et formant

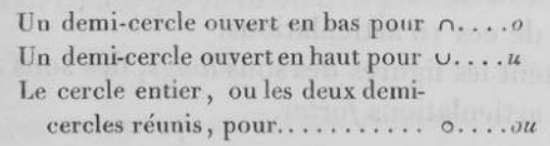

Charles-Louis Marle, Manuel de de la diagraphie, np.

9 Le signe horizontal - représente le a de ami, mais aussi de drap et a de mangea ou d'orgeat, en un mot tout ce qui se prononce en a, quelque soient les lettres qui indiquent la prononciation usuelle. Lorsque le signe - est surmonté d'un point, il remplace non seulement le an de plan mais aussi le and de gland, le ant de diamant... Le signe 7 est destiné à remplacer le c (dur) de cap mais aussi le ch de chrétien ou le q de cinq. En un mot tout ce qui dans la langue phonique a valeur phonique. Le.I accompagné d'un point en bas à gauche est destiné à remplacer le s de sel, mais aussi le c de cigale...

«Les signes révèlent les mots sans faire connaitre l'orthographe, ils lui peignent les sons de la voix comme s'ils entendaient une dictée: ils lui présentent une succession de problèmes orthographiques que l'enfant doit continuellement résoudre comme dans une dictée où chaque mot est une épreuve pour son savoir si bien qu'en signes diagraphiques un livre imprimé serait toujours une dictée continue et toujours bien dite. En réunion ou isolé, il suffit d'ouvrir un livre diagraphique pour jouir des avantages de cette dictée perpétuelle. »

Marle donne comme exemple la phrase : en négligeant de remplir les devoirs de chrétien on néglige son bonheur de ce monde et de l'autre. 
Fig. 3. - Usage du système.

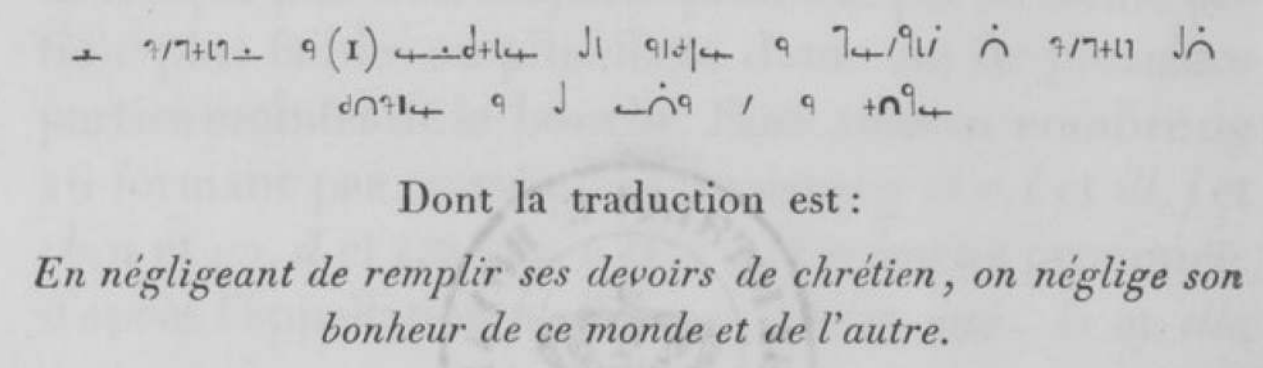

Charles-Louis Marle, Manuel de la diagraphie, p. 18.

Par la diagraphie on peut obtenir phonétiquement : an néglijan de rhamplir les devoirs de qurétiains, on néglije son bonheur, puis: an néglijan de remplir ces devoirs de kretien on négligent son bonheur, et ainsi de suite.

Fig. 4. - Usage du système.

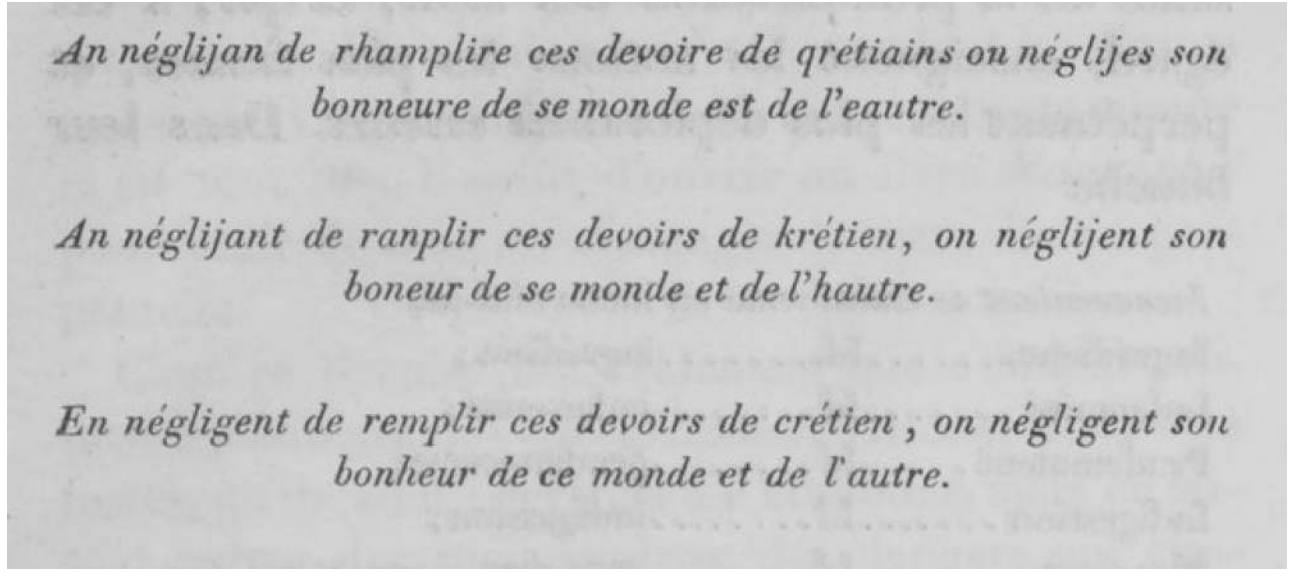

Charles-Louis Marle, Manuel de la diagraphie, p. 20.

Dans son exemple, il veut démontrer que l'on peut aboutir à la bonne orthographe. Au cours du procès, Lacordaire avait malencontreusement qualifié de sténographie (saisir pour soi à la hâte un texte pris au vol) le procédé, or la diagraphie ne suit pas la parole, son but est la pratique de l'orthographe, elle diffère de la tachygraphie (la tachygraphie devance les sons) et de la sténographie.

\section{De la diagraphie à la numérisation}

Si la diagraphie est un moyen de transcription des phonèmes dans le but de découvrir l'orthographe après l'avoir ignorée - son inventeur dira qu'elle peint les sons - et modélise une nouvelle forme d'écriture, elle conserve, comme la numérisation, son fond. L'instituteur connaît l'orthographe du texte qu'il dicte, alors que l'élève l'ignore. Le but de Charles-Louis était donc de dévoiler le fond du texte et, à partir des transcriptions puis des interprétations personnelles et fantaisistes de l'auditeur, de conduire peu à peu ce dernier, avec l'aide de l'instituteur, à découvrir l'orthographe, ce qu'il ne dit pas dans son manuel mais que l'on pressent dans son Manuel de grammaire théorique pratique, didactique ou Titre primitif de la grammaire diagraphique. 

auquel on accède via des moyens précis: le balisage du texte. Exemple : c'était à Mégara faubourg de Carthage, dans les jardins d'Hamilcar, devient après balisage : $<\mathbf{p}>$ C'était à $<\mathbf{i}>$ Mégara</i $>$ faubourg de Carthage, dans les jardins d'Hamilcar. $</ p>$

Il s'agit de dissocier le fond de la forme grâce à un « langage pivot » : il s'agit de partir d'un fichier word, et le transformer en fichier XML (Extented Markup Language). Le texte ainsi balisé est transformé en fichier XHTML (Extensible Hypertext Markup Language) fichiers constitutifs de l'ePub. Le fichier XML est garant de la pérennité du texte et permet - après transformation - l'interopérabilité entre les différents systèmes de lecture, que ce soit de l'imprimé ou du livre numérique. Dans les deux cas, l'objectif est de conduire à la transmission du savoir, de permettre à tous d'avoir accès à la culture, à l'enrichissement personnel.

\section{Louis-Georges Marle}

16 L'idée de la numérisation, contribuant à la lecture rapide, ergative et dont l'avantage est d'être de $20 \%$ à $60 \%$ moins cher que le livre papier ne pouvait que séduire LouisGeorges Marle. Grâce à sa capacité d'adaptation à ce mode de diffusion requérant un bagage scientifique, Louis-Georges après des études au Lycée Louis-le-grand puis au Lycée Fénelon, en classes de Mathématiques supérieures se rend compte que sa véritable vocation est l'édition. Il entreprend alors des études d'Histoire, avec option latin à la Sorbonne, tout en travaillant comme responsable de production aux éditions Les Belles lettres. Sollicité par le Groupe Albin Michel, cinquante et unième des grands groupes d'édition mondial, et sixième groupe d'édition en France, comme responsable de production du département sciences humaines, il va à partir de 2010 s'orienter vers la numérisation et entrer au Syndicat national de l'Édition. Ce qui a séduit LouisGeorges ce n'est pas le fait que la lecture soit considérée comme étant rapide et ergative, mais l'aspect technique qui consiste à trouver des passerelles entre les différents formats et de pouvoir créer des outils qui permettent de passer facilement de l'un à l'autre.

\section{La numérisation chez Albin Michel}

Les éditions ont commencé à numériser quelques ouvrages à partir de 2010, en particulier les romans. Depuis, sa production numérique n'a cessé d'augmenter et représente aujourd'hui 5 à $6 \%$ du chiffre d'affaires. Maison mère, elle n'est pas éditrice scientifique comme sa filiale Vuibert, et va s'orienter vers les secteurs porteurs que sont le roman contemporain, ou la fiction dont elle est l'un des leader. Depuis mai 2016, elle entre dans le dispositif PNB (prêt numérique entre bibliothèques) avec 4500 titres disponibles pour les bibliothèques numériques, élargissant ainsi son lectorat. C'est donc à un vaste projet auquel va participer Louis-Georges Marle en tant que responsable de la numérisation

\section{La technique}

Dès le début des années 2010, Louis-Georges Marle s'est orienté vers les langages de description de page plutôt que vers les logiciels de PAO (publication assistée par 
ordinateur), pour deux raisons: la première étant que les logiciels présents sur le marché ne permettaient pas de réaliser des mises en pages fluides pour les sciences humaines (il fallait créer systématiquement deux blocs de texte par page pour le texte et pour les notes de bas de pages, les notes étant déconnectées du texte), le système WYSYWYG - what you see is what you get avait des limites, en particulier pour la transcription des équations mathématiques. La seconde est qu'une fois le texte mis en page, on ne pouvait plus le transformer dans d'autres formats (formats de poche en l'occurrence à l'époque).

19 La découverte du langage LaTeX, langage de description de page (inventé en 1978 par Donald Knuth et amélioré par Leslie Lamport en 1992), toujours utilisé aujourd'hui, qui respecte les formules mathématiques, les règles typographiques et les polices modernes, permet, en dissociant le fond de la forme d'obtenir à peu près toutes les formes de mise en page propres aux sciences humaines, y compris plusieurs niveaux de notes de bas de page et plusieurs façons de les présenter - à la ligne ou en paragraphe.

Exemple : composition du texte de Johannes Kepler, Contra Ursum (in La Guerre des astronomes, volume II : La querelle au sujet de l'origine du système géo-héliocentrique à la fin $d u$ XVI e siècle, Paris, Les Belles Lettres, 2008)

Fig. 5. - Le texte balisé en langage LaTeX.

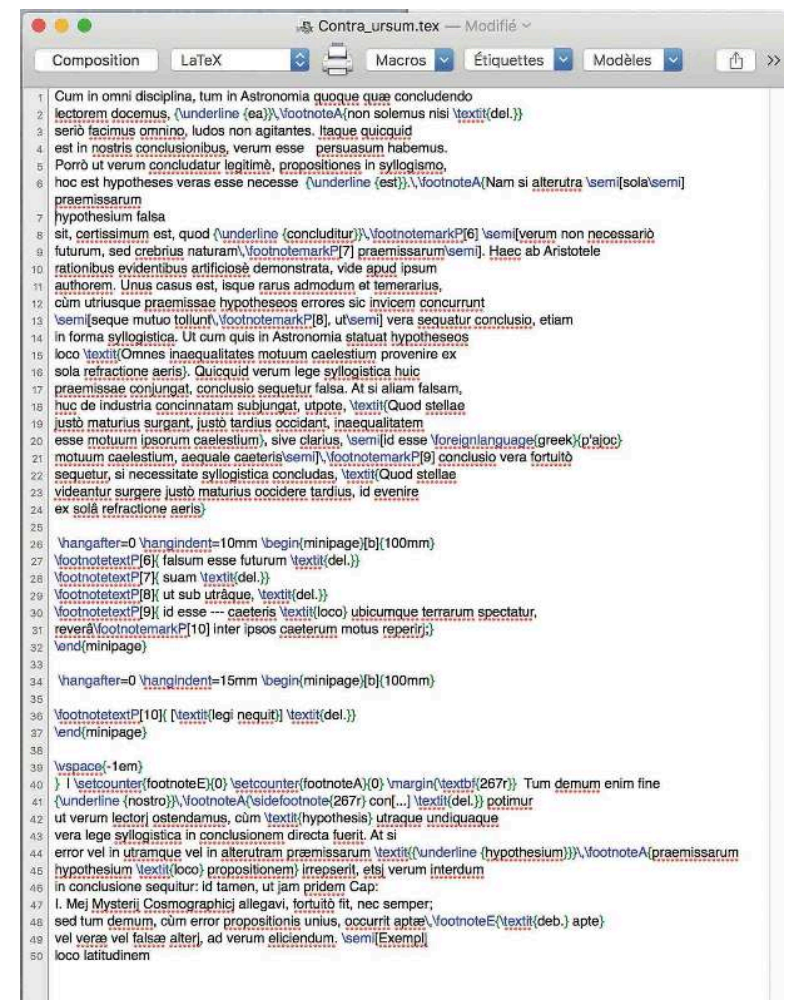

Louis-Georges Marle, Arch. priv. 
Fig. 6. - Le rendu de la page en PDF.

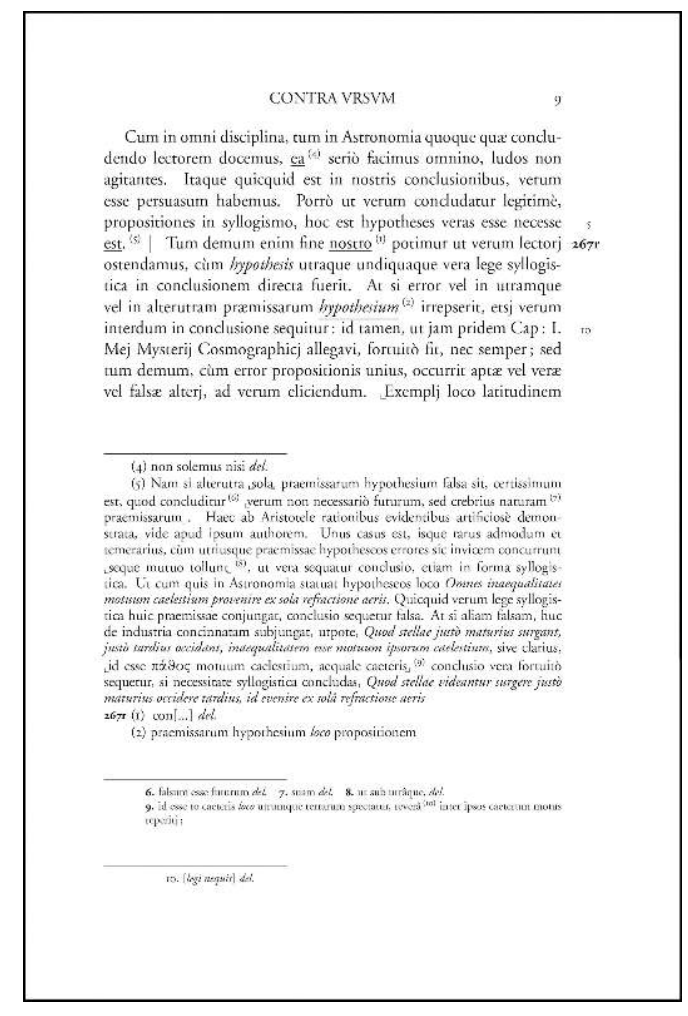

Louis-Georges Marle, Arch. priv.

21 Bien que ce langage soit semi-balisé puisqu'il colporte encore des indications de mise en pages, il permet de comprendre la distinction entre le fond d'un texte et la forme qu'on veut lui donner, si bien que passer aux langages de balisages devient un jeu d'enfant.

\section{Le langage de balisage}

Les langages de balisage sont relativement anciens : le premier GML Generalized Markup Langage est créé en 1969, puis SGML Standard Generalized Markup Language en 1984. Ces langages ont pour principe fondamental la séparation complète entre la structure logique d'un document (titres, chapitres, paragraphes, illustrations...) qui est identifié par des balises insérées dans le document lui-même, et sa mise en page qui dépend du support de présentation (livre, journal écran, graphique...) définie en dehors du document dans une ou plusieurs feuilles de style (police, style, taille et couleur des caractères, alignement et espacement des paragraphes...). La lourdeur et le prix très élevés des équipements informatiques pour faire tourner ces langages étaient tels qu'ils étaient réservés à l'industrie (Boeing, Airbus, Snecma, et l'armée).

C'est à partir du SGML Standard Generalized Markup Language que le Britannique Tim Berners-Lee inventa le WWW. Word Wild Web a développé le concept de HTML Hypper Text Markup Language et le langage internet. La baisse des coûts des systèmes informatiques, la démocratisation de la micro-informatique, l'apparition d'internet et la lourdeur du langage SGML ont poussé les informaticiens à trouver un langage plus souple permettant l'interopérabilité: le XML Extensible Markup Language (dont la première version date de 1995). 
24 Exemple : François Crouzet, Histoire de l'économie européenne, 1000-2000, Paris, Albin Michel, 2001.

Fig. 7. - Le texte balisé en XML.

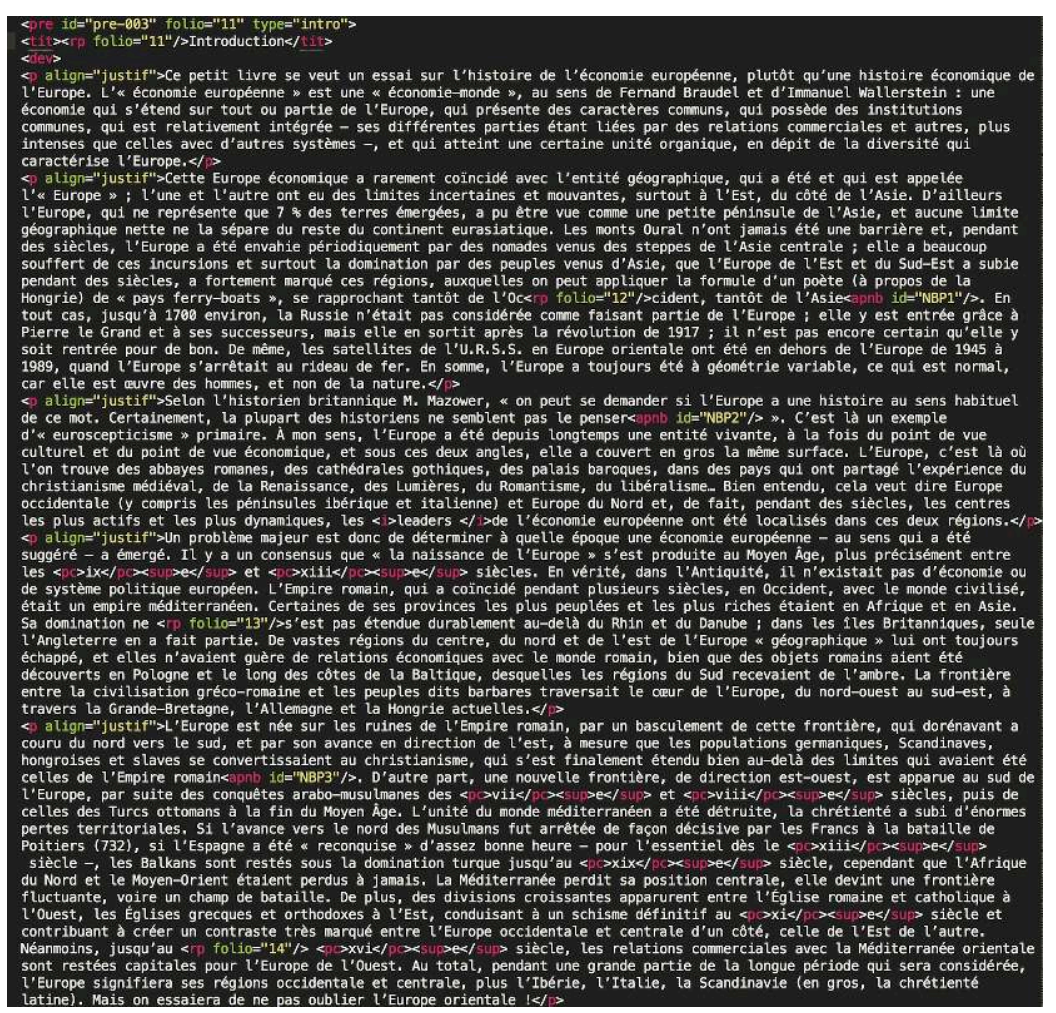

Louis-Georges Marle, Arch. priv.

25 Toute la structure du livre, que ce soit la hiérarchie logique des titres ou les enrichissements typographiques, est notée par des balises : <tit> pour un titre, $<$ p $>$ pour un paragraphe, $<\mathrm{i}>$ pour italique, $<\mathrm{pc}>$ pour les petites capitales... On obtient alors un fichier qui s'affranchit de la forme qu'on veut lui donner qui est pérenne et surtout interopérable. C'est-à-dire qu'on peut le transformer en d'autres types de fichiers en fonction des besoins. 
Fig. 8. - Le texte dans sa version XHTML.

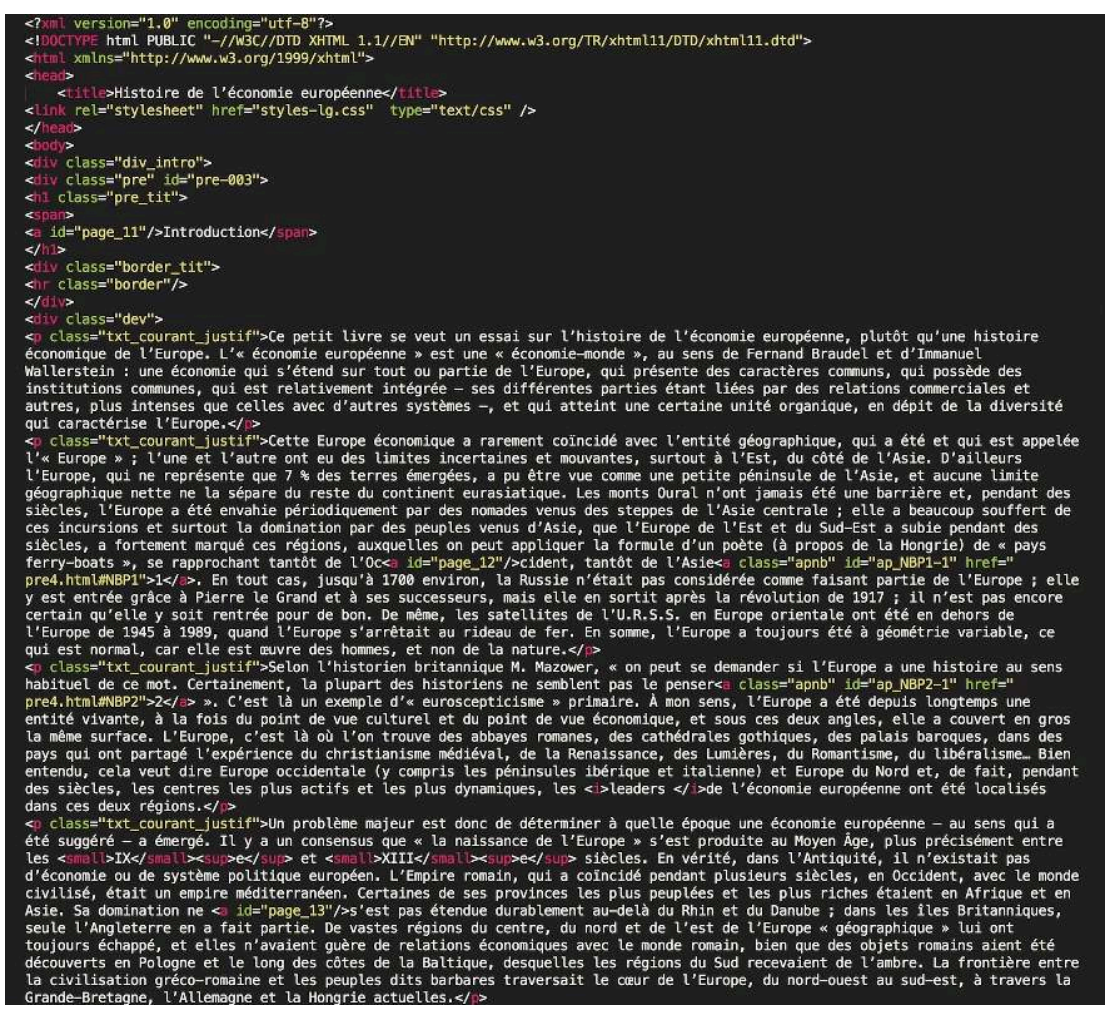

Louis-Georges Marle, Arch. priv.

Fig. 9. - Le rendu en epub.

\section{Introduction}

Ce petit livre se veut un essai sur l'histoire de l'économi européenne, plutôt qu'une histoire économique de l'Europe L'« économie européenne » est une " économie-monde », au sens de Fernand Braudel et d'Immanuel Wallerstein : une économie qui s'étend sur tout ou partie de l'Europe, qui présente des caractères communs, qui possède des institutions communes, qui est relativement intégrée - ses différentes parties étant liées par des relations commerciales et autres, plus intenses que celles avec d'autres systèmes -, et qui atteint une certaine unité organique, en dépit de la diversité qui caractérise l'Europe.

Cette Europe économique a rarement coïncidé avec l'en tité géographique, qui a été et qui est appelée l'« Europe » l'une et l'autre ont eu des limites incertaines et mouvantes, surtout à l'Est, du côté de l'Asie. D'ailleurs l'Europe, qui ne représente que $7 \%$ des terres émergées, a pu être vue comme une petite péninsule de l'Asie, et aucune limite géographique nette ne la sépare du reste du continent eurasiatique. Les monts Oural n'ont jamais été une barrière et, pendant des siècles, l'Europe a été envahie périodiquemen par des nomades venus des steppes de l'Asie centrale; elle a beaucoup souffert de ces incursions et surtout la domination par des peuples venus d'Asie, que l'Europe de l'Est et du Sud-Est a subie pendant des siècles, a fortement marqué ces régions, auxquelles on peut appliquer la formule d'un poète (à propos de la Hongrie) de "pays ferry-boats », se rapprochant tantôt de l'Occident, tantôt de l'Asie'. En tout cas, jusqu’à 1700 environ, la Russie n'était pas considérée comme faisant partie de l'Europe ; elle y est entrée grâce à Pierre le Grand et à ses successeurs, mais elle en sortit après la révolution de 1917 ; il n'est pas encore certain qu'elle y soit rentrée pour de bon. De même, les satellites de l'U.R.S.S. en Europe orientale ont été en dehors de l'Europe de 1945 à 1989, quand l'Europe s'arrêtait au rideau de fer. En somme, l'Europe a toujours été à géométrie variable, ce qui est normal, car elle est œuvre des hommes, et non de la nature.

Selon l'historien britannique M. Mazower, « on peut se demander si l'Europe a une histoire au sens habituel de ce mot. Certainement, la plupart des historiens ne semblent pas le penser ${ }^{2}$ ". C'est là un exemple d'« euroscepticisme " primaire. À mon sens, l'Europe a été depuis longtemps une entité vivante, à la fois du point de vue culturel et du point de vue économique, et sous ces deux angles, elle a couvert en gros la même surface. L'Europe, c'est là où l'on trouve des abbayes romanes, des cathédrales gothiques, des palais baroques, dans des pays qui ont partagé l'expérience du christianisme médiéval, de la Renaissance, des Lumières, du 


\section{L'implication sociale : le SNE (Syndicat National de l'Édition)}

Fidèle à sa vocation de transmettre à tous, Louis-Georges a rejoint le Syndicat National de l'Édition dans le Groupe normes et standards ${ }^{21}$ : production d'EPUB nativement accessible aux publics empêchés de lire, ciblant les $18 \%$ de personnes en situation de handicap en France (handicap visuel, auditif, Dys (langage), moteur, psychique, intellectuel et cognitif). Le but de l'atelier du SNE consiste à faire comprendre à l'éditeur qu'il existe des moyens pour fabriquer des livres numériques " accessibles » en se basant sur les langages informatiques existant sans que cette transformation n'engendre un coût rédhibitoire. L'information contenue dans les pages (X) html provenant de l'EPUB (Electronic PUBlication ou publication électronique), comportera de l'information sémantique lisible aussi bien par les liseuses classiques que par celles adaptées au public malvoyant.

Fig. 10. - Version XHTML avec balisage d'accessibilité conforme aux normes EPUB 3 et WCAG 2.0 (Web Content Accessibility Guidelines) du livre de Louise Erdrich, La Décapotable rouge.

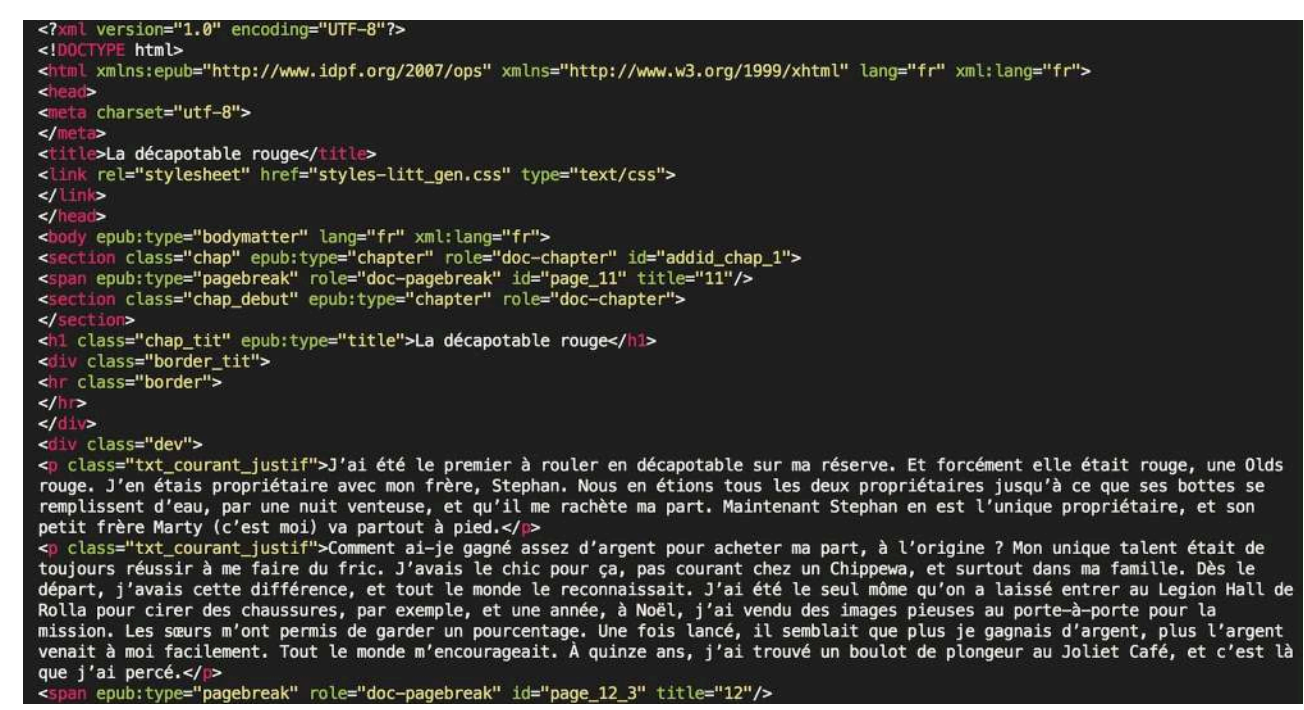

Louis-Georges Marle, Arch. priv.

Le fichier commence par une balise «body " indiquant où se situe le fichier en question: devant (frontmatter), page de titre, dédicace, exergue..., dans le corps du texte (bodymatter) ou à la fin de l'ouvrage (backmatter), dans la même collection, du même auteur <. Le fichier est découpé en sections ayant un rôle précis : EPUB : type = « chapter $»$ role = « doc-chapter » indiquant que l'on se trouve dans un chapitre et que cette information est lisible aussi bien par une liseuse EPUB type que par un dispositif pour malvoyants (role $=$ " doc-chapter »). Bien qu'il existe déjà des éditions adaptées par le biais des associations nationales, cela ne suffit pas à la demande. Le groupe propose de passer de l'édition adaptée répondant à des besoins spécifiques (lecture en braille, bibliothèque sonore) à l'édition inclusive répondant aux besoins les plus simples (romans, essais) : par le biais d'Epub destinés aux handicaps auditifs, visuels, moteurs, psychiques... Le but de l'atelier de recherche dont les assises se tiennent régulièrement sous forme de tables rondes est d'améliorer ce système permettant une numérisation plus large. 
Les objectifs sont semblables pour les deux idéalistes, mais, si le premier, agissant en cavalier seul à une époque où l'enseignement de la lecture, la refonte de la grammaire font l'objet de nombreuses expériences et innovations plus ou moins évidentes, est conduit à l'échec à cause de ses options politiques, son descendant, travaille en équipe dans l'Atelier groupe de recherche du SNE, en dehors de toute implication politique. Pour lui, seule compte l'amélioration d'un système destiné à transmettre et procurer aux plus démunis le plaisir du savoir et de la distraction.

\section{BIBLIOGRAPHIE}

Assises professionnelles du livre à l'heure du numérique, groupe normes et standards du numérique,

l'adéquation du langage ONIX au métier d'éditeur, groupe normes et standards du numérique, Paris, 2015. CHADOURNE Jacques, « Aujourd'hui comme hier, Charles-Louis Marle, premier directeur de l'école normale de Mâcon », dans Bulletin d'information pédagogique, nº 4, CDDP Mâcon, 1981.

CHERVEL André, Les grammaires françaises 1800-1914, Répertoire chronologique, Paris, Institut national de Recherches pédagogiques, Service, Histoire de l'Éducation, 1982.

BOUSTANY Joumana, BOUDROUX Evelyne, CHARTRON Ghislaine, La médiation numérique : renouvellement et diversification des pratiques, actes du colloque Documents numériques et société, Zagreb 2013, Louvain-la-Neuve, De Boeck supérieur, 2014.

M. MARLE aîné, Manuel de la diagraphie. Découverte qui simplifie l'étude de la langue, Paris, imprimerie administrative Paul Dupont et Cie, 1839.

MARLE Louis, De la Bourgogne à la bataille d'Alger, Paris, La Pensée universelle, 1973.

SANOSWSKI Jean-Claude, Marle, Charles Louis dit Marle aîné, Association d'Études et Cahiers Charles Fourier, Cléron, mai 2017.

VON HOOLAND Seth, GILLET Florence, HENCHEN Simon, Introduction aux activités numériques, Méthodes et pratiques de Sciences humaines et sociales, Louvain-la-Neuve, De Boeck supérieur, 2016.

\section{NOTES}

1. Olivier Marle, instituteur, fonda une école d'enseignement mutuel à Mâcon en 1818.

2. Ils ont 18 et 20 ans lors de leur arrestation.

3. Archives Privées Marle, (APM), dossier Louis et Jérôme (condamnations, déportation, transportation en Algérie. Création d'une imprimerie et de journaux politiques, liens avec Proudhon).

4. L'Émancipation de l'enseignement, publié de mai 1848 à novembre 1849. 
5. Charles et Guillaume, où les parfaits élèves d'une école d'enseignement mutuel, par C. Marle, directeur de l'école d'enseignement mutuel de Mâcon, à Mâcon, chez l'auteur, 1822.

6. Parue en 1784.

7. APM, dossier Charles-Louis, J. Chadourne, «Aujourd'hui comme hier, Charles-Louis Marle, premier directeur de l'école normale de Mâcon ».

8. Bourrousse de Lafforre, Brevet d'invention pour un procédé de lecture, Statilégie lecture immédiate. Art de lire au plus haut degré de simplicité, prospectus, 1827.

9. Appel aux Français, « Combat », Paris, Corréard jeune, éditeur, 21 rue Richelieu, $\mathrm{n}^{\circ} 21$, 1829, chap. III, p. 49.

10. J. Chadourne, « Aujourd'hui comme hier, Charles-Louis Marle, premier directeur de l'école normale de Mâcon ", p. 80.

11. L. Marle, De la Bourgogne à la bataille d'Alger, p. 65.

12. Ibid., Le 27 août 1838 , à l'école primaire du $10^{e}$ arrondissement de Paris, 4 septembre 1838 à l'école communale du 6e arrondissement, p. 65-66.

13. Ordre de saint Dominique ordre prêcheur.

14. La loi Guizot de 1833 prévoyait trois types d'écoles: l'école publique avec enseignement public, l'école publique avec enseignement congrégationiste et l'école privée avec enseignement congrégationiste.

15. J.-Cl. Sonowski, « Marle, Charles louis dir Marle aîné », Propriété littéraire, discours religieux, contrefaçon, Cour royale de Lyon, Le journal du palais, Jurisprudence française, vol. 45, p. 433-436. avocat, Pezzani, (fourieriste) Jean-Claude Sosnowski, in Association d'études et Cahiers Charles Fourier, 2017.

16. APM, Dossier Charles-Louis, L'Émancipation de l'enseignement, samedi 4 novembre $1848, \mathrm{n}^{\circ} 27$.

17. APM, Dossier Charles-Louis, La Constitution. République du Présent et de l'Avenir, rédacteur gérant Ch. Martin, rédacteur en chef, Marle Aîné, numéro spécimen, 28 février 1849, nº 17, 11 août 1849, 37, rue des Vieux-Augustin, Bureau, rue Saint Marc, $\mathrm{n}^{\circ} 39$, Paris.

18. L. Marle, De la Bourgogne à la bataille d'Alger, « Louis (il n'écrit pas Charles-Louis), est allé mener une vie assez misérable à Lyon où il a trouvé un emploi de maître de pension, et où il va mourir en 1860 », p. 245. Il semble qu'il y ait confusion avec un autre Louis Marle frère puîné de Charles. Une autre version est donnée dans les dossiers fouriéristes : il aurait quitté la France pour les États-Unis puis le Venezuela; version plausible. Les bâtiments de l'École normale de Mâcon furent légués à une congrégation de religieuses du Venezuela, qui l'occupait toujours en 1980. Une autre version le fait mourir à Saint Domingue en 1884. Généalogie Joël Marle par Geneanet.

19. Texte de la page de couverture accompagnant le titre.

20. M. Marle â̂né, Manuel de la diagraphie. Découverte qui simplifie l'étude de la langue.

21. Arts de la visibilité, et epub 3, pour une excellence de lecture numérique, Les assises du livre numérique, Paris, 23 novembre 2017. 


\section{RÉSUMÉS}

La vocation de Charles-Louis Marle tout comme celle de Louis-Georges est de transmettre le savoir au plus grand nombre. Si Charles-Louis né en 1799, fils d'un instituteur de Saône-et-Loire, s'engage dans un processus politique qu'il transmettra à ses deux fils Louis-Georges se consacre uniquement aux moyens de diffuser par le biais de la numérisation le savoir et la culture à une population privilégiant le côté pratique de la lecture sur l'outil informatique liseuse, tablette ou smartphone... ou bien à une population handicapée, les textes numérisés étant parfois restitués oralement.

\section{AUTEUR}

\section{ANNE MARLE-KOLLE}

Docteur en Histoire moderne et contemporaine, doctorante en littérature comparée, Université Paris IV - Paris-Sorbonne, chercheur hébergé Centre Roland Mousnier UMR 8596 Paris Sorbonne, CNRS 


\title{
Les laboratoires de recherche européens Kodak et la circulation interne du savoir scientifique durant l'entre-deux-guerres
}

\author{
Nicolas Le Guern
}

1 Les produits fabriqués par l'industriel américain Eastman Kodak ont été les plus utilisés dans le cadre des pratiques de photographie et de cinématographie dite amateur au vingtième siècle, et nous sommes nombreux à conserver au sein de nos archives familiales des supports négatifs, positifs ainsi que des tirages de la marque Kodak. Jusqu'à présent, nous n'avions pas accès à la compréhension de l'organisation interne de la production et de la recherche par les managers de Kodak. L'accès récent aux archives industrielles des filiales anglaises et françaises de la firme américaine permet désormais d'étudier non seulement le développement et la structure des différents sites de production, mais aussi la nature du savoir scientifique et technique produit par les chercheurs, ainsi que sa circulation interne. Dans une première partie, nous clarifierons le contexte de création des trois laboratoires de recherche Kodak aux ÉtatsUnis, en France et en Angleterre. Après avoir détaillé la structure des rapports de recherche anglais et français contenant le savoir des chercheurs, nous analyserons alors l'échange et la circulation interne de ce savoir dans le cadre de pratiques bureaucratiques progressivement instituées.

\section{La création des trois principaux laboratoires de recherche d'Eastman Kodak}

En 1912, George Eastman, le fondateur de Kodak Eastman, décida de créer un laboratoire de recherche au sein de Kodak Park, son important site de production au cœur de Rochester, près de New York. Les raisons en étaient multiples. Cette méthode de recherche industrielle par le biais du laboratoire de recherche privé était quasi 
inexistante au début du $\mathrm{xx}^{\mathrm{e}}$ siècle aux États-Unis. Or Eastman fut informé par ses agents européens des bénéfices de cette organisation au sein de l'industrie chimique des colorants en Allemagne, novatrice dans le domaine avant même la fin du xix $x^{e}$ siècle $^{1}$. Pour Eastman, il fallait d'une part renforcer la recherche dans la photographie couleur : malgré des collaborations avec quelques photochimistes indépendants, Kodak n'avait pas réussi à développer de procédés en couleurs naturelles et dans les années 1910, seul l'autochrome des frères Lumière était disponible sur le marché. Eastman souhaitait aussi protéger la production du savoir par le biais d'un laboratoire où seraient employés des chercheurs, dont la fonction serait justement de produire des connaissances scientifiques et techniques, propriété intellectuelle de Eastman Kodak ${ }^{2}$. Un laboratoire de recherche représentait également un outil de communication pour affirmer le prestige de la marque, par le biais de chercheurs travaillant à décrypter la nature physicochimique du procédé photographique, et à publier leurs résultats dans des revues scientifiques. Enfin, la nouvelle organisation de la recherche permettait à Eastman le moindre recours à une stratégie d'intégration horizontale, à savoir le rachat de concurrents directs, dans le contexte d'une politique américaine de plus en plus hostile aux situations de monopole industriel ${ }^{3}$. Eastman recruta un photochimiste talentueux, l'anglais Kenneth Mees, pour créer et diriger le laboratoire de recherche à Rochester. Mees, bien qu'assez jeune, avait l'expérience de l'entreprise et de la recherche dans le secteur privé : son premier poste était celui de directeur commercial dans une petite entreprise fabriquant des plaques sèches et des filtres photographiques dans la banlieue de Londres. Eastman accorda à Mees que la première décennie du laboratoire soit non seulement dévolue à la résolution de problèmes techniques liés à la production du film, mais aussi à la compréhension des mécanismes du procédé photographique, en pratiquant une recherche dite fondamentale.

Justement, environ quinze ans plus tard, c'est toujours Kenneth Mees qui décida de créer un nouveau laboratoire de recherche en Angleterre sur le site industriel de Kodak Limited, à Harrow. L'idée initiale de Mees était de valoriser la production des rapports de recherche du laboratoire américain, dont une partie indéterminée était envoyée à la filiale anglaise de Kodak, mais insuffisamment exploitée. La nouvelle entité de recherche, constituée d'experts des différents domaines scientifiques étudiés, serait un moyen de valoriser et de faire fructifier en deux endroits la recherche industrielle de Kodak $^{4}$. Le laboratoire de recherche de Harrow commença ses activités en 1928, avec des moyens et des effectifs limités. En 1930, les chercheurs employés n'étaient que 7, et 31 en $1938^{5}$. Pour en trouver le directeur, Kenneth Mees utilisa la méthode de George Eastman, qui avait abouti à sa propre embauche en 1911: il approcha et réussit à recruter Walter Clark, un photochimiste expert qui avait débuté une carrière prometteuse au sein de la British Photographic Research Association, une institution anglaise de recherche publique, dont les actions étaient limitées par la pratique du secret industriel et la brevetabilité des inventions dans l'industrie photographique ${ }^{6}$. Dès 1929, l'équipe de chercheurs anglais commença à consigner ses recherches techniques et scientifiques sous la forme de rapports de recherche à la rédaction codifiée, un corpus précieusement conservé et relié dans 99 gros volumes par un ou plusieurs archivistes de Kodak Limited. À Londres, l'archive Kodak de la British Library possède ces 2900 rapports. Couvrant la période 1929-1964, ils constituent une source primaire fondamentale pour les chercheurs en histoire des techniques. Nous en avons réalisé l'étude pour la période 1929-1935, soit 350 rapports. 
Quant à la création du laboratoire de recherche de Kodak-Pathé à Vincennes, le contexte historique et économique était radicalement différent. L'industriel Charles Pathé, qui avait tout d'abord été l'un des clients les plus importants de Eastman pour l'achat de stocks de films cinématographiques, avait décidé dès 1906 d'entreprendre des études techniques pour pouvoir fabriquer lui-même, un jour, de la pellicule cinématographique. Les premières recherches menées par les managers et les photochimistes français l'avaient été en Angleterre, au nez et à la barbe de Eastman En 1909, la production de film était lancée à Vincennes, ce qui provoqua les foudres de l'industriel américain. Mais au final, quand il fut temps pour Charles Pathé de se retirer des affaires, il se rapprocha d'Eastman et finit par lui vendre une partie de la société Pathé Frères qui était en charge de la production du support et de l'émulsion à l'usine de Vincennes. Le rachat aboutit en 1927 à la nouvelle entité Kodak-Pathé9. Que faire avec les scientifiques expérimentés de l'époque Pathé, qui avaient été en grande partie repris par Kodak? George Eastman et surtout Kenneth Mees décidèrent finalement de créer une structure équivalente à un laboratoire de recherches, afin d'exercer une recherche appliquée et fondamentale, et de collaborer avec son homologue anglais dans un premier temps. Ainsi, le laboratoire français possédait à sa création une longue expérience de la recherche et des techniques de production de supports photosensibles, à l'inverse du laboratoire anglais, qui débuta pour ainsi dire de zéro. Afin de diriger la nouvelle entité française, Kenneth Mees eut la prudence de choisir Marcel Abribat, un chimiste qui avait été le directeur commercial de la filiale française de Kodak avant le rachat de Pathé Cinéma. Un français, certes, mais un ancien concurrent que les managers et photochimistes de Pathé connaissaient bien.

\section{Le savoir scientifique : les rapports de recherche et leur contenu}

5 Ayant contextualisé la création des deux laboratoires de recherche européens de Kodak, nous allons brièvement clarifier la nature des rapports de recherche produits par les chercheurs anglais et leurs homologues français, dont nous allons étudier la circulation interne. Une remarque préalable : les rapports de recherche ne constituent qu'une partie du savoir scientifique et technique produit par les chercheurs, et ces derniers possédaient également d'autres éléments de conservation des savoirs comme des cahiers de notes personnels ou des artefacts qui n'ont pas été conservés dans les archives, sans compter la connaissance intellectuelle de chacun sur sa spécialité de recherche. Cependant, les rapports de recherche constituent le produit tangible de l'activité quotidienne des chercheurs, et un matériau que l'on peut transmettre à d'autres acteurs et auquel il est possible de se référer afin que les connaissances produites circulent dans les différents laboratoires.

6 Chez Kodak Limited, le rapport de recherche est un document à la constitution et au contenu très codifiés. Après une brève introduction qui contextualise la thématique de recherche, on trouve l'étude principale, suivie d'un résumé ou d'une conclusion. Chaque rapport possède une couverture standardisée comportant un emplacement pour le titre de la recherche, l'identité du ou des auteurs, la date de création, la classification et la numérotation du sujet traité ainsi que la mention Research Laboratory KODAK LTD. Wealdstone, Middx. Au dos de la couverture se trouvent les noms des personnes à qui a été adressée une copie du rapport. Cependant, cette information 
n'était en général pas renseignée, et les quelques indications présentes de temps à autre ne peuvent être jugées comme pertinentes. Les thèmes techniques et scientifiques sont variés, incluant par exemple l'étude du comportement de composés physicochimiques, le test de nouveaux colorants, l'étude de problèmes liés à la production du film, l'étude de produits concurrents par rétro-ingénierie, mais aussi l'analyse de nouvelles technologies développées par des individuels ou d'autres entreprises, ainsi que des visites en Europe afin de développer des partenariats technologiques ${ }^{10}$.

7 En 2013, la récente découverte de l'archive industrielle de Kodak-Pathé à Chalon-surSaône nous a permis l'analyse d'une partie des rapports de recherche français, et un regard complémentaire sur les activités de la recherche Kodak au sein des trois laboratoires de recherche ${ }^{11}$. Chez Kodak-Pathé, la méthode de classification des rapports de recherche est ainsi différente. Ces derniers sont classés par auteur dans un cahier, selon un processus d'archivage vraisemblablement à long terme. Si le chercheur était un scientifique prolifique, l'archiviste non identifié créait plusieurs cahiers à son nom. On remarque également qu'il n'y a pas de partition entre la période de Pathé jusqu'en 1927 et la période de Kodak-Pathé à partir de 1927. Pour les physicochimistes travaillant déjà chez Pathé, leurs rapports ont continué à être classés sans distinction et selon la même méthode, principalement par ordre chronologique. D'autres rapports, moins nombreux, ne sont pas liés à un chercheur spécifique, mais concernent une activité précise, comme les cahiers sur les activités du laboratoire de recherches. Quant au contenu des rapports, il est assez variable. Moins structurés que les rapports anglais, les rapports de Vincennes sont en général tapuscrits avec quelquefois des tracés de courbes sensitométriques, des tableaux ou des microphotographies (fig. 1). On trouve aussi de la correspondance originale ou copiée à la machine. 
Fig. 1. - Première page du rapport de recherche du chimiste Boussu sur la synthèse d'un produit chimique nécessaire à la fabrication du support photographique.

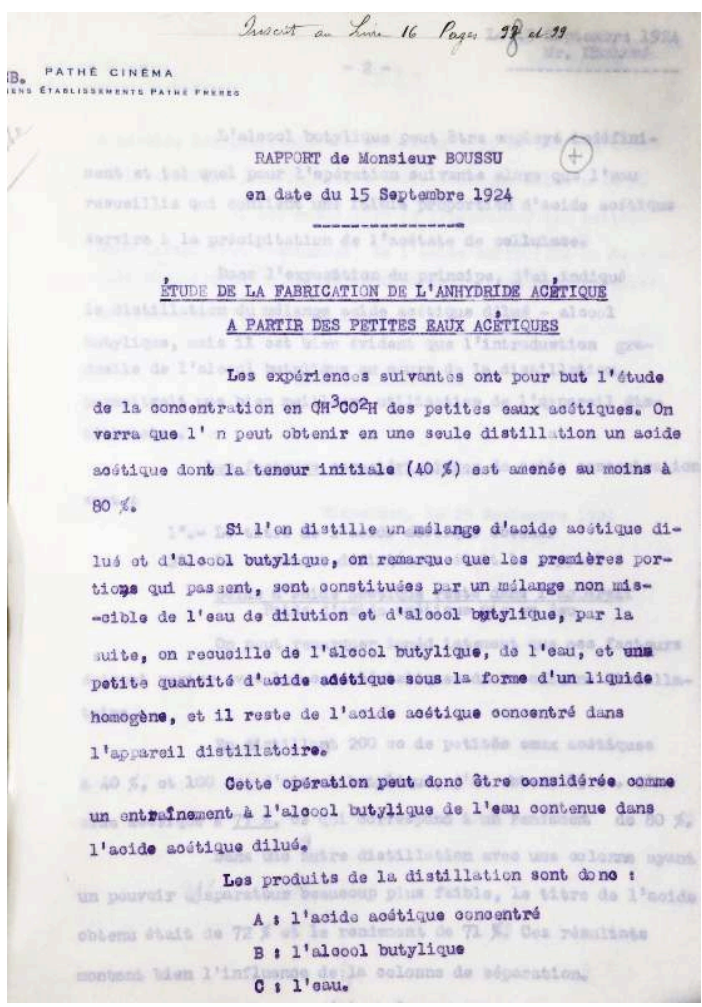

Association CECIL, archive Kodak-Pathé, 33154, dossier nº 1, «Étude de la fabrication de l'anhydride acétique à partir des petites eaux acétiques », 15 septembre 1924, fol. 1 .

8 Ce contenu hétérogène permet d'ailleurs le constat suivant: les chercheurs de Pathé travaillaient de manière plus indépendante que leurs futurs homologues de Kodak Limited, et avaient plus de latitude dans les axes de recherches. À l'origine, dans les années 1910, ils entamaient leurs études techniques directement à la demande de Charles Pathé. Au final, on note avec intérêt que les rapports jugés les plus pertinents étaient insérés au « livre de fabrication » pendant la période de Pathé, et cette tradition a perduré sous Kodak-Pathé. Outre les rapports de recherche, le livre de fabrication comportait des rapports d'activités industrielles et des comptes-rendus d'assemblées générales. Cette sélection des rapports fournit des indices importants sur la transmission interne du savoir chez Pathé et Kodak-Pathé.

\section{Circulation interne du savoir scientifique}

Dans notre thèse, une analyse préalable des rapports de recherche de Kodak en Angleterre clarifie le rôle du laboratoire de recherche anglais, sa mission et ses échanges avec le laboratoire principal de Rochester près de New York ${ }^{12}$. Avec la nouvelle archive française, il est possible de répondre aux questions suivantes: comment a été restructurée l'activité de recherche de Kodak-Pathé à partir du laboratoire de recherche initial de Pathé-Cinéma? Comment la nouvelle entité de recherche a-t-elle été incorporée à la stratégie d'innovation chez Eastman Kodak ? Au cours de nos recherches doctorales, plusieurs collectes de données dans l'archive industrielle de Kodak-Pathé, et plus particulièrement dans les cahiers de laboratoire 
des chercheurs français, nous permirent de mieux appréhender la méthodologie de la recherche employée par la filiale française de Kodak. Au cours de ces recherches, la découverte fortuite de plusieurs archives liées à la transmission du savoir entre les laboratoires de recherche Kodak a créé de nouvelles stratégies d'analyse des données. Suite à cette découverte, nous avons décidé de ne pas réaliser de comparaison entre la production des rapports de recherche anglais et français, comme nous l'avions envisagé initialement, mais de privilégier l'étude à partir des données françaises de la méthode utilisée par le laboratoire de Kodak-Pathé pour collaborer avec ses homologues anglais et américains, ainsi que la circulation des savoirs scientifiques entre les trois laboratoires.

Le premier document que nous allons utiliser est un inventaire sommaire des rapports de recherche réalisés par le directeur de la recherche Kodak-Pathé, Marcel Abribat, pour la période 1928-1935 (fig. 2).

Fig. 2. - Extrait de l'inventaire des rapports de recherche produits par le directeur Marcel Abribat pour la période 1928-1935.

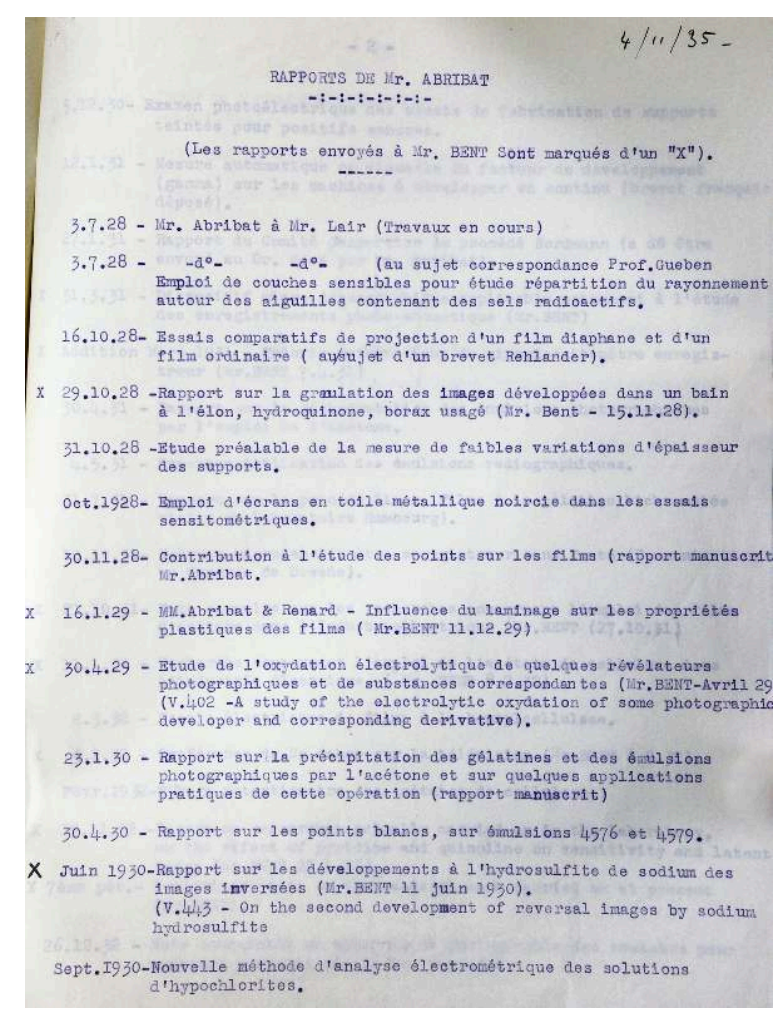

Association CECIL, archive Kodak-Pathé, 33498, «Liste des rapports de Monsieur Abribat de 1928 à $1935 », 4$ novembre 1935.

11 Ce document manuscrit et tapuscrit a été découvert dans le cahier de recherche d'un autre scientifique sans lien direct avec Abribat ${ }^{13}$. L'inventaire est constitué de plusieurs feuilles agrafées, ce qui tranche avec les rapports de recherche types, qui sont reliés dans des cahiers de recherche. Ainsi ce document n'a très certainement jamais été classifié et sa découverte tient surtout à la chance. La caractéristique majeure du document est que chaque rapport de recherche transmis à Kodak Limited est identifié par une marque " $\mathrm{X}$ » spécifique, rendant ainsi possible l'identification du savoir scientifique qui était partagé avec les chercheurs anglais, et dans quelle proportion. L'analyse de cet inventaire sommaire permet d'obtenir des données statistiques sur la 
fréquence du partage des savoirs créés en France avec la filiale anglaise de Kodak (tabl. 1).

Tabl. 1. - Liste des rapports de recherche produits par Marcel Abribat et transmis à Walter Bent ${ }^{14}$ pour la période 1928-1935. Le texte en italique représente la citation du document original.

\begin{tabular}{|c|c|c|}
\hline Année & Date & Rapports de recherche envoyés à Walter Bent \\
\hline \multirow{3}{*}{1928} & $29 / 10 / 1928$ & $\begin{array}{l}\text { Rapport sur la granulation des images développées dans un bain à l'élon, } \\
\text { hydroquinone, borax usage (Mr. Bent-15.11.28) }\end{array}$ \\
\hline & \multicolumn{2}{|c|}{ Autres rapports de recherche qui n'ont pas été transmis à Kodak $\mathrm{L}^{\mathrm{td}}: 6$} \\
\hline & \multicolumn{2}{|c|}{ Pourcentage de rapports de recherche transmis à Bent pour l'année : $14 \%$} \\
\hline \multirow{4}{*}{1929} & $16 / 01 / 1929$ & $\begin{array}{l}\text { MM. Abribat \& Renard - Influence du laminage sur les propriétés plastiques des } \\
\text { films (Mr. Bent 11.12.29) }\end{array}$ \\
\hline & $30 / 04 / 1929$ & $\begin{array}{l}\text { Étude de l'oxydation électrolytique de quelques révélateurs photographiques et } \\
\text { de substances correspondantes (Mr. Bent - Avril 29) (V.402 - A study of the } \\
\text { electrolytic oxydation of some photographic developer and corresponding } \\
\text { derivative). }\end{array}$ \\
\hline & \multicolumn{2}{|c|}{ Autres rapports de recherche qui n'ont pas été transmis à Kodak $\mathrm{L}^{\text {td }}: 0$} \\
\hline & \multicolumn{2}{|c|}{ Pourcentage de rapports de recherche transmis à Bent pour l'année : $100 \%$} \\
\hline \multirow{3}{*}{1930} & $06 / 1930$ & $\begin{array}{l}\text { Rapport sur les développements à l'hydrosulfite de sodium des images inversées } \\
\text { (Mr. Bent } 11 \text { juin 1930). (V.443 - On the second development of reversal images } \\
\text { by sodium hydrosulfite). }\end{array}$ \\
\hline & \multicolumn{2}{|c|}{ Autres rapports de recherche qui n'ont pas été transmis à Kodak $\mathrm{L}^{\text {td }}: 4$} \\
\hline & \multicolumn{2}{|c|}{ Pourcentage de rapports de recherche transmis à W. Bent pour l'année : $20 \%$} \\
\hline \multirow{5}{*}{1931} & $31 / 03 / 1931$ & $\begin{array}{l}\text { Dispositif micro densographique applicable notamment à l'étude des } \\
\text { enregistrements photo-acoustique (Mr. Bent) }\end{array}$ \\
\hline & $\begin{array}{l}\text { Addition } \\
\text { Mars } 1931\end{array}$ & Théorie mathématique du microdensitomètre enregistreur (Mr. Bent 7.4.31) \\
\hline & $21 / 10 / 1931$ & $\begin{array}{l}\text { Note sommaire sur les recherches concernant l'emploi des films d'acétate dans } \\
\text { l'Industrie électrique (Mr. Bent 27.10.31) }\end{array}$ \\
\hline & \multicolumn{2}{|c|}{ Autres rapports de recherche qui n'ont pas été transmis à Kodak Ltd : 6} \\
\hline & \multicolumn{2}{|c|}{ Pourcentage de rapports de recherche transmis à W. Bent pour l'année : 33 \% } \\
\hline \multirow{6}{*}{1932} & $11 / 01 / 1932$ & $\begin{array}{l}\text { Recherche concernant l'emploi de l'acétate de cellulose dans l'industrie } \\
\text { électrique (Mr. Bent 8.2.32) }\end{array}$ \\
\hline & $5 / 02 / 1932$ & Conférence de Mr. Cahen sur la télévision (Mr. Bent 8.2.32) \\
\hline & $29 / 11 / 1932$ & $\begin{array}{l}\text { Report on researches actually carried on in the laboratory, on the effect of } \\
\text { pyridine and quinoline on sensitivity and latent image (Mr. Bent 27.1.33). }\end{array}$ \\
\hline & $7^{\text {ème }}$ pér. & Preliminary note on the research work carried on at present (Mr. Bent). \\
\hline & \multicolumn{2}{|c|}{ Autres rapports de recherche qui n'ont pas été transmis à Kodak $\mathrm{L}^{\text {td }}: 3$} \\
\hline & \multicolumn{2}{|c|}{ Pourcentage de rapports de recherche transmis à W. Bent pour l'année : 57 \% } \\
\hline 1933 & - & - \\
\hline
\end{tabular}




\begin{tabular}{|c|c|c|}
\hline & \multicolumn{2}{|c|}{ Autres rapports de recherche qui n'ont pas été transmis à Kodak $\mathrm{L}^{\mathrm{td}}: 2$} \\
\hline & \multicolumn{2}{|c|}{ Pourcentage de rapports de recherche transmis à W. Bent pour l'année : $0 \%$} \\
\hline \multirow{3}{*}{1934} & - & - \\
\hline & \multicolumn{2}{|c|}{ Autres rapports de recherche qui n'ont pas été transmis à Kodak $\mathrm{L}^{\mathrm{td}}: 2$} \\
\hline & \multicolumn{2}{|c|}{ Pourcentage de rapports de recherche transmis à W. Bent pour l'année : $0 \%$} \\
\hline \multirow{3}{*}{1935} & $30 / 01 / 1935$ & $\begin{array}{l}\text { (Abribat-Pinoir) - Appareil pour la mesure de la conductivité électrique des } \\
\text { films émulsionnés (Mr. Bent) }\end{array}$ \\
\hline & \multicolumn{2}{|c|}{ Autres rapports de recherche qui n'ont pas été transmis à Kodak $\mathrm{L}^{\mathrm{td}}: 1$} \\
\hline & \multicolumn{2}{|c|}{ Pourcentage de rapports de recherche transmis à W. Bent pour l'année : $50 \%$} \\
\hline \multirow{2}{*}{$1928-1935$} & \multicolumn{2}{|c|}{ Nombre total de rapports de recherche produits sur la période : 36} \\
\hline & \multicolumn{2}{|c|}{ Nombre total de rapports de recherche envoyés à W. Bent sur la période : 12 (33 \%) } \\
\hline
\end{tabular}

Association CECIL, archive Kodak-Pathé, 33498, « Liste des rapports de Monsieur Abribat de 1928 à $1935 », 4$ novembre 1935.

12 On constate que sur la période une moyenne d'un rapport sur trois était transmis à Harrow, et que les thèmes de recherche étaient très variés : étude physique des films en fonction du révélateur, conférence sur la télévision, analyse théorique d'un instrument de laboratoire, etc. Abribat dirigeant le laboratoire, sa propre production en était réduite d'où le nombre assez faible de rapports. Chez Kodak Limited, le destinataire était Walter Bent, le directeur des usines. Cependant, on ne sait pas comment Bent redistribuait l'information au sein du laboratoire de recherche anglais. Du moins peuton supposer que tout ou partie des chercheurs de Harrow pouvait consulter les rapports qu'Abribat avait décidé de transmettre. On observe aussi qu'un seul rapport semble avoir été transmis à Kenneth Mees, donc au laboratoire de recherche américain. La raison de cette très faible communication avec Rochester pourrait provenir du fait que, dans ses premières années, le laboratoire de recherche de Vincennes devait uniquement rendre compte à son homologue anglais. Cette situation changea progressivement, notamment à partir de la fin de la Seconde Guerre mondiale, comme nous le verrons bientôt.

Un second inventaire, trouvé également dans l'archive française de Kodak au côté du premier inventaire, possède une portée plus importante afin de déterminer la circulation du savoir entre Vincennes et Harrow. Il ne concerne certes que l'année 1935, mais tous les chercheurs français sont cités (fig. 3) ${ }^{15}$. L'ensemble de leurs rapports est inventorié, et dans le cas où le rapport a été transmis à Kodak Limited, une lettre placée à côté du titre du rapport permet d'identifier le destinataire anglais. Les notes des chercheurs de Vincennes sont également mentionnées, il s'agit de documents techniques de moindre importance qui ne sont pas transmis à Kodak Limited. 
Fig. 3. - Extrait des rapports du chercheur H. Renard identifiant les éléments transmis à d'autres chercheurs et responsables de Kodak.

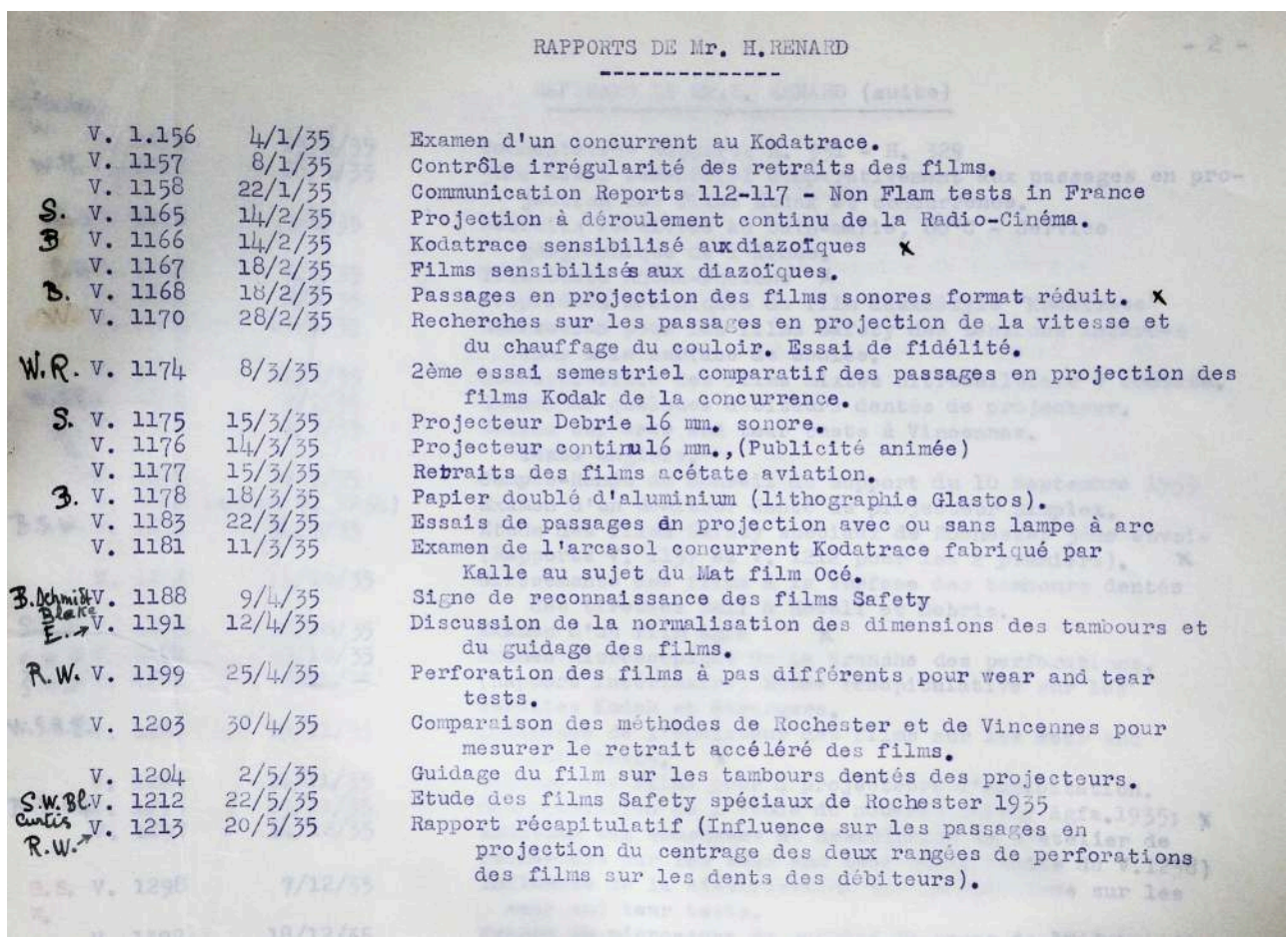

Association CECIL, archive Kodak-Pathé, 33498, « Liste des rapports (par noms d'auteurs) faits à Vincennes au cours de l'année 1935 », n.p.

Le tableau 2 suivant synthétise les informations fournies par cet inventaire en indiquant le résumé de la quantité de rapports produits par chercheur pour l'année 1935, ainsi que le nombre total de rapports transmis aux destinataires, en précisant leur identité et profession. Par exemple, si l'on considère Monsieur et Madame Cuissard, on constate que Monsieur a produit 7 rapports et qu'il n'en a transmis aucun à ses collègues étrangers. Par contre, Madame a produit 29 rapports et en a transmis 5 distincts à 4 interlocuteurs, soit $17 \%$ : le premier en ayant reçu 3 , le second 4 , le troisième un seul et le quatrième 2 pour l'année 1935. 
Tabl. 2. - Inventaire des rapports de recherche français produits et partagés avec les équipes de Kodak à Harrow ainsi que d'autres responsables de Kodak dans le monde pour l'année $1935^{16}$.

\begin{tabular}{|c|c|c|c|c|c|c|c|c|c|c|c|c|c|c|}
\hline 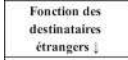 & Chercheurs franceais - & Abribat & Bousquet & Clément & \begin{tabular}{|l|} 
Mme. \\
cuissard \\
\end{tabular} & $\begin{array}{l}\text { Mr. } \\
\text { Cuissard } \\
\end{array}$ & Guillab & Landucei & Launte & Marrin & Pinoir & Renard & sykestre & Zelger \\
\hline+ & Rapports produits (total) & 1 & 5 & 10 & 29 & 7 & 4 & 4 & 10 & 1 & 8 & 52 & 10 & 17 \\
\hline $\begin{array}{c}\text { Directeur génicral } \\
\text { des usines Kodatk en } \\
\text { Eurrope }\end{array}$ & \begin{tabular}{|c|c|} 
Rapports transmis à \\
Beatt
\end{tabular} & $1\left(n^{0} 1\right)$ & $3(2,3,5)$ & $10(1 \mathrm{a}$ a 10$)$ & $3(3,1,1,167$ & & 1(4) & $3(1,3,4)$ & & & (3) & 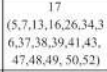 & $3(3,6,10)$ & 299,12 \\
\hline 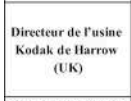 & $\begin{array}{c}\text { Rapports ransmis ì } \\
\text { Webb }\end{array}$ & & $20.5)$ & & $\mid 3.15,6,29)$ & & (2) & $2(2,3)$ & & & (3) & 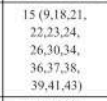 & & \\
\hline $\begin{array}{c}\text { Directerur general de } \\
\text { Kodak Prark ì } \\
\text { Rochester }\end{array}$ & $\begin{array}{c}\text { Rapporst rransmis à } \\
\text { Sulter }\end{array}$ & $1\left(n^{0} 1\right)$ & $2(3.5)$ & & (16) & & 1 (4) & $3(1,2,4)$ & & & (3) & 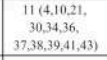 & $3(3,6,10)$ & 29,12 \\
\hline 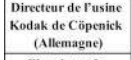 & \begin{tabular}{|c|c|} 
Rapports transmis à \\
Robinson
\end{tabular} & $1\left(n^{\circ} 1\right)$ & $2(1.5)$ & & $2(15,27)$ & & 1(3) & & 1(9) & & (3) & $\begin{array}{r}6(9,19,22,2, \\
24,39,41) \\
\end{array}$ & & 219,16 \\
\hline $\begin{array}{l}\text { Chercheur ru } \\
\text { labaratire de } \\
\text { recherche de Harrow }\end{array}$ & \begin{tabular}{|c|}
$\begin{array}{c}\text { Rapports transmis a } \\
\text { Eyles }\end{array}$ \\
\end{tabular} & & & & & & & & & & & $\mid 4(1730.313 .32)$ & & \\
\hline \begin{tabular}{|c}
$\begin{array}{c}\text { Directerar general de } \\
\text { Kodak Lud (IIK) }\end{array}$ \\
\end{tabular} & \begin{tabular}{|c|} 
Rapports transmis à \\
Blake
\end{tabular} & & & & & & & & & & & $2(16,21)$ & & \\
\hline \begin{tabular}{|l} 
Fanction inconnuc \\
\end{tabular} & \begin{tabular}{|c|c}
$\substack{\text { Rapports rtransmis a } \\
\text { schtmidt }}$ \\
\end{tabular} & & & & & & & & & & & $2(16.25)$ & & \\
\hline 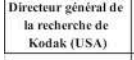 & $\begin{array}{c}\text { Rapports transmis à } \\
\text { Meses }\end{array}$ & & & $1(10)$ & & & & & & & & & & \\
\hline & $\begin{array}{c}\text { Nombre total de } \\
\text { rapports } \\
\text { rarasmis } \\
\text { Harrow par chercheur }\end{array}$ & $1 / 1$ & $4 / 5$ & 1010 & $5 / 29$ & a7 & 44 & 44 & 1/10 & $a / 1$ & 18 & 3052 & 310 & 3,17 \\
\hline & 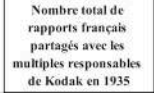 & \multicolumn{13}{|c|}{9 rapports (41\%) } \\
\hline
\end{tabular}

Doc. N. Le Guern

On voit donc que les Français communiquaient non seulement avec Kodak Limited, mais également avec le directeur général des usines de Kodak Park à Rochester, et le directeur de l'usine allemande de Kodak à Cöpenick. Cet exemple de production de savoirs technologiques pour l'année 1935 démontre l'importance de l'équipe de chercheurs de Kodak-Pathé, composée de 12 scientifiques experts de leur domaine et à qui il était demandé la production de rapports de recherche tout au long de l'année. Une information manque toutefois : l'inventaire ne précise pas la modalité de sélection des rapports à transmettre. Le directeur Abribat a pu être le seul décisionnaire à ce sujet. Ou bien chaque chercheur pouvait éventuellement sélectionner ses travaux qu'il jugeait important d'échanger. Ultime hypothèse, un comité de sélection se rassemblant régulièrement a pu s'acquitter de cette tâche.

Quant aux sujets de recherche, ils étaient très diversifiés et directement liés à la spécialité du chercheur. Si l'on considère à nouveau la chercheuse Madame A. Cuissard en guise d'exemple, on constate que ses états de services soulignent une carrière dans la recherche assez exceptionnelle. Chimiste analytique, elle réalisa en 1935 des études de nouvelles substances telles que le collodion fabriqué par Nobel, ou les résines de synthèse fabriquées par Albanol. Elle testa les propriétés mécaniques de supports expérimentaux, réalisa une veille technologique des films radiographiques de Lumière et Agfa, ainsi que des films positifs ciné. Elle étudia les nouveaux plastifiants, les adhésifs utiles pour réparer les films et la composition chimique des acétates de cellulose en fonction des fournisseurs ${ }^{17}$.

Mais qu'en est-il des rapports de recherche anglais ? Étaient-ils également transmis aux chercheurs de Vincennes ? Un troisième inventaire conservé dans le même cahier, à Chalon-sur-Saône, nous en fournit la réponse ${ }^{18}$. Ce document contient une liste de rapports anglais reçus entre 1929 et 1940 incluant la date de rédaction, le titre, les auteurs et le nombre de référence (fig. 4). Un comparatif de cet inventaire et de la liste des rapports de recherche dans l'archive anglaise à Londres confirme qu'il s'agit bien des mêmes rapports. La procédure d'inscription des rapports anglais reçus 
s'interrompit en 1940, très certainement en raison des conséquences de la Seconde Guerre mondiale sur le fonctionnement du laboratoire. L'inventaire nous permet de déterminer quel savoir scientifique était échangé entre Harrow et Vincennes. Mais à l'inverse du précédent, il ne nous fournit pas l'identité des expéditeurs et des destinataires. On peut supposer que le directeur Abribat collectait le premier ces rapports et qu'il les transmettait alors aux chercheurs (fig. 5) ${ }^{19}$.

Fig. 4. - Extrait de la liste des rapports de recherche anglais de l'année 1937 reçus par les chercheurs français de Kodak-Pathé.

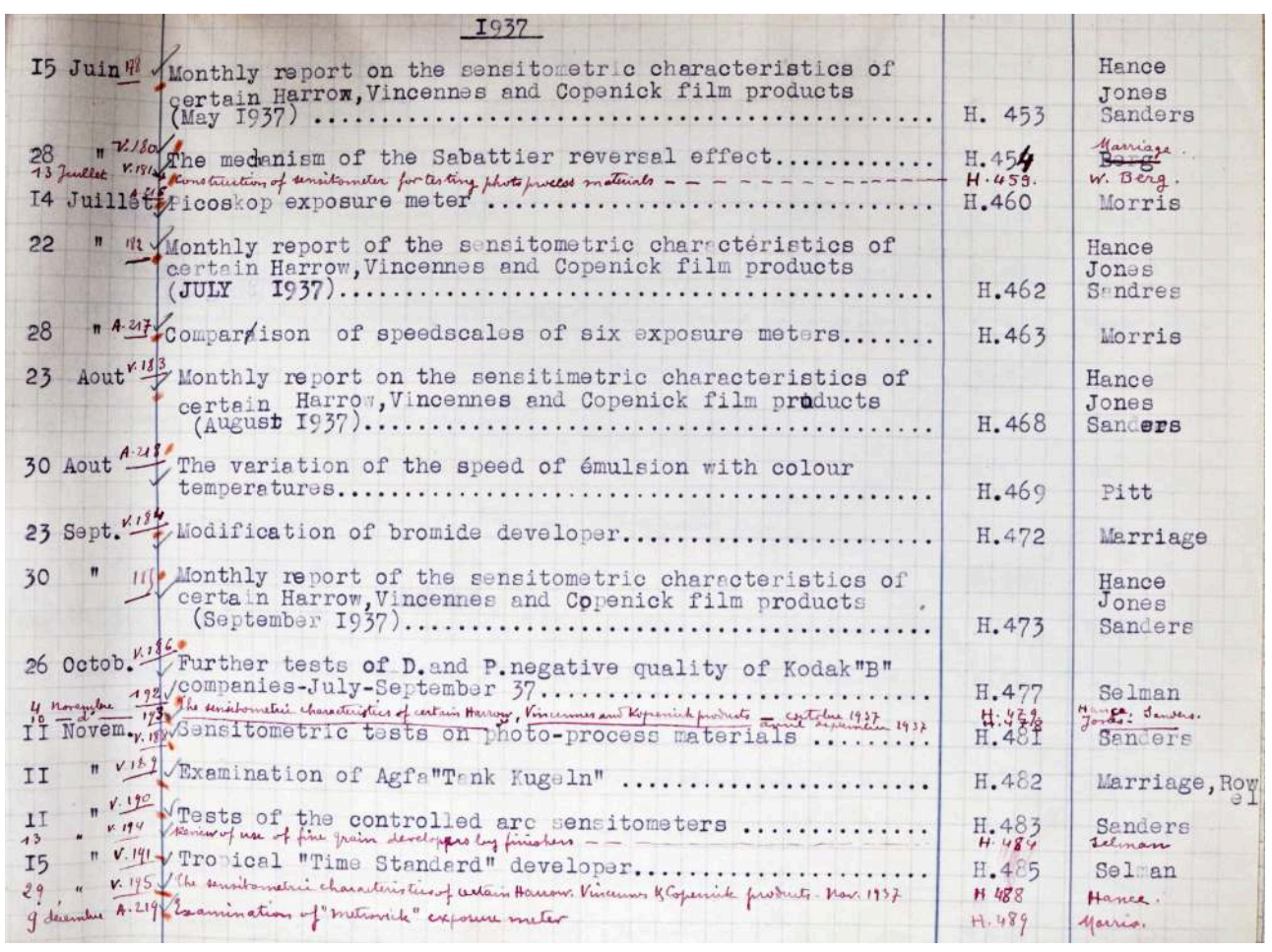

Association CECIL, archive Kodak-Pathé, 33498, inventaire, « Rapports Harrow reçus à Vincennes 1929 à $1940 »$ n.p. 
Fig. 5. - Proportion des rapports de recherche de Harrow transmis à Vincennes entre 1929 et 1936.

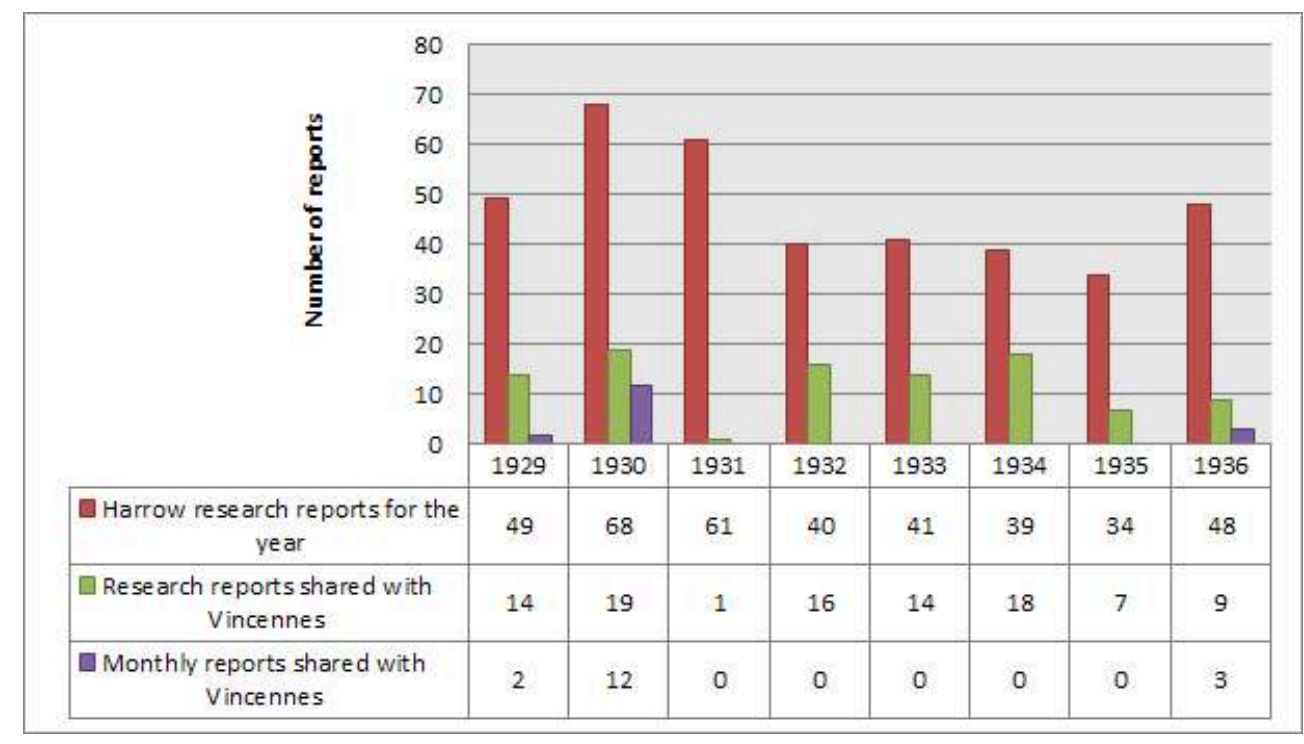

Sources : British Library, Kodak Historical archive, rapports de recherche, vol. 1 à 11, A2897.

Association CECIL, archive Kodak-Pathé, 33498, inventaire, « Rapports Harrow reçus à Vincennes 1929 à $1940 »$, n.p.

On apprend également que Vincennes recevait des rapports mensuels sur l'activité du laboratoire de recherche anglais. Cette information est importante, car ces rapports n'ont pas été trouvés, pour l'instant, dans l'archive de Kodak à Londres. On constate d'ailleurs que les modalités de leur production ont pu être modifiées après 1930, car leur réception est interrompue et ne reprend qu'en 1936. D'un point de vue statistique, c'est encore environ 1 rapport anglais sur 3 qui est transmis à Vincennes. Cette valeur constante fait écho à la nature incertaine de la recherche industrielle. Les résultats de la recherche fondamentale et appliquée n'étaient pas prévisibles, et certaines études et expérimentations ne valaient pas la peine d'être échangées avec les autres laboratoires de Kodak. De plus, cette incertitude créait le besoin de hiérarchiser l'importance de chaque rapport de recherche. Cette sélection était réalisée par un groupe indéterminé de scientifiques experts à Harrow, ainsi qu'à Vincennes. Au final, les sujets couverts par les Anglais et transmis aux Français étaient également très variés, comme dans le sens de transmission opposé. Cette variété souligne l'importance vitale du laboratoire de recherche au sein de l'industrie photographique. Il était utilisé pour développer la science photographique, améliorer les procédés de production et faire fructifier l'innovation. Et le fait d'échanger les savoirs créait une émulation et favorisait un esprit d'équipe entre les trois laboratoires de recherche de Kodak.

Nous allons citer un dernier rapport afin de conclure. Ce dernier prouve que les échanges de savoir technique et scientifique ont augmenté et se sont institutionnalisés après la Seconde Guerre mondiale entre les trois laboratoires. Ce rapport a été rédigé par un membre du laboratoire français en février 1951, et fournit le résumé des échanges de rapports entre les 3 laboratoires pour l'année $1950^{20}$. Le laboratoire anglais transmit $86 \%$ de ses rapports au laboratoire français (86 rapports sur 100). Les chercheurs français reçurent également $56 \%$ des rapports américains (106 sur 187). Et dans l'autre sens de circulation, les Français échangèrent $81 \%$ de leurs rapports avec leurs homologues anglais et américains. De plus, le rapport explicite les modalités de la circulation du savoir en amont, sur le plan décisionnel. Il confirme en effet l'existence 
d'un comité de sélection consultatif, ayant parcouru l'ensemble des rapports et décidant en fin de mois quels sont les rapports qu'il était opportun de partager (fig. 6).

Fig. 6. - Modalités de sélection des rapports de recherche jugés utiles pour être transmis à Rochester et Harrow, pour l'année 1950.

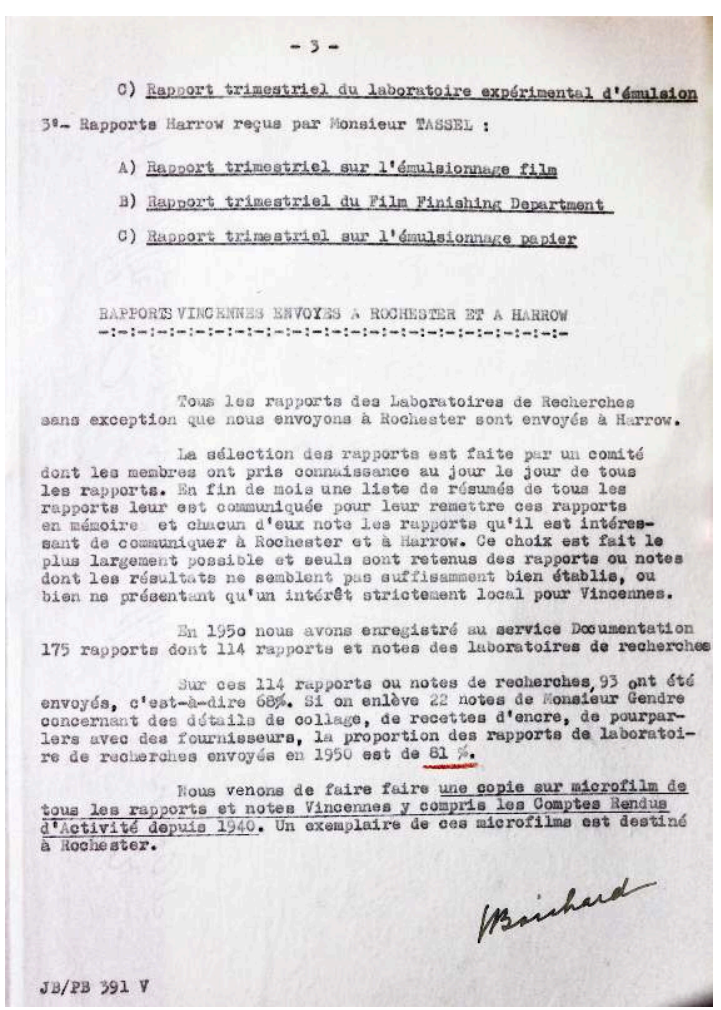

Association CECIL, archive Kodak-Pathé, 33023, «Échange de rapports entre Eastman Kodak \& Kodak-Pathé d'une part et entre Kodak-Limited \& Kodak-Pathé d'autre part », 13 février 1951, fol. 3.

Enfin, nous apprenons également que désormais, cette sélection de rapports les plus pertinents était transmise à l'identique au laboratoire de Rochester et au laboratoire de Harrow. Ainsi, non seulement le nombre de rapports de recherche augmentait et les sujets d'étude se diversifiaient au début des années 1950, mais la collaboration scientifique se renforçait aussi par l'intermédiaire des échanges multilatéraux du savoir scientifique et technique. Ces échanges ont progressivement créé de l'émulation au sein des trois laboratoires de recherche Kodak et, ce faisant, ont dynamisé les capacités de recherche et d'innovation de la firme dans le cadre d'une véritable recherche internationale.

Nous avons montré dans quel contexte Georges Eastman, le fondateur de Kodak, décida de créer un premier laboratoire de recherche à Rochester afin de prendre en charge l'organisation de l'innovation pour mettre au point de nouveaux produits photographiques et cinématographiques. Les activités de ce premier laboratoire ne suffirent pas et deux unités supplémentaires européennes furent créées en France et en Angleterre à la fin des années 1920. Ces nouveaux laboratoires utilisèrent les normes bureaucratiques de Kodak en conservant le savoir scientifique et technologique produit au sein de rapports de recherche. La disponibilité récente des archives industrielles de 
Kodak en Europe permet non seulement d'appréhender la nature de ce savoir, mais aussi de constater que les laboratoires instituèrent graduellement des échanges bilatéraux de rapports de recherche, après une sélection en fonction de leur pertinence scientifique. Ces échanges de savoirs s'accrurent après la Seconde Guerre mondiale en incluant également les comptes rendus d'activité liés plus directement à la production industrielle des différentes usines.

\section{BIBLIOGRAPHIE}

«Coal seen No. 1 Block for Britain », Kodakery, vol. V, n 12, 27 mars 1947, p. 1-5.

EDGERTON David E.H. et HORROCKS Sally M., « British Industrial Research and Development before 1945 », The Economic History Review, vol. XLVII, n² 2, 1994, p. 213-238.

FISK Catherine L., Working Knowledge Employee Innovation and the Rise of Corporate Intellectual Property, 1800-1930, Chapel Hill, University of North Carolina Press, 2009.

JENKINS Reese, Images and Enterprise: Technology and the American Photographic Industry, 1839 to 1925, Baltimore, John Hopkins University Press, 1975.

LE GUERN Nicolas, The Contribution of the European Kodak Research Laboratories to Innovation Strategy at Eastman Kodak, thèse en histoire de la photographie, études en culture visuelle, Leicester, Université de Montfort, 2017.

«Mr Sulzer honored on Thirty-Year Service Records », The Kodak Magazine, vol. XII, n 4-5, p. 16.

\section{NOTES}

1. R. Jenkins, Images and Enterprise: Technology and the American Photographic Industry, 1839 to 1925 , p. $305-306$.

2. En 1892, Eastman avait subi la concurrence déloyale de son photochimiste en chef Henri Reichenbach, responsable de la fabrication de l'émulsion, et avec qui il avait optimisé la composition du support photographique, désormais similaire à une base souple de type celluloïd. Eastman s'était rendu compte que Reichenbach avait créé une société parallèle avec deux autres de ses employés afin d'exploiter le nouveau support. Cette expérience formatrice le fit réfléchir sur le besoin impérieux de protéger le savoir technique et scientifique produit au sein de la firme. Vingt ans plus tard, la création du premier laboratoire de recherche de Kodak représenta l'une des meilleurs solutions à cette problématique. Voir, par exemple, C. Fisk, Working Knowledge Employee Innovation and the Rise of Corporate Intellectual Property, 1800-1930, p. 188.

3. N. Le Guern, «The Contribution of the European Kodak Research Laboratories to Innovation Strategy at Eastman Kodak », p. 117. 
4. Université de Montfort, Kimberlin Library, Kodak Collection, KC 338.4777 HAR, rapport tapuscrit, « The Harrow Research Laboratory. Origins and Growth 1928-1976. A Retrospective Album », fol. 8.

5. D. Edgerton et S. Horrocks, «British Industrial Research and Development before 1945 », p. 233.

6. Université de Montfort, Kimberlin Library, Kodak Collection, KC 338.4777 HAR, rapport tapuscrit, « The Harrow Research Laboratory. Origins and Growth 1928-1976. A Retrospective Album », fol. 10.

7. L'archive Kodak à la British Library (Kodak historical archive) contient l'intégralité des rapports de recherche du laboratoire anglais de Harrow de 1929 à 1964, conservés reliés en 99 volumes. Source: British Library, Kodak Historical archive, A2897, rapports de recherche, 99 volumes.

8. Association CECIL, archive Kodak-Pathé, 33014, rapport de recherche, Mr Löbel, «Commentaires faits à la suite d'un voyage à Foots Cray », 26 juin 1906, n.p.

9. N. Le Guern, ibid., p. 148.

10. Ibid., p. 166.

11. L'archive Kodak-Pathé est conservée à Fragnes, près de Chalon-sur-Saône, par l'association CECIL (Cercle des Conservateurs de l'Image Latente). [URL:http:// www.argentiquececil-kodak.fr/]

12. N. Le Guern, ibid., p. 155-192.

13. Association CECIL, archive Kodak-Pathé, 33498, inventaire, «Liste des rapports de Monsieur Abribat de 1928 à 1935 », 11 novembre 1935. Cet inventaire se trouvait dans le cahier de recherche (dossier 53) du photochimiste H. Renard.

14. Walter G.Bent, un ingénieur chimiste diplômé du Massachusetts Institute of Technology travailla pendant quinze ans à Kodak Park et sur le site industriel de Kodak à Toronto. En 1920, il fut nommé directeur de l'usine Kodak à Harrow, près de Londres. En 1930, il fut remplacé par William R. Webb et devint directeur général des sites de production de Kodak en Europe. Source : British Library, Kodak Historical archive, A2891, rapport tapuscrit, Margaret D. Gauntlett, "A History of Kodak Limited», p. 33, p. 57, p. 65, p. 152.

15. Association CECIL, archive Kodak-Pathé, 33498, «Liste des rapports (par noms d'auteurs) faits à Vincennes au cours de l'année 1935 », n.p.

16. Les chiffres entre parenthèse, qui suivent le nombre de rapports transmis au destinataire concerné, précisent le ou les numéros de ces rapports. Concernant les chercheurs français, H. Renard envoya également la copie de deux de ses rapports à deux autres collègues anglais (le $n^{\circ} 21$ à Curtis et le $n^{\circ} 23$ à Mc Master). À Rochester, Albert J. Sulzer, précédemment directeur général de la fabrication du film, fut promu directeur général de Kodak Park en 1929. Voir «Mr. Sulzer honored on Thirty-Year Service Records ", p. 16. En Angleterre, Ernest Edgar Blake travailla chez Kodak L ${ }^{\text {td }}$ dès 1903. Il fut promu responsable du département du film cinéma en 1911 et directeur général en 1930. En 1946, il devint président du conseil d'administration de Kodak $\mathrm{L}^{\text {td }}$ et directeur général des filiales européennes de Kodak. Voir M. D. Gauntlett, « A History of Kodak Limited », rapport tapuscrit, Kodak Historical archive, British Library, A2891, p. 56, p. 85. "Coal seen No. 1 Block for Britain », p. 1-5. Franck Robinson, le directeur de l'usine de Kodak A.G. près de Berlin ne doit pas être confondu avec Charles Thomas 
Robinson, qui fut responsable de production à Pathé-Cinéma puis à Kodak Limited à partir de 1927.

17. Association CECIL, archive Kodak-Pathé, 33262, 33271, 33235, 33263, 33273, 33274, $33143,33428,33440,33441,33255,33427$ et 33078.

18. Association CECIL, archive Kodak-Pathé, 33498, inventaire, « Rapports Harrow reçus à Vincennes 1929 à 1940 », n.p.

19. Sources: British Library, Kodak Historical archive, rapports de recherche, vol. 1 à 11, A2897. Association CECIL, archive Kodak-Pathé, 33498, inventaire, « Rapports Harrow reçus à Vincennes 1929 à 1940 ", n.p.

20. Association CECIL, archive Kodak-Pathé, 33023, "Échange de rapports entre Eastman Kodak \& Kodak-Pathé d'une part et entre Kodak-Limited \& Kodak-Pathé d'autre part », 13 février 1951, fol.1-3. Il n'a pas été possible d'identifier l'auteur du rapport à partir de sa seule signature.

\section{RÉSUMÉS}

À la fin des années 1930, Eastman Kodak décida d'augmenter sa capacité de recherche industrielle en ouvrant deux laboratoires de recherche additionnels, en France et au RoyaumeUni. Cet article, issu de ma thèse de doctorat, considère tout d'abord la structure de ces deux laboratoires ainsi que leurs scientifiques, acteurs véritables de la production, transmission et protection des savoirs. L'étude des archives inédites de Kodak $\mathrm{L}^{\text {td }}$ et de Kodak-Pathé montre que les chercheurs conservaient le savoir scientifique produit au sein de rapports de recherche, qui n'étaient transmis en interne qu'à une liste restreinte de responsables. De plus, des documents de l'archive française fournissent les modalités de transmission des savoirs scientifiques contenus dans les rapports entre les trois principaux laboratoires de Kodak. Cet article met en lumière cette circulation très codifiée du savoir de la firme, pour laquelle toute technologie d'avenir représentait un atout potentiellement exploitable.

\section{AUTEUR}

\section{NICOLAS LE GUERN}

Chercheur indépendant, responsable R \& D, diplômé du Photographic History Research Centre, Université de Montfort, Leicester, Royaume-Uni 


\section{Petre (Pierre) Sergescu (1893-1954) : historien des sciences et promoteur de la discipline}

\section{Alexandre Herlea}

Fig. 1. - Portrait de Petre (Pierre) Sergescu.

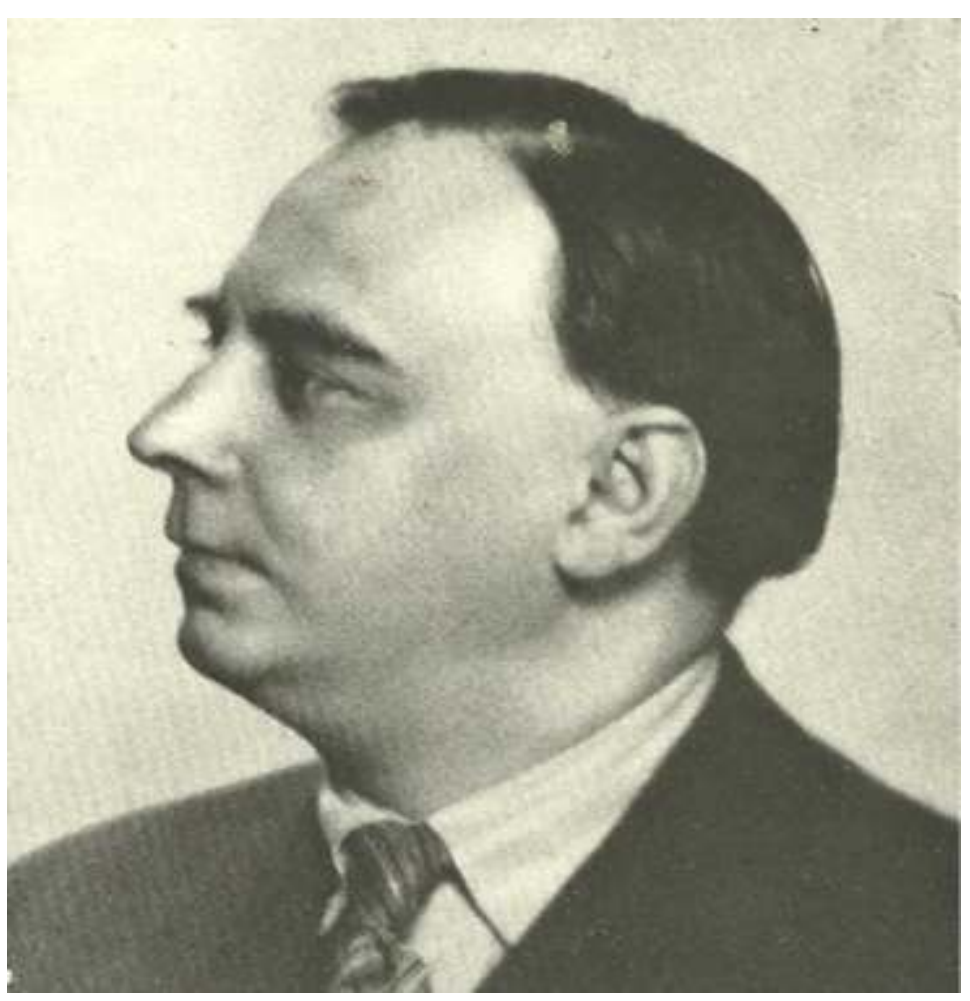

Archives de Mme Magda Stavinschi

Né en Roumanie le 17 décembre 1893, Petre Sergescu a fait une partie de ses études en France où il a vécu de longues années : il appartient à cette pléiade de Roumains dont 
l'œuvre fait partie intégrante de la culture française et européenne. Son attachement à la France s'est affirmé dès son plus jeune âge. Lors de la Grande Guerre, P. Sergescu mène une action pro-française pour laquelle, après l'occupation de Bucarest par les Allemands, il est interné pendant 18 mois dans des camps, par ces derniers. Lors de la Seconde Guerre mondiale, il prend contact en Suisse avec la Résistance française et en 1946, après l'occupation de la Roumanie par les Soviétiques, il se réfugie en France.

\section{8 : cent vingt-cinq ans depuis la naissance de Petre Sergescu}

2 Nous avons célébré en 2018 le cent vingt-cinquième anniversaire de sa naissance qui fut l'occasion de rappeler, au CTHS, ses engagements et réalisations, son rôle et son œuvre. D'ailleurs d'autres initiatives liées à cet anniversaire ont été aussi prises, telle l'organisation à l'Académie Roumaine d'une session d'hommages ${ }^{1}$ et la republication, par Mme Magda Stavinschi, de son livre Gândirea Matematică (La Pensée Mathématique), précédé d'une large présentation de sa vie et son œuvre ${ }^{2}$. Petre Sergescu est né à Turnu Severin, ville portuaire, située sur les rives du Danube près des Portes de Fer, dans une famille d'intellectuels dont les aïeuls ont pris part, au xIX siècle, à la lutte d'émancipation nationale et sociale du peuple roumain ${ }^{3}$. Esprit encyclopédique, après avoir passé son baccalauréat, au lycée de sa ville natale, il poursuit ses études, en mathématiques et en philosophie, à l'Université de Bucarest où il obtient les licences dans ces deux disciplines en 1916. La même année, il est diplômé du Conservatoire de Musique. Après la Guerre, il obtient une bourse et poursuit ses études à Paris de 1919 à 1923. Il suit les cours de l'École normale supérieure et ceux de la faculté des sciences de la Sorbonne, où il obtient, en 1922, une deuxième licence en mathématiques et commence à travailler à sa thèse de doctorat. Il suit aussi l'enseignement en histoire des sciences de Pierre Boutroux au Collège de France ${ }^{4}$.

En 1923, de retour en Roumanie, P. Sergescu passe l'agrégation en mathématiques à l'Université de Bucarest et soutient une brillante thèse, avec un sujet relevant du domaine des équations intégrales, intitulée "Sur les noyaux symétrisables» devant un jury prestigieux formé de Traian Lalescu (directeur de thèse), David Emmanuel (président du jury) et Gheorghe ȚiȚeica ${ }^{5}$. Paul Montel précise :

"L'étude des noyaux symétrisables des équations intégrales avait déjà attiré beaucoup de chercheurs, dont les principaux furent Joseph Marty, Traian Lalesco, Hermann Weyl. Sergescu introduit la notion nouvelle de noyau fermable qui lui permet de regrouper les résultats antérieurs au sein d'une théorie générale en utilisant la suite supposée fermée de solutions fondamentales et de leurs associées et la notion de genre due à Lalesco. Ces résultats et d'autres ont paru, en 1923 et l'année suivante, dans les Comptes rendus de l'Académie des sciences de Paris et dans le Bulletin de la Société des sciences de Cluj. ${ }^{6}$ ”

Immédiatement après, Sergescu commence sa carrière comme professeur suppléant à l'Université de Bucarest et à l'Institut Polytechnique de cette ville pour être nommé, en 1926, professeur agrégé de géométrie analytique à l'Université de Cluj, où, en 1930, il devient professeur titulaire (fig. 2). Il occupe ce poste jusqu'en 1943. À cette date, il retourne à Bucarest où il est nommé titulaire d'une même chaire à l'Institut Polytechnique. Entre-temps, il est élu membre correspondant de l'Académie Roumaine le 25 mai 1937 et membre de l'Académie des Sciences de Roumanie le 5 juin 19437. 
Fig. 2. - L'Université de Cluj - carte postale 1926.

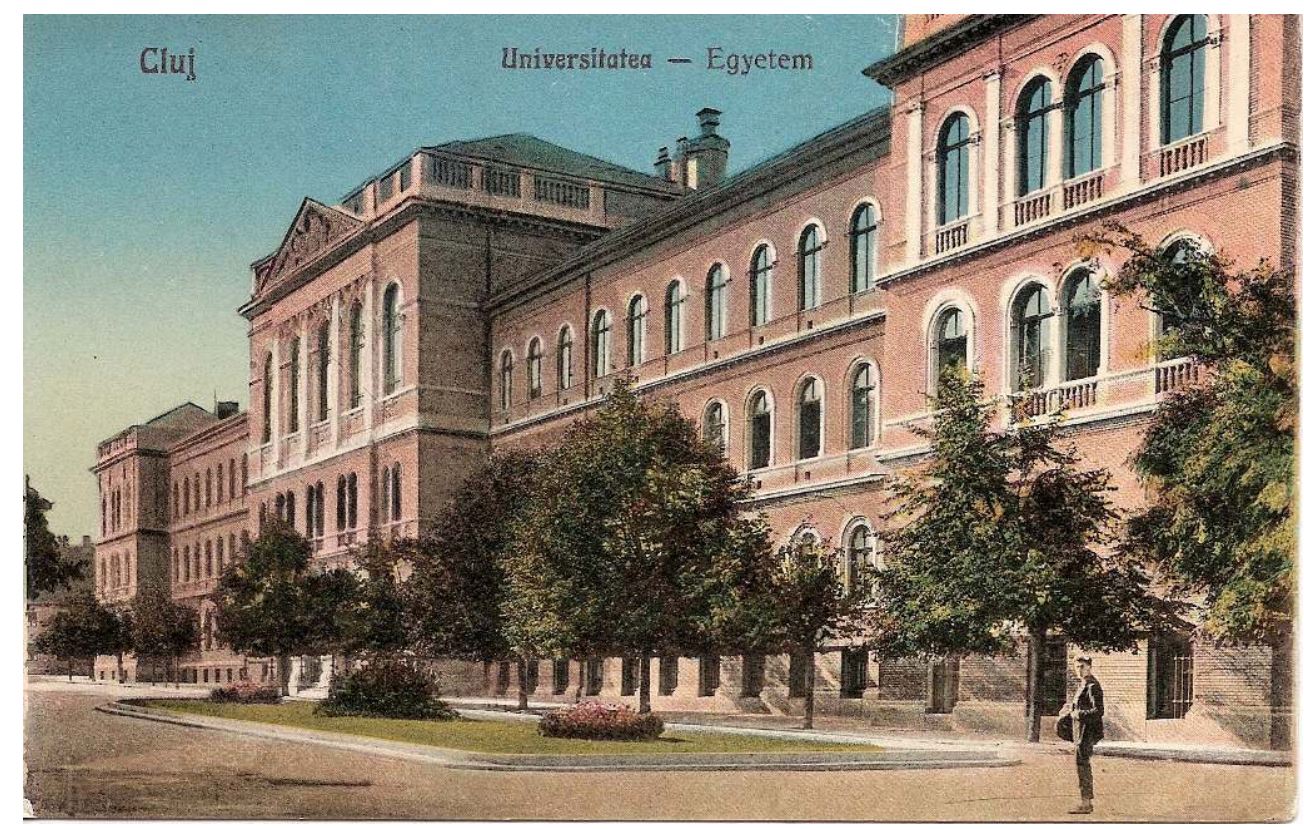

Archives du Musée de l'Université Babes-Bolyai de Cluj-Napoca.

5 Durant l'entre-deux-guerres, P. Sergescu développe une riche activité, aussi bien en mathématiques pures que dans l'histoire et la philosophie de celles-ci. Cela se produit tant au niveau de la recherche et de l'enseignement que de la promotion et de la diffusion du savoir. Il est actif au niveau de l'organisation de diverses manifestations scientifiques et participe à la vie des institutions du domaine. C'est surtout le cas de l'Académie internationale d'histoire des sciences (AIHS), dont l'idée fut lancée en 1928 lors du Congrès international des sciences historiques d'Oslo par Aldo Mieli et un groupe d'historiens des sciences et des techniques dont George Sarton, Charles Singer, Abel Rey. Son siège se situera, à partir de 1929, à Paris, 12 rue Colbert, dans l'Hôtel de Nevers, où elle est hébergée par le Centre international de synthèse ${ }^{8}$.

\section{Petre Sergescu : le mathématicien}

Dans le domaine des mathématiques contemporaines, P. Sergescu s'intéresse aux équations intégrales, sujet qu'il a abordé dans sa thèse de doctorat, à l'algèbre, domaine dans lequel il a le plus publié, à la théorie des nombres, à la géométrie des polynômes, à l'analyse combinatoire, à la théorie des fonctions, etc. Ses travaux sont publiés dans des revues scientifiques prestigieuses aussi bien en Roumanie (Mathematica, Bulletin scientifique de l'École polytechnique de Timişoara, etc.) qu'à l'étranger (Comptes-rendus de l'Académie des sciences de Paris, Les Annales de la Société polonaise de mathématique, etc.) $)^{9}$

7 Sergescu fonde en 1929, sous le patronage de ses maîtres les professeurs Gheorghe ȚiȚeica et Dimitrie Pompeiu, la revue Mathematica (revue bilingue franco-roumaine), dont il est le rédacteur en chef et le principal sponsor. Sa contribution financière est essentielle, assurant souvent la moitié du budget; 23 volumes vont sortir jusqu'en $1948^{10}$. À cette revue collaborent non seulement des Roumains mais aussi de nombreux étrangers de haut niveau tels les Français Paul Montel, Emil Picard, Maurice Fréchet et 
le Polonais Waclaw Sierpiński dont la revue Fondamenta Mathematicae a été un modèle au moment de la création de Mathematica. Elle atteste, dit René Taton :

« À la fois le haut niveau atteint par la science roumaine et la riche collaboration internationale que Pierre Sergescu a su lui attirer. ${ }^{11}$ »

Elle a été, affirme Paul Montel :

"Une revue fondamentale des Sciences mathématiques..$^{12}$ "

Petre Sergescu organise les deux premiers congrès de mathématiques, qui ont eu lieu en Roumanie, à Cluj en 1929, et à Turnu Severin en 1932. Y prennent part des mathématiciens réputés, français, polonais, belges, tchécoslovaques, bulgares, yougoslaves et d'autres nationalités (fig. 3). À ce propos, Paul Montel déclare :

«Le congrès de Cluj fut un grand succès. Celui de Turnu Severin par sa haute tenue scientifique, par la perfection de son organisation... se termine d'une manière brillante. ${ }^{13}$ »

Fig. 3. - Palais de la culture de Turnu Severin où s'est déroulé le congrès. En médaillon, les participants au congrès dans la salle du restaurant.

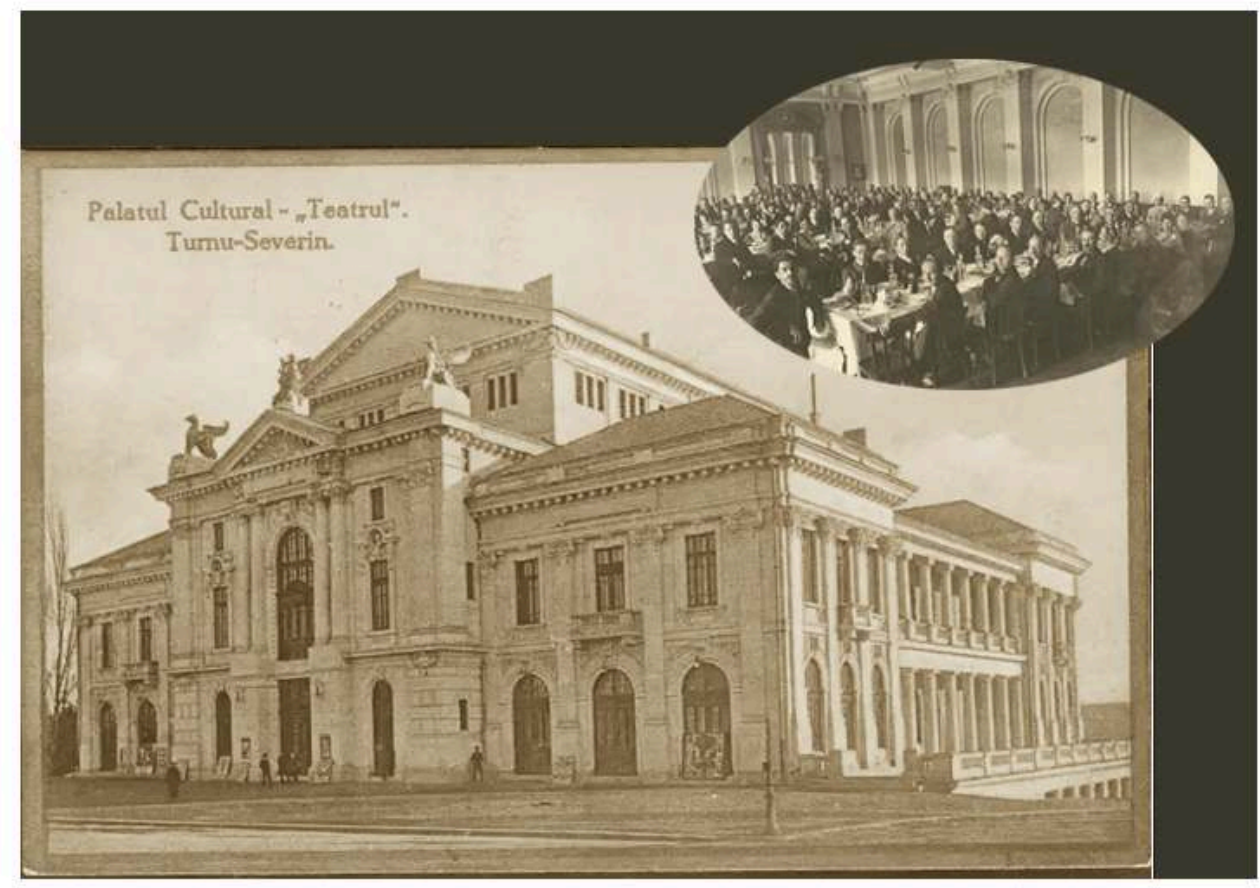

Archives de Mme Magda Stavinschi.

10 Désormais connu au plan international, P. Sergescu est souvent invité à l'étranger, principalement en France et en Pologne, pays avec lesquels il entretient des relations privilégiées. C'est un grand francophile qui a fait ses études en France et sa femme, l'écrivaine Maria Kasterska épousée à Paris en 1922, est polonaise avec des ancêtres français $^{14}$ (fig. 4). 
Fig. 4. - Petre Sergescu \& Maria Kasterska lors de leur mariage à Paris.

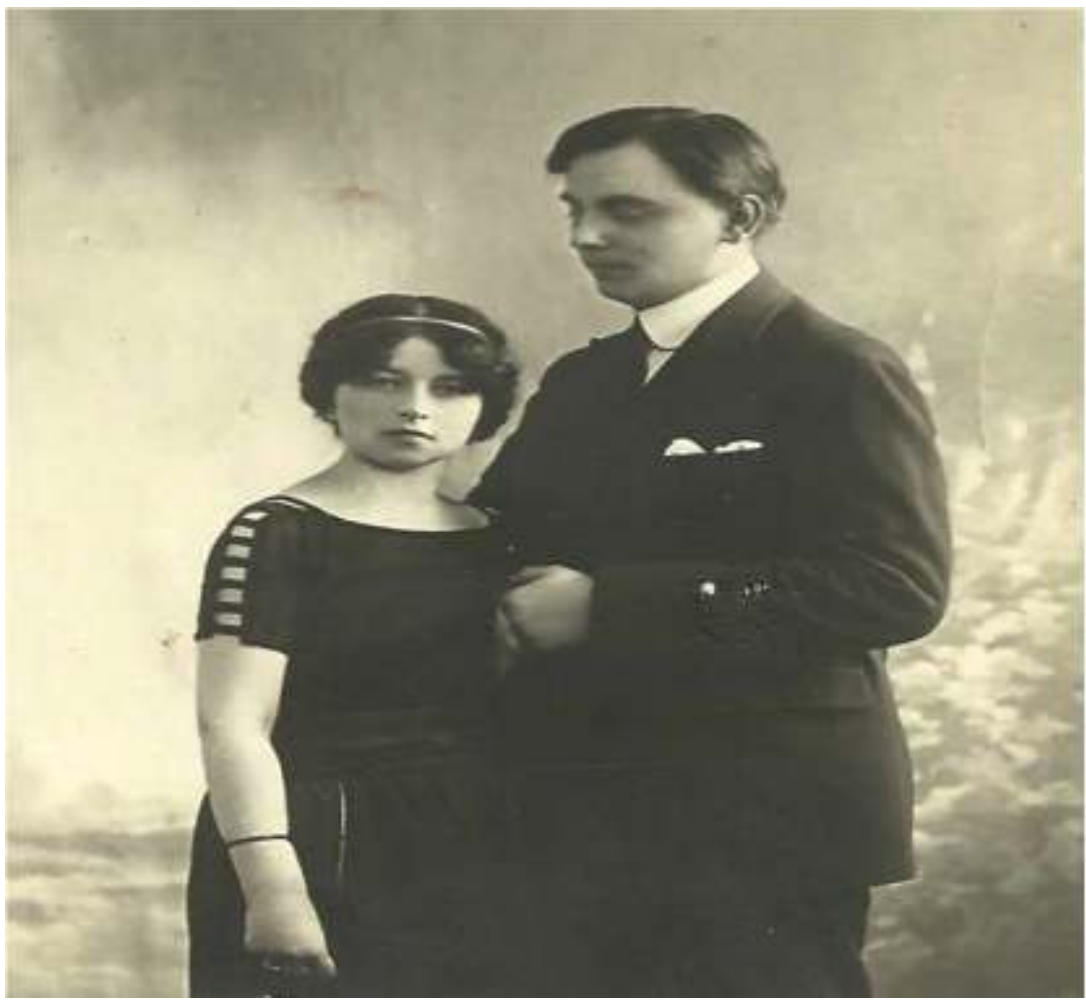

Archives de Mme Magda Stavinschi.

11 En France, où il séjourne souvent dans l'entre-deux-guerres, P. Sergescu est membre de la Société mathématique de France depuis 1920. À partir du début des années 1930, il s'intéresse aux activités de l'AIHS, dont le siège est à Paris; nous allons y revenir. Il participe aux congrès de l'Association française pour l'avancement des sciences (AFAS) dont il est membre actif à partir de 1924, notons ceux d'Alger en 1930 et de Chambéry en 1933. En 1937, à Paris, il est président du deuxième congrès des «Récréations Mathématiques", ce qui met en évidence le prestige dont il jouit déjà parmi les mathématiciens français. Dans les années 1930, et au début des années 1940, il donne des cours et fait des conférences et des séminaires dans plusieurs universités françaises: Paris, à la Sorbonne, Clermont-Ferrand, Poitiers, Montpellier, ainsi que dans des universités francophones de Belgique (Bruxelles, Liège) et de Suisse (Genève, Lausanne, Neuchâtel, Fribourg, Zurich) ${ }^{15}$. En 1932, la France lui décerne, en tant que professeur à l'université de Cluj où l'influence française est très forte, la Légion d'honneur ${ }^{16}$.

12 En Pologne, en tant que membre de la Société Polonaise de Mathématiques P. Sergescu est nommé président d'honneur des deuxième et troisième Congrès des Mathématiciens Polonais, qui eurent lieu à Vilnius en 1931 et à Varsovie en 1937. Il parle polonais et entretient des relations privilégiées avec ses collègues. Il tient des cours et conférences dans les universités de Lwow, Vilnius, Poznan et Varsovie; il est membre correspondant de la Societas Scientarum Varsoviensis et membre de la Société historique et littéraire polonaise (SHLP). Il est décoré de l'ordre Polonia Restituta ${ }^{17}$.

13 Mais ses relations et collaborations ne se limitent pas à la France et la Pologne. P. Sergescu participe également à des congrès et colloques dans d'autres pays 
européens, tels le Congrès international des mathématiciens de Zurich, en 1932 et en 1937 à celui des mathématiciens des pays slaves à Prague.

\section{Petre Sergescu : I'historien des sciences}

Au début des années 1930, quand il commence à fréquenter les milieux de l'histoire des sciences à Paris, son intérêt pour celle-ci va prendre une place de plus en plus importante dans ses préoccupations. En 1934, il devient membre de l'AIHS et s'implique dans sa vie; par la suite il participera à tous ses congrès. En cette même année, le troisième congrès a lieu à Coimbra, au Portugal.

C'est d'abord dans le domaine de l'histoire des sciences que P. Sergescu s'est imposé sur la scène internationale. En 1933, à Varsovie, il est élu président de la section d'Histoire des Sciences du Congrès des Sciences Historiques. En 1936, à l'occasion de la réunion en Roumanie du Comité International des Sciences Historiques et avec l'appui du grand historien Nicolae Iorga, il organise à Cluj et à Bucarest, du 11 au 16 avril, une rencontre des historiens des sciences d'une dizaine de pays, dont Aldo Mieli, Charles Singer, Arnold Reymond, Mario Gliozzi. En 1937, lors du quatrième congrès de l'AIHS qui a lieu à Prague, il est élu vice-président de cette institution, responsabilité qu'il gardera jusqu'en 1947 quand il en prend la présidence ${ }^{18}$.

16 Dans le domaine de l'histoire et de la philosophie des mathématiques et plus généralement des sciences, il aborde une grande variété de thèmes, publie plusieurs livres et de nombreuses études. Les principaux sujets abordés concernent l'évolution de la pensée scientifique au Moyen Âge, en mettant l'accent sur l'œuvre de l'école scientifique parisienne (Paul Tannery, Pierre Duhem) et les travaux des mathématiciens $d u \mathrm{XVII}^{\mathrm{e}}$ siècle et $d u$ début $d u$ XVIII ${ }^{\mathrm{e}}$ siècle, notamment la naissance $d u$ calcul infinitésimal, la polémique Rolle-Saurin au sujet du calcul différentiel (qu'il trouve dans le Journal des Savants) et autres aspects de l'école française dans la seconde moitié du XVII siècle ; la science à l'époque de la Révolution; le développement des sciences en Roumanie ${ }^{19}$.

Parmi ses livres, je citerai Gândirea Matematică, paru à Cluj en 1928, qui porte sur l'histoire et la philosophie des mathématiques depuis la Grèce antique au $\mathrm{xx}^{\mathrm{e}}$ siècle, couronné du prix de l'Académie Roumaine; Les sciences mathématiques en France au XIX siècle et au début $d u x^{e}$ siècle, paru chez Denoël \& Steele à Paris en 1933, dans la collection "Tableau du $\mathrm{xx}^{\mathrm{e}}$ siècle", élogieusement présenté par Emile Borel à l'Académie des sciences de Paris qui lui décerne un prix; le chapitre sur les mathématiques françaises au xiXe siècle dans le volume L'évolution des sciences mathématiques et physiques, paru chez Flammarion en 1935.

Parmi les études, je mentionne celle réalisée pour le Pavillon français à l'Exposition universelle de New York de 1939, intitulée : Some important dates in the evolution of French mathematics, publiée en des dizaines de milliers d'exemplaires; "L'évolution des principes de la mécanique de Newton à Laplace ", publiée en 1929 dans les Annales de la Société polonaise des mathématiques; « La vie contemporaine des mathématiques » (Revue de l'Université de Bruxelles, 1937), "Le développement des sciences mathématiques en Roumanie » (La vie scientifique en Roumanie, Bucarest, 1937) ; « Dernières batailles pour le triomphe du calcul infinitésimal» (Sphinx, Bruxelles, 1938); "Un soldat de la mécanique cartésienne au début du xviII ${ }^{\mathrm{e}}$ siècle : Antoine Parant » (Sphinx, Bruxelles, 
1938); «Les mathématiques au Moyen Âge» (Le Flambeau, Bruxelles, 1939); « Mathématiciens révolutionnaires » (Sphinx, Bruxelles, 1939) ${ }^{20}$.

19 La Seconde Guerre mondiale, avec ses horreurs et ses lourdes conséquences, bouleverse la vie de P. Sergescu. À l'été 1940, suite au pacte Hitler-Staline (Pacte germanosoviétique) et aux Diktats de Vienne (arbitrages de Vienne), la Roumanie perd la moitié de la Moldavie et de la Transilvanie, dont la ville de Cluj où se trouve son université. La faculté des sciences est transférée à Timișoara où Sergescu la suit et reste 3 ans. En novembre 1940, le grand historien Nicolae Iorga, personnalité dont Sergescu était très proche, est assassiné. Face à ce désastre, il retrouve le militantisme qui l'a caractérisé lors de la Grande Guerre. Il dénonce les crimes, vilipende les totalitarismes « rouge et brun ", fait une propagande active en faveur des alliés, il porte secours aux réfugiés polonais, etc. En 1943, invité en Suisse par son ami Arnold Reymond, professeur à l'Université de Lausanne, président de l'AIHS, il prend contact avec les milieux de la Résistance française ${ }^{21}$.

20 La fin de la Seconde Guerre mondiale le trouve, comme je l'ai déjà mentionné, professeur de géométrie analytique à l'Institut polytechnique de Bucarest dont il est élu président (recteur) en janvier 1945. Il remplit cette fonction jusqu'en août 1946, quand il se réfugie à Paris avec son épouse pour ne plus jamais retourner en Roumanie. En 1945 , il participe encore au troisième congrès des mathématiciens roumains à Bucarest (fig. 5).

Fig. 5. - Congrès des mathématiques de Bucarest, 1945. P. Sergescu, $1^{\mathrm{er}}$ rang, $5^{\text {ème }}$ à partir de la gauche.

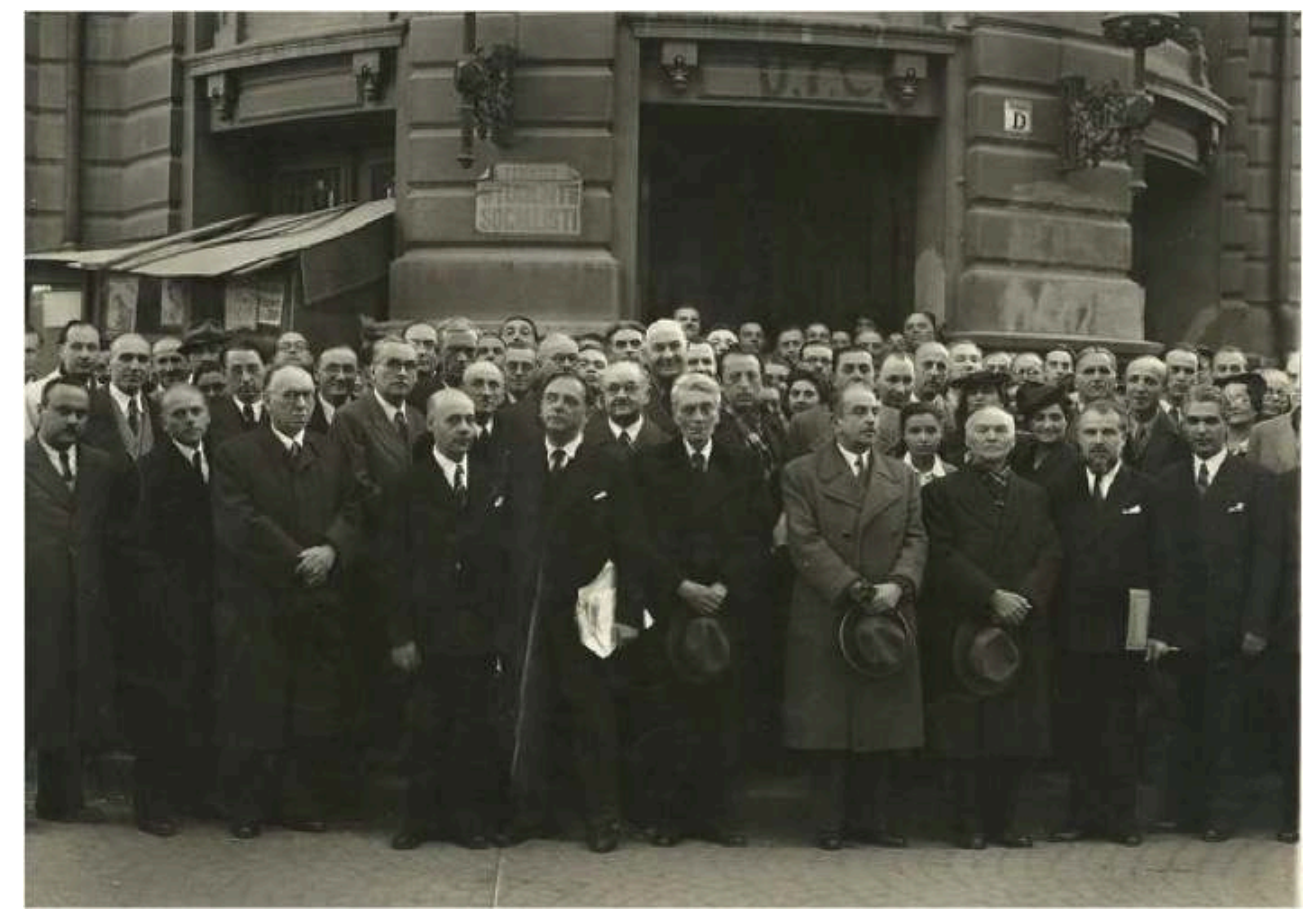

Archives de Mme Magda Stavinschi.

21 À Paris, P. Sergescu se consacre à la science, surtout à l'histoire des sciences et à la Roumanie. Il passera des années dans des conditions matérielles difficiles; il ne deviendra chargé de recherches au CNRS qu'à partir de 1952. 


\section{Petre Sergescu à Paris : le principal artisan de la collaboration internationale en histoire des sciences après la Seconde Guerre mondiale}

Au niveau de l'histoire des sciences, son action est prodigieuse : il devient le principal artisan de la collaboration internationale dans ce domaine. Il faut préciser qu'à la fin de la guerre, l'AIHS, principale institution de cette discipline, reprend ses activités. Elle souhaite bénéficier de l'aide que l'Unesco, créée en novembre 1945, pourrait lui apporter et sait que celle-ci soutiendra The International Council of Scientific Unions (ICSU) qui regroupe plusieurs grandes organisations internationales dont l'objectif est la promotion de l'activité scientifique. Il semble que Joseph Needham ait eu le premier l'idée d'introduire l'histoire des sciences et des techniques dans le cadre de l'ICSU ${ }^{22}$.

L'AIHS doit faire les démarches nécessaires et entamer des négociations. Mais, à l'automne 1946, sa situation est difficile: son secrétaire perpétuel Aldo Mieli est en Argentine, très malade, le président, Arnold Reymond, habite Lausanne et le secrétairetrésorier J. A. Vollgraff réside à Leyde. Seuls deux responsables sont à Paris, où se déroulent les négociations, le vice-président, Petre Sergescu qui vient d'arriver en août 1946 et le secrétaire-adjoint Pierre Brunet archiviste-bibliothécaire. Ce sont eux qui entament, fin 1946, les négociations avec l'Unesco, représenté par Joseph Needham et Armando Cortesão, et l'ICSU représenté par A. Establier. La solution qui se dégage est de créer une structure calquée sur le modèle des autres organismes membres de l'ICSU. Celle-ci devient l'Union internationale d'histoire des sciences (UIHS). Fin décembre, le Conseil de l'Académie approuve cette solution qui bénéficie du support des personnalités les plus connues du domaine telles que Ch. Singer, A. Reymond, G. Sarton, R. Taton ou M. Daumas ${ }^{23}$.

Peu de temps après, $P$. Brunet tombe malade et $P$. Sergescu reste seul en première ligne. C'est lui qui, à partir de décembre 1946, va jouer le rôle clef dans l'organisation institutionnelle de la discipline. Passionné d'histoire et de philosophie des sciences et des techniques, P. Sergescu n'a pas seulement l'enthousiasme du créateur mais aussi la compréhension de la situation institutionnelle et la capacité de définir une stratégie pour agir efficacement. Il mène avec succès les négociations qui aboutiront à la création des statuts de l'UIHS et à la modification de ceux de l'AIHS, afin que les deux institutions existent et collaborent étroitement ${ }^{24}$.

$\mathrm{Au}$ cinquième Congrès international de l'histoire des sciences qui a lieu à Lausanne du $1^{\mathrm{er}}$ au 4 octobre 1947, la décision de créer l'UIHS est entérinée (fig. 6). Sergescu est désigné secrétaire exécutif par l'Assemblée constitutive (2 octobre 1947). Un jour auparavant, toujours à ce Congrès, lors de l'assemblée générale de l'AIHS, il avait été élu président, s'établissant ainsi une liaison statutaire entre les deux institutions. L'UIHS devient membre de l'ICSU et Sergescu sera nommé délégué auprès de ce dernier. En cette qualité, il sera également membre du conseil de l'ICSU ${ }^{25}$. 
Fig. 6. - $5^{\text {ème }}$ Congrès international de l'histoire des sciences à Lausanne du $1^{\mathrm{er}}$ au 4 octobre 1947. P. Sergescu en $2^{\mathrm{e}}$ ligne $4^{\mathrm{e}}$ à partir de la gauche.

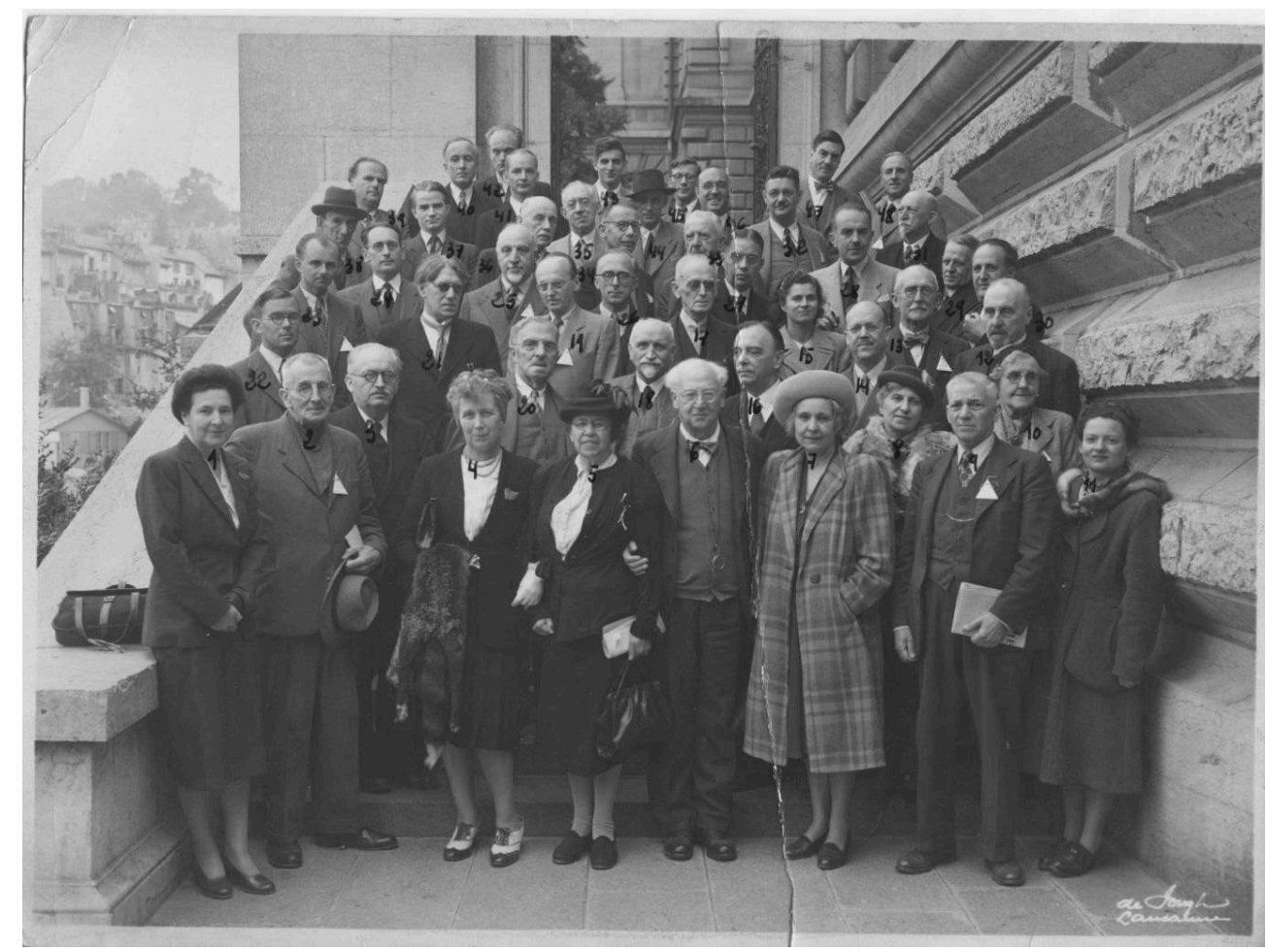

Archives René Taton.

une revue spécialisée voit le jour. Archeion, le périodique de l'AIHS, créé par Aldo Miel en 1929, avait cessé sa parution en 1943. Le premier numéro de la nouvelle revue qui porte le titre Archives Internationales d'Histoire des Sciences et le sous-titre Nouvelle Série d'Archeion apparaît en octobre 1947. Il s'agit de la publication trimestrielle de l'UIHS et en même temps le continuateur d'Archeion, l'ancien périodique de l'AIHS. Sergescu sera rédacteur des Archives et, à partir de mars 1950, son directeur, fonction qu'il avait exercée de fait au moins depuis mars $1948^{26}$.

En effet, à partir de cette date, Pierre Brunet gravement malade ne peut plus exercer ses fonctions de directeur adjoint aux Archives, ni remplir ses obligations qu'il avait en tant que représentant d'Aldo Mieli (directeur des Archives et secrétaire général de l'UIHS); ainsi Sergescu assume seul l'administration et le bon fonctionnement des activités de l'AIHS, de l'UIHS et des Archives ${ }^{27}$.

, survenu un mois plus tôt, Sergescu est élu secrétaire perpétuel de l'AIHS, fonction qu'il assume officiellement après le sixième Congrès international de l'histoire des sciences qui a lieu à Amsterdam du 14 au 21 août. Il deviendra également secrétaire général de l'UIHS et, comme déjà dit, directeur des Archives. C'est la consécration. Il avait espéré pouvoir organiser ce sixième Congrès à Bucarest, mais la situation politique en Roumanie a rendu ce projet irréalisable. Le septième Congrès International de l'Histoire des Sciences, le dernier auquel Sergescu participe, a lieu à Jérusalem en août 1953 : à ce congrès, il est le délégué de la France. exerçait au niveau de l'histoire des sciences ont été confiées à trois personnalités 
différentes: Alexandre Koyré est élu secrétaire perpétuel de l'AIHS, René Taton secrétaire général de l'UIHS et Jean Pelseneer directeur des Archives.

J'ai insisté sur le déroulement du processus de création des institutions pour l'histoire des sciences et des techniques pour mettre en évidence tant le rôle joué par P. Sergescu que ses qualités d'organisateur hors pair. Les articles et les comptes rendus concernant ces négociations, parus dans les Archives internationales d'histoire des sciences, donnent un aperçu du rôle joué par ce dernier. L'étude des fonds d'archives de l'AIHS pourrait fournir d'autres détails et jeter de nouveaux éclairages ${ }^{28}$.

\section{Autres activités de Petre Sergescu dans les sciences, leur histoire et diffusion}

31 Après 1945, P. Sergescu développe aussi une série d'autres activités à Paris dans le domaine des sciences, de leur histoire, enseignement et diffusion. Il organise ainsi, à partir de 1946, les réunions annuelles de la section d'Histoire des Sciences de l'Association Française pour l'Avancement des Sciences (AFAS). Jusqu'à son décès, en 1954, il participera à tous les congrès AFAS (à Nice, Biarritz, Genève, Clermont Ferrand, etc.) dans le cadre desquels il joue un rôle très actif; par exemple, au congrès de Biarritz, il est le président du département d'Histoire des sciences (fig. 7).

Fig. 7. - Congrès AFAS, Genève, 1948. De gauche à droite : René Taton, $X$, Juliette Taton, Maria Karsterska, Pierre Sergescu, Suzanne Delorme (directrice de la Revue d'histoire des sciences).

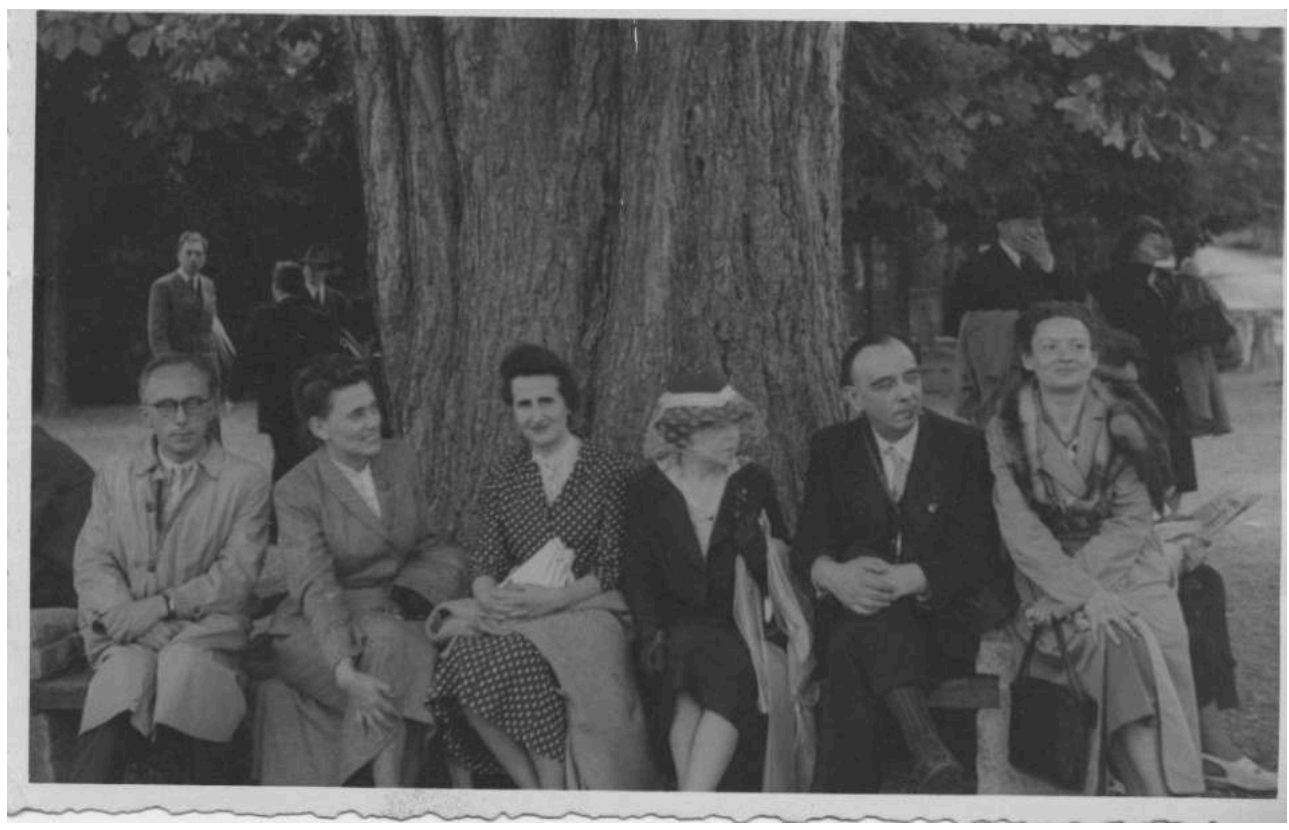

Archives René Taton.

Sergescu fonde le séminaire d'histoire des mathématiques à l'Institut Henri Poincaré et suscite la mise en place, dans le cadre de la Sorbonne, des cycles de conférences mensuelles d'histoire des sciences qui se déroulent au Palais de la découverte. Il participe également à la réalisation des expositions aussi bien 

écoutés et jouissent d'une vraie notoriété. En 1950, par exemple, il a fait quatorze
émissions à la Radiodiffusion française sur les origines de la science exacte moderne,
qui seront publiées dans un volume intitulé Coup d'œil sur les origines de la science exacte
moderne, livre à succès, paru à Paris en $1951^{30}$ (fig. 8). Petre Sergescu a beaucoup écrit,
plus de 160 titres, surtout dans le domaine des mathématiques, de l'histoire et de la
philosophie des sciences ${ }^{31}$.

Fig. 8. - Livre dédicacé : coup d'œil sur les origines de la science exacte moderne. Paris, 1951.

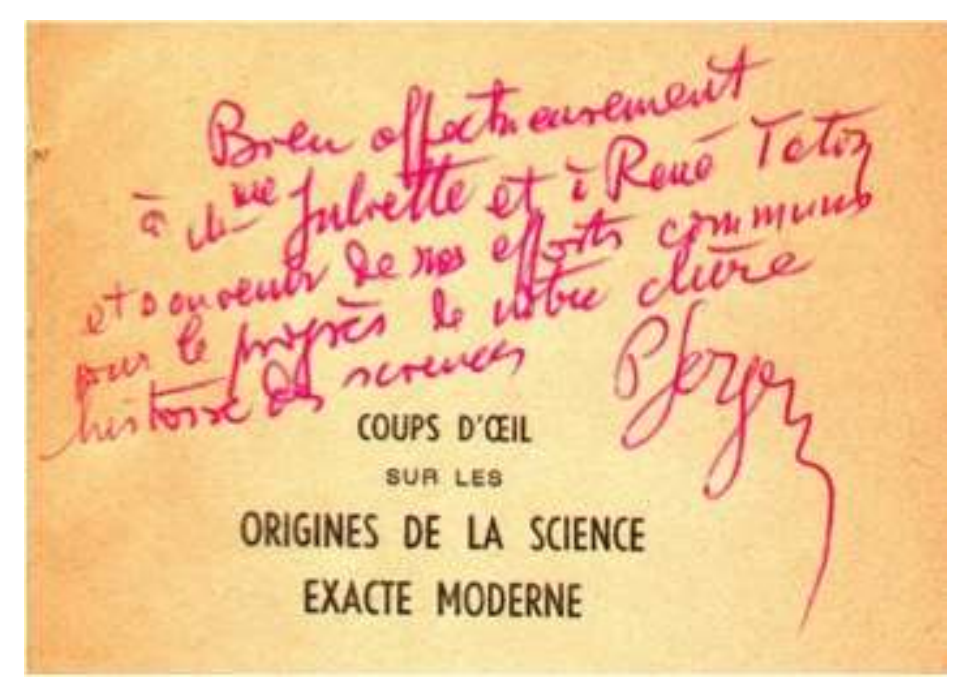

Archives René Taton.

\section{Petre Sergescu et la Roumanie}

En dehors de la science, P. Sergescu s'intéresse aussi à la Roumanie et aux exilés roumains. Ce n'est pas une nouveauté, car ce n'est pas un chercheur enfermé dans sa tour d'ivoire ; c'est un intellectuel engagé, charismatique, bon organisateur, défenseur des valeurs européennes, grand francophile et grand patriote roumain. À Paris, après la Seconde Guerre mondiale, il dénonce l'occupation soviétique, la terreur instaurée par les communistes et met en évidence le caractère profondément européen de la Roumanie. Il est président de l'Association des Roumains professeurs des universités à Paris et président-directeur général de la FundaŢia Regală Universitară Carol I, connue à Paris sous le nom de l'Institut universitaire Roumain Charles I. Il est présent aussi dans les longs et difficiles débats concernant l'organisation politique de l'exil, notamment la structure, la composition et le rôle du Comité national roumain. Il se dévoue à l'assistance des réfugiés en tant que membre de l'association d'entraide des réfugiés roumains Caritatea Romaneasca (CAROMAN) ${ }^{32}$. Mais ces aspects de la vie de P. Sergescu dépassent le cadre de cet article et nous n'allons pas les développer.

Rappelons seulement que son épouse Marya Kasterska et lui-même, dans leur modeste appartement du Quartier latin, au 7, rue Daubenton, (fig. 9) animent un salon culturel 
où se retrouvent les samedis soir des personnalités parisiennes de la vie culturelle et scientifique, telles Henry de Montherlant, les mathématiciens Paul Montel et Émile Borel, les historiens des sciences René Taton et Maurice Daumas, à côté des réfugiés des pays de l'Europe de l'Est, notamment de Roumanie, tels Mircea Eliade ou Nicolae Herescu et de jeunes étudiants. Mais on rencontre également de nombreux Polonais et des personnalités de passage à Paris venant des quatre coins du monde ${ }^{33}$.

Fig. 9. - Dernière photo du couple Sergescu.

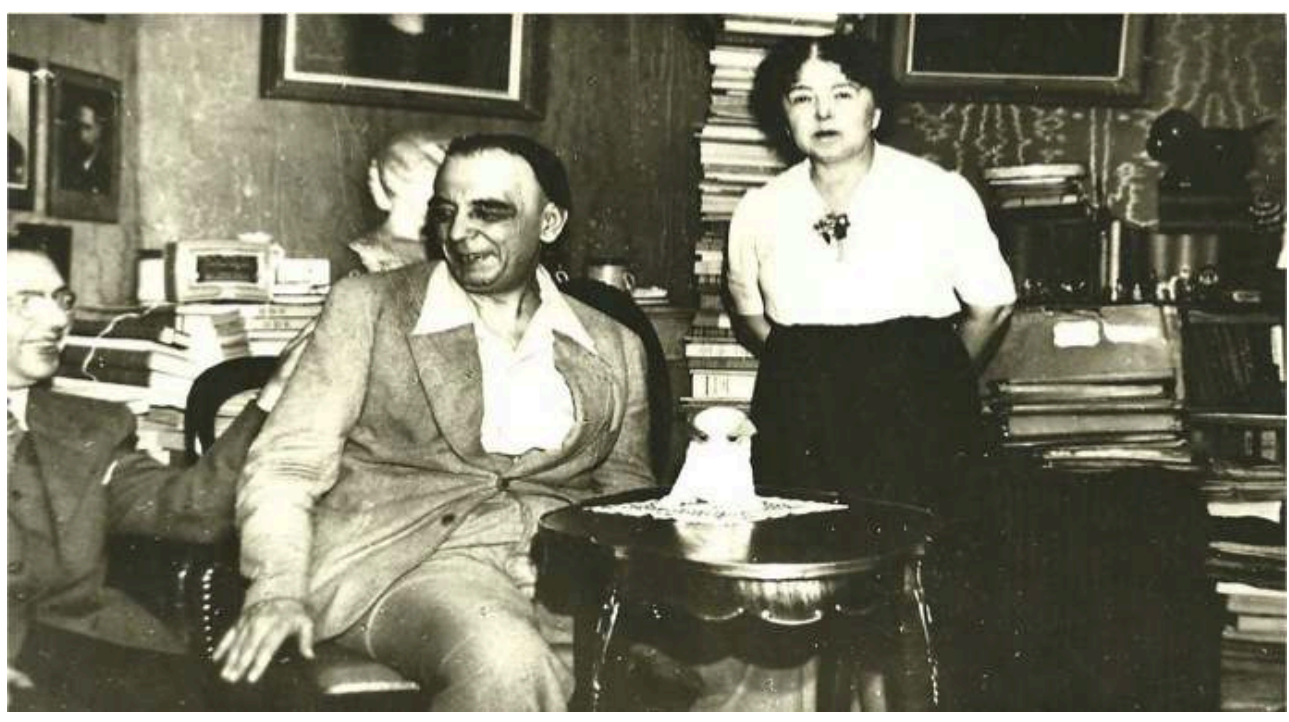

Archives de Mme Magda Stavinschi.

Dans cet appartement, Maria Kasterska fonde la Bibliothèque roumaine en 1961. En décembre 1969, immédiatement après son décès, le juriste Petre Mircea Cârjeu, son légataire testamentaire et celui qui a fait le plus pour la mémoire du couple Sergescu, désignera cette dernière sous le nom de «Bibliothèque roumaine Pierre Sergesco Marya Kasterska ». En 1978, il pose une plaque commémorative sur l'immeuble (fig. 10). Par la suite, il transfère cette bibliothèque, dont il était le directeur, au 39 rue Lhomond et la complète avec un petit musée. Elle existait encore en 1994 quand fut célébré le centenaire de la naissance de Petre Sergescu. Je l'ai bien connue ainsi que son directeur Petre Mircea Cârjeu, grand admirateur de Sergescu et de son épouse. La dernière fois je l'ai rencontré, en 1994, quand je glanais des informations pour l'article d'hommages que j'ai publié à l'occasion du centenaire de la naissance de Sergescu ${ }^{34}$. 
Fig. 10. - Immeuble 7, rue Daubenton, Paris $5^{\text {ème }}$ avec la plaque commémorative.

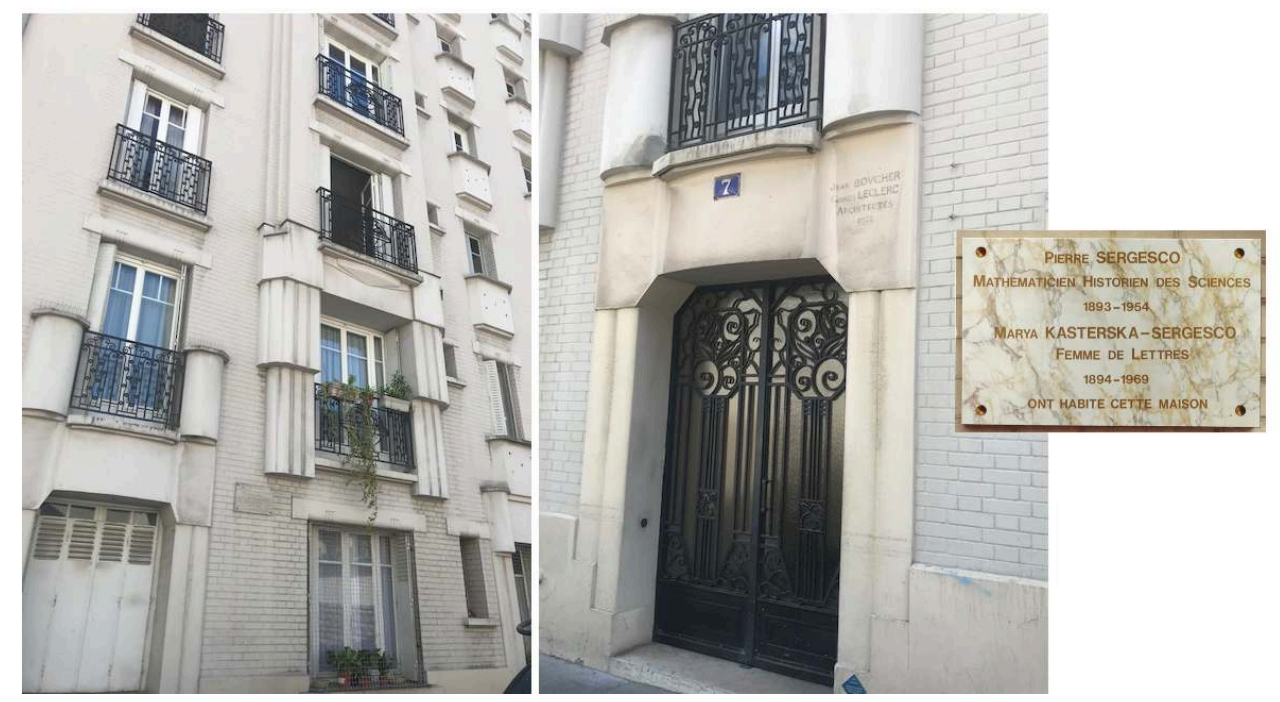

Archives Alexandre Herlea. et unième anniversaire, en pleine force de travail. René Taton a eu avec lui un entretien " confiant et détendu », comme il le caractérise quelques heures seulement avant sa mort, dans lequel Petre Sergescu :

« [lui] Exposait ses projets qui comme toujours, s'intéressaient beaucoup plus à l'avenir de l'Union Internationale et au développement des études d'histoire des sciences qu'à sa situation personnelle..$^{35}$ " $C^{\prime}$ est une perte durement ressentie par tous ceux
cimetière de Montmorency et sur sa tombe est écrit :

«J'ai ce que j'ai donné. » Pour conclure, je cite trois de ses admirateurs : les professeurs Paul Montel, René Taton et Maurice Daumas. Paul Montel achève son discours aux funérailles de Pierre Sergescu par ces paroles :

«La Roumanie perd un de ses savants et historiens universellement appréciés, la

France un de ses amis les plus fidèles et les plus généreux. ${ }^{36}$ " 


\section{BIBLIOGRAPHIE}

ANDONIE George ștefan, « Petre Sergescu (1893-1954) », Istoria Matematicii în România, Bucureşti, Ed. ştiinŢifică, 1965-1971, vol. II, p. 373-393.

ANDONIE George ștefan, « Pierre Sergescu (1893-1954) », Pierre Sergescu (1893-1954), Leiden, Ed. J. Brill, 1968, p. 3-12. (Janus, t. 55, 1968).

BoUligand G., « Le directeur d'études et son rayonnement », Pierre Sergescu (1893-1954), Leiden, Ed. J. Brill, 1968, p. 50-53. (Janus, t. 55, 1968).

CALAFETEANU Ion, Exilul românesc. Erodarea speranŢei. Documente (1951-1975), Ed. Enciclopedică, Bucureşti, 2003, 493 p.

CAPITAINE (TATON) Nicole, Pierre Sergescu collaboration scientifique avec René Taton et liens d'amitié avec la famille Taton. Conférence - Centre culturel Roumain, 22 janvier 2014.

CORTESAO Armando, « Adresse », Pierre Sergescu (1893-1954), Leiden, Ed. J. Brill, 1968, p. 61, (Janus, t. 55, 1968).

COSTABEL Pierre, «Pierre Sergescu, directeur des Archives internationales d'histoire des sciences ", Pierre Sergescu (1893-1954), Leiden, Ed. J. Brill, 1968, p. 30-37. (Janus, t. 55, 1968).

DUCA Dorel, PETRUşEl Adrian, « Petre Sergescu - Profesor la Universitatea din Cluj », Academica, nº 5-6, mai-iunie 2018, p. 75-80.

HALEUX Robert, SEVERYNS Benoit, Twenty-Five Years of International Institutions. LLULL, S.E.H.C.Y.T, Facultad de Ciencias (Matematicas), Zaragoza, Ciudad Universitaria, vol. 26, n 55,2003 , p. 315-321. 
HERLEA Alexandre, « Petre (Pierre) Sergescu (1893-1954), un artisan de la coopération internationale en Histoire des sciences ", Bulletin de la Société Française d'Histoire des Sciences et des Techniques, $\mathrm{n}^{\circ} 35$, février 1994, p. 14-19.

HERLEA Alexandru, « Petre Sergescu, personalitate reprezentativă a exilului romanesc », Academica, $\mathrm{n}^{\circ}$ 5-6, mai-iunie 2018, p. 81-86.

HERLEA Alexandre, «ICOHTEC, 50 Years. Tribute to Maurice Daumas and Petre Sergescu », ICON : The Journal of the International Committee for the History of Technology, vol. 24, 2018-2019, p. 12-32. HERLEA Alexandru, « Petre Sergescu : personalitate luminoasă a Exilului românesc », Buletinul Bibliotecii Române din Freiburg, seria noua 6 (2019), p. 128-135.

ISSARESCU Victor, « Pierre Sergescu (1893-1954). Son adolescence à Turnu Severin », Pierre Sergescu (1893-1954), Leiden, Ed. J. Brill, 1968, p. 12-19, (Janus, t. 55, 1968).

ITARD Jean, « Pierre Sergescu, historien des mathématiques », Pierre Sergescu (1893-1954), Ed. J. Brill, Leiden, 1968, p. 37-45. (Janus, t. 55, 1968).

MONTEL Paul, « Discours prononcé aux funérailles de Pierre Sergescu », Archives internationales d'histoire des sciences, $\mathrm{n}^{\circ}$ 30, 1955, p. 3-7.

MONTEL Paul, « Pierre Sergescu, mathématicien », Pierre Sergescu (1893-1954), Leiden, Ed. J. Brill, 1968, p. 46-50, (Janus, t. 55, 1968).

NICOLAIDIS Efthymios, « Petre Sergescu, président et secrétaire perpétuel de l'Académie internationale d'histoire des sciences ", Academica, $\mathrm{n}^{\circ}$ 5-6, mai-iunie 2018, p. 71-74.

NICOLESCu Basarab, «Un cuplu mitic : Petre Sergescu - Marya Kasterska », București, Magazin Istoric, decembrie 2013, p. 29-32.

SERGESCO Petre, Sur les noyaux symétrisables, Bucarest, Impr. de l'État, 1924, 47 p.

SERGESCU Petre, «L'Université roumaine de Cluj en exil », Archeion XXIV, 1942, p. 284-288.

SIERPINSKI Waclaw Franciszek, « Témoignage », Pierre Sergescu (1893-1954), Leiden, Ed. J. Brill, 1968, p. 62-63. (Janus, t. 55, 1968).

STAVINSCHI Magda, «René Taton et Pierre Sergescu, une collaboration au bénéfice de l'histoire des sciences », Archives Internationales d'Histoire des Sciences, $n^{\circ}$ 159, 2007, p. 553-562.

STAVINSCHI Magda, Petre Sergescu şi Gândirea Matematică, Bucureşti, Ed. Eikon, 2018, 356 p.

STAVINSCHI Magda, « Petre Sergescu şi Gândirea Matematică », Academica, nº 5-6, mai-iunie 2018, p. 63-70.

ŞTEFĂNESCu Doru, « Moştenirea matematică a lui Petre Sergescu ». Academica, nº 5-6 mai-iunie 2018, p. 86-90.

TATON René, « Pierre Sergescu (1893-1954) », Revue d'Histoire des Sciences, Paris, t. VIII, 1955, p. 77-80.

TATON René, « Petre Sergescu (1893-1954) », Bulletin Scientifique Roumain, Paris, Ed. Institut Universitaire Roumain Charles I ${ }^{\text {er }}$, t. III, 1955, p. 3-12.

TATON René, « Pierre Sergescu, artisan de la collaboration internationale en Histoire des sciences » Pierre Sergescu (1893-1954), Leiden, Ed. J. Brill, 1968, p. 20-30. (Janus, t. 55, 1968). 
TATON René, « Pierre Sergescu, son œuvre en Histoire des sciences et son action pour la renaissance des Archives internationales d'histoire des sciences", Archives internationales d'histoire des sciences, vol. 37, 1987, p. 104-119.

\section{NOTES}

1. La session d'hommages intitulée: Petre Sergescu, artizan al cooperării internaţionale în domeniul istoriei ştiinţei (Petre Sergescu, artisan de la coopération internationale dans le domaine de l'histoire des sciences), initiée par Mme M. Stavinschi, a eu lieu le 24 mai 2018. Les neuf communications présentées sont publiées dans la revue Academica, $\mathrm{n}^{\circ}$ 5-6, mai-juin 2018. Un prix annuel «Petre Sergescu », pour les meilleures publications en histoire des sciences et des techniques, a été également créé à cette occasion.

2. M. Stavinschi, Petre Sergescu şi Gândirea Matematică.

3. V. Issarescu, « Pierre Sergescu (1893-1954). Son adolescence à Turnu Severin », p. 12.

4. A. Herlea, «Petre (Pierre) Sergescu (1893-1954), un artisan de la coopération internationale en histoire des sciences », p. 14.

5. P. Sergesco, Sur les noyaux symétrisables.

6. P. Montel, « Pierre Sergescu, mathématicien », p. 46-47.

7. G. S. Andonie, «Petre Sergescu (1893-1954)», p. 374 et p. 378 ; D. Duca, A. Petruşel, «Petre Sergescu - Profesor la Universitatea din Cluj », p. 75-77.

8. E. Nicolaidis, «Petre Sergescu, président et secrétaire perpétuel de l'Académie internationale d'histoire des sciences», p. 71-74; R. Taton, "Pierre Sergescu, son œuvre en histoire des sciences et son action pour la renaissance des Archives internationales d'histoire des sciences ", p. 114-115.

9. P. Montel, «Pierre Sergescu, mathématicien », p. 47 ; G. S. Andonie, «Petre Sergescu (1893-1954)», p. 381-383; D. ştefănescu, « Moştenirea matematică a lui Petre Sergescu », p. 87-89.

10. P. Montel, "Pierre Sergescu, mathématicien», p. 48; G. S. Andonie, «Petre Sergescu (1893-1954)», p. 374-375 ; D. Duca, A. Petruşel, «Petre Sergescu-Profesor la Universitatea din Cluj», p.77-78 ; M. Stavinschi, «Petre Sergescu şi Gândirea Matematică », p. 21-22.

11. R. Taton, «Pierre Sergescu (1893-1954)», p. 78.

12. P. Montel, «Pierre Sergescu, mathématicien », p. 48.

13. P. Montel, « Discours prononcé aux funérailles de Pierre Sergescu », p. 5-6.

14. M. Stavinschi, Petre Sergescu şi Gândirea Matematică, p. 16-17. Les deux parents de Maria Kasterska avaient des origines françaises. La famille paternelle, originaire du Gers, s'appelait Casterra et avait émigré en Pologne au xvII siècle ; le grand-père maternel était un officier de Napoléon.

15. N. Capitaine (Taton), Pierre Sergescu collaboration scientifique avec René Taton et liens d'amitié avec la famille Taton; G. S. Andonie, «Pierre Sergescu (1893-1954)», p. 7 et 9.

16. M. Stavinschi, Petre Sergescu şi Gândirea Matematică, p. 38.

17. W. F. Sierpiński, « Témoignage », p. 62-63. 
18. R. Taton, «Pierre Sergescu, artisan de la collaboration internationale en histoire des sciences ", p. 20- 21.

19. J. Itard, "Pierre Sergescu, historien des mathématiques", p. 40-44. R. Taton; «Petre Sergescu (1893-1954) », Bulletin Scientifique Roumain, p. 11 ; G. S. Andonie, «Petre Sergescu (1893-1954)», p. 384.

20. Pierre Sergescu (1893-1954), "Bibliographie des publications de Pierre Sergescu ", p. 64-73 ; A. Herlea, « Petre (Pierre) Sergescu (1893-1954), un artisan de la coopération internationale en histoire des Sciences », p. 17.

21. P. Sergescu, "L’Université Roumaine de Cluj en Exil», Archeion XXIV, 1942, p. 284-288; R. Taton, « Petre Sergescu (1893-1954)», p. 6.

22. R. Haleux, B. Severyns, Twenty-Five Years of International Institutions, p. 315-316.

23. R. Taton, « Pierre Sergescu, son œuvre en histoire des sciences et son action pour la renaissance des Archives internationales d'histoire des sciences", p. 108-110; A. Herlea, «Petre (Pierre) Sergescu (1893-1954), un artisan de la coopération internationale en Histoire des Sciences ", p. 16.

24. R. Taton, «Pierre Sergescu, artisan de la collaboration internationale en histoire des sciences », p. 23; Documents officiels, Archives internationales d'histoire des sciences, $\mathrm{n}^{\circ} 1$, octobre 1947, p. 132-140.

25. Documents officiels, Archives internationales d'histoire des sciences, $\mathrm{n}^{\circ} 2$, janvier 1947, p. 312-316 ; R. Taton, « Pierre Sergescu, artisan de la collaboration internationale en histoire des sciences », p. 25-26.

26. P. Costabel, « Pierre Sergescu, directeur des Archives internationales d'histoire des sciences », p. 30- 37 ; R. Taton, «Pierre Sergescu, son œuvre en histoire des sciences et son action pour la renaissance des Archives internationales d'histoire des sciences", p. 112-119.

27. R. Taton. «Pierre Sergescu, artisan de la collaboration internationale en histoire des sciences ", p. 26.

28. http://caphes.ens.fr/IMG/file/InventaireAIHS.pdf. Voir aussi : Archives internationales d'histoire des sciences numéros parus dans les années 1947-1954.

29. R. Taton, «Pierre Sergescu, artisan de la collaboration internationale en histoire des sciences », p. 24 ; J. Itard, « Pierre Sergescu, historien des mathématiques », p. 42.

30. J. Itard, «Pierre Sergescu, historien des mathématiques », p. 38-39; G. Bouligand, "Le directeur d'études et son rayonnement », p. 52.

31. G. S. Andonie, «Petre Sergescu (1893-1954)», p. 381. Marya Kasterska a réalisé la bibliographie de l'œuvre mathématique et d'histoire des sciences de Petre Sergescu pour laquelle elle a reçu, en 1961, le prix d'Aumale de l'Académie Française des sciences, rapporteur Louis de Broglie.

32. A. Herlea, "Petre Sergescu, personalitate reprezentativă a exilului romanesc", p. 81-86; I. Calafeteanu, Exilul românesc. Erodarea speranţei. Documente (1951-1975), p. 105-166.

33. R. Taton, "Pierre Sergescu, artisan de la collaboration internationale en Histoire des sciences », p. 24 ; M. Stavinschi, Petre Sergescu şi Gândirea Matematică, p. 43-45; B. Nicolescu, « Un cuplu mitic : Petre Sergescu-Marya Kasterska », p. 29-32. 
34. A. Herlea, «Petre (Pierre) Sergescu (1893-1954), un artisan de la coopération internationale en Histoire des Sciences»; A. Herlea, «Petre Sergescu : personalitate luminoasă a Exilului românesc ».

35. R. Taton, « Petre Sergescu (1893-1954)», Bulletin Scientifique Roumain, p. 12.

36. P. Montel, « Discours prononcé aux funérailles de Pierre Sergescu », p. 7.

37. R. Taton, «Pierre Sergescu, son œuvre en histoire des sciences et son action pour la renaissance des Archives internationales d'histoire des sciences », p. 112.

38. A. Herlea, «ICOHTEC, 50 Years. Tribute to Maurice Daumas and Petre Sergescu ", p. 25.

\section{RÉSUMÉS}

Mathématicien de haut niveau qui a joué un rôle de premier plan dans le développement institutionnel de l'histoire des sciences et des techniques, Petre (Pierre) Sergescu a été tout au long de sa vie un intellectuel engagé, promoteur des grandes valeurs européennes et attaché à ses origines. L'article porte sur la vie et l'œuvre de cette personnalité lumineuse des milieux intellectuels de l'Europe d'avant et surtout d'après la Seconde Guerre mondiale quand elle a été coupée en deux par le rideau de fer installé par les Soviétiques. Il concerne davantage, le titre l'indique, les réalisations de Petre Sergescu en tant qu'historien des sciences et promoteur de la discipline que les autres aspects de sa prodigieuse activité, dont celle de farouche opposant à tous les totalitarismes et grand Roumain. Son rôle dans la création et le développement des institutions qui ont permis aux jeunes disciplines d'histoire des sciences et d'histoire des techniques de se développer et d'être reconnues comme disciplines académiques a été de la plus grande importance. Ceci est mis en évidence par les responsabilités exercées par P. Sergescu de président puis secrétaire perpétuel de l'Académie Internationale de l'Histoire des Sciences - AIHS et de secrétaire exécutif puis secrétaire général de l'Union internationale d'histoire des sciences - UIHS.

\section{AUTEUR}

\section{ALEXANDRE HERLEA}

Professeur émérite à l'Université de technologie Belfort Montbéliard (UTBM), membre de l'Académie Internationale d'Histoire des Sciences (AIHS), membre émérite du Comité des travaux historiques et scientifiques (CTHS), ancien président de l'ICOHTEC (International Committee for the History of Technology) 


\title{
Le « moment
}

\section{vulgarisateur français »: enjeux et modalités de la diffusion du savoir scientifique à la Libération}

\author{
Robert Belot
}

1 La fin de la Seconde Guerre mondiale et de l'Occupation n'est pas seulement pour la France une libération politique; c'est aussi l'avènement d'un nouveau rapport à la science et à la technique qui traduit une réconciliation de la société avec les vertus de la raison et avec la foi dans le " progrès ». La diffusion de la science alliée à la technique devient la source principale de la rééducation de la Nation et la garantie de sa renaissance. Le point de cristallisation est la révolution atomique révélée dramatiquement par Hiroshima et Nagasaki.

2 Ce qui étonne, au lendemain de la guerre, c'est la rapidité et la profondeur de la croyance en la capacité de la France de faire de l'atome l'arme absolue de son redressement industriel et de la préservation de son «rang». Une prescience largement partagée par le monde scientifique, relayée par les auteurs vulgarisateurs et diffusée par les médias de l'époque. Un large consensus s'organise autour de l'intérêt qu'il y a à créer une industrie nucléaire, comme si le relèvement et l'avenir de la France en dépendaient. On assiste à un engouement dans la société pour la modernité et la science en général qui indique une confiance en l'homme et son imagination créatrice. La puissance mythico-poétique de la science fait rêver et suscite une abondante et précoce littérature.

3 Le souci de démystifier et de populariser la découverte de l'énergie atomique est traversé par un élan utopique qui a pour effet de magnifier la "mystérieuse " énergie. La vulgarisation scientifique connaît un essor jamais vu. Le souci du "grand public", comme on dit alors, domine. Les savants ont la passion de la transmission et de la médiation ; les non-savants, journalistes et essayistes, découvrent dans la vulgarisation une nouvelle source d'inspiration et de revenus. C'est ce phénomène paradoxal qui préside à la naissance du mythe nucléariste et à sa diffusion dans le corps social. 
L'attitude de Frédéric Joliot-Curie, alors nouveau directeur du CNRS et bientôt HautCommissaire au Commissariat à l'énergie atomique, résume bien cette foi en la science malgré l'horreur. Le 18 décembre 1945, devant l'Académie nationale de Médecine dont il est membre, il déclare :

«Hélas! C'est par le fracas de l'explosion de Hiroshima que cette nouvelle conquête de la science nous fut révélée. En dépit de cette apparition terrifiante, je suis convaincu que cette conquête apportera aux hommes plus de bien que de mal. C'est pourquoi je me sens plus à l'aise pour en parler dans cette maison, devant cette compagnie dont l'idéal et l'action ont une si haute valeur humaine. »

Il semble que l'opinion éclairée soit sur la même ligne, si l'on en juge par le journal Le Monde qui annonce, le 20 décembre 1945 :

«Que le monde fasse confiance aux physiciens, l’ère atomique commence seulement. »

La techno-science allait sauver la France, et Frédéric Joliot-Curie prend figure de prophète.

\section{Les savants répondent à une volonté de savoir}

7 La dimension extraordinaire de la révélation atomique suscite une évidente fascination et provoque une envie de savoir et de faire savoir. Il y a une réelle attente sociale sur les nouvelles possibilités de la science.

\section{Une volonté de savoir}

C'est pourquoi Joliot-Curie, avec son statut de grand maitre du nouveau monde, est accablé de sollicitations en tout genre. Les grands éditeurs parisiens le sollicitent, soucieux à la fois de bénéficier de son prestige et de vulgariser une science nouvelle. Gaston Gallimard en personne l'entreprend, comme en témoigne cette lettre écrite 10 jours après Hiroshima par lui-même :

«Ce n'est pas un éditeur qui vous écrit, mais quelqu'un qui n'a aujourd'hui qu'une passion, celle de faire connaître par les moyens dont il dispose, ce qui dans tous les domaines témoigne encore d'une suprématie française. »

Charles Flammarion, fondateur de la maison d'édition du même nom, l'invite à déjeuner pour le convaincre de publier un livre «sur la physique nucléaire » dans la célèbre collection "Bibliothèque de philosophie scientifique "; le directeur de celle-ci lui envoie un contrat (que Joliot ne signera pas) :

«Ce serait, j'en suis sûr, un succès considérable qui ajouterait au lustre de la science française et à son prestige dans le monde... »

Dans les années d'après-guerre, la presse se l'arrache. Les invitations de conférences, de France et d'ailleurs, pleuvent. Un des leaders du Parti communiste français, résistant authentique, Étienne Fajon, écrit à Joliot pour lui demander de faire une conférence pour les cadres du Parti (8 novembre 1945). Les associations d'anciens résistants cherchent sa présence et son onction pour rehausser leurs comités d'honneur. De nombreuses autres associations et sociétés, aux raisons sociales les plus inattendues, tentent aussi de le séduire, comme, par exemple, la Société des amis de l'école de Bergeries de Rambouillet, sur l'amicale pression de Waldeck Rochet, directeur de la revue La Terre... 
11 Cette appétence de vulgarisation et ce désir de transmission/médiation ont également pour source la croyance selon laquelle la nouvelle science, soucieuse de réalisations techniques, peut avoir un effet bienfaisant sur la vie des gens. Il y a une impatience à connaître ce qui va pouvoir changer la vie. La science et la technique ne sont plus le monopole d'un cénacle coupé de la société. L'autre élément à prendre en compte pour comprendre ce phénomène est à chercher du côté des savants qui bénéficient alors d'un capital social de confiance inédit. Les savants, à l'image d'un Joliot, sont alors très engagés politiquement, et ils croient en leur mission citoyenne.

\section{Les savants et les ingénieurs vulgarisent et dédramatisent}

12 Les chercheurs sortent de leur laboratoire pour faire œuvre de pédagogie. Dès 1945, Jean Thibaud, directeur de l'Institut de physique atomique de Lyon, publie Énergie atomique et Univers. Du microscope électronique à la bombe atomique, et il réédite la même année Vie et transmutations des atomes (collection A. George, Albin Michel, Paris, $3^{\mathrm{e}}$ édition, 1945). Il met en valeur les perspectives bienfaisantes de l'utilisation pacifique de l'énergie nucléaire qui va transformer l'économie, renouveler le problème de la distribution d'énergie et de son accessibilité (quasi-gratuité du chauffage, des transports publics, de la force motrice dans les ateliers...). De nombreux autres livres suivront, techniques et didactiques: L'atome, source d'énergie (Marcel Boll, 1946); Les rayons cosmiques: les mésotons (Louis Leprince-Ringuet, 1946) ; La matière, sa constitution, sa désintégration. La Bombe atomique, l'énergie atomique (René Coppens, 1946); La physique du noyau dans certains pays d'Europe, durant la période 1939-1945 (Charles Manneback, 1946); De l'atome à l'énergie nucléaire (André Berthelot, 1947, préface de F. Joliot) ; Dix ans d'application de la radioactivité artificielle (Pierre Süe, 1948, préface de F. Joliot). En 1948, l'éditeur Hermann, dans sa célèbre collection "Actualités scientifiques et industrielles ", consacre un volume à la question patronné par le CNAM : Structure des atomes et énergie atomique. Les plus grands chimistes et physiciens du moment apportent leur contribution : Francis Perrin, Louis de Broglie, Irène Curie, René Dubrisay, Louis le Prince-Ringuet... Un des collaborateurs de Joliot, chef du laboratoire de radiobiologie à Marseille, Maurice E. Nahmias, met à la disposition du public français les principaux textes américains (qu'il traduit) sur la question de la bombe atomique avec L'Énergie atomique et son utilisation militaire, documents officiels (1946). Les jeunes chercheurs ne sont pas en reste. Ainsi, Léopold Vigneron et Raymond Chastel, tous deux attachés de recherche au laboratoire de Joliot au Collège de France, publient en 1949: L'énergie atomique, promesse ou calamité?

La presse s'en fait généreusement l'écho, car elle croit aussi à sa mission de médiation et d'éducation. Elle salue l'effort pédagogique des savants, comme celui de Marcel Boll dans son livre L'Atome, source d'énergie :

«L'historique des recherches alliées sur la fission de l'uranium et sur la fabrication $\mathrm{du}$ plutonium, ainsi que quelques considérations sur les possibilités d'avenir terminent cet ouvrage rédigé sous une forme accessible au grand public et illustré de graphiques suggestifs.»

14 Les ingénieurs apportent leurs lumières aux profanes. Ils vulgarisent la nouvelle science et n'hésitent pas à se faire futurologues. Marcel Demontvignier, ingénieur de l'ESPCI, publie L'Énergie nucléaire et ses applications récentes (1946). Daniel Florentin écrit La Bombe atomique et l'Énergie nucléaire (1946), préfacé par Joliot-Curie et édité par l'Union des ingénieurs et techniciens français. En général, le ton des ingénieurs est 
confiant mais sobre. Mais certains se laissent emporter par la course aux anticipations. $\mathrm{Au}$ Grand Orient de France, l'ingénieur André Samuel intervient sur le sujet suivant: "Où en est la question de l'énergie atomique? Son influence sur l'avenir de l'homme». Sa conférence est éditée sous forme de brochure en 1947.

L'occasion de se laisser aller à des vues anticipatrices voire délirantes: les marchandises à «vil prix » seront transportées par «fusées transcontinentales»; on pourra modifier la croûte terrestre, faire disparaître des collines; des "paysages artificiels grandioses " passeront du rêve à la réalité ; les déserts ne seront plus une fatalité ; les courants aériens pourront être modifiés ; les saisons seront à façon... Tout se passe comme si l'utopie était à présent à la portée des hommes. Le physicien René Coppens, dans son livre La matière, sa constitution, sa désintégration. La Bombe atomique, l'énergie atomique (1946), croit dur comme fer que l'uranium pourra servir le chauffage domestique :

«Les provisions de bois et de charbon ne seront plus nécessaires car les architectes auront soin d'encastrer dans les murs des maisons quelques morceaux d'uranium qui, en se désintégrant, produiront une douce chaleur... »

On imagine le pire, pour croire que le meilleur arrivera. Comme Daniel Declos avec La Bombe atomique: Vers la destruction totale ou le paradis terrestre? (1945) ou Édouard Jacquet avec Vers la fin du monde par la bombe atomique ou vers un avenir merveilleux? (1945). Maurice Déribéré résume la problématique dans le titre de son livre : L'énergie atomique. La bombe atomique annonce-t-elle notre fin prochaine ou le début d'une nouvelle ère? (1945). Il penche vers la deuxième hypothèse. Car pour lui, Hiroshima a rendu «la guerre impossible » :

"C'est peut-être à cette splendeur que nous convie la menace de la bombe atomique.»

Déribéré publie deux autres livres sur le sujet: Expériences atomiques (éditions Elzévir, 1946) et L'Uranium - la clef et la source des énergies nucléaires (Elzévir, 1946). C'est un ingénieur chimiste, qui a travaillé essentiellement dans l'industrie. Il est spécialiste de la couleur... Il incarne parfaitement le type du technologue humaniste affamé de vulgarisation sur les sujets les plus divers. Tout rationaliste qu'il est, il proclame sa foi en l'avenir de l'atome ("réservoir inépuisable ) annonciateur de paix et de prospérité, qui va " ouvrir une ère nouvelle de notre civilisation », malgré la bombe, voire grâce à elle.

\section{Répandre dans l'opinion publique la bonne parole de la félicité technoscientifique}

18 Jamais les "savants » n'ont été autant sollicités pour conférencier et causer afin de répondre à cette appétence nouvelle pour la science et la technique. La science sort des cénacles académiques. Les papiers privés de Joliot-Curie montrent qu'il est harcelé et se trouve dans l'obligation de renoncer très souvent. Mais il donne de très nombreuses conférences, devant des publics très divers avides de connaissances.

19 "Il existe actuellement en France un mouvement en faveur de la science qui facilite notre travail » reconnaît Joliot dans une lettre du 4 décembre 1945 à son collègue danois Niels Bohr. Il confirme cette tendance à un autre collègue, Bruno Pontecorvo, le 20 février 1946 :

«En ce qui concerne le développement de la recherche scientifique en France, un progrès très sensible a déjà été obtenu depuis la Libération grâce à l'ardeur de tous et à l'opinion publique favorable ${ }^{1} . .$. " 
cespondance des savants, et notamment celle de Joliot (le plus médiatique donc le plus sollicité), témoigne de cet engouement pour la modernité que représente l'énergie atomique. Deux jeunes lyonnais lui envoient, dix jours après Hiroshima, le manuscrit d'un roman qui aurait dû s'intituler : Jean Brédy, l'homme nouveau. Dans l'avant-propos, on lit ceci :

« La science avance, à pas de géant, et semble vouloir livrer aux savants les derniers secrets de la Nature [...] Ainsi, la bombe atomique, employée pour réduire le Japon, ouvre les yeux aux plus incrédules, aux plus arriérés... Ainsi, la désintégration de la matière, la captation de l'énergie intra-atomique, mettent une puissance formidable, inouie, entre les mains des hommes. ${ }^{2}$ "

Le fait marquant est que les savants et les enseignants s'impliquent intensément dans cette campagne de popularisation de la nouvelle science. Il y a ceux qui sont peu connus. À l'instar de A. Fouillé, professeur de physique et d'électricité à l'École nationale des Arts et Métiers qui prononce une conférence le 16 décembre 1945, publiée sous le titre : La désintégration atomique (Angers, 1945, 47 p.). Il commence par rappeler une évidence : depuis la bombe, l'atome est devenu le grand sujet de conversation des Français, dont on parle dans la rue ou dans les salons. Il est persuadé que la révolution atomique se présente comme «le plus heureux événement de l'histoire de l'humanité ». Dans cette conférence, l'orateur rappelle que la première intervention publique de Joliot-Curie sur ce thème a provoqué une quasi-émeute à la Sorbonne où l'on a vu « un véritable embouteillage de piétons et de voitures». Les professeurs provinciaux connaissent aussi du succès. C'est le cas de Marcel Laporte, chargé de cours en électricité et magnétisme à l'École supérieure de la métallurgie et de l'industrie des mines de Nancy. Dans l'introduction au fascicule qui reprend ses conférences prononcées à Nancy en 1946, il s'étonne que :

«La curiosité du public s'est montrée si avide qu'il a fallu répéter trois fois cet exposé dans une des plus grandes salles de la ville.»

La question est mise sur la place publique dans le cadre des "Grands Débats de Paris » qui, le 12 mars 1949, au palais de la Mutualité, organisent une discussion sur le thème : «L'énergie atomique: promesse ou calamité ?» Des noms prestigieux sont sollicités pour ce débat : Louis de Broglie, Raymond Chastel, Leprince-Ringuet, Léopold Vigneron, Yves Farge, Goldschmidt, André Labarthe «qui ont participé aux expériences de Bikini ", l'ingénieur Jacques Labeyrie pour la revue Atomes, et Rougeron pour la revue Sciences et Vie. Francis Crémieux dirige le débat. Il s'agit en fait de faire un sort à l'angoisse de la «calamité», en désamorçant l'image d'apprentis sorciers que pourraient avoir les physiciens, nouveaux maîtres du monde moderne. Évoquant certains augures imaginant l'anéantissement de la terre suite à une réaction en chaîne non contrôlée, ils posent la question : «Les physiciens feront-ils sauter la terre ? » Une expérience peut-elle transformer la terre en une supernova? C'est pour aussitôt s'inscrire en faux contre ce mythe négatif et purement fantasmatique :

«Le physicien moderne n'est pas plus un danger public que ne le fut l'inventeur du feu, il y a quelque vingt mille ans. Celui-ci risquait de faire un incendie de forêt ou de prairie qui aurait grillé sa tribu, mais n'aurait pas incendié toute la terre et détruit toute vie à sa surface. »

Conclusion :

«Il n'y a aucun risque d'accident pour la planète. » 


$$
\begin{aligned}
& \text { pourtant réputé austère et peu abordable pour le profane. On pourrait même affirmer } \\
& \text { qu'une nouvelle catégorie de journalistes et d'essayistes se constitue et occupe le } \\
& \text { champ éditorial : les vulgarisateurs scientifiques. }
\end{aligned}
$$

\section{Les non-spécialistes à la quête du grand public}

29 La question énergétique en général attise la plume de certains citoyens éclairés qui, bien que non-experts, cherchent à comprendre et qui pensent que leur avis peut être utile. L'enjeu est difficile, comme le mentionne le journal La Vie française en commentant le livre de Géraud-Jouve (L'âge atomique) en 1946 :

«Devant le mystère atomique, les auteurs sont parfois embarrassés : s'ils optent pour l'exposé scientifique, les profanes ne les suivent pas; s'ils transigent sur le chemin de la vulgarisation, ils déroutent à la fois les lecteurs sérieux et les lecteurs superficiels. »

Outre la question de la reconstruction politique de la France, c'est l'enjeu énergétique qui occupe les esprits, les médias et les intellectuels. Comment le pays peut-il renaître et retrouver son indépendance et sa puissance?

31 Henri Géraud-Jouve, agrégé d'allemand devenu journaliste puis député SFIO du Cantal, publie un petit livre entièrement consacré à l'atome : Voici l'âge atomique (Franc-Tireur, 1946). Il entend modestement faire œuvre de "chroniqueur" pour raconter à ses « arrière-petits-enfants » « la chronique des premières semaines de l'âge atomique ». Il s'adresse au " grand public » pour qu'il puisse « avoir plaisir et profit à relire d'un trait les pensées et les controverses qui ont agité le monde au début de cette ère nouvelle ». Seront convoqués «savants ", « industriels ", « illuminés et même charlatans ». Il faut que les Français soient sensibles à la "place» et à "la chance de la France dans le monde à l'âge atomique ». Son livre se termine ainsi :

« Ruinée et peu apathique, la France, qui n'a encore perdu ni le sens ni le goût de la grandeur, aspire, au seuil de l'âge atomique, à reparaître dans le cercle des grandes nations par la grande porte de la science. » 
a question du nucléaire, trois vulgarisateurs à succès émergent du lot: Pierre Rousseau, Albert Ducroq et Georges Sadoul. La présence de ce dernier sur le front de la vulgarisation de l'énergie atomique étonne : il est en effet connu aujourd'hui pour avoir été un grand historien du cinéma. Évoluant dans les milieux communistes, le quotidien L'Humanité lui demande de lancer une grande enquête sur la conquête atomique qui se déploie en feuilletons au printemps 1946. Dès les premiers mots de cette longue enquête (" Mystère et puissance de l'atome »), Georges Sadoul salue le retour en grâce de cette figure maudite de notre mythologie et veut en donner une interprétation positive :

«Mais la légende de Prométhée dérobant aux dieux la première étincelle de feu pour animer une statue d'argile et créer notre espèce, n'a-t-elle pas pour sens que l'homme n'est vraiment devenu un homme que le jour où il l'a domestiqué pour se chauffer et faire cuire ses aliments? L'homme, ce fut d'abord pendant des millénaires, le maître du feu, et le feu contribua à le distinguer définitivement des animaux. »

La science est à nouveau sagesse et promesse. Ces articles, très bien documentés, sont réunis en 1947 dans un livre qui connaît un grand succès sous le même titre. L'auteur «reconnaît volontiers n'avoir aucune connaissance particulière en physique atomique » et " prétend avoir seulement fait œuvre de vulgarisateur ». Sadoul apporte à Joliot-Curie un soutien médiatique de première importance. La conclusion de ce livre est qu'il faut que la France se lance totalement dans la mise en place d'une «industrie atomique» pour des raisons d'indépendance et de puissance et pour assurer «la supériorité des Européens » :

«Ce qui est dire que, si la France veut rester au rang des grandes puissances, elle doit créer au plus vite son industrie atomique, reprendre, avec des moyens puissants, les travaux que l'invasion a brusquement interrompus en 1940. »

Il faut donc arrêter de " maudire Prométhée ». Un autre vulgarisateur prolifique publie une Histoire de la Science (Fayard, 1945) qui connaît un grand succès. Il s'agit Pierre Rousseau, qui signale la confiance que le public place en la science et reconnaît que celle-ci « jouit d'une si grosse influence ${ }^{3}$ ». Il peint un tableau idyllique et irénique de l'humanité arrivée à l'âge atomique. C'est tout le rapport à l'énergie qui en est bouleversé :

«L'énergie ne coûte plus que les frais d'installation des centrales ou le prix d'achat des moteurs. Du coup, personne ne veut plus de charbon, le prix du pétrole est tombé à quelques centimes et les grands barrages ont été abandonnés. »

L'impact est aussi social. L'auteur est convaincu qu'une énergie si peu chère va transformer le monde capitaliste et la vie des ouvriers :

«L'ouvrier ne fait plus qu'une heure de travail par jour; encore ce travail ne consiste-t-il le plus souvent qu'à monter la garde devant une aiguille oscillant sur un cadran."

Deux ans après, les éditions Fayard éditent du même auteur une Histoire de l'atome en 359 pages, qui sera plusieurs fois rééditée. Les éditeurs et les vulgarisateurs ont compris qu'il fallait s'engager sur la vague de la «technophanie » et répondre à l'attente du public.

37 C'est dans cette atmosphère qu'un journaliste d'exception entame une brillante carrière de chroniqueur scientifique : Albert Ducroq. Les plus anciens se souviennent de sa voix caverneuse sur Europe 1. Il publie chez Calmann-Lévy : Les horizons de l'énergie atomique en 1948. L'éditeur présente ainsi l'ouvrage : 
«Pour l'homme cultivé qui a oublié ses mathématiques, comme pour le théoricien

le plus exigeant, voici le tout dernier état des recherches atomiques. »

\section{Démystifier, populariser, vulgariser, mais aussi éduquer}

faut vulgariser, mais aussi éduquer. Les cours que donnent Joliot-Curie et Francis Perrin au Collège de France ne touchent qu'une infime partie de la population. Il faut élargir la «zone de chalandise ». Le 21 mars 1946, avec la bénédiction du ministre de l'Éducation nationale, le comité de direction du Palais de la Découverte décide d'organiser une exposition sur «l'Atome ", où une salle entière sera dédiée à l'œuvre de Jean Perrin. Le ministère de l'Information (Radiodiffusion française) relaie une initiative de l'Alliance française qui souhaiterait que Frédéric Joliot enregistre sur disque « une déclaration de 3 minutes » sur ses travaux, destinée à être écoutée par les élèves des collèges français à l'étranger :

«Je me permets d'insister sur l'importance de cet enregistrement pour la propagande française à l'étranger... »

La direction de l'Enseignement du second degré organise en mai 1947 un « congrès des humanités scientifiques » au cours duquel des inspecteurs généraux et des professeurs de lycées discuteront de la nouvelle orientation à donner à l'enseignement des sciences, "ainsi que des rapports entre la culture humaniste et la culture scientifique». Des spécialistes du nucléaire y sont invités. On demande à Joliot de présider cette manifestation. En cette même année 1947 est divulgué le Plan Langevin-Wallon. Ce projet de réforme en profondeur du système éducatif français, inspiré par le programme du Conseil national de la Résistance, est la traduction concrète d'une nouvelle politique qui vise deux objectifs principaux: l'éducation comme valeur démocratique, la science comme vecteur du progrès humain et l'école comme "agent actif» de la «modernisation ». Ce Plan reprend certaines idées des projets de réforme de Jean Zay, au moment du Front populaire, qui tendait à mettre fin à l'hégémonie des Humanités.

Le directeur de la Documentation et de la diffusion du ministère de l'Information, JeanLouis Crémieux-Brilhac, lance un appel le 3 janvier 1946 aux chercheurs :

«Étant chargé par le ministère de l'Information d'organiser des conférences d'information dans la province française, l'utilisation de l'énergie atomique me semble être un des grands enjeux sur lesquels le public désire être renseigné. »

41 Le milieu associatif est très réceptif à cet engouement pour la science et il sert de vecteur de diffusion. La Confédération française des forces culturelles, économiques et sociales (Science, Action, libération), mouvement moderniste créé en 1944, organise une conférence à Oyonnax, à l'été 1946, sur le thème: "La science au service de l'homme». Un certain Georges Bardonnet explique que ce sujet est «le plus passionnant de l'heure ", car il annonce tout simplement la venue d'un "âge d'or » fondé sur des ressources énergétiques illimitées capables de «chasser de la face du monde l'inquiétude, la peur du lendemain, et cette plaie honteuse qui s'appelle la misère ». Les savants français, assure-t-il, apprendront à libérer l'énergie atomique de n'importe quelle substance, comme l'eau, la viande ou les épluchures de pommes de terre... « Anticipations? Oui sans doute. Utopie? Pas du tout! La bombe atomique est un fait, le premier de la série des réalisations pratiques ». Lors de cette conférence, il pressent ce que pourrait être la « dissuasion nucléaire » (que l'on n'appelle pas ainsi) : 
"Heureusement, avec la bombe atomique, les savants ont mis aux mains de gouvernements un élément d'une puissance telle qu'il les place face à un dilemme ultime, définitif : ou bien tuer tout le monde, ou bien vivre avec tout le monde. » Mouvement de jeunesse et d'éducation populaire prononce trois conférences (" L'atome, l'énergie atomique, la bombe atomique »), fin 1945 et début 1946, à Limoges, Pont-St-Étienne et Bellac. Sa conférence inaugurale, organisée par l'Université populaire de Limoges, sera publiée en une petite brochure de 12 pages (Limoges, Impr. nouvelle, 1946) préfacée par Jean Chaintron, un communiste préfet de Haute-Vienne et futur homme politique.

\section{L'engouement médiatique : presse, radio et revues}

Les médias œuvrent en faveur d'une meilleure information sur l'atome et n'hésitent à mettre en débat ce nouveau sujet d'actualité. Ainsi, début 1946, Radio Monte Carlo cherche des intervenants susceptibles de «pouvoir préciser, pour ses auditeurs, certaines questions touchant la désintégration atomique et l'emploi dont elle est susceptible. Nous voudrions notamment pouvoir leur dire, à côté des destructions dont cette nouvelle énergie est capable, les bienfaits qu'elle est susceptible d'apporter à l'Humanité. Nous désirerions également pouvoir leur préciser la part de vérité qui peut exister à l'heure actuelle dans les prétendues applications sensationnelles qui seraient sur le point d'en être faites, tels par exemple, prochain voyage (sic) dans la lune ${ }^{4}$ ».

Il est même arrivé qu'un homme de radio comme Jean Nocher, ancien résistant, alias "professeur Helium », imagine un canular radiophonique dans son émission diffusée sur la «Chaîne Parisienne » simulant une attaque nucléaire. On s'étonne aujourd'hui d'une telle légèreté ! Or, ce n'est pas un cas unique. Pour le quotidien La Voix de Paris, la jeune journaliste et future écrivaine Dominique Desanti est chargée fin août 1945 d'une étrange enquête. À un panel impressionnant de personnalités en vue (Georges Duhamel, Paul Léautaud, général Billotte, général de Lattre de Tassigny, Frédéric Joliot, pasteur Riou, Édouard Herriot, Jean-Paul Sartre et Picasso), elle envoie une lettre. Tout en leur demandant de l'excuser de les « déranger pour un aussi futile propos », elle ose leur poser la question suivante :

«Si vous aviez à votre disposition une bombe atomique, quel usage en feriezvous?»

L'opinion, habituée au pire, était-elle devenue indifférente ? L'atome s'était-il à ce point banalisé qu'il avait réussi à s'émanciper de la bombe? Cependant, des quotidiens lancent de vraies enquêtes pour démystifier la science atomique. À l'instar de L'Humanité et de sa "grande enquête » intitulée : «Mystère et puissance de l'atome ». L'enjeu est le suivant :

«Le grand public considère généralement l'énergie atomique comme une invention diabolique bonne à faire sauter la terre et détruire l'humanité. Nous sommes pareils aux hommes primitifs qui fuyaient le feu considéré seulement comme un fléau. » [Et d'annoncer que] «nous pouvons déjà construire des centrales atomiques qui produiront autant d'électricité que les plus grands barrages hydrauliques. »

Les journaux, assez unanimement, de L'Humanité au Figaro en passant par Le Monde, soulignent les bienfaits à attendre de «la puissance atomique». Parfois, une note dissonante apparaît, comme une exception qui confirme la tendance générale. Ainsi en est-il de Combat, journal créé sous l'occupation par un groupe de résistants et dirigé par 
Camus. Dans le numéro du 8 août 1945, l'éditorial marque sa « réserve » à l'égard du consensus ambiant qui se forme autour, note-t-il avec ironie, $\mathrm{du}$ " roman d'anticipation que les journaux nous proposent »; n'y a-t-il pas quelque « indécence » à célébrer ainsi "une découverte qui se met d'abord au service de la plus formidable rage de destruction dont l'homme ait fait preuve depuis des siècles"? Les revues les plus généralistes sont touchées par cette fièvre techno-scientiste. À l'instar de la revue Les Élites Françaises. Son directeur croit utile d'écrire le 28 août 1945 à Joliot-Curie pour lui annoncer que son équipe s'engage à :

« Donner une place prépondérante à tout ce qui touche la science, persuadés que le public français est plus avide que jamais de pénétrer dans un domaine qui le passionne. "

47 Les revues de vulgarisation scientifique (il s'en crée de nombreuses à cette époque et il faudrait les étudier) s'intéressent à cette nouvelle énergie, comme Sciences et Vie ou Constellations. Les revues spécialisées s'emparent du sujet, comme La Revue d'Optique, ou Atomes, nouveau-né en mars 1946 dans le paysage revuiste (qui devient La Recherche en 1970). Atomes est un peu l'organe officieux du réseau Joliot. Son directeur n'est autre que Pierre Süe, sous-directeur au Collège de France. Ainsi, en 1950, Raoul Dautry, administrateur du CEA, demande d'acheter 200 exemplaires de la revue, qu'il diffusera par ses propres moyens ${ }^{5}$. La publicité présente ainsi Atomes :

"Cette revue traite tous les aspects scientifiques du nouvel âge que nous vivons. Toutes ces questions sont traitées sous une forme compréhensible au grand public, par des spécialistes éminents des questions qui passionnent le monde actuellement. "

Le tirage de lancement est de 35000 exemplaires. L'enjeu est aussi éducatif. La revue Atomes veut soutenir "l'immense effort à accomplir» pour que, de l'école au laboratoire, cette nouvelle science imprègne la société :

«Toutes les disciplines scientifiques et leurs développements techniques y feront l'objet d'études conçues pour éveiller les qualités et le goût inventifs. "

Les articles seront de " qualité », car il s'agit d'une « revue éducative » :

«Les animateurs d'Atomes se sont refusés à prendre la voie facile que trop de marchands d'amusettes scientifiques choisissent. Tout à leur foi de consacrer leur activité à une œuvre des plus utile à notre pays, ils font aussi confiance au lecteur, persuadés que le public éprouve le besoin de s'initier sérieusement, même au prix d'un effort, au merveilleux progrès de son siècle. "

Les revues du champ intellectuel questionnent cette révolution technoscientifique. Comme Esprit, déjà citée, où l'on cultive le scepticisme. Comme aussi la revue Études où domine une franche approbation au développement de la civilisation atomique. Les jésuites sont en phase avec la position de l'Union sociale des ingénieurs catholiques (USIC). Celle-ci, après avoir salué les avancées de la science et «l'élan intellectuel sans précédent » que permet l'aventure atomique, s'exclame :

«Et nous assisterions, nous chrétiens, en spectateurs, sympathiques sans doute, mais en un sens inactifs, à cette incontestable ascension de l'humanité ! »

51 Le 10 décembre 1945, la revue consacre un article à « La bombe atomique ». Teilhard de Chardin s'exclame :

"Comme si le devoir de tout homme ne consistait pas en définitive à pousser jusqu'au bout toutes les puissances créatives de la connaissance et de l'action! [...] Comment, en face de ce succès, ne se sentirait-il pas exalté, comme jamais depuis sa naissance?» 


\section{Le domaine artistique et cinématographique}

La littérature populaire s'en empare rapidement. Jacques Dasit, par exemple, ouvre la veine en publiant en 1946 avec On a volé la bombe atomique. Il n'est pas jusqu'au chansonnier Christian Vebel qui écrit et joue au Théâtre des Deux-Ânes à Paris en 1947 : La Bombe atomique à la portée de tous, comédie en 1 acte. L'univers candide de la bande dessinée participe à cet engouement technologique et atomique. De Cyclone, l'homme atomique à Atome Kid en passant par L'Atomic, « le magazine dynamique ».

Les artistes ne restent pas insensibles au renouveau des formes picturales que cette libération massive et spectaculaire d'énergie peut provoquer. Peintres et sculpteurs semblent moins sensibles. Salvador Dali est le premier d'entre eux. Il abandonne le surréalisme au profit d'une "mystique atomique ", dès 1948, à travers des toiles majeures, dont Leda atómica. Au même moment, en Italie notamment, des artistes s'intéressent à la désintégration des formes. À l'instar de l'Italien antifasciste Enrico Baj, séduit par cette rencontre de l'atomisme et de l'automatisme permettant d'explorer des "infra-mondes ». Il fonde le "Mouvement nucléaire » (1951), où l'on retrouve Yves Klein et Asger Jorn, et il publie en 1952 avec Sergio Dangelo le Manifeste de la peinture nucléaire. Ce mouvement, d'obédience surréaliste, doit beaucoup à l'œuvre du peintre Voltolino Fontani, intitulée « Dinamica di assestamento e mancata estasi » (1948), où l'artiste tente de représenter le phénomène de radiations au lendemain d'une explosion nucléaire.

4 Les photographes sont intrigués par le phénomène. Le magazine Regards, émanation du Parti communiste français, soucieux d'occuper le créneau du photojournalisme, s'y intéresse régulièrement. Robert Doisneau y publie des photos des Joliot-Curie et des mystérieuses machines atomiques.

5 Le cinéma, qui devient au lendemain de la guerre une source populaire de savoir et de distraction, participe à l'engouement atomique et à la diffusion de l'idée de progrès. Par lui, cette énergie énigmatique accède à la notoriété et au grand public. Dès 1945 , le Conservatoire national des Arts et Métiers engage le grand réalisateur de documentaires scientifiques, Jean Painlevé, pour animer son Centre de production de films scientifiques et techniques. Painlevé entend achever la réalisation d'un documentaire, Solutions françaises, dont l'idée initiale, en 1939, était de contrebattre la propagande nazie qui présentait la France comme un pays rural impropre à une activité scientifique de haut niveau. C'est tout naturellement qu'il fait appel à Joliot en mai 1945 pour présenter son laboratoire de Synthèse atomique. La société de production « TadiéCinéma » se propose de réaliser «un film de vulgarisation scientifique sur l'énergie atomique qui exposerait au public les véritables données de la question et rétablirait les erreurs diffusées par une certaine presse ».

Un film est réalisé par Jacques Dréville: La Bataille de l'eau lourde (1947). Cette présentation héroïque et épique permet de rappeler la part qui revient aux physiciens français (dont Joliot-Curie, qui joue son propre rôle...) dans la naissance de la bombe atomique. Pas l'ombre d'une mauvaise conscience dans cette œuvre qui consacre l'énergie atomique comme l'arme absolue au service de la lutte contre les fascismes et les nationalismes.

Deux ans plus tard, un autre film met à nouveau en scène l'inventeur de la fission, avec d'autres figures du monde scientifique et artistique de la fin des années 1940. Ce docu- 
fiction de Nicole Védrès lui a été inspiré par le livre d'André Labarthe, publié en 1947 : La vie commence demain. Labarthe joue également son propre rôle. Il convie un touriste à laisser de côté les musées pour découvrir les hommes qui vont inventer l'avenir : on trouve Sartre, le psychiatre Lagache qui évoque l'inconscient, Joliot-Curie l'initie aux sciences atomiques; Jean Rostand explique la parthénogenèse; Le Corbusier lui fait visiter la «cité de demain» et Joliot-Curie, ami de Labarthe, initie à la science atomique. Le livre est un hymne au progrès. Il annonce le déferlement en Europe de la modernité américaine: l'automobile, la production automatisée, la société de consommation...

Un consensus se forme à la Libération pour développer la conscience scientifique de l'opinion et pour diffuser les vertus du nucléaire dans l'imaginaire collectif.

Rarement la France a connu un tel rassemblement et un tel engouement autour de la science. L'aube de l'âge atomique français est le point de convergence d'un double mouvement apparemment contradictoire de vulgarisation et de mythification. Le souci de vulgarisation vise à faire comprendre un phénomène qui paraît relever de l'extrahumain, mais aussi à désarmer les peurs qui pourraient s'attacher à cette mystérieuse technologie. Il faut vaincre l'inexplicable face à la révélation d'un monde infiniment petit et dépasser l'innommable que représentent Hiroshima et Nagasaki. Il faut que la fascination soit plus forte que la répulsion. La promesse d'un nouveau monde et de nouvelles prouesses techniques permet cette réconciliation. Le mythe démiurgique de la modernité doit faire oublier «l'enfantement tragique " d'où elle vient.

Le savant veut transmettre, vulgariser, faire aimer la science, et il n'hésite pas à descendre dans l'arène. Il entend donner des perspectives concrètes à ses recherches. L'opinion veut savoir et rêver à un avenir meilleur. Les médias et les éditeurs ont compris qu'ils étaient l'intermédiaire obligé entre ces deux mondes et qu'ils avaient un rôle nouveau de transmission à jouer. Il n'y a pas mieux que les ouvrages de vulgarisation pour révéler le large assentiment social que cette ambition rencontre. La transmission de la science est devenue aussi importante que la science elle-même dans la nouvelle société française qui succède à la guerre, à la défaite, à l'exclusion, à la compromission.

\section{BIBLIOGRAPHIE}

BELOT Robert, L'Atome et la France. Aux origines de la technoscience française, Paris, Odile Jacob, 2015.

BENSAUDE-VINCENT Bernadette, Langevin. Science et vigilance, Paris, Belin, 1987.

BERTHELOT Jean-Michel, MARTIN Olivier, COLLINET Cécile, Savoirs et savants. Les études sur la science en France, PUF, 2005. 
BIQUARD Pierre, Frédéric Joliot-Curie et l'énergie atomique, Paris, L'Harmattan, 2003.

BlocQ-Mascart Maxime, Chroniques de la Résistance, Paris, Corréa, 1945.

BOYER Paul, By the Bomb's Early Light. American Thought and Culture at the Dawn of the Atomic Age, New York, Pantheon Books, 1985.

COHEN Francis, DESANTI Jean, GUYOT Raymond, VASSAILS Gérard, Science bourgeoise et science prolétarienne, Éditions de la Nouvelle Critique, 1950.

DAGOGNET François, L'Essor technologique et l'idée de progrès, Armand Colin, 1997.

Doisneau chez les Joliot-Curie. Un photographe au pays des physiciens, éditions du Musée des arts et métiers/CNAM, 2005.

DUBOIS Joseph, GÉRAUD-JOUVE Henri, La Bataille de l'électricité. Chantages et anticipation atomique, Paris, édité par la S.E.I., 1946.

FAVEZ Jean-Claude et MYSYROWICZ Ladislas, Le nucléaire en Suisse : jalons pour une histoire difficile, L’Âge d'Homme, 1987.

GÜNTHER Anders, Hiroshima est partout, Paris, Seuil, 2008.

LABARTHE André, Statu quo de la peur, éd. Défense de la France, 1946.

PESTRE Dominique, Physique et physiciens en France 1918-1940, Paris, Éditions des Archives contemporaines, 1984.

ROYER Jean-Marc, La science, creuset de l'inhumanité. Décoloniser l'imaginaire occidental, L'Harmattan, 2012.

ROUSSEAU Pierre, Histoire de la Science, Fayard, 1945.

TAGUIEFF Pierre-André, L'effacement de l'avenir, Galilée, 2000.

THIBAUD Jean, Énergie atomique et Univers, Lyon, Audin éditeur, 1945.

TOPÇU Sezin, La France nucléaire. L'art de gouverner une technologie contestée, Paris, Seuil, 2013.

VIGNERON Léopold et CHASTEL Raymond, L'énergie atomique, promesse ou calamité ? Paris, éd. France Documentation, 1949.

WEART Spencer R., Scientists in Power, Cambridge (Massachusetts), Harvard University Press, 1979.

\section{NOTES}

1. Source pour ces deux lettres : Institut Curie, Fonds F. Joliot, F 152.

2. Lettre de Victor Boisson (journal La Voix du Peuple), à F. Joliot, Lyon, 14 août 1945. Institut Curie, Fonds F. Joliot, F 152.

3. P. Rousseau, Histoire de la Science, p. 799.

4. Lettre de Radio Monte Carlo à Frédéric Joliot-Curie, 6 février 1946. Archives du Musée Curie, fonds Frédéric Joliot-Curie (BNF), F 153.

5. Fonds privé Raoul Dautry, Arch. Nat., 307 AP/203 (juillet/décembre 1950). 


\section{RÉSUMÉS}

À la Libération de la France, dès 1945, la science et la technique, à nouveau réunies, sont investies d'une triple mission rédemptrice: tourner la page de la guerre, reprendre confiance et se réconcilier avec l'idée de progrès. En dépit du drame qui l'a enfantée, on salue l'avènement de l'ère atomique et de la technoscience qui doit permettre à la France de renouer avec l'indépendance et la puissance. Les grandes figures qui emblématisent ce tournant «technophanique », comme Frédéric Joliot-Curie, ne doivent pas cacher le mouvement social et politique de grande ampleur qui vise à démocratiser la science et la technique, à éduquer le peuple, mais aussi à ré-enchanter le rapport à la nation et au progrès. Les « savants " acceptent de faire œuvre de vulgarisation; des revues de sensibilisation sont créées ; les bandes dessinées trouvent une nouvelle source d'inspiration; une corporation de «vulgarisateurs " professionnels se constitue. C'est ce «moment vulgarisateur», qui occupe une place si mince dans l'historiographie française, que nous souhaitons évoquer.

\section{AUTEUR}

\section{ROBERT BELOT}

Université Lyon-Saint-Étienne, chaire européenne Jean Monnet « Politiques européennes du patrimoine », UMR CNRS EVS 5600, membre titulaire du CTHS, Section Sciences, histoire des sciences et des techniques et archéologie industrielle 


\title{
Le Plan comme vecteur de
} transmission des connaissances sur l'économie : l'exemple des commissions de modernisation à l'époque des quatre premiers plans (1946-1965)

\author{
Chunhua Chu
}

1 Imaginé par Jean Monnet et entériné par le général de Gaulle, le Plan est établi par le décret du 3 janvier 1946 en France par souci de reconstruire et de moderniser le pays. Une fois la reconstruction achevée, il est orienté vers une meilleure répartition des fruits de croissance. En tant qu'instrument de politique économique, le Plan dessine les grandes lignes de la stratégie adoptée pour développer l'économie dans son ensemble. Sous la forme d'un projet de loi, le Plan informe les agents sur les orientations économiques du pays, mais aussi sur les prévisions à moyen terme.

Ce rôle spécifique d'information et de transmission des connaissances du Plan sur l'économie tient pour une large part aux efforts déployés par les commissions de modernisation. Dès le début, le processus français de la planification repose sur deux innovations bien connues, produits autant de l'imagination de Jean Monnet que des nécessités économiques et sociales de la reconstruction : le Commissariat général du Plan, administration permanente du travail du Plan, et les commissions de modernisation, groupes de travail se réunissant autour du Plan ${ }^{1}$.

Chargées d'élaborer les propositions concernant un secteur déterminé, propositions qui seront soumises au commissaire général qui devra les synthétiser, les commissions de modernisation constituent l'originalité et la base même de la planification française $e^{2}$. Leur composition tripartite permet une confrontation fructueuse des divers points de vue et une collaboration étroite entre pouvoirs publics et catégories socioprofessionnelles. Au cours de leurs travaux, les commissions deviennent autant des 
sources d'information pour le Plan que les instruments de cette pédagogie qu'il faut inculquer aux responsables de l'économie et à travers eux au public ${ }^{3}$.

\section{Organisation et fonctionnement des commissions}

4 À l'initiative de Jean Monnet et avec l'idée d'introduire une triple représentation, les commissions de modernisation du Plan sont créées pour la préparation du $\mathrm{I}^{\mathrm{er}} \mathrm{Plan}$ et ensuite pour chaque Plan. Ouvertes à tous les agents économiques, les commissions de modernisation deviennent une table ronde où les informations sont rassemblées, où les perspectives sont confrontées. C'est ainsi que se met progressivement au point et se perfectionne la méthode de planification souple et incitative, dite « à la française ».

\section{Le choix non décisionnel des membres}

5 En tant que constituantes du pôle consultatif, les commissions de modernisation sont instituées, à l'occasion de la préparation de chaque Plan, par la nomination du gouvernement sur proposition du Commissaire général. Les commissions de modernisation comprennent généralement entre 30 et 80 membres, dont les origines sont bien différentes.

6 Le système du Plan français repose sur la compétence personnelle des hommes qui siègent. Le choix des membres des commissions vise alors à réunir des personnalités à la fois compétentes et représentatives au sens large, mais sans recherche de dosages numériques ${ }^{4}$. L'expérience confirme l'intérêt qu'il y a à associer, dans le cadre des commissions de modernisation, ceux qui, par leurs connaissances ou par leur autorité dans leur profession, peuvent contribuer non seulement à l'élaboration, mais aussi à l'exécution du Plan.

7 Le caractère non décisionnel des membres permet aux différents intéressés de conserver une liberté d'analyse et de jugement d'autant plus grande qu'aucun vote ne vient sanctionner les débats. Cette procédure est parallèle à celle employée dans les réunions syndicales d'entreprise en Union Soviétique ${ }^{5}$. En outre, elle présente l'avantage d'empêcher que les débats ne s'enrayent dès le début sur des positions de principe : parce que les partenaires sont tentés d'approfondir la discussion jusqu'à ce qu'un accord, au moins partiel, se manifeste. L'ambiance est telle que la recherche des concordances l'emporte sur celle des divergences.

8 Étant le lieu même des confrontations de perspectives, les commissions de modernisation représentent au total un effectif nécessairement plus nombreux que le Commissariat général du Plan. Afin de pousser les responsables du Plan aux collaborations extérieures et de les détourner des tentations de la gestion directe, ce dernier ne dispose que d'une quarantaine de «chargés de mission », dont la plupart en outre, n'y font pas carrière. Jusqu'au départ de Jean Monnet, le Commissariat lui-même ne compte pas plus de cent personnes. Alors, pour la préparation du Plan, les commissions de modernisation comptent un effectif total qui est passé d'environ mille participants pour le I ${ }^{\text {er }}$ plan, à un chiffre de l'ordre de 3137 personnes pour le IV ${ }^{e}$ Plan. 


\section{La structure tripartite : la recherche de décision commune}

Conformément à l'idée d'associer l'ensemble des partenaires sociaux à la préparation du Plan, les commissions de modernisation sont essentiellement composées, selon une structure tripartite, des représentants des trois interlocuteurs principaux: les membres des administrations intéressées; les chefs d'entreprise et les représentants des organisations professionnelles; les représentants et les délégués des syndicats ouvriers. Dans certaines conditions, les commissions s'adjoignent des experts, universitaires et techniciens, des représentants des consommateurs, ainsi que des représentants des régions et des municipalités ${ }^{6}$.

Cette composition permet une confrontation des perspectives diverses, une mobilisation des compétences différentes et enfin un rassemblement des renseignements d'ordre économique. En écoutant les points de vue des interlocuteurs sur les grands problèmes, les commissions élaborent des analyses de la situation de leurs domaines respectifs et publient leurs rapports en vue de proposer des orientations pour l'action.

11 C'est au sein des commissions que les hommes s'informent des multiples aspects d'une même réalité ; c'est là que les décisions sont prises. Ces commissions constituent des " tables rondes ", où se réunissent et où discutent des personnes qui, à un titre ou à un autre, sont intéressées à tel ou tel sujet. Les décisions des commissions sont prises à l'unanimité. C'est dans la mesure où les commissions fonctionnent bien que le Plan sera exécuté. Les commissions ne sont pas de petits parlements, mais des groupes de personnalités individuellement responsables : les avis divergents sont notés à la suite des rapports.

\section{Les compétences déterminées et le système décentralisé}

Les commissions sont des organes assez lourds. En vue d'améliorer le traitement des problèmes et de réduire les contradictions, les commissions de modernisation sont toutes organisées selon un système décentralisé.

Organisés et animés par les chargés de mission du Commissariat général du Plan, de nombreux va-et-vient sont établis entre les différentes commissions? ${ }^{7}$. En vue d'améliorer le traitement des problèmes et de réduire les contradictions, les commissions sont assistées par des sous-commissions et des intergroupes ou des comités spécialisés. Cependant, les décisions concernant la préparation du Plan et le rapport final sont prises par la commission elle-même. Prenons l'exemple de la Commission des industries de transformation: elle ne comporte que dix-huit groupes de travail pour le $\mathrm{II}^{\mathrm{e}}$ Plan, mais en compte soixante-quatre dans le III ${ }^{\mathrm{e}} \mathrm{Plan} .^{8}$

Deux sortes de commissions travaillent en même temps. Plus précisément, les commissions verticales, spécialisées par branches ou par secteurs d'activité, tels qu'agriculture, sidérurgie, chimie, habitation, transports, santé, éducation, aménagement, etc., et les commissions horizontales, occupées à des problèmes généraux relevant d'un grand domaine économique ou social, telles que les commissions de la recherche, de la main-d'œuvre, ou de l'aménagement du territoire. 
desquels le Commissariat général du Plan établit ensuite une esquisse globale. Et les secondes s'attaquent aux problèmes de leurs compétences. Par exemple, la commission de la main-d'œuvre veille à l'équilibre de l'emploi par qualification et par région. La commission de l'économie générale et du financement se préoccupe de l'ajustement de l'investissement et de l'épargne, des recettes et des dépenses publiques, des entrées et des sorties de devises ${ }^{9}$. En somme, les commissions et leurs groupes de travail jouissent de la plus grande latitude pour inventorier les problèmes et les difficultés affectant leur champ de compétence, pour expliciter dans leurs rapports les perspectives propres à chaque branche et les objectifs particuliers qui en résultent pour l'année terminale du Plan, et pour suggérer les mesures conditionnant la réalisation des objectifs retenus ou améliorant la capacité compétitive de la branche.

De plus, le nombre des commissions de modernisation s'accroît de manière régulière au fur et à mesure que les problèmes des méthodes de planification permettent d'intégrer dans le plan un plus grand nombre de problèmes de développement, et que les services publics ou les professions estiment eux-mêmes souhaitable qu'ils s'en créent de nouvelles. Par conséquent, on en compte 18 pour le $1^{\text {er }}$ Plan, 25 pour le $\mathrm{II}^{\mathrm{e}}$ et 28 pour le $\mathrm{IV}$, dans lequel siègent 991 personnes. Si l'on ajoute à ce chiffre les personnes siégeant à titre consultatif dans les groupes de travail créé par chaque commission, on arrive à un effectif total qui est passé d'environ mille pour le Ir Plan à un chiffre de l'ordre de 3100 personnes pour le IV ${ }^{\text {e }}$ Plan (tabl. 1).

\begin{tabular}{|l|l|}
\hline & Nombre de commissions \\
\hline I $^{\text {er } P l a n ~(1947-1953) ~}$ & 18 \\
\hline II $^{e}$ Plan (1954-1957) & 25 \\
\hline III $^{e} P l a n(1958-1961)$ & 22 \\
\hline IVe Plan (1962-1965) $^{2}$ & 28 \\
\hline
\end{tabular}

Tableau établi par l'auteur d'après l'Annuaire du Commissariat général du Plan, 1987.

\section{La vertu de la participation des groupes sociaux}

La participation des intérêts économiques et des forces sociales au Plan est une caractéristique essentielle de la planification française. Ce principe, adopté par Jean Monnet en 1946 et conservé par la suite, constitue un facteur essentiel qui explique le succès des plans français ${ }^{10}$. Selon Pierre Massé :

«Planifier, c'est établir le programme d'ensemble du développement d'un pays. ${ }^{11}$ "

Le rôle que la planification est appelée à jouer consiste non pas à supprimer la lutte des groupes, mais à l'orienter dans le sens d'un progrès collectif ${ }^{12}$. La planification modifie les normes de la concurrence et de la coordination inter-administrative : elle les rend plus intenses et plus fondées sur des dossiers argumentés.

\section{À la recherche des origines}

L'idée de faire participer les forces vives correspond aux visions des relations sociales inspirées en particulier par l'expérience du New Deal ${ }^{13}$. Il faut remarquer que l'idée d'un 
Plan de Jean Monnet prend ses racines dans les négociations de l'aide américaine à la France $^{14}$ : l'objectif étant de faciliter l'obtention de l'aide des États-Unis. Celui-ci constitue aussi un facteur très important dans le démarrage du Plan Monnet ${ }^{15}$. D'après le témoignage d'Étienne Hirsch:

\footnotetext{
"Monnet a à surmonter une difficulté sérieuse. La prétention, surtout de la part du Ministère de la Production industrielle, est que chaque commission soit présidée par le fonctionnaire responsable, ce à quoi Monnet objecte qu'il est essentiel d'éviter de donner aux membres des commissions l'impression qu'ils sont sous la tutelle de l'administration. L'accord se fait sur l'attribution de la vice-présidence au fonctionnaire responsable. ${ }^{16}$ »
}

Par conséquent, sur les dix-huit commissions de modernisation en 1946, quatre commissions sont présidées par des militants CGT, quatre par des patrons et les autres par des syndicalistes en majorité ${ }^{17}$.

21 En outre, cette idée de regrouper les forces vives autour du Plan constitue aussi un reflet de l'aspiration à la démocratie économique et sociale issue de la Résistance et de la Libération, de l'héritage du planisme ensuite, et des rapports de forces politiques établis en décembre 1945-janvier 1946 enfin ${ }^{18}$. En fait, la planification à la française cherche la source d'inspiration auprès des modèles de planification des autres pays. Elle apparaît comme un compromis entre deux extrêmes - la planification soviétique et celle des États-Unis - et s'adapte aux réalités économiques et politiques de la France. À la fois souple, indicative et démocratique, la planification française lie l'État, les entreprises et les syndicats. Elle collecte des informations auprès des acteurs économiques et les redistribue comme des références. Par là, la planification française dessine un paysage spécifique, en direction d'une économie concertée.

\section{La transmission et la cohérence des informations}

Créées à l'instigation de Jean Monnet lors du $\mathrm{I}^{\text {er }}$ Plan, ces commissions de modernisation ne visent non seulement à confronter les points de vue, à mobiliser les compétences, mais encore à rassembler des renseignements d'ordre économique sur lesquels les statistiques courantes font défaut.

La procédure d'élaboration du Plan comprend plusieurs phases qui entremêlent étroitement travaux techniques et choix d'objectifs avec des moyens de politique économique. Par leur nature, le Commissariat général du Plan et les commissions de modernisation constituent une interface entre le gouvernement, l'organisme de décision, et l'extérieur, les agents publics et privés. Son rôle principal est d'informer. Ils collectent de l'information auprès de l'extérieur et la communiquent au gouvernement pour fixer des politiques appropriées. Simultanément, parce que proche du gouvernement, ils diffusent de l'information de l'intérieur vers les agents économiques extérieurs. En fait, grâce à sa structure tripartie, le Plan engage la participation de tous les agents socio-économiques. Son élaboration constitue un processus d'études et d'échanges d'informations entre les groupes sociaux.

Les marges d'indépendance et d'autonomie constituent la richesse propre des commissions. D'une part, les représentants d'organisations patronales sont à l'origine de la planification, dont ils sont les sources essentielles d'information statistique. D'autre part, les acteurs sont aussi les interlocuteurs réunis au sein des commissions de modernisation. À travers celles-ci, se réalise par étapes, un apprentissage de la 
concertation entre représentants des administrations techniques et financières, représentants des organisations professionnelles d'employeurs et leurs homologues des syndicats de salariés. Par exemple, la fonction de pédagogie économique est très présente dès le $\mathrm{I}^{\text {er }}$ Plan. Certaines commissions, surtout les deux commissions horizontales du Plan Monnet (de la main-d'œuvre et de la consommation sociale), ont parmi leurs missions celle d'initier les participants au vocabulaire et aux concepts de la macro-économie.

Les commissions de modernisation sont un lieu central qui légitime l'existence du Commissariat général du Plan. Les travaux des commissions de modernisation sont les plus importants. Ils peuvent s'étendre sur une ou deux années en vue de l'élaboration d'un plan. Chaque commission est chargée de préparer un rapport. À partir de ces rapports, le Commissariat général du Plan prépare une synthèse sur les grandes options qui constitueront la base du Plan. Logiquement, les rapports des commissions aboutissent à des conclusions bien différentes, même controversés. Dans ces conditions, la cohésion définitive des rapports des commissions est assurée par le SEEF et l'INSEE, en accord avec le Commissariat général du Plan, qui procède à la rédaction définitive du projet de Plan et le soumet à l'avis d'organismes représentatifs des intérêts socio-professionnels. On assure ainsi un minimum de cohérence entre les divers rapports des commissions. Ceux-ci font l'objet de rectifications au fur et à mesure de leur élaboration afin d'éviter des incompatibilités entre secteurs, puis synthétisés dans le rapport général du Commissaire du Plan.

\section{La conscience de la solidarité}

Le Plan, une fois doté d'objectifs communs, implique une œuvre collective. L'exécution d'un Plan de redressement économique n'est possible que si tous les intéressés, industriels, ouvriers, agriculteurs et techniciens, apportent au gouvernement et aux administrations publiques leur concours actif et participent de façon étroite à l'élaboration des décisions comme à leur mise en œuvre ${ }^{19}$.

Le Plan n'est pas directement opérationnel. Formuler le Plan est une chose, le mettre en œuvre en est une autre, bien plus difficile ${ }^{20}$. La tâche de l'exécution du Plan incombe sur l'ensemble des agents économiques. L'État et ses administrations sont les premiers responsables de l'exécution du Plan. Les décisions prises de manière directe par l'État en fonction du Plan ont une valeur morale et exemplaire pour tous les agents économiques, tant publics que privés. Défini comme "cadre de programmes d'investissement et instrument d'orientation de l'expansion économique et du progrès social » selon la loi, le Plan fixe les grandes lignes des politiques à suivre pour sa période d'application. En fonction de ces dernières, le gouvernement définit ses politiques économiques et sociales et intervient dans l'économie. Quant au secteur privé, l'État profite de manière large des moyens d'incitation disponibles pour infléchir les actions des agents économiques vers une voie aboutissant à l'exécution du Plan.

En participant aux commissions, les groupes d'industriels et d'agriculteurs marquent leur intérêt pour ces perspectives à long terme, et modifient leur comportement: les syndicats de salariés ont relativement modéré leurs revendications. Le Plan fait franchir un pas aux divers groupes, qui, sans renoncer à leur autodéfense, sentent l'intérêt d'une véritable coordination de la politique économique, d'une organisation qui dépasserait le cadre de leur lutte. 


\section{L'action du plan : persuader et informer} essaye de l'éclairer. Le Plan représente un gisement d'information de telle sorte que les organisations syndicales aient une idée générale sur les projets de l'administration, les entreprises sur les perspectives du développement ${ }^{21}$.

\section{La force de la persuasion}

participation à l'élaboration donne aux entreprises privées ou publiques, firme ou individuel, le sentiment d'une communauté d'intention et d'engagement. Elles sont sujettes à croire que le Plan est le leur. Le concert réalisé dans l'élaboration du Plan tend spontanément à se prolonger par un concert dans l'exécution ${ }^{22}$. Enfin, à partir des études scientifiques réalisées pour son compte, le Plan fournit une image cohérente et rassurante de l'avenir. Les agents y voient l'intérêt à suivre grâce à la voie tracée par le Plan.

31 La force de persuasion des commissions de modernisation s'agrandit. Les discussions dans les commissions de modernisation sur l'élaboration ou le respect des perspectives du Plan pénètrent une partie de l'opinion publique de l'intérêt que présente un développement harmonieux. Les entreprises publiques donnent l'exemple à suivre. L'État et les entreprises publiques, par leurs investissements ou commandes, sont en mesure d'exercer un effet d'entraînement sur certains secteurs en aval ${ }^{23}$. Les agents économiques privés sont persuadés d'avoir un meilleur développement en suivant les perspectives du Plan. Le Plan a pour rôle de proposer, au-delà des pouvoirs et des limites du marché, une vue commune du développement économique éclairant les comportements individuels. Toutes les décisions étendant leurs effets sur une longue période, notamment les décisions d'investir, reposent sur une représentation de l'avenir.

\section{Le sondage de l'avenir}

Le rôle du Plan ne consiste plus alors à orienter de manière volontariste des secteurs industriels jugés prioritaires dès le $\mathrm{II}^{\mathrm{e}} \mathrm{Plan}$, mais à définir des prévisions indicatives par branche - et non par entreprises ou par produits.

La préoccupation dominante des planificateurs est de dessiner la cohérence de l'ensemble des perspectives de production, en fonction d'une projection de croissance globale et d'une ventilation délibérée du revenu national, et de la répartition des investissements dans les divers secteurs économiques. La seule existence de la projection est "réductrice d'incertitude». L'existence d'une projection globale constitue en effet un certain garde-feu contre la création de capacités de production trop largement excédentaires, et semble ainsi devoir permettre de réaliser le même taux de croissance à meilleur compte, c'est-à-dire avec un moindre investissement global ${ }^{24}$.

34 Certes, les entreprises peuvent essayer elles aussi de sonder l'avenir en effectuant des études de marché leur permettant de mieux connaitre l'évolution future de leurs approvisionnements et de leurs débouchés avant de lancer un programme de 
fabrication ou d'investissement. Mais de telles études sont forcément incomplètes dans la mesure où elles ne portent que sur les secteurs d'activité avec lesquels l'entreprise est directement en contact. Cependant le Plan, travail des commissions de modernisation et du Commissariat général du Plan, peut réduire l'incertitude économique par un effort systémique de prévision tenant compte de toutes les relations de fournisseurs à clients qui unissent les unes aux autres les branches d'activité et les différents agents économiques.

Ainsi informés des tendances de la demande, le Plan fournit un cadre général propre à influer sur les décisions individuelles des entrepreneurs, et à leur assurer comme un horizon de certitude d'une croissance équilibrée. De plus, le Plan n'annonce pas seulement le probable, il exprime aussi le souhaitable. Il ajoute l'affirmation de volonté. Il ne formule pas seulement des prévisions, il donne au développement économique des objectifs. De même que le passage du marché à l'étude du marché, partielle mais généralisée, introduit la conscience, le passage des prévisions aux objectifs introduit la volonté. Il répond ainsi à une aspiration profonde de notre époque, la participation des hommes à la création de la société de demain ${ }^{25}$. Au total, il importe que le Plan :

«Serve de référence constante à l'action du gouvernement, des entreprises et des particuliers. ${ }^{26}$ "

Le rapport sur les principales options du V ${ }^{\mathrm{e}}$ Plan résume les diverses utilités du Plan comme telles :

«Instrument d'éducation, le Plan tend à donner à tous ceux qui participent de près ou de loin son élaboration et à son exécution, une compréhension plus nette des mécanismes économiques, une vision plus juste des pouvoirs de l'homme et des limites de ces pouvoirs. Instrument d'ordre, il introduit plus de cohérence dans les projets des agents économiques et dans ceux de l'état lui-même, en leur permettant de se situer dans une perspective équilibrée du développement économique et social. Instrument de progrès, il encourage les initiatives en les épaulant les unes par les autres, prévient les gaspillages et élargit les limites de la croissance. ${ }^{27}$ »

Le Plan ne remplace pas le marché, il s'efforce de faire une prévision selon les règles du marché. Il s'agit d'un effort pour connaitre le réel. Sa portée sur les agents économiques est indicative. En fournissant un cadre dans lequel chacun devait trouver une large autonomie d'action et fixer ses propres barrières, le Plan ne dirige pas l'économie, il la conduit. Il suggère aux agents économiques d'atteindre certains objectifs d'intérêt national et les oriente en ce sens par un ensemble de stimulations appropriées. C'est ainsi que s'est progressivement mise au point et perfectionnée la méthode de planification souple et incitative, dite «à la française » et qu'ont été transmises des connaissances plus larges sur l'économie. 


\section{BIBLIOGRAPHIE}

ATREIZE, DUBOIS Paul (dir.), La planification française en pratique, Paris, Éditions Économie et humanisme, 1971.

BAUCHET Pierre, La planification française, quinze ans d'expérience, Paris, Éditions du Seuil, 1962.

BOUCHARD Julie, Comment le retard vient aux Français ?, Lille, Presses universitaires du Septentrion, 2008.

FOURASTIÉ Jean, COURTHÉOUX Jean-Paul, La planification économique en France, Paris, PUF, 1963.

HIRSCH Étienne, Ainsi va la vie, Lausanne, Fondation Jean Monnet pour l'Europe - Centre de Recherches Européennes, 1988.

KUISEL Richard F., Le capitalisme et l'État en France. Modernisation et dirigisme au XXe siècle, Paris, Gallimard, 1984.

MAC ARTHUR John H., SCOTT Bruce R., L'industrie française face aux plans, Paris, Les Éditions d'organisation, 1970.

MARGAIRAZ Michel, Rousso Henry, « Le Plan, une affaire d'État? Les responsables des commissions du $\mathrm{I}^{\mathrm{er}}$ au IX $\mathrm{e}^{\mathrm{e}}$ Plan », dans Rousso Henry et alii. La planification en crise 1965-1985, Paris, Édition du CNRS, 1987.

MASSÉ Pierre, « planification et prévision », dans BERGER Gaston, BOURBON-BUSSET Jacques de, MASSÉ Pierre (dir.), De la prospective, textes réunis et présentés par Philippe DURANCE, Paris, L'Harmattan, $2^{\mathrm{e}}$ édition, 2008

MASSÉ Pierre, Histoire, méthodes et doctrine de la planification française, Paris, Documentation française, 1962.

MINC Alain, « La planification en question », CFDT Aujourd'hui, janvier 1984.

MIOCHE Philippe, «Syndicats et CNPF dans le Plan : l'amorce d'un consensus », dans Rousso Henry et alii, La planification en crise 1965-1985, Paris, Édition du CNRS, 1987.

MIOCHE Philippe, Origines et démarrage de la planification en France, 1941-1946, Thèse de troisième cycle, Université de Paris 1, 1983.

MONNET Jean, Mémoires, Paris, Fayard, 1976, tome I.

PAGÉ Jean-Pierre, « Les institutions économiques et la planification », dans PAGÉ Jean-Pierre et alii, Profil économique de la France au seuil des années 80, Paris, La Documentation française, 1981.

PASCALLON Pierre, La planification de l'économie française, Paris, Masson et C $C^{\mathrm{ie}}, 1974$.

\section{NOTES}

1. M. Margairaz, H. Rousso, «Le Plan, une affaire d'État? Les responsables des commissions du I ${ }^{\text {er }}$ au IX ${ }^{\mathrm{e}}$ Plan », p. 19-78.

2. É. Hirsch, Ainsi va la vie, p. 90-91.

3. J. Monnet, Mémoires, p. 292. 
4. P. Pascallon, La planification de l'économie française, p. 30.

5. J. H. Mac Arthur, B. R. Scott, L'industrie française face aux plans, p. 119.

6. J. Fourastié, J.-P. Courthéoux, La planification économique en France, p. 96.

7. Atreize, La planification française en pratique, p. 46.

8. Archives nationales (Arch. nat.), 80AJ/93, Commissariat général du Plan, Troisième Plan de Modernisation et d'équipement (1958-1961), p. 9.

9. P. Massé, Histoire, méthodes et doctrine de la planification française, p. 8.

10. Ibidem, p. 3.

11. P. Massé, « planification et prévision », p. 197.

12. P. Bauchet, La planification française, quinze ans d'expérience, p. 279.

13. P. Mioche, «Syndicats et CNPF dans le Plan : l'amorce d'un consensus », p. 79-103.

14. J. Bouchard, Comment le retard vient aux Français?, p. 100.

15. Voir R. F. Kuisel, Le capitalisme et l'État en France. Modernisation et dirigisme au XXe siècle

16. É. Hirsch, Ainsi va la vie, p. 98-99.

17. P. Mioche, «Syndicats et CNPF dans le Plan : l'amorce d'un consensus », p. 79-103.

18. $P$. Mioche, Origines et démarrage de la planification en France, 1941-1946, p. 252-278.

19. Arch. nat., $80 \mathrm{AJ} / 1$, Documents relatifs à la première session du Conseil du Plan introduction, le 16-19 mars 1946, $1^{\text {ère }}$ session du Conseil du Plan.

20. É. Hirsch, Ainsi va la vie, p. 96.

21. A. Minc, «La planification en question », CFDT Aujourd'hui, janvier 1984.

22. P. Massé, Histoire, méthodes et doctrine de la planification française, p. 9.

23. J.-P. Pagé, «Les institutions économiques et la planification », p. 20.

24. Arch. nat., 19930277/14, loi $\mathrm{n}^{\circ}$ 64-1265 du 22 décembre 1964, Préparation du Ve Plan, Rapport sur les principales options - (Texte publié au Journal Officiel), p. 7.

25. Ibidem, p. 7.

26. Arch. nat., 19960048/2, Conférence Prospective et orientation économique de Pierre Massé (15 mars 1962), 8 p.

27. Arch. nat., 19930277/14, loi $\mathrm{n}^{\circ}$ 64-1265 du 22 décembre 1964, Préparation du V Plan, Rapport sur les principales options, Texte publié au Journal Officiel, p. 7-8.

\section{RÉSUMÉS}

La planification est introduite en France dans l'immédiat après-guerre et connaît son âge d'or à partir de l'avènement du général de Gaulle en 1958, et durant les années 1960. En tant qu'instrument de politique économique, le Plan informe les agents sur les orientations stratégiques du pays, mais aussi sur les prévisions économiques à moyen terme. Ce rôle d'information et de transmission des connaissances sur l'économie s'explique par l'action des 
commissions de modernisation, groupes de travail réunissant autour du Plan des représentants des différentes branches. En écoutant les points de vue des interlocuteurs sur les grands problèmes, ces commissions élaborent des analyses de la situation de leurs domaines respectifs et simultanément, deviennent autant des sources d'information pour le Plan que les instruments de cette pédagogie qu'il faut inculquer aux responsables de l'économie, pour atteindre, à travers eux, le public.

\section{AUTEUR}

\section{CHUNHUA CHU}

Docteure en Histoire de Sorbonne Université Lettres, professeure associée au département de Langues et civilisations étrangères de l'Université des sciences sociales de Nanjing (Jiangsu, Chine) 
De l'information scientifique et technique aux transferts de technologie : un défi pour notre temps

\section{Importance des transferts de technologie}




\title{
Transmission des savoirs
} technologiques de la France à la Russie : le cas de l'industrie textile au XIX ${ }^{\mathrm{e}}$ siècle

\author{
Olga Melnichenko
}

1 Les Français ont joué un rôle considérable dans la transmission des savoirs technologiques nécessaires au développement de l'industrie textile en Russie au cours $\mathrm{du}$ xIX ${ }^{\mathrm{e}}$ siècle. Cette transmission n'a pas été organisée dans le cadre classique de contrats de transfert de technologie, de licences d'exploitation de procédés de fabrication. En fait, elle résulte de multiples efforts et d'initiatives individuelles, ce qui lui confère un caractère original. Elle est essentiellement l'œuvre de professionnels ambitieux, d'entrepreneurs audacieux, de constructeurs d'équipements spécialisés. Elle s'étend aussi à la construction de bâtiments industriels modernes. Elle profite enfin de la diffusion de «la mode de Paris» en favorisant le développement de l'industrie textile.

2 Le sujet des transferts des spécialistes entre les pays européens et la Russie prérévolutionnaire suscite un grand intérêt des historiens. Cependant, la majorité des recherches porte sur l'activité des ingénieurs en industrie lourde, construction et exploitation des chemins de fer, travaux publics, des banquiers ${ }^{1}$, tandis que dans le domaine de l'industrie textile, il n'existe que quelques articles $^{2}$ dont la plupart sont consacrés au secteur cotonnier ${ }^{3}$. En s'appuyant sur les sources inexploitées des archives françaises et russes, et en abordant l'historiographie existante, il est possible de révéler quels acteurs participaient à ce transfert, comment il était organisé et quelles connaissances étaient transmises pour l'industrie textile. 


\section{Le contexte socio-économique international et les acteurs principaux de transfert des connaissances dans l'industrie textile}

Le 9 octobre 1847 «Sa Majesté l'Empereur de toute la Russie ${ }^{4}$ » édite un oukase sur l'ordre d'entrée des contremaitres, ouvriers et des personnes de " couche inférieure " embauchés pour le travail dans les fabriques et ateliers d'artisans ${ }^{5}$. Ce document indique que, avant de franchir la frontière, le candidat devait avoir, d'abord, un contrat signé avec un employeur (à condition que ce dernier le confirme dans le commissariat de police), puis il demandait de faire viser son passeport étranger dans le Consulat russe, enfin il imposait de présenter tous ses documents à la douane russe.

Le problème du manque de cadres reste actuel dans les années 1870, quand l'économie nationale entre dans une phase d'industrialisation. Des institutions de l'enseignement professionnel sont créées mais la demande dépasse l'offre. Étant donné le caractère de l'équipement et des technologies mises en œuvre, l'industrie émergente a besoin de spécialistes de tous niveaux : des entrepreneurs capables d'organiser la production, des spécialistes (architectes, ingénieurs, mécaniciens, chimistes, coloristes, dessinateurs, maîtres, contremaîtres, ouvriers qualifiés) et des intermédiaires comme des compagnies de transport, des commerçants, des agents de recrutement (fig. 1).

Fig. 1. - Acteurs principaux de la transmission des savoirs dans l'industrie textile.

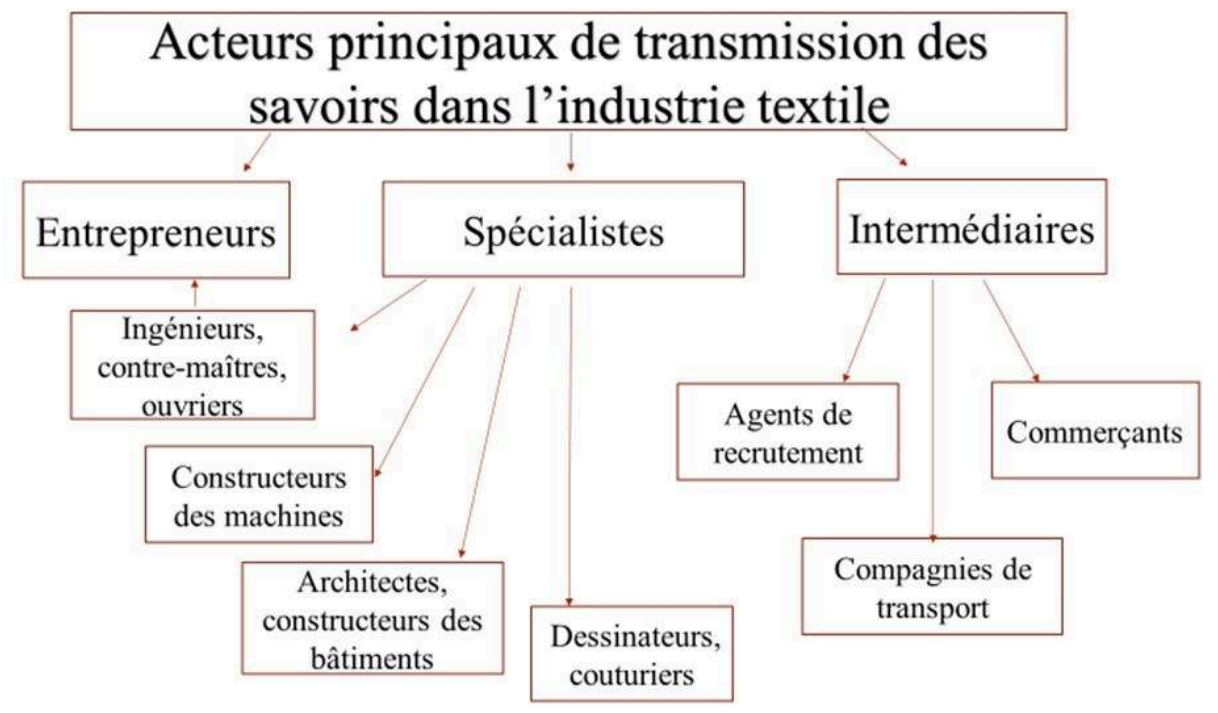

Doc. O. Melnichenko.

\section{La transmission des savoirs technologiques de production}

Comment se passe le transfert des spécialistes ? Certains sont invités par des fabricants russes d'après leurs renseignements dans les chambres de commerce ou auprès des autorités locales, d'autres sont recrutés par des agents intermédiaires ou par les industriels eux-mêmes. Quelques spécialistes viennent d'eux-mêmes et, déjà sur place, 
trouvent un emploi, tandis que d'autres écrivent des lettres aux dirigeants des fabriques et se rendent en Russie à l'invitation de ces derniers.

De nombreux spécialistes arrivent en tant que contremaîtres, sans moyens, comme Georges Steinbach (arrivé en 1833), Émile Zundel $(1838)^{6}$, Théodore Watremez (1848) ${ }^{7}$, Claude Giraud et Jean-Pierre Moussy (1860), ou comme les entrepreneurs lyonnais Joseph Marie Gabriel Bouvier et Ernest Gustelle. Ces deux derniers, en fondant en 1897 la fabrique de filature de soie à Mojaisk, à $100 \mathrm{~km}$ de Moscou, déclarent :

«MM. Bouvier et Gustelle apportent à la Société, leurs noms, leurs intelligences, leurs aptitudes, leurs relations commerciales, leurs connaissances spéciales, le projet d'établissement de moulinage, tel qu'ils l'ont conçu et les diverses représentations qui leur sont promises. ${ }^{8}$ "

7 Cette déclaration des entrepreneurs lyonnais pourrait s'appliquer aussi à d'autres Français venus en Russie avec les mêmes idées. Enfin certains industriels, comme l'Alsacien Albert Hubner (1844) $)^{9}$, les Lyonnais Hector Simonod $(1880)^{10}$, les Gillet ${ }^{11}$, la "Société Anonyme de Filature de Schappe (1886) ainsi que la "Société Anonyme Cotonnière Russo-Française » (fondée à Paris-1898) arrivent avec un capital ${ }^{12}$.

\section{Théodore Watremez, teinturier de Cambrai}

8 Un bon exemple de franchissement de la frontière russe et de développement de la carrière professionnelle d'un spécialiste étranger est celui du contremaître teinturier Théodore Watremez. Il est embauché en juin 1848 par le fabricant belge Achille Favart, installé avec son frère Charles en Russie. Favart vient spécialement à Cambrai (Nord) pour le recrutement de spécialistes pour la fabrique de laine à Pouchkino près de Moscou. Watremez arrive en Russie par le port de Kronstadt, durant l'année de la révolution de 1848 en France. Il est retenu pendant quelques semaines à la frontière avec d'autres spécialistes venus d'Europe sur le même bateau ${ }^{13}$. En effet, l'Empereur et le gouvernement considéraient l'arrivée des étrangers, particulièrement de France, comme un danger pouvant menacer la stabilité du pouvoir de l'Empire Russe. Ils voulaient s'assurer se protéger contre la propagation des idées de la dernière révolution française ${ }^{14}$. Il fallait fournir de nombreux documents pour justifier la nécessité de la présence de cette personne en Russie et confirmer le bon comportement dans le pays de sa résidence ${ }^{15}$. Les archives conservent les échanges savoureux entre les services de l'amiral commandant du port militaire de Kronstadt, F. Bellingshausen, le ministre des finances F. Vrontchenko, le gouverneur de Moscou A. Zakrevskiy, le ministre des Affaires Intérieures L. Perovskiy et le chef du troisième département de la Chancellerie de l'Empereur (police politique) A. Orlov.

9 À la demande du Ministre des Affaires Intérieures, le gouverneur de Moscou répond que :

«L'arrivée de cet étranger a vraiment une grande importance pour le fabricant Favart dont la fabrique de teinture de laine est très connue à Moscou, elle emploie jusqu'à 450 ouvriers et fait 250 mille roubles argent par an..$^{16}$ "

10 Le Ministre des finances, quant à lui, considère aussi l'arrivée de ce spécialiste important, et demande au comte Orlov de contribuer à l'entrée de Watremez dans la ville :

«Au gracieux souverain comte Alexei Fedorovich,

Me référant au message du 27 juillet de votre lumière, j'ai l'honneur de vous 
notifier que, comme l'indique le Gouverneur civil de Moscou, l'usine de teinture Favart est l'une des plus célèbres dans la province, et que la difficulté de trouver en Russie un maître expérimenté peut mettre Favart dans l'incapacité de poursuivre son activité au niveau de la réputation qu'elle a déjà acquise ;

Par conséquent, je crois que sa demande d'autoriser l'embauche de maître Watremez mérite satisfaction.

En vous retournant les papiers que vous m'avez envoyés, je prie humblement votre Seigneurie d'accepter l'assurance de ma profonde estime et de mon parfait dévouement. ${ }^{17}$ »

11 Ce n'est que le 13 août que Théodore Watremez est enfin autorisé à entrer à SaintPétersbourg et poursuivre son voyage jusqu'à Moscou, après la demande du Consul de France à Saint-Pétersbourg, Famen, et une intervention personnelle du comte Orlov auprès de l'amiral F. Bellingshausen. Il est intéressant d'étudier le contrat, signé par Théodore Watremez ${ }^{18}$ avec son employeur ${ }^{19}$ :

«M. Théodore Watremetz engage par les présentes son industrie et son soin, en qualité de contremaître en teinturerie, au service de MM. Favart pour un terme de quatre années à compter du $1^{\mathrm{er}}$ juillet prochain, c'est-à-dire jusqu'au $1^{\mathrm{er}}$ juillet 1852 . En conséquence, il s'oblige à partir incessamment pour la Russie et à se rendre à Pouchkino (près de Moscou) dans les ateliers de MM. Favart pour y surveiller la teinture de toute espèce de tissus en laine. Il s'oblige, jusqu'au terme ci-dessus désigné à consacrer entièrement et exclusivement son temps et ses connaissances aux besoins des établissements de MM. Favart en ladite qualité, sans pouvoir les quitter sans motif de mécontentement grave. Par suite, il ne pourra durant ledit espace de temps former en Russie pour son compte personnel aucune entreprise particulière [...]. M. Favart en son nom s'oblige à payer à $\mathrm{M}$. Watremetz une somme de 8000 francs, à titre d'appointements pour chacune des 4 années du présent engagement. [...] Fait en double original à Cambrai, le 22 juin $1848 .{ }^{20}$ »

12 En acceptant les conditions du contrat, le jeune spécialiste en teinturerie part pour la Russie. Les Favart achetaient à bon compte les savoirs technologiques pour la teinture de la laine. La somme de 32000 francs pour 4 années était payée pour les connaissances acquises par T. Watremez en Europe ${ }^{21}$. La confiance n'était pas totale; les Favart retenaient $50 \%$ du traitement mensuel; les parties s'en remettaient à la sagesse des consuls pour régler un différend éventuel. En outre, les Favart s'assuraient contre le départ de contremaître invité en s'appuyant sur leur propre expérience : avant de fonder sa propre entreprise en 1844, Charles Favart dirigea pendant quelques années l'atelier de teinture chez les fabricants russes Goutchkov ${ }^{22}$.

La fabrique des Favart est rachetée en 1854 par Louis Eugène Armand. Théodore Watremez continue à travailler pour le nouveau propriétaire, et dans un prospectus édité pour l'exposition manufacturière à Varsovie en 1857, il est désigné comme :

«[un] spécialiste, qui perfectionne constamment les processus de teinture, et les tissus dont il teint, ont des couleurs très vives et stables. ${ }^{23}$ "

En 1875, Jules Watremez, le fils de Théodore, fonda la fabrique de teinture, à Ivanteyevka, près de Pouchkino. Elle jouera un rôle important, notamment, dans le fonctionnement de la fabrique de soieries Simonod et Cie. Comme elle ne possédait pas d'atelier de teinture, elle faisait teindre ses tissus chez Watremez ${ }^{24}$. Vers 1900 , la société est rachetée par la maison Gillet de Lyon, l'un des leaders des techniques de teintures depuis leur création du noir impérial ${ }^{25}$.

De même, Claude Giraud, contremaître en tissage, fut recruté en 1860 dans un café lyonnais par le fabricant d'étoffes russe Istomine. En 1875, il fonda la fabrique de soieries C. Giraud à Moscou, qui deviendra quelques années plus tard la plus grande 
fabrique de soieries en Russie ${ }^{26}$. En outre, Claude Giraud était actionnaire de la société Watremez et Cie. Jean-Pierre Moussy, futur fondateur de la manufacture de soieries (1872), commence également sa carrière russe comme un simple employé de fabrique ${ }^{27}$.

Ces quelques exemples illustrent la diversité des vecteurs de transmission des savoirs technologiques de production français dans l'industrie textile russe. Cette transmission est essentiellement l'œuvre d'hommes du métier, des contremaîtres, des "petits patrons » armés d'un esprit d'entrepreneur conquérant. Ces hommes n'apparaissent pas comme des inventeurs ou des capitalistes, mais comme d'excellents techniciens et manageurs qui ont transmis leurs secrets de fabrique développés durant plusieurs générations en France.

17 Mais ils ne furent pas les seuls, les architectes, les ingénieurs, les entrepreneurs de génie civil ont accompagné le développement de l'industrie textile et transmis leurs savoir-faire en matière de constructions industrielles. Les constructeurs européens de machines textiles modernes ont diffusé le dernier cri de la technique et favorisé le développement d'ateliers russes de construction mécanique.

\section{Transmission des savoirs dans les constructions industrielles}

Au début de leur activité industrielle les entrepreneurs français louaient des bâtiments chez les fabricants moscovites. Au fur et à mesure du développement des fabriques naquit la nécessité d'agrandissement. D'abord, il fallait faire un projet des bâtiments selon la quantité, le volume et le poids de l'équipement ainsi que les processus technologiques, puis trouver le réalisateur de ce projet, c'est-à-dire la compagnie de construction, enfin, trouver des capitaux et signer le contrat. Vers la fin des années 1880 une grande partie des bâtiments était réalisée par l'architecte Roman Klein, issu d'une famille allemande installée en Russie au milieu du XIX ${ }^{e}$ siècle. En 1882, après avoir terminé l'Académie des Arts de Saint-Pétersbourg, il continua à étudier en Europe et, notamment, travailla dans un atelier de Charles Garnier sur son projet de l'Exposition universelle à Paris de 1889. Parmi les ouvrages créés par Romain Klein pour l'industrie textile, on compte la manufacture de soieries Moussy-Goujon (1900), la fabrique de filature de la soie Catoire (1900), la maison de Hector Simonod (1898), les bâtiments de la fabrique Simonod $(1890)^{28}$, les immeubles de rapport de C. Giraud (1907-1908, 1911-1914), huit bâtiments de la fabrique C. Giraud (1907-1914) et une maison pour les employés de la fabrique Giraud (1911-1914)29(fig. 2. et fig. 3). 
Fig. 2. - Un des bâtiments de la fabrique Goujon-Moussy.

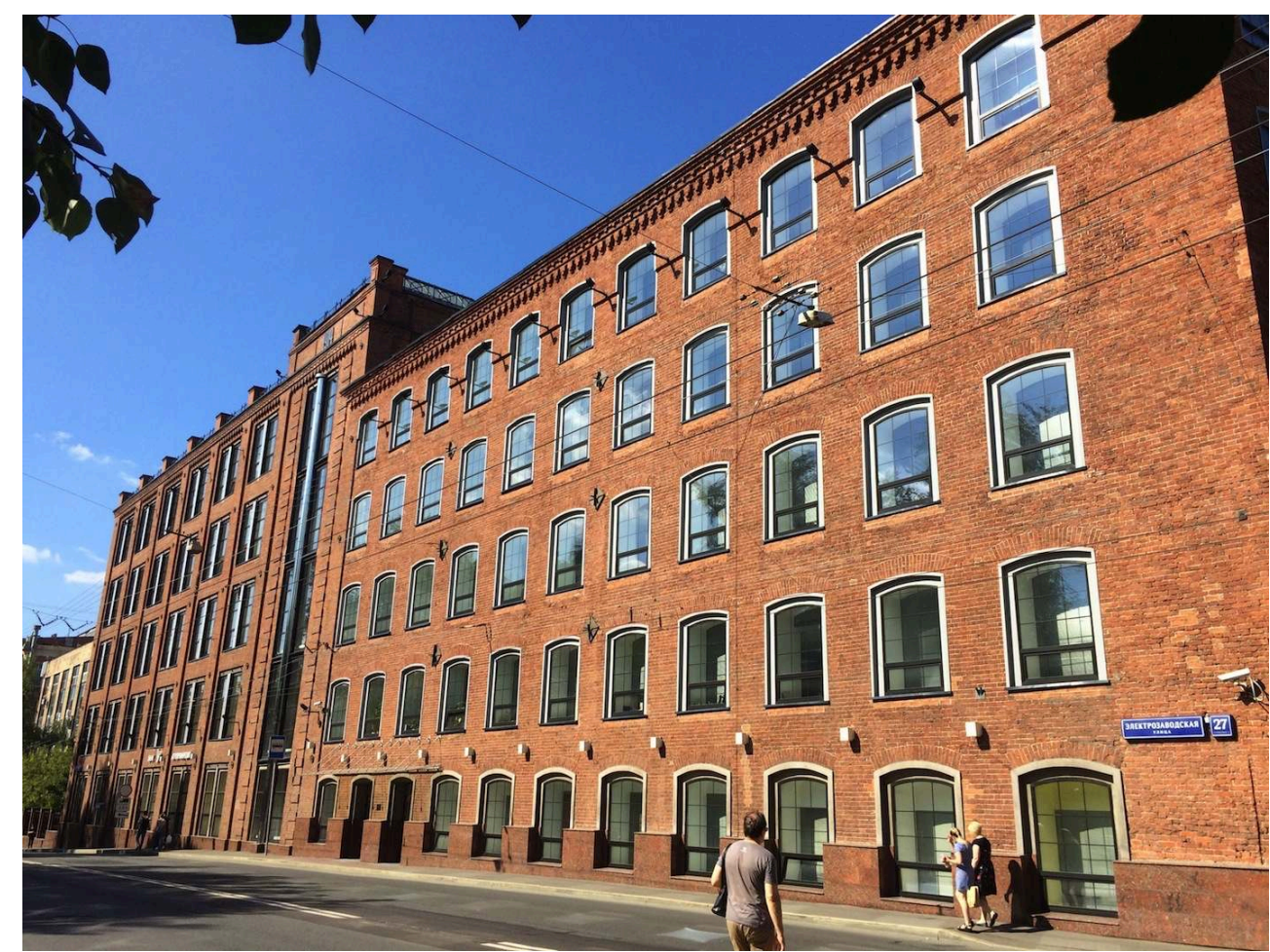

Doc. O. Melnichenko.

Fig. 3. - Un des bâtiments de la fabrique Giraud.

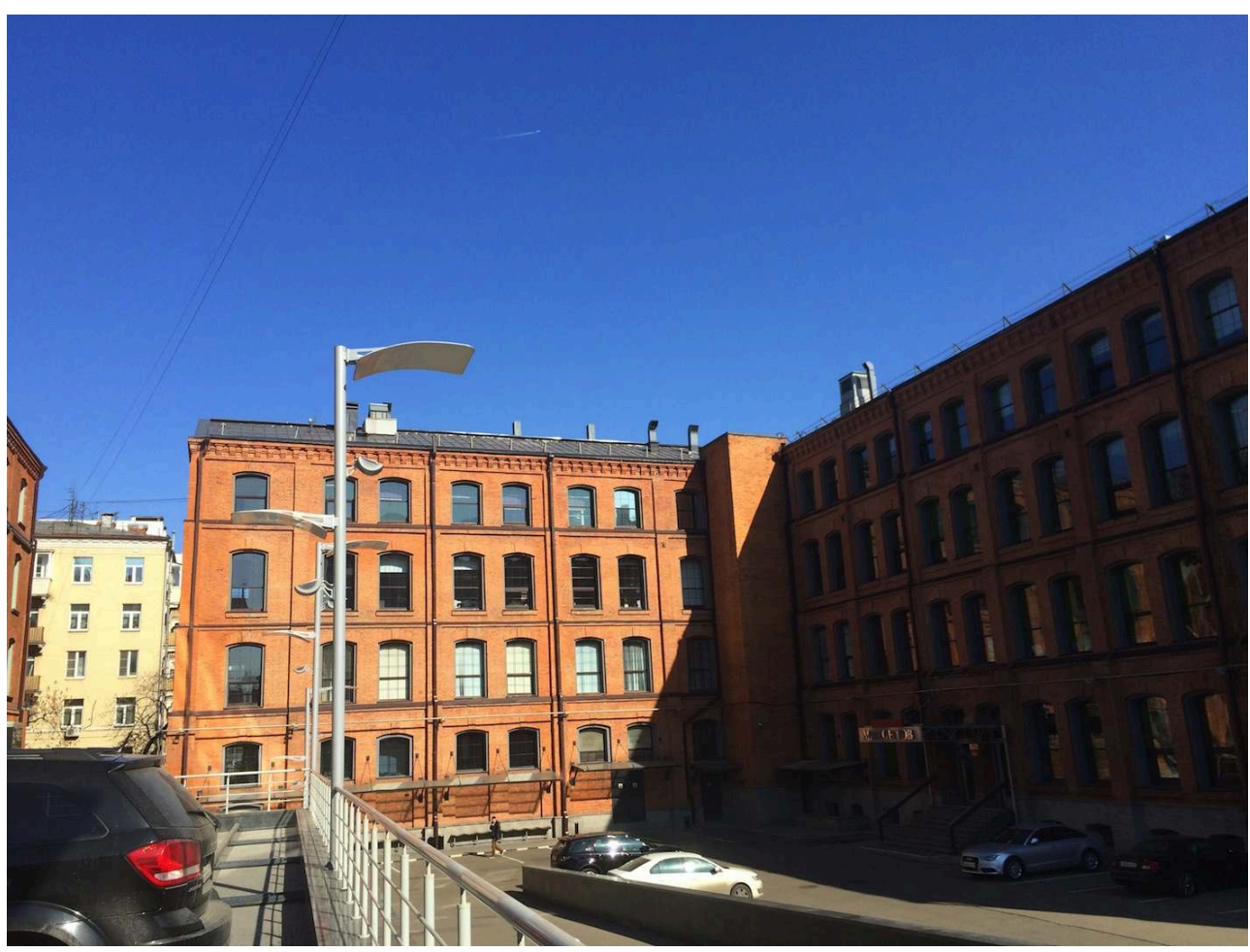

Doc. O. Melnichenko. 
Les travaux de construction des bâtiments, dirigés par les architectes, sont réalisés soit par des artisans russes, soit par des comptoirs fondés par des ressortissants européens. Parmi ces derniers on trouvait, notamment, la société Steffen et Lehmann ${ }^{30}$. Cette entreprise possédait une usine mécanique à Moscou. Elle représentait des firmes européennes comme celle du docteur Hipp de Neuchâtel, en Suisse, la fabrique téléphonique Mixt et Genst de Berlin, en Allemagne, la fabrique de câble Franz Tobich de Vienne, en Autriche et d'autres ${ }^{31}$.

En particulier, cette dernière entreprise effectua en 1889 l'agrandissement des bâtiments et une installation complète de l'équipement pour la fabrique Simonod et Cie, notamment :

- l'installation d'une machine à la vapeur « Piquet » transportée de Lyon ;

- l'installation de mécanismes de transmission: contre-arbre pour la machine «Dynamo »; contre-arbre pour la pompe ; transmission allant de la salle de la machine à vapeur dans le dévidage, et dans la salle des machines à côté du comptoir; les arbres de transmission (graisseur de brevet Stauffer) ;

- tous les travaux liés à la construction de bâtiment supplémentaire pour la chaudière et la machine à vapeur: démolition des vieux murs, construction des nouveaux murs pour agrandir le bâtiment ; reconstruction du toit et de sa charpente ; changement des fenêtres et des portes ainsi que la construction de nouvelles fenêtres et portes, les peintures intérieure et extérieure du bâtiment :

«Installation de tous les tuyaux et leurs accessoires, qui regardent l'installation de l'eau dans les logements, ainsi que pour amener l'eau par la cour pour aller se rejoindre aux tuyaux ou réservoirs des water-closets et de la pompe au réservoir dans les 4 étages de la fabrique; les poutrelles en fer ou en bois qui pourraient devenir nécessaires pour fortifier le plafond, sur lequel sera posé le réservoir dont le poids peut atteindre 2500 pouds $[. . .]^{32}$ »

La Société anonyme de constructions J. Vernet et Cie, une société ayant son siège social à Paris et un comptoir à Moscou introduisit de nouvelles techniques de construction industrielle ${ }^{33}$. Parmi les clients de cette entreprise se trouvaient les fabriques textiles C. Giraud et Fils, Émile Zundel, Alfred Hubner ainsi que la fabrique de teinture Watremez et $\mathrm{Cie}^{34}$. Une partie importante des travaux d'agrandissement de la fabrique des soieries Simonod et Cie était réalisée par cette entreprise dans les années $1890-1916^{35}$.

\section{La transmission des savoirs technologiques par les constructeurs de machines textiles modernes}

Pour assurer le bon fonctionnement de leurs fabriques, les « soyeux » devaient avoir un équipement moderne. Comment était-il possible de satisfaire cette exigence qui évoluait sans cesse? Comment s'informer correctement et trouver un bon fournisseur de machines? Premièrement, les entrepreneurs et les spécialistes venant d'Europe occidentale possédaient les connaissances techniques nécessaires dans leur domaine avant leur arrivée en Russie. Deuxièmement, ils gardaient un étroit contact avec leurs fournisseurs français de matières premières et de machines, qui les informaient régulièrement sur les tendances de l'industrie textile et l'apparition de nouvelles inventions. Enfin, ils profitaient à leur tour de l'expérience occidentale des 
contremaîtres et des spécialistes qu'ils faisaient venir. Claude Giraud notamment, renouvelait ses contremaîtres français tous les trois ans ${ }^{36}$.

Sous l'impulsion de ces patrons énergiques, les technologies de l'industrie textile firent un bond en avant en employant le nouvel équipement créé en Europe. Pour montrer la diversité des machines utilisées par les entrepreneurs français, il convient de prendre l'exemple de Claude Giraud. Il fonda les usines C. Giraud à Moscou avec 200 métiers à bras en $1875^{37}$. "Prévoyant l'avenir des articles à bon marché, plus à la portée de la masse de la population indigène ${ }^{38} "$, et l'avantage qu'il $\mathrm{y}$ aurait à les traiter mécaniquement et en grande quantité, Claude Giraud fit un premier essai de tissage mécanique et installa une cinquantaine de métiers en 1883. Pour l'entrepreneur, c'était une période d'expérimentation industrielle entravée par bien des difficultés, et surtout par d'extraordinaires préjugés tant de la part des ouvriers russes, qui craignaient de perdre leur emploi, que de celle de la clientèle qui avait une méfiance instinctive des étoffes tissées mécaniquement. « Il a fallu une persévérance et une énergie infatigable ${ }^{39}$ " pour triompher tour à tour de tous ces obstacles. Néanmoins, le nombre de métiers mécaniques tripla en trois ans. Il continua à progresser rapidement pour atteindre 2100 métiers mécaniques en 1900 . Cela nécessita dès 1886 la construction de nouveaux ateliers et d'une centrale thermoélectrique contenant les générateurs de vapeur, les machines motrices et les dynamos pour l'éclairage électrique ${ }^{40}$.

Vers 1900, la fabrique de soieries C. Giraud utilisait tous les systèmes des métiers mécaniques. Après plusieurs années, $C$. Giraud en conservait deux types principaux : le système français Diederichs, de Bourgoin et le système suisse Honegger, de Rüti [...] qui se répartissaient ainsi :

« 1880 métiers simples ou jumelles travaillant en uni ; 200 pour façonnés, avec mécaniques Jacquard et Verdol; 20 métiers à velours [...] 30 métiers à la main ont été conservés afin de pouvoir satisfaire la clientèle qui demande parfois des articles inexécutables mécaniquement. ${ }^{41}$ "

L'installation d'apprêt comprenait également une grande variété de machines exportées d'Europe ou fabriquées en Russie selon les dernières technologies :

«Machines à métrer, à enrouler, des polisseuses, tambours, calandres, rames sans fin ordinaires et à dérailler, dérompeuses, flambeuses, presses hydrauliques, raseuses pour le velours, foulards mouilleurs à 2, 3 et 4 rouleaux, gaufrage, moirage et apprêts dissimulés ; formant un ensemble de 58 machines. ${ }^{42}$ "

Le tableau 1 présente ainsi l'équipement de la fabrique C. Giraud en 1900. Étant donné la quantité d'équipements utilisés dans la fabrique Giraud vers la fin du XIX siècle, et la diversité des processus technologiques, cette entreprise a pu prendre la première place parmi les producteurs de soieries en Russie.

Tabl. 1. - Équipement de la fabrique C. Giraud en 1900.

\begin{tabular}{|l|l|}
\hline Équipement & Quantité \\
\hline Machines motrices à vapeur, puissance totale 975 chevaux & 10 \\
\hline Petites machines et moteurs divers, puissance totale 225 chevaux & 20 \\
\hline Pompes à vapeur au débit totale 293200 litres par heure & 5 \\
\hline Dynamos génératrices, puissance totale 299 250 watts & 14 \\
\hline Métiers mécaniques & 2100 \\
\hline
\end{tabular}




\begin{tabular}{|l|l|}
\hline Métiers à la main & 30 \\
\hline Broches de moulins & 12000 \\
\hline Tavelles de moulinage & 2144 \\
\hline Tavelles de dévidage & 6420 \\
\hline Détrancanoirs & 31 \\
\hline Cannetières & 28 \\
\hline Bobinoirs & 35 \\
\hline Ourdissoirs mécaniques pour chaines & 89 \\
\hline Ourdissoirs à la main & 4 \\
\hline Ourdissoirs pour cordon & 9 \\
\hline Machines d'apprêt & 58 \\
\hline Barques ordinaires de teinture & 160 \\
\hline Barques mécaniques & 40 \\
\hline Appareils mécaniques divers pour la teinture & 17 \\
\hline Lampes à incandescence & 4700 \\
\hline Lampes à arc & 12 \\
\hline
\end{tabular}

Industrie de la soie en Russie. Usines Claude Giraud à Moscou, 1875-1900, Paris, 1900, p. 31-32.

À partir des données des Archives d'État de Moscou, il est possible d'observer la progression du développement quantitatif et qualitatif de l'équipement des fabriques textiles de Moscou fondées par les Français durant la période 1842-1900. Cependant le cadre de cet article ne permet pas d'aborder ce sujet plus largement ni celui de la participation des autres acteurs dans le processus de transfert des connaissances dans l'industrie textile : agents de recrutement des spécialistes ; commerçants qui font venir l'équipement et les matières premières; compagnies de transport qui sont, en outre, chargées de régler les problèmes avec la douane et sont responsables de la marchandise ; enfin, les créateurs de mode diffusant « la mode de Paris » et favorisant la prospérité de l'industrie textile. L'étude approfondie de ces questions sera l'objet de prochaines publications.

Au cours du XIX ${ }^{e}$ siècle, l'arrivée de spécialistes européens en Russie, notamment français, contribue au développement des manufactures de textiles franco-russes. Ce sont les connaissances techniques, le fameux "savoir-faire" français qui étaient apportées par les acteurs majeurs du processus de l'échange. Les contremaîtres étaient capables d'organiser la production et de transmettre leurs connaissances aux ouvriers, et même de créer leurs propres fabriques. Les visionnaires et créatifs entrepreneurs français, en attirant des capitaux français et russes, en utilisant toutes les innovations de l'industrie textile occidentale ont formé le personnel russe aux nouvelles technologies et obtenu rapidement des résultats impressionnants. La participation à ce processus de transmission des savoirs des constructeurs de machines et de bâtiments 
industriels, des compagnies de transport et des créateurs de mode, a favorisé l'émergence d'une industrie moderne mettant en œuvre le dernier cri de la technique.

\section{BIBLIOGRAPHIE}

BRANDT André, « Essai sur les Mulhousiens en Russie au XIX siècle », Bulletin du musée historique de Mulhouse, 1959, vol. 68.

DARTEVELLE Raymond, « Paysages et risques industriels en Russie en 1906, l'expertise d'un réassureur français ", dans CHARON Annie, DELMAS Bruno, LE GOFF Armelle, La France et les Français en Russie. Nouvelles sources et approches (1815-1917), Paris, École des chartes, 2011, p. 411-442.

Electritcheskiy signalnyi kolokol dlia jeleznyh dorog, Katalog, Moscou, typo-lithographie « Tehnik », 1887.

GASNAUlT François, KISELEV Alexei (dir.), Paris-Moscou, un siècle d'échanges, 1819-1925. Documents inédits des Archives de Paris et de Moscou, Paris, Musées, 1999.

Industrie de la soie en Russie. Usines Claude Giraud à Moscou, 1875-1900, Paris, 1900.

JoLY Hervé, Les Gillet de Lyon. Fortunes d'une grande dynastie industrielle (1838-2015), Librairie Droz S.A., Genève, 2015.

MELNICHENKo Olga, «Les Français dans la vie économique russe : le cas des entrepreneurs textiles (fin du XIX ${ }^{\mathrm{e}}$-début du Xx $\mathrm{e}^{\mathrm{e}}$ siècle) ", Revue française d'histoire économique, $\mathrm{n}^{\circ}$ 7-8, Paris, 2017, p. 86-103.

MORIN Jean, Souvenirs d'un banquier français, Paris, Denoël, 1983.

NACHOKINA Maria, Arhitectory moskovskogo moderna. Tvorctheskie portrety. Moscou, Giraffe, 2005.

STOSKOPF Nicolas, «Quitter l'Alsace pour faire fortune. Le cas des entrepreneurs du XIX siècle », Diasporas. Histoire et Sociétés, $n^{\circ}$ 9, « Chercher fortune », 2006.

TCHOUBARIAN Alexandre, LIECHTENHAN Francine-Dominique, RJÉOUTSKI Vladislav, OKOUNEVA Olga, Les Français dans la vie intellectuelle et scientifique en Russie (XVIII $-\mathrm{XX}^{e}$ siècles), Moscou, Académie des Sciences de Russie/Institut d'Histoire universelle/Centre Roland Mousnier (Paris-Sorbonne CNRS)/Archives de l'Académie des Sciences de Russie, 2010.

TCHOUBARIAN Alexandre, LIECHTENHAN Francine-Dominique, RJÉOUTSKI Vladislav, oKouNEVA Olga, Les Français dans la vie intellectuelle et scientifique en Russie au XIX siècle, Moscou, Académie des Sciences de Russie, l'Institut d'Histoire universelle, 2013.

BRANDT André, « Essai sur les Mulhousiens en Russie au XIX siècle », Bulletin du musée historique de Mulhouse, 1959, vol. 68.

DARTEVELLE Raymond, « Paysages et risques industriels en Russie en 1906, l'expertise d'un réassureur français », dans CHARON Annie, DELMAS Bruno, LE GOFF Armelle, La France et les Français en Russie. Nouvelles sources et approches (1815-1917), Paris, École des chartes, 2011, p. 411-442. 
Electritcheskiy signalnyi kolokol dlia jeleznyh dorog, Katalog, Moscou, typo-lithographie « Tehnik », 1887.

GASNAULT François, KISELEV Alexei (dir.), Paris-Moscou, un siècle d'échanges, 1819-1925. Documents inédits des Archives de Paris et de Moscou, Paris, Musées, 1999.

Industrie de la soie en Russie. Usines Claude Giraud à Moscou, 1875-1900, Paris, 1900.

JoLY Hervé, Les Gillet de Lyon. Fortunes d'une grande dynastie industrielle (1838-2015), Librairie Droz S.A., Genève, 2015.

MELNICHENKO Olga, « Les Français dans la vie économique russe : le cas des entrepreneurs textiles (fin du XIX ${ }^{\mathrm{e}}$-début du Xx $\mathrm{e}^{\mathrm{e}}$ siècle) », Revue française d'histoire économique, $\mathrm{n}^{\circ}$ 7-8, Paris, 2017, p. 86-103.

MORIN Jean, Souvenirs d'un banquier français, Paris, Denoël, 1983.

NACHOKINA Maria, Arhitectory moskovskogo moderna. Tvorctheskie portrety. Moscou, Giraffe, 2005.

SтоSкорғ Nicolas, «Quitter l'Alsace pour faire fortune. Le cas des entrepreneurs du XIX siècle », Diasporas. Histoire et Sociétés, $n^{\circ}$ 9, « Chercher fortune », 2006.

TCHOUBARIAN Alexandre, LIECHTENHAN Francine-Dominique, RJÉouTSKI Vladislav, oKounEVA Olga, Les Français dans la vie intellectuelle et scientifique en Russie (XVIII $-\mathrm{XX}{ }^{e}$ siècles), Moscou, Académie des Sciences de Russie/Institut d'Histoire universelle/Centre Roland Mousnier (Paris-Sorbonne CNRS)/Archives de l'Académie des Sciences de Russie, 2010.

TCHOUBARIAN Alexandre, LIECHTENHAN Francine-Dominique, RJÉOUTSKI Vladislav, oKouNEVA Olga, Les Français dans la vie intellectuelle et scientifique en Russie au XIX $X^{e}$ siècle, Moscou, Académie des Sciences de Russie, l'Institut d'Histoire universelle, 2013.

\section{Annexes}

1/Contrat d'embauche de M. Théodore Watremetz à Pouchkino, Russie ${ }^{43}$

Timbre royal

Entre les soussignés

M. Achille Favart, négociant à Pouchkino, Russie, se trouvant momentanément à Cambrai, descendu à l'Hôtel de France,

Agissant aux présentes tant pour lui et en son nom personnel qu'au nom et comme le faisant de la part et pour le besoin de M. Charles Favart, son frère et associé, demeurant avec lui au dit Pouchkino, qu'il déclare obliger solidairement avec lui à l'effet ci-après.

Qu'au nom et comme étant l'un des gérants de la société de commerce qui existe entre lui et le dit Sieur, son frère, et comme ayant pouvoir de stipuler pour et au nom de ladite société,

D'une part ;

M. Théodore Watremetz, contremaître teinturier, demeurant à Cambrai, rue des Capucines chez M. Liboire Watremetz

D'autre part ;

A été fait et convenu ce qui suit :

$\underline{\text { Article }}^{\mathrm{er}} \mathrm{M}$. Théodore Watremetz engage par les présentes son industrie et son soin, en qualité de contremaître en teinturerie, au service de MM. Favart pour un terme de quatre années à compter du $1^{\text {er }}$ juillet prochain, c'est-à-dire jusqu'au $1^{\text {er }}$ juillet 1852. En conséquence, il s'oblige à partir incessamment pour la Russie et à se rendre à Pouchkino (près de Moscou) dans les ateliers de MM. Favart pour y surveiller la teinture de toute espèce de tissus en laine. 
Il s'oblige, jusqu'au terme ci-dessus désigné à consacrer entièrement et exclusivement son temps et ses connaissances aux besoins des établissements de MM. Favart en ladite qualité, sans pouvoir les quitter sans motif de mécontentement grave. Par suite, il ne pourra durant ledit espace de temps former en Russie pour son compte personnel aucune entreprise particulière, ni d'accepter de place dans aucun autre établissement de quelque genre que ce soit.

Néanmoins dans le cas où M. Watremetz ne pourrait s'habituer au séjour dans la Russie, il lui sera loisible de se retirer dans le terme de deux années, c'est-à-dire au $1^{\mathrm{er}}$ juillet 1850 , mais il devra prévenir MM. Favart 6 mois à l'avance. Dans le cas où il voudrait user de cette faculté, il devra recevoir dans l'intervalle du dit terme de 6 mois la personne qui lui sera présentée pour le remplacer et la mettre au courant de l'industrie qu'il exercera chez MM. Favart.

$\underline{\text { Article }} 2^{\text {ème }} \mathrm{M}$. Favart en son nom s'oblige à payer à M. Watremetz une somme de 8000 francs, à titre d'appointements pour chacune des 4 années du présent engagement. Cette somme sera exigible par douzième à raison de 666,66 francs par mois, nette et franche de toute retenue pour change ni à tout autre titre quelconque.

Les frais de voyage de $\mathrm{M}$. Watremetz de Cambrai à Pouchkino, compris ceux de passeport, seront à la charge de M. Favart, aussi bien que le logement, la nourriture, le chauffage, l'éclairage et le blanchissage de M. Watremetz aussi longtemps que sera exécuté le présent contrat.

M. Watremetz sera autant que possible admis à la table de MM. Favart qui s'obligent en cas de maladie à lui fournir les soins et médicaments nécessaires.

MM. Favart ne pourront congédier M. Watremetz avant l'expiration du terme de 4 années cidessus fixé, sauf le cas d'infirmité, de délit ou d'infraction grave et réitérée de M. Watremetz au règlement de leur maison, sauf aussi au cas où par évènement fortuit ou de force majeure les établissements de MM. Favart se trouveraient fermés sans espoir de reprise prochaine. En cas de clôture des établissements de MM. Favart, les frais de retour de M. Watremetz depuis Pouchkino jusqu'à Cambrai seraient à la charge de M. Favart. Il en sera de même au cas où à l'expiration du terme de 4 années ci-dessus fixé, MM. Favart n'offriraient pas à M. Watremetz la prorogation du présent acte à des conditions semblables sinon plus avantageuses pour

M. Watremetz.

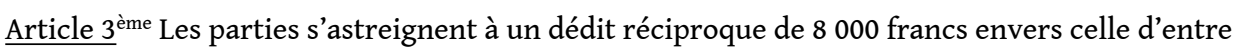
elles qui viendrait à transgresser les présentes et se refuserait à en continuer l'exécution. À cet effet et pour leurs garanties respectives, les parties conviennent ce qui suit :

MM. Favart, aussitôt l'arrivée de M. Watremetz en Russie, seront tenus de placer à la banque de Russie ou tout autre caisse ou établissement public, au nom du dit M. Watremetz une somme équivalente à 8000 francs dont le titre sera déposé entre les mains d'une personne tierce amiablement choisie entre les parties, pour être remise à $\mathrm{M}$. Watremetz à titre d'indemnité en cas d'inexécution par MM. Favart de leurs engagements. Ce placement aura lieu aux risques et périls de MM. Favart, qui seront garants du remboursement envers M. Watremetz et qui percevront les intérêts et revenus quelconques que ladite somme pourra produire, si tant est qu'elle en produise.

De leur côté MM. Favart pourront exiger, mais seulement après avoir préalablement justifié du dépôt fait par eux de la somme de huit mille francs qu'une retenue de moitié soit faîte sur les appointements mensuels de $\mathrm{M}$. Watremetz, pour être placée de la même manière au nom de MM. Favart dans une caisse publique jusqu'à ce que par l'accumulation successive des dites retenues et intérêts qu'elles pourront produire, il eut été complété une pareille somme de 8000 francs dont le titre sera également déposé entre les mains d'un tiers désigné d'un commun accord pour être remis à MM. Favart à titre d'indemnité si nonobstant les engagements présentement contractés, $M$. Watremetz venait à quitter leur établissement, sauf pour les cas cidessus présentés.

Toutefois, le dédit stipulé ne pourra être encouru de part ni d'autre que sur la décision préalable des consuls de la nation respective des contractants, lesquels apprécieront les faits et les 
prétentions des parties.

Fait en double original à Cambrai, le 22 juin 1848.

Approuvé l'écriture ci-dessus A. Favart

J'approuve l'écriture ci-dessus Th. Watremez

2/Lettre de P. Chevalier à comte Orlov ${ }^{44}$

À Son Excellence Monsieur le Comte Orloff Ministre de l'Empire

Monsieur le Comte,

Absent depuis quelque temps de Saint-Pétersbourg, j'apprends à mon retour que mon parent Georget et sa femme venant en Russie pour mon établissement, sont retenus à Cronstadt, sans pouvoir obtenir l'autorisation de se rendre à Saint-Pétersbourg, quoique munis d'un passeport recouvert d'un visa de l'ambassade de Russie à Paris et sur la foi duquel ils se sont mis en route.

[Certain] de pouvoir répondre de leurs principes et de leur moralité j'ai l'honneur d'exposer à Votre Excellence que l'ordre rigoureux qui met un obstacle à leur arrivée jusqu'ici, me jette dans le plus cruel embarras, n'ayant entrepris mon commerce que dans la ferme croyance d'être secondé par eux. Plein de confiance dans la noblesse et la générosité de votre caractère, j'ose venir supplier Votre Excellence d'avoir la bonté de prendre part à ma position en donnant l'ordre de faire visiter les effets et les papiers de mon parent Georget et sa femme et de leur permettre, d'après le résultat de cette vérification, de se rendre après de moi à Saint-Pétersbourg.

Dans l'espoir que vous daignerez accueillir ma demande.

P. Chevalier, naturalisé sujet russe et marchand de la $3^{\text {ème }}$ guilde.

SP, le 13 août 1848

\section{NOTES}

1. A. Tchoubarian, F.-D. Liechtenhan, V. Rjéoutski, O. Okouneva, Les Français dans la vie intellectuelle et scientifique en Russie ( $\mathrm{XVIII}{ }^{e}-\mathrm{XX} \mathrm{X}^{e}$ siècles).

2. Notamment, R. Dartevelle, "Paysages et risques industriels en Russie en 1906, l'expertise d'un réassureur Français », p. 411-442.

3. A. Brandt, «Essai sur les mulhousiens en Russie au xix ${ }^{e}$ siècle », N. Stoskopf, « Quitter l'Alsace pour faire fortune. Le cas des entrepreneurs du XIX ${ }^{\mathrm{e}}$ siècle ».

4. Archive d'État de la ville de Moscou (Gosudarstvennyi Arhiv goroda Moskvy), désormais GAM, f. 17, op. 21, d. 3, p. 1 .

5. Ibid.

6. R. Dartevelle, « Paysages et risques industriels en Russie en 1906 », art. cit.

7. Archive d'État de Fédération de Russie (Gosudarstvennyi Arhiv Rossiyskoi Federatsii, désormais GARF), f. 109, op. 133, d. 427.

8. Archives Départementales du Rhône, f. 242J, d. 120, p. 118.

9. F. Gasnault, A. Kiselev (dir.), Paris-Moscou, un siècle d'échanges, 1819-1925. Documents inédits des Archives de Paris et de Moscou, p. 59. 
10. O. Melnichenko, "Les Français dans la vie économique russe: le cas des entrepreneurs textiles (fin du XIX ${ }^{\mathrm{e}}$-début du Xx ${ }^{\mathrm{e}}$ siècle) », p. 86-103.

11. H. Joly, Les Gillet de Lyon. Fortunes d'une grande dynastie industrielle (1838-2015), p. 251.

12. F. Gasnault, A. Kiselev (dir.), Paris-Moscou, un siècle d'échanges, 1819-1925, ibidem, p. 65.

13. Parti de Londres, et passé par Le Havre, le navire «Camilla » arrive dans le port de Kronstadt le 25 juillet 1848. Hormis Théodore Watremez, sont retenus dans la forteresse de Kronstadt : 1) Louisa Watkins avec sa fille Lyuza (Louiza), 17 mois, sans passeport, qui accompagne son époux Robert Watkins, ressortissant de GrandeBretagne, arrivé pour enseigner au lycée de l'Église Anglaise ; 2) Pierre Jaubert, citoyen français de Segonzac (Charente), précepteur, 3) Just Georget, tailleur, citoyen français de Montbrun, avec sa femme Élise. (GARF, f. 109, op. 133, d. 427, p. 11). Les passagers arrivés sur le "Vladimir» (ou "Nicolas I» selon d'autres documents) également retenus à Kronstadt sont : 1) $\mathrm{M}^{\text {lle }}$ Cécile Matile, Suisse de Locle, canton Neuchâtel, qui rentre à Moscou chez ses frères, Alexandre et Paul Matile, fabricants d'indiennes. À Saint-Pétersbourg, elle a son cousin Charles Verner, marchand d'horlogerie ; 2) Pierre "Piotr» Eger, ressortissant danois, graveur, qui rentre chez son beau-père, petit bourgeois, Helming ; 3) Friedrich Miller, ressortissant prussien, apprenti orfèvre, qui rentre chez son père, l'orfèvre Miller ; 4) $\mathrm{M}^{\text {lle }}$ Anne Singer, ressortissante saxonne, qui se rend à Moscou pour se marier avec le lithographe Bachmann. (GARF, f. 109, op. 133, d. 427, p. 32). Le document est traduit du russe.

14. GARF, f. 109, op. 133, d. 427.

15. Par exemple, le général-lieutenant Maurice Destrem, ingénieur, académicien, demande pour tailleur Just Georget : « [...] $\mathrm{M}^{\mathrm{r}}$ Georget tailleur qui a travaillé pour moi lors de mon dernier séjour à Paris, m'a communiqué, aussitôt après les [...] journées de Février, son intention de fuir un pays où dominait l'anarchie et de venir s'établir en Russie. C'est un homme de caractère doux et sérieux et d'une excellente moralité, et qui n'ayant pour but que de vivre de son travail, et, par cela même, ennemi du désordre et de toute [...] qui pourrait menacer la propriété, et je le connais assez pour me porter garant de sa bonne conduite [...] ». (GARF, f. 109, op. 133, d. 427, p. 37.) En outre, pour Georget prie Marie Narychkine, née comtesse Dolgoroukova, et le parent de Georget P. Chevalier (voir sa lettre, annexe 2).

16. GARF, f. 109, op. 133, d. 427, p. 2. Un autre fonctionnaire ajoute son commentaire en bas de ce document : «Bien sûr, cela pourrait être considéré comme un cas, ayant une grande importance commerciale, mais il me semble que la fabrique ne disparaitra pas sans ce Français ».

17. GARF, f. 109, op. 133, d. 427, p. 4 (manuscrit traduit du russe).

18. Dans les documents, ce nom s'écrit différemment: Watremetz, Watremest, Vaternest, Watremez. Théodore signe le contrat comme Watremez.

19. Voir le texte complet dans les annexes.

20. GAM, f. 109, op. 133, d. 427, p. 15-16v.

21. Le pouvoir d'achat d'un franc 1848 était équivalent à environ 4 euros de 2018. En effet, le franc germinal équivalait à $290 \mathrm{mg}$ d'or fin de 1803 à 1914 et selon l'INSEE, 1 franc or 1901 aurait un pouvoir d'achat équivalent à 3,86 euros 2017, compte tenu de l'inflation observée entre les deux années. 
22. A. Alpatov, Fabrika kupza Favara, http://pushkino.tv/news/kray-rodnoy/132824/, consulté en juillet 2018.

23. Ibid.

24. O. Melnichenko, «Les Français dans la vie économique russe », art. cit.

25. http://www.bm-lyon.fr/expositions-en-ligne/une-fabrique-de-l-innovation/ exposition/portraits/article/gillet-joseph, consulté en aout 2018.

26. O. Melnichenko, «Les Français dans la vie économique russe », art. cit.

27. Ibid.

28. GAM, f. 349, op. 1, d. 81, p. 31-32v.

29. M. Naschokina, Arhitectory moskovskogo moderna. Tvorctheskie portrety, p. 254-274.

30. Pour le moment, les origines des fondateurs ne sont pas déterminées. Le tampon de ce comptoir parle des origines germanophones (Steffen \& Lehmann am Kanal in Moscau) (GAM, f. 349, op.1, d. 81, p. 41), la correspondance avec des clients est en français.

31. Electritcheskiy signalnyi kolokol dlia jeleznyh dorog, Katalog.

32. GAM, f. 349, op. 1, d. 81, p. 37-41.

33. En 1911, le comptoir J. Vernet et $C^{\text {ie }}$ se transforma en Société anonyme de constructions $\mathrm{J}$. Vernet et $\mathrm{C}^{\mathrm{ie}}$, avec un capital réservé à la filiale russe d'un montant de 1 million de francs (GAM, f. 51, op. 10, d. 48, p. 1). L'assemblée générale des actionnaires du 27 juin 1912, réunie au siège social à Paris, 115 rue du Faubourg Poissonnière, mentionne le capital de deux millions de francs (GAM, f. 51, op. 10, d. 48, p. 16).

34. GAM, f. 51, op. 10, d. 48, p. 12.

35. GAM, f. 349, op. 1, d. 168.

36. J. Morin, Souvenirs d'un banquier français.

37. L'historiographie parle de la fabrique C. Giraud, fondée avec 250 métiers dans le quartier Khamovniki. En revanche, les documents découverts dans les Archives d'État de Moscou témoignent que Claude Giraud crée sa première fabrique dans les bâtiments loués chez Solodovnikov, dans le quartier Serpoukhov de Moscou, où il monte 200 métiers à bras. (GAM, f. 16, op. 25, d.870, p. 4). C'est en 1879, en partenariat avec son maître et parent du coté de sa femme Marie Kakouchkine, le fabricant Istomine, C. Giraud, en demandant (d'après le gouvernement de Moscou en novembre 1878) l'autorisation d'ouverture d'une nouvelle fabrique avec un atelier de teinture, s'installe à Khamovniki. (GAM, f. 16, op. 25, d.1789, p. 1-3).

38. Industrie de la soie en Russie. Usines Claude Giraud à Moscou, 1875-1900, p. 6.

39. Ibid., p. 7.

40. Ibid.

41. Ibid., p. 29.

42. Ibid., p. 29-30.

43. GAM, f. 109, op. 133, d. 427, p. 15-16v.

44. GAM, f. 109, op. 133, d. 427, p. 42. 


\section{RÉSUMÉS}

Jusqu'en 1880 l'industrie de la soie en Russie se trouve dans un état archaïque très dépendant de la demande de la clientèle riche: noblesse, clergé, bourgeoisie naissante. Les raisons sont: manque d'innovations, manque de ressources humaines qualifiées, défaut de la politique protectionniste du gouvernement russe. L'arrivée des spécialistes français, notamment lyonnais, avec leur savoir-faire contribue au développement des manufactures franco-russes. Les visionnaires et créatifs entrepreneurs français, en attirant des capitaux français et russes, en utilisant toutes les innovations de l'industrie textile, tout en conservant et développant leur savoir-faire lyonnais, obtiennent des résultats impressionnants quelques années seulement après leur arrivée. Les relations avec les fournisseurs français de matières premières, l'importation d'équipement performant européen, et l'utilisation de l'électricité et de la force de la vapeur ont procuré aux fabricants français un avantage certain sur leurs concurrents russes.

\section{AUTEUR}

\section{OLGA MELNICHENKO}

Doctorante en histoire, Sorbonne Université, UMR 8596 Centre Roland Mousnier 


\title{
L'URSS et le CoCom (Comité de coordination pour le contrôle multilatéral des exportations) : transferts de technologie et guerre froide économique
}

\author{
Irina Sheveleva
}

1 Le contrôle par les alliés occidentaux des exportations vers le bloc soviétique, institutionnalisé au sein du Comité de coordination pour le contrôle multilatéral des exportations (CoCom), est un aspect de la guerre froide rarement analysé et mal compris. L'une des raisons est le secret qui a entouré l'organisation depuis sa création. Cela a entraîné une faible attention du public et des chercheurs, ce qui rend l'analyse difficile. Un autre facteur est probablement l'autorité conférée à une seule étude empirique majeure sur les contrôles des exportations américaines et le CoCom, Western Economic Warfare $^{1}$ de Gunnar Adler-Karlsson (1968). Aujourd'hui, cela semble quelque peu regrettable, car cette monographie, malgré sa valeur indéniable en tant que réussite scientifique pionnière, est dépassée sur les plans conceptuel et empirique.

2 L'historiographie du CoCom s'est en grande partie formée vers les années 1990 durant l'existence de cette structure ${ }^{2}$. Ce thème fait à l'époque partie du mainstream scientifique avec tous les attributs emblématiques, à savoir des colloques, des tables rondes organisées sur « le contrôle des exportations de [...] technologie vers les pays de l'Est ${ }^{3}$ ». La chute de l'URSS a suscité un nouvel intérêt pour le CoCom au début des années 2000. Des publications ont eu pour but de faire le point ex post sur cette structure secrète de la guerre froide ${ }^{4}$. La présente analyse a pour but de montrer les facteurs explicatifs des particularités du CoCom durant ces années de fonctionnement 1945-1991. Pour parvenir à cet objectif, on va étudier le modèle d'emprunt des technologies occidentales par l'URSS ainsi que les premiers pas de l'organisation du CoCom. 


\section{Le modèle d'emprunt des technologies occidentales par l'URSS : I'Occident a-t-il acheté la paix ou plutôt "vendu la corde »?}

3 Malgré le remplacement du CoCom en 1996 par l'arrangement de Wassenaar ${ }^{5}$ sur le contrôle des exportations d'armes conventionnelles et de biens et technologies à double usage, les mécanismes de l'embargo stratégique restent pertinents jusqu'à nos jours. Reprenant Hegel, Marx fait référence au philosophe allemand au début de son livre Le 18 brumaire de Louis Bonaparte :

«Tous les grands événements et personnages historiques se répètent pour ainsi

dire deux fois [...] la première fois comme tragédie, la seconde fois comme farce. ${ }^{6}$ "

Mais ce qui se passe aujourd'hui dans le domaine des sanctions économiques entre les pays occidentaux et la Russie est-il vraiment une farce?

5 Pas du tout. En effet, à l'été 2018, la Douma, la chambre basse du Parlement russe, en réponse aux sanctions économiques des États-Unis et de ses alliés, a proposé, pour ces derniers, d'instaurer «l'épuisement des droits exclusifs sur la marque». Cette déclaration signifie que, si le projet de loi était adopté, la Russie pourrait se permettre la production de biens sans la permission des titulaires de droits, par exemple sur la base de prototypes américains. En pratique, cela pourrait signifier un piratage légalisé dans la sphère technologique.

6 Pourquoi cette réponse? Parce que cela s'est déjà vu par le passé de l'URSS. Pour illustrer ce constat, prenons l'exemple de l'origine de la sidérurgie en URSS dans les années 1930. Staline a mis l'accent sur le fer et l'acier comme bases de l'économie socialiste. Ses plans comprenaient la reconstruction et l'expansion d'une vingtaine d'usines érigées à l'époque prérévolutionnaire, et la construction de trois gigantesques usines à Magnitogorsk, Kuznetsk et Zaporozhe. Il était bien évident pour son dirigeant que l'Union soviétique manquait totalement des ressources techniques nécessaires pour construire les nouvelles usines métallurgiques et reconstruire les anciennes. En ce qui concerne les ingénieurs russes compétents, la plupart d'entre eux avaient quitté la Russie. Les usines d'équipement métallurgique tsaristes telles que Sormovo et Kramatorsk, qui avaient produit certains types de fer et l'équipement des aciéries à la fin du XIX ${ }^{e}$ siècle, avaient été dépouillées de leurs ressources techniques. Les ingénieurs allemands qui dirigeaient les usines métallurgiques en 1927-1928 étaient politiquement suspects.

7 En même temps, les ambitions étaient énormes. Près de la montagne Magnitnaya par exemple, il était prévu de construire une usine d'une capacité de production de 570000 tonnes de fonte, avec la possibilité d'une nouvelle expansion à 660000 tonnes. Puis, au début des années 1930, alors que la construction était déjà en cours, le Comité Central du Parti Communiste a fixé l'objectif de capacité de production à 2,5 millions de tonnes de fonte avec une expansion ultérieure à 4 millions de tonnes par an ${ }^{7}$. Initialement, de 1918 à 1921, l'élaboration de la conception de l'usine sidérurgique de Magnitogorsk fut confiée à la Société Sibérienne des Ingénieurs de Tomsk et rattachée au Combinat Oural-Kouznetsk. Après 1925, ce fut l'Uralprojectbureau de Sverdlovsk qui poursuivit le développement du projet. Cependant, dans le cadre de la création du Guipromez (Institut d'État pour la construction des usines métallurgiques), le travail de conception de Sverdlovsk fut transféré à Leningrad. Ces agissements ministériels, 
autour du développement du concept de l'industrie métallurgique dans son ensemble et des entreprises individuelles en particulier, étaient dus à l'incapacité de l'URSS de jeter par ses propres forces les bases techniques de la métallurgie soviétique.

C'est le bureau d'études d'Arthur G. Mc Kee à Cleveland qui a été invité, en 1928, pour concevoir le design de l'usine métallurgique à Magnitogorsk ${ }^{8}$. La conception de Mc Kee était basée sur l'usine de Gary, Indiana, United States Steel Corp., la plus grande usine de fer et d'acier intégrée dans le monde à cette époque. On ne sait pas pourquoi ce fut Mc Kee qui fut chargé de concevoir l'usine. La préhistoire de la conclusion du contrat avec Mc Kee n'est pas encore assez claire ni bien étudiée 9 . La Société «Mc Kee » ne possédait pas ses propres responsables d'ateliers. Le lendemain de la signature de l'accord, la direction de Mc Kee a publié dans les journaux et les magazines des annonces pour recruter des employés. Pour réaliser ce projet, 450 ingénieurs américains travaillaient jour et nuit. Le témoignage de R.W. Stuck décrit cette conception de Mc Kee comme complète :

«Jusqu'au dernier écrou avant le début de la construction; rien de cette taille et ampleur n'avait déjà été fait avant... C'est le meilleur design d'usine sidérurgique... ${ }^{10}$

")

9 Pour diriger le design de Magnitostroy, Mc Kee a invité Thayer, qui fut pendant 13 ans l'ingénieur en chef de la plus grande usine métallurgique de Gehry ${ }^{11}$. Ce dernier fut chargé de toute la gestion du développement du projet. De plus, de célèbres professeurs américains, faisant autorité sur les questions minières, Swart et Davies, de l'Université du Minnesota ont été impliqués dans le projet.

10 Une commission de Magnitostroy a été envoyée aux États-Unis. Elle comprenait le chef de la construction V.A. Smolyaninov, l'ingénieur en chef V.A. Gasselblat, le spécialiste en hauts fourneaux V.A. Sorokin, l'ingénieur I.D. Semikin et d'autres spécialistes ${ }^{12}$. Les lettres du chef adjoint de la construction de Magnitostroy, Chingiz Ildrim, qui représentait les intérêts de Magnitogorsk aux États-Unis, donnent une idée sa haute considération pour l'expérience de la Société Mc Kee. En août 1931, Ildrim écrivait à Ordjonikidze :

« Je considère nécessaire de mettre les ingénieurs américains à la tête de l'affaire et de leur donner notre aide. Seulement dans ce cas nous pouvons compter sur le succès du travail. ${ }^{13}$ "

11 Les Américains ont accusé les ingénieurs soviétiques d'aventurisme. En réponse à une réplique sur le rythme du travail extrêmement lent, Arthur Mc Kee a répondu à Ildrim :

«Voulez-vous vous précipiter? Avancer trop vite ou agir à la hâte? La plus grande usine métallurgique du monde, Gehry, américaine, a été construite pendant douze ans et demi. Ajouter onze autres années pour son design. Et vous espérez mettre en marche le combinat en trois ans $!^{14} »$

M. S. Gugel se rappelait ses conversations avec W. Haven, le vice-président de Mc Kee :

«M. Haven est certainement un métallurgiste compétent. Il perçoit facilement l'échelle de Magnitostroy; il aime même Magnitostroy; c'est une personne polyvalente, éduquée, cultivée. J'ai aimé discuter avec lui au bureau pendant des heures et à la maison. La principale chose que n'a pas comprise M. Haven était notre rythme bolchevique. Il ne comprenait pas pourquoi il fallait se dépêcher. À son avis, l'aboutissement est quand tout est prêt. À notre avis, l'aboutissement est quand le projet devrait être prêt. ${ }^{15}$ "

13 Le contrat avec Mc Kee fut finalement rompu en mai 1932, après le lancement de la première étape de l'usine, alors que le travail de conception principale était déjà 
terminé. La véritable raison de la rupture peut être considérée comme le désir des dirigeants soviétiques d'économiser de l'argent et de l'assistance technique. Le contrat avec Mc Kee coûta cher au Trésor soviétique (2 800000 dollars), sans compter les salaires versés au personnel envoyé en URSS. Naturellement, la partie soviétique se réservait le droit de résilier unilatéralement l'accord. La plupart des employés de Mc Kee ne voulaient pas jouer le jeu selon les règles soviétiques, s'adapter aux méthodes de travail soviétiques, en les considérant comme de l'aventurisme ou de la folie. Plus les Américains discutaient avec les ingénieurs soviétiques, plus ces faits furent utilisés dans un but de propagande. Les représentants de la compagnie américaine pensaient qu'ils étaient invités en URSS pour former le personnel russe aux méthodes adoptées dans la pratique américaine, mais, chez Magnitostroy, on exigeait d'eux non seulement une qualification élevée et une expérience de la production considérable, mais aussi une capacité de suivre les règles du jeu à la soviétique.

14 L'histoire de la relation entre Magnitostroy et la Société Mc Kee peut être considérée comme l'un des points les plus controversés concernant l'efficacité du transfert de l'expérience technique occidentale à l'industrie soviétique. Les sources ne nous permettent pas de comprendre clairement qui avait raison au bout du compte. Il est encore difficile de savoir pourquoi la Société Mc Kee a accepté de réaliser le projet dans un aussi bref délai pour ensuite déclarer son irréalité. Dans l'historiographie soviétique, le projet mené par Mc Kee a fait l'objet de critiques. À cet égard, l'opinion de l'ingénieur M. Litichevsky - qui a travaillé à Magnitogorsk dans la filiale du Guipromez depuis la fin des années 1950 et qui a fait face au projet américain de l'atelier des hautsfourneaux est intéressante :

« La compagnie Mc Kee, qui a construit notre usine, est critiquée par beaucoup. En fait, son projet, derrière certains inconvénients inévitables, est un exemple de professionnalisme. ${ }^{16}$ »

15 L'autre usine métallurgique de Kouznetsk a été construite en coopération avec la Freyn Engineering Company de Chicago. Le journal américain de 1927 présente ce contrat comme l'un des plus grands jamais négociés dans le domaine des services de conseil en ingénierie ${ }^{17}$. L'accord d'entreprise pour la construction de l'usine de Kuznetsk, d'une capacité de 1 million de tonnes par an, a été signé le 4 juin 1930 avec Novostal. Un contemporain a écrit :

«Entre cinquante et soixante ingénieurs américains constitueront l'organisation qui dirigera l'exécution de la conception, des spécifications, de la fabrication, et supervisera l'érection et le fonctionnement initial de l'aciérie Kuznetsky. Freyn, pour sa part, avait réussi à traduire ce potentiel (le marché de l'Union soviétique qui devait être capturé) dans un contrat dans le secteur stratégique de la métallurgie ferreuse. ${ }^{18}$ "

16 Les recherches montrent qu'au fur et à mesure du développement de l'URSS, un modèle d'emprunt des technologies occidentales a été formé. Le trait caractéristique de ce modèle était l'usage de tous les moyens possibles pour saturer l'économie par les technologies modernes, même par des moyens non « conventionnels ». Ce modèle a été institutionnalisé en 1931 par la loi sur les inventions. La nouvelle loi a encouragé le "plagiat» en autorisant le rejet du principe de nouveauté et "l'application des inventions déjà décrites dans la littérature étrangère ${ }^{19}$ ", soi-disant "sans l'aide de licence ». On montrera que le choix de ce modèle d'emprunt de la technologie occidentale est devenu un point de déformation. Il a entrainé une déformation des 
approches des normes d'emprunts technologiques par rapport à celles en vigueur dans le monde occidental.

17 La déformation professionnelle est une phraséologie qui indique une attitude apprise à l'occasion de l'exercice d'un métier et qui a été progressivement appliquée aussi au monde de la vie privée. Selon une phrase attribuée au psychologue américain Abraham Maslow :

"Pour qui ne possède qu'un marteau, tout ressemble à un clou. »

$\mathrm{Au}$ fil des années, le coût de la politique «copier et ne pas payer» a augmenté, condamnant le pays à l'arriération. Une solution semblant simple et facile s'est avérée être une impasse. C'est peut-être la véritable raison de la lutte internationale contre le piratage. Parfois, cela est dû non seulement à des considérations éthiques, mais aussi à des considérations pragmatiques. Pour les industries technologiques, le piratage est comme une drogue : offrant une détente temporaire, il ferme l'avenir. À cet égard, il semble opportun de citer l'historien Hédi Ennaji. Après avoir donné un bref descriptif des secteurs militaires soviétiques clés ayant bénéficié des transferts technologiques occidentaux - permettant d'établir l'échec du CoCom - il écrit :

« [que] nous aurions pu rajouter à ce bilan les secteurs agricoles et chimiques, mais ces données suffisent pour établir que le commerce avec l'URSS, que certains avaient promu dans l'optique «d'adoucir» les Soviétiques, eut les effets inverses:

l'Occident loin d'avoir acheté la paix a plutôt « vendu la corde ${ }^{20}{ }^{20}$ "

19 Quant au début des années 1950, l'embargo est instauré, la vulnérabilité de l'économie de l'URSS au plan technologique était bien évidente, au moins pour les structures étroitement intéressées. Toute une série de documents, consacrés aux questions de la "Vulnérabilité du bloc soviétique à la guerre économique ${ }^{21}$ ", a été produite par la CIA. Selon ces notes, chacun des plans quinquennaux en URSS avait pour objet principal de renforcer la capacité de production soviétique dans les zones déficitaires, même au prix d'un gaspillage considérable de ressources et d'énergies ${ }^{22}$. L'URSS et ses satellites avaient constamment besoin de pièces de rechange pour la grande quantité d'équipements étrangers acquis au cours des années précédentes. Une grande partie de l'inventaire de l'équipement industriel dans la sphère soviétique était de fabrication étrangère. Par exemple, on estimait qu'environ $35 \%$ des machines-outils en service en URSS étaient de construction occidentale. La majeure partie de ces équipements avait plus de cinq ans ${ }^{23}$, et commençait déjà à présenter de sérieux problèmes de maintenance en raison de la non-disponibilité des pièces de rechange et de la difficulté de les fabriquer en URSS ou dans ses satellites ${ }^{24}$.

\section{CoCom : guerre économique ou sanctions économiques?}

20 La consolidation d'un modèle, dans lequel l'URSS agit en tant que destinataire des technologies occidentales, conduirait à ce que l'Occident, à son tour, agisse en tant que donateur ou, tôt ou tard, en refusant ce rôle, introduise des sanctions contre le destinataire. La page la moins étudiée de la guerre froide a commencé à la fin de 1947 quand l'administration Truman a été mise sous pression par des groupes d'intérêt nationaux qui voulaient que les États-Unis restreignent leurs exportations stratégiques vers l'Europe de l'Est. Cette pression extérieure a coïncidé avec une initiative au sein de l'administration pour empêcher que le commerce Est-Ouest augmente le potentiel de 
guerre du bloc soviétique. Les États-Unis ont appliqué un embargo unilatéral à l'encontre de l'Europe de l'Est à partir de mars 1948. Cela devait se faire sans acte manifeste de discrimination arbitraire à l'encontre de l'Europe de l'Est, mais par le moyen d'une procédure commencée le $1^{\mathrm{er}}$ mars 1948 pour exiger des licences pour toutes les exportations vers l'Europe.

21 La principale motivation de l'administration Truman était probablement une crainte réelle que les exportations occidentales vers l'Europe de l'Est ne renforcent le potentiel de guerre soviétique. En même temps, l'administration Truman a conclu à la nécessité d'une politique occidentale concertée. Le point de départ qui a conduit l'Europe occidentale à rejoindre l'embargo stratégique était le câble Torep $816^{25}$. Il a été envoyé à l'ambassadeur W. Averell Harriman avec pour l'objectif d'entamer des négociations avec les pays du plan Marshall sur les contrôles stratégiques à l'exportation correspondant à la politique d'embargo des États-Unis. En 1949, au cours des négociations préalables, des divergences sont apparues concernant le degré de rigueur de la structure de coordination qui devrait diriger l'organisation éventuelle et sur les produits eux-mêmes qui devaient être sous embargo ou exportés en quantités limitées. Les Néerlandais en particulier n'acceptaient rien de plus que la poursuite du mécanisme de coordination informel déjà établi. Tandis que les négociateurs américains préféraient une organisation plus permanente, allant même jusqu'à vouloir faire de l'organisation naissante de contrôle des exportations un organe de l'OTAN.

En fin de compte, malgré une série de réunions à Paris, les Américains ont obtenu que la plus grande partie de la structure organisationnelle soit proche de ce qu'ils voulaient. Washington devait se contenter cependant d'assurances écrites secrètes des alliés, indiquant leur volonté de coopérer au sein du CoCom. Ce n'est qu'à l'automne 1949 que la majorité des pays d'Europe occidentale continentale ont accepté d'instituer des contrôles à l'exportation et de coordonner leur politique de façon permanente avec les autres pays du plan Marshall et les États-Unis. Une structure multilatérale de contrôle des exportations, sans précédent en temps de paix, a été créée en janvier 1950. Elle comportait deux organes : le Groupe consultatif (GC) et le Comité de coordination (CoCom). Le Groupe consultatif devait avoir des fonctions d'élaboration des politiques, tandis que le Comité de coordination était chargé des tâches administratives quotidiennes. Elle était composée par les États-Unis, le Canada, la Grande-Bretagne, la France, l'Italie, les Pays-Bas, la Belgique, le Luxembourg, le Danemark, la Norvège et l'Allemagne de l'Ouest. Par la suite, le nombre de participants a été monté à 18.

Il existe différentes approches dans la littérature scientifique pour déterminer la stratégie du CoCom. Certains chercheurs la définissent comme la déclaration d'une guerre économique, les autres comme un régime de sanctions. Dans les documents de la CIA, ce terme de guerre économique, a été appliqué aux mesures prises en temps de paix pour «diminuer et neutraliser le potentiel de guerre du bloc soviétique ${ }^{26}$. L'absence d'opérations militaires a été considérée comme faisant partie du mandat ${ }^{27}$ ». La plupart des références au CoCom se trouvent dans la littérature sur les sanctions économiques. Dans ce cas, l'embargo du CoCom est mis en regard de sanctions unilatérales ou internationales telles que celles en vigueur envers la Russie depuis l'annexion de la Crimée. Il s'agit des cas où un groupe de pays transmet un message public de désapprobation de sa conduite à un pays "cible ", en le punissant - ou en menaçant de le punir - économiquement pour conduite incorrecte. 

contexte. Aucun message n'avait été envoyé au Kremlin. Les dirigeants soviétiques n'ont pas été visés pour s'être mal conduits selon le droit international ou la morale ; l'embargo n'a pas été institué comme une forme de punition. Certes, le Kremlin avait conscience de l'existence du CoCom. Il est également vrai que les perceptions occidentales de l'Union soviétique en tant que menace étaient liées à ce que l'Occident considérait comme un mépris de Moscou pour les normes de droit international. La distinction entre sanctions économiques et CoCom est mieux décrite dans la terminologie développée par Knorr ${ }^{28}$. Selon ses termes, les sanctions représentent des tentatives de contraindre un pays en influençant ses décideurs. L'embargo du CoCom, visant à affaiblir (ou du moins à ne pas améliorer) le potentiel militaire soviétique, a été une tentative d'exercer directement une pression par la force pure (économique) plutôt que d'essayer d'influencer les acteurs du Kremlin ${ }^{29}$. L'objectif était de «modifier la capacité d'un État sans persuader ses décideurs ${ }^{30}$ \%. Ceci, bien sûr, a eu des conséquences sur la façon dont l'embargo fut mené. Il pourrait être, par exemple, tenu secret, comme le CoCom l'a été pendant plusieurs décennies.

Plutôt que de considérer le CoCom comme un système de sanctions, nous tentons ici de le considérer comme un outil de guerre économique en temps de paix. Dans ces guerres (comme dans les conflits antérieurs) l'ennemi était combattu aussi bien par des moyens économiques que militaires: le but de la guerre économique était de limiter les capacités de guerre de l'ennemi en lui refusant des matières premières ou en affamant son peuple et ses armées. Le moyen de guerre économique privilégié était le blocus : c'est-à-dire le siège de la côte ennemie, en veillant à ce qu'il n'y ait pas de ravitaillement. Pendant la Première et la Seconde Guerres mondiales, les Britanniques et les Américains en particulier ont perfectionné les techniques de guerre économique, entre autres par la mise sur liste noire d'entreprises connues ou soupçonnées de commercer avec l'ennemi.

Les historiens sont donc en désaccord sur la question de savoir si la guerre économique devrait inclure toutes les activités visant à entraver l'économie de l'ennemi, ou seulement des moyens économiques pour la réalisation d'un objectif national. Pour l'étude du CoCom, il semble mieux adapté de baser l'analyse sur un concept de guerre économique comme celui de Knorr ${ }^{31}$, visant à affaiblir le fondement économique du pouvoir ennemi par tous les moyens: économiques, propagandistes, législatifs, diplomatiques ou militaires. En temps de paix, le dernier terme serait généralement inutilisé, sauf en tant qu'action secrète.

L'exemple de l'industrie métallurgique montre qu'après avoir été largement dépendant de l'Occident pour l'aide économique, l'URSS a adopté le rôle de destinataire des technologies occidentales. En dépit des efforts acharnés pour développer son autosuffisance, l'URSS ne manque pas d'institutionnaliser ce modèle d'emprunt en autorisant en particulier le plagiat. On peut dire que cela est un point de non-retour. Comme l'aide occidentale a joué un rôle clé dans les progrès économiques de l'Union soviétique, la capacité de l'URSS à se développer a été considérablement affectée par la disponibilité irrégulière d'une telle assistance. Ce que démontre la guerre économique, dont l'élément marquant était le coCom. 


\section{BIBLIOGRAPHIE}

ADLER-KARLSSON Gunnar, Western Economic Warfare: a case study in foreign economic policy, (Guerre économique occidentale : une étude de cas en politique économique étrangère), Stockholm, Almqvist \& Wiksell, 1968, 1 vol. (XVI-320 p.).

BAILES Kendall E., Technology and Society under Lenin and Stalin, Princeton University Press, 1976. CHANTEBOUT Bernard et WARUSFEL Bertrand (dir.), Le contrôle des exportations de haute technologie vers les pays de l'Est, actes du colloque organisé par le Centre Droit et Défense, Ed. Masson, 1988.

ENNAJI Hédi, «Le camp occidental à l'épreuve de l'arme économique : les leçons du CoCom », Stratpole (Centre d'analyses politico-stratégiques)

[URL : https://stratpol.com/]

ELLINGS Richard J., Embargoes and world power: lessons from American foreign policy, Boulder, Westview, 1985.

FORLAND Tor Egil, cold Economic Warfare: CoCom and the forging of Strategic Export Controls, 1948-1954, New York, London, Routledge, 2017.

GUGEL' Yakov, « Vospominaniya o Magnitke », Ural'skij literaturno-kraevedcheskij sbornik, Chelyabinsk: Rifej, 1980.

HEHJVEN V., « Zamechaniya o razrabotke proekta Magnitogorskogo metallurgicheskogo zavoda i rudnika », Magnitostroy, $n^{\circ}$ 9-12, 1931.

KNORR Klaus Eugen, The power of nations: the political economy of international relations, New York, Basic Books, 1975.

LABBÉ Marie-Hélène, La politique américaine de commerce avec l'Est : 1969-1989, Paris, Presses Universitaires de France, 1990.

MASTANDUNO Michael, Economic Containment, CoCom and the politics of East-West trade, Ithaca, N.Y, Cornell University Press, New York, 1992.

MARX Karl, Le 18 Brumaire de Louis Bonaparte, Paris, Les Éditions sociales, 1969.

Magnitostroj: stroitel'stvo i ehkspluataciya Magnitogorskogo metallurgicheskogo kombinata imeni tov. Stalina. 1929-1933, (Magnitostroy : construction et exploitation de l'usine métallurgique de Magnitogorsk portant le nom d'un camarade Staline. 1929-1933), Sverdlovsk - Magnitogorsk, p. 1-3, 1934 .

POLONSKIJ Lev, Magnitka zovet, Baku, 1972.

RIGNARD Henrie, «L'URSS et le renseignement scientifique, technique et technologique », Défense Nationale, $\mathrm{n}^{\circ}$ 438, p. 107-121, décembre 1983.

SPASOVA L.P., « Magnitostroy i « Mak-Ki » : stolknoveniye delovykh kultur », Vestnik Permskogo universiteta, vol. 1 (12), p. 107, 2010.

scotT John, Derrière l'Oural, Cambridge, The Riverside Press, 1942.

Vulnerability of the Soviet Bloc to Existing and Tightened Western Export Controls, Division of Research for the USSR and Eastern Europe, Office of Intelligence Research, OIR 5447 of 26 January 1951 https://www.cia.gov/library/readingroom/docs/CIA-RDP79R01012A000500030013-1.pdf 
wooD Juntos B., « Special Cable to The Cornell Dally Sun and Chicago Dally News from Moscow,

May $14 »$, The Cornell Daily Sun, Volume XLVII, Number 180, 25 May 1927.

http://cdsun.library.cornell.edu/cgi-bin/cornell?a=d\&d=CDS19270525.2.24

\section{NOTES}

1. G. Adler-Karlsson, Western Economic Warfare: a case study in foreign economic policy.

2. H. Rignard, «L'URSS et le renseignement scientifique, technique et technologique ", p. 107-121. M.-H. Labbé, La politique américaine de commerce avec l'Est : 1969-1989.

3. B. Chantebout, B. Warusfel, Le contrôle des exportations de haute technologie vers les pays de l'Est. Ce livre résume les interventions du colloque organisé en 1986 par le Centre Droit et Défense. Pour la première fois en France, on a présenté un panorama complet des problèmes stratégiques, diplomatiques, industriels et juridiques que posait le contrôle des exportations de haute technologie vers les pays de l'Est.

4. M. Mastanduno, Economic Containment, CoCom and the politics of East-West trade. T. E. Forland, cold Economic Warfare: CoCom and the forging of Strategic Export Controls, 1948-1954.

5. L'arrangement de Wassenaar est un régime multilatéral de contrôle des exportations mis en place par 33 États afin de coordonner leurs politiques en matière d'exportations d'armements conventionnels et de biens et technologies à double usage.

6. K. Marx, Le 18 Brumaire de Louis Bonaparte, p. 9.

7. Magnitostroj: stroitel'stvo i ehkspluataciya Magnitogorskogo metallurgicheskogo kombinata imeni tov. Stalina. 1929-193, p. 1-3.

8. Un accord entre le Novostal Trust (auquel Magnitostroy était subordonné) et la société américaine Arthur J. McKee and Co de Cleveland (nommée pour la conception et la gestion responsable de la construction de l'usine de Magnitogorsk) a été conclu le 14 mars 1930.

9. En novembre 1930, dans un mémorandum adressé à Akulov, le commissaire adjoint de l'industrie lourde de l'URSS, et au président de la société, Vostokostal Kosior, on rapportait que selon les données des agents du Guépéou, la société « Mc Kee » était considérée comme très modeste sur le marché américain, n'ayant jamais réalisé de grands travaux de construction.

10. J. Scott, Derrière l'Oural, p. 68. John Scott a fait remarquer qu'il était nécessaire de confier ce contrat à un entrepreneur en raison de l'incapacité évidente de toute organisation soviétique pour faire le travail.

11. V. Hehjven, «Zamechaniya o razrabotke proekta Magnitogorskogo metallurgicheskogo zavoda i rudnika », p. 27.

12. Les archives de la ville de Magnitogorsk (MU MGA), f. 99, op. 11, d. 5, p. 6.

13. RGASPI (Russian State Archive of Socio-Political History), f. 85. op. 28. d. 71. p. 28.

14. L. Polonskij, Magnitka zovet, p. 80.

15. Y. Gugel', « Vospominaniya o Magnitke », Ural'skij literaturno-kraevedcheskij sbornik, p. 18-19.

16. L.P. Spasova, « Magnitostroy $\mathrm{i}$ « Mak-Ki » : stolknoveniye delovykh kultur », p. 107. 
17. J.-B. Wood, «Special Cable to The Cornell Dally Sun and Chicago Dally News from Moscow, May $14 »$, p. 4.

18. GARF, f. 7952, op. 5, d. 140, p. 7-8.

19. K. E. Bailes, Technology and Society under Lenin and Stalin, p. 345.

20. H. Ennaji, «Le camp occidental à l'épreuve de l'arme économique: les leçons du CoCom ", Stratpole (Centre d'analyses politico-stratégiques).

21. Vulnerability of the Soviet Bloc to Existing and Tightened Western Export Controls, Division of Research for the USSR and Eastern Europe, Office of Intelligence Research.

22. Ibid, p. 5.

23. Au moment de la publication du rapport en 1951.

24. Vulnerability of the Soviet..., p. 8.

25. Torep, 816, Aug 27, 1948, Foreign Relations of the United States, 1948, Eastern Europe; The Soviet Union.

26. Le bloc soviétique est défini comme suit: URSS, Chine, Albanie, Bulgarie, Tchécoslovaquie, Allemagne de l'Est, Hongrie, Pologne et Roumanie. La Corée, la Mongolie extérieure et certaines zones contrôlées par les communistes en Asie du Sud ne sont pas prises en compte, en raison de l'insignifiance relative des économies dans ces régions. Cependant, il est supposé que les mesures de guerre économique s'appliquent également à ces régions. Puisque la Chine était engagée dans des opérations militaires contre les forces des Nations Unies en Corée, il est nécessaire d'évaluer l'effet de la guerre économique sur la Chine.

27. National Intelligence Estimate. Vulnerability of the Soviet Bloc to Economic Warfare. Published 19 February 1951 of CIA. Vulnerability of the Soviet Bloc....

28. K. E. Knorr, The power of nations: the political economy of international relations.

29. Ibid, p. 5, 142.

30. R. J. Ellings, Embargoes and world power: lessons from American foreign policy, p. 3.

31. K. E. Knorr, The power of nations: the political economy of international relations.

\section{RÉSUMÉS}

L'un des facteurs explicatifs de la vulnérabilité du bloc soviétique à la guerre économique est le modèle d'emprunt des technologies occidentales au sein duquel l'URSS est le destinataire des technologies, tandis que l'Occident est son donateur. Ce modèle s'est formé pendant la période de l'entre-deux-guerres de sorte que, après la Seconde Guerre mondiale, avec l'apparition du bloc socialiste et le déclenchement de la guerre froide, alors que l'URSS prônait l'idéologie de la séparation plutôt que de la convergence des deux mondes, ce choix l'a conduit à une impasse. 
AUTEUR

IRINA SHEVELEVA

Doctorante en histoire, Paris I - Panthéon-Sorbonne, UMR 8 138-SIRICE 


\title{
Transferts de technologie entre l'URSS et la France (1954-1991)
}

\author{
Boris Vinogradov
}

1 Après la Seconde Guerre mondiale, l'URSS s'est montrée très intéressée dans l'accès aux technologies modernes occidentales. Dans cet article, on se concentrera sur le transfert de technologie entre la France et l'URSS. On décrira les principaux axes et tendances de transferts de technologie entre l'URSS et la France dès les années 1950 et jusqu'à la chute de l'Union soviétique. Les travaux des historiens français, Dominique Barjot ${ }^{1}$ et Marie-Pierre Rey ${ }^{2}$ par exemple, abordent la thématique de transfert de technologie entre la France et l'URSS pendant la "détente » des années 1964-1974. Ces travaux démontrent l'importance des aspects diplomatiques, financiers et commerciaux du transfert des technologies et permettent de comprendre que la France a joué un rôle majeur dans les transferts de technologie vers l'Union soviétique durant les années $1970^{3}$.

Pour évaluer les transferts, il est indispensable de préciser ce que nous entendons par «transfert de technologie». Il s'agit «d'un processus par lequel des innovations (nouveaux produits ou savoir-faire) réalisées dans un pays sont transmises dans un autre pour y être utilisées ${ }^{4}$ ». Il s'agit essentiellement d'une transmission de savoir qui implique un rôle actif de celui qui transfère comme de celui qui reçoit. Dans le présent article, le transfert de technologie entre la France et l'URSS est étudié comme une composante de la coopération économique entre ces deux pays. En restant dans le cadre de l'histoire économique, l'analyse du transfert de technologies a été réalisée en s'appuyant sur les sources essentiellement économiques et politiques, telles que les archives françaises du Ministère de l'Économie, des Finances et de l'Industrie, dossiers économiques des archives présidentielles de France, également sur les volets économiques des archives du Ministère des Affaires Étrangères de l'URSS. Une telle approche a, bien évidemment, ses limites, dont la principale se trouve dans le fait qu'en travaillant avec les sources économiques, il est difficile d'avoir une bonne vision de la coopération scientifique, tandis que le transfert de technologie se réalise souvent grâce à la coopération des chercheurs, des universités et des organismes de recherche. En même temps, dans le contexte de la guerre froide, il est clair cependant que le transfert 
de technologies entre la France et l'URSS est davantage le résultat d'une coopération économique et politique.

\section{Avant le transfert de technologies, laborieux rétablissement de relations commerciales (1950-1966)}

3 L'étude du cas franco-soviétique présente un intérêt particulier. En effet, au cours des années 1960-1980, la France a joui d'un statut privilégié dans les relations extérieures de l'Union soviétique ${ }^{5}$. À ce choix, on peut voir trois raisons. Premièrement, à la différence des États-Unis, "citadelle de l'impérialisme » et de l'Allemagne fédérale, foyer supposé de revanchisme, la France a une image et une audience internationales telles qu'elle peut fournir une caution morale à la politique de détente. Ceci est d'autant vrai que ce pays a été le premier en Occident à s'engager dans cette politique. Deuxièmement, la politique d'indépendance poursuivie par la France intéresse Moscou dans la mesure où elle s'écarte de l'orthodoxie atlantiste, par conséquent, elle apparaît aux Soviétiques comme un moyen d'affaiblir la cohésion du monde occidental ou d'empêcher la naissance d'une Europe supranationale. Troisièmement, de façon plus fondamentale, la situation géographique de la France fait de ce pays le contrepoids inévitable à l'Ouest de la puissance allemande.

Pendant la période de la guerre froide, le volume du commerce Est-Ouest est réduit au minimum. En 1949, le CoCom, comité de l'OTAN créé sur l'initiative américaine, est chargé de dresser une liste des produits dits stratégiques que les pays membres s'engagent à ne pas exporter vers l'Est. En 1952, cette liste comprend $50 \%$ des biens entrant dans les échanges internationaux. Il n'est donc pas surprenant que le commerce de l'URSS avec l'Ouest qui représentait le cinquième de son commerce extérieur total en 1948 soit réduit au dixième en 1950. Ce déclin se répercute à l'échelon franco-soviétique lorsque les échanges sont extrêmement faibles dans les années 1950-1951, malgré l'accord commercial du 29 décembre 1945 qui marque leur reprise. Cet accord, renouvelé en 1951, définit les conditions générales présidant aux relations commerciales entre les deux pays ${ }^{6}:$ il réaffirme la clause de la nation la plus favorisée et le principe du tarif douanier minimum pour les importations et les exportations soviétiques.

En 1952, avec la conférence économique internationale de Moscou, apparaît le premier signe d'une évolution des rapports Est-Ouest. Cette conférence est significative de la volonté soviétique d'augmenter son commerce extérieur, en particulier avec l'Ouest. À titre d'exemple, le président de la Chambre de commerce soviétique y déclarait :

« [que] les Soviétiques seraient prêts à augmenter leurs achats en France de cinq à six fois ainsi que leurs exportations, dépassant ainsi le chiffre d'affaires maximum d'avant-guerre. " "

6 Après la mort de Staline, la conférence de Genève confirme cette nouvelle orientation (avril 1953). Sur le plan franco-soviétique, le résultat direct de la conférence de Moscou est la signature, le 14 juillet 1953, d'un accord commercial et d'un accord de paiement qui marquent un tournant dans les relations des deux pays. Le volume des échanges commence à s'accroître rapidement : de 37 millions de dollars en 1953, ces échanges passent à 94 millions de dollars en 1955. 
7 À partir de 1955, l'accélération de l'industrialisation soviétique d'une part, les exigences de la coexistence pacifique d'autre part poussent à un accroissement des échanges considérés par l'Est comme un moyen politique de détente vis-à-vis des pays industrialisés de l'Ouest. Jusqu'aux années 1965-1966, les relations purement commerciales se développent à un rythme assez rapide. Les principaux instruments du commerce Est-Ouest sont des accords bilatéraux qui stipulent les biens à échanger pour une période pluriannuelle, et qui concrétisent le désir des États de voir au moins un minimum d'échanges s'établir entre eux. Ces accords sont révisés par des protocoles commerciaux qui permettent de rééquilibrer les échanges au cas où ceux-ci sont effectués plus rapidement d'un côté que de l'autre.

Ce bilatéralisme est indissociable de deux autres éléments: monopole d'État du commerce extérieur et inconvertibilité du rouble. Le commerce extérieur est un monopole d'État, d'après l'article 14 de la Constitution de l'URSS. La réalisation des opérations de commerce avec l'étranger, négociations et signatures de contrats est déléguée à des organisations spécialisées par type de produits et par secteur géographique, communément appelées "Centrales du Commerce Extérieur" ». Le contrôle des changes par l'État va de pair avec le monopole du commerce extérieur. L'État se réserve le droit exclusif de réaliser toutes les opérations en devises et en or. Il est le seul à disposer des moyens de paiement acceptés par les pays étrangers.

Enfin, ce monopole des ventes et achats de devises est lié à l'inconvertibilité du rouble. Cette dernière a pour conséquence la conclusion d'accords bilatéraux, strictement équilibrés, dans lesquels les exportations soviétiques servent à obtenir les moyens de paiement nécessaires aux importations.

10 À cela s'ajoutent les difficultés de commercialisation des marchandises soviétiques qui ne conquièrent pas "aisément» le marché occidental en raison de leur qualité moyenne et de leur "technicité » insuffisante. Il en résulte, pour l'URSS, une faible capacité de paiement en devises qui est un frein au développement de ses échanges avec les pays occidentaux. Néanmoins, dès le milieu des années 1960, le commerce, au sens traditionnel d'un échange de biens matériels équilibré à double sens, n'est plus considéré comme un cadre suffisant pour les relations économiques. Aux accords commerciaux se superposent des accords de "coopération ", dont le plus complet est celui mis en place par l'accord franco-soviétique du 30 juin 1966.

\section{Débuts et approfondissement du transfert des technologies (1966-1979)}

11 C'est à ce moment que commence le transfert des technologies entre la France et l'URSS. Les relations franco-soviétiques ont, en effet, une configuration particulière que n'ont pas celles des autres pays occidentaux commerçant avec l'URSS. L'inspiration en faveur de la coopération entre les deux pays a été avant tout politique. On part fréquemment de la position privilégiée que la France aurait dans ses rapports avec l'URSS; l'un des meilleurs exemples est l'adoption par les Soviétiques du procédé français SECAM de TV couleur en $1965^{\circ}$. Ceci s'explique par le désir qu'avait l'URSS de voir la France jouer le rôle d'arbitre de la détente en Europe, tout en conservant le leadership en ce qui concerne les rapports Est-Ouest, et maintenir le statu quo européen, en empêchant la RFA, en particulier, de prendre la place dominante. 

un approfondissement au-delà du commerce en direction de la coopération économique, scientifique et technique ${ }^{10}$. Cette nouvelle approche s'est concrétisée par la signature, le 27 octobre 1971, «de l'accord sur le développement de la coopération économique, technique et industrielle » pour une période de dix ans. L'objectif du doublement des échanges que s'étaient fixé les deux gouvernements français et soviétique lors de la signature tant du premier accord commercial (1965-1969) que du second (1970-1974) a été largement dépassé. Le volume du commerce est ainsi passé de 1,5 milliard de francs en 1964 à près de 6 milliards en $1974^{11}$. La balance commerciale entre la France et la Russie est en outre excédentaire depuis 1968. En 1974, l'évolution du commerce extérieur était particulièrement satisfaisante pour les Soviétiques. Leurs exportations en France ont augmenté de plus de $50 \%$, grâce à l'accroissement des prix du pétrole et des matières premières, alors que leurs achats en France n'ont progressé que de $10 \%$ environ - si l'on fait abstraction des ventes de céréales réalisées en 1973 (572 millions de francs) qui ne se sont pas poursuivies en 1974. L'augmentation s'élève à $25 \%$. Il faut noter toutefois que les ventes de machines françaises à l'URSS ont triplé et représentent en 1974, presque la moitié des exportations ${ }^{12}$.

Lors des années 1970, la France et l'URSS poursuivent leurs efforts en vue de développer et de renforcer la coopération entre les deux pays. En matière politique, les relations franco-soviétiques n'ont cessé de devenir plus confiantes et plus régulières ${ }^{13}$. Parallèlement les échanges économiques et commerciaux marquaient une croissance très rapide et s'approfondissaient pour s'étendre largement jusqu'à la coopération industrielle et scientifique. Cette coopération se réalise en priorité dans les domaines de l'économie qui offrent les perspectives les plus favorables à son développement, par exemple, l'industrie lourde et chimique.

14

Dans le cadre des accords de la coopération économique et technique, les entreprises françaises ont été invitées à réaliser de nombreux projets industriels en URSS. Il s'agit des contrats de fourniture d'équipements, usines clés en main aux industries chimiques, pétrochimiques, métallurgiques, légères et alimentaires. La forme la plus efficace du transfert de technologie, c'est la livraison d'usines "clés en main », qui s'accompagne le plus souvent d'une aide de démarrage, et fréquemment de stages de formation. Ce type de transfert s'est fortement développé au cours des années 1970, notamment dans l'industrie chimique, la sidérurgie et l'industrie automobile. Le montant des contrats est souvent considérable. Pour les pays de l'Est, les usines « clés en main » ont essentiellement pour avantage de présenter une technologie supérieure et de pouvoir être mises en marche rapidement ${ }^{14}$.

15 En ce qui concerne le transfert des technologies soviétiques à destination de la France, ces transferts existaient aussi. Les organismes et les spécialistes soviétiques ont participé à la réalisation d'installations industrielles en France; des contrats ont été conclus en particulier pour la fourniture d'équipements destinés aux hauts-fourneaux de la première tranche du complexe sidérurgique de Fos-sur-Mer, aux installations de raffinage primaire du pétrole, ainsi que pour la livraison des presses hydrauliques de haut tonnage (65 000 tonnes). 


\section{Vers la crise du transfert de technologies françaises à destination de l'URSS (1979-1991)}

16 économique et de transfert de technologie entre la France et l'URSS. Débute alors «le repli des années 1970 ", analysé par Georges Sokoloff ${ }^{16}$. Il s'engage à partir du XXV congrès du Parti, qui s'est tenu en 1976. Le coup de frein affecte notamment les exportateurs occidentaux : leur part du marché soviétique des équipements atteint en 1976 son niveau le plus haut depuis 1947. Il s'agit en effet pour les autorités soviétiques de stabiliser, puis diminuer l'endettement net en devises convertibles de l'URSS. Y contribue aussi le facteur politique, notamment la crise polonaise et, surtout, l'invasion de l'Afghanistan, survenue fin décembre 1978. Celle-ci entraîne une politique de sanctions de la part des États-Unis. La France ne peut échapper au mouvement. Dès avril 1978, N. Patolitchev admet «la nécessité d'une pause des commandes de l'URSS ${ }^{17}$ ".

De manière globale, les achats soviétiques de biens d'équipement aux principaux pays occidentaux ont diminué de façon importante depuis 1981 (diminution de près de $50 \%$ en 1983 par rapport à 1981). En France, le ministère de l'Économie et des Finances a observé la diminution des contrats de très grande taille (supérieur à $1 \mathrm{Md}$ de $\mathrm{F}$ ) ou même, de façon moins accentuée, de grande taille (supérieur à $100 \mathrm{MF}$ ). Dans une Note datant de mars 1984, il constate que l'Union soviétique s'est orientée vers la construction d'unités moins grandes, ou la modernisation d'installations existantes. Cette tendance reflète une modification des priorités sectorielles. Dans les secteurs de l'industrie pétrolière, chimique et pétrochimique, les grands projets sont en diminution. En revanche, les achats soviétiques se maintiennent, sinon se développent dans le secteur prioritaire de l'industrie agro-alimentaire, ainsi que pour les machinesoutils et les équipements pour l'industrie légère.

Les années 1980 ont révélé une stagnation et un manque de perspectives des liens commerciaux et économiques bilatéraux existants, principalement sur le plan structurel. En 1986, l'échange des marchandises est tombé en dessous de 3 milliards de roubles, niveau de 1979 ${ }^{18}$. En 1986, la France, en tant que partenaire de l'Union soviétique (parmi les pays capitalistes industrialisés), ne s'est classée que cinquième, perdant sa position traditionnelle au profit du Japon ${ }^{19}$. La diminution des contrats de biens d'équipement conclus par la France avec l'URSS pendant les années 1980 est donc due à trois facteurs :

- la baisse des commandes de biens d'équipement passées de façon générale avec les pays occidentaux;

- la tendance à l'accroissement du taux d'échec des sociétés françaises, due, notamment, aux difficultés de financement ;

- la mauvaise adaptation de l'offre française, qui, incontestablement, joue un rôle très important.

Une telle situation a révélé les limites évidentes au développement du commerce franco-soviétique "asymétrique ", où l'on échange les matières premières contre les produits finis. Cette situation contrevient aux principes modernes de la participation des pays industrialisés dans la division internationale du travail ${ }^{20}$. La circonstance la plus négative est que cette tendance continue à se développer. En même temps, les difficultés économiques internes et externes des États socialistes se font sentir. Il existe 
toujours une dette extérieure significative dans les pays du CAEM. Le transfert de technologie est étroitement lié à la « santé » des échanges économiques. Or, les années 1980 ont été marquées par des crises dans le processus de transfert de technologies françaises à destination de l'Union soviétique.

L'exemple le plus marquant d'une telle crise est la situation créée autour de la société française Thomson- $\operatorname{CSF}^{21}$. Conformément au contrat signé en 1979, Thomson-CSF devait livrer à l'URSS l'équipement pour la production de centraux téléphoniques automatiques électroniques en vue du fonctionnement des systèmes de contrôle de gazoduc d'exportation Ourengoï-Pomary-Uzhgorod. Ce contrat prévoyait également le transfert de la production de circuits intégrés monolithiques. En avril 1982, probablement sous la pression de l'administration Reagan, le gouvernement français décida de suspendre l'exécution des obligations, par la société Thomson-CSF, aux termes de ce contrat, dans sa partie concernant le transfert de la production de circuits intégrés monolithiques, ce qui rendait impossible l'organisation de la production de centraux téléphoniques automatiques électroniques. Les approvisionnements reprirent en septembre 1982, grâce aux démarches actives de la représentation commerciale soviétique à Paris.

21 Il convient d'abord de constater, que le transfert de technologies entre la France et l'URSS a bien existé tout au long de la période de la guerre froide. Il a connu des hauts et des bas, étant fortement lié au climat politique de l'époque. L'Union soviétique et la France en ont tiré leur profit. En plus de revenus considérables issus de la vente de ses technologies et des biens technologiques à l'URSS, la France a assuré l'orientation de certains domaines de l'industrie soviétique vers la technologie française. Le résultat de cette tendance est que, même plusieurs années après les livraisons et le montage de l'équipement français, l'URSS a continué à acheter des pièces de rechange en France, et à inviter des spécialistes français pour l'entretien de machines. L'URSS a su construire une industrie compétitive et développée en s'appuyant sur le transfert des technologies occidentales, y compris Françaises.

Un autre résultat en a été l'instauration des contacts permanents entre les industriels russes et leurs homologues en France. La confiance en technologies françaises a garanti l'avenir prometteur de la coopération industrielle franco-russe pour les décennies à suivre. L'exemple le plus significatif de la continuité du transfert de technologies françaises a été l'industrie automobile. Grâce aux contacts entamés au début des années 1950 et poursuivis jusqu'aux années 1980, la marque française Renault a réussi à s'implanter avec beaucoup de succès en Russie des années 1990-2010. 


\section{BIBLIOGRAPHIE}

BADEL Laurence, Diplomatie et grands contrats. L'État français et les marchés extérieurs au $\mathrm{XX}^{e}$ siècle, Paris, Publications de la Sorbonne, 2010.

BARJOT Dominique, «Les débuts de la Cinquième République, âge d'or des relations franco-russes ? L'exemple de la télévision en couleur ", dans Les Français dans la vie intellectuelle et scientifique en Russie (XVIII ${ }^{e}-\mathrm{XX}^{e}$ siècles), TCHOUBARIAN Alexandre, LIECHTENHAN Francine-Dominique (dir.), OKUNEVA Olga (coord.), Moscou, Olma Media Group, 2010, p. 355-365.

BARJOT Dominique, PARK-BARJOT Rang-Ri, « Transferts de technologie entre France et URSS du milieu des années 1960 à celui des années 1970 : entre coopération scientifique et technologique, choix des politiques étrangères et montages financiers à long et moyen termes » dans TCHOUBARIAN Alexandre, LICHTENHAN Francine-Dominique, CÆEURÉ Sophie et OKOUNEVA Olga (dir.), Les Français dans la vie intellectuelle et scientifique en URSS au XXe siècle, Moscou, Institut d'histoire universelle de l'Académie des sciences de Russie, 2014, p. 101-116.

BLANCHEMAISON-KARANOV Isabelle, 1991, la fin de l'URSS, Paris, Sciences Po, 2011.

BORNSTEIN Morris, Transferts de technologie entre l'Est et l'Ouest : le transfert de technologie occidentale à l'URSS, Paris, OCDE, 1985.

BRAND Denis, L'expérience soviétique, Paris, Sirey, 1993.

CARRIÈRE Pierre, L'économie de l'URSS, Paris, Masson, 1974.

DOUBININE Youri, Moscou-Paris dans un tourbillon diplomatique : témoignage d'ambassadeur, Paris, Broché, 2002.

DYSON Kenneth, European détente : case studies of the politics of east-west relations, Londres, Frances Pinter, 1986.

ESCARGUEL Olivier, Mitterrand devant la fin de l'URSS : perceptions, réactions, répercussions 1985-1991, thèse de doctorat en histoire, Paris, Sciences Po, 2006.

GEORGE Pierre, L'économie de l'URSS, Paris, PUF, 1990.

GOMART Thomas, Double détente : les relations franco-soviétiques de 1958 à 1964, Paris, Publications de la Sorbonne, 2003.

GRANTU Claude, «1988-1992 : la France et l'URSS/Russie : la période des occasions perdues », Relations internationales et stratégiques, $\mathrm{n}^{\circ}$ 9, Villetaneuse, 1993, p. 172-180.

LHOMEL Édith, Transitions économiques à l'Est : 1989-1995, Paris, la Documentation française, 1995.

MOULLEC Gaël, Pour une Europe de l'Atlantique à l'Oural : les relations franco-soviétiques (1956-1974), Paris, les Éditions de Paris-Max Chaleil, 2016.

NARINSKIJ Mihail, VAïsSE Maurice, Les crises dans les relations franco-soviétiques : 1954-1991, Paris, A. Pedone, 2009.

NYGREN Bertil, Cooperation between the Soviet Union and three Western great powers, 1950-1975, Stockholm, The Swedish institute of international affairs, 1981. 
OBERDIECK Annette, «Le rôle des banques françaises et allemandes dans les relations économiques avec l'Union soviétique entre 1970 et 1990 », thèse de doctorat en histoire, Paris, Institut d'études politiques, 1996.

SERANNE Catherine, « Les relations économiques franco-soviétiques de 1955 à 1970 », Notes et Études Documentaires, La Documentation française, 19 juillet 1971.

SEUROT François, Les causes économiques de la fin de l'Empire soviétique, Paris, Presses universitaires de France, 1996.

SокоLOFF Georges, L'économie de la détente : l'URSS et le capital occidental, Paris, Presses de la FNSP, 1983.

sоUтоU Georges-Henri, La guerre de cinquante ans. Les relations Est-Ouest 1943-1990, Paris, Fayard, 2001.

REY Marie-Pierre, La tentation du rapprochement France et URSS à l'heure de la détente (1964-1974), Paris, Publications de la Sorbonne, 1991.

REY Marie-Pierre, «La détente franco-soviétique et les relations économiques et financières, 1960-1974 ", dans Études et documents, Comité pour l'histoire économique et financière de la France, V, 1993, p. 307-355.

VAïsSE Maurice, De Gaulle et la Russie, Paris, CNRS, 2012.

VAïsSE Maurice, «Les crises dans les relations franco-soviétiques dans les années 1970 », Revue d'histoire diplomatique, $\mathrm{n}^{\circ}$ 2, 2006, p. 99-187.

WERTH Nicolas, Histoire de l'Union soviétique : de l'Empire russe à la Communauté des États indépendants : 1900-1991, Paris, PUF, 2012.

WIENERT Helgard, ZALESKI Eugène, Transfert de techniques entre l'Est et l'Ouest, Paris, OCDE, 1980.

\section{NOTES}

1. D. Barjot, R.-R. Park-Barjot, "Transferts de technologie entre France et URSS ... », p. 101-116.

2. M.-P. Rey « La détente franco-soviétique et les relations économiques et financières, 1960-1974 », p. 307-355.

3. M. Bornstein, Transfert de technologie entre l'Est et l'Ouest: le transfert de technologie occidentale à l'U.R.S.S, p. 162-173.

4. Technology Transfer and Scientific Co-operation Between the United States and the Soviet Union: A Review, préparé pour le Sub-Committee on International Security and Scientific Affairs du Committee on International Relations par le Congressional Research Service, 1977.

5. Archives nationales, Archives présidentielles de Valery Giscard d'Estaing, (dorénavant Arch. prés. VGE), AG/5(3)/1089, lettre, le 7 octobre 1974.

6. C. Seranne, « Les relations économiques franco-soviétiques de 1955 à 1970 », p. 5.

7. Idem, p. 6.

8. Archives du ministère de l'Économie, des Finances et de l'Industrie (MINEFI), B4451, Conseils aux exportateurs. L'organisation du commerce extérieur en URSS, mai 1975. 
9. D. Barjot, «Les débuts de la Cinquième République, âge d'or des relations économiques franco-russes ? L'exemple de la télévision en couleur ", p. 355-365.

10. Arch. prés. VGE, AG/5(3)/1089, lettre, le 7 octobre 1974.

11. Archives MINEFI, B4451, mai 1975.

12. Ibid.

13. Arch. prés. VGE, AG/5(3)/1089, lettre, le 7 octobre 1974.

14. H. Wienert, E. Zaleski, Transfert de techniques entre l'Est et l'Ouest, p. 23.

15. D. Barjot, R.- P. Barjot, « Transferts de technologie entre France et URSS ... », p. 101.

16. G. Sokoloff, L'économie de la détente :l'URSS et le capital occidental, p. 181.

17. L. Badel, Diplomatie et grands contrats. L'État français et les marchés extérieurs au $\mathrm{XX}^{e}$ siècle, p. 370.

18. Archives du Ministère de la politique extérieure de la Russie (AVPRF), 71/188/18, "Problèmes de la coopération économique franco-soviétique ", note analytique, 1987.

19. Archives du Ministère de la politique extérieure de la Russie (AVPRF), 71/188/18, «Problèmes de la coopération économique franco-soviétique », note analytique, 1987.

20. Ibid.

21. Archives AVPRF, 68/174/19, «Relations commerciales et économiques entre l'URSS et la France », Note d'information, 3 janvier 1983.

\section{RÉSUMÉS}

Cet article étudie le transfert de technologie entre l'URSS et la France pendant la période de la guerre froide. La recherche s'appuie sur l'analyse des archives soviétiques et françaises de nature économique et politique. Le transfert de technologie est présenté comme une composante de la coopération économique entre l'URSS et la France. Il a connu des hauts et des bas, étant fortement lié au climat politique de l'époque. L'Union soviétique et la France en ont tiré profit: l'URSS a su construire une industrie compétitive et développée en s'appuyant sur le transfert des technologies occidentales, y compris françaises tandis que l'utilisation des technologies françaises a assuré l'orientation de certains domaines de l'industrie soviétique vers la technologie française. Un autre résultat est l'instauration de contacts permanents entre les industriels russes et français. La confiance portée aux technologies françaises a garanti un avenir prometteur dans la coopération industrielle franco-russe pour les décennies futures.

\section{AUTEUR}

\section{BORIS VINOGRADOV}

Doctorant contractuel, Sorbonne Université, Centre Roland Mousnier, UMR 8596 Sorbonne Université CNRS 
Le transfert de savoirs technique et industriel français au Pérou dans les années 1950 et 1960 : l'exemple de la sidérurgie de Chimbote et de la centrale hydroélectrique du Canyon del Pato

\author{
Getsiva Cayo Durand de Geist
}

1 Le transfert de know-how dans les relations Nord-Sud constitue une problématique complexe qu'il est essentiel d'aborder. La transmission de savoirs entre la France et les pays d'Amérique latine demeure en effet un sujet très peu étudié ${ }^{1}$. Alors que diverses entreprises françaises ont été chargées de mener à bien des projets industriels dans les pays latino-américains, le savoir-faire français, pourtant présent et mis en place au Pérou, n'est que très peu connu ${ }^{2}$. Cet article s'interroge ainsi sur le processus de transfert de savoirs technique et industriel entre la France et le Pérou dans les années 1950-1960. Dans quel contexte se déroulait ce transfert des savoirs? Quel était le rôle des différents acteurs? Quels projets industriels ont pu illustrer ces échanges? Cet article propose tout d'abord de contextualiser ces échanges et d'expliquer ensuite la place qu'a eu la transmission du savoir-faire français dans les relations diplomatiques et économiques entre ces deux pays. Pour ce faire, deux projets de génie civil ont été étudiés : la construction hydroélectrique du Canyon del Pato et de celle de l'aciérie de Chimbote. Il s'agit de démontrer que le transfert de savoir-faire français a eu un impact économique, industriel et social au Pérou. Les facteurs et les acteurs de ces deux grands projets seront présentés et explicités. Cette étude a cherché à mettre en valeur l'expertise française au Pérou en tenant compte de son potentiel et de ses limites. 


\section{La coopération technique franco-péruvienne : un domaine privilégié}

2 Il faut rappeler que depuis la Première Guerre mondiale, l'influence croissante des États-Unis a affecté les relations franco-péruviennes dans tous les domaines ${ }^{3}$. Pendant les Trente Glorieuses, la concurrence internationale croissante n'a fait que compliquer l'état des relations économiques entre ces deux pays. Après la création de la Communauté économique européenne (CEE), l'économie française est devenue plus performante. Néanmoins, ce pays a dû faire face aussi bien à la concurrence des pays européens qu'à celle du Japon ${ }^{4}$. La France et le Pérou ne pouvaient donc pas être considérés comme des partenaires commerciaux importants. Le pourcentage de la valeur des importations et des exportations françaises au Pérou représentait environ $2 \%$ par an. Cependant, dans ces relations bilatérales, d'autres domaines ont été privilégiés et eu un impact plus important. Parmi ceux-ci, on peut nommer la coopération technique ${ }^{5}$.

3 Après la Seconde Guerre mondiale, la coopération technique franco-péruvienne s'est développée et a été encouragée par l'action des diplomates, des fonctionnaires d'État ainsi que des hommes d'affaires et entrepreneurs. Les gouvernements et les ambassades constituaient deux piliers importants pour ce transfert de savoirs. Ces deux acteurs publics avaient donc un rôle majeur pour le développement, l'approbation et la réalisation des différents projets entrepris ${ }^{6}$. Ces deux partenaires avaient déjà noué de bonnes relations culturelles, diplomatiques et militaires ${ }^{7}$. Mais ils se sont rapprochés dans le cadre des relations Nord-Sud et pour des intérêts précis. Ces deux pays avaient certes des niveaux de développement économique asymétriques. Néanmoins, ils partageaient des intérêts communs: accroitre et développer leurs secteurs économiques. La coopération technique attira ainsi de plus en plus l'attention des présidents, fonctionnaires, diplomates, hommes d'affaires et notamment des entreprises publiques et privées 8 .

D’un côté, il y avait la France. Au cours des années 1950, celle-ci réussit à reconstruire son économie. Et elle fut reconnue comme étant un exportateur de savoirs techniques et industriels d'excellence ${ }^{9}$. De ce fait, la France chercha à renforcer ses échanges avec les pays latino-américains dans trois domaines: commercial, des services et des capitaux. La coopération technique était le moyen de renforcer sa présence dans ces trois secteurs. Plusieurs centres techniques français furent créés avec succès dans différents pays latino-américains comme l'Équateur, la Colombie ou le Brésil. En 1957, le Centre technique franco-péruvien (C.T.F.P.) fut ouvert à Lima. Dans ce contexte, le transfert de savoir-faire technique français au Pérou se déploya à travers un programme de coopération spécialisé aussi bien en matière technique que pédagogique ${ }^{10}$.

5 De l'autre côté, il y avait le Pérou qui était un importateur potentiel du savoir-faire étranger. Ce pays s'efforçait d'équiper et de moderniser ses secteurs de production ${ }^{11}$. Pendant les années 1950, le Pérou était vu comme un pays en voie de développement de taille moyenne. Son modèle de croissance économique était basé sur le libéralisme ${ }^{12}$. En conséquence, il devint l'un des pays les plus attractifs de la région pour les investisseurs étrangers. Tout au long de la période étudiée, le taux de croissance de son PIB était de $5.5 \%$ par an ${ }^{13}$. L'État péruvien joua un rôle fondamental dans la promotion de concessions, le contrôle des entreprises étrangères et l'incitation à la formation 
technique. Les différents gouvernements péruviens cherchèrent à accélérer la croissance économique nationale ${ }^{14}$. Les adjudications publiques pour la construction de nouvelles usines, la modernisation des secteurs industriels ainsi que la réalisation des travaux publics faisaient partie des programmes présidentiels ${ }^{15}$.

6 Dans les années soixante, la coopération technique et scientifique intéressait toujours les représentants de chaque État. Les bonnes relations politiques et diplomatiques furent symbolisées par deux visites présidentielles. En 1960, les inquiétudes concernant l'extrême dépendance à l'égard des États-Unis amenèrent le président péruvien Manuel Prado à visiter les pays européens. Lors de son passage à Paris, M. Prado chercha à renforcer les relations, aussi bien politiques qu'économiques, avec la France. La recherche d'investissements, l'offre d'adjudications ainsi que la vente de matières premières (charbon) étaient quelques exemples des démarches entreprises dans la ville lumière ${ }^{16}$.

7 Quelques années plus tard, le président Charles de Gaulle et ses propos sur la «latinité » liant la France et l'Amérique latine parvinrent aussi au Pérou. Pour de Gaulle, la coopération technique devait prendre plus d'importance afin de faire face à la perte d'influence française dans les pays de toute l'Amérique latine ${ }^{17}$. Son arrivée à Lima en 1964 fut à l'origine de la mise en place de projets industriels francopéruviens ${ }^{18}$. Pour ces deux présidents, l'innovation et le transfert de savoirs techniques et scientifiques constituèrent des sujets importants. Il faut noter que la sympathie, l'amitié et la fraternité franco-péruvienne proclamée par ces deux présidents, ne garantissait pas le succès de certains échanges commerciaux. En revanche, en matière de coopération technique, la France et le Pérou envisagèrent un ensemble de projets. Une liste de précontrats et de prospections industrielles vit ainsi le jour à la fin de chaque voyage présidentiel ${ }^{19}$.

8 Les acteurs économiques français essayèrent de remporter le plus grand nombre d'adjudications de projets industriels au Pérou, notamment dans le secteur du génie civil et des travaux publics. La coopération bilatérale, au sein de laquelle le transfert de savoirs fut mis en œuvre, avait pour objectif principal de permettre à la France d'être présente économiquement au Pérou. Les données consultées dans les archives ont montré que les secteurs du bâtiment et des travaux publics, de l'ingénierie, de l'électricité et de l'hydroélectricité, de l'industrie de l'armement et le secteur bancaire étaient parmi les plus sollicités par les établissements publics et privés péruviens ${ }^{20}$.

9 Les Péruviens s'intéressèrent ainsi à la technologie et à l'industrie française. Quant aux appels d'offres dans le secteur des grands projets, la concurrence internationale s'était fortement accrue au Pérou. En effet, pour la mise en œuvre des travaux de grande envergure, le Pérou évaluait aussi bien les capitaux humains que financiers. La France dut lutter pour remporter ces projets. De fait, même si les Français étaient souvent bien placés, un grand nombre de projets n'ont pas abouti pour des raisons financières, politiques, voire climatiques. Toutefois, lors de la prise de décisions, quelques projets d'importance furent, tout de même, confiés aux Français ${ }^{21}$.

10 La transmission de savoirs technique et industriel français ne s'effectua pas sans difficultés. La sidérurgie de Chimbote et la centrale hydroélectrique du Canyon del Pato illustrent bien les potentiels et les limites de la diffusion de ce savoir-faire au Pérou. La réalisation de ces deux ouvrages est connue sous le nom de "l'affaire Santa ${ }^{22}$ ». Ce contrat a été vu comme le symbole du resserrement commercial et industriel francopéruvien ${ }^{23}$. Pour comprendre la mise en place des savoirs français au Pérou, il sera donc 
important de connaître les actions mises en place par les entreprises publiques et privées aussi bien péruviennes que françaises. Celles-ci ont été les acteurs principaux du transfert de compétences françaises au Pérou lors de la mise en œuvre de ces deux grands projets industriels.

\section{La construction du Canyon del pato : le rôle de la corporación peruana del santa (CPS)}

11 La Corporación peruana del santa était un organisme industriel de l'État péruvien ${ }^{24}$. Dès 1912, l'ingénieur péruvien Santiago Antunez de Mayolo a été le promoteur de la création de l'usine sidérurgique de Chimbote ainsi que de la centrale hydroélectrique du Canyon del Pato. Diplômé de l'Institut électronique de Grenoble en 1909, S. Antunez de Mayolo contribua de manière notable à la prise de décision concernant la collaboration des sociétés financières et industrielles françaises en $1954^{25}$. En effet, depuis de longues années, cet illustre ingénieur péruvien avait accompli des travaux dans le domaine technique pour faciliter l'industrialisation du Pérou. Dès la création de la CPS, S. Antunez participa aux nombreux efforts mis en œuvre pour le développement industriel de la ville de Chimbote. L'installation d'ateliers d'outillage, de locomotives, de services auxiliaires du chemin de fer, du port, de l'industrie charbonnière à Chimbote a permis de développer l'indépendance économique du Pérou ${ }^{26}$.

En tant que partenaire commercial et industriel potentiel pour les firmes françaises au Pérou, la CPS était chargée de gérer les ressources industrielles de la vallée du Santa au nord du pays. Parmi les projets envisagés au début des années cinquante, l'usine électro-sidérurgique de Chimbote et l'usine hydroélectrique du Canyon del Pato ont été les plus importants. En 1952, Pierre Burthe-Mique, l'attaché commercial de l'ambassade de France à Lima, avait averti les autorités françaises que la différence entre les prix français et étrangers au Pérou, en ce qui concerne l'adjudication d'une petite centrale hydroélectrique, était surprenante ${ }^{27}$.

En effet, parmi les cinq propositions faites par une firme américaine, trois allemandes et une Française, le prix le plus élevé était celui des Français. Face à une telle réalité, les chances de remporter les adjudications péruviennes se sont trouvées réduites. Par ailleurs, M. Aisner, le conseiller commercial, s'exprimait sur le sujet en ces termes :

«Nous sommes ici très peu de Français sincèrement animés par le sentiment des possibilités de notre pays, notre devoir est de nous entendre et de nous épauler pour faire réussir nos projets. ${ }^{28}$ "

Il est vrai que la colonie française au Pérou était très peu nombreuse. En outre, le conseiller commercial relevait que la cohésion du groupe, dès lors que des opportunités économiques se présentaient, était un élément primordial.

La construction du complexe hydroélectrique Canyon del Pato fut réalisée afin de permettre le bon fonctionnement de l'usine sidérurgique de Chimbote. Cette centrale hydroélectrique se trouvait située à environ $138 \mathrm{~km}$ à l'est de Chimbote. L'histoire de sa construction remontait au début des années quarante. La réalisation de cette entreprise fut longue et complexe. La construction des ouvrages de génie civil et l'achat des principaux matériels électromécaniques français se trouvèrent soudainement interrompus en octobre 1950 à la suite d'une inondation. Les travaux reprirent en novembre 1954, et la mise en service de la Centrale eut lieu en mars $1958^{29}$. 

Conseil National du Patronat Français, s'était rendue au Pérou. À la fin de cette visite, des facilités de financement en vue de l'achèvement de l'ensemble des travaux hydroélectriques restant à effectuer furent proposées à la CPS. Un groupe de constructeurs français, chargé de fournir le matériel d'équipement de l'usine sidérurgique de Chimbote, s'y intéressait d'ailleurs énormément ${ }^{30}$. La CPS accepta la proposition faite par le «groupe français » en mars 1955. Le chef de file de ce nouveau projet était la Société Générale d'exploitations industrielles (SOGEI), associé au Consorcio de Ingenieros Contratistas Generales (CICG). Leur mission était d'achever les travaux de génie civil. Ces travaux permettaient d'utiliser les deux premiers groupes de $25000 \mathrm{~kW}$ du Canyon del Pato pour assurer le bon fonctionnement de l'usine sidérurgique de Chimbote ${ }^{31}$. œuvre de ce projet. Concernant les tâches des sociétés françaises, il a été possible d'en repérer les principaux aspects. La SOGEI s'occupa principalement des études à réaliser, ainsi que de la coordination et du contrôle des travaux. La société Neyrpic fut chargée de fournir et de monter le matériel mécanique de la prise d'eau et du déssableur, des vannes du tunnel et de la vanne papillon. Les firmes Alsthom et Jeumont se chargèrent du matériel électromécanique de complément, à haute et basse tension. La société Constructions Métalliques et Entreprises (CME) pourvut à l'approvisionnement et au montage des blindages du tunnel et des puits. Elle fournit également les pylônes de complément. Quant au financement, il avait été mis sur pied par la Banque de Paris \& des Pays-Bas prenant ainsi en charge l'acheminement des travaux relatifs à l'aménagement de la centrale hydroélectrique du Canyon del Pato ${ }^{32}$.

Le Consorcio de Ingenieros Contratistas Generales participa également à cette réalisation. Il fut chargé des finitions des ouvrages de génie civil et des montages pour les revêtements du tunnel et des puits avec l'assistance de la Société Générale d'Entreprises $(\mathrm{SGE})^{33}$. Les entreprises françaises durent faire face à des problèmes techniques et financiers lors de la transmission de leur savoir-faire. Néanmoins, l'expertise française faisait partie des compétences étrangères les plus recherchées par le Pérou.

\section{L'usine sidérurgique de Chimbote : « le début de l'ère de l'acier au Pérou ${ }^{34}$ »}

Lorsque l'on considère l'industrie sidérurgique au Pérou, la création de l'usine de Chimbote a été perçue comme une étape historique majeure. Son achèvement fut considéré comme un grand pas vers l'indépendance industrielle en matière d'acier visà-vis d'autres pays d'Amérique latine ${ }^{35}$. Elle permit d'accélérer l'accroissement de la population, et de ses activités, par la création de moyens et de grands complexes industriels dans la ville de Chimbote, dans les départements d'Ancash et de la Libertad. L'inauguration de cette usine industrielle a été sans doute un événement important au niveau national. Un futur très optimiste pouvait être ainsi envisagé ${ }^{36}$.

La première étape de la construction de cette usine fut réalisée avec succès. En 1951, le «groupe français» formé par la Banque de Paris et des Pays-Bas (BPP) et les Établissements Delattre et Frouard Réunis signa un contrat avec la CPS, en vue de l'établissement de l'usine sidérurgique de Chimbote. Toutefois, des difficultés 
administratives retardèrent la mise en œuvre du projet ${ }^{37}$. Après la mission économique présidée par M. Villiers en mars-avril 1954, le représentant de la BPP, M. Bedin, précisa que :

«L'administration a finalement donné toute latitude aux négociations du groupe pour offrir, si la nécessité les y oblige, les conditions de crédit exceptionnelles permettant d'améliorer les propositions françaises. ${ }^{38}$ ”

Il s'agissait d'un financement sur 7 ans et $1 \frac{1}{2}$. La BPP faisait un emprunt de 10 millions de dollars. Ainsi, les avantages des propositions françaises et les modalités de crédit s'avérèrent décisifs pour remporter ce projet pour lequel les Américains étaient les principaux concurrents.

22 En juillet 1954, le contrat de la construction de cette usine, signé entre la CPS, le "groupe français » et le Consorcio de Ingenieros Contratistas Generales, fut relancé. Ces deux derniers furent chargés de la construction des bâtiments, des fondations, des services directs (direction et organisation des travaux, ingénieurs résidents, personnel ouvrier) ainsi que des installations de montage de matériels d'équipement de l'usine (appareils de chantier, fournitures) ${ }^{39}$.

Les conditions d'exécution des travaux, les plans généraux et de détails, les dessins et les spécifications techniques avaient été préparés par le "groupe français » et le Consortium péruvien. Les Établissements Delattre et Frouard Réunis, la Société Générale d'exploitations industrielles (SOGEI, vide supra) et la Banque de Paris et des Pays-Bas avaient été les sociétés françaises engagées dans cette affaire. D’autre part, sur les 3 millions de dollars prévus pour les travaux et les fournitures, le "groupe français » finança le projet à hauteur de 670000 dollars ${ }^{40}$.

En 1955, cette négociation était considérée comme la plus grande entreprise française réalisée au Pérou. Selon Pierre d'Huart, le chargé d'affaires, ce projet était perçu comme une première étape permettant d'envisager d'autres possibilités favorables à l'expansion économique française au Pérou ${ }^{41}$. Nonobstant l'enthousiasme présent, il fallut faire face à certains problèmes. La première commande de produits sidérurgiques (23000 tonnes) effectuée par les importateurs péruviens, ne fut pas livrée dans les temps ${ }^{42}$. En effet, l'accord concernant l'aciérie de Chimbote n'avait pas encore été approuvé par le gouvernement péruvien. Il ne le fut qu'à partir de décembre 1954. De fait, les industriels français se montrèrent réticents à poursuivre leurs livraisons ${ }^{43}$. Pour autant, cette difficile négociation fut conclue le 9 mars 1955 lors de la signature du contrat.

L'installation et la mise en service de l'usine sidérurgique de Chimbote ont été vues comme un "succès total de prestige français ${ }^{44}$ ». L'inauguration de l'usine sidérurgique et celle de la centrale hydroélectrique eurent lieu le 21 avril 1958 à la demande spéciale du président péruvien Manuel Prado. Pour des raisons politiques et personnelles, il voulut que cet événement soit " revêtu d'un éclat tout particulier ${ }^{45}$ ». Cette décision ne faisait cependant pas l'unanimité. En effet, les représentants de la CPS auraient préféré attendre un an pour que les installations soient correctement mises en marche ${ }^{46}$.

Les techniciens français furent forcés de faire fonctionner les différentes sections de l'usine précipitamment. Toutefois, lors de l'inauguration, aucun incident ne permit de se rendre compte des contraintes auxquels les techniciens avaient dû faire face. Des personnalités politiques influentes ainsi que des représentants de diverses sociétés françaises étaient attendus pour les cérémonies. Le directeur de la SOGESA, Jules Thérry, un expert dans l'industrie de la sidérurgie, dirigeait cette mission. Lors de cette 
manifestation d'amitié franco-péruvienne, la délégation française fut présidée par M. Jacques Roux, directeur adjoint des affaires politiques du Quai d'Orsay. Ses membres furent bien accueillis par les autorités péruviennes ${ }^{47}$.

Néanmoins, des contraintes à l'échelle internationale contrarièrent les perspectives à long terme. La baisse des prix de minerais sur les marchés mondiaux et la conjoncture internationale créèrent une atmosphère défavorable au Pérou. Ces incidents affectèrent le règlement des créances étrangères. Des négociations difficiles entre les représentants des sociétés engagées eurent lieu ${ }^{48}$. La difficulté financière de la CPS, dirigée par Max Peña Prado, dut être étudiée avant d'établir de nouveaux accords entre le "groupe français » et le consortium péruvien. L'esprit entrepreneurial du ministre des Finances au Pérou, M. Gallo Porras et les voyages de M. de Margerie, représentant de la Banque de Paris et des Pays-Bas, permirent de poursuivre cette grande entreprise franco-péruvienne ${ }^{49}$.

La seconde étape de la construction de l'usine s'avéra néanmoins plus compliquée. En avril 1959, les activités de l'usine de Chimbote et de la centrale hydroélectrique du Canyon del Pato, qui lui fournissait l'énergie, se trouvèrent paralysées. Les dettes de la CPS et les difficultés de trésorerie de la SOGESA constituaient de graves obstacles. Les pertes enregistrées par la nouvelle usine en fonctionnement eurent pour conséquence d'en épuiser les fonds de trésorerie. Ce fait n'était pas si étonnant étant donné qu'elle n'en était qu'à l'étape de démarrage. Cependant, un article de La Prensa intitulé : "L'usine sidérurgique del Santa a eu pendant sa première année 10 millions de perte " remit en cause le succès de l'entreprise franco-péruvienne. Aux problèmes financiers s'ajoutèrent les problèmes sociaux : une grève des ouvriers réclamant leurs salaires se produisit en avril $1959^{50}$.

Il est vrai que la réalisation de cette usine avait été parsemée d'incidents. Mais pour les comprendre, il faut les replacer dans le contexte, à la fois économique et politique du Pérou. Il faut également prendre en compte le fait que la construction de cette usine et celle de la centrale hydroélectrique du Canyon del Pato contribuèrent de manière considérable au développement économique de Chimbote. L'aciérie employait à elle seule plus de 1000 ouvriers. Ainsi, la population de la ville augmenta beaucoup, passant de 10000 à près de 60000 habitants en moins de trois ans ${ }^{51}$. Quelques mois plus tard, la commande de 2000 tonnes de fer à béton effectuée par les États-Unis (valeur de 180000 dollars) était suivie d'une autre commande, cette fois pour l'Argentine. De fait, ces deux achats encouragèrent l'avenir de l'usine ${ }^{52}$.

En mars 1961, afin d'achever l'extension de l'industrie lourde sidérurgique, la société SOGESA fut réorganisée. La CPS y était encore le principal actionnaire. Mais la société étrangère Thesaurus avait aussi la possibilité de souscrire 15 millions de dollars avec ses propres bénéfices commerciaux. Pour le Journal El Comercio, cette affaire n'avait pas été favorable à la CPS, parce que le patrimoine des minerais de Marcona en avait été affecté s3. $^{3}$.

31 D'autre part, en dépit des attentes, en 1965 l'usine ne produisait que 60000 tonnes d'acier électrique par an, quantité insuffisante pour le rendement normal d'une aciérie. Le fonctionnement de l'usine était meilleur lorsque les techniciens français étaient encore sur place. Cependant, une fois rentrés en France, des incidents techniques se produisirent. Les laminoirs semi-automatiques n'arrivaient pas à tourner correctement $^{54}$. 
gouvernement de M. Prado, l'adjudication de l'extension de l'usine de Chimbote fut octroyée à la firme allemande Ferrostal. Toutefois, un scandale politique se produisit en raison de prix trop élevés. Une fois de plus, les circonstances de la politique interne du Pérou retardèrent ce grand projet. Quelques mois après l'élection du nouveau président Belaunde Terry (1963), un appel d'offres fut lancé sur la base des études faites par une firme anglaise. Cette société conseilla l'installation de laminoirs capables de traiter 180000 tonnes par an, capacité suffisante pour la consommation locale. Les Anglais étaient très bien placés dans cette affaire, mais l'industrie française représentée par Delattre \& Schneider, était davantage disposée à proposer du matériel français ${ }^{55}$.

Lanalyse de la transmission de savoirs technique et industriel est étroitement liée à la problématique de la coopération technique et scientifique franco-péruvienne. Il a été démontré que le transfert du savoir-faire français a eu un impact social et économique au Pérou. Si ces deux pays n'ont pas été des partenaires commerciaux privilégiés, ils l'ont été dans le domaine de la coopération technique.

Les acteurs du transfert de compétences techniques et industrielles françaises au Pérou ont été identifiés. Les gouvernements ont joué un rôle déterminant au moment de mener des politiques de coopération bilatérale. Les diplomates et les fonctionnaires publics ont géré les conditions administratives de la mise en œuvre de l'échange de savoir-faire français au Pérou. L'expertise des entreprises françaises au Pérou ainsi que celle de leurs partenaires péruviens ont assuré la mise en œuvre de ces projets industriels. Cependant, le rôle des individus a tout autant été important car ce sont eux qui ont mené à bien, de manière directe, le transfert de savoirs industriels. L'expertise des ingénieurs, des techniciens, ainsi que celle d'hommes d'affaires français, ont contribué au processus d'industrialisation de l'économie péruvienne. Il faut noter que pour mener à bien ces projets, la participation des collaborateurs péruviens était aussi un facteur d'optimisation.

Pendant les années 1950 et 1960, les apports des Français au Pérou ont été renforcés dans le domaine du génie civil. L'exemple de l'aciérie de Chimbote et de la centrale hydroélectrique Canyon del Pato a mis en évidence les potentiels du savoir-faire français au Pérou. Ces deux projets n'ont pas donné uniquement un nouvel élan aux relations financières. Certes, cet élan a été essentiellement visible au niveau de la coopération technique et industrielle franco-péruvienne. Mais il a donné naissance à l'ère de l'acier au Pérou. La construction de l'aciérie de Chimbote a eu un impact considérable dans la société péruvienne, notamment dans la ville de Chimbote. Ces travaux furent en effet réalisés par des capitaux et par des techniciens français.

37 Cette entreprise franco-péruvienne n'a pas été exempte de critiques. Le transfert du savoir-faire français au Pérou a rencontré des obstacles d'ordres financier, politique et 
technique. En dépit d'un contexte assez favorable, quelques incidents se sont produits. Le problème le plus grave tenait au fonctionnement de l'usine sidérurgique, surtout sensible après le départ des Français : ces problèmes étaient liés à l'énergie électrique et au personnel péruvien. Mais le bilan est positif. En effet, les vecteurs de la mise en œuvre de la transmission de savoirs techniques furent, dans une large mesure, les missions de différents hommes d'affaires, ministres et ingénieurs français envoyés au Pérou. De plus, la presse locale péruvienne et les conférences des experts français aidèrent à diffuser le savoir-faire français au profit du développement économique péruvien.

\section{BIBLIOGRAPHIE}

BARJOT Dominique et CAYO DURAND Getsiva, «Les échanges économiques entre la France et l'Amérique latine pendant la présidence du général de Gaulle : une première approche », dans VAïsSE Maurice (dir.) De Gaulle et l'Amérique latine, 2014, p. 177-196.

BARJOT Dominique, La trace des bâtisseurs : histoire du Groupe Vinci, Vinci, Rueil-Malmaison, 2003.

BARJOT Dominique, BELTRAN Alain, HAU Michel (éds.), Industrialisation et sociétés en Europe occidentale du début des années 1880 à la fin des années 1960 : France, Allemagne-RFA, Italie, Royaume-Uni et Benelux, France, Éd. CNED-SEDES, 1997.

BARJOT Dominique, Travaux publics de France : un siècle d'entrepreneurs et d'entreprises, 1883-1992, Paris, Presses de l'École Nationale des Ponts et Chaussées, 1993.

CAYO DURAND DE GEIST Getsiva, «El reforzamiento de las relaciones franco-peruanas en el campo de la diplomacia económica (1945-1975) », Agenda Internacional, Lima, PUCP, vol. 24, n 35, 2017, p. 193-212.

CAYO DURAND DE GEIST Getsiva, « Moderniser le Pérou sous l'influence française : la coopération technique et scientifique franco-péruvienne (1945-1975) », La Clé des Langues (éd. num), Lyon, ENS de LYON/DGESCO, ISSN 2017-7029, août 2016

[http//cle.ens-lyon.fr:espagnol:civilisation:histoire-latino-americaine:relationsinternationales:moderniser-le-perou-sous-l-influence-francaise-la-cooperation-technique-etscientifique-franco-peruvienne-1945-1975-]

CAYO DURAND Getsiva, Les relations économiques et financières entre la France et le Pérou : l'exemple des entreprises industrielles et commerciales (1945-1975), mémoire de master 2 d'histoire, Paris, Université Paris-Sorbonne, sous la direction de Dominique BARJOT, 2011.

C.C.A.L., « Presencia francesa en América latina », Revue de la Chambre de Commerce FranceAmérique latine, Edición Especial para América Latina, nº 1, Febrero, 1965, p. 18.

C.C.A.L., «L'économie d'un grand pays : le Pérou », Revue de la Chambre de Commerce FranceAmérique Latine, volume IX, $\mathrm{n}^{\circ}$ 5, novembre 1955, p. 7.

COMPAGNON Olivier, L'Adieu à l'Europe : L'Amérique latine et la Grande Guerre, Paris, Fayard, 2013. 
HAMPE MARTINEZ Teodoro, El Banco Central de Reserva y la economía peruana (1922-1980), Lima, BCRP, 1995.

INEI, Comportamiento de la economía peruana 1950-2015. Panorama de la Economía Peruana 1950-2015, Lima, INEI, 2016, 17-18.

NOVAK TALAVERA Fabian, Las relaciones entre Perú y Francia (1827-2004), Lima, PUCP, 2005.

TEMPLE SEMINARIO Winston, Guía Económica del Perú, Lima, Instituto Peruano de Administración de Empresas (IPAE), 1968.

SHEAHAN John, La economía peruana desde 1950 : buscando una sociedad mejor, Lima, Instituto de Estudios peruanos, vol. 19, 2001.

\section{NOTES}

1. D. Barjot et G. Cayo Durand, «Les échanges économiques entre la France et l'Amérique latine pendant la présidence du général de Gaulle: une première approche », p. 177-196.

2. D. Barjot, La trace des bâtisseurs : histoire du Groupe Vinci, p. 133. Voir aussi : D. Barjot, Travaux publics de France : un siècle d'entrepreneurs et d'entreprises, p. 58-60 et p. 219-231.

3. O. Compagnon, L'adieu à l'Europe : l'Amérique latine et la Grande Guerre.

4. D. Barjot, A. Beltran, M. Hau (éds.), Industrialisation et sociétés en Europe occidentale du début des années 1880 à la fin des années 1960 : France, Allemagne-RFA, Italie, Royaume-Uni et Benelux, 1997.

5. Voir partie I : La coopération technique : un domaine privilégié dans G. Cayo Durand de Geist, « Moderniser le Pérou sous l'influence française : la coopération technique et scientifique franco-péruvienne (1945-1975)».

6. G. Cayo Durand de Geist, «El reforzamiento de las relaciones franco-peruanas en el campo de la diplomacia económica (1945-1975) », p. 193-212.

7. Voir F. Novak Talavera, Las relaciones entre Perú y Francia (1827-2004).

8. Archivo Central del Ministerio de Relaciones Exteriores del Perú, Cote-5-14-A/359, «Circular referente a la promoción económica », Paris, 11 de noviembre de 1968, fol. 1.

9. D. Barjot, La trace des bâtisseurs : histoire du Groupe Vinci, p. 123-140.

10. C.C.A.L., « Presencia francesa en América latina », p. 18.

11. W. Temple Seminario, Guía Económica del Perú, p. 226.

12. T. Hampe Martinez, El Banco Central de Reserva y la economía peruana (1922-1980), p. 77.

13. Capítulo I : Comportamiento de la economía peruana 1950-2015. Panorama de la Economía Peruana 1950-2015, p. 17-18.

14. J. Sheahan, La economía peruana desde 1950 : buscando una sociedad mejor, p. 33.

15. T. Hampe Martinez, El Banco Central de Reserva y la economía peruana (1922-1980), p. 103-109.

16. Centre des Archives Économiques et Financières, Cote F, B-0061 428, Pérou, situation financière 1944-1971, lettre $n^{\circ} 273$, «Amérique latine. Discours du président du Pérou », Buenos Aires, 26 mai 1960, fol. 1. 
17. Archivo Central del Ministerio de Relaciones Exteriores del Perú, Cote-5-14-A / 48, "Viaje de técnicos franceses, Paris, 12 agosto de 1964, fol. 1-2.

18. Centre des Archives Économiques et Financières, "Communiqué franco-péruvien ", Télégramme n ${ }^{\circ} 179$, B-0061 432/1, Lima, $1^{\text {er }}$ octobre 1964, p. 3.

19. D. Barjot, G. Cayo Durand, "Les échanges économiques entre la France et l'Amérique latine pendant la présidence du général de Gaulle: une première approche », p. 195.

20. G. Cayo Durand, «Les relations économiques et financières entre la France et le Pérou : l'exemple des entreprises industrielles et commerciales (1945-1975)», p. 209.

21. G. Cayo Durand de Geist, «Moderniser le Pérou sous l'influence française: la coopération technique et scientifique franco-péruvienne (1945-1975)».

22. Archivo Central del Ministerio de Relaciones Exteriores del Perú, Cote-5-14-A/84, «Plan hidroeléctrico de Cañon del Pato », Paris, setiembre de 1962, fol. 1.

23. "L'économie d'un grand pays: le Pérou », Revue de la Chambre de Commerce FranceAmérique Latine, p. 7.

24. Organisme créé en juillet 1942 en vue de l'exploitation hydro-électrique de la vallée du fleuve Santa. Archives diplomatiques, Série B-Amérique (1952-1963), Pérou (36), 13.9.1. Questions économiques (1952-1957), lettre $n^{\circ} 916$, «Réorganisation de la Corporation du Santa », Lima, 20 octobre 1952, fol. 1.

25. Archives diplomatiques, Série B-Amérique, Pérou (37), Questions économiques avec la France (1958-1963), lettre $\mathrm{n}^{\circ} 320$, « Usine sidérurgique de Chimbote », Lima, 24 avril 1958, fol. 1.

26. Archives Paribas, Cote ET 1204/Santa, 1955-1966, lettre s.n., « Le Docteur Antunez de Mayolo fait une conférence sur la Corporación del Santa », 1955, fol. 1-2.

27. Archives diplomatiques, Série B-Amérique (1952-1963), Pérou (36), 13.9.1. Questions économiques (1952-1957), lettre $n^{\circ} 1654$, «Prix français et étrangers », Lima, 2 octobre 1952, fol. 1.

28. Archives Paribas, Divers I Pérou, Boîte 209, lettre $n^{\circ}$ A-127/53, « Extrait d'une lettre de M. Aisner, conseiller commercial, à M. Jozon ", 3 août 1953, fol. 1-2.

29. Le Canyon del Pato se situe au confluent de la rivière Santa et de la rivière Los Cedros. Il s'étend sur une distance d'environ $9 \mathrm{~km}$. La rivière Santa coulait entre des parois rocheuses de plus de 1000 mètres de hauteur. La dénivellation entre l'entrée et la sortie du Canyon del Pato était de 450 mètres environ dont 420 mètres étaient utilisés pour la production d'énergie électrique. L'usine du Canyon del Pato était reliée au centre de consommation de Chimbote par une ligne haute tension de $90 \mathrm{~km}$ de longueur, passant à $4650 \mathrm{~m}$ d'altitude. Archives Paribas, Divers I Pérou, Boîte 210, «Corporación Peruana del Santa, Rapport Général, Aménagement hydroélectrique du Canyon del Pato-S.O.G.E.I. », 1958, fol. 1.

30. Ibidem, fol. 2.

31. L'expression "groupe français» est extraite du document original. Archives Paribas, Divers I Pérou, Boîte 210, «Corporación Peruana del Santa, Rapport général, aménagement hydroélectrique du Canyon del Pato. S.O.G.E.I. », 1958, fol. 1-3.

32. Ibidem.

33. Archives Paribas, Divers II Pérou, Boîte 209, «Le financement des réalisations industrielles de la France à l'étranger ", 10 mai 1957, fol. 1-2. 
34. Expression extraite de l'annexe I: La inauguración de la Planta Siderúrgica de Chimbote: un acontecimiento histórico, Archives diplomatiques, Série B-Amérique, Pérou (37), Questions économiques avec la France (1958-1963), lettre $n^{\circ} 318$, « Inauguration du Centre Sidérurgique de Chimbote ", fol. 1.

35. G. Cayo Durand, «Les relations économiques et financières entre la France et le Pérou : l'exemple des entreprises industrielles et commerciales. 1945-1975 ", p. 185.

36. Archives diplomatiques, Série B-Amérique, Pérou (37), Questions économiques avec la France (1958-1963), «La inauguración de la Planta Siderurgica de Chimbote: un acontecimiento histórico », fol. 1-3.

37. Ibidem, fol. 2-3.

38. Archives diplomatiques, Série B-Amérique (1952-1963), Pérou (36), 13.9.1. Questions économiques (1952-1957), Télégramme $n^{\circ} 165 / 6$, «Affaire du Santa», Paris, 30 juin 1954, fol. 1.

39. Archives Paribas, Divers I Pérou, Boîte 210 « Corporación Peruana del Santa, Contrat ", 1954, fol. 2-4.

40. Ibidem., fol. 4-6.

41. Archives diplomatiques, Série B-Amérique (1952-1963), Pérou (36), 13.9.1. Questions économiques (1952-1957), lettre $n^{\circ} 660$ «Passage de M. Corniglion-Molinier», 1955, fol. 1-2.

42. Archives diplomatiques, Série B-Amérique (1952-1963), Pérou (36), 13.9.1. Questions économiques (1952-1957), Télégramme $\mathrm{n}^{\circ} 6$, "Communiqué DIRELATEX », Lima, 17 décembre 1954, fol. 1.

43. Archives diplomatiques, Série B-Amérique (1952-1963), Pérou (36), 13.9.1. Questions économiques (1952-1957), Télégramme $\mathrm{n}^{\circ} 1 / \mathrm{DET}$, «À Monsieur l'Ambassadeur de France à Lima », Paris, 23 décembre 1954, fol. 1.

44. Archives diplomatiques, Série B-Amérique, Pérou (37), Questions économiques avec la France (1958-1963), lettre ${ }^{\circ}$ 336, « Nouvel accord financier », Lima, 2 mai 1958, fol. 1.

45. Archives diplomatiques, Série B-Amérique, Pérou (37), Questions économiques avec la France (1958-1963), lettre $n^{0} 283$, «Inauguration des ensembles sidérurgiques et hydro-électriques du Santa », Lima, 11 avril 1958, fol. 1.

46. Archives diplomatiques, Série B-Amérique, Pérou (37), Questions économiques avec la France (1958-1963), lettre $n^{\circ} 891$, "Inauguration des ensembles sidérurgiques et hydro-électriques du Santa AMAE, Lima, 10 avril 1958, fol. 1.

47. Archives diplomatiques, Série B-Amérique, Pérou (37), Questions économiques avec la France (1958-1963), lettre $n^{\circ} 318$, "Inauguration du Centre Sidérurgique de Chimbote », Lima, 10 avril 1958, fol. 1-2.

48. Ibidem.

49. Archives diplomatiques, Série B-Amérique, Pérou (37), Questions économiques avec la France (1958-1963), lettre $n^{\circ} 509$, «Difficultés financières de la Corporation du Santa », Lima, 21 juillet 1958, fol. 1.

50. Archives diplomatiques, Série B-Amérique, Pérou (37), Questions économiques avec la France (1958-1963), lettre n 281, « Aciérie de Chimbote », Lima, 15 avril 1959, fol. 1-3. 51. Archives diplomatiques, Série B-Amérique, Pérou (37), Questions économiques avec la France (1958-1963), lettre $n^{\circ} 605$, «Présence française à Chimbote », l'Ambassadeur 
de France au Pérou à M. le Ministre des Affaires Étrangères, Direction Amérique, Lima, 19 septembre 1959, fol. 1.

52. Archives diplomatiques, Série B-Amérique, Pérou (37), Questions économiques avec la France (1958-1963), lettre $n^{\circ} 369$, «Exportation acier Chimbote États-Unis », Lima, 21 mai 1959, fol. 1.

53. « Le Contrat de l'acier », El Comercio, Lima, jeudi 2 mars 1961, p. 1.

54. Archives diplomatiques, Boîte 13-9-1, lettre $\mathrm{n}^{\circ}$ 55, « Affaires économiques en cours ", 29 janvier 1965, fol. 5.

55. Ibidem., fol. 6 .

56. Archives diplomatiques, Boîte 13-9-1, lettre $n^{\circ} 343 / 347$, "Affaire Chimbote", 13 novembre 1966, fol. 1-2.

57. Centre des Archives Économiques et Financières, Boîte 54915, Pérou, lettre $\mathrm{n}^{\circ} 6942$, "Groupe Consultatif pour le Pérou et politique de crédit vis-à-vis de ce pays », $\mathrm{n}^{\circ} 8$, août 1966, fol. 4.

\section{RÉSUMÉS}

Cet article s'interroge sur la transmission du savoir-faire technique et industriel français au Pérou pendant les années 1950 et 1960. Il s'agit d'expliquer comment ce transfert de compétences était mis en place par deux pays différents par leurs économies, mais aux intérêts communs. Il a donc été important de s'intéresser brièvement aux actions diplomatiques et économiques menées pour encourager cette coopération technique. Pour illustrer quels sont les problèmes et les réussites de cette coopération, deux projets industriels ont été étudiés: la centrale hydroélectrique du Canyon del Pato et l'aciérie de Chimbote. Cet article cherche à mettre en évidence l'expertise française au Pérou dans le domaine du génie civil. Les acteurs et les facteurs de ce processus de transmission des savoirs ont été identifiés et problématisés.

\section{AUTEUR}

\section{GETSIVA CAYO DURAND DE GEIST}

Docteure en histoire de l'Université Paris Sorbonne, chercheure associée au Centre Roland Mousnier, UMR 8596 Sorbonne Université CNRS 